Frithjof Netzer

\title{
Strategische Allianzen im Luftverkehr
}

Nachfragerorientierte Problemfelder ihrer Gestaltung 


\section{Frithjof Netzer}

\section{Strategische Allianzen im Luftverkehr}

Unternehmen gehen strategische Allianzen mit anderen Unternehmen derselben Marktstufe ein, um ihre eigene Wettbewerbsfähigkeit zu verbessern. Maßgeblich gefördert durch eine zunehmende Deregulierung und Privatisierung, haben sich auch im internationalen Luftverkehr Allianznetzwerke von Fluggesellschaften herausgebildet. Die Arbeit untersucht, wie sich die Partner-Airlines bei der gemeinsamen Leistungserstellung abstimmen und welche Implikationen sich hieraus für die Flugreisenden ergeben. Dabei werden allianzspezifische Nutzenvorteile, aber auch Nutzennachteile diskutiert und Handlungsempfehlungen für die Ausgestaltung des Marketing-Mix von Allianzen gegeben. In die Überlegungen fließen Ergebnisse einer internationalen Focusgruppenbefragung von Vielfliegern ein.

Frithjof Netzer wurde 1967 in Gehrden/Hannover geboren. Von 1988 bis 1993 studierte er Betriebswirtschaftslehre an der Universität Münster mit den Schwerpunkten Marketing und Internationales Management. Nach seinem Diplom arbeitete er bis zum Januar 1999 am Institut für Marketing bei Prof. Dr. Dr. h.c. H. Meffert als Wissenschaftlicher Mitarbeiter. Schwerpunkte seiner Tätigkeit bildeten das Marketing im Luftverkehrsbereich sowie das internationale Management. 
Strategische Allianzen im Luftverkehr 


\section{SCHRIFTEN ZU \\ MARKETING UND MANAGEMENT}

Herausgegeben von Prof. Dr. Dr. h.c. Heribert Meffert

Band 37

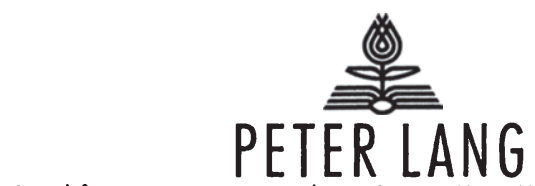

Frankfurt am Main - Berlin - Bern - New York - Paris - Wien 


\section{Frithiof Netzer}

\section{Strategische Allianzen im Luftverkehr}

Nachfragerorientierte Problemfelder

ihrer Gestaltung

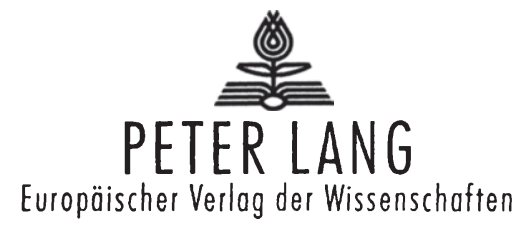


Die Deutsche Bibliothek - CIP-Einheitsaufnahme

Netzer, Frithjof:

Strategische Allianzen im Luftverkehr : nachfragerorientierte Problemfelder ihrer Gestaltung / Frithjof Netzer. - Frankfurt am Main ; Berlin ; Bern ; New York ; Paris ; Wien : Lang, 1999

(Schriften zu Marketing und Management ; Bd. 37)

Zugl.: Münster (Westfalen), Univ., Diss., 1998

ISBN 3-631-34768-5

Open Access: The online version of this publication is published on www.peterlang.com and www.econstor.eu under the international Creative Commons License CC-BY 4.0. Learn more on how you can use and share this work: http://creativecommons. org/licenses/by/4.0.

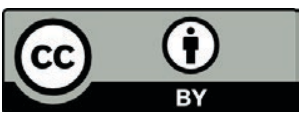

This book is available Open Access thanks to the kind support of ZBW - Leibniz-Informationszentrum Wirtschaft.

Gedruckt auf alterungsbeständigem, säurefreiem Papier.

\author{
D 6 \\ ISSN 0176-2729 \\ ISBN 3-631-34768-5 \\ ISBN 978-3-631-75092-6 (eBook) \\ (C) Peter Lang GmbH \\ Europäischer Verlag der Wissenschaften \\ Frankfurt am Main 1999 \\ Alle Rechte vorbehalten.
}

Das Werk einschließlich aller seiner Teile ist urheberrechtlich geschützt. Jede Verwertung außerhalb der engen Grenzen des

Urheberrechtsgesetzes ist ohne Zustimmung des Verlages unzulässig und strafbar. Das gilt insbesondere für Vervielfältigungen, Übersetzungen, Mikroverfilmungen und die Einspeicherung und Verarbeitung in elektronischen Systemen.

Printed in Germany 124567 
Für Irene, Nils Bastian, Nele Marie und meine Eltern 
Frithjof Netzer - 978-3-631-75092-6

Downloaded from PubFactory at 01/11/2019 08:28:25AM

via free access 


\section{Vorwort des Herausgebers}

Mit der Absicht, die eigene Wettbewerbsfähigkeit zu verbessern, gehen Unternehmen in wachsendem Maße Kooperationen mit anderen Unternehmen derselben Marktstufe ein. Entwicklungen wie die Deregulierung von Märkten, die Verkürzung von Produktlebenszyklen bei gleichzeitig steigenden Investitionsvolumina, aber auch eine größere Offenheit des Management gegenüber neuartigen Lösungen haben das Entstehen derartiger "strategischer Allianzen“ in vielen Branchen nachhaltig gefördert. Nach einer anfänglichen Erfolgseuphorie stellte sich jedoch bald heraus, daß strategische Allianzen eine Reihe besonderer Herausforderungen bergen, die in dem Spannungsfeld einer gleichzeitigen unternehmerischen Unabhängigkeit und Abhängigkeit der beteiligten Partner begründet liegen.

Für den organisationstheoretisch geprägten Zweig der Betriebswirtschaftslehre sowie für managementorientierte Arbeiten ergaben sich bisher unterschiedliche Problemstellungen, die im Themenbereich strategischer Allianzen diskutiert wurden. Im Vordergrund standen Überlegungen aus einer inside-out-Perspektive, die sich mit der Planung, Organisation und Kontrolle von Allianzaktivitäten auseinandersetzten. Behandelt wurden u.a. Fragen der allianzspezifischen Zielsetzungen, der Partnerwahl, der gemeinsamen Strategie, der Leitungsgliederung, der unternehmensübergreifenden Prozeßorganisation und der Ergebnisrealisation. Viele Beiträge erkannten, daß der Erfolg strategischer Allianzen nur in begrenztem Umfang planbar ist und in hohem Maße von "weichen“ Faktoren wie gegenseitigem Vertrauen und gemeinsamem Commitment abhängt.

Überlegungen zu den Auswirkungen allianzintern abgestimmter Prozesse auf die Empfänger von Leistungen wurden dagegen bisher nur in sehr begrenztem Umfang von der wissenschaftlichen Diskussion aufgegriffen. Dieser Befund lenkt die Aufmerksamkeit in besonderem Maße auf den Dienstleistungsbereich, in dem sich die Leistungserstellung unter Einbeziehung des externen Faktors vollzieht und unternehmensübergreifende Abstimmungsprozesse im Rahmen strategischer Allianzen somit potentiell der Wahrnehmung und dem Erleben von Konsumenten unterliegen. Die zentrale Zielsetzung der vorliegenden Arbeit liegt daher in der Erarbeitung von Ansatzpunkten zur Erfassung der Wahrnehmung, des Erlebens und der Beurteilung strategischer Allianzen und ihrer Leistungen durch Nachfra- 
ger, um Empfehlungen für die Ausgestaltung des Marketing-Instrumentariums der beteiligten Partner-Unternehmen ableiten zu können. Als Branchenfokus wurde der Personenluftverkehr zugrunde gelegt, der sich einerseits durch eine verhältnismäßig hohe Intensität von Allianzaktivitäten auszeichnet und andererseits das Merkmal der Integration des externen Faktors erfüllt.

Bei der Bearbeitung der Problemstellung steht zunächst eine Untersuchung der allianzinternen Abstimmungsprozesse zwischen Fluggesellschaften im Mittelpunkt, die sich vor dem Hintergrund der luftverkehrsspezifischen Rahmenbedingungen vollzieht. Darüber hinaus werden die marketingstrategische Ausrichtung am Beispiel der zur Zeit dominanten internationalen Allianz-Netzwerke diskutiert und das von Partner-Airlines gewählte Vorgehen bei der Arbeitsteilung, Konfiguration und Ergebnisverteilung exemplarisch erörtert.

Entlang der von Fluggästen durchlaufenen "Reisekette", die mit der Informationssuche und der Flugbuchung beginnt und mit dem Verlassen des Zielflughafens endet, werden die Auswirkungen strategischer Allianzen auf die einzelnen Kontaktpunkte diskutiert und gewürdigt. Die konzeptionellen Überlegungen und Erkenntnisse aus dokumentierten empirischen Studien bereiten den Grund für die Erläuterung von Ergebnissen einer Focusgruppenbefragung, in der Vielflieger zur Beurteilung strategischer Luftverkehrsallianzen und ihrer Leistungen befragt wurden. Im Mittelpunkt stehen dabei die Fragen, inwieweit strategische Allianzen von Fluggesellschaften in der Lage sind, die Bedürfnisse von Vielfliegern zu erfüllen und für welche Nutzenerwartungen sich ggf. allianzspezifische Nutzenvorteile oder Nutzennachteile gegenüber einzeln agierenden Fluggesellschaften feststellen lassen. Verbesserungsbedarf wird demnach insbesondere bei der Sicherstellung eines nahtlosen Reiseprozesses gesehen, während bei der weltweiten Anerkennung des Vielfliegerstatus in Verbindung mit gewährten Privilegien ein besonders hohes Differenzierungspotential festgestellt werden kann.

Auf der Basis von fünf zentralen Nutzendimensionen wird schließlich eine Idealpositionierung für eine strategische Luftverkehrsallianz vorgeschlagen, welche die Grundlage für die Ausrichtung des Marketing-Instrumentariums bildet. Im Bereich der Allianz-Markenpolitik ist die Ausgestaltung einer Dachmarkenstrategie an luftverkehrsrechtliche Gegebenheiten gebunden und erfordert eine sorgsame Abschätzung von Markenimagetransfereffekten der einbezogenen Partner-Flug- 
gesellschaften, während die zentrale Herausforderung bei der gemeinsamen Leistungspolitik in der harmonischen Gestaltung der Schnittstellen von Flugplänen, Produkt- und Serviceangeboten liegt.

Die Eignung alternativer Vertriebsformen zur Erreichung der allianzmarkenspezifischen Zielsetzungen und die damit verbundenen Koordinationserfordernisse werden anschließend einer kritischen Würdigung unterzogen. Bei der Abstimmung der gemeinsamen Preis- und Konditionenpolitik erweisen sich internationale Kartellgesetzgebungen und unternehmensindividuell bestehende Optimierungsansätze in Form von Yield Management-Systemen als Grenzen einer allianzweiten Harmonisierung. Länderspezifische kulturelle Besonderheiten sind von wesentlicher Bedeutung für die Ausgestaltung einer gemeinsamen Kommunikationspolitik, während sich im Bereich der marketingrelevanten Personalpolitik vor allem bestehende Loyalitätsschemata der Mitarbeiter als Herausforderung für eine integrierte Leistungserstellung im Rahmen strategischer Allianzen erweisen.

Die vorliegende Untersuchung stellt insgesamt eine Bereicherung der wissenschaftlichen Arbeiten auf dem Gebiet der marktorientierten Unternehmensführung dar und gibt Hinweise auf die Erfassung des spezifischen positiven, aber auch negativen Nutzenbeitrags, den strategische Allianzen für Nachfrager aufweisen können. Die Stärke der Arbeit liegt in der strukturierten, kontextbezogenen Aufarbeitung der outside-in-Perspektive eines komplexen Forschungsgegenstandes. Es bleibt zu wünschen, daß die Publikation in Wissenschaft und Praxis auf eine möglichst breite Resonanz stößt. 


\section{Vorwort des Verfassers}

Den Ausgangspunkt der vorliegenden Untersuchung bildete die Fragestellung, ob und ggf. welche Auswirkungen sich von anbieterseitig initiierten strategischen Allianzen auf die Nachfrager gemeinschaftlich erbrachter Leistungen ergeben. Als besonders geeignet für die Bearbeitung dieser Frage schien der Branchenkontext des internationalen Personenluftverkehrs, in dem einerseits eine hohe Allianzintensität der Fluggesellschaften feststellbar ist und in dem sich andererseits die Leistungserstellung unter Einbeziehung der Fluggäste und ihrer Verfügungsobjekte vollzieht.

Um zunächst Aufschluß über das anbieterseitige Leistungspotential und die gemeinsamen Prozesse der Leistungserstellung zu erhalten, wird zunächst das Design strategischer Luftverkehrsallianzen erörtert. Die Koordination der Schnittstellen in der Reisekette von Fluggästen stellt hohe Anforderungen an Strukturen, Systeme und Personal der Allianzpartner. Auf Basis der hieraus gewonnenen Erkenntnisse verlagert sich die Untersuchungsperspektive daraufhin auf die Nachfragerseite. Im einzelnen wird diskutiert, mit welchen kundenseitigen Änderungen Allianzflugdienstleistungen einhergehen und ob sich diese Änderungen eher als Nutzenvorteile oder -nachteile für Fluggäste erweisen. Anhand der Ergebnisse einer weltweit durchgeführten Focusgruppenbefragung von Vielfliegern kann für eine bedeutende Luftverkehrsallianz belegt werden, daß gerade bei dem von vielen Fluggesellschaften angestrebten "nahtlosen Reisen" Nutzennachteile konstatiert werden, während die zunehmende Größe des Streckennetzes durch den Verbund sowie erweiterte Vielfliegerprivilegien als besonders positive allianzspezifische Nutzendimensionen gewertet werden.

Die aus der Focusgruppenstudie abgeleitete Soll-Positionierung für die strategische Allianz bildet daraufhin die Grundlage für die Diskussion des Marketing-Mix. Die gemeinsame Gestaltung der Marketing-Instrumente bewegt sich im Spannungsfeld von Eigeninteressen und Harmonisierungsbedürfnissen der Partner-Airlines, ohne daß der rechtliche Kontext zu vernachlässigen ist. Mit einem Vorschlag zur konkreten Erhebung allianzspezifischer Nutzendimensionen schließt die Untersuchung ab. 
Ohne die Unterstützung einiger Personen wäre die Erstellung der Arbeit nicht möglich gewesen. Mein Dank gilt zunächst meinem akademischen Lehrer, Herrn Prof. Dr. Dr. h.c. Heribert Meffert. Er regte bereits frühzeitig die Themenstellung an und gab ihr die entscheidenden Impulse. Zudem förderte er in umfassender Weise die Fertigstellung der Arbeit. Herrn Prof. Dr. Klaus Backhaus möchte ich für die Übernahme des Zweitgutachtens danken. Für die Diskussionen und Anregungen in der mühsamen "ersten Hälfte" danke ich meinen Kollegen, Herrn Dr. Helmut Schneider und Dr. Jesko Perrey. Mein Dank gilt auch den Managern der in die Untersuchung einbezogenen Fluggesellschaften, die mir trotz eigener Zeitknappheit für persönliche Gespräche zur Verfügung standen und damit maßgeblich zum Gelingen des Werkes beigetragen haben.

Ganz besonders möchte ich meinen Eltern danken, die durch ihre liebevolle Erziehung und umfassende Förderung die Grundlage für die Erstellung der Arbeit gelegt haben. Meinem Sohn Nils Bastian verdanke ich viele Stunden der Heiterkeit und Entspannung sowie das notwendige Maß an Distanz zur eigenen Arbeit. Auch allen Freunden sei gedankt, die mir während dieser anstrengenden Phase zur Seite gestanden haben.

Der ohne Zweifel größte Dank gilt schließlich meiner wunderbaren Frau Irene, die in nimmermüdem Eifer trotz ihrer vielfältigen eigenen Belastungen gleich in mehrfacher Weise das Gelingen der Arbeit gefördert hat. Ihre kompetenten fachlichen Anmerkungen, die vorbildliche, zügige Erstellung der Abbildungen sowie der persönliche, motivierende Zuspruch waren ebenso unersetzbar wie ihre eigene Erfahrung im Umgang mit einer Dissertation. Ohne ihre Hilfe wäre die Arbeit in dieser Form nicht möglich gewesen. 
Frithjof Netzer - 978-3-631-75092-6

Downloaded from PubFactory at 01/11/2019 08:28:25AM

via free access 


\section{Inhaltsverzeichnis}

Abbildungsverzeichnis $X V I$

Abkürzungsverzeichnis XVIII

\section{A. Strategische Allianzen als Herausforderung an das Management von} Fluggesellschaften .......................................................................................... 1

1. Bedeutung strategischer Allianzen für die Unternehmensführung ................ 1

2. Entwicklungslinien von Forschungsaktivitäten im Bereich strategischer Allianzen.

3. Strategische Allianzen bei Flugdienstleistungen ....................................... 12

4. Problemstellung und Gang der Untersuchung ........................................... 27

B. Absatzmarktgerichtete Gestaltung strategischer Luftverkehrsallianzen .............................................................................. 33

1. Design strategischer Luftverkehrsallianzen..............................................33

1.1 Theoretische Grundlagen strategischer Allianzen und Rahmenbedingungen des internationalen Luftverkehrs ...................... 33

1.1.1 Ansätze zur Erklärung und Gestaltung strategischer

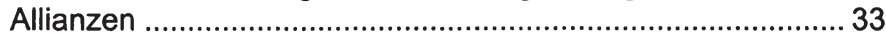

1.1.2 Rahmenbedingungen strategischer Luftverkehrsallianzen ..... 40

1.2 Ziele für strategische Luftverkehrsallianzen..................................... 48

1.2.1 Absatzmarktgerichtete Wirkungsziele .................................5 50

1.2.2 Unternehmensgerichtete Effizienzziele ................................. 59

1.3 Marketingstrategische Ausrichtung von Luftverkehrsallianzen ............62 62

1.3.1 Räumliche Marktabdeckung ................................................. 63

1.3.2 Strategische Positionierung von Luftverkehrsallianzen........... 67

1.4 Organisationale Gestaltung strategischer Luftverkehrsallianzen ......... 69

1.4.1 Arbeitsteilung zwischen den Partnerfluggesellschaften .......... 69

1.4.2 Konfiguration der strategischen Luftverkehrsallianz................ 78

1.4.3 Ergebnisverteilung zwischen den beteiligten Fluggesellschaften

2. Strategische Luftverkehrsallianzen im Spannungsfeld zwischen

Endnutzern, Absatzmittlern und Wettbewerbern 89 
2.1 Strategische Luftverkehrsallianzen aus Sicht des vertikalen Marketing

2.1.1 Auswirkungen strategischer Luftverkehrsallianzen auf die Beziehungen zu Reisebüros

2.1.2 Auswirkungen strategischer Luftverkehrsallianzen auf die Beziehungen zu Firmenkunden

2.2 Konzeptionelle Überlegungen und empirische Befunde zu den endnutzergerichteten Beziehungen strategischer

Luftverkehrsallianzen

2.2.1 Wahrnehmung strategischer Luftverkehrsallianzen am Beispiel ausgewählter Informationsinhalte

2.2.2 Erleben strategischer Luftverkehrsallianzen im Teilprozeß der Informationssuche.

2.2.3 Ausgewählte empirische Befunde zum nutzenbasierten Auswahl- und Kaufverhalten bei Flugdienstleistungen unter besonderer Berücksichtigung strategischer Luftverkehrsallianzen

2.3 Ergebnisse einer Focusgruppenbefragung zur Beurteilung strategischer Luftverkehrsallianzen durch das Endnutzersegment der "Vielflieger"

2.3.1 Anlage der empirischen Untersuchung 139

2.3.2 Fundamentale Bedürfnisse von Vielfliegern und ihre Einstellungen gegenüber strategischen Luftverkehrsallianzen

2.3.3 Erfüllung zentraler Nutzenerwartungen von Vielfliegern durch strategische Luftverkehrsallianzen.

2.3.4 Ableitung und Überprüfung alternativer Positionierungsansätze am Beispiel einer fiktiven Luftverkehrsallianz

2.3.5 "Worldwide Recognition“ als vielfliegerorientierter Positionierungsansatz für eine strategische Luftverkehrsallianz.... 150

3. Nachfragergerichtete Ausgestaltung strategischer Luftverkehrsallianzen.. 156

3.1 Ausgestaltung der allianzspezifischen Markenpolitik 158

3.1.1 Markenpolitische Zielsetzungen und rechtliche Rahmenbedingungen der Allianz-Markenpolitik 158

3.1.2 Festlegung der Markenstrategie für die Luftverkehrsallianz.. 162

3.2 Leistungspolitik der strategischen Luftverkehrsallianz 
3.2.1 Allianzseitiges Netzmanagement durch gegenseitige Abstimmung von Flugplänen.

3.2.2 Produkt- und Servicemanagement der strategischen Luftverkehrsallianz 176

3.3 Ausgestaltung der Distributionspolitik der strategischen Luftverkehrsallianz. 184

3.4 Absatzmarktorientierte Ausrichtung der AllianzKontrahierungspolitik 189

3.4.1 Problemfelder einer gemeinsamen Preissetzung 189

3.4.2 Abstimmung der Konditionenpolitik in der Luftverkehrsallianz 198

3.5 Ausgestaltung der Allianz-Kommunikationspolitik 199

3.5.1 Festlegung einer Kommunikationsstrategie für die Luftverkehrsallianz 199

3.5.2 Problemfelder der Budgetierung 208

3.6 Relevanz der Personalpolitik für die absatzmarktgerichtete Gestaltung der Luftverkehrsallianz

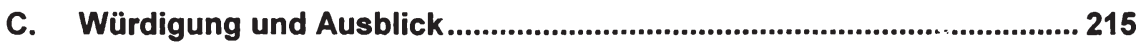

1. Zusammenfassende Würdigung der Untersuchungsergebnisse................215

2. Ansatzpunkte zur Erfassung des Nutzenbeitrags von AllianzFlugdienstleistungen

3. Implikationen für das Management von Allianzen unter besonderer Berücksichtigung von Controlliņ̣ aspekten 226

4. Ausblick 231

Anhang 233

Literaturverzeichnis. 274 


\section{Abbildungsverzeichnis}

Abb. 1: Entwicklung strategischer Allianzen im Luftverkehr........................ 8

Abb. 2: Begriffsmerkmale und Bedeutungsinhalte strategischer Allianzen.. 17

Abb. 3: $\quad$ Schwerpunkte der Untersuchung ……........................................... 29

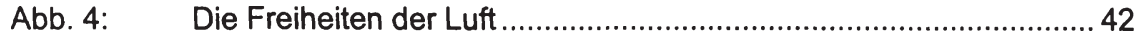

Abb. 5: Marktabdeckung ausgewählter internationaler strategischer

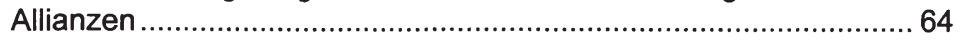

Abb. 6: Arbeitsteilung bei strategischen Luftverkehrsallianzen.................... 71

Abb. 7: Beispielhafte Konfiguration einer strategischen Allianz ................... 80

Abb. 8: $\quad$ Strukturelle Anbindung einer strategischen Allianz an bestehende Organisationsstrukturen.......................................... 82

Abb. 9: $\quad$ Vertikale Absatzkanalstruktur im Personenluftverkehr .................... 90

Abb. 10: Wahrnehmungstypen bei strategischen Luftverkehrsallianzen ..... 102

Abb. 11: Werbeanzeige der Star Alliance ................................................. 106

Abb. 12: Inhalte von Presseberichten zu strategischen

Luftverkehrsallianzen................................................................ 112

Abb. 13: Vereinfachtes Modell des Auswahlverhaltens und der

Beurteilung von Flugdienstleistungen durch Endnutzer ................ 115

Abb. 14: Kennzeichnung von Code Sharing-Flugdienstleistungen in großen Computerreservierungssystemen.................................. 118

Abb. 15 a): Vergleich zwischen Nutzenerwartungen und AllianzLeistungsangebot am Beispiel ausgewählter Studien ................... 129

Abb. 15 b): Vergleich zwischen Nutzenerwartungen und AllianzLeistungsangebot am Beispiel ausgewählter Studien

Abb. 15 c): Vergleich zwischen Nutzenerwartungen und AllianzLeistungsangebot am Beispiel ausgewählter Studien

Abb. 15 d): Vergleich zwischen Nutzenerwartungen und AllianzLeistungsangebot am Beispiel ausgewählter Studien

Abb. 16: Konzeptualisierung kaufverhaltensrelevanter Merkmale und Merkmalsausprägungen von Flugdienstleistungen in der Studie von Gellman Research Associates

Abb. 17: Beurteilung des allianzbezogenen Erfüllungsgrads zentraler Nutzenerwartungen durch Vielflieger

Abb. 18: Kerneigenschaften der Allianzmarke (Idealposition) 
Abb. 19: Ansatzpunkte für die Positionierung am Beispiel einer strategischen Luftverkehrsallianz 154

Abb. 20: Markenhierarchie der strategischen Luftverkehrsallianz ............... 164

Abb. 21: Nutzenorientierte Ausgestaltung der Allianz-Leistungspolitik ........ 171

Abb. 22: Ermittlung des Harmonisierungsbedarfs am Beispiel gemeinsamer Vielfliegerprogramme.

Abb. 23: Geeignetheit alternativer Vertriebsformen zur Erreichung allianzmarkenpolitischer Zielsetzungen ....

Abb. 24: Aufbau eines Yield Management-Systems..................................... 194

Abb. 25: Exemplarisches Profil der Allianz-Kommunikationsstrategie......... 207

Abb. 26: Typologisierung von Mitarbeitern ................................................ 213

Abb. 27: Konzeptualisierung von Nutzendimensionen zur Erfassung allianzspezifischer Nutzenbeiträge ............................................ 223

Abb. 28: Aufbau des Allianz-Controlling ………....................................... 227

Abb. 29: Erfassungsraster für das Allianz-Controlling................................. 229 


\section{Abkürzungsverzeichnis}

a.a.O.

a.M.

Abb.

Anm. d. Verf.

Ans.

Art.

Aufl.

BA

Bd.

bspw.

bzw.

ca.

CRS

D.C.

d.h.

DBW

Deutsche BA

DM

e.V.

etc.

EU

EWGV

f., ff.

F.hafen

Flugdienstl.

ggf.

ggs. am angegebenen Ort

am Main

Abbildung

Anmerkung des Verfassers

Ansett

Artikel

Auflage

British Airways

Band

beispielsweise

beziehungsweise

circa

Computerreservierungssystem

District of Columbia

das heißt

Die Betriebswirtschaft

Deutsche Besondere Angelegenheiten

Deutsche Mark

eingetragener Verein

et cetera

Europäische Union

Europäische Wirtschaftsgemeinschaft Vertrag

folgende, fortfolgende

Flughafen

Flugdienstleistung

gegebenenfalls

gegenseitig(es) 


$\begin{array}{ll}\text { H. } & \text { Heft } \\ \text { Hrsg. } & \text { Herausgeber } \\ \text { i.d.R. } & \text { in der Regel } \\ \text { i.S. } & \text { im Sinne } \\ \text { IATA } & \text { International Air Transport Association } \\ \text { ICAO } & \text { International Civil Aviation Organization } \\ \text { IMP } & \text { Industrial Marketing and Purchasing Group } \\ \text { insb. } & \text { insbesondere } \\ \text { IT } & \text { Informationstechnologie } \\ \text { IVW } & \text { Internationales Verkehrswesen } \\ \text { Jg. } & \text { Jahrgang } \\ \text { KLM } & \text { Koninklyke Luchtvaart Maatschappij } \\ & \\ \text { LH } & \text { Lufthansa } \\ \text { max. } & \text { maximal } \\ \text { Mio. } & \text { Million(en) } \\ \text { Mrd. } & \text { Milliarde(n) } \\ \text { Multimediaanwend. Multimediaanwendungen }\end{array}$

No. Number

Nr. Nummer

o.V. ohne Verfasser

PC Personal Computer

persönl. persönlicher

QF Qantas

S. Seite

SAS Scandinavian Airline System 


\section{$X X$}

sog.

$\mathrm{Sp}$.

St.

str.

TAP

TV

u.a.

UA

US

USA

vgl.

Vol.

vs.

WiSt

z.B.

z.T.

z.Zt.

zfbf

ZFP sogenannte

Spalte

Sankt

strategische

Air Portugal

Television

und andere, unter anderem

United Airlines

United States

United States of America

vergleiche

Volume

versus

Wirtschaftswissenschaftliches Studium

zum Beispiel

zum Teil

zur Zeit

Zeitschrift für betriebswirtschaftliche Forschung

Zeitschrift für Forschung und Praxis 


\section{A. Strategische Allianzen als Herausforderung an das Management von Fluggesellschaften}

\section{Bedeutung strategischer Allianzen für die Unternehmensführung}

Unternehmen sehen sich einer Reihe unterschiedlicher Herausforderungen gegenüber, von denen seit längerer Zeit vor allem die Globalisierung an Bedeutung gewinnt. ${ }^{1}$ Unter diesem Begriff werden verschiedene Entwicklungen subsumiert. Zu beobachten ist die Herausbildung wirtschaftlicher Integrationsräume, innerhalb derer institutionelle Hemmnisse für Unternehmensaktivitäten abgebaut und eine Annäherung der beteiligten Staaten angestrebt werden. ${ }^{2}$ Staatliche Deregulierungs- und Privatisierungsbemühungen führen in zahlreichen Branchen zu einer Veränderung bislang gültiger „Spielregeln“ und öffnen den Zutritt zu verschlossenen Märkten. ${ }^{3}$ Unternehmen verbinden mit der Globalisierung sowohl eine Bedrohung angestammter Heimatmarktpositionen durch global agierende Wettbewerber als auch Chancen zur Erschließung neuer Wachstumspotentiale auf Inlands- und Auslandsmärkten. Nachfragerseitig stehen eine wachsende Mobilität sowie eine weltweite informationstechnologische Vernetzung im Vordergrund der Globalisierungsdiskussion, ${ }^{4}$ die einerseits auf die Angleichung von Bedürfnissen schließen lassen, zugleich jedoch mit einer sozio-kulturellen Fragmentierung einher gehen. ${ }^{5}$ Vor dem Hintergrund dieser Spannungsfelder bilden

1 Vgl. Perlmutter, H.V., The Tortuous Evolution of the Multinational Corporation, in: Columbia Journal of World Business, No. 4, 1969, S. 9-18; Levitt, T., The Globalization of Markets, in: Harvard Business Review, Vol. 61, No. 6, 1983, S. 92-102; Ohmae, K., Die Macht der Triade, Wiesbaden 1985; Meffert, H., Globalisierungsstrategien und ihre Umsetzung im internationalen Wettbewerb, in: DBW, 49.Jg., Heft 4, 1989, S. 445-463; Pfeiffer, R. (Hrsg.), Systemdenken und Globalisierung: Folgerungen für die lernende Organisation im internationalen Umfeld, Berlin 1997.

2 Vgl. z.B. Meffert, H., Bolz, J., Internationales Marketing-Management, 3., überarb. und erg. Aufl., Stuttgart, Berlin, Köln 1998, S. 49-53.

3 Hier sei beispielhaft auf die Auswirkungen der Deregulierung in der Europäischen Union verwiesen. Vgl. EU-Kommission (Hrsg.), Wirkung und Wirksamkeit des Binnenmarktes, Dokumenten-Nr. MEMO/96/103, Luxemburg 1996. Das Beispiel der Privatisierung von neun südafrikanischen Flughäfen, für die fünf ausländische Flughafengesellschaften bieten, belegt darüber hinaus die globalisierungsfördernde Wirkung veränderter politisch-rechtlicher Rahmenbedingungen. Vgl. o.V., Flughafen Frankfurt bietet in Südafrika, in: FAZ vom 29.11.1997, S. 21.

4 Vgl. Backhaus, K., Büschken, J., Voeth, M., Internationales Marketing, 2., überarb. und erg. Aufl., Stuttgart 1998, S. 33. Daten der Lufthansa prognostizieren z.B. eine weltweite Zunahme der Anzahl Auslandsreisen von 550 Millionen im Jahre 1996 auf 800 Millionen im Jahre 2006. Vgl. Klein, H., Dienstleistung als Hidden Champion, Vortragsunterlagen der Deutschen Lufthansa AG am 5. November 1997 an der Westfälischen Wilhelms-Universităt Münster.

5 Vgl. Steinmann, H., Scherer, A.G., Die multinationale Unternehmung als moralischer Aktor Bemerkungen zu einigen normativen Grundlagenproblemen des interkulturellen Managements, 
Unternehmen insbesondere seit Anfang der achtziger Jahre in wachsendem Umfang Partnerschaften mit Wettbewerbern, um ihre Wettbewerbsposition innerhalb der veränderten Rahmenbedingungen zu sichern und auszubauen oder um neue Wettbewerbspositionen zu besetzen und gestalterisch auf die Rahmenbedingungen einzuwirken. ${ }^{6}$ Diese auch als "strategische Allianzen ${ }^{67}$ bezeichneten Partnerschaften sollen als Instrument der strategischen Unternehmensführung idealerweise die Stärken der Partnerunternehmen vereinigen und deren Schwächen kompensieren. ${ }^{8}$

Mit dem Eingehen strategischer Allianzen verbinden Unternehmen die Erwartung, eigene Ziele effektiver und effizienter als im Fall eines Alleingangs erreichen zu können. ${ }^{9}$ Die Bedeutung strategischer Allianzen ergibt sich aus ihren Erfolgswirkungen, zu deren Erfassung verschiedene Indikatoren herangezogen werden können, die sich wiederum aus den Zielsetzungen der Partner ableiten. Neben globalen Erfolgseinschätzungen, die zumeist aus Expertenurteilen resultieren, finden marktorientierte, kostenrechnerische und finanzwirtschaftliche Erfolgsindikatoren Anwendung. ${ }^{10}$ Der Stellenwert strategischer Allianzen wird in empirischen Studien beispielsweise an einem wachsenden Anteil allianzspezifischer Umsatzerlöse am Gesamtumsatz von Unternehmen belegt. Eine Untersuchung

in: Interkulturelles Management: Theoretische Fundierung und funktionsbereichsspezifische Konzepte, Hrsg.: Engelhard, J., Wiesbaden 1997, S. 24.

6 Vgl. Perlmutter, H.V., Heenan, D.A., Cooperate to compete globally, in: Harvard Business Review, Vol. 64, No. 2, 1986, S. 136-138.

7 Der Begriff der strategischen Allianz wurde vermutlich von der Unternehmensberatung McKinsey\&Company im deutschen Sprachraum eingeführt und hat in der Folge unterschiedliche terminologische Auslegungen erfahren. Vgl. Schäfer-Kunz, J., Strategische Allianzen im deutschen und europäischen Kartellrecht, Frankfurt am Main u.a. 1995, S. 25.

8 Vgl. z.B. Taucher, G., Der dornige Weg strategischer Allianzen, in: Harvard Manager, 10. Jg., Nr. 3, 1988, S. 89.

9 Gahl, A., Die Konzeption strategischer Allianzen, Berlin 1991, S. 2. „Effektiv“ ist ein Instrument, wenn es grundsätzlich zur Erreichung eines festgelegten Zieles geeignet ist, "effizient", wenn sich das Ziel möglichst treffsicher erreichen läßt. Vgl. Schulte-Zurhausen, M., Organisation, München 1995, S. 4.

10 Vgl. Eisele, J., Erfolgsfaktoren des Joint-Venture-Management, Wiesbaden 1995, insb. S. 8595; Altwegg, R., Strategiebewertung und Ermittlung des Synergiewertes bei Kooperationen: ein strategischer Kooperationsbewertungsansatz, Basel 1995, insb. S. 57-60. In jüngerer Zeit werden strategische Allianzen auch verstärkt als Instrument zum Austausch von erfolgsrelevantem Wissen zwischen Unternehmen thematisiert. Vgl. Mowery, D.C., Oxley, J.E., Silverman, B.S., Strategic Alliances and Interfirm Knowledge Transfer, in: Strategic Management Journal, Vol. 17, Winter Special Issue, 1996, S. 77-91; Khanna, T., Gulati, R., Nohria, N., The Dynamics of Learning Alliances: Competition, Cooperation, and Relative Scope, in: Strategic Management Journal, Vol. 19, No. 3, 1998, S. 193-210. 
der 1.000 größten US-amerikanischen Aktiengesellschaften kommt zu dem Ergebnis, daß der Umsatzanteil strategischer Allianzen von ca. 3 Prozent im Jahre 1985 auf 14,8 Prozent im Jahre 1995 angewachsen ist. ${ }^{11}$ Eine solche Betrachtung belegt zwar die grundsätzliche Bedeutung strategischer Allianzen für die erfaßten Unternehmen, vermag jedoch keine Aufschlüsse über die erfolgreiche Gewinnung zusätzlicher Umsatzpotentiale durch dieses Instrument zu geben. Die Bewertung strategischer Allianzen anhand kostenrechnerischer Daten unterliegt i.d.R. der Geheimhaltung der betreffenden Unternehmen. Gewisse Aufschlüsse können jedoch aus Produktivitätskennzahlen gewonnen werden, die für Luftverkehrsallianzen beispielsweise eine Zunahme des sogenannten Sitzladefaktors (Anzahl verkaufte Sitzkilometer geteilt durch Anzahl angebotene Sitzkilometer) auf Strecken verzeichnen, die von zwei Fluggesellschaften gemeinsam bedient werden. Flüge, die von United Airlines und Lufthansa auf bestimmten Strecken gemeinschaftlich angeboten werden, weisen für die Lufthansa einen um 12 Prozent gestiegenen Sitzladefaktor aus. ${ }^{12}$ Die Isolierung der allianzbedingten Zuwächse ist jedoch auch in diesem Fall mit Restriktionen aufgrund verschiedenartiger Einflußvariablen verbunden.

Die Bewertung strategischer Allianzen anhand finanzwirtschaftlicher Größen wirft ähnliche Probleme auf. Allianzpartner bemühen sich i.d.R. um eine Quantifizierung des zu erwartenden Synergiepotentials aus der Allianz, ${ }^{13}$ das dann teilweise um einen pauschalen Risikoabschlag korrigiert wird. Als Basis der Berechnungen dient z.B. der getrennt berechnete diskontierte Cash-Flow der beteiligten Unternehmensbereiche. ${ }^{14}$ Aufgrund der innen beigemessenen Erfolgsrelevanz und ungeachtet der kurz erörterten Bewertungsprobleme haben sich strategische Allianzen in vielen Branchen fest etabliert und sind zum Gegenstand zahlreicher wissenschaftlicher und populärwissenschaftlicher Veröffentlichungen geworden.

11 Vgl. Harbison, J.R., Pekar, P., Cross-Border Alliances in the Age of Collaboration, Working Paper, Hrsg.: Booz, Allen \& Hamilton, Los Angeles 1997, S. 4.

12 Vgl. Klein, H., Internationaler Wettbewerb der Allianzen, in: Allianzen bei Verkehrsdienstleistungen - Perspektiven vor dem Hintergrund sich ändernder Wirtschaftsbedingungen, Dokumentationspapier Nr. 112 der Wissenschaftlichen Gesellschaft für Marketing und Unternehmensführung e.V., Hrsg.: Meffert, H., Backhaus, K., Münster 1997, S. 17.

13 Auf den Begriff der Synergie wird in Kapitel B 1. näher eingegangen.

14 Vgl. z.B. Pierer, H. von, Mirow, M., Das Management strategischer Allianzen, in: Handbuch Unternehmungsführung, Hrsg.: Corsten, H., Reiß, M., Wiesbaden 1995, S. 715f. Die Autoren beziffern den Risikoabschlag in Höhe von 50 Prozent der angesetzten Synergie, die ihrerseits durch konkrete Maßnahmen zu begründen ist. 


\section{Entwicklungslinien von Forschungsaktivitäten im Bereich strate- gischer Allianzen}

Volkswirtschaftliche Beiträge zum Themenbereich strategischer Allianzen widmen sich einerseits der Diskussion ihrer Entstehung, die vornehmlich unter Rückgriff auf Gedankengut der Neuen Institutionenökonomik erklärt wird. ${ }^{15}$ Zum anderen nehmen die Auswirkungen von strategischen Allianzen auf die Funktionsfähigkeit des Wettbewerbs einen relativ breiten Raum unter den volkswirtschaftlichen Veröffentlichungen ein. ${ }^{16}$ In betriebswirtschaftlichen Arbeiten stehen dagegen hauptsächlich Fragen der Organisation und des Management von Kooperationen zwischen Unternehmen im Vordergrund, wobei nicht selten auf Theorieansätze der Volkswirtschaftslehre zurückgegriffen wird. ${ }^{17}$

Erste betriebswirtschaftlich geprägte Publikationen setzten sich mit strategischen Allianzen im internationalen Wettbewerb auseinander und entstanden vornehmlich im angloamerikanischen Sprachraum. ${ }^{18}$ Während der Schwerpunkt dieser frühen Untersuchungen noch auf den Beziehungen zwischen international expandierenden Großunternehmen („Multinational Corporations“) und überwiegend lokal operierenden, oftmals deutlich kleineren Partnern lag, wurde der Fokus nicht zuletzt durch den Beitrag von Porter und Fuller (1986) auf horizontale Allianzen zwischen

15 Vgl. z.B. Bonus, H., The Cooperative Association as a Business Enterprise: A Study in the Economics of Transactions, in: Zeitschrift für die gesamte Staatswissenschaft, 142. Jg., 1986, S. 310-339.

16 Vgl. stellvertretend Blancke, W., Evolution und strategische Allianzen: Der Einfluß von strategischen Allianzen auf den Wettbewerb, Bayreuth 1994.

17 Meyer erforscht z.B. die Organisation von Kooperationen zwischen Herstellern und Zulieferern in der Automobilindustrie. Anwendung findet vor allem die Transaktionskostentheorie als Erklärungsansatz für die entstehenden "Netzwerkarrangements“. Vgl. Meyer, M., Ökonomische Organisation der Industrie: Netzwerkarrangements zwischen Markt und Unternehmung, Wiesbaden 1995.

Preiss, Goldman und Nagel untersuchen demgegenüber das Management von Kooperationen aus einer wettbewerbsstrategischen Sichtweise. Vgl. Preiss, K., Goldman, S.L., Nagel, R.N., Cooperate to Compete: Building Agile Business Relationships, New York u.a. 1996.

18 Dem Einsatz von Gemeinschaftsunternehmen (Joint Ventures) zur internationalen Expansion widmete sich bereits relativ frühzeitig Franko, L.G., Joint venture survival in multinational corporations, New York 1971; später dann auch Killing, J.P., How to make a global joint venture work, in: Harvard Business Review, Vol. 60, No. 3, 1982, S. 120-127 sowie Harrigan, K.R., Strategies for joint ventures, Lexington, Mass. 1985. 
Großunternehmen erweitert, die sich vor dem Eingehen der Allianz in einem weltweiten Wettbewerb gegenüberstanden. ${ }^{19}$

Unter besonderer Berücksichtigung der Koexistenz kooperativer und kompetitiver Beziehungen zwischen den Partnern („Co-opetition") ${ }^{20}$ richtete sich in der Folge das Augenmerk der Untersuchungen in der Tradition strategieorientierter Forschung auf den Erfolg strategischer Allianzen. ${ }^{21}$ Konzeptionelle Arbeiten, Fallstudienanalysen und größerzahlige empirische Erhebungen kamen dabei bisher zu verschiedenen Erfolgsfaktoren, die in der Unternehmensstrategie, -struktur, -kultur und in den Unternehmenssystemen der Partner geortet wurden. ${ }^{22}$

Mit einiger Verzögerung wurde strategischen Allianzen gegen Ende der achtziger bzw. Anfang der neunziger Jahre auch in der deutschsprachigen Literatur wachsende Aufmerksamkeit zuteil. Es zeichneten sich ähnliche Forschungsschwerpunkte wie im angloamerikanischen Bereich $a b .{ }^{23}$ In den USA erhielt die Diskus-

19 Vgl. Porter, M.E., Fuller, M.B., Coalitions and global strategy, in: Competition in global industries, Hrsg.: Porter, M.E., Boston, Mass. 1986, S. 315-343.

20 Die Bezeichnung „co-opetition“ geht vermutlich auf die Autoren Nalebuff und Brandenburger zurück, die mit dieser Begriffsschöpfung die Parallelităt von Wettbewerb und Kooperation pointieren wollen. Vgl. Nalebuff, B., Brandenburger, A., Coopetition - kooperativ konkurrieren: mit der Spieltheorie zum Unternehmenserfolg, Frankfurt am Main, New York 1996.

21 Dussauge und Garrette bezeichnen die Konzeptualisierung und Operationalisierung des Erfolgs von Allianzen als komplexe Aufgabe, die ihrer Meinung nach noch immer nicht zufriedenstellend gelöst wurde. Vgl. Dussauge, P., Garrette, B., Determinants of success in international strategic alliances: evidence from the global aerospace industry, in: Journal of International Business Studies, Third Quarter 1995, S. 506.

22 Die Unterschiedlichkeit der Ergebnisse liegt vor allem in den verschiedenen zugrunde liegenden theoretischen Ansätzen, dem gewählten Branchenkontext und dem methodischen Vorgehen begründet. Borys und Jemison (1989) diskutieren in ihrer konzeptionellen Arbeit formale und prozeßorientierte Erfolgsdeterminanten, wăhrend Hamel, Doz und Prahalad anhand von 15 Fallstudien über internationale strategische Allianzen Vorgehensweisen zum Aneignen von Technologien und Know-how vorschlagen. Kogut (1988) schließlich validiert auf empirischer Basis Ursache-Wirkungszusammenhänge zwischen Branchenmerkmalen und Kooperationsfeldern mit dem Erfolg. Vgl. Borys, B., Jemison, D.B., Hybrid arrangements as strategic alliances: Theoretical issues in organizational combinations, in: Academy of Management Review, Vol. 14, 1989, S. 234-249; Hamel, G., Doz, Y.L., Prahalad, C.K., Collaborate with Your competitors - and Win, in: Harvard Business Review, Vol. 67, No. 1, 1989, S. 133-139; Kogut, B., A study of the life cycle of joint ventures, in: Cooperative strategies in international business, Hrsg.: Contractor, F.J., Lorange, P., Lexington, Mass. 1988, S. 169-186.

1990 erschien das von Backhaus und Piltz herausgegebene Sonderheft Nr. 27 "Strategische Allianzen" der zfbf, in dem konzeptionelle Fragen insbesondere aus wettbewerbsstrategischer Perspektive am Beispiel unterschiedlicher Branchen erörtert wurden. Joint Ventures wurden später als internationale Unternehmenskooperation im Ergänzungsheft 1/1991 der ZfB thematisiert; im selben Jahr veröffentlichten Bronder und Pritzl einen praxisorientierten 
sion wiederum Aufschwung durch Entwicklungen im Bereich moderner Informations- und Kommunikationstechnologien, die den Äußerungen einiger Autoren zufolge zur Auflösung von Unternehmens-, Branchen- und Ländergrenzen beitrugen. ${ }^{24}$ Daraus resultierte eine stärker strukturorientierte Erforschung von Möglichkeiten zur organisatorischen Umsetzung strategischer Allianzen. Zurückgegriffen wurde dabei vor allem auf bereits vorliegendes Gedankengut von Autoren, die eine Vernetzung von Unternehmensaktivitäten problematisierten. ${ }^{25}$

Im Jahre 1992 schien in der Form von virtuellen Unternehmen („virtual corporations") im angloamerikanischen Sprachraum eine Lösung für die interaktive, multimedial unterstützte Gestaltung von Beziehungen zwischen kooperierenden Unternehmen, ihren Abnehmern und der Gesellschaft gefunden zu sein. ${ }^{26}$ Im Gegensatz zu diesen stark praxis- und managementorientierten Überlegungen griffen deutschsprachige Arbeiten in diesem Zeitraum auf unterschiedliche strategie- und organisationsbezogene Theorieansätze zurück, um sie auf gestaltungsbezogene Problemstellungen horizontaler strategischer Allianzen zu übertragen. ${ }^{27}$ Das Konzept des virtuellen Unternehmens hielt jedoch wenig später auch Einzug in die

"Leitfaden" zur Gestaltung strategischer Allianzen, und Gahl legte eine branchenübergreifende empirische Studie vor, in der 33 Unternehmen auf Eigentumsrechte, Managementprozesse und Erfolgsrealisation untersucht werden. Vgl. Bronder, C., Pritzl, R., Leitfaden für strategische Allianzen, in: Harvard Manager, 13. Jg., Nr. 1, 1991, S. 44-53; Gahl, A., Die Konzeption strategischer Allianzen, Berlin 1991.

Vgl. stellvertretend Grenier, R., Metes, G., Enterprise networking: working together apart, o.O., 1992, S. 4-8.

25 Hervorzuheben sind insbesondere das Sammelwerk von Nystrom und Starbuck (1981) sowie die Aufsätze von Miles und Snow (1986) und Jarillo (1988). Vgl. Nystrom, P.C., Starbuck, W.H. (Hrsg.), Handbook of organizational design, Vol. 1, Oxford u.a. 1981; Miles, R.E., Snow, C.C., Organizations: New Concepts for New Forms, in: California Management Review, Vol. 28, No. 3, 1986, S. 62-73; Jarillo, J.C., On Strategic Networks, in: Strategic Management Journal, Vol. 9. No. 1, 1988, S. 31-41. Nohria und Eccles (1992) schließlich gelingt eine verhaltenswissenschaftlich fundierte Zusammenstellung von unterschiedlichen Beiträgen zu diesem Thema. Vgl. Nohria, N., Eccles, R.G. (Hrsg.), Networks and organizations: structure, form, and action, Boston 1992.

Vgl. Davidow, W.H., Malone, M.S., The virtual corporation: Structuring and revitalizing the corporation for the $21^{\text {st }}$ century, New York 1992.

27 Lutz (1993) baut ihre Arbeit auf einem "Multiperspektivenansatz" auf, da ihrer Ansicht nach die Verwendung nur einer Theorie den vielschichtigen Fragestellungen strategischer Allianzen nicht gerecht wird. Vgl. Lutz, V., Horizontale strategische Allianzen: Ansatzpunkte zu ihrer Institutionalisierung, Hamburg 1993, insb. S. 65-92.

Schwamborn (1994) diskutiert u.a. wettbewerbs-, transaktionskosten- und spieltheoretische Ansätze und bemüht den Netzwerkansatz als Integrationskonzept. Vgl. Schwamborn, S., Strategische Allianzen im internationalen Marketing: Planung und portfolioanalytische Beurteilung, Wiesbaden 1994, S. 23-74. 
deutschsprachige Diskussion und stellte mit zeitlicher Verzögerung den Anschluß an die angloamerikanische Entwicklung her. ${ }^{28}$

Seit Mitte der neunziger Jahre greifen Forschungsarbeiten zu strategischen Allianzen vor allem Austauschprozesse zwischen Unternehmen auf, deren Objekte vornehmlich Technologien, Know-how und Rechte sind. ${ }^{29}$ Der internationale Bezug ist dank der vorausgegangenen Forschung mittlerweile oft integrierter Bestandteil der Untersuchungen, ${ }^{30}$ die i.d.R. einen spezifischen Branchenkontext unterstellen. Beachtung hat in diesem Zusammenhang die Luftverkehrsbranche gefunden, die sich - gemessen an der Anzahl strategischer Allianzen im Verhältnis zu der Anzahl erfaßter Fluggesellschaften - durch eine hohe Allianzintensität auszeichnet (vgl. Abbildung 1). Rein rechnerisch ergeben sich im Jahr 1998 für jede Fluggesellschaft rund fünf Allianzbeziehungen zu anderen Fluggesellschaften, da wechselseitige Beziehungen zwischen zwei Partnern in der Statistik nur einfach gezählt werden. ${ }^{31}$ Die Entwicklung der absoluten Anzahl strategischer Allianzen schien den Daten zufolge im Jahr 1997 auf eine Konsolidierung hinzudeuten, während 1998 mit einer Zunahme um 38 Prozent ein erneuter Wachstumsschub zu verzeichnen ist.

28 Nach der 1993 erschienenen Übersetzung des Werkes von Davidow/Malone (s.o.) setzt sich Schräder (1996) mit Umsetzungsaspekten der Virtualisierung am Beispiel strategischer Allianzen auseinander. Picot, Reichwald und Wigand (1996) vermeiden zwar weitgehend die Begrifflichkeit der Virtualităt, diskutieren aber den Einfluß von Informations- und Kommunikationstechnologien auf Unternehmensstrukturen. Meffert (1997) nimmt eine marktorientierte Sichtweise virtueller Unternehmen ein. Vgl. Schräder, A., Management virtueller Unternehmungen: organisatorische Konzeption und informationstechnische Unterstützung flexibler Allianzen, Frankfurt/M., New York 1996; Picot, A., Reichwald, R., Wigand, R.T., Die grenzenlose Unternehmung: Information, Organisation und Management, 2. Aufl., Wiesbaden 1996; Meffert, H., Die virtuelle Unternehmung: Perspektiven aus der Sicht des Marketing, in: Marktleistung und Wettbewerb, Festschrift zum 65. Geburtstag von W.H. Engelhardt, Hrsg.: Backhaus, K., u.a., Wiesbaden 1997, S. 115-141.

Vgl. z.B. Kotabe, M., Swan, K.S., The role of strategic alliances in high-technology new product development, in: Strategic Management Journal, Vol. 16, No. 8, 1995, S. 621-636; Khanna, T., Gulati, R., Nohria, N., The Dynamics of Learning Alliances: Competition, Cooperation, and Relative Scope, a.a.O., S. 193-210; Rao, A.R., Qu, L., Ruekert, R.W., Brand Alliances as Information About Unobservable Product Quality, MSI Working Paper No. 97-100, Cambridge, Mass. 1997.

Kutschker und Mößlang (1996) beispielsweise würdigen unterschiedliche Kooperationsformen als Bestandteil von Internationalisierungsstrategien im Dienstleistungsbereich. Vgl. Kutschker, M., Mößlang, A., Kooperationen als Mittel der Internationalisierung von Dienstleistungsunternehmen, in: DBW, 56. Jg., Nr. 3, 1996, S. 319-337.

31 Die Aufstellung umfaßt nur Fluggesellschaften, deren Flotte mehrheitlich aus Fluggerăt mit einer Kapazität von mindestens 100 Sitzplätzen besteht. Vgl. Gallacher, J., Partners for now, in: Airline Business, No. 6, 1997, S. 27. 


\begin{tabular}{|l|c|c|c|c|c|}
\cline { 2 - 6 } \multicolumn{1}{c|}{} & 1994 & 1995 & 1996 & 1997 & 1998 \\
\hline $\begin{array}{l}\text { Anzahl Allian- } \\
\text { zen }\end{array}$ & 280 & 324 & 390 & 363 & 502 \\
\hline $\begin{array}{l}\text { davon } \\
\text { mit Kapital- } \\
\text { beteiligung } \\
\text { ohne Kapi- } \\
\begin{array}{l}\text { talbetei- } \\
\text { ligung }\end{array}\end{array}$ & $58(20,7 \%)^{1)}$ & $58(17,9 \%)$ & $63(16,1 \%)$ & $54(14,8 \%)$ & $56(11,1 \%)$ \\
$\begin{array}{l}\text { neu gebildet } \\
\text { neury }\end{array}$ & $(-)$ & $50(15,4 \%)$ & $71(18,2 \%)$ & $72(19,8 \%)$ & $121(24,1 \%)$ \\
\hline $\begin{array}{l}\text { Anzahl erfaßter } \\
\text { Fluggesell- } \\
\text { schaften }\end{array}$ & 136 & 153 & $327(83,9 \%)$ & $309(85,2 \%)$ & $446(88,9 \%)$ \\
\hline
\end{tabular}

1) Die Werte in Klammern geben die Prozentwerte in bezug auf die Anzahl Allianzen an.

\section{Abb. 1: Entwicklung strategischer Allianzen im Luftverkehr}

(Quelle: Gallacher, J., Partners for now, in: Airline Business, No. 6, 1997, S. 26; dieselbe, Hold your horses, in: Airline Business, No. 6 , 1998, S. 43.)

Die Ursachen der hohen Allianzintensität im Luftverkehr liegen zum einen in den institutionellen Rahmenbedinungen begründet. Umfangreiche Deregulierungsmaßnahmen im US-amerikanischen Luftverkehrsmarkt seit 1978 sowie Liberalisierungsbestrebungen im europäischen Luftverkehr ab Mitte der achtziger Jahre führten zu einer Intensivierung des Wettbewerbs und damit zu einer Bedrohung etablierter Marktpositionen. ${ }^{32}$ In diesem Zuge fanden sich Fluggesellschaften sowohl auf nationaler als auch internationaler Ebene zu strategischen Allianzen zusammen, um ihre Wettbewerbsposition gemeinsam zu stärken. Das Fortbestehen institutioneller und marktseitiger Markteintrittsbarrieren ließ strategische Allianzen zudem als ein vorteilhaftes Instrument zu ihrer Überwindung erscheinen. ${ }^{33}$

32 Zu einem Überblick vgl. Jäckel, K., Kooperationsstrategien im Linienluftverkehr vor dem Hintergrund zunehmender Integrationsentwicklung in Europa, Bergisch Gladbach, Köln 1991, S. 156-166 sowie 219-232.

33 Vgl. Beyhoff, S., Ehmer, H., Wilken, D., Code-Sharing im internationalen Luftverkehr der Bundesrepublik Deutschland, Forschungsbericht Nr. 95-23 der Deutschen Forschungsanstalt für Luft- und Raumfahrt e.V., Köln 1995, insb. S. 27-30. 
Zum anderen ist die Ursache der hohen Allianzintensität im Luftverkehr in der ausgeprägten Erfolgsrelevanz strategischer Allianzen zu sehen. Schätzungen zufolge beläuft sich der Gewinnbeitrag für Lufthansa aus den strategischen Allianzen mit United Airlines, SAS, South African Airways, Thai Airways, Air Canada und Varig auf 340 Millionen DM. Diese Summe entspricht - bezogen auf den 1997 ausgewiesenen Konzerngewinn vor Steuern - einem Anteil von 21,25 Prozent. ${ }^{34}$ In ähnlicher Weise veranschlagen Marktanalysten den potentiellen Gewinn aus der strategischen Allianz zwischen British Airways und American Airlines auf jährlich rund 100 Millionen britische Pfund für British Airways, entsprechend einem Anteil von umgerechnet rund 18 Prozent des Betriebsergebnisses. ${ }^{35}$ Diese Befunde haben strategische Luftverkehrsallianzen wiederholt zum Gegenstand unterschiedlicher Forschungsbemühungen werden lassen. Im Sinne einer anbietergerichteten „inside-out"-Forschungstradition setzen sich nahezu alle Arbeiten mit Fragestellungen der Planung, Organisation und Kontrolle von strategischen Luftverkehrsallianzen auseinander. ${ }^{36}$ Konzeptionelle Arbeiten sowie Fallstudienanalysen überwiegen hier.

34 Vgl. Klein, H., Internationaler Wettbewerb der Allianzen, a.a.O., S. 20. Die Höhe des Konzerngewinns wurde entnommen aus o.V., Lufthansa will höhere Dividende zahlen, in: Handelsblatt vom 12.3.1998, S. 1.

Vgl. Cohen, A., Transatlantische Allianz zwischen British Airways und American Airlines, Neuer Vorstoß auf Europas Luftverkehrsmärkte, in: fvw, Nr. 14, 1996, S. 70. Zugrunde gelegt wurde das Betriebsergebnis von British Airways für $1996 \mathrm{gem}$. dem World Airline Report, in: Air Transport World, No. 7, 1997, S. 62.

Jăckel beispielsweise stellt die Realisierung des Kooperationsziels „System-Synergie“ vor dem Hintergrund der EU-Integration in den Mittelpunkt seiner strategieorientierten Analyse. Drei Beispielfälle zur Strategie ergänzen die Arbeit. Vgl. Jäckel, K., Kooperationsstrategien im Linienluftverkehr vor dem Hintergrund zunehmender Integrationsentwicklung in Europa, Bergisch Gladbach, Köln 1991.

Youssef fokussiert seine Forschungsarbeit auf Allianzformen mit Kapitalbeteiligungen. Vgl. Youssef, W.A.H., Causes and Effects of International Airline Equity Alliances, Ann Arbor 1992.

Schmidt legt eine Arbeit zu Gemeinschaftsunternehmen (Joint Ventures) als Form strategischer Allianzen vor, die mittels einer Inhaltsanalyse von 187 vertraglichen und kapitalbasierten Joint Ventures aus dem Luftverkehr unterstützt wird. Vgl. Schmidt, S., Strategische Allianzen im Luftverkehr - Erfolgsorientiertes Management europäischer FlugCarrier, Trier 1993.

Frentz schildert den zeitlichen Ablauf des Entstehens und der Auflösung von Konzentrationen und Allianzen im US-amerikanischen Luftverkehr und leitet Schlußfolgerungen für den europäischen Markt ab. Frentz, M. H., Ex Occidente Nox? „Corporate Restructuring, Mergers \& Acquisitions" vs. strategischer Allianzen in der liberalisierten Luftverkehrsindustrie, in: Journal für Betriebswirtschaft , 43. Jg., Nr. 1, 1993, S. 20-44.

Klein erläutert den Stellenwert strategischer Allianzen für die strategische Entwicklung der Deutschen Lufthansa. Vgl. Klein, H., Allianzen - Herausforderungen und Strategien aus Sicht der Deutschen Lufthansa AG, in: Internationales Verkehrswesen, Nr. 12, 1996, S. 12-16. 
Auf der Nachfragerseite („outside-in-Perspektive“) sehen sich strategische Luftverkehrsallianzen durchaus kritischen Stimmen gegenüber. Gegenstand der Kritik sind vornehmlich sogenannte Code Share-Flüge, die gemeinsam von den Allianzpartnern vermarktet und arbeitsteilig durchgeführt werden. ${ }^{37}$ Die nachfragerseitigen Vorwürfe gegenüber den Fluggesellschaften reichen von einer mangelhaften Koordination zwischen den beteiligten Airlines bei der Ausführung der Flugdienstleistung über eine unzureichende Informationspolitik bezüglich der gemeinsamen Aktivitäten bis hin zu absichtsvoller Irreführung und bewußt eingegangenen Sicherheitsrisiken. ${ }^{38}$ Im Bereich der wissenschaftlichen Literatur wurde nachfragerbezogenen Aspekten strategischer Luftverkehrsallianzen bislang verhältnismäßig wenig Aufmerksamkeit zuteil. Für diesen Befund sind verschiedene Ursachen verantwortlich. Zum einen handelt es sich bei strategischen Allianzen um ein stark anbieterseitig dominiertes Instrument, dessen Einsatz durch Fluggesellschaften bei Beachtung einiger Grundregeln der Integration von Flugplänen, Vertriebssystemen und Produkten derzeit noch einen "Erfolgsautomatismus" aufweist. Mit einer derartigen Anbietergetriebenheit ist die Gefahr einer nicht hinreichenden Kundenorientierung verbunden, die den Ruf nach einem Ausgleich des Forschungsdefizits um so lauter werden läßt, zumal der Stellenwert der Kundenorientierung für die Unternehmenssicherung und das Unternehmenswachstum heute unbestritten ist. ${ }^{39}$

Zum anderen liegt ein nur zögerliches Aufgreifen nachfragerorientierter Problemstellungen strategischer Luftverkehrsallianzen in einem Mangel an Informationen und empirischem Datenmaterial begründet. Angesichts einer hohen Reaktions-

${ }^{37}$ In vielen Allianzen bilden Code Share-Flüge den Kern der Zusammenarbeit zwischen Fluggesellschaften. Vgl. Wilken, D., Code-Sharing im Luftverkehr Deutschlands, in: Internationales Verkehrswesen, Nr. 7/8, 1996, insb. S. $25 f$.

38 Kiani-Kress kritisiert z.B. Sicherheitsrisiken und Servicemängel als mögliche Folgeerscheinung, wenn sich etwa ein Passagier von Japan Airlines auf dem Flug nach Saigon nach einem Zwischenstopp in Osaka in einem Flugzeug der durch mehrere Unfälle in Verruf geratenen Vietnam Airlines wiederfindet. Vgl. Kiani-Kress, R., Heineken-Effekt, in: Wirtschaftswoche, Nr. 49, 1996, S. 134. In die populäre Sachbuchliteratur hat das Code Sharing u.a. Eingang gefunden als „übles Spiel, (...) bei dem [die großen Airlines, Anm. d. Verf.] Passagiere einfach an eine andere Fluggesellschaft weiterreichen und dafür Provisionen kassieren." Ogger, G., König Kunde: angeschmiert und abserviert, Hamburg 1996, S. 233.

39 Vgl. Meffert, H., Marketing: Grundlagen marktorientierter Unternehmensführung: KonzepteInstrumente-Praxisbeispiele, 8., vollst. neubearb. und erw. Aufl., Wiesbaden 1998, S. 258. Neben der Kundenorientierung werden in diesem Kontext auch die Wettbewerbs- und Anspruchsgruppenorientierung hervorgehoben. 
verbundenheit im oligopolistischen Luftverkehrsmarkt unterliegen die Ergebnisse von Marktforschungsstudien, die im Auftrag von Fluggesellschaften durchgeführt werden, i.d.R. der Geheimhaltungspflicht. ${ }^{40}$ Daten, die über die Beurteilung strategischer Allianzen durch Fluggäste Auskunft geben, könnten von erfolgskritischer Relevanz und dementsprechend von einer Publikation ausgeschlossen sein. Diese Vermutung wird von Erfahrungen gestützt, die staatliche Institutionen sowie von innen beauftragte Forschungsinstitute in zurückliegenden Projekten gewinnen konnten. So gab das US-amerikanische Department of Transportation 1994 eine Studie in Auftrag, die sich mit dem nachfragerseitigen Wahlverhalten zwischen Fluggesellschaften bei bestehenden Code Sharing-Angeboten auseinandersetzen sollte. Die Untersuchungsergebnisse wurden mit einem ausdrücklichen Hinweis auf eine kaum ausreichende Datenbasis veröffentlicht, die aus einer zurückhaltenden Informationspolitik seitens der Fluggesellschaften resultierte. ${ }^{41}$ Ähnliche Einschränkungen hob das US-amerikanische General Accounting Office in einer 1995 veröffentlichten Studie zu den Auswirkungen strategischer Luftverkehrsallianzen u.a. auf die Tarifstruktur und den Angebotsumfang hervor. ${ }^{42}$

1995 gab der deutsche Bundesminister für Verkehr eine Studie in Auftrag, die sich konzeptionell und empirisch mit den Auswirkungen strategischer Luftverkehrsallianzen auf den Wettbewerb im allgemeinen und auf den Verbraucherschutz im speziellen auseinander setzen sollte. Die Studie wurde von der Deutschen Forschungsanstalt für Luft- und Raumfahrt e.V. durchgeführt und legte unter Einbeziehung einer Telefonbefragung von 40 Reisebüros im Großraum Köln/Bonn/Düsseldorf z.T. gravierende Informationsmängel offen, die als ein nachfragerseitiges Hauptproblem erkannt wurden. ${ }^{43}$ In diesem Zusammenhang darf jedoch nicht übersehen werden, daß auch beim Vorliegen der relevanten Informationen Koordinationsmängel zwischen den Partnern von

40 Zu einer Aufarbeitung der Marktforschungsinhalte von 13 Fluggesellschaften vgl. Söderlund, M., Market orientation and the firm's market intelligence: an empirical study of what airlines want to know about their customers, in: Marketing Today and for the 21st Century, Proceedings of the 24th Annual Conference of the European Marketing Academy, CergyPontoise Cedex 1995, S. 959-978.

41 Vgl. Gellman Research Associates (Hrsg.), A Study of International Airline Code Sharing, Washington 1994, S. 109.

42 Vgl. General Accounting Office (Hrsg.), International Aviation: Airline Alliances Produce Benefits, but Effect on Competition is Uncertain, Report No. 95-99, Washington 1995, S. 25.

43 Vgl. Beyhoff, S., Ehmer, H., Wilken, D., Code-Sharing im internationalen Luftverkehr der Bundesrepublik Deutschland, Forschungsbericht Nr. 95-23 der Deutschen Forschungsanstalt für Luft- und Raumfahrt e.V., Köln 1995, insb. S. 89-96. 
Luftverkehrsallianzen auftreten können, die eine gewichtige Ursache für ein unzureichend kundenorientiertes Verhalten sein können.

Am Beispiel des Luftverkehrs soll im folgenden versucht werden, dem deutlich gewordenen Forschungsdefizit der outside-in-Perspektive strategischer Allianzen wirksam zu begegnen. ${ }^{44}$

\section{Strategische Allianzen bei Flugdienstleistungen}

Um den Untersuchungsgegenstand der strategischen Allianz abzugrenzen, ist zunächst die Erarbeitung einer Definition notwendig. ${ }^{45}$ Aus einer tiefergehenden Interpretation der beiden Begriffskomponenten "strategisch" und „Allianz" ergeben sich wertvolle Aufschlüsse über konstitutive Merkmale strategischer Allianzen. Mit dem Merkmal "strategisch" wird die langfristige Ausrichtung des Verhaltens der Allianzpartner umschrieben. ${ }^{46}$ Die gemeinsame, langfristige Ausrichtung des Verhaltens bezieht sich i.d.R. auf ein oder mehrere strategische Geschäftsfelder, in denen die Partner-Unternehemen - im vorliegenden Fall die Fluggesellschaften aktiv sind. ${ }^{47}$ Strategische Geschäftsfelder werden durch die Kombination unter-

44 Die Ergebnisse einer mit internatonalen Fluggästen durchgeführten Focusgruppenbefragung zur Beurteilung strategischer Luftverkehrsallianzen werden an den betreffenden Punkten in die Ausführungen der vorliegenden Arbeit einfließen.

45 Die Definition von Begrifflichkeiten ist vor allem an ihrer Zweckmäßigkeit zu messen, da die Auswahl oder Ausgrenzung bestimmter Merkmale sowie ihre Erhebung zu konstitutiven Merkmalen stets mehr oder weniger subjektiv sind. Vgl. Lode, R., Formen der Unternehmenskooperation: Betriebswirtschaftliche Überlegungen und Ansätze zur Begriffsbildung der Unternehmenskooperation und zur Systematisierung ihrer Formen, Münster 1974, S. 12. Die Zweckmäßigkeit der hier zu erarbeitenden Definition ist an der eindeutigen und sinnvollen Bestimmung des Untersuchungsobjekts "strategische Allianz" zu bemessen.

46 In seiner ursprünglichen Bedeutung geht das Wort „strategisch“ auf griech. strategòs „Heerführer, Leiter“ zurück und wurde dann substantivisch als „Kunst der Heerführung, Kampfplanung" verwendet. Das Merkmal der Langfristigkeit wird von vielen Autoren als konstitutiv für die Begriffe „strategisch“, „Strategie“ angesehen. Vgl. stellvertretend Kreikebaum, H., Strategische Unternehmensplanung, 6., überarb. und erw. Aufl., Stuttgart, Berlin, Köln 1997, S. 19.

47 Das Konzept der Geschäftsfeldabgrenzung hat insbesondere in der deutschsprachigen Literatur Akzeptanz zur definitorischen Bestimmung strategischer Allianzen gefunden. Vgl. stellvertretend Backhaus, K., Piltz, K., Strategische Allianzen - eine neue Form kooperativen Wettbewerbs? In: Strategische Allianzen, Hrsg.: Backhaus, K., Piltz, K., Sonderheft Nr. 27 der zfbf, 1990, S. 2f. Angloamerikanische Beiträge wählen oftmals ein eher pragmatisches Vorgehen und kennzeichnen das Bezugsobjekt strategischer Allianzen z.B. als "tasks associated with the alliance" oder "common purpose“. Vgl. Rangan, U.S., Yoshino, M.Y., Forging Alliances: A Guide to Top Management, in: The Columbia Journal of World Business, Fall 1996, S. 7; Hwang, P., Burgers, W.P., The Many Faces of Multi-Firm Alliances, in: California Management Review, Vol. 39, 1997, No. 3, S. 101. 
schiedlicher Dimensionen gebildet, von denen die Dimensionen "Zielgruppe“, "Bedürfnisse", "Technologie" sowie "Raumbezug" besondere Beachtung in der betriebswirtschaftlichen Diskussion erfahren haben. ${ }^{48}$ Demnach können sich strategische Allianzen zwischen Fluggesellschaften beispielsweise auf das strategische Geschäftsfeld des Personenluftverkehrs beziehen. Als Zielgruppe sind Endnutzer, d.h. Fluggäste, sowie Reisebüros und Firmenkunden denkbar, ${ }^{49}$ das Bedürfnis besteht vereinfacht in einem Wunsch nach Ortsveränderung, mit der Technologie kann das eingesetzte Fluggerät eingegrenzt werden (z.B. Turbopropvs. Jetflugzeuge) und der Raumbezug kennzeichnet die geographische Flugstrecke (z.B. innerhalb Europas). ${ }^{50}$

Weiterhin impliziert das Merkmal "strategisch“ die Ausrichtung des Verhaltens der Allianzpartner auf die Erreichung eines oder mehrerer Ziele. ${ }^{51} \mathrm{Zu}$ unterscheiden

48 Für Meffert steht die Bildung strategischer Geschäftsfelder am Anfang der strategischen Unternehmensplanung und beinhaltet ein „Aufbrechen des Gesamtmarktes in intern homogene Segmente, die sich in ihren abnehmerbezogenen Anforderungen und anderen erfolgsrelevanten Charakteristika, wie zum Beispiel der Intensität und Struktur des Wettbewerbs, deutlich voneinander unterscheiden." Meffert, H., Marketing: Grundlagen marktorientierter Unternehmensführung, a.a.O., S. 225. Damit besteht eine enge Verknüpfung der Geschäftsfeldabgrenzung mit der Marktsegmentierung. Auf Strategischen Geschäftsfeldern sind sog. Strategische Geschäftseinheiten tătig, welche die unternehmensinterne, organisatorische Verankerung als Gegenpol der marktbezogenen Geschäftsfelddefinition darstellen. Im Ursprung geht das Konzept der strategischen Geschäftsfeldabgrenzung auf Abell (1980) zurück, dessen Ausführungen ein Industriegüter-Kontext zugrunde liegt. Vgl. Abell, D.E., Defining the Business: The Starting Point of Strategic Planning, Englewood Cliffs 1980.

49 Die Trennung zwischen Endnutzern und Reisebüros/Firmenkunden beruht auf dem Kriterium der tatsächlichen Inanspruchnahme von Flugdienstleistungen. Endnutzer sind Fluggäste, die eine Flugdienstleistung selbst in Anspruch nehmen, während Reisebüros und Firmenkunden Flugscheine von Fluggesellschaften erwerben, um sie an Endnutzer weiterzuveräußern oder weiterzugeben. Bedeutende Abnehmer von Flugdienstleistungen sind darüber hinaus Reiseveranstalter, die Flugdienstleistungen mit anderen Teilleistungen zu einem touristischen Leistungspaket zusammenstellen und dieses wiederum über Reisebüros vertreiben. Vgl. z.B. Pompl, W., Touristikmanagement 2: Qualitäts-, Produkt-, Preismanagement, Berlin u.a. 1996, S. 3.

50 Ein anderes strategisches Geschäftsfeld für strategische Allianzen zwischen Fluggesellschaften ergibt sich in der Flugzeugwartung und -instandhaltung. Hier bestünde die Zielgruppe in Fluggesellschaften, das Bedürfnis in der Sicherstellung technischer Funktionsfähigkeit des Fluggeräts, die Technologie in dem Umfang technischer Arbeiten und der Raumbezug im Standort der Wartungs- und Instandhaltungsaktivităten.

Dieser Interpretation wohnt eine präskriptive Sichtweise der Strategie inne, die bereits früh von Chandler (1962/1984) vertreten wurde und im deutschen Sprachraum maßgeblich im entscheidungsorientierten Ansatz von Heinen (1978) ihren Niederschlag fand, der Strategie als Inhalt derjenigen Entscheidungen definiert, die zur "Festlegung langfristiger Unternehmensziele und globaler Maßnahmen zu ihrer Erreichung dienen“. Vgl. Chandler, A., Strategy and Structure: Chapters in the History of the Industrial Enterprise, Cambridge, Mass., London 1984, 
sind unternehmensindividuelle Ziele, zu deren Erreichung die strategische Allianz beitragen soll, und unternehmensübergreifende Allianzziele, die in der Literatur oft als gemeinsame Erhaltung und/oder Erzielung von Wettbewerbsvorteilen bezeichnet werden. ${ }^{52}$ Aus der geschäftsfeldbezogenen Zusammenarbeit ergeben sich Zielinterdependenzen zwischen den beteiligten Partnerunternehmen, die sich im Spannungsfeld zwischen erhaltenen Anreizen und zu leistenden Beiträgen bewegen. ${ }^{53}$ In einer weiteren Deutung des Begriffs "strategisch“ zeichnet sich eine strategische Allianz durch die Orientierung an Erfolgspotentialen aus, ${ }^{54}$ die mittels Partnerschaft gesichert oder in Form von neuen Erfolgspotentialen erschlossen werden sollen. ${ }^{55}$

Weitere konstitutive Merkmale erschließen sich aus dem Begriff der „Allianz“, mit dem im ursprünglichen Sinne ein "Bündnis“ oder eine „Verbindung" impliziert

S. 13 (13., unveränd. Nachdruck der 1. Aufl. von 1962) sowie im wörtlichen Zitat Heinen, E., Führung als Gegenstand der Betriebswirtschaftslehre, in: Betriebswirtschaftliche Führungslehre: ein entscheidungsorientierter Ansatz, Hrsg.: Heinen, E., Wiesbaden 1978, S. 34.

Dem präskriptiven steht der deskriptive Strategiebegriff gegenüber, demgemäß sich in Anlehnung an Mintzberg (1978) eine strategische Allianz als Muster in einem Strom von Entscheidungen herausbilden müßte. Die Zielabhängigkeit von Strategien tritt hier stärker in den Hintergrund. Vgl. Mintzberg, H., Patterns in Strategy Formulation, in: Management Science, Vol. 24, No. 9, 1978, S. 935.

52 Vgl. z.B. Rangan, U.S., Yoshino, M.Y., Forging Alliances: A Guide to Top Management, a.a.O., S. 7; Gahl, A., Die Konzeption strategischer Allianzen, a.a.O., S. 9. Backhaus und Voeth (1995) sprechen von Koalitionsrenten, die zur Bildung strategischer Allianzen führen. Vgl. Backhaus, M., Voeth, M., Strategische Allianzen - Herausforderungen neuer Kooperationsformen, in: Stabilităt und Effizienz hybrider Organisationsformen, Hrsg.: Wagner, H., Jäger, W., Münster 1995, S. 77.

53 Vgl. Lutz, V., Horizontale strategische Allianzen: Ansatzpunkte zu ihrer Institutionalisierung, a.a.O., S. 44.

54 Zu Knyphausen-Aufseß definiert den „Begriff des Strategischen“ als "die Erfolgspotentiale (signifikant) betreffend“. Erfolgspotentiale gehen demnach auf einen Fit zwischen System und Umwelt zurück, der bestimmte Făhigkeiten wie beispielsweise Handlungsfähigkeit, Lernfähigkeit und Responsiveness von Unternehmen erfordert. Größen wie Marktposition oder Position auf der Erfahrungskurve sind nach seiner Erkenntnis mit finanzwirtschaftlichen Größen wie Return on Investment oder Return on Assets "zirkulär verbunden“ und geben Aufschluß auf bestehende Erfolgspotentiale. Vgl. Knyphausen-Aufseß, D. zu, Theorie der strategischen Unternehmensführung: state of the art und neue Perspektiven, Wiesbaden 1995, S. 360f. Offen bleibt bei inm jedoch die Konkretisierung des nicht im statistischen Sinne gebrauchten Attributs „signifikant".

55 Vgl. z.B. Gahl, A., Die Konzeption strategischer Allianzen, a.a.O., S. 9-11 sowie Hamel, G., Competition for Competence and Interpartner Learning Within International Strategic Alliances, in: Strategic Management Journal, Summer Special Issue, 1991, S. 83-103. 
wird. ${ }^{56}$ Ein Bündnis kann als gegenseitige Bindung von Unternehmen aneinander verstanden werden, die sich mittels dieses Bündnisses gegenüber anderen Unternehmen abgrenzen. Diese Interpretation berührt zum einen die Beziehungen zwischen den Partnern der strategischen Allianz („Intraallianz-Beziehungen“) und zum anderen die Beziehungen zu der Umwelt außerhalb der strategischen Allianz. Unter der Annahme einer rechtlichen Selbständigkeit und weitgehender wirtschaftlicher Selbständigkeit der beteiligten Allianzpartner ${ }^{57}$ bewegen sich Intraallianz-Beziehungen angesichts des Geschäftsfeldbezugs im Spannungsfeld von Kooperation und Wettbewerb. ${ }^{58}$ Die Schwierigkeit, dieses Spannungsfeld zu überbrücken, wird teilweise als Ursache für relativ niedrige "Überlebensraten“ von Luftverkehrsallianzen gewertet. Für rein vertragliche Luftverkehrsallianzen ergab eine Studie die "Überlebensrate" von 26 Prozent (1992-1995), während kapitalbasierte Allianzen in 73 Prozent der beobachteten Fälle Bestand hatten. ${ }^{59}$ Wichtige Parameter der Intraallianz-Beziehungen liegen in der Zieldefinition und organisationalen Gestaltung durch die Allianzpartner. ${ }^{60}$

56 „Allianz“ hat sich aus dem lat. alligare „anbinden, verbinden“ entwickelt und wird im Deutschen häufig als "Staatenbündnis“ gebraucht.

57 Über dieses Merkmal strategischer Allianzen besteht in der Literatur weitgehender Konsens. Zur Grenzziehung zwischen wirtschaftlicher Selbständigkeit und Unselbständigkeit der Allianzpartner schlägt Schäfer-Kunz (1995) vor, die Freiheit der Eintritts- und Austrittsentscheidung als Indikator zu verwenden. Am Beispiel der zwischen Singapore Airlines und Lufthansa offiziell am 24.11.1997 vereinbarten strategischen Allianz wird die Freiheit der Eintrittsentscheidung deutlich, da vor der Allianz quasi keine Abhängigkeiten und kein politischer Zwang zur Allianzbildung vorlagen. Die Trennung von USAir und British Airways zum Mărz 1997 relativiert dagegen die Freiheit von Austrittsentscheidungen aus strategischen Allianzen. Die Allianz der beiden Fluggesellschaften war durch die geplante Partnerschaft zwischen British Airways und American Airlines unter Druck geraten und führte zum Verkauf der Kapitalanteile, die British Airways an USAir hielt. Vgl. Schäfer-Kunz, J., Strategische Allianzen im deutschen und europäischen Kartellrecht, a.a.O., S. 37; o.V., Lufthansa und Singapore Airlines kooperieren, in: FAZ vom 25.11.1997, S. 29; o.V., Streit um die Allianz von British Airways und American Airlines, in: FAZ vom 16.1.1997, S. 17.

58 Hwang und Burgers (1997) schlagen in Abhängigkeit von vier unterschiedlichen Situationen bzw. Konstellationen bestimmte Verhaltensweisen im Umgang mit derartigen dynamischen Allianzbeziehungen vor. Zugrunde liegt ein spieltheoretischer Ansatz, der den engen Fokus von 2-Personen-Spielen verläßt und sich auf "multi-firm alliances“ anwenden läßt. Vgl. Hwang, P., Burgers, W.P., The Many Faces of Multi-Firm Alliances, a.a.O., S. 101ff.

$59 \mathrm{Vgl}$. Lindquist, J., The role of alliances in the strategy of airlines, Präsentationsunterlagen der Boston Consulting Group im Rahmen der Airline Alliances Conference, London 1995, S. 13 u. 20. Eisele (1995) kritisiert die eingeschrănkte Aussagefähigkeit derartiger „Überlebensraten“, da sie bei ex ante vereinbartem Auflösungsdatum als Erfolgsmaßstab ebenso untauglich sind wie als Einflußgrőße von Entscheidungen, da sie lediglich eine ex post-Kennzahl bereits gescheiterter Allianzen darstellen. Vgl. Eisele, J., Erfolgsfaktoren des Joint-VentureManagement, a.a.O., S. 86.

60 Hierauf wird im Kapitel B 1. näher eingegangen. 
Wesentliche Bedeutung für die Gestaltung der Beziehungen zwischen der strategischen Allianz und ihrer Umwelt wird der Strategie beigemessen, welche die Allianz verfolgt. ${ }^{61}$ In Abhängigkeit von der jeweiligen Bezugsgruppe und den Marktteilnehmern unterscheidet Meffert (1994) abnehmer-, absatzmittler-, konkurrenzund sonstige anspruchsgruppengerichtete Strategien, ${ }^{62}$ die ein systemtheoretisch geprägtes Verständnis der Umwelt widerspiegeln. Diese wird oft in eine globale Umwelt (z.B. politisch-rechtliche, sozio-kulturelle Umwelt) und eine Aufgabenumwelt (z.B. Beschaffungs- und Absatzmarkt, Wettbewerb) unterteilt. ${ }^{63} \mathrm{Im}$ Mittelpunkt der vorliegenden Arbeit stehen abnehmer- und absatzmittlergerichtete Strategien von strategischen Luftverkehrsallianzen, da sie den Rahmen für die Interaktion zwischen strategischer Allianz und Nachfragern festlegen.

Die damit erörterte Bindung von Unternehmen aneinander, die aus der wortgetreuen Interpretation des Allianzbegriffs abgeleitet wurde, beinhaltet schließlich noch die gemeinsame Verbindung von Aktivitäten im Rahmen der strategischen Allianz. ${ }^{64}$ Unter Zugrundelegung des Kontextes "Personenluftverkehr" stimmen mindestens zwei Fluggesellschaften im Zuge einer Flugdienstleistung ihre Aktivitäten aufeinander ab. Grundsätzlich kann es sich um Aktivitäten handeln, die im Bereich der "line of visibility" liegen und daher von den Nachfragern wahrgenommen und/oder erlebt werden, oder um „backoffice"-Aktivitäten, die den Nachfragern verborgen bleiben.

61 Mintzberg bezeichnet eine Strategie allgemein als "Vermittlungsinstanz" zwischen einer Organisation und ihrer Umwelt. Vgl. Mintzberg, H., Opening up the Definition of Strategy, in: The Strategy Process: Concepts, Contexts, \& Cases, Hrsg.: Quinn, J.B., Mintzberg, H., James, R.M., Englewood Cliffs, New Jersey 1988, S. 17 ( $\ldots$... strategy becomes the mediating force (...) between organization and environment, ...").

62 Vgl. Meffert, H., Marketing-Management: Analyse, Strategie, Implementierung, Wiesbaden 1994, S. 124. Später subsumiert Meffert (1998) die drei erstgenannten Bezugsgruppen dann ebenfalls unter die Anspruchsgruppen und kennzeichnet diese generell als „Einfluß ausübende Gruppen mit konkreten Erwartungen an das Unternehmen." Meffert, H., Marketing: Grundlagen marktorientierter Unternehmensführung, a.a.O., S. 30.

63 Diese Einteilung geht auf Dill zurück. Vgl. Dill, W.R., Environment as an influence on managerial autonomy, in: Administrative Science Quarterly, Vol. 3, 1958, S. 409ff.

64 Porter und Fuller (1986) unterscheiden zwischen sogenannten Y-Allianzen, bei denen die Partner eine Aktivităt gemeinsam betreiben, und X-Allianzen, in deren Rahmen sich die Partner die Durchführung der Wertaktivitäten teilen. Porter, M.E., Fuller, M.B., Coalitions and global strategy, in: Competition in global industries, Hrsg.: Porter, M.E., Boston, Mass. 1986, S. $315 \mathrm{ff}$. 
Abbildung 2 verdichtet die erarbeiteten konstitutiven Merkmale strategischer Allianzen und bereitet den Weg für eine Definition, die dann auf den Kontext des Personenluftverkehrs übertragen werden kann.

\begin{tabular}{|l|l|}
\hline Begriffskomponenten & Bedeutungsinhalt \\
\hline strategisch & $\begin{array}{l}\text { langfristige Ausrichtung des gemeinsamen Verhaltens } \\
\text { Geschäftsfeldbezug }\end{array}$ \\
\hline Allianz & Ausrichtung auf ein Ziel oder mehrere Ziele \\
& $\begin{array}{l}\text { Orientierung an Erfolgspotentialen } \\
\text { nungsfeld zwischen Kooperation und Wettbewerb } \\
\text { nemeinsame Beziehungen zur Umwelt } \\
\text { gechtliche Selbständigkeit } \\
\text { weitgehende wirtschaftliche Selbständigkeit } \\
\text { Verbindung und gemeinsame Abstimmung von Aktivitä- } \\
\text { ten }\end{array}$ \\
\hline
\end{tabular}

\section{Abb. 2: Begriffsmerkmale und Bedeutungsinhalte strategischer Allianzen}

Strategische Allianzen beinhalten demnach

die langfristig angelegte, zielorientierte und geschäftsfeldbezogene Abstimmung von Aktivitäten zwischen mindestens zwei Unternehmen. Die Allianzpartner bewegen sich als rechtlich selbständige und wirtschaftlich weitgehend selbständige Unternehmen in einer gemeinsamen Umwelt und gewähren sich in einem Spannungsfeld zwischen Kooperation und Wettbewerb gegenseitig Zugang zu Erfolgspotentialen. ${ }^{65}$

65 Gegenüber dem im deutschsprachigen Bereich insbesondere von Sydow (1992) geprägten Begriff des strategischen Netzwerks grenzen sich hier strategische Allianzen durch einen wesentlich engeren Fokus ab, der sich vornehmlich aus dem Geschäftsfeldbezug ergibt. Vgl. Sydow, J., Strategische Netzwerke: Evolution und Organisation, Wiesbaden 1992, S. 82.

Backhaus und Meyer (1993) sowie Meyer (1995) subsumieren (horizontale) strategische Allianzen und (vertikale bzw. diagonale) strategische Netzwerke unter sogenannte Netzwerkarrangements, die sich wesentlich durch die Wertschöpfungsbeziehung unterscheiden. Vgl. Backhaus, K., Meyer, M., Strategische Allianzen und strategische 
Übertragen auf den Luftverkehr, ergeben sich für die Untersuchung strategischer Allianzen im Sinne dieser Definition einige Eigenheiten, die vor allem aus dem Produkt "Flug" erwachsen. Luftverkehr umfaßt zunächst in einem allgemeinen Verständnis die "Gesamtheit aller Vorgänge, die der Ortsveränderung von Personen, Fracht und Post auf den Luftwegen dienen (...) und alle damit unmittelbar

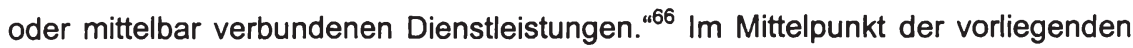
Arbeit steht der Personenluftverkehr, der sich nach dem Kriterium des Transportobjektes gegenüber dem Luftfracht- und -postverkehr abgrenzt. ${ }^{67}$ Wird der Definitionsbestandteil "Gesamtheit aller Vorgänge" auf einen einzelnen Vorgang reduziert, ergibt sich ein einzelner Flug als Produkt des Luftverkehrs, der von Fluggesellschaften produziert und im Personenluftverkehr von Fluggästen nachgefragt wird. ${ }^{68}$ Die mit diesem Produkt definitionsgemäß „unmittelbar oder mittelbar verbundenen Dienstleistungen" legen nahe, den Begriff des Fluges auf den Begriff der Flugdienstleistung auszudehnen, deren Kern der eigentliche Flug

Netzwerke, in: WiSt, Nr. 7, 1993, S. 332; Meyer, M., Okonomische Organisation der Industrie: Netzwerkarrangements zwischen Markt und Unternehmung, Wiesbaden 1995, S. 156-161.

Des weiteren wird mit der obigen Definition der Unterschied zwischen strategischen Allianzen und strategischen Gruppen deutlich. Ursprünglich von Hunt (1972) in industrieökonomischer Tradition empirisch erforscht, zeichnen sich strategische Gruppen durch ein homogenes wettbewerbsstrategisches Verhalten in einer vergleichbaren Umfeldsituation aus. Vgl. Hunt, M.S., Competition in the major home appliance industry, Diss., Harvard University 1972, S. 57ff. Damit fehlen entscheidende Begriffsmerkmale, die für die strategische Luftverkehrsallianz konstitutiv sind. Nichtsdestoweniger könnte dieser Ansatz vereinzelt Aufschluß über die anbieterseitige Gestaltung strategischer Luftverkehrsallianzen geben (z.B. Auswahl von Partnern aus derselben strategischen Gruppe).

Die Abgrenzung zum Kartell macht Schäfer-Kunz vor allem an der wettbewerbsbeschränkenden Wirkung fest, die zwar so nicht explizit in obiger Definition enthalten ist, implizit jedoch oft einen Nebeneffekt strategischer Allianzen bildet. In der Koexistenz von Wettbewerb und Kooperation besteht jedoch ein greifendes Abgrenzungsmerkmal der strategischen Allianz zum Kartell. Vgl. Schäfer-Kunz, J., Strategische Allianzen im deutschen und europäischen Kartellrecht, a.a.O., S. 62; ăhnlich auch Kartte, W., Wettbewerbspolitische und wettbewerbsrechtliche Probleme Strategischer Allianzen, in: Wegweiser für Strategische Allianzen: Meilen- und Stolpersteine bei Kooperationen, Hrsg.: Bronder, C., Pritzl, R., Wiesbaden 1992, S. $403 \mathrm{ff}$. Rößger, E., Hünermann, K., Einführung in die Luftverkehrspolitik, Zürich 1965, S. 3.

67 In den Personenluftverkehr ist die gleichzeitige fakultative Beförderung von Verfügungsobjekten der Fluggäste (z.B. Haustiere, Gepäck) eingeschlossen. Da für Lufttrachtund -postverkehr im Vergleich zum Personenluftverkehr unterschiedliche Angebots- und Nachfragebedingungen vorliegen, ist an dieser Stelle eine Eingrenzung der Ausführungen auf den Personenluftverkehr notwendig. Vgl. z.B. O'Connor, W.E., An introduction to airline economics, 5. Aufl., Westport 1995, S. 151ff. Diese Eingrenzung ermöglicht eine konzentrierte Auseinandersetzung mit den spezifischen Problemstellungen, die Gegenstand der Arbeit sind.

68 Vgl. Pompl, W., Luftverkehr: eine ökonomische Einführung, 2., aktualisierte und erw. Aufl, Berlin u.a. 1991, S. 33. 
bildet. Aus diesem Grund erscheint es sinnvoll, unter Bezugnahme auf die Literatur zum Dienstleistungsmarketing einige Besonderheiten von Flugdienstleistungen herauszustellen.

Zunächst ist zu überprüfen, inwieweit die für Dienstleistungen allgemein erarbeiteten Spezifika auf Flugdienstleistungen zutreffen. ${ }^{69}$ Meffert und Bruhn (1997) legen eine Dienstleistungsdefinition vor, die aus der kritischen Bewertung unterschiedlicher, in der Literatur vorhandener Definitionsansätze entwickelt wird. Dienstleistungen sind demnach „selbständige, marktfähige Leistungen, die mit der Bereitstellung (...) und/oder dem Einsatz von Leistungsfähigkeiten (...) verbunden sind (Potentialorientierung). Interne (zum Beispiel Geschäftsräume, Personal, Ausstattung) und externe Faktoren (also solche, die nicht im Einflußbereich des Dienstleisters liegen) werden im Rahmen des Erstellungsprozesses kombiniert (Prozeßorientierung). Die Faktorenkombination des Dienstleistungsanbieters wird mit dem Ziel eingesetzt, an den externen Faktoren, an Menschen (zum Beispiel Kunden) oder deren Objekten (...) nutzenstiftende Wirkungen (...) zu erzielen (Ergebnisorientierung)."70

Übertragen auf den Personenluftverkehr, sind an der Bereitstellung bzw. dem Einsatz von Leistungsfähigkeiten mehr als ein Unternehmen bzw. mehr als eine Institution beteiligt. ${ }^{71}$ Die Fluggesellschaft betreibt - z.T. in enger Abstimmung mit

69 An dieser Stelle soll auf eine Diskussion des Für und Wider einer Dienstleistungsdefinition im Sinne einer Abgrenzung zur Sachleistung verzichtet werden. Die von Engelhardt, Kleinaltenkamp und Reckenfelderbäumer (1993) vorgeschlagene Leistungstypologie hat zwar einen möglichen Weg aus dieser Zweiteilung gewiesen, läßt aber die geforderte Vermeidung des Dienstleistungsbegriffs nicht zwingend notwendig erscheinen. Die Mehrzahl von Autoren nähert sich dem Dienstleistungsbegriff nach wie vor über die Erarbeitung bestimmter Merkmale, die z.T. als konstitutiv angesehen werden. Vgl. Engelhardt, W.H., Kleinaltenkamp, M., Reckenfelderbäumer, M., Leistungsbündel als Absatzobjekte: Ein Ansatz zur Überwindung der Dichotomie aus Sach- und Dienstleistungen, in: zfbf, 45. Jg., Nr. 5, 1993, S. 404ff.; Meffert, H., Bruhn, M., Dienstleistungsmarketing: Grundlagen-Konzepte-Methoden, mit Fallbeispielen, 2., überarb. und erw. Aufl., Wiesbaden 1997, S. 23-46 sowie die dort angegebene Literatur.

Meffert, H., Bruhn, M., Dienstleistungsmarketing: Grundlagen-Konzepte-Methoden, mit Fallbeispielen, a.a.O., S. 27 (Original in grau schattiertem Kasten).

Dieser Sachverhalt ist grundsätzlich nicht neu. Dieckmann etwa erläutert die Problemstellungen, die aus der Kombination mehrerer Produktkomponenten zu "Güterbündeln“ entstehen, insbesondere unter dem Blickwinkel der Preiswahrnehmung. Für ihn entsteht ein „komplexes Produkt" aus der Zusammenfassung von Leistungen zu einem Bündel, das als zusammenhängende Einheit verkauft wird. Dies trifft auch auf Flugdienstleistungen zu, die damit als komplexe Dienstleistung bezeichnet werden können. Vgl. Dieckmann, R., Optimale Preis- und Angebotspolitik für komplexe Produkte: eine Analyse der Güterbündelung unter Berücksichtigung der Verarbeitung von Preisen durch Konsumenten, Frankfurt am Main u.a. 1993, S. 4f. Birkelbach nimmt sich der durch mehrere Dienstleistungsanbieter erstellten 
anderen Fluggesellschaften - Fluggerät, das von Flugzeugbauunternehmen hergestellt wird, ${ }^{72}$ der Staat vergibt die Rechte zur Nutzung der über seinem Hoheitsgebiet liegenden Verkehrswege an Fluggesellschaften, Flugsicherungsgesellschaften gewährleisten die Überwachung des Flugbetriebs, und Flughäfen stellen den Fluggesellschaften Start- und Landerechte („Slots“) zur Verfügung. Flughäfen bilden zudem die physische Schnittstelle zur Nutzung von Flügen durch Nachfrager und können auch als (Verkehrs-)Systemzugangspunkt bezeichnet werden. ${ }^{73}$

Die Prozeßorientierung der Flugdienstleistung kommt in der sog. Reisekette zum Ausdruck, die in Abhängigkeit von der Zeit ${ }^{74}$ die verschiedenen Phasen bzw. Prozeßstufen im Verlauf einer Flugdienstleistung wiedergibt. ${ }^{75}$ Entlang der Reise-

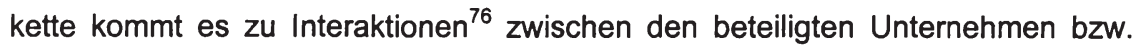

Verbundangebote aus Sicht des Qualitătsmanagement an. Ein Beispielfall ist bei inm u.a. das Dienstleistungsangebot von Verkehrsflughäfen. Vgl. Birkelbach, R., Qualitătsmanagement in Dienstleistungscentern: Konzeption und typenspezifische Ausgestaltung unter besonderer Berücksichtigung von Verkehrsflughäfen, Frankfurt am Main u.a. 1993.

72 Dabei befindet sich ein Teil der Flugzeuge nicht im Eigentum der Fluggesellschaften: 1993 ergab die Analyse eines britischen Flugzeugversicherers, daß von einem weltweiten Bestand von ca. 10.000 Jet-Flugzeugen 1.629 Flugzeuge geleast waren. Vgl. Verchère, I., The air transport industry in crisis: solving over-capacity and financing new equipment, London 1994, S. 95.

73 Diese Darstellung ist bewußt vereinfacht und erfaßt die einer Flugdienstleistung zugrunde liegenden Leistungsfähigkeiten daher nicht vollständig. Sterzenbach (1996) subsumiert die genannten Leistungsfähigkeiten unter ein "System der Produktionsfaktoren" im Gutenbergschen Sinne und bleibt damit einer anbieterzentrierten Sichtweise verhaftet. Vgl. Sterzenbach, R., Luftverkehr: betriebswirtschaftliches Lehr- und Handbuch, München, Wien 1996, insb. S. 71-138. Pompl stellt dagegen Fluggesellschaften, Agenturen, Reiseveranstalter und Verbraucher in den Mittelpunkt des "System[s] Luftverkehrswirtschaft" und bezieht sich auf einen systemtheoretischen Hintergrund. Vgl. Pompl, W., Luftverkehr: eine ökonomische Einführung, a.a.O., S. 9.

74 Strenggenommen bestimmt sich die Abfolge der Phasen nach rechtlichen ( $z$.B. Ausweiskontrolle), technischen (z.B. Gepäckaufgabe vor Betreten des Flugzeugs) und ökonomischen (z.B. Ticketkauf vor Flug) Notwendigkeiten, die den zeitlichen Ablauf des Flugdienstleistungsprozesses festlegen.

75 Eine Darstellung der Reisekette im Luftverkehrsbereich findet sich z.B. bei Laakmann (1995), der auf das von Shostack (1982) entwickelte Verfahren des Blueprinting zurückgreift und es auf die systematische Erfassung des Dienstleistungserstellungs- und -konsumtionsprozesses einer Flugdienstleistung überträgt. Vgl. Laakmann, K., Value added services als Profilierungsintrument im Wettbewerb: Analyse, Generierung und Bewertung, Frankfurt am Main u.a. 1995, S. 145. Siefke (1998) unterteilt eine Bahnreise in vier unterschiedliche Reisephasen und untersucht den Einfluß phasenspezifischer Teilerlebnisse auf die Kundenzufriedenheit. Vgl. Siefke, A., Zufriedenheit mit Dienstleistungen: ein phasenorientierter Ansatz zur Operationalisierung und Erklärung der Kundenzufriedenheit im Verkehrsbereich auf empirischer Basis, Frankfurt am Main u.a. 1998, insb. S. 126-129.

76 Der Begriff der Interaktion hat insbesondere durch die Human-Relations-Forschung Einzug in den verhaltenswissenschaftlich geprägten Teil der betriebswirtschaftlichen Diskussion 
Institutionen (anbieterseitige Abstimmungsprozesse) einerseits und zwischen diesen Unternehmen bzw. Institutionen und den Nachfragern andererseits. Die zwischen Dienstleistungsanbietern und -nachfragern stattfindenden Interaktionen werden in der Literatur auch als "moments of truth" oder "service encounter" bezeichnet, während deutschsprachige Arbeiten von "Augenblicken der Wahrheit" oder „Kundenkontaktpunkten“ sprechen. ${ }^{77}$ Damit wird folglich ein Teil der anbieterseitigen Abstimmungsprozesse von den Nachfragern wahrgenommen und/oder erlebt. ${ }^{78}$ Bei diesem Teil handelt es sich um diejenigen Abstimmungsprozesse, die in dem für den Nachfrager relevanten Kontaktbereich der Dienstleistung, der „line of visibility", ablaufen. ${ }^{79}$ Vor der Inanspruchnahme einer Flugdienstleistung können Nachfrager beispielsweise Werbeanzeigen wahrnehmen, die über die strate-

gehalten. Staehle definiert Interaktion als „zweckgerichtete wechselseitige soziale Beziehungen zwischen mindestens zwei Interaktionspartnern“ und betont den ihr immanenten Aspekt des Handelns. Staehle, W.H., Management: eine verhaltenswissenschaftliche Perspektive, 7. Aufl., München 1994, S. 288. Jeschke stellt stärker auf den Inhalt bzw. Ablauf der Interaktion ab und bestimmt Interaktionen als "gegenseitige Abhängigkeiten und Beeinflussungen in menschlichen Austauschbeziehungen". Jeschke, K., Nachkaufmarketing: Kundenzufriedenheit und Kundenbindung auf Konsumgütermärkten, Frankfurt am Main u.a. 1995, S. 149.

Vgl. stellvertretend Shostack, G.L., Planning the Service Encounter, in: The Service Encounter: Managing Employee/Customer Interaction in Service Businesses, Hrsg.: Czepiel, J.A., Solomon, M.R., Surprenant, C.F., Lexington 1985, S. 243ff.; Stauss, B., "Augenblicke der Wahrheit" in der Dienstleistungserstellung, in: Dienstleistungsqualität: Konzepte, Methoden, Erfahrungen, Hrsg.: Bruhn, M., Stauss, B., 2. Aufl., Wiesbaden 1995, S. 382; Siefke, A., Zufriedenheit mit Dienstleistungen, a.a.O., insb. S. 101-104.

78 Meffert definiert Wahrnehmung im Sinne einer kaufverhaltensrelevanten Determinante als Prozeß der Aufnahme, Selektion, Organisation und Interpretation von Informationen durch ein Individuum. Aktivität, Subjektivität und Selektivität sind wichtige Merkmale dieses kognitiv orientierten Prozesses. Vgl. Meffert, H., Marketingforschung und Käuferverhalten, 2., vollst. überarb. und erw. Aufl., Wiesbaden 1992, S. 61. In der Dienstleistungsliteratur findet sich in deutschsprachigen Übersetzungen des von Zeithaml, Parasuraman und Berry (1985) im Rahmen ihres GAP-Modells verwendeten Begriffes "perceived service“ der Terminus "wahrgenommene Dienstleistung". Das zugrunde liegende Verb "to perceive" ist zwar auch im Englischen eher kognitiv besetzt (wahrnehmen; erkennen i.S. von to understand, realize, recognize), wird aber in der betreffenden Dienstleistungsliteratur nichtsdestoweniger eher als "Erleben“ einer Dienstleistung verwendet. Vgl. Parasuraman, A., Zeithaml, V.A., Berry, L.L., A Conceptual Model of Service Quality and Its Implications for Future Research, in: Journal of Marketing, Vol. 49, No. 4, 1985, S. 44; Siefke, A., Zufriedenheit mit Dienstleistungen, a.a.O., S. 73ff. Zur Übersetzungsproblematik vgl. Pons-Großwörterbuch Deutsch-Englisch, EnglischDeutsch, 2. Aufl., Stuttgart, Dresden 1993, S. 493. Es erscheint daher sinnvoll, die Wahrnehmung von Bildern, Schrift und akustischen Signalen im Vorfeld der Dienstleistungsinanspruchnahme vom Prozeß des eigentlichen Erlebens der Dienstleistung zu trennen.

79 Der interne Erstellungsbereich der Flugdienstleistung wird demgegenüber oft als „backoffice“ bezeichnet und ist für den Nachfrager i.d.R. nicht einsehbar. Vgl. Shostack, G.L., Planning the Service Encounter, in: The Service Encounter: Managing Employee/Customer Interaction in Service Businesses, Hrsg.: Czepiel, J.A., Solomon, M.R., Surprenant, C.F., Lexington 1985, S. 243ff. 
gische Allianz bestimmter Fluggesellschaften informieren. Das Flugticket für eine gemeinsam von zwei Allianzpartnern angebotene Flugdienstleistung wird dann z.B. auf Fluggesellschaft $A$ ausgestellt, der Eincheckvorgang findet an einem Schalter dieser Gesellschaft statt, der eigentliche Flug und die Betreuung während des Fluges werden hingegen von der Partner-Fluggesellschaft B durchgeführt. ${ }^{80}$

Besondere Beachtung erlangt die am Nachfrager ${ }^{81}$ während der Flugdienstleistung vollzogene Nutzenstiftung als ein Ergebnis der Interaktion zwischen Nachfrager und Dienstleistungsanbietern. ${ }^{82}$ Demnach kann lediglich das Urteil des Nachfragers darüber entscheiden, ob und ggf. in welchem Umfang ein bestimmtes Bedürfnis befriedigt und folglich ein Nutzen gestiftet wurde. Quellen der Nutzenstiftung liegen grundsätzlich in allen unmittelbar und mittelbar mit der Flugdienstleistung in Zusammenhang stehenden Bedürfnissen. Denkbar sind beispielsweise das Bedürfnis nach persönlichem Kontakt zu einem weit entfernt lebenden Verwandten oder das Bedürfnis, im Zuge komplizierter Vertragsverhandlungen persönlich mit einem Geschäftspartner zusammenzuarbeiten, der an einem anderen Ort lebt. Das Bedürfnis "Mangel an persönlichem Kontakt" wird daraufhin in das Motiv „Wunsch, in persönlichen Kontakt zu treten“ übersetzt und bildet einen Erklärungsansatz für den Antritt eines Fluges. ${ }^{83}$ Im Verlauf der Flugdienstleistung

80 Dieser Fall tritt beispielsweise auf dem Flug Nr. 6300 der Deutschen Lufthansa AG von Frankfurt nach Bangkok ein. Der Flug ist im Flugplan der Lufthansa enthalten und wird auf einem Flugschein der Lufthansa ausgestellt, der Fluggast checkt im Frankfurter Flughafen am Schalter der Lufthansa ein, wird dann jedoch von Lufthansas thailändischem Partner Thai Airways von 14.10 Uhr bis 07.00 Uhr in einem Flugzeug der Thai Airways (Boeing 747-400) befördert und kann am Ankunftsort den Warteraum der Thai Airways nutzen, sofern er Business oder First Class gebucht hatte (Beispielflug entnommen aus dem Flugplan der Deutschen Lufthansa AG, gültig vom 26.10.97-28.03.1998, S. 96).

$81 \mathrm{Zu}$ kritisieren ist an dieser Stelle die objektgerichtete Formulierung, in welcher der Nachfrager eher einseitig als Empfänger denn wechselseitig als Interaktionspartner aufgefaßt wird.

82 Mit dem Begriff des Nutzens wird in der ökonomischen Theorie das nach subjektiven Maßstäben bewertbare und damit intersubjektiv kaum überprüfbare $M a ß$ an Bedürfnisbefriedigung verstanden. Eine anspruchsvolle Aufgabe besteht in der meßtechnischen Erfassung des Nutzens, so daß in der Literatur verschiedene Konstrukte und Methoden zu diesem Zweck Anwendung finden. Zu einer ausführlichen Diskussion und Abgrenzung des Nutzenbegriffs sowie einem empirischen Meßansatz im Verkehrsdienstleistungsbereich vgl. Perrey, J., Nutzenorientierte Marktsegmentierung: ein integrativer Ansatz im Verkehrsdienstleistungsbereich, Wiesbaden 1998, S. $12 \mathrm{ff}$.

83 In der Literatur werden die zur Deutung menschlichen Verhaltens herangezogenen Begriffe „Bedürfnis“ und „Motiv“ z.T. synonym verwendet. Vgl. z.B. Meffert, H., Marketingforschung und Käuferverhalten, a.a.O., S. 52. Diese Auffassung unterstellt ein quasi gleichzeitiges Auftreten des Mangelgefühls mit der Suche nach Mitteln zur Beseitigung desselben, so daß die ggf. vorhandene Bereitschaft zum Erdulden eines empfundenen Mangels unterschlagen wird. Es erscheint demnach sinnvoller, Motive rangmäßig als den Bedürfnissen nachgelagert zu 
können aufgrund des oben erörterten Prozeßcharakters weitere Bedürfnisse wie „Mangel an Information oder Unterhaltung“ sowie physiologische Bedürfnisse wie Durst oder Hunger auftreten und daher Ansatzpunkte zur Nutzenstiftung durch die Dienstleistungsanbieter aufzeigen. ${ }^{84}$

Dem Nutzen sind indes die kundenseitigen Kosten einer Dienstleistung gegenüberzustellen, die einerseits als zu entrichtendes Entgelt (Preis) das "Opfer" des Nachfragers für die Inanspruchnahme der Dienstleistung widerspiegeln. ${ }^{85}$ Andererseits entstehen dem Fluggast auch während einer Flugdienstleistung Kosten, wenn beispielsweise Wartezeiten, Verspätungen oder Gepäckschäden in Kauf zu nehmen sind. ${ }^{86}$ Das Auftreten derartiger prozeßbezogener Kosten, deren Bewertung strenggenommen aus Sicht des einzelnen Fluggastes zu erfolgen hat, mindert den Nutzen der Flugdienstleistung. Die eingangs erwähnten Koordinationsund Informationsmängel, die sich zwischen Allianzpartnern bei der gemeinsamen Erbringung von Code Share-Flugdienstleistungen ergeben können, sind daher nachfragerseitig als nutzenmindernde Faktoren in die Überlegungen zum Nutzen strategischer Luftverkehrsallianzen einzubeziehen.

Der Grund für die besondere Berücksichtigung der Nutzenkomponenten einer Flugdienstleistung liegt in der Kaufverhaltensrelevanz, die der mit einem Dienstleistungsangebot zusammenhängenden Nutzenerwartung zugesprochen wird. Diese entsteht in der Psyche des Nachfragers als Ergebnis eines situationsbezo-

interpretieren. Bedürfnisse zeigen demnach ein generelles Mangelgefühl an und versetzen Menschen in allgemeine Handlungsbereitschaft, während Motive eine inhaltliche Klassifikation von angestrebten Zielzuständen darstellen, die sich personenspezifisch im Laufe der Sozialisation herausgebildet haben. Vgl. Staehle, W.H., Management: eine verhaltenswissenschaftliche Perspektive, a.a.O., S. 152.

84 Informationsstände in Flughäfen, Zeitschriftenangebote am Flugsteig oder an Bord von Flugzeugen sowie Getränke- und Speisenangebote im Flughafen oder an Bord von Flugzeugen sind Beispiele für die Reaktion der Dienstleistungsanbieter auf diese Bedürfnisse. Ein Teil dieser Angebote wurde bewußt „proaktiv“ geschaffen, um sich gegenüber Wettbewerbern mit einem breiten Leistungsspektrum abzugrenzen.

Vgl. Simon, H., Preismanagement: Analyse-Strategie-Umsetzung, 2. Aufl., Wiesbaden 1992, S. 4.

86 Jäckel (1991) berichtet, daß europäische Fluggesellschaften im Jahre 1988 insgesamt rund 68.500 Flugstunden durch Luftraum- und Flughafenüberlastung verloren haben. Für die Passagiere ergab sich infolge der resultierenden Verspätungen und Wartezeiten ein Verlust von 4,7 Mio. Arbeits- und Freizeitstunden, deren monetăres Äquivalent auf rund $5 \mathrm{Mrd}$. DM geschätzt wird. Vgl. Jäckel, K., Kooperationsstrategien im Linienluftverkehr vor dem Hintergrund zunehmender Integrationsentwicklung in Europa, Bergisch Gladbach, Köln 1991, S. 174. 
genen, merkmalsorientierten Beurteilungsprozesses und bildet die Basis für die Festlegung einer individuellen Präferenzrangfolge zwischen alternativen Flugdienstleistungsangeboten. ${ }^{87}$ Dementsprechend sind Fluggesellschaften, die im Rahmen einer horizontalen strategischen Allianz kooperieren, bestrebt, das Augenmerk auf die nutzenorienterte Gestaltung ihres gemeinschaftlich erbrachten Angebots zu richten. Diese Nutzenorientierung gilt jedoch auch für die einzeln, d.h. ohne Allianzpartner erbrachten Flugdienstleistungen im "regulären Geschäft" der Fluggesellschaft. Zentrale Herausforderungen an die strategische Allianz erwachsen daher aus der Identifikation, Umsetzung und Kommunizierung allianzspezifischer Nutzendimensionen, die den Nachfragern die möglichst höchste Nutzenerwartung beim Vergleich mit konkurrierenden Angeboten versprechen. ${ }^{88}$ Gleichzeitig besteht eine wichtige Herausforderung in der Vermeidung negativer Auswirkungen von Koordinations- und/oder Informationsmängeln auf die nachfragerseitige Wahrnehmung und/oder das Erleben von Flugdienstleistungen. ${ }^{89}$

Nutzenerwartungen sind der eigentlichen Inanspruchnahme der Flugdienstleistung als Ergebnis der Kaufentscheidung durch den Nachfrager zeitlich vorgelagert und setzen keinen Kontakt mit den Dienstleistungsanbietern voraus. Erfahrungen mit einer Flugdienstleistung, die dann bei der nächsten Kaufentscheidung in die Nutzenerwartung einfließen können, erwirbt der Nachfrager erst durch die

87 Dem hier nur ansatzweise wiedergegebenen Kaufverhaltensprozeß liegen neobehavioristische Grundannahmen des menschlichen Verhaltens zugrunde. Zu einer detaillierteren Aufarbeitung vgl. Perrey, J., Nutzenorientierte Marktsegmentierung: ein integrativer Ansatz im Verkehrsdienstleistungsbereich, a.a.O., S. 15ff.

88 Nachfragerseitige Nutzenerwartungen stellen aus diesem Grund ein wichtiges Kriterium zur Segmentierung des relevanten Marktes dar. Die US-amerikanische Fluggesellschaft United Airlines beispielsweise segmentiert ihre Passagiere zunächst nach dem Reiseanlaß in Geschäftsreisende (40\%) und Privatreisende $(60 \%)$. Innerhalb dieser "Makrosegmente“ werden die Passagiere nach ihren Nutzenerwartungen in fünf bzw. drei „Mikrosegmente“ aufgeteilt. Unter den geschäftich bedingt Reisenden machen die service- und komfortorientierten, relativ preisunsensiblen "road warriors" zwar lediglich $6 \%$ aller Kunden aus, stehen jedoch für $37 \%$ des Gesamtumsatzes des Unternehmens. Vor allem auf diese Kernzielgruppe der Vielflieger konzentriert United Airlines ihre Marketingaktivităten. Als zentrale Nutzenerwartungen der Vielflieger wurden Sicherheit, Zuverlässigkeit und ein angemessenes Preis-/Leistungs-Verhältnis identifiziert. Weitere Nutzenerwartungen bestehen in einem unbegrenzten, weltweiten Streckennetzzugang, störungsfreien Reiseabläufen und in dem Wunsch nach Aufmerksamkeit für individuelle Bedürfnisse. Vgl. Flint, P., Will the real United please stand up? In: Air Transport World, No. 8, 1997, S. 31 f.

89 Negative Auswirkungen könnten beispielsweise aus einer mangelhaften Koordination von Reservierungsvorgängen, Gepäckmitnahmevorschriften oder Verständigungsproblemen mit dem Servicepersonal zwischen den Allianzpartnern resultieren. 
Inanspruchnahme der Dienstleistung. ${ }^{90}$ Dementsprechend groß sind die Anstrengungen der Fluggesellschaften, die Flugdienstleistung so auszugestalten, daß die (Wieder-)Kaufwahrscheinlichkeit steigt. Einen zentralen Stellenwert hat in diesem Zusammenhang das Konstrukt der Zufriedenheit erlangt. ${ }^{91}$ Verstanden als „Ergebnis eines psychischen Ist-Vergleichs über Konsumerlebnisse“, ${ }^{92}$ gibt die Zufriedenheit eines Fluggastes folglich Auskunft darüber, inwieweit die mit der Flugdienstleistung verbundenen subjektiven Erwartungen (Soll-Komponente) in der Situation des tatsächlichen Erlebens bzw. nach erfolgter Inanspruchnahme der Flugdienstleistung (Ist-Komponente) erfüllt wurden. ${ }^{93}$

In der Praxis des Personenluftverkehrs wenden einige Fluggesellschaften Meßinstrumente an, die in unterschiedlicher Form Auskunft über die Zufriedenheit der Fluggäste geben. Oft unter der Bezeichnung "Qualitätsmanagement" subsumiert, ${ }^{94}$ werden z.T. regelmäßige Kundenbefragungen, technische Kennziffern

90 Die vom Nachfrager vor Inanspruchnahme der Dienstleistung gesammelten Informationen über dieselbe betreffen gemäß dem informationsökonomischen Ansatz die Sucheigenschaften der Dienstleistung. Die Informationssuche dient der Senkung von Unsicherheit, die subjektiv und situationsabhängig besteht. Weiterhin existieren Erfahrungseigenschaften, die erst nach der Nutzung der Dienstleistung beurteilt werden können. Vertrauenseigenschaften - bei einer Flugdienstleistung z.B. die technische Sicherheit des Fluggeräts - lassen sich weder vor noch nach Inanspruchnahme der Dienstleistung oder nur zu prohibitiv hohen Kosten prüfen. Vgl. z.B. Meffert, H., Marketing: Grundlagen marktorientierter Unternehmensführung, a.a.O., S. 23-25; Kaas, K.-P., Marketing als Bewältigung von Informations- und Unsicherheitsproblemen im Markt, in: Die Betriebswirtschaft, 50. Jg., 1990, S. 539-548.

91 Eine ausführliche Zusammenstellung zentraler Entwicklungen in der Kundenzufriedenheitsforschung sowie eine Begriffsdiskussion finden sich bei Siefke, A., Zufriedenheit mit Dienstleistungen, a.a.O., S. 21-29.

Kaas, K.-P., Runow, H., Wie befriedigend sind die Ergebnisse der Forschung zur Verbraucherzufriedenheit? In: DBW, 44. Jg., Nr. 3, 1984, S. 452.

Die größte Verbreitung unter den Meßmodellen der Kundenzufriedenheit hat das sogenannte Confirmation/Disconfirmation-Paradigm gefunden, gemaßß dem sich vor der Inanspruchnahme der Dienstleistung ein Vergleichsstandard bildet, der im Zuge des Erlebens der Dienstleistung bestätigt bzw. nicht bestätigt wird. Vgl. Churchill, G.A., Suprenaut, C., An Investigation into the Determinants of Customer Satisfaction, in: Journal of Marketing Research, Vol. 19, No. 11, 1982, S. 491-504. Unbestreitbar besteht hier eine gewisse Năhe der Soll-Komponente der Zufriedenheit (Dienstleistungserwartung) zu der oben erörterten Nutzenerwartung.

Die Abgrenzung der Begriffe "Dienstleistungsqualität" und „Zufriedenheit" wird in Literatur und Praxis nicht einheitlich gehandhabt. Während Hentschel (1992) zwischen der Informationsseite (=Zufriedenheit) und Aktionsseite (=Qualitătsgestaltung) unterscheidet und damit einen pragmatischen Weg beschreitet, schlägt Siefke (1998) eine integrierte Sichtweise vor. Demgemăß stellt die wahrgenommene Dienstleistungsqualităt eine zentrale Einflußgröße der Kundenzufriedenheit dar, die ihrerseits als zeitlich weniger stabiles, transaktionsbezogenes Konstrukt Einfluß auf künftige Qualitätsbeurteilungen nimmt. Vgl. Hentschel, B., Dienstleistungsqualităt aus Kundensicht: vom merkmals- zum ereignisorientierten Ansatz, Wiesbaden 1992, S. 120 sowie Siefke, A., Zufriedenheit mit Dienstleistungen, a.a.O., S. 63. 
und Kundenbeschwerden ausgewertet und in Berichtsbänden verdichtet. ${ }^{95}$ Auch Bewertungen der Wettbewerber finden häufig als Vergleichsgröße Eingang in derartige Untersuchungen. Diese Daten sind im Fall von strategischen Allianzen mit anderen Fluggesellschaften in zweierlei Hinsicht von Relevanz. Zum einen bieten sie ein marktorientiertes Entscheidungskriterium, anhand dessen sich mögliche Partner a priori auf ihre Eignung für eine Kooperation prüfen lassen. Zum anderen können durch eine kontinuierliche Überprüfung der Daten im Verlauf der Partnerschaft eventuelle Qualitäts- bzw. Zufriedenheitslücken aufgedeckt werden. ${ }^{96}$

Es liegt insgesamt nahe, die Komplexität der Flugdienstleistung sowie situativ und subjektiv unterschiedliche, mit einer Flugdienstleistung verbundene Nutzenerwartungen und eine bzw. ein ebenso situativ und subjektiv unterschiedliche(s) Wahrnehmung bzw. Erleben derselben als nachfragerseitige Bestimmungsfaktoren der Gestaltung strategischer Allianzen aufzufassen. ${ }^{97}$ Zugleich rücken Arbeiten, die in Tradition der inside-out-Forschung stehen, unternehmens- und wettbewerbsbezogene sowie politisch-rechtliche Faktoren in den Mittelpunkt und sprechen innen eine zentrale Bedeutung für die Ausgestaltung strategischer Luftverkehrsallianzen zu. ${ }^{98}$ In diesem Spannungsfeld bewegen sich daher die im folgenden zu konkretisierenden Problemstellungen der vorliegenden Arbeit.

95 Vgl. z.B. Klein, H., Qualitătsmanagement der Deutschen Lufthansa AG, in: Dienstleistungsqualităt: Konzepte-Methoden-Erfahrungen, Hrsg.: Bruhn, M., Stauss, B., 2. Auflage, Wiesbaden 1995, insb. S. 488f. Zufriedenheitsrelevante Merkmale wie Pünktlichkeit und Gepäckbehandlung lassen sich z.B. als "Anteil Flüge, die nicht später als 15 Minuten nach Plan gestartet oder gelandet sind“ und "Anzahl Gepäckschäden/-fehlleitung pro 1.000 Gepäckstücke“ als Kennzahl erfassen. In den USA wird alljährlich die „triple crown“ an diejenige Fluggesellschaft verliehen, die beide obengenannten Kriterien am besten erfült und zugleich die geringste Anzahl Beschwerden pro 1.000 Fluggäste aufweist.

Ein Manager einer Regionalfluggesellschaft gab an, daß der wesentlich großere Allianzpartner - eine global operierende Fluggesellschaft - zur Überprüfung der Zufriedenheitswerte des kleineren Partners anfänglich den Einsatz eines Kundenzufriedenheitsindex verlangt hatte. Die Überprüfung wurde von Juni bis Dezember 1996 mit dem Meßinstrument des großen Partners auf dessen Kosten durchgeführt. Aufgrund guter bis sehr guter Ergebniswerte wurde dann auf eine Fortführung dieses Monitoring seitens der großen Fluggesellschaft verzichtet. Fünf Monate später setzte die von der Wirkung eines solchen Meßinstruments überzeugte Regionalfluggesellschaft die Messung auf eigene Kosten fort. Vgl. Anhang 4.

97 Der Begriff „Bestimmungsfaktor" suggeriert eine einseitige Beeinflussungsrichtung und birgt daher die Gefahr, den gestalterischen Einfluß der strategischen Allianz auf die Komplexität sowie auf die Nutzenerwartungen und die Zufriedenheit der Nachfrager zu vernachlässigen. Die Verwendung dieses Begriffes geht daher im folgenden stets von einer immanenten Wechselseitigkeit aus, um den Vorwurf eines zu starren Determinismus abzuschwăchen.

98 Jäckel etwa betont den zentralen Stellenwert unternehmensspezifischer „Ausstattungen und Fähigkeiten“ für die Effizienz von Kooperationen zwischen Fluggesellschaften. Vgl. Jäckel, K., 


\section{Problemstellung und Gang der Untersuchung}

Die vorliegende Arbeit verfolgt die Hauptzielsetzung, Ansatzpunkte zur Erfassung der Wahrnehmung, des Erlebens und der Beurteilung strategischer Allianzen durch Nachfrager aufzuzeigen und auf Basis dieser Erkenntnisse Empfehlungen für die Ausgestaltung der Marketinginstrumente strategischer Allianzen abzuleiten. Die Ausführungen bewegen sich am Kontext des Personenluftverkehrs, in dem strategische Luftverkehrsallianzen durch die Interaktion mit Nachfragern in besonderem Maße mit dieser Problemstellung konfrontiert sind. Um der Hauptzielsetzung gerecht zu werden, ist die Formulierung und Bearbeitung aufeinander aufbauender Teilziele notwendig.

1. In einer ersten Teilzielsetzung soll aus organisationstheoretischem Blickwinkel ergründet werden, warum sich strategische Allianzen im Personenluftverkehr bilden und welche Entscheidungstatbestände von zentraler Bedeutung für ihre Ausgestaltung sind. Durch den Aufbau von Beziehungen untereinander und zu ihrer Umwelt schaffen Fluggesellschaften als Allianzpartner die Grundlage für die gemeinsame Erstellung von Flugdienstleistungen (inside-out-Perspektive), die damit potentiell für die Nachfrager wahrnehmbar und ggf. erlebbar werden. Im Fall der tatsächlichen Wahrnehmung bzw. des tatsächlichen Erlebens unter-

Kooperationsstrategien im Linienluftverkehr vor dem Hintergrund zunehmender Integrationsentwicklung in Europa, a.a.O., S. 95.

Schmidt hebt die Bedeutung der Wettbewerbsstruktur im Luftverkehr hervor und sieht die Entstehung strategischer Allianzen in industrieökonomischer Tradition als verhaltensbezogene Reaktion auf die oligopolistische Marktstruktur. Vgl. Schmidt, S., Strategische Allianzen im Luftverkehr - Erfolgsorientiertes Management europäischer Flug-Carrier, Trier 1993, insb. S. 36-38.

Der Einfluß politisch-rechtlicher Faktoren auf die Form strategischer Luftverkehrsallianzen wird deutlich, wenn beispielsweise das gemeinschaftliche Angebot von Flügen (Code Sharing) zwischen Partner-Fluggesellschaften der EU nach Art. 851 EWGV im Einzelfall für kartellrechtlich problematisch erachtet und untersagt wird. Vgl. Reckewerth, S., Die Zusammenarbeit der Luftverkehrsgesellschaften nach europäischem und US-amerikanischen Recht, Köln u.a. 1993, S. 288. Diese Rechtsnorm findet nach dem sog. Auswirkungsprinzip auch auf Allianzen zwischen EU- und Nicht-EU-Fluggesellschaften Anwendung (vgl. ebenda, S. 388). Die Auswirkungen zeigen sich u.a. am Beispiel der strategischen Allianz zwischen British Airways und American Airlines, die erst nach ausführlichen Prüfungen und mit Auflagen durch die EU-Kommission genehmigt wurde. Vgl. o.V., Streit um die Allianz von British Airways und American Airlines, in: FAZ vom 16.1.1997, S. 17 sowie o.V., Vehemente Kritik an Auflagen der EU, in: Handelsblatt vom 9.7.1998, S. 14. Zum Einfluß nationalstaatlicher Politik auf die Allianzgestaltung vgl. Jegminat, G., Strategische Allianzen nur die zweitbeste Lősung, in: fvw, Nr. 25, 1997, S. 78. 
liegen diese gemeinsam erstellten Flugdienstleistungen der Beurteilung von Nachfragern.

2. Aus einer outside-in-orientierten Sichtweise gilt es daher in einer weiteren Teilzielsetzung der Arbeit, Ansatzpunkte zur Erfassung der Wahrnehmung, des Erlebens und der Beurteilung strategischer Luftverkehrsallianzen durch die Nachfrager zu entwickeln. Entlang der Reisekette ist zu prüfen, ob und ggf. welche Merkmale von Flugdienstleistungen sich als "allianztypisch" erweisen, d.h. ob und ggf. welche positiven und/oder negativen Abweichungen zu Flugdienstleistungen bestehen, die von einer Fluggesellschaft allein erbracht werden. Gleichzeitig ist zu überprüfen, welche Kaufverhaltensrelevanz die betreffenden Merkmale für den Nachfrager besitzen. Gestützt auf verhaltenswissenschaftliche Erkenntnisse, soll die Diskussion von Ergebnissen einer internationalen Fluggastbefragung zum Thema strategische Allianzen in die Ausführungen einfließen.

3. Aufbauend auf der Untersuchung der nachfragerseitigen Wahrnehmung, des Erlebens und der Beurteilung strategischer Luftverkehrsallianzen verfolgt die vorliegende Arbeit das Teilziel, am Beispiel einer bestimmten Luftverkehrsallianz Empfehlungen für die Gestaltung des Marketing-Instrumentariums der beteiligten Fluggesellschaften abzuleiten und damit exemplarisch einen Weg zur Erhöhung der Kundenorientierung aufzuzeigen. Dabei werden die Marketinginstrumente von den Allianzpartnern auf Absatzmittler und Endnutzer (Fluggäste) ausgerichtet. Im Fokus der Betrachtung stehen die Kontaktpunkte im Verlauf der Reisekette, an denen die Interaktion zwischen den Partnerfluggesellschaften in eine Interaktion zwischen strategischer Allianz und Nachfrager mündet. Neben den Ergebnissen einer internationalen Fluggastbefragung finden die Inhalte mehrerer Expertengespräche eines Allianznetzwerks Eingang in die Überlegungen.

Der Gang der Arbeit ergibt sich aus der Bearbeitung der Teilzielsetzungen und wird durch Abbildung 3 verdeutlicht, welche die Schwerpunkte der Untersuchung wiedergibt. In Kapitel B 1 wird zunächst literaturgestützt-konzeptionell ein Erklärungsansatz für die Entstehung strategischer Allianzen im Luftverkehr entwickelt, um den Untersuchungsgegenstand in seinem spezifischen Kontext zu konkretisieren und eine Grundlage für die sich anschließenden Überlegungen zu schaffen. Aufbauend auf der Erörterung der zentralen Zielsetzungen, die Fluggesellschaften mit dem Eingehen strategischer Allianzen verfolgen, wird die marketingstrategische Ausrichtung von Luftverkehrsallianzen diskutiert. 


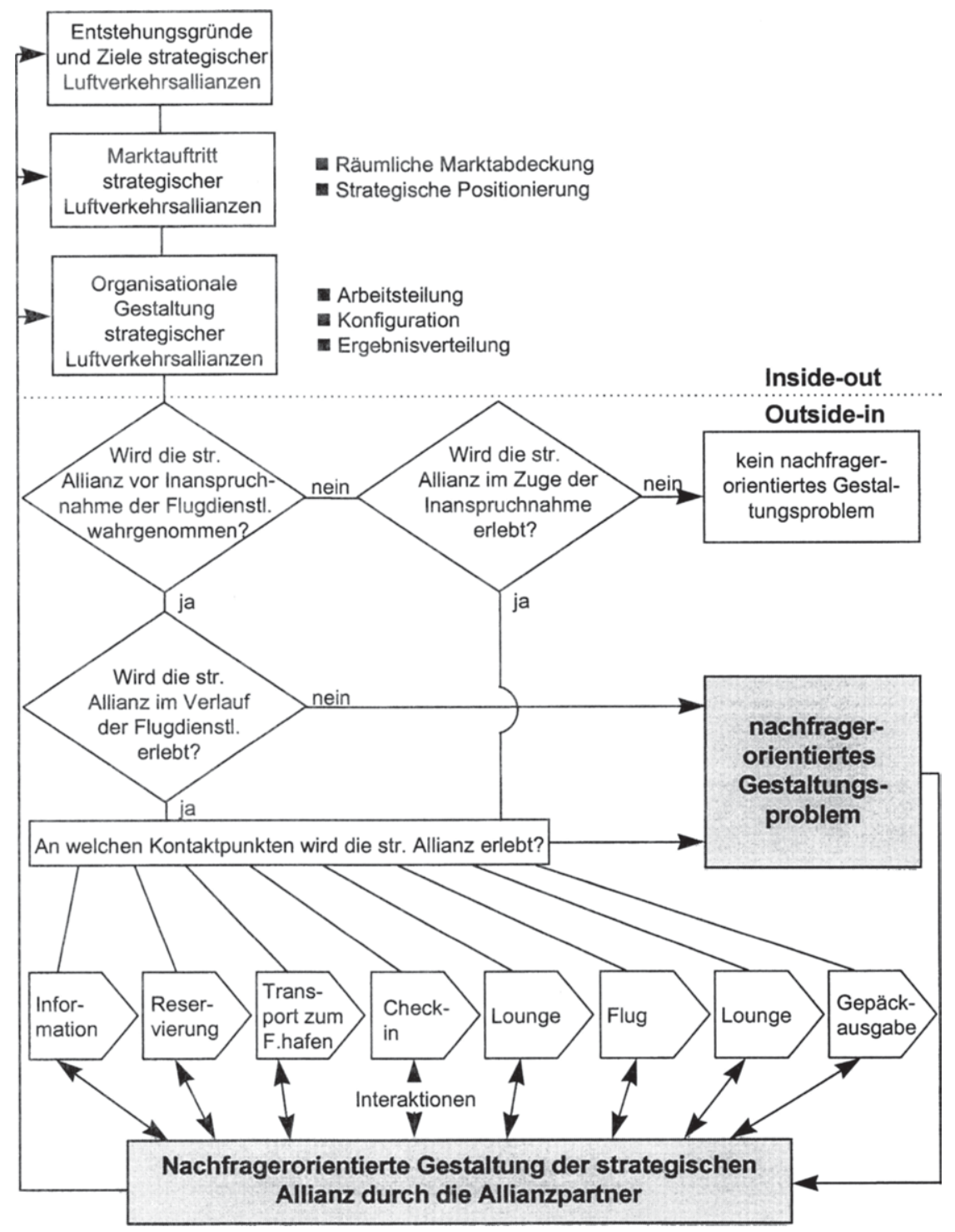

\section{Abb. 3: Schwerpunkte der Untersuchung}


Ausführungen zur organisationalen Umsetzung über die Festlegung der Arbeitsteilung, der Konfiguration und der Ergebnisverteilung schließen sich an. Ergebnisse der Expertengespräche unterstützen die Darlegungen.

Kapitel B 2. geht zunächst der Frage nach, welche Nutzenerwartungen die Nachfrager an Flugdienstleistungen haben. Da im Zuge der Reisekette häufig auch Reisebüros und Firmenkunden in die Interaktion zwischen Fluggesellschaft und Endnutzer (Fluggast) einbezogen sind, schließen die Ausführungen Überlegungen zu den Nutzenerwartungen dieser Marktpartner ein. Bei der sich anschließenden Untersuchung der Endnutzerperspektive kann auf empirische Studien zur nationalen und internationalen Nutzensegmentierung von Flugdienstleistungen zurückgegriffen werden, die in der Literatur dokumentiert sind.

Zudem wird im speziellen untersucht, ob Fluggäste entlang der Reisekette allianzspezifische Leistungsmerkmale wahrnehmen und/oder erleben und wie sie diese ggf. beurteilen. Zur Beantwortung dieser Fragestellungen finden die Ergebnisse einer 1996 von einer strategischen Luftverkehrsallianz in Auftrag gegebenen Marktforschungsstudie Eingang in die wissenschaftliche Auseinandersetzung. In unterschiedlichen Ländern wurden Focusgruppeninterviews mit Fluggästen durchgeführt, die über ihre Erfahrungen mit strategischen Luftverkehrsallianzen befragt wurden. Auf Basis dieser Erkenntnisse wird darüber hinaus geprüft, auf welche Weise sich strategische Luftverkehrsallianzen gegenüber einzeln agierenden Fluggesellschaften und konkurrierenden Allianzen positionieren können.

Im sich anschließenden Kapitel B 3 gilt es, die zuvor herausgearbeiteten Ergebnisse als Grundlage für die absatzmarktorientierte Ausrichtung des MarketingInstrumentariums am Beispiel einer strategischen Luftverkehrsallianz zu nutzen. Daraus ergibt sich die Notwendigkeit einer kritischen Auseinandersetzung mit strategischen und operativen Marketingaktivitäten bestehender Luftverkehrsallianzen im Hinblick auf ihre Positionierung im Wettbewerb. Zu diesem Zweck flieBen die Ergebnisse der Expertengespräche und Focusgruppeninterviews in die Ausführungen ein.

Kapitel C setzt sich nach einer Zusammenfassung der zentralen Ergebnisse abschließend mit Controllingaspekten strategischer Luftverkehrsallianzen auseinander und beendet die Arbeit mit einem zusammenfassenden Ausblick auf weitergehenden Forschungsbedarf. 
Bei der theoretischen Grundlegung der vorliegenden Arbeit ist die Grundsatzentscheidung zu fällen, die Teilzielsetzungen entweder unter Bezugnahme auf eine möglichst umfassende Theorie im Sinne eines Theoriemonismus zu bearbeiten oder parallel mehrere Theorien („Theoriepluralismus“) zu diesem Zweck heranzuziehen. ${ }^{99}$ Angesichts der Tatsache, daß die Problemstellung der nachfragerorientierten Gestaltung strategischer Allianzen eine Betrachtung sowohl der Makro- als auch der Mikroebene verlangt, ${ }^{100}$ erscheint an dieser Stelle die Anwendung mehrerer Theorien statthaft. ${ }^{101}$ Unabdingbare Voraussetzung hierfür

99 Vgl. Kieser, A., Anleitung zum kritischen Umgang mit Organisationstheorien, in: Organisationstheorien, Hrsg.: Kieser, A., 2., überarb. Aufl., Stuttgart, Berlin, Köln 1995, S. If. Für die Beschreitung des auf nur einer Theorie aufbauenden Weges spricht die Tatsache, daß sich im Zuge der historischen Entwicklung in den Wirtschaftswissenschaften eine Vielzahl unterschiedlicher Theorieansätze herangebildet hat, die aufgrund ihres z.T. außerst heterogenen forscherischen Kontextes nicht miteinander vergleichbar sind (Inkommensurabilität). Die Anwendung nur einer Theorie auf das hier betrachtete Erkenntnisobjekt "strategische Allianz" mit den Teilproblemen der organisationalen und absatzmarktgerichteten Gestaltung wäre daher mit Ergebnissen verbunden, die auf konsistenten Grundannahmen und einer ebensolchen Disziplintradition aufbauen. Ein derartiges Vorgehen sähe sich jedoch zugleich dem Vorwurf einer nicht ausreichend differenzierten Untersuchung der Problemstellung ausgesetzt. Dieser Kritikpunkt kann mit den unterschiedlichen Forschungsperspektiven untermauert werden, die den Theorieansätzen zugrunde liegen und jeweils nur ausgewählte Teilaspekte eines Erkenntnisobjekts zu erfassen vermögen. Backhaus (1992) konstatiert beispielsweise, daß die Erkenntnisse der klassischen mikroökonomischen Theorie für die Erklärung realer Marktphänomene wenig brauchbar sind und leitet aus dieser Erkenntnis die Zuwendung des Marketing zu verhaltenswissenschaftlichen Ansätzen ab. Vgl. Backhaus, K., Investitionsgüter-Marketing Theorieloses Konzept mit Allgemeinheitsanspruch? in: zfbf, 44. Jg., Nr. 9, 1992, S. 777. Zugleich wirft er die Suggestivfrage auf, ob nicht die "Pluralismusforderung der Forschungskonzeptionen doch fruchtbarer [ist, Anm. d. Verf.] als mancher denkt? “. Ebenda, S. 788.

Die Makroebene ist zur Untersuchung der gestaltungsbezogenen inside-out-Fragestellung, die Mikroebene zur Analyse der outside-in-gerichteten Problemfelder strategischer Luftverkehrsallianzen einzunehmen. Vgl. zur Unterscheidung von forscherischen Aggregationsebenen Pfeffer, J., Organizations and Organization Theory, Boston u.a. 1982, S. 13.

101 Als Nachteil dieser Vorgehensweise ist das Fehlen einer Meta-Theorie mit präzisierender, einengender Funktion anzumerken. Da eine solche Meta-Theorie bisher nicht entwickelt wurde, wird dem auf Vielfalt beruhenden Erkenntnisgewinn durch die Anwendung mehrerer Theorien der Vorzug gegeben. Vgl. auch Kieser, A., Anleitung zum kritischen Umgang mit Organisationstheorien, a.a.O., S. 3.

Steinmann und Schreyögg (1997) legitimieren für die Managementlehre die disziplinenübergreifende Heranziehung von wissenschaftlichen Erkenntnissen durch das Prinzip der Problemorientierung. Nach ihrer Argumentation treten in der betrieblichen Praxis Probleme auf, die keine Rücksicht auf Disziplinen nehmen („a-disziplinär") und daher die Generierung eines interdisziplinären Wissens oder die Heranziehung bereits bestehenden Grundlagenwissens aus verschiedenen Theorien rechtfertigen. Vgl. Steinmann, H., Schreyögg, 
ist gleichwohl die Beachtung der den Theorien zugrunde liegenden Prämissen und der eingesetzten Methoden. ${ }^{102}$

G., Management: Grundlagen der Unternehmensführung; Konzepte - Funktionen - Fallstudien, 4., überarb. und erw. Aufl., Wiesbaden 1997, S. 37.

102 Vgl. Schanz, G., Methodologie für Betriebswirte, 2., überarb. u. erw. Aufl., Stuttgart 1988, S. 82. 


\section{B. Absatzmarktgerichtete Gestaltung strategischer Luftverkehrsallianzen \\ 1.}

\section{esign strategischer Luftverkehrsallianzen}

\subsection{Theoretische Grundlagen strategischer Allianzen und Rahmenbedin- gungen des internationalen Luftverkehrs}

\subsubsection{Ansätze zur Erklärung und Gestaltung strategischer Allianzen}

Die zu untersuchenden nachfragerorientierten Problemfelder der Gestaltung strategischer Luftverkehrsallianzen können einerseits aus einer ressourcenorientierten Perspektive bearbeitet werden, in deren Mittelpunkt die Abstimmungsprozesse und der "Fit" zwischen den beteiligten Allianzpartnern stehen. Theorien, die im Gefolge dieser Perspektive Anwendung finden, sollten in der Lage sein, anbieterseitige Ursachen der nachfragerrelevanten Probleme zu identifizieren und Ansätze zu ihrer Lösung bereit zu halten. Angesichts der zentralen Fragestellung der Nachfragerorientierung liegt es zum anderen nahe, die in Beziehungen zwischen strategischen Luftverkehrsallianzen und Nachfragern auftretenden Problemfelder unter Einnahme der Nachfragerperspektive zu untersuchen und Theorien zur Erklärung des Nachfragerverhaltens heranzuziehen, aus denen ein Erkenntnisgewinn für die nachfragerorientierte Allianzgestaltung abgeleitet werden kann.

Die in der ersten Teilzielsetzung der Arbeit enthaltene Untersuchung der Entstehung und organisationalen Gestaltung strategischer Luftverkehrsallianzen, die für das Verständnis der gemeinsamen Leistungserbringung und damit bereits für mögliche nachfragerrelevante Schnittstellenprobleme bedeutsam ist, betrifft die Beziehungen zwischen den Organisationen der Allianzpartner. Angesichts dieser Aggregationsebene engt sich das Suchfeld in Frage kommender Theorien auf sogenannte Makro-Theorien der Organisationsforschung ein. ${ }^{103}$ Ökonomische Makro-Theorien, zu denen beispielsweise die Transaktionskostenökonomik sowie die Koalitionstheorie zu rechnen sind, versuchen, die Entstehung und Veränderung von Organisationen aus dem Verhalten nutzenmaximierender Individuen

103 In der US-amerikanischen Forschung hat sich auf dieser Ebene analog der Forschungszweig des Macro Organizational Behaviour, auch als Organization Theory bezeichnet, herausgebildet. Inhalte betreffen die Struktur und das Verhalten sozialer Systeme, inre Beziehungen zur Umwelt sowie Prozesse von Macht und Konflikt. Vgl. Staehle, W.H., Management: eine verhaltenswissenschaftliche Perspektive, a.a.O., S. $137 f$. 
abzuleiten und bedienen sich damit eines methodologischen Individualismus. ${ }^{104}$ Verhaltenswissenschaftliche Theorieansätze wie beispielsweise die soziologische Systemtheorie oder der Netzwerkansatz stellen dagegen die sozialen Interaktionen zwischen den Mitgliedern von Organisationen in den Mittelpunkt ihrer Überlegungen. ${ }^{105}$

Übertragen auf den hier zugrunde liegenden Untersuchungskontext, vermag die Koalitionstheorie Aufschlüsse über die Entstehung und auch Gestaltung strategischer Allianzen zu geben. Während die Transaktionskostentheorie durch ihre Austauschorientierung auf die Erklärung vertikaler Vertragsprobleme gerichtet ist, ${ }^{106}$ interpretiert die Koalitionstheorie den Transaktionsbegriff im Sinne von Interaktionen zwischen Wirtschaftssubjekten und schließt auf diese Weise grundsätzlich auch strategische Allianzen als potentielle Erkenntnisobjekte in die Betrachtung ein. ${ }^{107}$ In ihrem Kern besagt sie, daß Unternehmen immer dann eine Koalition - hier: strategische Allianz - eingehen, wenn die in die Partnerschaft eingebrachten Ressourcen einen höheren Ertrag erwirtschaften als ohne eine solche Koalition. Der Unterschiedbetrag zwischen dem Ressourcenertrag im Koalitionsfall und demjenigen im Nicht-Koalitionsfall wird als koalitionsspezifische Quasirente bezeichnet. $^{108}$

Die organisationale Gestaltung einer Koalition oder strategischen Allianz hat sich gemäß den Aussagen dieser Theorie an einer Maximierung der Koalitionsrente auszurichten, die im Sinne einer Zielfunktion koordinierenden Einfluß auf die gemeinsamen Aktivitäten nimmt. Zugleich bedarf es institutioneller Regelungen zur Verteilung der Quasirente, von der die innere Stabilität der Allianz in hohem

$104 \mathrm{Vgl}$. Ebers, M., Gotsch, W., Institutionenökonomische Theorien der Organisation, in: Organisationstheorien, Hrsg.: Kieser, A., 2., überarb. Aufl., Stuttgart, Berlin, Köln 1995, S. 185.

Vgl. Staehle, W.H., Management: eine verhaltenswissenschaftliche Perspektive, a.a.O., S. 388.

Gemäß Williamson (1985) ist der dauerhafte Übergang eines Gutes oder einer Dienstleistung in den Verfügungsbereich eines anderen Wirtschaftssubjekts konstitutiv für eine Transaktion. Vgl. Williamson, O.E., The Economic Institutions of Capitalism, New York 1985, S. 1.

$107 \mathrm{Vgl}$. zu dieser Interpretation Backhaus, M., Voeth, M., Strategische Allianzen Herausforderungen neuer Kooperationsformen, a.a.O., S. 76.

108 Vgl. Alchian, A.A., Specifity, Specialization, and Coalitions, in: Journal of Institutional and Theoretical Economics, Vol. 140, 1984, S. 36. Heinen spricht - allerdings auf der Mikro-Ebene von "Netto-Vorteilen“, deren Evaluation einer Koalitionsentscheidung vorausgeht, und läßt damit Berührungspunkte zwischen ökonomischer und verhaltenswissenschaftlicher Forschungstradition erkennen. Vgl. Heinen, E., Führung als Gegenstand der Betriebswirtschaftslehre, a.a.O., S. 25. 
Maße abhängt. ${ }^{109}$ Die gemeinsame Realisierung von Quasirente setzt zudem voraus, daß die Koalitions- bzw. Allianzpartner bereit sind, koalitionsspezifische Investitionen zu leisten, die verwendungszweck- und/oder aufgabenspezifisch gebunden sind. Beispiele sind gemeinsame Investitionen der Allianzpartner in die Schulung von Bord- und Bodenpersonal, die Erweiterung der eigenen Flugkapazität eines Partners für andere Partner sowie wechselseitige Investitionen in einen gemeinsamen Markennamen. ${ }^{110}$ Aus derartigen Investitionen entstehen wiederum gegenseitige Abhängigkeiten, die ihrerseits Einfluß auf die Stabilität der Koalition bzw. Allianz nehmen. ${ }^{111}$

Die nur in ihren Grundzügen erörterte Koalitionstheorie stellt mit der Quasirente einen überzeugenden ökonomischen Erklärungsansatz für die Entstehung strategischer Allianzen zur Verfügung, der als Grundlage für die Darlegung der Ziele dienen soll, die Fluggesellschaften mit dem Eingehen strategischer Allianzen verfolgen (Kapitel B 1.2). Koalitionsspezifische Investitionen geben in Verbindung mit der koordinierenden Wirkung der Ziele Aufschluß über allianzinterne Abstimmungsprozesse und die daraus resultierende Leistungserstellung, die auf dieser Basis in den Kapiteln B 1.3 sowie B 1.4.1 und B 1.4.2 untersucht werden sollen. Die in der Koalitionstheorie zum Ausdruck kommende Notwendigkeit geeigneter Rahmenbedingungen für die Verteilung der gemeinsam erwirtschafteten Quasirente wird in Kapitel B 1.4.3 diskutiert.

Wenngleich damit bereits ein Leitfaden zur Bearbeitung der ersten Teilzielsetzung der vorliegenden Arbeit zur Verfügung steht, läuft eine ausschließliche Fokussierung auf die Koalitionstheorie Gefahr, die Bedeutung sozialer Beziehungen für die Stabilität strategischer Allianzen zu übersehen. ${ }^{112}$ Einem solchen Defizit kann durch die Einbeziehung des netzwerktheoretischen Gedankenguts wirksam

109 Backhaus, M., Voeth, M., Strategische Allianzen - Herausforderungen neuer Kooperationsformen, a.a.O., S. 77.

110 Vgl. Klein, B., Leffler, K., The Role of Market Forces in Assuring Contractual Performance, in: Journal of Political Economy, Vol. 90, 1981, S. 626.

111 Die exemplarisch aufgeführten Investitionen haben beim Austritt aus der Koalition Kosten für den austretenden Partner zur Folge, die im Sinne von „sunk costs“ nicht rückgängig gemacht und nicht als freie Ressourcen in einer alternativen Verwendung eingesetzt werden können.

112 Meffert (1997) akzentuiert die Bedeutung monetärer Anreize für die Entstehung temporärer Koalitionen, während er für ihre Stabilität vor allem nicht-monetäre Anreize in Form der Qualităt sozialer Beziehungen verantwortlich sieht. Vgl. Meffert, H., Die virtuelle Unternehmung: Perspektiven aus der Sicht des Marketing, in: Marktleistung und Wettbewerb, Festschrift zum 65. Geburtstag von W.H. Engelhardt, Hrsg.: Backhaus, K., U.a., Wiesbaden 1997, S. 121. 
begegnet werden, das in in jüngerer Zeit verstärkte Beachtung gefunden hat. ${ }^{113}$ Der Begriff des Netzwerks wurde von Jay (1964) als Summe aller Einheiten, die durch einen bestimmten Typ von Beziehung verbunden sind, definiert. ${ }^{114}$ In Weiterentwicklung der Systemtheorie, deren definitorische Nähe des Systembegriffs zum Netzwerkbegriff auffällt, sollen die Handlungsweisen von Organisationen anhand ihrer Position in Beziehungsnetzwerken erklärt werden, so daß eher die soziale Interaktion und Kommunikation im Mittelpunkt der Untersuchung stehen denn formalisierte leistungswirtschaftliche Austauschprozesse. Die Handlungen der Akteure gestalten in diesem Verständnis die Netzwerke und werden zugleich durch die Netzwerke in bestimmte Bahnen gelenkt. ${ }^{115}$

Von entscheidender Bedeutung für die Handlungsmöglichkeiten innerhalb solcher Beziehungsnetzwerke ist die Netzwerkposition eines Unternehmens, für deren Konzeptualisierung in der Literatur verschiedene Ansätze vorliegen. Mattsson (1987) diskutiert beispielsweise vier Dimensionen: (1) die im Netzwerk ausgeübte Funktion des Unternehmens, (2) die Bedeutung des Unternehmens im Netzwerk im Vergleich zu den anderen Unternehmen, (3) die Stärke der Beziehungen zu den anderen Netzwerkunternehmen sowie (4) die Identität der Unternehmen. ${ }^{116}$

113 Schrittmachende Werke im angloamerikanischen Bereich sind z.B. Miles, R.E., Snow, C.C., Organizations: New Concepts for New Forms, in: California Management Review, Vol. 28, No. 3, 1986, S. 62-73; Jarillo, J.C., On Strategic Networks, in: Strategic Management Journal, Vol. 9, No. 1, 1988, S. 31-41; Nohria, N., Eccles, R.G. (Hrsg.), Networks and organizations: structure, form, and action, Boston 1992. Darüber hinaus sind die Weiterentwicklungen und Anwendungen der Uppsala-Schule um Håkansson hervorzuheben (vgl. stellvertretend die perspektivenhafte Einführung, Håkansson, H., Easton, G., Markets as networks: Editorial introduction, in: International Journal of Research in Marketing, Vol. 13, 1996, S. 407-413). Im deutschsprachigen Raum hat insbesondere das Werk von Sydow eine Reihe von Forschungsarbeiten unter der Netzwerkperspektive nach sich gezogen. Vgl. Sydow, J., Strategische Netzwerke: Evolution und Organisation, Wiesbaden 1992; Kloyer, M., Management von Franchisenetzwerken: eine Resource-Dependence-Perspektive, Wiesbaden 1995.

114 Vgl. Jay, 1964, zitiert in: Aldrich, H., Whetten, D.A., Organization-sets, action-sets, and networks: making the most of simplicity, in: Handbook of organizational design, Vol. 1, Hrsg.: Nystrom, P.C., Starbuck, W.H., Oxford u.a. 1981, S. 387.

Vgl. Nohria, N., Is a Network Perspective a Useful Way of Studying Organizations? In: Networks and organizations: structure, form, and action, Hrsg.: Nohria, N., Eccles, R.G., Boston 1992, S. 7. Sydow (1995) bezeichnet diesen Zusammenhang als „Theorem der Dualität von Struktur" und bezieht sich auf die Strukturationstheorie im Sinne Giddens'. Vgl. Sydow, J., Finanzdienstleistungsnetzwerke: zur Organisation einer ökonomischen Institution des Kontraktgütermarketings, in: Kontrakte, Geschäftsbeziehungen, Netzwerke - Marketing und Neue Institutionenökonomik, Sonderheft der zfbf, Nr. 35, 1995, Hrsg.: Kaas, K.-P., S. 149.

Vgl. Mattsson, L.-G., Management of strategic change in a "markets-as-networks" perspective, in: The management of strategic change, Hrsg.: Pettigrew, A.M., Oxford 1987, S. 237. Nohria (1992) nimmt Anleihen bei Burt und unterscheidet auf der Ebene von Individuen die 
Dieser Ansatz liefert damit Erklärungsmöglichkeiten für das Bestehen unterschiedlicher Formen von Allianzen, für Entscheidungsabläufe innerhalb von Allianzen sowie für Problemfelder, die sich aus unterschiedlichen Beziehungsstrukturen und Identitätsdifferenzen der Partnerunternehmen ergeben. ${ }^{117}$ Diesbezügliche Überlegungen fließen in die angebotsorientierten Ausführungen der vorliegenden Arbeit ein.

Der Netzwerkansatz bietet sich darüber hinaus zur Untersuchung der Beziehungen zwischen strategischen Luftverkehrsallianzen und Reisebüros sowie Firmenkunden an, die dem direkten Kontakt zum Endnutzer - dem Fluggast - vorgelagert sind. ${ }^{118}$ Aufgrund der aus Sicht von Fluggesellschaften hohen mengenund wertmäßigen Bedeutung von Reisebüros und Firmenkunden als Absatzmittler bzw. Abnehmer von Flugscheinen erscheint es angebracht, die Auswirkungen strategischer Allianzen auf die Geschäftsbeziehungen zu diesen Marktpartnern zu überprüfen (Kapitel B 2.1). Dem Netzwerkansatz folgend, entstehen aus AnbieterAbnehmer-Geschäftsbeziehungen durch wiederholte Kontakte langfristige, stabile, interdependente und relativ geschlossene Beziehungen („Netzwerke“), die sich durch hohe Kosten des Partnerwechsels auszeichnen. Diese Beziehungen ent-

Dimensionen „cohesion“, „equivalence“, „prominence“, „range“ und „brokerage“. Vgl. Nohria, N., Is a Network Perspective a Useful Way of Studying Organizations? In: Networks and organizations: structure, form, and action, Hrsg.: Nohria, N., Eccles, R.G., Boston 1992, S. 6 f. Eine Operationalisierung der genannten Dimensionen bleibt jedoch bei beiden Autoren offen. Als Methode zur Erfassung und Untersuchung von Netzwerkbeziehungen hat sich die Netzwerkanalyse etabliert, die z.B. bei Aldrich und Whetten (1982) beschrieben ist. Aldrich, $H$., Whetten, D.A., Organization-sets, action-sets, and networks: making the most of simplicity, in: Handbook of organizational design, Vol. 1, Hrsg.: Nystrom, P.C., Starbuck, W.H., Oxford u.a. 1981, S. 397-401.

Die Positionsverteilung zwischen den Partner-Fluggesellschaften einer Allianz läßt sich anhand unterschiedlicher Dimensionen erklären - in der Allianz zwischen British Airways und ihren Franchisepartnern übernimmt British Airways beispielsweise die Vertriebs- und Marketingfunktion und erwirbt durch die Kontrolle dieser absatzmarktgerichteten Funktionen eine dominante Position gegenüber den rein durchführenden Partnern. Innerhalb der durch eher ebenbürtige Mitgliedsgesellschaften getragenen Star Alliance erweist sich die Deutsche Lufthansa als überaus „kontaktfreudiges“ Unternehmen, das im September 1997 und im März 1998 strategische Allianzen mit zwei großen asiatischen Fluggesellschaften geschlossen hat Singapore Airlines und All Nippon Airways - und durch die Stärkung ihres Beziehungskapitals zugleich ihre dominante Position innerhalb der Star Alliance unterstreicht.

Die Anwendung des Netzwerkansatzes auf sowohl horizontale als auch vertikale Unternehmensbeziehungen kommt auch in der Systematisierung von Backhaus und Meyer (1993) zum Ausdruck, die horizontale strategische Allianzen sowie vertikale/diagonale strategische Netzwerke als Typen von Netzwerkarrangements einordnen. Vgl. Backhaus, K., Meyer, M., Strategische Allianzen und strategische Netzwerke, a.a.O., S. 332. 
wickeln sich durch ein gemeinsam motiviertes Handeln und/oder die Ausübung von Macht ${ }^{119}$ und benötigen einen wechselseitigen Ressourceneinsatz. ${ }^{120}$

Im Zuge der zweiten Teilzielsetzung gilt es, unter Kenntnis der Beziehungen von Allianzen zu Reisebüros und Firmenkunden die Wahrnehmung, das Erleben und die Beurteilung von Allianz-Flugdienstleistungen durch Endnutzer zu untersuchen. Dieses Vorgehen erfordert den Rückgriff auf verhaltenswissenschaftliche Erkenntnisse, die auf der Mikro-Ebene ${ }^{121}$ ihren Niederschlag in unterschiedlichen Kaufverhaltenstheorien und Modellen des Kaufverhaltens gefunden haben. ${ }^{122}$ Ein tragfähiges theoretisches Aussagensystem steht mit dem auf Erkenntnissen des Neo-Behaviorismus beruhenden Stimulus-Organism-Response-Modell des Kaufverhaltens zur Verfügung. ${ }^{123}$ Dieses Modell ist um die Erklärung des Kaufverhaltens von Individuen bemüht, indem die beobachtbaren Größen „Stimulus" (Reiz) und "Response" (Reaktion) mit nicht beobachtbaren Vorgängen im Individuum verknüpft werden. ${ }^{124}$ Die nicht beobachtbaren Vorgänge werden als intervenierende Variablen bezeichnet, die über theoretische Begriffe und Konstrukte

Vgl. Turnball, P.W., Valla, J.-P., Strategies for industrial marketing, London 1986, S. 2.

Håkansson formuliert beispielsweise: „Relationships are one of the most valuable resources that a company possesses." Håkansson, H., Industrial technological development: A network approach, London u.a. 1987, S. 10. Die sogenannte Uppsala-Gruppe um Håkansson setzt sich unter der Bezeichnung "Industrial Marketing and Purchasing Group" (IMP) mit Problemstellungen interagierender Organisationen im Industriegüterbereich auseinander. Vgl. Håkansson, H. (Hrsg.), Industrial marketing and purchasing of industrial goods - An interaction approach, New York 1982; im deutschsprachigen Raum vgl. Backhaus, K., Industrial marketing - State of the art in Germany, in: Industrial Marketing, Hrsg.: Backhaus, K., Wilson, D.T., Berlin 1986, S. 3-14.

Staehle ordnet die auf der Individualebene ansiedelnde Verhaltensforschung als "Micro Organizational Behavior" (Organisationspsychologie) in den US-amerikanischen Forschungskontext ein. Vgl. Staehle, W.H., Management: eine verhaltenswissenschaftliche Perspektive, a.a.O., S. $139 f$.

Eine Theorie kann als System von Gesetzesaussagen definiert werden, die auf allgemeine und bewährte Hypothesen zurückgehen. Modelle hingegen sind als Abbildung von Objektsystemen zu verstehen und müssen bestimmte Ähnlichkeitserfordernisse bezüglich des realen Erfahrungsobjekts erfüllen (Isomorphie/Homomorphie). Sie bauen i.d.R. auf TheorieErkenntnissen auf. Vgl. Schanz, G., Methodologie für Betriebswirte, 2., überarb. u. erw. Aufl., Stuttgart 1988, S. 24 u. 63. Zu Theorieansätzen und Modellen des Kaufverhaltens vgl. z.B. Meffert, H., Marketingforschung und Käuferverhalten, a.a.O., S. $47 \mathrm{ff}$.

$\mathrm{Zu}$ einer Diskussion verschiedener Ansätze zur Modellierung des Produkwahlverhaltens vgl. Herrmann, A., Produktwahlverhalten: Erläuterung und Weiterentwicklung von Modellen zur Analyse des Produktwahlverhaltens aus marketingtheoretischer Sicht, Stuttgart 1992, S. $79 \mathrm{ff}$.

124 Vgl. Kroeber-Riel, W., Weinberg, P., Konsumentenverhalten, 6., völlig überarb. Aufl., München 1996, S. $29 f$. 
zur Erklärung des menschlichen Verhaltens herangezogen werden sollen. ${ }^{125}$ Die bereits in Kapitel A 3. hervorgehobene Kaufverhaltensrelevanz von Nutzenerwartungen und Präferenzen läßt diese beiden Größen als geeignete intervenierende Variablen erscheinen, um Unterschiede zwischen den individuellen Bewertungsund Entscheidungsprozessen von aktuellen und potentiellen Fluggästen herauszustellen. ${ }^{126}$ Den Auslöser eines Kaufverhaltensprozesses ${ }^{127}$ stellt ein durch einen Stimulus aktiviertes Bedürfnis dar, zu dessen Befriedigung alternative Leistungsangebote in Frage kommen. ${ }^{128}$ Die Informationssuche führt daraufhin zu einer Wahrnehmung der subjektiv relevanten Leistungsangebote - hier: Flugdienstleistungen -, die als Bündel von Merkmalen und deren Ausprägungen aufgefaßt werden können. Im Zuge eines Bewertungsprozesses erfolgen anschließend der Vergleich und die Beurteilung der Alternativen durch die Endnutzer, deren Ergebnis der angebotsbezogene erwartete Nutzen darstellt. Auf der Grundlage der angebotsbezogenen Nutzenerwartungen wird schließlich die alternativenbezogene Präferenzrangfolge gebildet, die auch in Auswahl- bzw. Kaufwahrscheinlichkeiten ausgedrückt werden kann. ${ }^{129}$

Dieses Modell ebnet den Weg für die Anwendung von Methoden, durch die eine Ermittlung von Nutzendimensionen und ihren Wichtigkeiten möglich ist. ${ }^{130}$ Zur Bearbeitung der zweiten Teilzielsetzung der vorliegenden Arbeit werden einerseits in der Literatur dokumentierte Studien herangezogen, die in diesem Modellkontext zu sehen sind (Kapitel B 2.2). Andererseits bildet das auf Nutzenerwartungen und Präferenzen aufbauende Kaufverhaltensmodell den Bezugspunkt für eine interna-

125 Vgl. Meffert, H., Marketingforschung und Käuferverhalten, a.a.O., S. 26. Als Konstrukt wird der Versuch bezeichnet, nicht direkt beobachtbare Sachverhalte durch Indikatoren einer meßtechnischen Erfassung zugänglich zu machen. Vgl. Andritzky, K., Die Operationalisierbarkeit von Theorien zum Konsumentenverhalten, Berlin 1976, S. 23.

Perrey, J., Nutzenorientierte Marktsegmentierung: ein integrativer Ansatz im Verkehrsdienstleistungsbereich, a.a.O., S. $15 f$.

Neben Prozeßmodellen werden auch Strukturmodelle des Kaufverhaltens diskutiert, die sich um die Abbildung der wesentlichen Bestimmungsfaktoren der nicht beobachtbaren Variablen bemühen. Vgl. z.B. Manzanec, J., Strukturmodelle des Konsumentenverhaltens, Wien 1978.

Zur Beschreibung eines Kauf- bzw. Auswahlprozesses vgl. z.B. Gutsche, J., Produktpräferenzanalyse. Ein modelltheoretisches und methodisches Konzept zur Marktsimulation mittels Präferenzerfassungsmodellen, Berlin 1995, S. 42.

Vgl. Perrey, J., Nutzenorientierte Marktsegmentierung: ein integrativer Ansatz im Verkehrsdienstleistungsbereich, a.a.O., S. 16f. Perrey weist gleichwohl darauf hin, daß die Vorziehenswürdigkeit einer Alternative nicht zwangsläufig mit einer tatsächlichen Auswahl derselben gleichzusetzen ist. 
tionale Fluggastbefragung zu strategischen Luftverkehrsallianzen, deren Ergebnisdiskussion den Kern des Kapitels B 2.3 darstellt. ${ }^{131} \mathrm{Im}$ Mittelpunkt dieser Untersuchung steht die Analyse des Erfüllungsgrades der an den Nutzenerwartungen der Endnutzer ausgerichteten Flugdienstleistung einer bestimmten Allianz. Der in der Studie vollzogene Vergleich zwischen der Idealausprägung bestimmter Kerneigenschaften von Allianz-Flugdienstleistungen und ihrer Realausprägung orientiert sich dabei an der Nähe zwischen dem Konstrukt der Nutzenerwartung und demjenigen der Einstellung. ${ }^{132}$ Die im Trommsdorff'schen Idealmodell der Einstellungsmessung zugrunde liegende Idealausprägung entspricht der SollKomponente der Nutzenerwartung, während die Realausprägung mit dem Grad der endnutzerseitig empfundenen Leistungserfüllung gleichzusetzen ist. ${ }^{133}$

In der dritten Teilzielsetzung spiegelt sich schließlich der Versuch einer Integration von Makro- und Mikro-Ebene wider, wenn sich die Ausgestaltung der Marktbearbeitung durch strategische Luftverkehrsallianzen zwischen den allianzseitigen Ressourcen und den absatzmarktbezogenen Bedürfnissen aufspannt. Um zunächst die Bedingungen, unter denen jedwede Ausgestaltung strategischer Allianzen zu erfolgen hat, zu verdeutlichen, werden im folgenden die relevanten Kontextfaktoren des internationalen Luftverkehrs erörtert.

\subsubsection{Rahmenbedingungen strategischer Luftverkehrsallianzen}

Mit der Aufnahme des ersten Linienflugdienstes ${ }^{134}$ am 8. Februar 1919 zwischen London und Paris wurde ein Abkommen ausgearbeitet, dessen Ziel die Kodifizie-

131 Die Befragung wurde nicht von dem Verfasser selbst durchgeführt. Dennoch ist die Kenntnis der Grundannahmen, die der Untersuchung zugrunde liegen, für das Verständnis und die Interpretation der Ergebnisse von hoher Bedeutung.

Eine Einstellung kann als Zustand einer gelernten und relativ dauerhaften Bereitschaft definiert werden, in einer entsprechenden Situation gegenüber einem Objekt regelmäßig mehr oder weniger stark positiv oder negativ zu reagieren. Vgl. Trommsdorff, V., Konsumentenverhalten, 3., überarb. und erw. Aufl., Stuttgart, Berlin, Köln 1998, S. 143.

133 Aus diesem Grunde wird die Nutzensegmentierung bisweilen auch als Variante der produktspezifischen Einstellungsmessung bezeichnet. Vgl. hierzu Meffert, H., Perrey, J., Nutzensegmentierung im Verkehrsdienstleistungsbereich - theoretische Grundlagen und empirische Erkenntnisse am Beispiel des Schienenpersonenverkehrs, in: Tourismus Journal, 1. Jg., Nr. 1, 1997, S. 21; Stegmüller, B., Internationale Marksegmentierung als Grundlage für internationale Marketing-Konzeptionen, Bergisch Gladbach, Köln 1995, S. 220. Zu den Annahmen des Trommsdorff-Modells vgl. Trommsdorff, V., Konsumentenverhalten, 3., überarb. und erw. Aufl., Stuttgart, Berlin, Köln 1998, S. 142ff.

134 Linienflugdienst bzw. Linienluftverkehr ist im Sinne des deutschen Luftverkehrsgesetzes (LuftVG § 21 Abs. 1, 2) durch Gewerbsmäßigkeit, Öffentlichkeit, Regelmäßigkeit, Linienbindung, Betriebspflicht, Beförderungs- und Tarifpflicht gekennzeichnet. Im 
rung von Regeln für den Luftverkehr war. ${ }^{135}$ Die Gründe für diese Bemühungen lagen in der Notwendigkeit von Sicherheitsstandards ${ }^{136}$ für den Betrieb von Flugzeugen und den Transport von Personen, Fracht und Post sowie in politischen Interessen der Staaten, zwischen denen der Luftverkehr stattfand. Mit der im Pariser Abkommen enthaltenen und noch heute herrschenden Lufthoheitstheorie, die den Luftraum der Gebietshoheit des Bodenstaates unterwirft, wurde damit eine entscheidende Rahmenbedingung für den internationalen Luftverkehr gesetzt. Im Abkommen von Chicago räumten sich 1944 die meisten der 52 teilnehmenden Nationalstaaten in einer multilateralen Übereinkunft die Rechte des Überflugs und der technischen Landung ein, die verkehrsrechtlich auch als „erste" bzw. „zweite Freiheit der Luft" bezeichnet werden (vgl. Abbildung 4). ${ }^{137}$ Die Gewährung der für den gewerblichen Luftverkehr bedeutsamen Rechte zum Absetzen, Aufnehmen und Befördern von Passagieren, Fracht oder Post zwischen Vertragsstaaten (dritte bis achte Freiheit) blieb demgegenüber einer Vielzahl von bilateralen Luftverkehrsabkommen zwischen einzelnen Staaten vorbehalten. Eine multilaterale Regelung scheiterte an der Verschiedenartigkeit der politisch-rechtlichen Vorstellungen. ${ }^{138}$ Neben der Verabschiedung des Chicagoer Abkommens mündete das Treffen der Staatsvertreter in der Gründung der ICAO (International Civil Aviation Organization), die bis heute ein Forum für die Vertretung luftverkehrsspezifischer Interessen der Mitgliedsstaaten bildet.

internationalen Luftverkehrsabkommen von Chicago wird Linienluftverkehr als any scheduled air service performed by aircraft for the public transport of passengers, mail or cargo“ definiert. Übernommen aus Pompl, W., Luftverkehr: eine ökonomische Einführung, a.a.O., S. 21.

Auch als Pariser Luftverkehrsabkommen oder CINA-Abkommen bekannt (Convention Internationale portant Réglementation de la Navigation Aérienne). Vgl. Reckewerth, S., Die Zusammenarbeit der Luftverkehrsgesellschaften nach europäischem und US-amerikanischen Recht, a.a.O., S. 29.

Vor dem zweiten Weltkrieg wurden noch in 21,6 Prozent der Falle Mängel am Flugzeug als Unfallursache angegeben, während sich nach dem zweiten Weltkrieg eine Verlagerung der Unfallursache in Richtung menschlichen Versagens abzeichnete. Vgl. Leonhardt-Weber, B., Die Entwicklung der Qualitătsmerkmale im Verkehr: eine Analyse vor dem Hintergrund der technischen, wirtschaftlichen und gesellschaftlichen Entwicklung, München 1990, S. $368 f$.

Neben der Verabschiedung des Chicagoer Abkommens mündete das Treffen der Staatsvertreter in der Gründung der ICAO (International Civil Aviation Organization), die sich vornehmlich mit staatlichen Aspekten des Luftverkehrs auseinandersetzt.

138 Vgl. Doganis, R., Flying off Course: The Economics of International Airlines, 2. Aufl., London, New York 1991, S. 27. 


\begin{tabular}{|c|c|}
\hline 1. Freiheit & $\begin{array}{l}\text { Recht, das Hoheitsgebiet der Vertragsstaates zu } \\
\text { überfliegen. }\end{array}$ \\
\hline 2. Freiheit & $\begin{array}{l}\text { Das Recht zur nicht-gewerblichen Zwischenlandung (technische } \\
\text { Landung) im Vertragsstaat, d.h. es dürfen weder Fracht noch } \\
\text { Passagiere aufgenommen oder abgesetzt werden. }\end{array}$ \\
\hline 3. Freiheit & $\begin{array}{l}\text { Beinhaltet das Recht, im Vertragsstaat zu landen, um dort } \\
\text { Passagiere, Fracht und Post abzusetzen. }\end{array}$ \\
\hline 4. Freiheit & $\begin{array}{l}\text { Zusătzlich zum Recht der } 3 \text {. Freiheit dürfen im Vertragsstaat } \\
\text { auch Passagiere, Fracht und Post aufgenommen werden. }\end{array}$ \\
\hline 5. Freiheit & $\begin{array}{l}\text { Es dürfen Passagiere, Fracht und Post zwischen zwei auslän- } \\
\text { dischen Staaten befördert werden. Bedingung ist, daß der Flug } \\
\text { im Heimatland der Luftverkehrsgesellschaft beginnt oder endet. }\end{array}$ \\
\hline 6. Freiheit & $\begin{array}{l}\text { Ergibt sich als Kombination von Rechten der } 3 \text {. und } 4 \text {. Freiheit im Verhältnis zu zwei } \\
\text { verschiedenen Vertragsstaaten. (Beforderung von einem Vertragsstaat über den } \\
\text { Heimatstaat in einen anderen Vertragsstaat oder umgekehrt). }\end{array}$ \\
\hline 7. Freiheit & $\begin{array}{l}\text { Recht zum Verkehr ausschließlich zwischen Drittstaaten, ohne Verbindung mit dem } \\
\text { Heimatstaat der Luftverkehrsgesellschaft. }\end{array}$ \\
\hline $\begin{array}{c}\text { 8. Freiheit } \\
\text { (Kabotagerecht) }\end{array}$ & $\begin{array}{l}\text { Recht zur Beförderung von Passagieren, Fracht und Post innerhalb eines Staates. (Wird } \\
\text { in der Regel nur einheimischen, nicht aber ausländischen Gesellschaften gewährt). }\end{array}$ \\
\hline
\end{tabular}

\section{Abb. 4: Die Freiheiten der Luft}

(Quelle: Diegruber, J., Erfolgsfaktoren nationaler europäischer Linienluftverkehrsgesellschaften im Markt der 90er Jahre, St. Gallen 1991)

Zur Umsetzung der Inhalte bilateraler Verträge bedienten sich die Staaten in der Folge sogenannter designierter Fluggesellschaften, die für die Bedienung der entsprechenden Strecken bestimmt (designiert) wurden. ${ }^{139}$ Neben dem Austausch von Verkehrsrechten sahen die bilateralen Abkommen Regelungen bezüglich der anzubietenden Kapazitäten, der festzulegenden Tarife sowie mitunter der Beteiligung der Fluggesellschaften an den Kosten und/oder Erlösen des gemeinschaftlichen Betriebs von Flugstrecken („Poolabkommen") vor. ${ }^{140}$ Dementsprechend fanden auf der Ebene der Fluggesellschaften Kooperationen statt, die den Charakter einer staatlich induzierten Zwangspartnerschaft trugen.

139 Nahezu alle bilateralen Luftverkehrsabkommen schreiben vor, daß sich die zu designierenden Fluggesellschaften im Eigentum oder unter überwiegender Kontrolle von Anghörigen des Designierungsstaates befinden müssen. Vgl. Reckewerth, S., Die Zusammenarbeit der Luftverkehrsgesellschaften nach europäischem und US-amerikanischen Recht, a.a.O., S. 41.

Zu einer ausführlichen Darstellung der vertraglichen Ausgestaltung bilateraler Abkommen des Typs Chicago, Bermuda I und Bermuda II vgl. Reckewerth, S., Die Zusammenarbeit der Luftverkehrsgesellschaften nach europäischem und US-amerikanischen Recht, a.a.O., S. 4457. 
Um ein Gegengewicht zu den politischen Interessen der Staaten zu schaffen, gründeten die Vertreter der führenden Luftverkehrsgesellschaften im Jahre 1945 einen Dachverband, die International Air Transport Association (IATA). Neben der Wahrnehmung der Interessenvertretung kam und kommt der IATA vor allem die Aufgabe zu, die Zusammenarbeit zwischen den Fluggesellschaften zu fördern. Zu diesem Zweck wurden ihr die Festsetzung der Flugtarife, die Vereinheitlichung von Beförderungsbedingungen und die Abrechnung von Flugscheinen im Rahmen von Interline-Vereinbarungen zwischen den Fluggesellschaften übertragen. ${ }^{141}$ Das Interlining eröffnet den Kunden von IATA-Fluggesellschaften über die gegenseitige Anerkennung von Flugscheinen den direkten Zugang zum weltweiten Strekkennetz, das durch die Zusammenarbeit der IATA-Mitglieder entsteht. Damit ist es den Passagieren möglich, mit nur einem Flugschein Verbindungsflüge unterschiedlicher Fluggesellschaften im IATA-System wahrzunehmen oder kurzfristige Umbuchungen zwischen verschiedenen IATA-Airlines mit demselben Ticket zu realisieren. ${ }^{142}$

Das Chicagoer Abkommen in Verbindung mit der Gründung der ICAO und die später gebildete IATA führten nach dem zweiten Weltkrieg zu einer ersten Phase administrativ geregelten Wettbewerbs bzw. staatlich induzierter, kartellgleicher Kooperation unter den IATA-Fluggesellschaften, die 1976 ca. $90 \%$ des Weltluftverkehrs repräsentierten. ${ }^{143}$

Aus der Produktion und Verbreitung von großvolumigen Langstrecken-Verkehrsflugzeugen mit Jet-Antrieb entstanden gegen Ende der sechziger bzw. Anfang der siebziger Jahre Überkapazitäten, die sich zu einem zentralen Problem der Luftverkehrsindustrie verdichteten und die innere Stabilität der IATA schwächten. ${ }^{144}$

141 Die Aufgaben leiten sich wesentich aus den Zielen der IATA ab, die in der Gründungsakte (Art. 3 des Act of Incorporation) niedergelegt sind, und sind hier nur reduziert wiedergegeben.

1421994 waren ca. 280 Fluggesellschaften in das IATA-System integriert. Vgl. Göpfert, I., Interlining, Code-Sharing und Trucking, in: WiSt, Heft 9, 1994, S. 460.

$143 \mathrm{Vgl}$. Jăckel, K., Kooperationsstrategien im Linienluftverkehr vor dem Hintergrund zunehmender Integrationsentwicklung in Europa, a.a.O., S. 168.

$144 \mathrm{Vgl}$. Verchère, I., The air transport industry in crisis: solving over-capacity and financing new equipment, London 1994, S. VI. Das Problem der Überkapazitaten verleitete IATAMitgliedsgeselischaften zur Aufgabe der Kartell-Disziplin und zur Initiierung eines Preiswettbewerbs zwecks Flugzeugauslastung, der ein Fünftel bis ein Drittel der Umsätze an der IATA vorbeifließen ließ. Vgl. Henard, J., Das Tarifkorsett reißt an allen Năhten, in: FAZ vom 
Zugleich stellten Charter-Fluggesellschaften ${ }^{145}$ und asiatische Airlines, die nicht Mitglieder der IATA waren, die Tarifkoordination der IATA durch flexible, günstige Preisangebote zusehends in Frage, da die Preisbildungsverfahren der IATA als starr galten und den dynamischen Wettbewerbserfordernissen nicht mehr gerecht zu werden schienen. ${ }^{146}$

Hieran schloß sich eine zweite Phase des Kapazitäts- und Preiswettbewerbs an. Deregulierungsaktivitäten in den USA, die im Airline Deregulation Act von 1978 ihren Anfang nahmen, ${ }^{147}$ sowie die Privatisierung und Liberalisierung des Luftverkehrs in Europa markierten weitere Schritte in Richtung einer Reduzierung staatlichen Einflusses. Eine Betrachtung der Entwicklungen auf dem US-amerikanischen Luftverkehrsmarkt offenbart in dieser Phase ein wechselhaftes Bild. Während Preiskämpfe und Neuordnungen der inneramerikanischen Streckennetze zwischen 1978 und etwa 1982 in Ergebniseinbußen der Airlines resultierten, führte ab 1983 eine steigende Nachfrage nach Flügen bei zahlreichen Neugründungen von Fluggesellschaften daraufhin zu einer Branchenexpansion mit abnehmendem Konzentrationsgrad. ${ }^{148}$ Ab 1985 waren Fusionen und Akquisitionen die Folge eines fortdauernden inneramerikanischen Verdrängungswettbewerbs, der vornehmlich über den Parameter des Preises ausgetragen wurde und der bis 1990 zum Marktaustritt von insgesamt ca. 200 Fluggesellschaften führte. ${ }^{149}$ Das Ergebnis dieses Konzentrationsprozesses drückt sich u.a. in dem

13.06.1986, S. R 7. Derartige Preisangebote wurden und sind bei den Kunden als „GraumarktTickets" oder "special rates" bekannt.

145 Als Charter-Fluggesellschaften gelten im Sinne einer Negativabgrenzung diejenigen Fluggesellschaften, die sich nicht dem Linienluftverkehr zurechnen lassen. Besondere Bedeutung erlangt diese Form des Luftverkehrs im Touristik-Bereich. Durch hohe Wachstumsraten und ein in geringerem Maße saisonal orientiertes Reiseverhalten entwickeln sich viele Charter-Fluggesellschaften $\mathrm{zu}$ touristisch orientierten Linienfluggesellschaften, welche die Grenzziehung zum Linienflugverkehr teilweise verwischen lassen. Vgl. Sterzenbach, R., Luftverkehr, a.a.O., S. 41 f.

Vgl. Jäckel, K., Kooperationsstrategien im Linienluftverkehr vor dem Hintergrund zunehmender Integrationsentwicklung in Europa, a.a.O., S. 167. Vgl. United States of America (Hrsg.), Public Law 95-504, Oct. 24, 1978, S. 1705-1754.

Wăhrend die zehn grőßten US-amerikanischen Fluggesellschaften 1977 noch 88 Prozent aller verkauften Passagiermeilen auf sich vereinigten, lag dieser Wert 1984 bei 75,3 Prozent. Vgl. Laaser, C., Ausländische Erfahrungen mit Deregulierungsexperimenten im Verkehrswesen, Kieler Arbeitspapiere Nr. 270, Kiel 1986, S. 30.

149 Vgl. Frentz, M. H., Ex Occidente Nox? "Corporate Restructuring, Mergers \& Acquisitions“ vs. strategischer Allianzen in der liberalisierten Luftverkehrsindustire, in: Journal für Betriebswirtschaft , 43. Jg., Nr. 1, 1993, S. 22. 
Marktanteil von 92,5 Prozent aus, den die 10 größten US-amerikanischen Airlines 1989 im US-Binnenmarkt auf sich vereinigten. ${ }^{150}$

Ein weiteres Resultat bestand in der Herausbildung sogenannter Drehkreuze („hubs") an bedeutenden Verkehrsflughäfen, die den großen Fluggesellschaften zur Sammlung und Verteilung von Verkehrsaufkommen dienten und zu einer Kooperation mit kleineren Zubringer-Fluggesellschaften führten. ${ }^{151}$ Diese Zubringer-Fluggesellschaften operierten mit ihrem kleinerem und kostengünstigerem Fluggerät auf Strecken mit geringerem Verkehrsaufkommen unter gemeinsamer Flugnummer ihres größeren Kooperationspartners, so daß es sich um ein Code Sharing handelte. ${ }^{152}$

Als der US-amerikanische Luftverkehrsmarkt nach der "Marktbereinigung" in eine vergleichsweise stagnative Phase eingetreten war, gerieten strategische Allianzen mit europäischen Fluggesellschaften in den Blickpunkt insbesondere der großen US-amerikanischen Airlines. In Europa sahen sie sich einer Wettbewerbslandschaft gegenüber, die vornehmlich von in staatlichem Besitz befindlichen nationalen Fluggesellschaften, sogenannten flag carriers, geprägt war. ${ }^{153}$

Politische und wirtschaftliche Entwicklungen führten in Europa jedoch ab der zweiten Hälfte der achtziger Jahre zu einer Liberalisierung und Neuordnung von Marktstrukturen, die in Verbindung mit den Ergebnissen der US-amerikanischen Deregulierung den Ausgangspunkt für eine dritte Phase des Wettbewerbs von Allianzen bildeten. Auf politischer Seite sind die Neuverhandlungen bilateraler

150 Vgl. Schmidt, S., Strategische Allianzen im Luftverkehr - Erfolgsorientiertes Management europäischer Flug-Carrier, Trier 1993, 37.

$151 \mathrm{Zu}$ einer ausführlichen Beschreibung und Bewertung derartiger "Nabe-Speichen-Systeme" (hub and spoke networks) vgl. Williams, G., The airline industry and the impact of deregulation, 2. Aufl., Aldershot 1996, S. 17-28.

152 Beyhoff, Ehmer und Wilken (1995) identifizieren das Allegheny Commuter System, das 1967 in den USA eingeführt wurde, als Anfangspunkt des Code Sharing. Allegheny Airlines, Vorgängergesellschaft von USAir, ging mit einer steigenden Zahl unabhängiger kleinerer Gesellschaften, die mit günstigeren Kostenstrukturen und kapazitătsadäquatem Fluggerăt operierten, Marketing- und Betriebskooperationen ein. Diese Kooperationen bezogen sich auf Strecken, die durch das von Allegheny erworbene Jet-Fluggerăt okonomisch nicht vorteilhaft zu bedienen waren. Vgl. Beyhoff, S., Ehmer, H., Wilken, D., Code-Sharing im internationalen Luftverkehr der Bundesrepublik Deutschland, a.a.O., S. $17 f$.

153 Zu einer gerafften Darstellung vgl. Sanz, F.L., The EC air transport in the international legal framework of civil aviation, in: Der Luftverkehr in der Europäischen Union: die Lücke im Binnenmarkt, Hrsg.: Rogalla, D., Schweren, K., Baden-Baden 1994, S. 15-21. 
Abkommen zwischen europäischen Staaten sowie die im Dezember 1987 verabschiedeten "Liberalisierungspakete" der Europäischen Gemeinschaft hervorzuheben. ${ }^{154}$ Auf unternehmerischer Ebene vollzogen sich in Europa dagegen Fusionsversuche, ${ }^{155}$ Akquisitionen und Beteiligungen, die vornehmlich der Absicherung von Marktpositionen dienten und zu einer kapital- und/oder vertraglich basierten Verflechtung von großen internationalen und kleinen bis mittelgroßen regionalen Fluggesellschaften führten. $\mathrm{Zu}$ unterscheiden sind zum einen hundertprozentige Tochtergesellschaften großer Fluggesellschaften mit regional ausgerichtetem Schwerpunkt wie beispielsweise die niederländische KLM Cityhopper, die skandinavische SAS Commuter oder die deutsche Lufthansa CityLine, ${ }^{156}$ zum anderen Franchisesysteme bzw. franchiseähnliche Kooperationssysteme wie Team Lufthansa oder British Airways Express, die Regionalfluggesellschaften wie Augsburg Airways oder CityFlyer unter sich vereinen, und schließlich sonstige kapitalbasierte oder vertragliche Abkommen zwischen Fluggesellschaften wie z.B. die 49prozentige Beteiligung von British Airways an der Deutschen BA oder das Abkommen zwischen KLM und Eurowings. ${ }^{157}$

Gegen Ende der achtziger Jahre markierten die strategischen Allianzen zwischen Continental Airlines und SAS sowie Northwest Airlines und KLM den Beginn weltweiter strategischer Luftverkehrsallianzen, in die bald auch Fluggesellschaften aus dem asiatisch-pazifischen Raum einbezogen wurden (Singapore Airlines formierte

154 Die Liberalisierungspakete betreffen im wesentlichen Regelungen bezüglich der Tarifgenehmigung, Kapazităten, des Marktzugangs, der Verkehrsrechte, Designierung und Wettbewerbsregeln. Einen präzisen und knappen Überblick über EU-spezifische Integrationsmaßnahmen liefert Ehmer, H., Ein Jahr Binnenmarkt im Luftverkehr, in: Internationales Verkehrswesen, Nr. 9, 1994, S. 512-517. Eine detaillierter Darstellung findet sich bei Piepelow, V., Die europäischen Linienfluggesellschaften im Wettbewerb: eine Analyse mittels des Konzepts strategischer Gruppen, Frankfurt am Main u.a. 1997, S. 94-110.

Beachtung haben die im Herbst 1991 zwischen British Airways und der niederländischen KLM geführten Fusionsverhandlungen sowie die 1993 geplante Fusion von SAS (Skandinavien), Swissair (Schweiz), KLM und Austrian Airlines (Österreich) gefunden. Gründe des Scheiterns wurden im ersten Fall überwiegend in Unstimmigkeiten der Kapitalanteilsregelung gesehen, während die zweitgenannten Planungen unter der Projektbezeichnung "Alcazar" vor allem an der Frage der zu bestimmenden US-Partner-Fluggesellschaft scheiterten (KLM war mit Northwest Airlines, Swissair mit Delta Air Lines und SAS mit Continental Airlines alliiert). Vgl. Ehmer, H., Ein Jahr Binnenmarkt im Luftverkehr, in: Internationales Verkehrswesen, Nr. 9, 1994, S. 515.

Eine hundertprozentige Kapitalbeteiligung erfüllt nicht das ex definitione vorgegebene Merkmal der weitgehenden wirtschaftlichen Selbständigkeit, so daß es sich nicht um eine strategische Allianz handelt.

157 Zu einer Übersicht vgl. Odell, M., Whitaker, R., A picture of health, in: Airline Business, No. 5, 1997, S. 38. 
sich mit Delta Air Lines und Swissair 1989 zur "Global Excellence"-Allianz, British Airways erwarb 1993 einen 25-Prozent-Anteil an der australischen Qantas). Eine zwischenstaatliche Politik, die unter der Bezeichnung "open skies“ eine weitgehende Liberalisierung des internationalen Luftverkehrs vorsah, räumte den Allianzpartnern in bilateralen Abkommen bis heute teilweise weitreichende Abstimmungsmöglichkeiten von Flugplänen, Kapazitäten und Tarifen ein. ${ }^{158}$

Diese Entwicklungen verdeutlichen die hohe Bedeutung der Wettbewerbsintensität für die Entstehung strategischer Allianzen, die damit als anbieterseitig initiiertes Instrument zur Erreichung unternehmerischer Zielsetzungen auftreten. Einer Erörterung des Designs strategischer Luftverkehrsallianzen ist vor diesem Hintergrund eine prinzipielle Überlegung voranzustellen. Es wurde deutlich, daß Fluggesellschaften unterschiedlicher Größe - gemessen am Umsatzvolumen und geographischer Marktabdeckung - an Allianzen beteiligt sind. Dabei sind sowohl Allianzen zwischen Airlines annähernd gleicher Größe als auch deutlich unterschiedlicher Größe zu beobachten. Um den im folgenden zu erarbeitenden Aussagen ein hinreichendes Maß an Vergleichbarkeit innerhalb des Luftverkehrskontextes zu verleihen, beschränken sich alle weiteren Aussagen auf strategische Allianzen zwischen Fluggesellschaften, die sich durch die gleichzeitige Erfüllung folgender Merkmale auszeichnen: ${ }^{159}$

- die beteiligten Partner zählen in ihrem Heimatmarkt zu den fünf umsatzstärksten Anbietern und

- fliegen Ziele an, die in mindestens vier von den sechs Hauptverkehrsmärkten ${ }^{160}$ der Welt liegen. ${ }^{161}$

158 Vgl. Jegminat, G., Die Airline-Allianz von KLM und Northwest: aus transatlantischer Achse globales Netz geformt, in: fvw, Nr. 13, 1996, S. 65.

159 Eine auf dem Merkmal des Umsatzvolumens beruhende Eingrenzung der Untersuchungsgegenstands nimmt auch Bongartz (1998) bei der Analyse strategischer Gruppen im US-amerikanischen Luftverkehrsmarkt vor. Als kritische Größe wird in Anlehnung an die Unterscheidung des Verkehrsministeriums der USA ein Jahresumsatz von 1 Mrd. USDollar herangezogen, dem 1994 zehn Fluggesellschaften, die sogenannten Majors, entsprachen. Vgl. Bongartz, U., Unternehmensspezifische Ressourcen und strategische Gruppen im US-Luftverkehrsmarkt, in: ZfB, 68. Jg., Nr. 4, 1998, S. 385.

160 Als Hauptverkehrsmärkte werden hier USAKKanada, Lateinamerika/Karibik, Europa, Afrika, Mittlerer Osten und Asien/Pazifik zugrunde gelegt. Die präzise Abgrenzung dieser Märkte lehnt sich an den World Airline Report 1997 der Fachzeitschrift Air Transport World an. Dort werden jedoch die USA und Kanada getrennt ausgewiesen. Vgl. o.V., The World Airline Report, in: Air Transport World, No. 7, 1997, S. 85-139. 
Diese Eingrenzung ist zwar der Kritik einer subjektiven Merkmalsauswahl und Grenzziehung ausgesetzt, vermag aber einen wertmäßig bedeutenden Anteil der in der Praxis zu beobachtenden strategischen Luftverkehrsallianzen operational zu erfassen. ${ }^{162}$ Zudem lassen die Merkmalsausprägungen einen ausreichenden Spielraum zur Berücksichtigung von Größenunterschieden und Marktpräsenz der Fluggesellschaften offen. Mit dieser Einordnung und Abgrenzung des Untersuchungsobjekts ist der Weg bereitet für eine konsistente Auseinandersetzung mit den Zielen der Allianzpartner, an denen sich die absatzmarktgerichtete und organisationale Gestaltung strategischer Luftverkehrsallianzen orientieren.

\subsection{Ziele für strategische Luftverkehrsallianzen}

Den Zielen ${ }^{163}$ für strategische Luftverkehrsallianzen liegt unter Bezugnahme auf das koalitionstheoretische Gedankengut ein erhoffter ökonomischer Vorteil durch das Zusammengehen mit anderen Fluggesellschaften zugrunde, der sich in Form einer koalitionsspezifischen Quasirente ausdrücken läßt. ${ }^{164}$ Diese Überlegung findet in der Definition strategischer Allianzen ihren Niederschlag, nach der sich die Allianzpartner wechselseitig Zugang zu Erfolgspotentialen gewähren. Durch die gegenseitige Nutzbarmachung von Erfahrungen im Umgang mit Märkten, Technologien, sozialen Strukturen und Prozessen, in denen sich bestehende Erfolgspotentiale ausdrücken, ${ }^{165}$ erhoffen die Partnerunternehmen eine Verbesse-

161 Ausdrücklich ausgegrenzt sind damit strategische Allianzen, an denen Regionalfluggesellschaften mit einer engen Verkehrsmarktabdeckung (z.B. nur Kontinentaleuropa) beteiligt sind. Die Expertengespräche mit Vertretern dieser Fluggesellschaften belegten deutliche Unterschiede nicht nur hinsichtlich der Zielsetzungsschwerpunkte, sondern auch der organisationalen Gestaltung. Vgl. Anhang 4.

Zur kritischen Reflexion der Leistungsfähigkeit von Systematisierungsansătzen vgl. z.B. Meurer, J., Führung von Franchisesystemen: Führungstypen - Einflußfaktoren - Verhaltensund Erfolgswirkungen, Wiesbaden 1997, S. 8 und die dort wiedergegebenen Fußnoten 27-30.

Nach Meffert sind Ziele im betriebswirtschaftlichen Kontext definiert als erstrebenswerte Vorzugszustände ("Imperative"), die durch den Einsatz unternehmerischer Maßnahmen erreicht werden sollen. Vgl. Meffert, H., Marketing: Grundlagen marktorientierter Unternehmensführung, a.a.O., S. 70.

Anwendung finden in der Praxis etwa finanzwirtschaftliche Indikatoren wie beispielsweise der allianzspezifische Cash-Flow, aber auch Produktivitätskennziffern (z.B. allianzinduzierte Veränderungen des Sitzladefaktors) und marktorientierte Größen (z.B. allianzspezifische Umsatzerlöse).

185 Vgl. Bleicher, K., Das Konzept integriertes Management, 4., rev. und erw. Aufl., Frankfurt/M., New York 1996, S. 75. Der Aufbau, die Pflege und die Ausbeutung von Erfolgspotentialen bilden im Verständnis des St. Galler Management-Konzepts den Kern des strategischen Management. Gälweiler (1976) betont die Funktion von Erfolgspotentialen als Steuerungsgröße für die strategische Planung, die er ihrerseits als Steuerungsinstrument 
rung ihrer Wettbewerbsposition zu erreichen. ${ }^{166}$ Ziele dienen der Präzisierung dieser "Leerformel“ und können bei der Bildung und im Zuge des Management strategischer Luftverkehrsallianzen in absatzmarktgerichtete Wirkungsziele und unternehmensgerichtete Effizienzziele unterschieden werden. ${ }^{167} \mathrm{Da}$ es sich aufgrund der weitgehenden Unabhängigkeit der Partner um individuelle Zielsetzungen der Allianzpartner handelt, kann hierbei zunächst nicht von Allianzzielen im Sinne von „Zielen der Allianz" gesprochen werden. Durch die Abstimmung von Einzelzielsetzungen ${ }^{168}$ der Partner-Fluggesellschaften im Rahmen interaktiver Prozesse können sich gleichwohl unternehmensübergreifende Allianz-Zielsetzungen herausbilden. Die im Rahmen der Arbeit geführten Expertengespräche bestätigen diesen Befund. ${ }^{169}$

auffaßt. Auf der Ebene der operativen Planung dienen der betriebswirtschaftliche Erfolg, auf der Ebene der Finanzplanung die Liquidität als Steuerungsgrößen. Vgl. Gälweiler, A., Unternehmenssicherung und strategische Planung, in: zfbf, 28. Jg., 1976, S. 375.

166

167

Die

Diskussion der flugdienstleistungsspezifischen Potential-, Prozeß- und Ergebnisorientierung hat deutlich gemacht, daß die hier unterschiedene Absatzmarkt- und Unternehmensdimension der Allianzziele in einem Spannungsfeld zueinander stehen. Dieses Spannungsfeld wird in der Grundlagendiskussion zum Marketingverstăndnis schwerpunktartig vertieft. Vgl. z.B. Dreher, A., Marketingorientierung als Unternehmensphilosophie: Phänomen und empirische Erfassung, Wiesbaden 1995, insb. S. 133-150; Bleicher, K., Marketing im Spannungsfeld von Wettbewerbs- und Potentialorientierung, in: Marktorientierte Unternehmensführung: Reflexionen - Denkanstöße - Perspektiven, Hrsg.: Bruhn, M., Steffenhagen, H., Wiesbaden 1997, insb. S. 50f.

Diese Ziele stehen in enger Beziehung zu Quellen von Synergievorteilen, die im Zuge strategischer Allianzen erschlossen werden sollen. Als formale Bedingung für das Vorliegen eines Synergievorteils wird in der Literatur folgende Ungleichung herangezogen: $U(A+B)>U$ $(A)+U(B)$ mit $U=U$ msatz, $A=F$ luggesellschaft $A, B=$ Fluggesellschaft $B$, das heißt der von zwei Fluggesellschaften im Zuge einer Allianz erwirtschaftete Umsatz ist ceteris paribus größer als der im Zuge eines Alleingang beider Airlines realisierte Umsatz. Auf der Kostenseite ließe sich

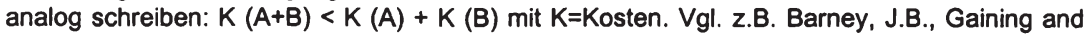
Sustaining Competitive Advantage, a.a.O., S. 286.

Hwang und Burgers (1997) vermerken dazu: „Indeed, companies have not simply the right, but also the obligation to their immediate stakeholders to pursue their own interests at the expense of others." Hwang, P., Burgers, W.P., The Many Faces of Multi-Firm Alliances, a.a.O., S. 102. Derartige Befunde lassen deutlich werden, daß die Überwindung loyalitătsbedingter Denkschemata, die mit dem arbeitgebenden Unternehmen verbunden sind, zugunsten eines Denkens in den Dimensionen der strategischen Allianz nicht ohne weiteres zur Realităt wird.

Vgl. die im Anhang dokumentierten Gesprăche. 


\subsubsection{Absatzmarktgerichtete Wirkungsziele}

Unter absatzmarktgerichteten Wirkungszielen werden im folgenden Umsatz- und Marktanteilssteigerung, Markterschließung, der Aufbau und/oder die Umgehung von Markteintrittsbarrieren, die Steigerung des Bekanntheitsgrads und die Verbesserung des Markenimage subsumiert. ${ }^{170}$ Dabei wird die strategische Allianz als Instrument eingesetzt, um den Zielerreichungsgrad der einzelnen Partnerunternehmen in diesen Zieldimensionen zu erhöhen. Sofern die strategische Allianz mit einer eigenen Marke im Markt etabliert ist, können die Ziele „Bekanntheitsgradsteigerung" und "Markenimageverbesserung“ auch für die Allianz definiert werden. Die Zieldiskussion ist dann aus einer Einzelunternehmensund Allianzsicht zu führen.

Zwischen den genannten absatzmarktgerichteten Wirkungszielen bestehen zahlreiche Interdependenzen. ${ }^{171}$ Die Zielgröße Umsatz bildet ein übergeordnetes Ziel, zu dem die übrigen Ziele in einer Mittel-Zweck-Relation stehen. Es erscheint daher sinnvoll, zunächst auf die untergeordneten Ziele einzugehen.

Durch die gemeinsame Abstimmung von Streckennetzplanung, Marketing- und Vertriebsaktivitäten streben die an einer Allianz beteiligten Fluggesellschaften eine Erhöhung ihrer Marktanteile in den zugrunde liegenden strategischen Geschäftsfeldern an. ${ }^{172} \mathrm{Im}$ Geschäftsfeld des Personenluftverkehrs ${ }^{173}$ mit großvolumigen Jet-Flugzeugen auf der Transatlantik-Route ${ }^{174}$ zwischen den USA und

170 Meffert (1998) faßt die ersten drei Zieldimensionen unter der Basiskategorie „Marktstellungsziele“ zusammen. Vgl. Meffert, H., Marketing: Grundlagen marktorientierter Unternehmensführung, a.a.O., S. 71. Damit handelt es sich bei den Allianzzielen nicht um grundsätzlich neue Ziele, sondern um die Erreichung höherer Zielerreichungsniveaus bei bestehenden Zielen.

171 Adam (1996) spricht von Interdependenzen, wenn das zielsetzungsgerechte Niveau einer Variablen - z.B. Marktanteil - vom Niveau einer anderen Variablen - z.B. Markteintrittsbarriere abhăngt. Vgl. Adam, D., Planung und Entscheidung: Modelle - Ziele - Methoden, 4., vollst. überarb. u. wesentlich enw. Aufl., Wiesbaden 1996, S. 175. Im Beispielfall könnte die Erreichung eines bestimmten Marktanteilsniveaus durch eine hohe Markteintrittsbarriere erschwert oder sogar verhindert werden.

172 Eine detaillierte Auseinandersetzung mit der Abstimmung gemeinsamer Aktivităten innerhalb strategischer Luftverkehrsallianzen erfolgt im anschließenden Kapitel.

173 An dieser Stelle wird bewußt auf eine weitere Untergliederung dieser Geschäftsfelddimension in Abnehmerbedürfnisse verzichtet, da der gesamthafte Eindruck im Vordergrund steht.

174 In der Luftverkehrspraxis wird i.d.R. von "Märkten" gesprochen, welche die răumliche Dimension von Start- und Zielpunkt in den Vordergrund stellen. Die kleinste Einheit eines „Marktes" stellt demnach eine Flugstrecke zwischen zwei Punkten dar. 
Europa gelang es beispielsweise Northwest Airlines und KLM, ihren Marktanteil von zusammengerechnet 7 Prozent vor der Allianz (1992) auf 11,5 Prozent nach der Allianz (1994) zu steigern. ${ }^{175}$ Eine Untersuchung des US-amerikanischen General Accounting Office kommt zu dem Ergebnis, daß Marktanteilsgewinne von Allianzen i.d.R. zu Lasten konkurrierender Fluggesellschaften gehen, ohne diesen Verdrängungseffekt jedoch quantifizieren zu können. ${ }^{176}$

Neben der Steigerung von Marktanteilen auf bestehenden Strecken streben Fluggesellschaften Marktanteilsgewinne auf neuen, bisher nicht beflogenen Flugstrekken an. Dieses Ziel steht damit in engem Zusammenhang mit dem Ziel der Markterschließung. Durch die Bündelung von Verkehrsaufkommen in einem gemeinsamen Allianz-Streckennetz ergeben sich teilweise Start-/Zielkonstellationen oder Anschlußmöglichkeiten, die eine Erschließung bisher nicht bedienter Flugstrecken ökonomisch vorteilhaft erscheinen lassen. ${ }^{177}$ Als ein Teil der strategischen Allianz zwischen Delta Air Lines und Austrian Airlines wurde z.B. die Flugverbindung zwischen Washington (D.C.) und Wien im Code Sharing etabliert, die zuvor von keiner Fluggesellschaft oder strategischen Luftverkehrsallianz angeboten worden war. Ähnliche Beispiele finden sich für die Verbindungen Houston-Rom (Continental Airlines/Alitalia) und Cincinnati-Zürich (Delta Air Lines/Swissair). ${ }^{178}$

Im Zuge eines sogenannten Drittland-Code Sharing zielen strategische Luftverkehrsallianzen auf die Erschließung von Märkten ab, die über diejenigen der Partnerländer hinausgehen. Northwest Airlines fliegt beispielsweise das Drehkreuz Amsterdam des Partners KLM an, der seinerseits Städteverbindungen nach Deutschland durch Code Share-Dienste an das Netz von Northwest anbindet. In

175 In inrem Bericht geht das US-amerikanische General Accounting Office von 1992 als "offiziellem" Startzeitpunkt der Allianz aus, da in diesem Jahr das Open Sky-Abkommen zwischen den USA und den Niederlande unterzeichnet und nur wenig spăter die Ausnahmegenehmigung von kartellrechtlichen Auflagen gewährt wurde ( Faktisch währte die Allianz bereits seit 1989 mit einer Kapitalbeteiligung von KLM an Northwest. Vgl. General Accounting Office (Hrsg.), International Aviation: Airline Alliances Produce Benefits, but Effect on Competition is Uncertain, a.a.O., S. 27 und Airline Business, No. 6, 1997, S. 67.

Vgl. General Accounting Office (Hrsg.), International Aviation: Airline Alliances Produce Benefits, but Effect on Competition is Uncertain, a.a.O., S. 30.

Zu Netzeffekten, die aus der Bündelung von Verkehrsaufkommen resultieren, vgl. z.B. Weber, G., Erfolgsfaktoren im Kerngeschäft von europăischen Luftverkehrsgesellschaften, Bamberg 1997, insb. S. 70-76. of International Airline Code Sharing, a.a.O., S. 22. 
ähnlicher Weise kann Verkehrsaufkommen aus Nachbarländern gebündelt und dann über das eigene Heimatland in das Partnerland verteilt werden. ${ }^{179}$

Strategische Luftverkehrsallianzen werden auch eingegangen, um Markteintrittsbarrieren gegenüber Wettbewerbern aufzubauen. ${ }^{180}$ Ein wichtiger Faktor, der im Sinne einer Markteintrittsbarriere den Eintritt anderer Airlines in Märkte erschwert oder verhindert, ${ }^{181}$ besteht im Aufbau sogenannter Wechselkosten für Fluggäste. ${ }^{182}$ Durch die Mitgliedschaft im Vielfliegerprogramm ${ }^{183}$ einer Fluggesellschaft kann ein Fluggast verschiedene Vorteile erlangen, die für inn ein Anreiz sind, Flüge möglichst nur bei der betreffenden Airline zu buchen. Da Allianzpartner i.d.R. ihre Vielfliegerprogramme miteinander abstimmen, brauchen Kunden bei Flügen mit Partnerfluggesellschaften nicht auf die gewohnten Vorzüge zu verzichten und können zudem schneller Meilen sammeln, auch wenn sie verschie-

179 Der erste Fall ist mit Verkehr der fünften, der zweite Fall mit Verkehr der sechsten Freiheit vergleichbar. Vgl. Beyhoff, S., Ehmer, H., Wilken, D., Code-Sharing im internationalen Luftverkehr der Bundesrepublik Deutschland, a.a.O., S. 29.

180 Der Begriff der Markteintrittsbarriere geht auf die industrieökonomische Forschung zurück, die durch Porter maßgeblich für betriebswirtschaftliche Fragestellungen der Wettbewerbsanalyse und -strategie nutzbar gemacht wurde. Gleichwohl verzichtet Porter in seinem grundlegenden Werk „Competitive Strategy“ auf eine explizite Definition; vielmehr setzt er sich mit den Quellen von Markteintrittsbarrieren auseinander. Vgl. Porter, M.E., Competitive Strategy: Techniques for Analyzing Industries and Competitors, New York, London 1980, S. 7-17.

Zur Definition von Markteintrittsbarrieren vgl. z.B. Meffert, H., Marketing-Management: Analyse, Strategie, Implementierung, a.a.O., S. 144.

182 Wechselkosten entstehen aus Sicht von Nachfragern durch den Wechsel des Anbieters einer bestimmten Leistung. Zugrunde liegt die Annahme, daß sich Geschäftsbeziehungen im Zeitablauf zu netzwerkartigen Beziehungen verfestigen (vgl. die Ausführungen zum Netzwerkansatz) und der Wechsel des Anbieters daher mit psychischem Unbehagen, aufwendigem Knüpfen neuer Beziehungen sowie der Einstellung auf verănderte Prozeßabläufe oder technische Gegebenheiten seitens des Nachfragers verbunden ist. Vgl. Porter, M.E., Competitive Strategy: Techniques for Analyzing Industries and Competitors, a.a.O., S. 10. In die neuere Literatur haben diese Überlegungen unter dem Aspekt der Kundenbindung Eingang gefunden. Vgl. z.B. Blattberg, R.C., Deighton, J., Aus rentablen Kunden vollen Nutzen ziehen, in: Harvard Business Manager, 19. Jg., Nr. 1, 1997, S. 24-32, die sich um eine Operationalisierung des Kundenbindungs-Konstruktes bemühen.

Dieses auch als "Frequent Flyer Program" oder Bonusprogramm bezeichnete Marketinginstrument besteht aus einer Kundendatenbank zu Erfassung von persönlichen und Reisedaten, einem Meilen- oder Punktesystem, mittels dessen ein moglicher Anspruch auf bestimmte Sach- oder Dienstleistungsprämien erworben werden kann, sowie einem Servicepaket, das bestimmte Zusatzdienstleistungen umfaßt. Vgl. Schmengler, H.J., Thieme, M., Die Bedeutung eines Bonusprogramms im Marketing einer Luftverkehrsgesellschaft, in: Marketing ZFP, Nr. 2, 1995, S. 130f. Petersen diskutiert den Stellenwert derartiger Programme im Rahmen strategischer Luftverkehrsallianzen. Vgl. Petersen, R., The price of loyalty, in: Airline Business, No. 8, 1997, S. 38-40. 
dene Airlines nutzen. ${ }^{184}$ Lufthansa erkennt beispielsweise das United Airlines "Mileage Plus"-Programm an, während United Airlines die "miles\&more"-Karte der Lufthansa-Kunden akzeptiert. Bei einem Wechsel zur Konkurrenz-Allianz KLM/Northwest Airlines entstünden Fluggästen demzufolge Kosten in Form von entgangenen Vorteilen, die daher den Markteintritt für $\mathrm{KLM} /$ Northwest in bestimmte Märkte erschweren oder gänzlich verhindern. ${ }^{185}$

Weiterhin können strategische Allianzen den Markteintritt von Wettbewerbern durch die gezielte Abstimmung von Preisen und Kapazitäten erschweren oder verhindern. Wenngleich die konkreten Auswirkungen strategischer Allianzen auf die Tarifstrukturen bis heute nicht eindeutig geklärt sind, stimmen Partnerfluggesellschaften unter der Prämisse kartellrechtlicher Genehmigung ihre Preis- und Konditionenpolitik in den betreffenden Geschäftsfeldern ab und bieten Flugdienstleistungen teilweise deutlich unter dem durchschnittlichen Marktpreisniveau an. ${ }^{186}$ In einem Expertengespräch kam zum Ausdruck, daß das Allianz-Ticket für umgerechnet DM 1.200,- angeboten wurde, während der reguläre IATA-Tarif bei DM 1.800,- lag. ${ }^{187}$ Grenzen sind derartigen Praktiken allerdings durch rechtliche Auflagen und das Verhalten der Wettbewerber gesetzt, die insbesondere bei hohen Preisunterschieden Einspruch bei nationalen Gerichten einlegen. ${ }^{188}$

Neben dem Aufbau zielt die Bildung strategischer Luftverkehrsallianzen auf die Überwindung von Markteintrittsbarrieren ab, indem beispielsweise Beschrän-

\footnotetext{
184 Vgl. Gallacher, J., Power to the plans, in: Airline Business, No. 8, 1997, S. 34.

Aus ökonomisch leicht nachvollziehbaren Gründen ist es für Flugreisende günstiger, das Sammeln von Meilen auf nur ein Bonussystem zu konzentrieren, da ein bestimmter Zeitraum für das Erreichen der kritischen Meilengrenze vorgegeben wird.

186 Flugtickets werden z.T. zu "Graumarkttarifen“ verkauft, welche die Tarifhoheit der IATA umgehen und daher in offiziellen Statistiken nicht erfaßt werden. Vgl. Weber, G., Erfolgsfaktoren im Kerngeschäft von europäischen Luftverkehrsgesellschaften, a.a.O., S. 108f. Ein weiteres Problem für die Untersuchung von Preiseffekten strategischer Allianzen ergibt sich aus der luftverkehrsspezifisch hohen Anzahl von Preisdifferenzierungen, die durch den Einsatz komplexer Preisoptimierungssysteme ermöglicht werden. Tarife haben im Luftverkehr oft eine geringe Zeitstabilität. Vgl. Z.B. Whitaker, R., A system approach, in: Airline Business, No. 1, 1996, S. 40ff. Vgl. Anhang 4.

188 Hinthorne (1996) zeichnet Gerichtsverfahren aufgrund von "Kampfpreisverhalten“ ausgewählter US-amerikanischer Fluggesellschaften detailliert in seinem Beitrag nach. Vgl. Hinthorne, T., Predatory Capitalism, Pragmatism, And Legal Positivism In The Airlines Industry, in: Strategic Management Journal, Vol. 17, No. 4, 1996, S. 251-270.
} 
kungen von Luftverkehrsrechten umgangen werden. ${ }^{189}$ Wenn zwischen Ländern restriktive bilaterale Abkommen existieren, die bestimmte Fluggesellschaften für die Bedienung der Flugstrecken designiert haben, ${ }^{190}$ bietet sich im Code Sharing für die nicht-designierten Airlines eine Möglichkeit, in Konkurrenz zu jenen zu treten. Einerseits können sie durch strategische Allianzen mit den designierten Fluggesellschaften am Flugverkehrsaufkommen partizipieren. Andererseits können die nicht-designierten Fluggesellschaften Nachbarländer anfliegen und mit dort ansässigen Airlines durch strategische Allianzen den Verkehr in das Zielland auf der Basis von Verkehrsrechten der dritten und vierten Freiheit aufnehmen (Drittland-Code Sharing). ${ }^{191}$

Mittels strategischer Allianzen versuchen Fluggesellschaften zudem, Zugang zu Start- und Landerechten zu erhalten, die an vielen großen Verkehrsflughäfen ein knappes Gut darstellen. Da Slots neben Luftverkehrsrechten unabdingbare Voraussetzung für den Zugang zu bestimmten Zielorten sind, bilden fehlende oder nur schwer erhältliche Start- und Landerechte eine Markteintrittsbarriere. ${ }^{192}$ Der Londoner Flughafen Heathrow beispielsweise darf nach dem derzeit gültigen Luftverkehrsabkommen im Verkehr zwischen den USA und Großbritannien nur von jeweils zwei Fluggesellschaften beider Länder angeflogen werden - United Airlines und American Airlines seitens der USA sowie British Airways und Virgin Atlantic seitens Großbritanniens. Für Delta Air Lines ergab sich aus dieser Situation eine

189 Krahn (1994) unterscheidet strukturelle und strategische Markteintrittsbarrieren, die privater (unternehmensseitiger) oder staatlicher (institutioneller) Natur sein können. Während die oben genannte Abstimmung der Vielfliegerprogramme sowie Preis- und Kapazitătsabsprachen strategisch-privat ausgeprägt sind, handelt es sich bei einer Regulierung des Marktzutritts in seinem Systematisierungsvorschlag um eine strategisch-staatliche Markteintrittsbarriere. Vgl. Krahn, H., Markteintrittsbarrieren auf dem deregulierten US-amerikanischen Luftverkehrsmarkt: Schlußfolgerungen für die Luftverkehrspolitik der Europäischen Gemeinschaft, Frankfurt am Main u.a. 1994, S. 43. Die Designierung ist i.d.R. mit der Gewăhrung von Luftverkehrsrechten der vierten oder fünften Freiheit verbunden.

191 Vgl. Beyhoff, S., Ehmer, H., Wilken, D., Code-Sharing im internationalen Luftverkehr der Bundesrepublik Deutschland, a.a.O., S. 27-30. Beide Fälle setzen voraus, daß Code SharingDienste nicht staatlich reguliert sind.

192 Grund für die Vergabe von Start- und Landerechten an Fluggesellschaften sind Kapazitätsrestriktionen seitens der Flughäfen. Slotquoten legen die maximale Anzahl an planmäßigen Flugzeugbewegungen innerhalb bestimmter Zeitintervalle fest. Die Vergabe an Fluggesellschaften richtet sich nach verschiedenen Allokationsmechanismen, von denen die sogenannte IATA-Regel (auch "grandfathering“ genannt) und das in den USA praktizierte buysell-Prinzip die wichtigste Bedeutung haben. Vgl. Langner, S.J., The Allocation of Slots in the Airline Industry: A Transaction Cost Economics Analysis, Baden-Baden 1996, S. $18 f$. 
Markteintrittsbarriere im Zielmarkt London Heathrow, welche die Fluggesellschaft durch ein Code Share-Abkommen mit Virgin Atlantic überwinden konnte. ${ }^{193}$

Weitere Ziele, die mit strategischen Luftverkehrsallianzen verfolgt werden, liegen in einer Steigerung des Bekanntheitsgrads und einer Verbesserung des Markenimage begründet. Die Bedeutung des Bekanntheitsgrades - verstanden als Anteil einer Personengesamtheit, der den Namen eines Produktes, einer Dienstleistung oder eines Unternehmens kennt - ergibt sich aus dem nachgewiesenen Einfluß desselben auf das Kaufverhalten. Demgemäß ist die Bekanntheit eines Unternehmens oder seiner Leistung Voraussetzung für den Aufbau eines Markenimage, das seinerseits präferenzbildend wirkt und einen Kaufakt zugunsten der betreffenden Leistung entscheiden kann. ${ }^{194}$ Die Verfolgung dieses Ziels im Sinne einer partnerindividuellen Zielsetzung ist nur möglich, wenn die Fluggesellschaften ihre eigene Marke ${ }^{195}$ im Rahmen der Allianz weiterführen und damit eindeutig identifizierbar sind. ${ }^{196}$ Als Beispiel ist die strategische Allianz von British Airways, American Airlines, Qantas und Canadian Airlines International zu nennen, in der jedes Unternehmen mit eigener Marke auftritt. Ein anderer Sachverhalt liegt im Fall der "Star Alliance“ vor. In dieser Allianz sind derzeit sechs Fluggesellschaften aus unterschiedlichen Ländern vereinigt, die ihre eigenen Unternehmensmarken parallel zur Allianzmarke „Star Alliance" führen. ${ }^{197}$ Daraus ergibt sich

193 Vgl. Gallacher, J., Airline Alliances: Partners for now, in: Airline Business, No. 6, 1997, S. $26 f$. und S. 65. Mittlerweile hat Virgin Atlantic diese Allianz zugunsten einer Allianz mit Continental Airlines aufgegeben.

$194 \mathrm{Vgl}$. zu diesem Wirkungszusammenhang Meffert, H., Bruhn, M., Dienstleistungsmarketing: Grundlagen-Konzepte-Methoden, mit Fallbeispielen, a.a.O., S. 144-146. Im Konzept der selektiven Markenauswahl führt ein hoher Bekanntheitsgrad dazu, daß sich die betreffende Marke im Kaufzeitpunkt bei einem großen Anteil der Konsumenten im sogenannten awareness set befindet. In Verbindung mit einem positiven Markenimage kann es gelingen, einen günstigen Rangplatz im sogenannten evoked set einzunehmen, aufgrund dessen der Konsument letztlich seine Kaufentscheidung trifft. Vgl. zu einer Darstellung des auf NarayanaMarkin zurückgehenden Modells Meffert, H., Marketingforschung und Käuferverhalten, a.a.O., S. $40 \mathrm{f}$.

195 Eine Marke kann als Vorstellungsbild von einem Produkt oder einer Dienstleistung bezeichnet werden, das in der Psyche von Konsumenten verankert ist. Das markierte Produkt oder die markierte Dienstleistung wird in einem möglichst großen Absatzraum über einen längeren Zeitraum in gleichartiger Erscheinung sowie konstanter oder verbesserter Qualităt angeboten. Vgl. Meffert, H., Marketing: Grundlagen marktorientierter Unternehmensführung, a.a.O., S. 785.

196 Fluggesellschaften fassen ihre Leistungen in der überwiegenden Zahl der Fälle unter dem Namen ihres Unternehmens zusammen, der dann als Dachmarke verwendet wird und dadurch u.a. eine Identifikationsfunktion für Fluggäste erfüllt. Zur Funktion von Marken unter besonderer Berücksichtigung von Dienstleistungsaspekten vgl. Meffert, H., Bruhn, M., Dienstleistungsmarketing: Grundlagen-Konzepte-Methoden, mit Fallbeispielen, a.a.O., S. 321.

197 Es handelt sich um Air Canada, Lufthansa, SAS, Thai Airways, United Airlines und Varig. 
die Notwendigkeit, im Zuge interaktiver Verhandlungsprozesse zu einem gemeinsamen Konsens zwischen partnerindividuellen Zielsetzungen - Erhöhung des Bekanntheitsgrades eines bestimmten Unternehmens $i$ - und einer partnerübergreifenden Zielsetzung - Erhöhung des Bekanntheitsgrades der Star Alliance - zu gelangen. ${ }^{198}$

Von strategischen Allianzen erhoffen sich einige Fluggesellschaften zudem, am Markenimage ihres Partners oder ihrer Partner partizipieren zu können. ${ }^{199}$ Unter einem Markenimage verstehen Meffert und Burmann (1996) die "Gesamtheit aller subjektiven Vorstellungen einer Person von der Marke hinsichtlich der wahrgenommenen Eigenschaften und der Eignung dieser Marke zur Befriedigung der rationalen und emotionalen Bedürfnisse des Individuums". ${ }^{200}$ Der Aufbau eines positiven Markenimage soll generell eine absatzfördernde Wirkung erzielen. Eigenschaften, die zu einem positiven Markenimage von Fluggesellschaften führen können, sind beispielsweise eine hohe Pünktlichkeit, hohe Zuverlässigkeit sowie ein zuvorkommender Umgang mit Fluggästen. Auch ein weltweites Strekkennetz sowie technische Kompetenz stellen positive Eigenschaften dar, die möglicherweise das Markenimage von Airlines prägen. ${ }^{201}$ Teile eines solchermaßen positiven Markenimage können durch einen gemeinsamen Marktauftritt von einem Allianzpartner auf den anderen im Zuge eines Marken- bzw. Imagetransfers über-

198 Die Beantwortung dieser Frage ist u.a. von Bedeutung für die Abstimmung der Marketinginstrumente im Rahmen der strategischen Allianz, da diese einen wichtigen unternehmensseitigen Parameter zur Beeinflussung von Marketingzielen wie des Bekanntheitsgrades darstellen.

199 Vgl. Beyhoff, S., Ehmer, H., Wilken, D., Code-Sharing im internationalen Luftverkehr der Bundesrepublik Deutschland, a.a.O., S. 31.

200 Meffert, H., Burmann, C., Identitătsorientierte Markenführung - Grundlagen für das Management von Markenportfolios, Arbeitspapier Nr. 100 der Wissenschaftlichen Gesellschaft für Marketing und Unternehmensführung e.V., Hrsg.: Meffert, H., Wagner, H., Backhaus, K., Münster 1996, S. 34. Gemäß dieser Definition handelt es sich beim Markenimage um ein multidimensionales Konstrukt, dessen Konzeptualisierung und Operationalisierung in Ansătzen der Markenwertforschung vertieft diskutiert werden. Vgl. weiterführend z.B. Sander, M., Die Bestimmung und Steuerung des Wertes von Marken. Eine Analyse aus Sicht des Markeninhabers, Heidelberg 1994; Sattler, H., Markenbewertung, in: Zeitschrift für Betriebswirtschaft, 65. Jg., Nr. 6, 1995, S. 663-682.

201 Weber (1997) unterscheidet bei Fluggesellschaften zwischen „Muß-Komponenten" des Markenimage wie Sicherheit, Zuverlässigkeit und Pünktlichkeit sowie "ZusatznutzenKomponenten“, die besondere Betreuungsleistungen wie beispielsweise Vielflieger-Lounges und Unterhaltungsangebote umfassen. Vgl. Weber, G., Erfolgsfaktoren im Kerngeschäft von europäischen Luftverkehrsgesellschaften, a.a.O., S. 84ff. 
tragen werden. ${ }^{202}$ Fluggesellschaften bemühen sich teilweise, das Markenimage ihrer Partnerairlines - unter Voraussetzung positiver Ausprägung - insbesondere in deren Heimatmärkten für die Stärkung des eigenen Markenimage nutzbar zu machen. ${ }^{203}$ Deutlich wird dabei die im Netzwerkansatz problematisierte Netzwerkposition, die hier in der starken Imageposition eines Unternehmens zum Ausdruck kommt und demensprechend die Handlungsmöglichkeiten desselben zu erweitern vermag. ${ }^{204} \mathrm{Zu}$ beachten ist in diesem Zusammenhang der sogenannte Herkunftslandeffekt, nach dem das Markenimage eines Unternehmens oder einer Leistung auch durch das Image des Landes mitgeprägt sein kann, aus dem das Unternehmen oder die Leistung stammen. ${ }^{205}$ Zudem ist davon auszugehen, daß das Markenimage einer Fluggesellschaft von Land zu Land variiert. ${ }^{206}$ Eine von Lufthansa im November 1995 durchgeführte Fluggastbefragung belegt, daß die Allianz zwischen United Airlines und Lufthansa zum Befragungszeitpunkt 14 Prozent der befragten US-amerikanischen Passagiere auf Transatlantikstrecken bekannt war. 74 Prozent dieser Personen urteilten, daß Lufthansa einen Markenimagegewinn durch die Allianz erfährt, während 13 Prozent von einem Markenimageverlust für

202 Vgl. z.B. Hätty, H., Der Markentransfer, Heidelberg 1989; Meffert, H., Heinemann, G., Operationalisierung des Imagetransfers, in: Marketing ZFP, Nr. 1, 1990, S. 5-10. Eine jüngere empirische Untersuchung bestătigt das Vorliegen positiver Markentransfereffekte bei strategischen Allianzen zwischen zwei Unternehmen. Vgl. Rao, A.R., Qu, L., Ruekert, R.W., Brand Alliances as Information About Unobservable Product Quality, MSI Working Paper No. 97-100, Cambridge, Mass. 1997, insb. S. 18 f.

Die Expertengespräche belegen die Relevanz dieses Ziels. Vgl. Anhang. Zudem unterstützen Dollinger, Golden und Saxton (1997) in ihrer empirischen Untersuchung die Bedeutung der Unternehmensreputation für den Auswahlprozeß von Partnern in horizontalen und vertikalen Allianzen. Vgl. Dollinger, M.J., Golden, P.A., Saxton, T., The Effect of Reputation on the Decision to Joint Venture, in: Strategic Management Journal, Vol. 18, No. 2, 1997, S. 127-140.

$\mathrm{Vgl}$. Mattsson, L.-G., Management of strategic change in a "markets-as-networks“ perspective, in: The management of strategic change, Hrsg.: Pettigrew, A.M., Oxford 1987, S. 237.

Empirische Forschungsarbeiten konnten zeigen, daß sich ein negatives Herkunftslandimage nachteilig auf den Absatz von Leistungen im Ausland auswirkt. In umgekehrter Weise können aus einem positiven Landesimage absatzfördernde Wirkungen für Leistungen von Unternehmen aus dem betreffenden Land resultieren. Vgl. z.B. Papadopoulos, N., What product and country images are and are not, in: Product-Country Images: Impact and Role in International Marketing, Hrsg.: Papadopoulos, N., Binghamton 1993, S. 3-38. Strategische Allianzen können folglich durch ausländische Fluggesellschaften genutzt werden, um eventuell bestehende Reaktanzen auf dem Heimatmarkt des Partners mittels gemeinsamen Marktauftritts abzubauen.

Nach Meffert und Burmann (1996) spannt sich zwischen den lăndermäßig unterschiedlich wahrgenommenen Markenimages ein Spannungsfeld auf, das durch ein "Management der Markenidentităt" zu überbrücken ist. Vgl. Meffert, H., Burmann, C., Identitătsorientierte Markenführung, in: International Brand Management, Dokumentationspapier Nr. 103 der Wissenschaftlichen Gesellschaft für Marketing und Unternehmensführung e.V., Hrsg.: Meffert, H., Wagner, H., Backhaus, K., Münster 1996, S. $37 f$. 
Lufthansa überzeugt waren. 8 Prozent blieben diesbezüglich unentschieden. ${ }^{207}$ Obwohl sich diese Daten auf einer relativ hoch aggregierten Ebene bewegen, lassen sie die Vermutung zu, daß sich die Allianz aus Sicht der Lufthansa über einen Transfer des positiven United Airlines-Markenimage vorteilhaft auf das Kaufverhalten auswirkt. ${ }^{208}$

Die Verbesserung des eigenen Markenimage durch die strategische Allianz setzt jedoch voraus, daß die zu erwartenden positiven Transfereffekte größer sind als die negativen. Damit ist diese partnerindividuelle Zielsetzung in hohem Maße abhängig von der Partnerfluggesellschaft oder den Partnerfluggesellschaften, da die Funktionsweise eines Transfereffekts ein Gefälle zwischen Markenimages voraussetzt. $^{209}$

Durch die Abstimmung ihrer marktgerichteten Unternehmensaktivitäten können Fluggesellschaften im Zuge strategischer Allianzen demzufolge sowohl die Mengenkomponente als auch - unter bestimmten wettbewerbsrechtlichen Bedingungen - die Preiskomponente des Umsatzes erhöhen. Das Ziel der Umsatzsteigerung als Produkt aus Mengeneinheiten und Preisen leitet sich daher aus den zuvor erörterten marktgerichteten Wirkungszielen ab.

207 Bei den Befragungspersonen handelte es sich um Fluggäste, die aus geschäftlichem Anlaß unterwegs waren und innerhalb der zurückliegenden 12 Monate mindestens einmal auf der Transatlantik-Route geflogen waren. Der Bekanntheitsgrad wurde ungestützt erfaßt. Vgl. Lufthansa AG (Hrsg.), Lufthansa Imagemonitor: Awareness and judgement of alliances between a US airline and a foreign airline, November 1995, S. 204 u. 216.

Einschränkend wirken sich der relativ geringe Bekanntheitsgrad der Allianz sowie die Beschränkung der Untersuchung auf einen bestimmten relevanten Markt auf diese Aussage aus.

Dem Anreiz für ein Unternehmen, Teile seines aus Nachfragersicht günstigeren Markenimage z.B. durch gemeinsame Kommunikationsaktivităten auf einen Partner mit einem ungünstigeren Markenimage zu übertragen, muß gemäß der oben dargestellten Koalitionstheorie ein geeigneter Beitrag gegenüberstehen, der zumeist in Verhandlungen bestimmt wird. 


\subsubsection{Unternehmensgerichtete Effizienzziele}

Unternehmensgerichtete Effizienzziele sind vor allem auf die optimale Ausnutzung vorhandener Ressourcen (Ressourceneffizienz) sowie die möglichst flexible, fehlerfreie und schnelle Abwicklung der Leistungserstellungsprozesse (Prozeßeffizienz) ausgerichtet. ${ }^{210}$ In der Ressourceneffizienz spiegelt sich die Potentialorientierung, in der Prozeßeffizienz die Prozeßorientierung von Flugdienstleistungen wider, die im Zuge strategischer Allianzen von den Partnerfluggesellschaften gemeinsam angeboten werden. ${ }^{211}$

Ansatzpunkte zur optimalen Ausnutzung von Ressourcen, die sich Fluggesellschaften im Zuge einer Allianz gegenseitig zugänglich machen, bestehen entlang der gesamten Werkette, die Unternehmen in strategisch relevante Tätigkeiten gliedert. ${ }^{212}$ Durch strategische Allianzen streben Fluggesellschaften beispielsweise die Realisierung von Kostensenkungspotentialen mittels koordinierter Beschaffungsaktivitäten in den Bereichen des Flugzeug- und Treibstoffeinkaufs sowie des Catering an. ${ }^{213}$ Durch größere Orders können tendenziell niedrigere Einstandspreise sowie günstigere Rabatte oder Boni durchgesetzt werden. Weiterhin lassen sich durch die Zusammenlegung von Bodenstationen, die dann von

210 Der Begriff der Effizienz wird in der Literatur nicht einheitlich definiert. In der Organisationstheorie wird zumeist der Effizienzbegriff im Sinne Barnards (1938, zitiert bei Schreyögg 1996) bemüht, der Organisationen als effizient bezeichnet, wenn es ihnen gelingt, bestimmte Ziele möglichst treffsicher zu erreichen ("doing things rightly ${ }^{*}$ ). Organisationen sind dagegen effektiv in dem Maße, in dem sie grundsătzlich die richtigen Maßnahmen zur Zielerreichung einsetzen („doing the right things“). Vgl. z.B. Schreyögg, G., Organisation: Grundlagen moderner Organisationsgestaltung, Wiesbaden 1996, S. 48. Schulte-Zurhausen (1995) unterscheidet zwischen der oben wiedergegebenen Ressourcen- und Prozeßeffizienz, die einen möglichst hohen Zielerreichungsgrad gewährleisten sollen. Vgl. Schulte-Zurhausen, M., Organisation, a.a.O., S. 4.

211 Im Effizienzziel tritt das okonomische Grundprinzip in beiden Ausprägungen zu Tage, d.h. ein definiertes Ziel ist mit minimalen Kosten zu erstellen - z.B. Beforderung einer vorgegebenen Anzahl Passagiere mit möglichst geringem Kapazitătseinsatz - oder mit gegebenen Kosten soll ein Maximum an Leistung erbracht werden (z.B. Steigerung der derzeitigen Kapazitätsauslastung von 73 Prozent auf 78 Prozent bei konstanten Kosten).

212 Porter (1985) schlägt die Werkette als Instrument zur systematischen Suche nach Kostensenkungs- und Differenzierungspotentialen vor, die für inn die Quelle von Wettbewerbsvorteilen bilden. Vgl. Porter, M.E., Competitive Advantage, New York, London 1985, S. 33.

213 Kosten der Finanzierung und Abnutzung von Flugzeugen haben gemäß einer empirischen Studie europäischer Fluggesellschaften einen durchschnittlichen Anteil von 8 Prozent, Treibstoffkosten einen Anteil von durchschnittlich 15 Prozent und Cateringkosten einen Anteil von 5 Prozent an den Gesamtkosten von Airlines. Vgl. Barton, C., u.a., is there a future for Europe's airlines? in: The McKinsey Quarterly, No. 4, 1994, S. 32. 
den Allianzpartnern gemeinsam genutzt werden, Miet- und Personalkosten senken. Lufthansa konnte z.B. durch die Kooperation mit United Airlines allein in den USA 44 Bodenstationen einsparen, die nun durch United Airlines betreut werden. $^{214}$

Im eigentlichen Flugbetrieb verfolgen Fluggesellschaften mit dem Eingehen strategischer Allianzen das Ziel, ihre Produktivität durch gemeinsame Code ShareFlüge zu steigern. Durch die Abstimmung von Flugplänen und Kapazitäten soweit wettbewerbsrechtlich zulässig - können Verkehrsströme so gebündelt und miteinander verbunden werden, daß der Auslastungsgrad des eingesetzten Fluggeräts, ausgedrückt als Quotient aus verkauften Sitzkilometern zu angebotenen Sitzkilometern (Produktivitätskennzahl „Sitzladefaktor"), steigt. ${ }^{215}$ Zunehmende Sitzladefaktoren auf Strecken, die von Allianzpartnern angeboten werden, scheinen die Relevanz dieses Ziels zu unterstreichen. Auf Nordatlantikrouten, die von Northwest Airlines und KLM im Rahmen ihrer Allianz beflogen werden, war Z.B. ein Anstieg des Sitzladefaktors in dem von beiden Unternehmen eingesetzten Fluggerät von 74,7 Prozent im Jahre 1993 auf 82,6 Prozent im Jahre 1995 zu verzeichnen. ${ }^{216}$ Bereits eingangs wurde kurz erwähnt, daß für die von United Airlines und Lufthansa gemeinschaftlich angebotenen 440 täglichen Code Share-Flüge seitens der Lufthansa ein um 12 Prozentpunkte gestiegener Sitzladefaktor auf den betreffenden Strecken angegeben wurde. ${ }^{217}$

214 Vgl. Klein, H., Internationaler Wettbewerb der Allianzen, a.a.O., S. 17. Bodenstationen von Fluggesellschaften übernehmen interne Aufgaben der Stationsorganisation sowie externe Aufgaben wie die Abstimmung der Airline mit dem Flughafen, der Flugsicherung, Behörden, Vertragspartnern wie Abfertigungs- und Cateringgesellschaften sowie anderen Fluggesellschaften. Vgl. Nickel, F., Stationsmanagement von Luftverkehrsgesellschaften - eine systemanalytische Betrachtung und empirische Untersuchung der StationsmanagementSysteme internationaler Luftverkehrsgesellschaften, Karlsruhe 1994, S. 28.

Vgl. Jäckel, K., Kooperationsstrategien im Linienluftverkehr vor dem Hintergrund zunehmender Integrationsentwicklung in Europa, a.a.O., S. 125-129.

Vgl. Dyer, D., Schlesinger, L., Northwest Airlines: Strategic Alliance and Strategic Position, Harvard Business School Case, Boston, Mass. 1997, S. 24. Betrachtet wurden die Streckenverbindungen Minneapolis/St. Paul-Amsterdam, Detroit-Amsterdam sowie BostonAmsterdam. Die Werte müßten jedoch den Durchschnittswerten von Fluggesellschaften auf diesen Strecken gegenübergestellt werden, um die Allianzeffekte năherungsweise isolieren zu können.

217 Klein, H., Internationaler Wettbewerb der Allianzen, a.a.O., S. 17. Dieser Wert wird an dem ohne Allianzpartner durch Lufthansa realisierten Sitzladefaktor gespiegelt und gibt dadurch mehr Aufschluß über den Allianzeffekt. 
In den Bereichen Marketing und Vertrieb, die rund 20 Prozent der Gesamtkosten von Fluggesellschaften verursachen, verfolgen Fluggesellschaften durch die Bildung von Allianzen ebenfalls die Ausschöpfung von Kostensenkungspotentialen. Zu nennen sind beispielsweise die Ausnutzung bestehender Beziehungsstrukturen des Partners zu Werbeagenturen, um günstigere Konditionen zu erhalten, sowie die Zusammenlegung von Außendienstpersonal, das mit der Wahrnehmung aller reisebüro- und firmengerichteten Aktivitäten für die Allianzpartner betreut wird. Die Verwertung lokaler Marktkompetenz durch langjährige Beziehungen zu Reisebüros und Firmenkunden wird auch kostenseitig als wichtiges Argument für die Vorteilhaftigkeit von Allianzen gesehen, da Allianzpartner auf diese Weise von niedrigeren Provisionen und bestehenden Rabattstaffeln profitieren können. ${ }^{218}$

Aus einer gemeinsamen Nutzung bestehender technischer Infrastruktur mit Partnern, die ihrerseits mit etablierten Systemen arbeiten, enwachsen neben vielversprechenden Effizienzvorteilen auch Herausforderungen in Form von informationstechnologischen Anpassungsmaßnahmen. ${ }^{219}$ Trotz Konzentrationstendenzen bei Computerreservierungssystemen greifen Allianzpartner z.T. noch auf unterschiedliche Systeme zu, die demnach Anpassungsbedarf erzeugen. Die Allianz zwischen Lufthansa und Thai Airways verzögerte sich beispielsweise um sechs Monate, da die Zusammenführung der EDV-Systeme für ein funktionsfähiges Code Sharing technische Schwierigkeiten bereitete. ${ }^{220}$ In diesem Kontext heben kritische Stimmen nachteilige Auswirkungen strategischer Allianzen auf die bestehende Prozeßeffizienz von Fluggesellschaften hervor. Demnach verursachen notwendige Abstimmungen einen hohen Koordinationsaufwand, der durch strategische, strukturelle und kulturelle sowie systembedingte Unstimmigkeiten zwischen den Partnern verschärtt werden kann. ${ }^{221}$

218 Vgl. Anhang 4.

Vgl. zu ausgewăhlten Detailproblemen Pommes, C. de, Are you IT-compatible? In: Airline Business, No. 7, 1998, S. 26ff.

Vgl. Hildebrandt, K., Thai Airways baut Kooperation mit Lufthansa und United aus und strukturiert um, in: fvw, Nr. 17, 1996, S. 49.

221

Beyhoff, S., Ehmer, H., Wilken, D., Code-Sharing im internationalen Luftverkehr der Bundesrepublik Deutschland, a.a.O., S. 32-34 sowie Bleicher, K., Der Strategie-, Struktur- und Kulturfit Strategischer Allianzen als Erfolgsfaktor, in: Wegweiser für Strategische Allianzen: Meilen- und Stolpersteine bei Kooperationen, Hrsg.: Bronder, C., Pritzl, R., Wiesbaden 1992, insb. S. 271-285. 
Diese Ausführungen lassen deutlich werden, daß sich die Grundannahme der koalitionsspezifischen Quasirente aus Sicht des einzelnen Partnerunternehmens in einem durch die Allianz erhöhten Zielerreichungsgrad ausdrückt. Dieser Effekt läßt sich beispielsweise durch den Vergleich des Bekanntheitsgrads der eigenen Marke vor und nach dem Eingehen einer strategischen Allianz messen, wenngleich ein derartiges Vorgehen mit statistischen Zurechnungsproblemen und Störgrößen behaftet ist. Als besonders schwierig erweist sich die Messung der koalitionsspezifischen Quasirente, die aus einer Bekanntheitsgradsteigerung oder Markenimageverbesserung einer Allianzmarke resultiert, da in diesem Fall Wirkungsverläufe von der Allianzmarke auf die Einzelmarke des Partners und vice versa zu erfassen sind. Einfacher stellt sich dagegen die Messung der allianzinduzierten Umsatzveränderungen dar, die anhand der entsprechend codierten Flugscheine als allianzspezifisch identifizierbar und den Partnern eindeutig zurechenbar sind. Sie bilden aus der Perspektive des einzelnen Allianzpartners eine koalitionsspezifische Quasirente, der gleichwohl die koalitionsspezifischen Investitionen gegenüberzustellen sind.

Um die mit der Bildung und dem „Betrieb“ der strategischen Allianz verfolgten Zielsetzungen im Sinne einer Maximierung der eigenen Quasirente zu erreichen, haben die beteiligten Partner-Fluggesellschaften im Hinblick auf den Absatzmarkt gemeinsame Entscheidungen zu treffen, die in der Marketingstrategie ihren Niederschlag finden.

\subsection{Marketingstrategische Ausrichtung von Luftverkehrsallianzen}

Als Leitbild einer nach „außen“ gerichteten Perspektive wird das Marketing im Sinne einer marktorientierten Unternehmensführung diskutiert, das sich im Spannungsfeld zwischen Kunden, Absatzmittlern und Wettbewerb bewegt. ${ }^{222}$ Die Festlegung der räumlichen Marktabdeckung bildet ebenso wie die strategische Positionierung gegenüber den Marktteilnehmern einen Entscheidungstatbestand

222 Meffert (1998) faßt Marketing als duales Führungskonzept auf, das sich zum einen im Marketing als gleichberechtigte Unternehmensfunktion und zum anderen im Marketing als Leitkonzept der Unternehmensführung ausdrückt, und erweitert die drei genannten Bezugsgruppen noch um die Anspruchsgruppen. Vgl. Meffert, H., Marketing: Grundlagen marktorientierter Unternehmensführung, a.a.O., S. 6. Dreher (1995) sieht die Erweiterung der Marketingorientierung von einer reinen Kundenorientierung hin zu einer zusätzlichen, parallelen Ausrichtung an Konkurrenten, Distributionsorganen und sozio-ökonomischen Faktoren, die auf den Absatzmarkt einwirken, als zentrales Verdienst des strategischen Marketing-Management an. Vgl. Dreher, A., Marketingorientierung als Unternehmensphilosophie: Phănomen und empirische Erfassung, Wiesbaden 1995, S. 117. 
innerhalb der strategischen Marketingplanung. ${ }^{223}$ Durch die Auswahl der gemeinsam zu bearbeitenden geographischen Absatzmärkte legen die Partnerunternehmen von strategischen Luftverkehrsallianzen fest, wo sie gemeinsam auftreten. Durch die strategische Positionierung bestimmen sie, wie sie gemeinsam gegenüber Kunden, Absatzmittlern und Wettbewerb auftreten. ${ }^{224}$ In operativer Hinsicht wird der Marktauftritt von Luftverkehrsallianzen schließlich durch den Einsatz der Marketinginstrumente geprägt.

\subsubsection{Räumliche Marktabdeckung}

Mit der Entscheidung, welche Flugstrecken im Sinne geographisch eindeutig definierter Verbindungen von Start- und Zielpunkten im Rahmen einer strategischen Allianz arbeitsteilig bedient werden, legen die Allianzpartner den Grad der räumlichen Marktabdeckung fest. Diese Entscheidung fällt bereits bei der Abgrenzung des strategischen Geschäftsfelds, auf das sich die Allianz ex definitione bezieht. ${ }^{225}$ Die Marktabdeckung ist unmittelbar mit den absatzmarktgerichteten Wirkungszielen verbunden, die mit der Allianz verfolgt werden. So könnte beispielsweise das Ziel einer europäischen Fluggesellschaft, neue Flugverbindungen im asiatisch-pazifischen Verkehrsmarkt zu erschließen, in der Abdeckung dieses Marktes durch einen Allianzpartner zum Ausdruck kommen, dessen Heimatmarkt in dem betreffenden Raum liegt. ${ }^{226}$

Die Bestimmung der räumlichen Marktabdeckung strategischer Luftverkehrsallianzen kann anhand einer Unterteilung des weltweiten Personenluftverkehrs in geographisch abgegrenzte Verkehrsmärkte erfolgen. Von Marktabdeckung soll im vorliegenden Fall nur dann gesprochen werden, wenn die Allianzpartner gegenseitig Code Share-Flüge in den betreffenden Märkten durchführen, da ohne die-

Meffert, H., Marketing-Management: Analyse, Strategie, Implementierung, a.a.O., S. $123 f$.

Die Definition der Positionierung findet sich in Kapitel B 1.3.2.

Vgl. Kapitel A 3., in dem der "Raumbezug" als eine Dimension der Geschäftsfeldabgrenzung erörtert wird, sowie Meffert, H., Marketing: Grundlagen marktorientierter Unternehmensführung, a.a.O., S. 228.

226

Nach der Auffassung Porters (1980) wird durch die Marktabdeckung eine Dimension der Wettbewerbsstrategie festgelegt - Gesamtmarkt vs. Nische -, auf welche die Dimensionen des von inm herausgearbeiteten Differenzierungs- und Preisvorteils ausgerichtet sein können. Er unterscheidet "industrywide" und "particular segment only" als Marktabdeckungsdimensionen (=strategic target). Vgl. Porter, M.E., Competitive Strategy: Techniques for Analyzing Industries and Competitors, a.a.O., insb. S. 38f. Als mögliche Kriterien zur Abgrenzung des Marktes erwăhnt Porter Kaufverhaltensmerkmale, technische Produktmerkmale und geographische Merkmale, ohne sich explizit für eines der genannten zu entscheiden.
} 
ses Merkmal nicht von einer gegenseitigen Anbindung der Streckennetze im engeren Sinne gesprochen werden kann.

\begin{tabular}{|l|l|l|l|l|l|l|}
\cline { 2 - 6 } \multicolumn{1}{c|}{} & \multicolumn{1}{c|}{$\begin{array}{c}\text { USA } \\
\text { Kanada } \\
44,85 \%\end{array}$} & $\begin{array}{l}\text { Lateiname- } \\
\text { rika/Karibik } \\
3,99 \%\end{array}$ & \multicolumn{1}{|c|}{$\begin{array}{c}\text { Europa } \\
25,20 \%\end{array}$} & $\begin{array}{c}\text { Afrika } \\
1,40 \%\end{array}$ & $\begin{array}{c}\text { Mittlerer } \\
\text { Osten } \\
2,44 \%\end{array}$ & $\begin{array}{c}\text { Asien/ } \\
\text { Pazifik } \\
22,12 \%\end{array}$ \\
\hline $\begin{array}{l}\text { Star } \\
\text { Alliance }\end{array}$ & $\begin{array}{l}\text { United } \\
\text { Airlines } \\
\text { Air Canada }\end{array}$ & Varig & $\begin{array}{l}\text { Lufthansa } \\
\text { SAS }\end{array}$ & & & Thai Airways \\
\hline $\begin{array}{l}\text { Allianz um } \\
\text { British } \\
\text { Airways }\end{array}$ & $\begin{array}{l}\text { American } \\
\text { Airlines } \\
\text { Canadian } \\
\text { Airlines }\end{array}$ & & $\begin{array}{l}\text { British } \\
\text { Airways }\end{array}$ & & & Qantas \\
\hline $\begin{array}{l}\text { Allianz um } \\
\text { Northwest/ } \\
\text { KLM }\end{array}$ & $\begin{array}{l}\text { Northwest } \\
\text { Airlines } \\
\text { Continental } \\
\text { Airlines }\end{array}$ & & $\begin{array}{l}\text { KLM } \\
\text { Alitalia }\end{array}$ & & & \\
\hline $\begin{array}{l}\text { Qualiflyer } \\
\text { Group }\end{array}$ & $\begin{array}{l}\text { Delta Air } \\
\text { Lines }\end{array}$ & & $\begin{array}{l}\text { Swissair } \\
\text { Austrian Airt., } \\
\text { Sabena } \\
\text { AOM, TAP }\end{array}$ & & Turkish & \\
\hline $\begin{array}{l}\text { Aillines } \\
\text { Singapore } \\
\text { Airlines }\end{array}$ & & & & & & \\
\hline
\end{tabular}

Markt durch ggs. Code Sharing abgedeckt Markt nicht durch ggs. Code Sharing abgedeckt

Die Prozentwerte geben den Mengenanteil der Fluggäste am Weltluftverkehr an. Vgl. o.V., The World Airline Report, in: Air Transport World, No. 7, 1997, S. 58.

Abb. 5: Marktabdeckung ausgewählter internationaler strategischer Allianzen

(Quelle: Gallacher, J., Airline Alliances: Partners for now, in: Airline Business, No. 6, 1997, S. 26ff. sowie aktuelle Zeitungsberichte.)

227 Die wettbewerbsrechtliche Genehmigung für die Allianz zwischen British Airways und American Airlines ist von der EU-Kommission unter Auflagen erteilt worden, hängt im endgültigen Ergebnis jedoch z.Zt. noch von den bilateralen Verhandlungen zwischen den USA und Großbritannien ab. Vgl. o.V., Vehemente Kritik an Auflagen der EU, in: Handelsblatt vom 9.7.1998, S. 14; Skapinker, M., BA-American air alliance faces a further setback, in: Financial Times vom 8.10.1998, S. 17. Neueren Berichten zufolge wird diese Allianz unter der Bezeichnung "Oneworld“ operieren und neben den oben aufgeführten Fluggesellschaften auch Cathay Pacific, Japan Airlines, Iberia, Finnair und Maersk Air sowie Dragonair, Deutsche BA und Air Liberté umfassen. Vgl. o.V., Lufffahrt: Neue weltweite Allianz, in: Spiegel Homepage, www.spiegel.de/homepage/home/link-network_wir.html vom 21.09.1998.

Die Bezeichnung "Qualiflyer Group" bezieht sich auf die Allianz zwischen allen Fluggesellschaften mit Ausnahme von Delta Air Lines. 
Die in Abbildung 5 für die derzeit bedeutendsten strategischen Allianzen wiedergegebene räumliche Marktabdeckung spiegelt deutlich den Stellenwert der Heimatmärkte der beteiligten Fluggesellschaften wider. Auf diesen haben die Airlines aufgrund politisch-rechtlicher und marktbezogener Faktoren jeweils die im Vergleich zu den Partnern stärkste Position im Allianznetzwerk inne.

Ein Vergleich der allianzspezifischen Marktabdeckung läßt zunächst eine deutliche Konzentration auf die Hauptverkehrsmärkte USA/Kanada, Europa und Asien/Pazifik erkennen, aus denen zusammen 92,17 Prozent des weltweiten Passagieraufkommens stammen. Eine solche konzentrierte Marktabdeckung ist auf seiten der Allianz um British Airways zu erkennen. Die Star Alliance ist zusätzlich zu diesen Märkten durch die Beziehungen zu ihrem brasilianischen Mitglied Varig in Lateinamerika/Karibik vertreten und erreicht damit die höchste Marktabdeckung (96,16 Prozent), so daß nahezu von einer Gesamtmarktabdeckung gesprochen werden kann. Die strategische Allianz um Singapore Airlines stellt sich dagegen als Nischen-Allianz im asiatisch-pazifischen Raum dar, während die Partner um KLM und Northwest Airlines eine selektive Marktabdeckung mit zwei geographischen Schwerpunkten verfolgen. ${ }^{229}$ Darüber hinaus sind viele Fluggesellschaften weitere bilaterale strategische Allianzen eingegangen, um aus ihrer Sicht bestehende "Lücken" in der Marktabdeckung zu schließen. Zu nennen seien exemplarisch die Allianzen zwischen Lufthansa und All Nippon Airways sowie Singapore Airlines, die Allianzen zwischen Northwest Airlines und Air New Zealand sowie zwischen KLM und Ansett Australia.

Aus Sicht einer einzelnen Fluggesellschaft, die Mitglied in einer der oben aufgeführten strategischen Allianzen ist, wird der marktbezogene Verflechtungsgrad zwischen eigenen und allianzbedingten Aktivitäten deutlich. ${ }^{230} \mathrm{Je}$ nach Höhe des

229 Die ebenfalls selektive Marktabdeckung der Swissair-Allianzen ist in der hier dargestellten Form durch jüngste Entwicklungen in Frage gestellt. United Airlines und Delta Air Lines haben am 1. Mai 1998 die Bildung einer strategischen Allianz bekanntgegeben, die bis Anfang 1999 in den USA umgesetzt und dann auch auf ausländische Streckenverbindungen ausgeweitet werden soll. Diese Partnerschaft könnte zu einer Auflösung der Allianz zwischen Swissair und Delta Air Lines führen, da Swissair in Europa in Konkurrenz zu den United-Partnern SAS und Lufthansa steht und die laterale Verflechtung von letzteren vermutlich als kritisch angesehen wird. Vgl. o.V., Trio kommt auf $80 \%$ Marktanteil, in: Handelsblatt vom 27.4.1998, S. 13.

Khanna, Gulati und Nohria (1998) verwenden den Begriff des „relative scope“, der sich unternehmensspezifisch als Maßzahl der im Rahmen der Allianz bearbeiteten Märkte im Verhältnis zu den einzeln vom Unternehmen bearbeiteten Märkten versteht. Vgl. Khanna, T., Gulati, R., Nohria, N., The Dynamics of Learning Alliances: Competition, Cooperation, and Relative Scope, a.a.O., S. 195. 
Verflechtungsgrades stellt sich für die einzelne Fluggesellschaft die Frage eines isolierten oder integrierten Marktauftritts. Liegt der marktbezogene Verflechtungsgrad beispielsweise bei 100 Prozent, d.h. alle bisher einzeln bearbeiteten Märkte werden auch im Rahmen der Allianz bearbeitet, könnte ein integrierter Marktauftritt sinnvoll erscheinen, da von relativ hohen Interdependenzen zwischen einzelunternehmens- und allianzbezogenen Aktivitäten auszugehen ist. Die Integration des eigenen Marktauftritts mit demjenigen der Allianzpartner entspräche folglich der konsequenten, marktgerichteten Umsetzung des Netzwerkgedankens. ${ }^{231}$ Im Fall eines niedrigen marktbezogenen Verflechtungsgrades wäre demgegenüber eher ein isolierter Marktauftritt zu erwägen, der Einzel- und Allianzaktivitäten stärker trennt.

Aus einer vergleichenden Analyse der Marktabdeckung geht zudem die Bedeutung des Wettbewerbs der Allianznetzwerke (Interallianz-Wettbewerb) hervor, der neben einen reinen Einzelwettbewerb der Fluggesellschaften tritt. ${ }^{232}$ Die Marktabdeckung verdeutlicht, auf welchen Märkten welche strategischen Allianzen als relevante Wettbewerber anzusehen sind. Die mittels einer strategischen Positionierung von Allianzen angestrebte Differenzierung gegenüber einzeln agierenden Airlines wird folglich um die Differenzierungsnotwendigkeit gegenüber konkurrierenden Allianzsystemen erweitert.

231 Henke (1995) beschreibt den Integrationsgrad eines Unternehmens als Ausmaß, in dem Interdependenzen durch horizontale Verflechtungen und vertikale Verknüpfungen von Unternehmensteilen berücksichtigt werden. Übertragen auf die vorliegende Problemstellung würde sich ein integrierter Marktauftritt folglich in der horizontalen Verflechtung der marktgerichteten Aktivităten der einzelnen Fluggesellschaft mit denjenigen der Partnerfluggesellschaften äußern. Vgl. Henke, N., Wettbewerbsvorteile durch Integration von Geschäftsaktivităten: ein zeitablaufbezogener wettbewerbsstrategischer Analyseansatz unter besonderer Berücksichtigung des Einsatzes von Kommunikations- und Informationssystemen (KIS), Frankfurt am Main u.a. 1995, S. 8.

Vgl. Gomes-Casseres, B., The alliance revolution: the new shape of business rivalry, Cambridge, London 1996, S. 3. 


\subsubsection{Strategische Positionierung von Luftverkehrsallianzen}

Das Konzept der Positionierung ${ }^{233}$ umfaßt im Kern zunächst die Darstellung einer aus Nachfragersicht wünschenswerten Vorstellung des Produkts oder der Dienstleistung im Sinne einer Idealposition, die sich in einem mehrdimensionalen Eigenschaftsraum abbilden läßt. Eine „ideale" Flugdienstleistung ist demnach in der Wahrnehmung des einzelnen Nachfragers durch bestimmte ideale Merkmale und deren Ausprägungen gekennzeichnet, die auch Aussagen über Preis-Leistungs-Relationen beinhalten. ${ }^{234}$ In einem solchen mehrdimensionalen Eigenschaftsraum kann daraufhin auch die Realposition einer Flugdienstleistung abgebildet werden, die von einer Fluggesellschaft angeboten wird. ${ }^{235}$ Den der Positionierung zugrunde liegenden Gedanken folgend sind die Partnerfluggesellschaften der Allianz bestrebt, mit ihrer Flugdienstleistung der aus Nachfragersicht wünschenswerten Idealposition möglichst nahezukommen ("Dominanz") und einen möglichst großen Abstand zu den nachfragerseitig wahrgenommenen Positionen der Konkurrenzangebote aufzubauen („Differenzierung“). ${ }^{236}$

Gelingt es den Unternehmen, im Zuge der strategischen Allianz bei einem für den Nachfrager wichtigen Dienstleistungsmerkmal wie beispielsweise "Verbindungsqualität von Anschlußflügen" eine wettbewerbsüberlegene Leistung anzubieten, deren Überlegenheit auch wahrgenommen wird und gegenüber den Konkurrenzangeboten für längere Zeit verteidigbar ist, liegt ein Wettbewerbsvor-

233 Die Analysetechnik der Positionierung geht auf Bass, Pessemier und Lehmann (1971) zurück und wird vor allem im Rahmen der strategischen Produkt- und Programmplanung eingesetzt. Vgl. Bass, F.M., Pessemier, E.A., Lehmann, D.R., An Experimental Study of Relationships between Attitudes, Brand Preference and Choice, Institute Paper No. 307, Krannert Graduate School of Industrial Administration, Purdue University, Layette 1971; ein knapper Überblick findet sich bei Meffert, H., Marketing: Grundlagen marktorientierter Unternehmensführung, a.a.O., S. 343-350.

Vgl. Freter, H., Marktsegmentierung, Stuttgart 1983, S. $34 f$.

235 Neben einer gemeinsamen Darstellung im Eigenschaftsraum, der von Brockhoff (1993) auch als "Produktmarktraum" oder "joint space" bezeichnet wird, ist eine getrennte Darstellung in Form einer Realproduktwahrnehmung (mperceptual map") und Idealproduktwahrnehmung („preference map“) gebräuchlich. Vgl. Brockhoff, K., Produktpolitik, 3. Aufl., Stuttgart u.a. 1993, S. 39.

236 Meffert (1994) diskutiert die Dominanz und Differenzierung als zentrale Aufgaben im Rahmen der Markenpositionierung, die bei erfolgreicher Umsetzung das zentrale Nutzenversprechen der Marke bilden. Vgl. Meffert, H., Entscheidungsorientierter Ansatz der Markenpolitik, in: Handbuch Markenartikel: Anforderungen an die Markenpolitik aus Sicht von Wissenschaft und Praxis, Hrsg.: Bruhn, M., Stuttgart 1994, S. $178 f$. 
teil vor. ${ }^{237}$ Zur Eruierung möglicher Quellen von Wettbewerbsvorteilen schlägt Porter (1985) die Zerlegung des Unternehmens in strategisch relevante Primäraktivitäten und unterstützende Aktivitäten vor, deren Ergebnis die sogenannte Wertkette ist. ${ }^{238}$ Die Wertkette soll als Analyseinstrument zur systematischen Suche nach Kostensenkungs- und Differenzierungspotentialen dienen, die für Porter die Quellen möglicher Wettbewerbsvorteile darstellen. ${ }^{239}$ Im Zuge der gemeinsamen Dienstleistungserstellung greifen die Wertketten der an einer Luftverkehrsallianz beteiligten Unternehmen ineinander. Die damit verbundenen Abstimmungsprozesse werden nachfragerseitig in Form der Reisekette wahrnehmbar und erlebbar und unterliegen damit auch der Beurteilung von Absatzmittlern, Firmenkunden und den Fluggästen, die mit den Fluggesellschaften interagieren.

Eine Übertragung dieser Überlegungen auf die marketingstrategische Ausrichtung von Luftverkehrsallianzen hat unter Berücksichtigung allgemeiner und spezieller Kritik an den Konzepten zu erfolgen. Die Anwendung des Positionierungskonzepts durch mehrere Wettbewerber gleichzeitig kann zu einer Angleichung der Marketingaktivitäten der betreffenden Fluggesellschaften führen. ${ }^{240}$ Diese Gefahr scheint gerade im oligopolistischen Markt des Luftverkehrs gegeben zu sein. Zudem ist davon auszugehen, daß die einer Kaufentscheidung zugrunde liegenden Kaufentscheidungskriterien in Abhängigkeit von den betrachteten Nachfragern stark variieren, so daß die Positionierung segmentspezifisch

237 Vgl. Porter, M.E., Competitive Advantage, a.a.O., S. 3; Ghemawat, P., Sustainable Advantage, in: Harvard Business Review, Vol. 64, No. 5, 1986, S. 53-58; Simon, H., Management strategischer Wettbewerbsvorteile, in: Wettbewerbsvorteile und Wettbewerbsfähigkeit, Hrsg.: Simon, H., Stuttgart 1988, S. 4.

Vgl. Porter, M.E., Competitive Advantage, a.a.O., S. 33. Als Zielgroße der Wertkette gibt Porter die Gewinnspanne an, die sich bekanntermaßen als Residualgröße aus Preis und Kosten ergibt. Daher ist die in der deutschen Literatur mitunter gebräuchliche Übersetzung von "value chain" als "Wertschöpfungskette“ irreführend, da sich die Wertschöpfung eines Unternehmens strenggenommen aus den Umsatzerlösen abzüglich der extern erbrachten Vorleistungen ("fremde Leistungen Dritter") errechnet. Vgl. z.B. Kreikebaum, H., Strategische Unternehmensplanung, 6., überarb. und erw. Aufl., Stuttgart, Berlin, Köln 1997, S. 136-138.

In Verbindung mit der Ausrichtung auf den Gesamt- oder Teilmarkt leitet er die Kostenführerschafts-, Differenzierungs- und Nischenstrategie als wettbewerbsstrategische Grundkonzeptionen ab. Vgl. Porter, M.E., Competitive Strategy: Techniques for Analyzing Industries and Competitors, a.a.O., S. 35ff.

240 Zur Kritik am Konzept der Positionierung vgl. auch Haedrich, G., Tomczak, T., Produktpolitik, Stuttgart u.a. 1996, S. 145. 
durchzuführen ist. ${ }^{241}$ Ein an der Positionierung geäußerter Kritikpunkt bezieht sich auf die statische Betrachtung von Ideal- und Realpositionen als "Momentaufnahme“, die um dynamische Aspekte geänderter Bedürfnisstrukturen zu ergänzen ist. Im speziellen ist bei strategischen Luftverkehrsallianzen zu prüfen, inwieweit gemeinsam angebotene Flugdienstleistungen auch als Gemeinschaftsleistung wahrgenommen und ggf. erlebt werden und ob sie sich damit als „Allianzleistung“ in einen Eigenschaftsraum einordnen lassen. Fluggesellschaften, die in einem Hauptverkehrsmarkt zugleich einzeln als auch im Rahmen der Allianz auftreten, stehen vor der Herausforderung, sich spagatähnlich mit ihrer Einzelleistung gegenüber der Allianzleistung abzugrenzen, wenn der Gedanke der Positionierung konsequent umgesetzt wird.

Im folgenden ist zu untersuchen, welche organisationalen Maßnahmen die an einer Allianz beteiligten Fluggesellschaften treffen, um dieser besonderen Herausforderung zu begegnen und um die marketingstrategische Ausrichtung der Allianz umzusetzen. Im Mittelpunkt steht dabei die möglichst effektive und effiziente Gestaltung der Beziehungen zwischen den Allianzpartnern. Die Effektivität bemißt sich aus der Eignung des Instruments „strategische Allianz" zur Erreichung der aufgezeigten Ziele, während die Effizienz den Grad der Zielerreichung angibt.

\subsection{Organisationale Gestaltung strategischer Luftverkehrsallianzen}

\subsubsection{Arbeitsteilung zwischen den Partnerfluggesellschaften}

Eine Strukturierung der Allianzbeziehungen entspringt der Notwendigkeit, die Komplexität, die sich aus der Vielzahl und Vielfalt im Allianznetzwerk zu treffender Entscheidungen und ihren Auswirkungen ergibt, handhabbar zu machen. ${ }^{242}$ In der

241 Unter "segmentspezifisch“ wird an dieser Stelle die Durchführung der Positionierung mit einem nach bestimmten Kriterien gebildeten Teilmarkt bzw. Teilsegment eines Gesamtmarkts verstanden. Es bietet sich beispielsweise an, Nachfrager mit ăhnlichen Idealanforderungen an ein Produkt oder eine Dienstleistung zu Marktsegmenten zusammenzufassen. Hierauf wird in Kapitel B 2. năher eingegangen.

242 Komplexităt wird durch den Begriff der Varietăt als Anzahl moglicher Zustănde definiert, die ein System annehmen kann. Vgl. Malik, F., Strategie des Managements komplexer Systeme, 5. Aufl., Bern 1996, S. 201. Akzeptiert man die definitorische Nahe des Systembegriffs zum Netzwerkbegriff, läßt sich diese Definition auf die vorliegende Problemstellung übertragen. Bliss (1998) unterscheidet zwischen einer Elementekomplexităt (Anzahl und Heterogenităt von Variablen, hier die Partnerunternehmen), einer Relationenkomplexităt (Verknüpfung, z.B. durch organisatorische Gestaltungsmaßnahmen) und einer dynamischen Komplexităt (Veränderlichkeit der Beziehungen, z.B. bei Austritt oder Neueintritt von Allianzpartnern). Komplexe Systeme sind in seinem Verständnis nicht "beherrschbar", vielmehr sind die komplexitätstreibenden Faktoren zu identifizieren und zu reduzieren. Vgl. Bliss, C., Integriertes 
Organisationsliteratur wird die Arbeitsteilung innerhalb einer einzelnen Organisation als Ausgangsproblem jeder organisatorischen Strukturierung angesehen. ${ }^{243}$ Verstanden als Zuteilung von Aufgaben nach Art und Menge auf mehrere Personen, ${ }^{244}$ sind bei der Übertragung dieses einzelorganisationsbezogenen Begriffsinhalts auf strategische Allianzen im Luftverkehr einige Besonderheiten zu beachten.

Innerhalb des strategischen Geschäftsfelds, auf das sich die Allianz bezieht, ist im ersten Schritt eine Zerlegung des Gesamtprozesses der Flugdienstleistungserstellung in verschiedene Teilprozesse vorstellbar. Zu diesem Zweck kann eine Reisekette herangezogen werden, die den Prozeßablauf der Flugdienstleistung aus Sicht eines Fluggastes wiedergibt. Aus jedem Teilprozeß der Reisekette ergeben sich bestimmte Aufgabenbereiche, die durch die Fluggesellschaften zu erfüllen sind. Richtet sich die Organisationsstruktur der Airlines an dem Prozeß der Flugdienstleistung aus, kann von Prozeßorganisation gesprochen werden. ${ }^{245}$ Der Teilprozeß der Information umfaßt z.B. die Zurverfügungstellung relevanter und aktueller Daten des eigenen Angebots an Reisebüros, Firmen-Reisestellen und Endnutzer. ${ }^{246}$ In einem zweiten Schritt ist das Bezugsobjekt der Arbeitsteilung festzulegen - in diesem Fall die Flugverbindung zwischen zwei geographisch eindeutig bestimmbaren Punkten. Die Durchführung einer Flugdienstleistung ist darüber hinaus auch tageszeitlich eindeutig abgrenzbar, so daß eine Flugdienstleistung auf einer bestimmten Strecke von $A$ nach $B$ mit der planmäßigen Abflugzeit $C$ und der planmäßigen Ankunftszeit $D$ das kleinste denkbare Bezugsobjekt einer Arbeitsteilung im Luftverkehr darstellt. ${ }^{247}$

Komplexitätsmanagement - Ansătze und Lösungsmöglichkeiten, Arbeitspapier $\mathrm{Nr} .115$ der Wissenschaftlichen Gesellschaft für Marketing und Unternehmensführung e.V., Hrsg.: Meffert, H., Backhaus, K., Münster 1998, S. 5 und 17.

Vgl. stellvertretend Kieser, A., Kubicek, H., Organisation, 3., völlig neu bearb. Aufl., Berlin, New York 1992, S. 74; Frese, E., Grundlagen der Organisation: Konzept - Prinzipien - Strukturen, 6., überarb. Aufl., Wiesbaden 1995, S. 6.

Vgl. Schulte-Zurhausen, M., Organisation, a.a.O., S. 116. Arbeitsteilung nach Art wird auch funktionale und Arbeitsteilung nach Menge segmentierende Arbeitsteilung genannt.

Osterloh und Frost (1996) sprechen von „structure follows process“. Vgl. Osterloh, M., Frost, J., Prozessmanagement als Kernkompetenz: wie Sie Business Reengineering strategisch nutzen können, Wiesbaden 1996, S. 31.

Zu den Kommunikationsaktivitäten von Fluggesellschaften vgl. z.B. Shaw, S., Airline Marketing\&Management, 3. Aufl., London 1990, S. 222ff.

247 Vgl. die Ausführungen von Weber, G., Erfolgsfaktoren im Kerngeschäft von europäischen Luftverkehrsgesellschaften, a.a.O., S. $55 f$. 
Werden nun die Teilprozesse der Reisekette mit den geographisch und zeitlich definierten Flugdienstleistungen, auf die sich die strategische Allianz erstreckt, kombiniert, entsteht eine Matrix (vgl. Abbildung 6), aus der die art- und mengenmäßige Arbeitsteilung hervorgehen.

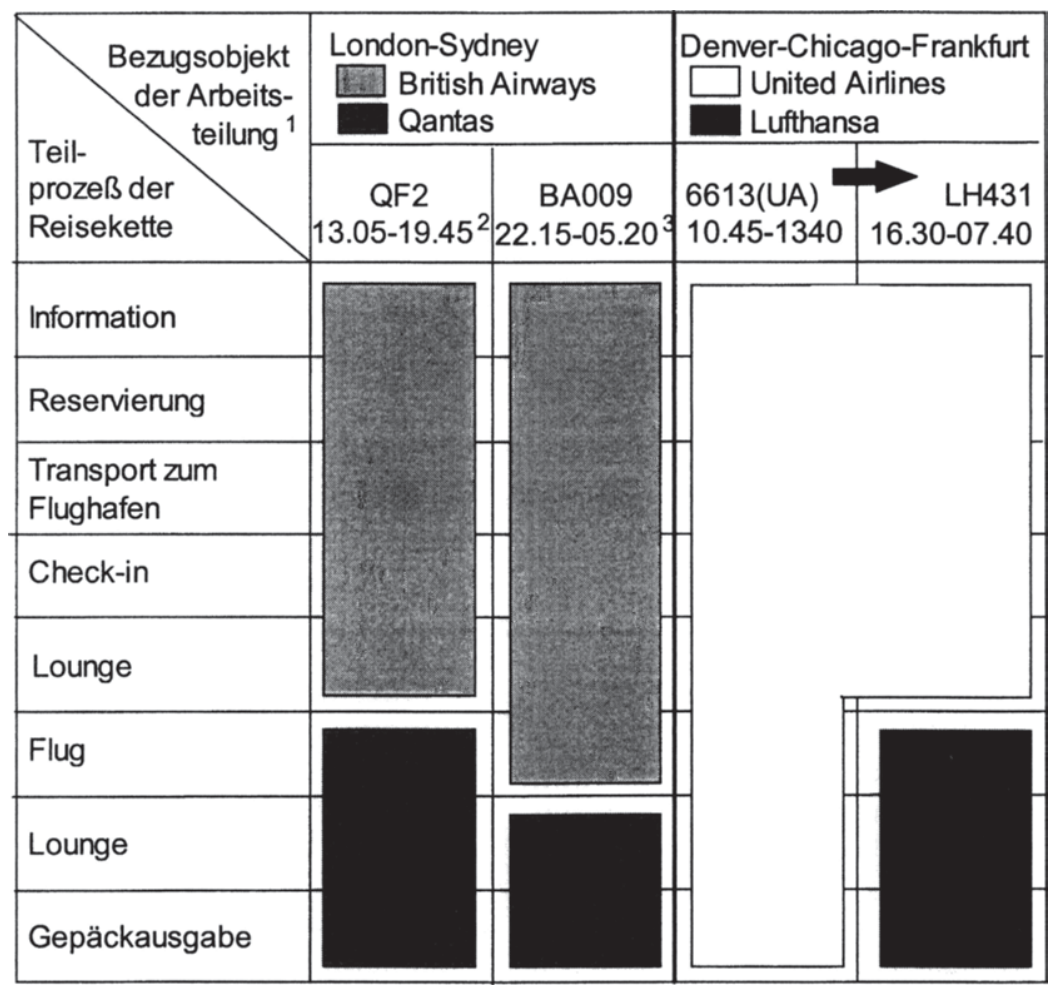

${ }^{1}$ Nur ausgewählte Verbindungen $\quad 2$ Einen Tag später $\quad{ }^{3}$ Zwei Tage spăter

\section{Abb. 6: Arbeitsteilung bei strategischen Luftverkehrsallianzen}

Unter der Verbindung zwischen London Heathrow - Sydney sind zwei Direktverbindungen aufgeführt, die von den Partnern British Airways und Qantas im Rahmen ihrer Allianz angeboten werden. Eine artmäßige Arbeitsteilung ergibt sich in den Teilprozessen "Information" bis "Lounge", die von British Airways vor dem Abflug in London durchgeführt werden, während Qantas die Teilprozesse 
"Lounge" und „Gepäckausgabe" am Ankunftsort übernimmt. ${ }^{248}$ Die mengenmäßige Arbeitsteilung wird aus dem Teilprozeß des eigentlichen Fluges ersichtlich: der $13.05 \mathrm{~h}$ - Flug wird von Qantas und der $22.15 \mathrm{~h}$ - Flug von British Airways übernommen. ${ }^{249}$ Im Flugplan erhält der British Airways-Flug die Flugnummer BA 009, während der Qantas-Flug mit QF2 bezeichnet ist. Die alphabetischen Kürzel geben den Airline-Code an und können mittels eines Abkürzungsverzeichnisses "entschlüsselt" und der jeweiligen Fluggesellschaft zugeordnet werden. ${ }^{250}$

Eine andere Form kombinierter art- und mengenmäßiger Arbeitsteilung kann aus einer Umsteigeverbindung zwischen Allianzpartnern resultieren. Lufthansa weist in ihrem Flugplan z.B. die Verbindung Denver-Frankfurt aus, die sich aus den Teilstrecken Denver-Chicago O'Hare und Chicago O'Hare-Frankfurt zusammensetzt. United Airlines führt im Sinne einer artmäßigen Arbeitsteilung die Teilprozesse von "Information" bis "Lounge" in Denver und Chicago durch. Zudem übernimmt die US-amerikanische Fluggesellschaft den eigentlichen Flug von Denver nach Chicago O'Hare mitsamt des Gepäckhandlings. Die mengenmäßige Arbeitsteilung kommt in dem von Lufthansa operierten Flug von Chicago O'Hare nach Frankfurt zum Ausdruck; am Zielort Frankfurt werden von ihr auch Loungebetreuung und Gepäckausgabe versehen. ${ }^{251}$ Im Lufthansa-Flugplan ist die Flugnummer 6613 (UA) in Verbindung mit Nr. 431 aufgeführt, und ein Fußnotenvermerk informiert über die Durchführung der Teilstrecke durch den Partner United. $^{252}$

248 Der Teilprozeß der Betreuung von Fluggästen in Lounges müßte bei getrennter Durchführung durch die Partner strenggenommen als mengenmäßige Arbeitsteilung bezeichnet werden. Die Tatsache, daß sich die Lounges jedoch in unterschiedlichen Städten und ggf. Ländern befinden, rechtfertigt insbesondere bei internationalen Streckenverbindungen aufgrund der Verschiedenartigkeit vor allem der rechtlichen und kulturellen Rahmenbedingungen die Bezeichnung „artmaßige Arbeitsteilung“.

Die Flugplaninformationen wurden dem British Airways Worldwide Timetable, gültig vom 29.03. bis 24.10.1998, S. 164 entnommen und spiegeln aus Gründen der Übersichtlichkeit nur die beiden Direktverbindungen auf der Strecke London Heathrow-Sydney wider.

Die Flugnummer QF2 ist in diesem Fall relativ ungewöhnlich, da Code Share-Verbindungen der beiden Partner im Flugplan von British Airways üblicherweise mit Flugnummer von BA7300 bis BA7499 gekennzeichnet sind. Vgl. British Airways Worldwide Timetable, S. 5. Das Abkürzungsverzeichnis für Airline-Codes findet sich auf S. 273.

Den Ausführungen liegen die Flugplaninformationen der Lufthansa zugrunde, der vom 29.03. bis zum 30.06.1998 gültig ist (S. 76). Es wurde bewußt nur ein Flug ausgewählt, um die Übersichtlichkeit zu wahren. Zeitliche Details dieser Strecke wurden bei einem großen münsterischen IATA-Reisebüro erfragt.

Der in Klammern gesetzte Airline-Code wird in einer Fußnote im Lufthansa-Flugplan zweisprachig erläutert: ") () Lufthansa Flight operated by Partner Airline " und ${ }_{n}()=$ 
Bei der vorliegenden Form der Arbeitsteilung werden die primären Geschäftsprozesse der Fluggesellschaften, d.h. die direkt am Kundennutzen orientierten Kernprozesse der Reisekette, zum zentralen Ansatzpunkt der Unternehmensorganisation. ${ }^{253} \mathrm{Da}$ diese Kernprozesse nicht an Unternehmensgrenzen enden, liegt eine Form der unternehmensübergreifenden Prozeßorganisation vor, die mit ihrer Flexibilität der z.B. durch Nachfrageschwankungen bedingten hohen Marktdynamik in besonderem Maße Rechnung trägt. ${ }^{254}$ Die Partner-Fluggesellschaften dieser Luftverkehrsallianzen bleiben Prozeß„Generalisten“, die in der Lage sind, alle Teilprozesse zu beherrschen. 255

Zwischen den Teilprozessen entstehen sowohl unternehmensbezogen als auch unternehmensübergreifend Schnittstellen, welche die Interdependenzen zwischen den Organisationseinheiten und den Allianzpartnern verdeutlichen. ${ }^{256}$ Eine mißlungene Gepäckweiterleitung durch United Airlines am Chicagoer Flughafen führt im obigen Beispiel dazu, daß der Prozeßablauf gestört wird und der betroffene Fluggast nach seiner Ankunft in Frankfurt möglicherweise eine Beschwerde an Lufthansa richtet, mit der er von Chicago nach Frankfurt geflogen ist. Das Ineinandergreifen der Wertketten, die für den Nachfrager in der Reisekette wahr-

Durchgeführt von einer Lufthansa Partner Airline“. Die Airline-Codes der Partner werden im Flugplan auf den Seiten 10-15 deutsch- und englischsprachig „entschlüsselt“.

Vgl. Picot, A., Dietl, H., Franck, E., Organisation: eine ökonomische Perspektive, Stuttgart 1997, S. 218.

254 Picot, Dietl und Frank (1997) halten die Prozeßorganisation immer dann für okonomisch sinnvoll, wenn die Flexibilitătsnachteile und Transaktionskosten einer funktionalen Arbeitsteilung ab einer bestimmten Marktdynamik die Spezialisierungsvorteile derselben übersteigen. Vgl. Picot, A., Dietl, H., Franck, E., Organisation: eine ökonomische Perspektive, Stuttgart 1997, S. 218. Spezialisierungsvorteile können z.B. in Form von Zeit- und Kostenersparnissen durch Großeneffekte sowie Übung, Erfahrung und Lernen im Zeitablauf entstehen. Als Nachteile der funktionalen Arbeitsteilung gelten z.B: die steigende Komplizierung des Informationsaustausches sowie die monotoniebedingte Arbeitsunzufriedenheit. Vgl. Bühner, R., Betriebswirtschaftliche Organisationslehre, 6., verb. und erg. Aufl., München und Wien 1992, S. 104.

Picot, Reichwald und Wigand (1996) stellen eine dynamische Zerteilung, Aufteilung und Zuteilung von Aufgaben auf hoch spezialisierte Problemlöser („kognitive Arbeitsteilung“) als ein Merkmal virtueller Organisationen heraus. Dieser Ansatz kann folglich nicht auf die vorliegende Problemstellung übertragen werden und läßt damit den in der Luftverkehrsindustrie bisweilen erhobenen Ruf nach Virtualităt zumindest derzeit verfehlt wirken. Vgl. Picot, A., Reichwald, R., Wigand, R.T., Die grenzenlose Unternehmung: Information, Organisation und Management, 2. Aufl., Wiesbaden 1996, S. 426.

$258 \mathrm{Vgl}$. allgemein zu dieser Problematik Bleicher, K., Organisation: Strategien - Strukturen Kulturen, 2., vollständig neu bearb. und erw. Aufl., Wiesbaden 1991, S. 58. 
nehmbar und ggf. erlebbar werden, erzeugt daher Koordinationsbedarf, d.h. die Ausrichtung der arbeitsteilig ausgeführten Prozesse auf ein oder mehrere übergeordnete(s) Gesamtziel(e). ${ }^{257}$ Die partnerindividuelle Verfolgung absatzmarktgerichteter Wirkungsziele und unternehmensgerichteter Effizienzziele durch Fluggesellschaften wirkt sich erschwerend auf eine Koordination der gemeinsamen Aktivitäten aus, wenn kein unternehmensübergreifend definiertes und akzeptiertes Gesamtziel existiert.

In der Literatur hat sich eine Zweiteilung von Koordinationsinstrumenten herausgebildet, die strukturelle und nicht-strukturelle Instrumente unterscheidet. ${ }^{258}$ Unter strukturellen werden wiederum personenorientierte und technokratische Koordinationsinstrumente subsumiert. ${ }^{259}$ Eine Koordination der Allianzaktivitäten über persönliche Weisungen, die ein personenorientiertes, strukturelles Instrument darstellen, ist nur dann in umfangreicher Weise möglich, wenn ein zentrales Allianzmanagement institutionalisiert wird, das mit den erforderlichen Entscheidungs- und Weisungsbefugnissen ausgestattet ist. ${ }^{260}$ Aufgrund der rechtlichen und weitgehenden wirtschaftlichen Selbständigkeit der Allianzpartner besitzen Manager einer Fluggesellschaft keine Entscheidungs- und Weisungsbefugnisse gegenüber den Managern der Partner-Fluggesellschaften, ${ }^{261}$ so daß dieses Instrument zur partnerübergreifenden Koordination hier nicht geeignet

257 Vgl. z.B. Kieser, A., Kubicek, H., Organisation, 3., völlig neu bearb. Aufl., Berlin, New York 1992, S. 95f. Im Fall der mißlungenen Gepäckweiterleitung könnte eine solche koordinierende Gesamtzielsetzung der Allianz z.B. in der gemeinsamen Verpflichtung zur Einhaltung von bestimmten Servicequalitätsstandards bestehen.

Schreyögg (1996) verwendet statt des Koordinationsbegriffs den Begriff der "Integration" und setzt sich kritisch mit Instrumenten auseinander, die der vertikalen Integration (= Abstimmung zwischen Planung und Durchführung von Aufgaben) und horizontalen Integration (= Abstimmung von Aufgaben, die auf einer organisatorischen Ebene ablaufen) dienen. Vgl. Schreyögg, G., Organisation: Grundlagen moderner Organisationsgestaltung, a.a.O., 151-201.

Vgl. Kieser, A., Kubicek, H., Organisation, a.a.O., S. 103-126; Schulte-Zurhausen, M., Organisation, a.a.O., S. 194-203; Schreyogg, G., Organisation: Grundlagen moderner Organisationsgestaltung, Wiesbaden 1996, S. 151-201; Meffert, H., Marketing: Grundlagen marktorientierter Unternehmensführung, a.a.O., S. 934-941.

Gahl, A., Die Konzeption strategischer Allianzen, a.a.O., S. 33.

Rechtliche Bestimmungen beschränken den Stimmrechtsanteil ausländischer Airlines an Fluggesellschaften in den USA beispielsweise auf maximal 25 Prozent und in Deutschland auf unter 50 Prozent. Durch Kooptation, d.h. die partielle Hereinnahme von Mitgliedern der Partnerorganisation in eigene Kontrollorgane, kann daher ein gewisser Einfluß auf die Entscheidungsfindung ausgeübt werden. Dieser dürfte aber für die kurzfristige Koordination der operativen Allianzaktivităten von eher geringer Bedeutung sein. Zur Relevanz bei Fluggesellschaften vgl. z.B. den Fall KLM/Northwest Airlines, dargestellt bei Jennings, M., Poisoned Pals? In: Airline Business, No. 7, 1996, S. 22-24. 
erscheint. ${ }^{262}$ Eine der persönlichen Weisung nahekommende Form der Koordination läßt sich jedoch bei einer Allianz feststellen, mit deren Beteiligten Expertengespräche geführt wurden. Zwischen den Partnern werden jeweils für ein Jahr gültige Entscheidungskompetenzen verteilt, die sich auf die Wahrnehmung allianzübergreifender Funktionen wie beispielsweise Streckennetzplanung, Marketingkommunikation und Produktgestaltung beziehen. Ein derartiges "Lead PartnerKonzept" soll durch die Rotation der Entscheidungskompetenzen zum einen Erfahrungswissen im unternehmensübergreifenden Allianzmanagement fördern und zum anderen dem Entstehen dauerhafter Machtungleichgewichte vorbeugen. ${ }^{263}$ Zugleich birgt dieses Konzept jedoch die Gefahr, Partneregoismen Vorschub zu leisten oder unbefriedigende Koordinationsergebnisse aufgrund mangelnder Managementkompetenz des Lead Partners entstehen zu lassen. Neben einer derartigen funktionsbezogenen Koordination haben dieselben Partner regionale Entscheidungskompetenzen definiert, die sich aus der jeweiligen Heimatmarktposition der Fluggesellschaften ergeben. ${ }^{264}$ Dieses Konzept wird in ähnlicher Weise als "Lead-Country-Konzept" in der Literatur zum internationalen Management diskutiert. ${ }^{265}$

Eine große Bedeutung für die Koordination von Allianzaktivitäten kommt der sogenannten Selbstabstimmung zu, die ebenfalls zu den personenorientierten, strukturellen Koordinationsinstrumenten zählt. Die von den Allianzentscheidungen betroffenen Organisationseinheiten innerhalb der beteiligten Fluggesellschaften stimmen sich unternehmensübergreifend unter Wahrung derselben Hierarchieebene (= horizontale Abstimmung) durch Interaktionen ab, um eine Koordination

262 Desungeachtet werden persönliche Weisungen innerhalb der jeweiligen PartnerOrganisationen zur Durchsetzung von Allianzentscheidungen als unternehmensinternes Koordinationsinstrument eingesetzt. Giesen-Netzer unterscheidet daher eine horizontale, unternehmensübergreifende und eine vertikale, unternehmensspezifische Dimension der Durchsetzung von Entscheidungen. Vgl. Giesen-Netzer, I., Implementierung von Rücknahmeund Recyclingsystemen bei Gebrauchsgütern, Frankfurt am Main u.a. 1998, S. 58.

Vgl. Anhang 3.

Vgl. Anhang 1.

Das Lead-Country-Konzept wurde 1987 erstmalig von Kreutzer im Zusammenhang mit der Steuerung von Landesgesellschaften im internationalen Kontext diskutiert. Die Auswahl einer Landesgesellschaft, die als "primus inter pares“ den Orientierungsrahmen für die Marketingaktivităten auf zuvor bestimmten internationalen Märkten festlegt, richtet sich u.a. nach ihrer Marketing-Kompetenz, der strategischen Bedeutung ihres Heimatmarktes sowie rechtlichen Bedingungen. Im Unterschied zum Fall strategischer Allianzen werden Landesgesellschaften als wirtschaftlich von ihren Muttergesellschaften abhängige Tochtergesellschaften definiert. Vgl. Kreutzer, R., Lead-Country-Konzept, in: Wirtschaftswissenschaftliches Studium, Nr. 8, 1987, S. 416-419. 
der gemeinsamen Aktivitäten zu erreichen. ${ }^{266} \mathrm{Da}$ die Beteiligung aller betroffenen Organisationsmitglieder an Zeit- und Qualifikationsrestriktionen scheitert, werden i.d.R. je nach Umfang der Allianzaktivitäten Gremien gebildet, deren Aufgabe in der Institutionalisierung allianzbezogener Koordination besteht. ${ }^{267}$ Durch die Interaktion zwischen den mit Allianzentscheidungen betrauten Managern der Partnerfluggesellschaften entstehen unternehmensübergreifende Netzwerke persönlicher Beziehungen, die durch Möglichkeiten moderner Informations- und Kommunikationstechnologien wie elektronische Post, Videokonferenzen und interaktive multimediale Systeme Unterstützung erfahren. ${ }^{268}$ Partnerfluggesellschaften bedienen sich aus leicht ersichtlichen Gründen zudem in intensiver Weise der Möglichkeit, persönliche Kontakte auch über weite Distanzen durch die Nutzung von Flugdienstleistungen zu pflegen und auszubauen. Jüngere Forschungsarbeiten zu strategischen Allianzen stellen nicht zuletzt in Anerkennung dieser Entwicklungen den Stellenwert vertrauensgeprägter Beziehungen in den Mittelpunkt ihrer Ausführungen und rekurrieren damit auf den oben dargestellten Netzwerkansatz. ${ }^{269}$

Als strukturelle, technokratische Koordinationsinstrumente werden in der Literatur vornehmlich Pläne und Programme diskutiert. ${ }^{270}$ Während Pläne koordinierende Vorgaben für eine bestimmte Periode enthalten, legen Programme bestimmte

266 Vgl. Schreyögg, G., Organisation: Grundlagen moderner Organisationsgestaltung, a.a.O., S. 169.

Vgl. Kieser, A., Kubicek, H., Organisation, a.a.O., S. $107 f$.

Vgl. Picot, A., Reichwald, R., Wigand, R.T., Die grenzenlose Unternehmung: Information, Organisation und Management, 2. Aufl., Wiesbaden 1996, insb. S. $411 \mathrm{ff}$. sowie Nitschke, U.K., Mediatisierte interne Kommunikation in internationalen Unternehmungen - Möglichkeiten und Auswirkungen von Informations- und Kommunikationssystemen für globale Netzwerkorganisationen, Bamberg 1996, insb. S. 122-151.

Vgl. Gulati, R., Alliances and Networks, in: Strategic Management Journal, Vol. 19, No. 4, 1998, S. 303; Aulakh, P.S., Kotabe, M., Sahay, A., Trust and Performance in Cross-Border Marketing Partnerships: A Behavioral Approach, in: Journal of International Business Studies, Special Issue, 1996, S. 1005-1032. Blankenburg Holm, Eriksson und Johanson (1996) bestătigen den Zusammenhang zwischen den Faktoren "commitment" und "business network connections" mit dem Kosten-/Nutzen-Verhältnis von Netzwerkbeziehungen im Rahmen ihrer empirischen Untersuchung von $n=136$ internationalen Zuliefererbeziehungen. Vgl. Blankenburg Holm, D., Eriksson, K., Johanson, J., Business Networks and Cooperation in International Business Relations, in: Journal of International Business Studies, Special Issue, 1996, insb. S. 1043 (Konstruktbeschreibung) und 1045-1047.

270 Kenter (1985) legt eine Unterteilung technokratischer Instrumente in Planung und Formalisierung vor. Planung gliedert sich bei inm wiederum in Ziel-, Maßnahmen- und Ressourcenplanung, wăhrend Formalisierung die Instrumente Programmierung, Standardisierung und Lenkpreissysteme umfaßt. Vgl. Kenter, M.E., Die Steuerung ausländischer Tochtergesellschaften: Instrumente und Effizienz, Frankfurt am Main, Bern, New York 1985, S. 105. 
Verfahren fest, die auf Dauer zur Bewältigung von Aufgaben anzuwenden sind. ${ }^{271}$ Pläne unterliegen in einer dynamischen Umwelt Störeinflüssen, zu denen beispielsweise das Ausscheiden alter oder der Eintritt neuer Partner zählen. Dennoch bedienen sich Fluggesellschaften bestimmter Vorgaben in Form quantitativer und qualitativer Sollgrößen, deren Abweichung von Istgrößen zu Korrekturmaßnahmen führt. Die kontinuierliche Auswertung von Flugscheinen gibt z.B. Auskunft über streckenbezogene Passagierzahlen und -umsätze, die anhand des AirlineCodes als einzelunternehmens- oder allianzbezogen identifiziert werden können und eine sehr kurzfristige Erkennung von Abweichungen ermöglichen. ${ }^{272}$ Die Koordination durch Programme dient bei Luftverkehrsallianzen in den Grenzen rechtlicher Rahmenbedingungen vor allem der Erzeugung eines einheitlichen Leistungsstandards, auf den sich die Partner geeinigt haben. Derartige Programme sind jedoch nur insoweit einsetzbar, als die mit innen verbundenen Anpassungskosten in einem von den Partnern als angemessen empfundenen Verhältnis zu dem daraus resultierenden Nutzen stehen.

Ein insbesondere seit Anfang der achtziger Jahre proklamiertes nicht-strukturelles Koordinationsinstrument besteht schließlich in der Schaffung einer gemeinsamen Organisationskultur, die eine Ausrichtung von Handlungen an gemeinsam getragenen Werten und Normen im Sinne von „shared values“ impliziert. ${ }^{273}$ Der eingangs der vorliegenden Arbeit kurz hervorgehobene international zu beobachtende Trend zur kulturellen Fragmentierung läßt insbesondere bei Allianzen, an denen Fluggesellschaften unterschiedlicher Nationalität beteiligt sind, die Frage nach der Eignung dieses Instruments zur Koordination von gemeinsamen Aktivitäten aufkommen. ${ }^{274}$ Dies gilt vor allem für die kurzfristige Einsetzbarkeit, ${ }^{275} \mathrm{da}$

271 Kieser, A., Kubicek, H., Organisation, 3., völlig neu bearb. Aufl., Berlin, New York 1992, S. 110 u. 114.

272 Vgl. Meffert, H., Netzer, F., Formen strategischer Netzwerke und Implikationen für das Marketing, Arbeitspapier Nr. 109 der Wissenschaftlichen Gesellschaft für Marketing und Unternehmensführung e.V., Hrsg.: Meffert, H., Backhaus, K., Münster 1997, S. 4.

Maßgeblichen Anteil an der Berücksichtigung der Organisationskultur hatte das Werk der Unternehmensberater Peters und Waterman: vgl. Peters, T., Waterman, R.H., Auf der Suche nach Spitzenleistungen. Was man von den bestgeführten US-Unternehmen lernen kann, Landsberg/Lech 1983.

Gahl (1991) setzt sich kritisch mit der Bedeutung der Unternehmenskultur für den Erfolg von strategischen Allianzen auseinander und schlägt bei ${ }_{n}$ inkompatiblen“ Kulturen der Partner eine möglichst getrennte Durchführung von Managementprozessen vor. Vgl. Gahl, A., Die Konzeption strategischer Allianzen, a.a.O., S. $49 f$. Marketing, Band 1, Hrsg.: Meyer, A., Stuttgart 1998, S. 132. 
sich eine entsprechende kulturelle „Meta-Identität" ${ }^{\text {"76 }}$ in Allianzen erst durch eine intensive, langfristige Zusammenarbeit entwickeln kann. ${ }^{277}$ Daher scheint es angemessen, das Konzept der gemeinsamen Organisationskultur im Zusammenhang mit der interaktionsorientierten Selbstabstimmung unter besonderer Berücksichtigung der Vertrauenskomponente als unterstützendes Instrument zu sehen.

Da jedes Koordinationsinstrument mit spezifischen Vor- und Nachteilen verbunden ist, setzen Allianzpartner i.d.R. eine Kombination verschiedener Instrumente i.S. eines "Koordinationsmix" ein. ${ }^{278}$ Die Gestaltung der Allianzbeziehungen über die gemeinsame Arbeitsteilung und den Einsatz von Koordinationsinstrumenten führt zu einem Gefüge aus Stellen, deren äußere Form als Konfiguration bezeichnet wird. ${ }^{279}$

\subsubsection{Konfiguration der strategischen Luftverkehrsallianz}

Aus der Konfiguration, die auch als Leitungsgliederung bezeichnet wird, geht die hierarchische Unter- und Überordnung von denjenigen Stellen hervor, die mit der Ausführung von allianzbezogenen Aktivitäten betraut sind. ${ }^{280}$ Sofern kein eigenständiges Unternehmen zur Durchführung aller allianzbezogenen Aktivitäten gegründet wird und die Leistungserstellung jeweils dezentral bei den Partnerfluggesellschaften erfolgt, handelt es sich um eine parallel zur Einzelorganisation der

276 Vgl. Meffert, H., Netzer, F., Formen strategischer Netzwerke und Implikationen für das Marketing, a.a.O., S. 10.

Unter Identităt werden hier diejenigen Eigenschaften strategischer Allianzen verstanden, die konstant bleiben, auch wenn die einzelnen Allianzmitglieder wechsein. Die Identităt manifestiert sich in geteilten Werten, Gefühlen und Überzeugungen, die aus gemeinsamen Erfahrungen und Lernen resultieren. Vgl. zu einer Diskussion des Identitătsbegriffs Meffert, H., Burmann, C., Identitătsorientierte Markenführung - Grundlagen für das Management von Markenportfolios, a.a.O., insb. S. 23-31.

Vgl. auch Schwamborn, S., Strategische Allianzen im internationalen Marketing: Planung und portfolioanalytische Beurteilung, a.a.O., S. 190.

Der Konfigurationsbegriff stammt aus der Organisationsforschung und geht ursprünglich auf Pugh u.a. (1968) zurück. Vgl. Pugh, D.S., u.a., Dimensions of Organization Structure, in: Administrative Science Quarterly, Vol. 13, No. 1, 1968, S. 65-105.

Bronder und Pritzl (1992) verstehen unter Konfiguration die Bestimmung des Kooperationsfelds und der Verflechtungsintensität sowie die Multiplikationsmöglichkeiten strategischer Allianzen und weichen damit vom "traditionellen" Begriffsverständnis im hier verwendeten Sinne ab. Vgl. Bronder, C., Pritzl, R., Ein konzeptioneller Ansatz zur Gestaltung und Entwicklung strategischer Allianzen, in: Wegweiser für strategische Allianzen: Meilen- und Stolpersteine bei Kooperationen, Hrsg.: Bronder, C., Pritzl, R., Frankfurt am Main 1992, S. 30. 
Partner bestehende Konfiguration. ${ }^{281}$ Die Stellen dieser Konfiguration werden dann mit Mitarbeitern der beteiligten Unternehmen "besetzt", welche die Allianzaktivitäten entweder zusätzlich zu ihrem bisherigen Aufgabengebiet übernehmen oder ausschließlich allianzspezifische Aufgaben durchführen. ${ }^{282}$

Eine in den Expertengesprächen erfaßte Konfiguration ist exemplarisch für den Fall von drei Allianzpartnern in Abbildung 7 dargestellt. Das Alliance Development Board setzt sich als multilaterales Gremium aus den Vorstandsvorsitzenden aller beteiligten Fluggesellschaften zusammen, entwickelt die Vision und trifft die strategischen Entscheidungen der Allianz. Diese werden auf der zweiten Ebene vom multilateralen Alliance Development Committee in umsetzbare Handlungsanweisungen überführt. Das Alliance Development Committee dient darüber hinaus als Instanz zur Klärung möglicher Konflikte, die auf Arbeitsebene entstehen. ${ }^{283}$

Neben dem multilateralen Alliance Development Committee bestehen auf der zweiten Führungsebene der strategischen Allianz sogenannte Joint Alliance Committees. Diese bilden sich aus den Beziehungen zwischen jeweils zwei Partnern, so daß bei drei Allianzpartnern rein rechnerisch drei Joint Alliance Committees existieren.

281 Vgl. Gahl, A., Die Konzeption strategischer Allianzen, a.a.O., S. 34 und Lutz, V., Horizontale strategische Allianzen: Ansatzpunkte zu ihrer Institutionalisierung, a.a.O., S. 190.

282 Die Besetzung erfolgt nicht durch die strategische Allianz, die in dem beschriebenen Fall keine eigene Rechtspersönlichkeit besitzt, sondern vielmehr in einer Art gedanklichen Konstruktion, die sich bei den jeweiligen Fluggesellschaften ggf. in einer formalisierten Modifikation oder Neuentwicklung einer Stellenbeschreibung für die betreffenden Mitarbeiter ăußert.

$283 \mathrm{Vgl}$. Anhang 1 sowie dort nicht wiedergegebene interne Informationen, die dem Verfasser in schriftlicher Form vorliegen. 


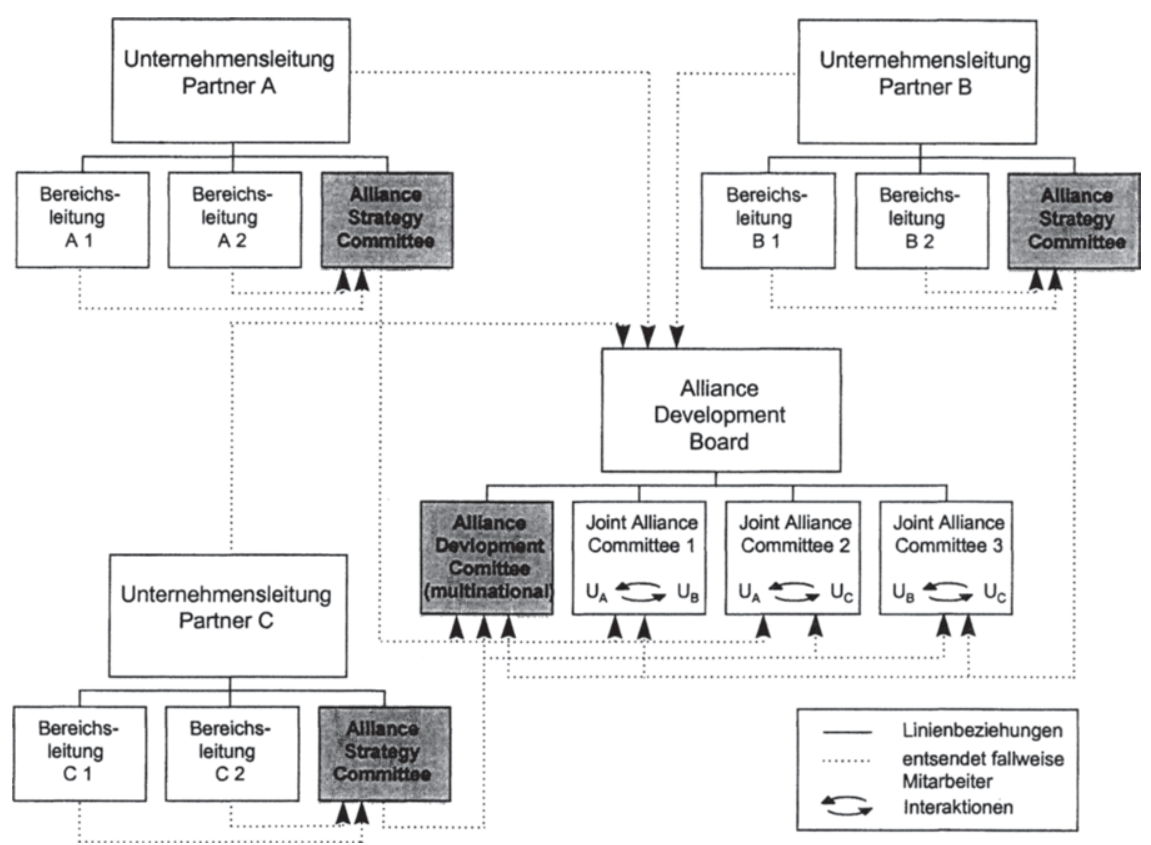

Abb. 7: Beispielhafte Konfiguration einer strategischen Allianz

Das Alliance Development Board, das Alliance Development Committee sowie die Joint Alliance Committees setzen sich jeweils - wie im oben dargestellten Fall der Swissair-Allianzen - aus Mitarbeitern der beteiligten Partnerfluggesellschaften zusammen, die fallweise an den Sitzungen teilnehmen. ${ }^{284}$ Die einzelnen Partnerfluggesellschaften bilden innerhalb ihrer eigenen Organisationen ein sogenanntes Alliance Strategy Committee. Es konstituiert sich nach Bedarf aus Mitarbeitern der zweiten (Bereichsleiter) und dritten Hierarchieebene (Hauptabteilungsleiter), ${ }^{285}$ die dann an den Sitzungen des Alliance Development Committee und der Joint Alliance Committees teilnehmen. Diesem Alliance Strategy Committee obliegen in den Einzelorganisationen die Aufgaben der Umset-

284 Nach Auskünften der Gesprächspartner richtet sich die Zusammensetzung der Meetings nach inhaltlichen Schwerpunkten, denen organisationsseitig bestimmte Zuständigkeiten gegenüberstehen. Vgl. Anhang 1 und 2.

285 Aus Gründen der Darstellbarkeit sind die Hauptabteilungen in Abbildung 6 nicht wiedergegeben. 
zung und Durchsetzung der Allianz innerhalb des Unternehmens, ${ }^{286}$ die sich u.a. in der Vorgabe von Meilensteinen und dem Monitoring der definierten Arbeitsprozesse äußern. Geleitet wird das Alliance Strategy Committee jeweils durch einen "Allianzkoordinator", der in keine weitere Linienfunktion eingebunden ist. Die Koordination und Steuerung dieser Vorgaben im Sinne einer Vermittlungsstelle zu den Fachbereichen erfolgt durch das Alliance Coordination Team (vgl. Abbildung 8), das in Form einer Matrix nach Allianzpartnern und Funktionen gegliedert ist. Es besitzt keine formalen Weisungskompetenzen, sondern setzt Entscheidungen durch Überzeugungsfähigkeit, persönliche Beziehungen sowie „geliehene Macht" von übergeordneten Linienmanagern, die am Alliance Strategy Committee beteiligt sind, gegenüber den Fachbereichen durch. ${ }^{287}$

Die Einflußmöglichkeiten der Alliance Coordination Team-Mitarbeiter zur Durchsetzung allianzbezogener Entscheidungen im Unternehmen bemessen sich zum einen aus der Bedeutung, welche die strategische Allianz für die Erfüllung der Gesamtunternehmensziele besitzt - z.B. Argumentation mit Nachweisbarkeit positiver Umsatzwirkung einer bestimmten Allianzmaßnahme für das Unternehmen -, und zum anderen aus den persönlichen Eigenschaften der Mitarbeiter wie beispielsweise Fachwissen oder Charisma. ${ }^{288}$

286 Meffert (1994) versteht unter Implementierung den Prozeß der Umwandlung theoretischer Konzepte in aktionsfăhige Aufgaben und unterteilt die Implementierung in Umsetzung und Durchsetzung. Umsetzung beinhaltet die Spezifizierung des Implementierungsobjekts und die Festlegung des Anpassungsbedarfs in der Unternehmensstruktur, -kultur und den Systemen, während Durchsetzung die Schaffung von Akzeptanz auf der Ebene der Mitarbeiter umfaßt. Vgl. Meffert, H., Marketing-Management: Analyse, Strategie, Implementierung, a.a.O., S. 362364.

Vgl. Anhang 1 sowie dort nicht wiedergegebene interne Informationen, die dem Verfasser in schriftlicher Form vorliegen.

Staehle (1994) spricht von „formalen, positionsbezogenen“ und „personalen Machtgrundlagen“, auf denen die soziale Einflußnahme beruht. Vgl. weiterführend Staehle, W.H., Management: eine verhaltenswissenschaftliche Perspektive, a.a.O., S. $377 \mathrm{ff}$. 


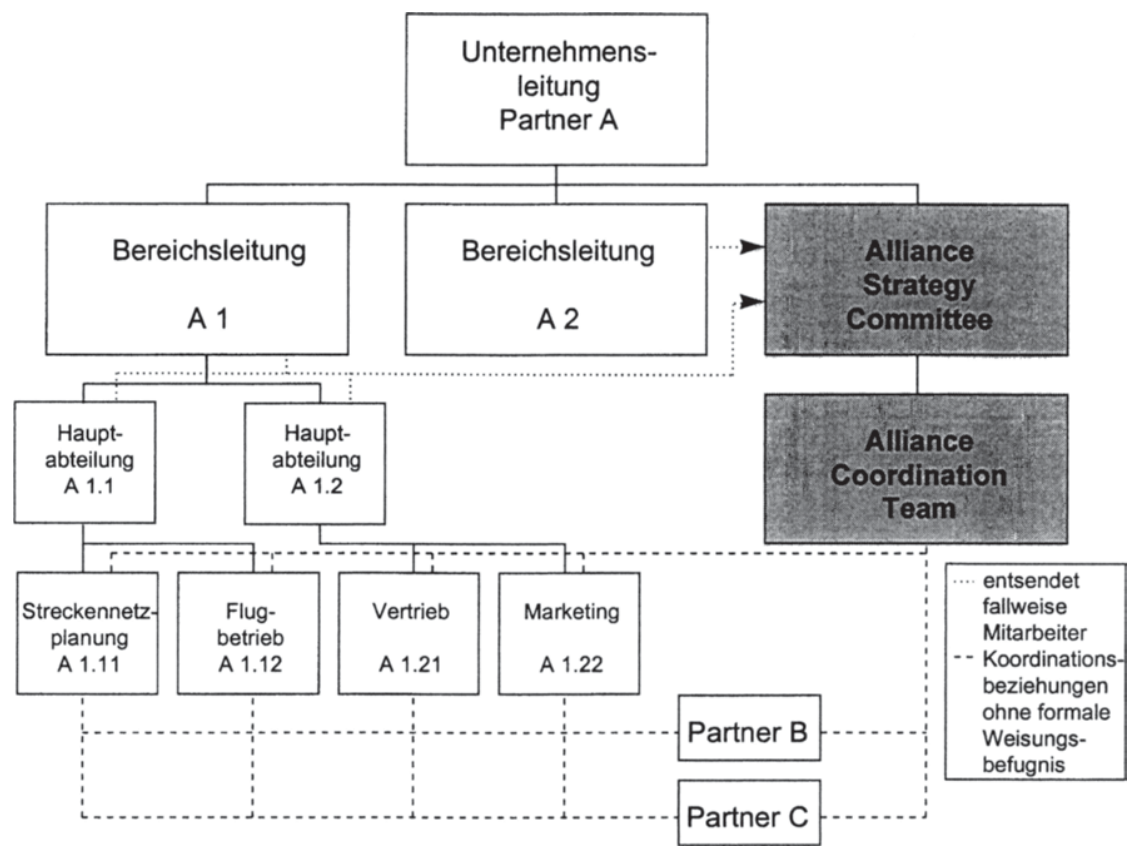

Abb. 8: Strukturelle Anbindung einer strategischen Allianz an bestehende Organisationsstrukturen

Gegenüber den Partnerfluggesellschaften besteht ein formal gleichgestelltes Verhältnis, das in Abhängigkeit von der zu treffenden Entscheidung variieren kann. Als Beispiel ist das im Rahmen der Koordinationsinstrumente erörterte Lead Partner-Konzept zu nennen, das zu einer temporären funktionsbezogenen Unterordnung der Partner unter den "Lead Partner" führt. Diese Unterordnung gründet angesichts der rechtlichen und weitgehenden wirtschaftlichen Selbständigkeit der Partner auf einem freiwilligen Verhalten, das durch die persönlichen Interaktionen zwischen den beteiligten Mitarbeitern zu tragen ist. Normative Wirkung zwischen den Allianzpartnern geht demzufolge weniger von Weisungsbefugnissen als vielmehr von vertrauensbasierten, persönlichen Beziehungen der Mitarbeiter aus. ${ }^{289}$

289 Vgl. Gulati, R., Alliances and Networks, a.a.O., S. 303. 
Die Konfiguration der hier exemplarisch erörterten Allianz läßt auf ein hohes Maß organisatorischer Komplexität schließen, ${ }^{290}$ die vor allem auf folgende Faktoren zurückgeht:

- Durch eine wachsende Zahl von Allianzpartnern steigt die Anzahl möglicher Beziehungen zwischen innen überproportional. Bei drei Partnern sind drei, bei vier bereits sechs, bei fünf Partnern zehn und bei sechs Partnern sogar fünfzehn Zweierbeziehungen möglich. ${ }^{291}$

- Je nach Problemstellung wechselt die Besetzung der Joint Alliance Committees mit Linienmanagern der Partner. Dies verlangt von den Mitarbeitern den Umgang mit flexiblen Beziehungskonstellationen auf dieser Ebene sowie zusätzlichen Ressourceneinsatz neben der Wahrnehmung übriger Aufgaben.

- Die Koordination von Entscheidungen in einer Matrix aus Partner- und Funktionszuständigkeiten erfordert von den Allianzmitarbeitern auf der Ebene des Alliance Coordination Team zugleich interne Durchsetzungsfähigkeiten, die eine fehlende fachliche Weisungskompetenz auszugleichen haben, und ein hohes Maß an unternehmensübergreifender, interkultureller Beziehungskompetenz. ${ }^{292}$ Zudem ist das Erkennen von Wirkungsinterdependenzen zwischen einzelunternehmens- und allianzbezogenen Entscheidungen bedeutsam für die Tätigkeit in diesem Gremium.

Während Arbeitsteilung und Konfiguration den Rahmen für die Durchführung der gemeinsamen Allianzaktivitäten festlegen, beziehen sich Regelungen der Ergebnisverteilung auf den „Output" dieser Tätigkeiten.

290 Zur organisationsbedingten Komplexităt vgl. Bliss, C., Integriertes Komplexitătsmanagement Ansätze und Lösungsmöglichkeiten, Arbeitspapier Nr. 115 der Wissenschaftlichen Gesellschaft für Marketing und Unternehmensführung e.V., Hrsg.: Meffert, H., Backhaus, K., Münster 1998, S. 24-26.

Zur Berechnung wird die Formel $\left[\mathrm{N}^{*}(\mathrm{~N}-1)\right] / 2$ verwendet. Vgl. Aldrich, H., Whetten, D.A., Organization-sets, action-sets, and networks: making the most of simplicity, in: Handbook of organizational design, Vol. 1, Hrsg.: Nystrom, P.C., Starbuck, W.H., Oxford u.a. 1981, S. 388.

Der interkulturelle Aspekt erweist sich als relevant, da strategische Allianzen Unternehmen miteinander verbinden können, die aus unterschiedlichen Kulturrăumen stammen. Mit den hieraus resultierenden Folgen für die Interaktion setzt sich Nitschke (1996) am Beispiel einer als Netzwerk organisierten Einzelunternehmung auseinander. Vgl. Nitschke, U.K., Mediatisierte interne Kommunikation in internationalen Unternehmungen - Möglichkeiten und Auswirkungen von Informations- und Kommunikationssystemen für globale Netzwerkorganisationen, Bamberg 1996, insb. S. 175ff. 


\subsubsection{Ergebnisverteilung zwischen den beteiligten Fluggesellschaften}

Die arbeitsteilige Erstellung von Flugdienstleistungen im Rahmen einer strategischen Allianz konfrontiert die beteiligten Fluggesellschaften mit der Frage, wie die generierten Umsatzerlöse und anfallenden Kosten auf die Partner verteilt werden sollen. Zunächst ist eine Unterscheidung in das „vermarktende Unternehmen“ und "durchführende Unternehmen“ (operating carrier) vorzunehmen. ${ }^{293}$ Das vermarktende Unternehmen verkauft die Flugdienstleistung am Absatzmarkt und erzielt für beide Partner Umsatzerlöse, während das "durchführende Unternehmen" den eigentlichen Flug durchführt und hierfür alle entstehenden Kosten für beide Partner zu tragen hat. ${ }^{294}$ Eine auf diese Weise getrennte Form der Ergebnisrealisation liegt z.B. im Fall der Flugverbindung London-Sydney, Flug-Nr. QF 2, zwischen British Airways (vermarktende Fluggesellschaft) und Qantas (durchführende Airline) vor. Qantas werden hier die Umsatzerlöse gutgeschrieben, British Airways erhält unter Umständen einen allianzinternen „Provisionsanteil“.

Die Ergebnisverteilung läßt sich in dieser verhältnismäßig einfachen Form nur für einen Punkt-zu-Punkt-Verkehr realisieren. Sobald die von den Allianzpartnern bediente Strecke Teil einer Umsteigeverbindung ist wie bei dem oben genannten Flug-Nr. 6613 (UA)/LH 431 von United Airlines und Lufthansa, in deren Zuge beide Partner einen Streckenabschnitt befliegen, sind die Umsatzerlöse nach einem anderen Verfahren aufzuteilen, da der Durchgangsflugpreis i.d.R. niedriger ist als die Summe der Teilstreckenabschnitte. ${ }^{295}$ Die Partner können folglich nicht den Normalpreis der Teilstrecken in voller Höhe verrechnen. Aus dem Verhältnis zwischen Durchgangspreis und der Summe der Teilstreckenpreise, die sich im internationalen Luftverkehr nach von der IATA festgelegten Sektoren richten, wird ein Ertragswert, auch „Prorate“ genannt, ermittelt. Mit dem Prorate kann anschließend der auf den jeweiligen Partner entfallende Umsatzwert ermittelt werden. ${ }^{296}$ Eine Beispielrechnung könnte folgendermaßen aussehen: ${ }^{297}$

293 Vgl. Gahl, A., Die Konzeption strategischer Allianzen, a.a.O., S. 35. Unterstellt wird hier eine aus zwei Unternehmen bestehende strategische Allianz. Von diesen soll zunächst aus Gründen der Übersichtlichkeit abstrahiert werden. Vgl. Pompl, W., Luftverkehr: eine ökonomische Einführung, a.a.O., S. $196 f$. 
Durchgangsflugpreis von $\mathrm{A}$ nach $\mathrm{C} \quad 1.800,-\mathrm{DM}$

Teilstrecken-/Sektorpreis A nach B 600,- DM (United Airlines)

+ Teilstrecken-/Sektorpreis B nach C 1.500,- DM (Lufthansa)

= Summe der Teilstreckenpreise $\quad 2.100,-$ DM

Prorate $=1.800: 2.100=0,857$

Umsatzanteil pro Ticket von A nach B

$0 * 0,857=514,29 \mathrm{DM}$ (United Airlines)

Umsatzanteil pro Ticket von B nach C

1.

$500 * 0,857=1.285,71 \mathrm{DM}$ (Lufthansa)

Eine getrennte Form der Ergebnisrealisation kann folglich bei einer Einzelfallbetrachtung zu einem Ungleichgewicht zwischen den Allianzpartnern führen. Selbst bei Zahlung einer Provision durch Qantas beispielsweise wäre der mit dem Code Sharing verbundene Gewinn für British Airways im obengenannten Fall wesentlich geringer als derjenige von Qantas. Über die Arbeitsteilung streben die Allianzpartner daher ein Gleichgewicht im Allianz-Netzwerk an, das durch eine kontinuierliche Gegenüberstellung von Umsatzerlösen und Kosten kontrolliert wird. Dauerhafte Abweichungen von diesem Gleichgewichtszustand werden in den Allianzgremien diskutiert und durch moralische Appelle auszuräumen versucht:

„Werden nicht vereinbarte Ungleichgewichte oder Abweichungen von Zielvereinbarungen festgestellt, wird die Methode der moral suasion angewandt, um den bzw. die Partner wieder entsprechend auszurichten. Die moral suasion muß in besonderer Weise Rücksicht auf die kulturellen Unterschiede der Partner nehmen, da gerade die Äußerung von Kritik von hoher Sensibilität ist. Wird die Abweichung eines einzel-

geringer Anteil der Flugscheine exakt berechnet und der Rest hochgerechnet wird. Vgl. Pompl, 297

W., Luftverkehr: eine ökonomische Einführung, a.a.O., S. 195-197.

Ticket- und Sektorenpreise sind hier fiktiv. 
nen Allianzpartners von den anderen Partnern in gleicher Weise diagnostiziert, erfolgt in multilateralen Gesprächen eine klare Adressierung des Problemgegenstandes, um zu einer Lösung desselben zu gelangen. ${ }^{2988}$

Aus diesen Ausführungen geht die besondere Relevanz der Ergebnisverteilung für die Stabilität strategischer Allianzen hervor. Die damit am Fallbeispiel bestätigten Überlegungen der Koalitionstheorie können als harter Faktor für die Stabilität von Luftverkehrsallianzen gewertet werden. Der ökonomische Kern einer Luftverkehrsallianz läßt sich demnach durch ein gewachsenes Beziehungsnetzwerk der Allianzpartner weder aufweichen noch gar ersetzen. Unter der Annahme, daß keiner der Allianzpartner mittel- bis langfristig weniger Gewinn aus der Partnerschaft ziehen möchte als die anderen, läßt sich als formale Bedingung für ein Gleichgewicht folgende Beziehung aufstellen:299

$\frac{\text { allianzbezogene Umsatzerlöse Partner A }}{\text { allianzbezogene Kosten Partner A }}=\frac{\text { allianzbezogene Umsatzerlöse Partner B }}{\text { allianzbezogene Kosten Partner B }}$

In der Praxis ist die Orientierung an einer derartigen Gleichung mit verschiedenen Problemen behaftet. Während die Umsatzzahlen der Partner durch Buchungssysteme mit gemeinsamem Zugriff in Verbindung mit den jeweils gültigen Preisen noch für die einzelnen Unternehmen nachvollziehbar sind, ergibt sich ein Zurechnungsproblem zwischen allianz- und nicht allianzbezogenen Kosten. Es stellt sich beispielsweise die Frage, inwieweit Degressionseffekte auf allianzinduzierte Zusatznachfrage zurückzuführen sind. Zudem erweist sich die Quantifizierung der partnerindividuellen Kosten aus Gründen der Geheimhaltung als schwierig, wenn nicht gar unmöglich. Ein weiteres Problem erwächst aus der Bewertung partnerschaftlicher Beiträge, die über Umsatz- und Kostenkomponenten hinausgehen. Ein vergleichsweise schlechteres Markenimage des Partners könnte die oben

298

298

Anhang 1.

Dieses Gleichheitsprinzip beruht auf verhaltenswissenschaftlichen Überlegungen, die sich auf der Ebene von Gruppen mit Interaktionen auseinandersetzen, die als Austauschprozesse aufgefaßt werden („Equity-Theorie“). Vgl. Staehle, W.H., Management: eine verhaltenswissenschaftliche Perspektive, a.a.O., S. 291-293. Da strategische Allianzen wesentlich durch die Beziehungen der Partnerunternehmen und deren Mitarbeiter zueinander gestaltet werden, erscheint die Übertragung dieses Gedankenguts an dieser Stelle zulässig. 
wiedergegebene Gleichung beispielsweise zuungunsten des Partners mit dem subjektiv positiveren Markenimage verändern. ${ }^{300}$

Eine gemeinsame Form der Ergebnisrealisation liegt dagegen vor, wenn Allianzpartner vereinbaren, sowohl Umsatzerlöse als auch Kosten des gemeinsam vermarkteten und betriebenen Streckennetzes in einem bestimmten Verhältnis zu teilen. Im Luftverkehr wird dann von einem "revenue/cost-pool" gesprochen, ${ }^{301} \mathrm{der}$ z.B. von zwei Fluggesellschaften, die in Expertengesprächen befragt wurden, im Rahmen einer Allianz vereinbart worden war. Die in diesem Fall im Verhältnis von 50:50 erfolgende Verteilung der Umsätze und Kosten auf die Partnerunternehmen setzt ein hohes Maß an gegenseitigem Vertrauen voraus („open book policy“). Die Allianzpartner gewähren sich Zugang zu Kosten- und Erlösinformationen, ohne eine eigenständige Gesellschaft zu gründen, und ermitteln auf dieser Basis das gemeinsame Ergebnis, das zu gleichen Teilen auf die Unternehmen verteilt wird. ${ }^{302}$ Eine solche Vereinbarung kann auch als vertragliches (=Contractual) Joint Venture bezeichnet werden, in dem grundsätzlich auch andere Verteilungsschlüssel denkbar sind. ${ }^{303}$ Voraussetzung für die Ergebnisverteilung in dieser Form ist jedoch eine kartellrechtliche Genehmigung. ${ }^{304}$

Von der Ergebnisverteilung sowie von der zuvor problematisierten Arbeitsteilung und indirekt auch von der Konfiguration strategischer Luftverkehrsallianzen sind

300 Die unter den absatzmarktgerichteten Wirkungszielen wiedergegebenen Marktforschungsergebnisse zur Bedeutung der Markenimageverbesserung als partnerindividuelles Ziel belegen die Relevanz dieser Überlegung. Zum Problem der Markenbewertung vgl. Sattler, H., Markenbewertung, in: Zeitschrift für Betriebswirtschaft, 65. Jg., Nr. 6, 1995, S. 663ff. sowie ausführlich Sander, M., Die Bestimmung und Steuerung des Wertes von Marken. Eine Analyse aus Sicht des Markeninhabers, Heidelberg 1994.

Pool-Abkommen bildeten bis zum Einsetzen der Deregulierung und Liberalisierung die wichtigste Form der (staatlich induzierten) Kooperation zwischen Fluggesellschaften. Die Festlegung von Anteilen an Umsatzerlösen und/oder Kosten hatte die Marktaufteilung bestimmter Strecken im Rahmen bilateraler Abkommen zum Inhalt. Vgl. Jäckel, K., Kooperationsstrategien im Linienluftverkehr vor dem Hintergrund zunehmender Integrationsentwicklung in Europa, a.a.O., S. 71-73.

Vgl. Anhang 3.

Schwamborn (1994) diskutiert den Aspekt der gemeinsamen Ergebnisrealisation im Zusammenhang mit kapitalbasierten (=Equity) Joint Ventures, deren Kapitalbeteiligungsverhältnis zugleich den Verteilungsschlüssel für den gemeinsamen Erfolg angibt. Vgl. Schwamborn, S., Strategische Allianzen im internationalen Marketing, a.a.O., S. 178f. Vgl. auch Eisele, J., Erfolgsfaktoren des Joint-Venture-Management, Wiesbaden 1995, insb. S. $13 f$.

Vgl. z.B. Reckewerth, S., Die Zusammenarbeit der Luftverkehrsgesellschaften nach europäischem und US-amerikanischen Recht, a.a.O., S. $287 \mathrm{ff}$. 
schließlich auch Absatzmittler, Firmenkunden und Endnutzer von Flugdienstleistungen betroffen. Die Ergebnisverteilung kann sich beispielsweise in sinkenden aber auch steigenden - Flugscheinpreisen ausdrücken, die Arbeitsteilung ist an den Kontaktpunkten der Flugdienstleistung unmittelbar erlebbar und die Qualität der Leistung läßt Rückschlüsse auf die Konfiguration im backoffice-Bereich der Allianz zu. Daher gilt es im folgenden, die Auswirkungen strategischer Allianzen auf Absatzmittler, Firmenkunden und Endnutzer zu untersuchen. 


\section{Strategische Luftverkehrsallianzen im Spannungsfeld zwischen End- nutzern, Absatzmittlern und Wettbewerbern}

\subsection{Strategische Luftverkehrsallianzen aus Sicht des vertikalen Marketing}

Fluggesellschaften können durch die Festlegung der vertikalen Absatzkanalstruktur bestimmen, wie lang der Absatzweg zwischen ihnen und den Endnutzern ist (vgl. Abbildung 9). ${ }^{305}$ Eine Zwischenschaltung von Absatzmittlern führt zu längeren, indirekten Absatzwegen. ${ }^{306}$ Reiseveranstalter beispielsweise kombinieren Flugdienstleistungen mit anderen Teilleistungen wie Hotelübernachtungen und Freizeitangeboten und setzen diese als Urlaubsreise an Endnutzer ab. Consolidators kaufen im Sinne von Zwischenhändlern Kapazitäten von Charterfluggesellschaften ein, um sie dann über Reiseveranstalter, Reisebüros oder direkt an Endnutzer weiterzuveräußern. Andere Fluggesellschaften fragen Flugdienstleistungen im Rahmen von strategischen Allianzen, Interline-Abkommen oder über Generalverkaufsagenturen nach. Als branchenfremde Unternehmen agieren beispielsweise Mietwagengesellschaften oder Hotels, bei denen Flugscheine gebucht werden können. Eigene Verkaufsbüros treten wie Reisebüros auf und verkaufen schwerpunktmäßig das Angebot derjenigen Fluggesellschaft, in deren Eigentum sie sich befinden. ${ }^{307}$

Da die Endnutzer von Flugdienstleistungen insbesondere in den Teilprozessen der Information und Reservierung überwiegend indirekt mit den Fluggesellschaften interagieren, erscheint es notwendig, zunächst die zwischengeschalteten Institutionen in die folgenden Überlegungen einzubeziehen, bevor auf die Endnutzerperspektive eingegangen wird.

305 Zur Systematisierung von Absatzkanälen vgl. stellvertretend Ahlert, D., Distributionspolitik. Das Management des Absatzkanals, 3. Aufl., Stuttgart 1996, S. $153 f$.

306 Die Trennung zwischen indirektem und direktem Vertrieb beruht auf dem Kriterium des Kontaktes zwischen Anbieter und Endnutzer (unmittelbar vs. mittelbar) und läßt gewisse Auslegungsspielräume offen. Die Übergänge zwischen unternehmenseigenen Verkaufsbüros und den aufgeführten Formen des Direktvertriebs sind fließend, da die Unmittelbarkeit des Kontaktes auch subjektivem Empfinden unterliegt. Das Kriterium des Eigentums des Anbieters an den Absatzmittlerorganisationen führt zur Unterscheidung in indirekten Fremd- und Eigenvertrieb. Zur Entscheidung über die Auswahl der Absatzkanäle vgl. Meffert, H., Marketing: Grundlagen marktorientierter Unternehmensführung, a.a.O., S. 602-608.

Vgl. Pompl, W., Luftverkehr: eine ökonomische Einführung, a.a.O., S. 173ff. 


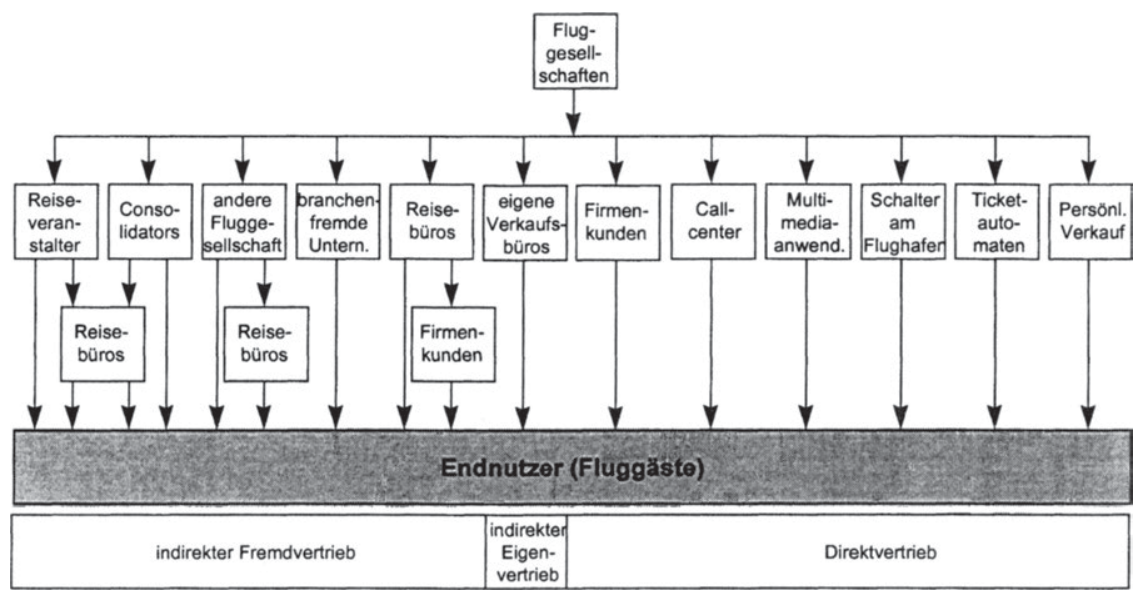

Abb. 9: Vertikale Absatzkanalstruktur im Personenluftverkehr

(Quelle: in Anlehnung an: Sterzenbach, R., Luftverkehr: betriebswirtschaftliches Lehr- und Handbuch, München, Wien 1996, S. 231.)

Dies gilt um so mehr, als Fluggesellschaften und Allianzen ihre Marketingaktivitäten im Sinne eines vertikalen Marketing ${ }^{308}$ auch an den Nutzenerwartungen der Absatzmittler ausrichten und damit indirekt Einfluß auf die endnutzerseitige Wahrnehmung, das Erleben und die Beurteilung des Angebots nehmen. Von besonderer Relevanz für den Kontakt und das situative Umfeld im Verlauf der ersten beiden Teilprozesse der Reisekette erweisen sich die Reisebüros, über die ein großer Anteil aller Flugbuchungen realisiert wird, ${ }^{309}$ sowie die Firmenkunden, die über Firmen-Reisestellen ${ }^{310}$ oder Reisebüros

308 Vertikales Marketing wird nach Meffert (1975) als die "über alle Distributionsstufen hinweg koordinierte Steuerung und Regelung marktgerichteter Unternehmensaktivitäten “ verstanden. Meffert, H., Vertikales Marketing und Marketingtheorie, in: Konflikt und Kooperation in Absatzkanälen: ein Beitrag zur verhaltensorientierten Marketingtheorie, Hrsg.: Steffenhagen, H., Wiesbaden 1975, S. 15.

309 In den USA machten Buchungen von Flugdienstleistungen über Reisebüros von 1984 bis 1994 etwa 85 Prozent aller Buchungen aus, wăhrend dieser Wert zwischen 1995 und 1997 auf etwa 80 Prozent gesunken ist. Trotz einer Zunahme des Direktvertriebs sind Reisebüros noch immer von zentraler Bedeutung für den Vertrieb von Flugdienstleistungen. Vgl. McGee, W.J., Reshaping the Relationship, in: Air Transport World, No. 12, 1997, S. 57. Die Deutsche Lufthansa AG realisiert ca. 95 Prozent aller Buchungen über den indirekten Vertriebsweg. Vgl. Spielberger, M., Reisebüro-Vertrieb wird an seinem Nutzen gemessen, in: fvw, Nr. 23, 1997, S. 12.

310 Reisestellen sind organisatorische Einrichtungen von Unternehmen oder bffentlichen Institutionen, die für Mitarbeiter Reisedienstleistungen vermitteln, beschaffen und selbst 
unternehmensspezifisches Geschäftsreisevolumen ihrer Mitarbeiter bündeln und von hoher wertmäßiger Bedeutung für Fluggesellschaften sind. ${ }^{311}$

\subsubsection{Auswirkungen strategischer Luftverkehrsallianzen auf die Beziehun- gen zu Reisebüros}

Reisebüros sind Unternehmen, deren Zweck in der Vermittlung von reise- und freizeitrelevanten Produkten und Dienstleistungen besteht. ${ }^{312}$ Hierzu zählen vor allem Reiseveranstaltungen (z.B. Pauschalreiseangebote), Hotels, Flugdienstleistungen, Bahnreisen, Mietwagen, Veranstaltungstickets (z.B. Theaterkarten) und Reiseliteratur. Für die Vermittlung von Flugdienstleistungen erhalten sie von den betreffenden Fluggesellschaften Provisionszahlungen, deren Betrag sich i.d.R. als Prozentsatz vom fluggesellschaftsspezifischen Umsatz berechnet. 1995 belief sich die durchschnittliche Provisionshöhe für Flüge innerhalb der USA beispielsweise auf 9,17 Prozent und sank 1996 auf 8,57 Prozent, während die Provisionen für internationale Flüge im selben Zeitraum von durchschnittlich 16,24 auf 15,37 Prozent fielen. ${ }^{313}$ Darüber hinaus gewähren viele Fluggesellschaften Rabatte an Reisebüros, deren Höhe von dem jeweils mit innen realisierten Umsatzvolumen abhängt, um das Empfehlungsverhalten der Reisebüros zu ihren eigenen Gunsten zu beeinflussen. ${ }^{314} \mathrm{Im}$ Gegenzug übernehmen die Reisebüros alle Vermitt-

erstellen. Vgl. Deiß, G., Aufgaben und Gestaltungsprobleme betrieblicher Reisestellen, in: Betriebswirtschaftliche Studien zum Personenverkehr und Tourismus, Hrsg.: Brauer, K.M., Berlin 1985, S. 83. Die Begriffe "Reisestelle" und "Firmen-Reisestelle werden im folgenden synonym verwendet.

Der Stellenwert von Firmenkunden kann exemplarisch an dem Verband Deutsches Reisemanagement verdeutlicht werden: $\mathrm{Er}$ repräsentiert nach eigenen Angaben 300 Wirtschaftsunternehmen, die jährlich jeweils einen Flugscheinumsatz in zweistelliger Millionenhöhe aufweisen. Vgl. Haas, S., Jetzt sind auch noch die Firmenkunden sauer, in: fvw, Nr. 27, 1997, S. 8. Die Bedeutung des Geschäftsreisevolumens geht auch aus einer Studie von United Airlines hervor, nach der nur 9 Prozent aller Fluggäste für 44 Prozent des Gesamtumsatzes verantwortlich zeichnen. Diese Gruppe reist aus geschäftichem Anlaß und überträgt die Buchungsabwicklung i.d.R. auf Firmen-Reisestellen oder Reisebüros. Vgl. Flint, P., Will the real United please stand up?, a.a.O., S. 31.

312 Vgl. z.B. Freyer, W., Tourismus: Einführung in die Fremdenverkehrsökonomie, 3., erg. und aktualisierte Aufl., München, Wien 1991, S. 41.

$313 \mathrm{Vgl}$. Levere, J., Agents of change, in: Airline Business, No. 8, 1997, S. 52. Auch in Europa ist insbesondere seit Anfang 1997 eine Tendenz zur Senkung der Provionshöhe zu beobachten. Die Fluggeselischaften Ryanair (Irland), KLM sowie SAS streben eine Senkung von 9 auf 7,5 Prozent an oder haben diese bereits umgesetzt. Vgl. Jones, L., Euro agents fight change, in: Airline Business, No. 5, 1997, S. 18.

Die Deutsche BA beispielsweise gewährt den Reisebüros neben den 5 Prozent Provisionen zusătzlich 2 Prozent Rabatt, wenn das Umsatzvolumen des Vorjahres erreicht wird. Vgl. Haas, S., Auch die Briten halten die Reisebüros jetzt kurz, in: fww, Nr. 27, 1997, S. 11 (in dieser Form 
lungsfunktionen, die an der Schnittstelle zwischen Fluggesellschaft und Endnutzer anfallen. Hervorzuheben sind die Information und Beratung von Kunden sowie die buchungstechnische Abwicklung von Flugdienstleistungen.

Aus solchen Beziehungen können entsprechend dem netzwerktheoretischen Gedankengut langfristige Bindungen zwischen Fluggesellschaften und Reisebüros erwachsen. Das Spannungsfeld zwischen Anreizen und Beiträgen kann jedoch zugleich in Diskussionen um die Höhe der Provisionen und Rabatte sowie um die Bewertung der Reisebüroleistungen ihren Niederschlag finden. Um Aufschluß über die Bewertung der Reisebüroleistungen zu gewinnen, wurde 1997 eine Prozeßkostenanalyse in 15 deutschen Reisebüros mit IATA-Lizenz ${ }^{315}$ durchgeführt. Für den Bereich der Vermittlung von Flugdienstleistungen lassen sich folgende Ergebnisse zusammenfassen: ${ }^{316}$

- Die Vermittlungstätigkeiten für Flugdienstleistungen nehmen 76,0 Prozent, Bahnfahrten 20,0 Prozent und Hotels/Mietwagen 4,0 Prozent des gesamten Vermittlungszeitvolumens in den Reisebüros ein.

- Innerhalb des Vermittlungsvorgangs für eine Flugdienstleistung verteilt sich der Zeitaufwand wie folgt: Buchung/Flugscheinausstellung 66,0 Prozent, Flugauskünfte 20,1 Prozent, Umbuchungen von Flugscheinen 7,2 Prozent, Rückerstattung 5,5 Prozent und Stornierungen 1,2 Prozent.

- Die Gesamtprozeßkosten einer einzelnen Flugbuchung belaufen sich bei Betrachtung der reinen Prozeßzeit auf 1,52 DM pro Minute. Zusatztätigkeiten und Warte-/Bereitschaftszeit erhöhen diesen Wert auf 3,22 DM pro Minute (Vollkostenbasis).

- Die durchschnittlichen Gesamtkosten einer Flugbuchung liegen bei 35,53 DM (reine Prozeßkosten) und bei 53,83 DM, wenn auf Vollkostenbasis gerechnet wird.

scheint ein ${ }_{n}$ Anreizsystem ${ }^{4}$ allerdings nur bei einem Marktrückgang okonomisch sinnvoll zu sein).

Die IATA-Lizenz ermächtigt ein Reisebüro, rechtlich als Handelsvertreter für alle Mitgliedsgesellschaften der IATA zu fungieren. Die Erteilung der Lizenz erfolgt nach einem festgelegten Zulassungsverfahren und ist an bestimmte Voraussetzungen gebunden. Vgl. weiterführend Pompl, W., Luftverkehr: eine ökonomische Einführung, a.a.O., S. 178-180.

Die Studie beruht auf 61 Beobachtungen und Interviews in den betreffenden Reisebüros und wurde vom Deutschen Reisebüro Verband sowie dem Berufsverband mittelständischer Reisebürounternehmen in Auftrag gegeben. Die Finanzierung übernahmen die Deutsche Luftahnsa, das Bundesministerium für Wirtschaft sowie die beteiligten Reisebüros. Vgl. Jahrfeld, M., Der Weg zum Mehrwert bleibt verschlungen, in: fww, Nr. 23, 1997, S. 15. 
Wenngleich diese Ergebnisse Einschränkungen unterliegen, die sich aus dem Design und der Methodik der Untersuchung ergeben, lassen sich aus Sicht der Reisebüros eine Verringerung des Zeitaufwands pro Buchung sowie eine im Vergleich zu den Prozeßkosten „angemessene“ Provisions- und Rabatthöhe grundsätzlich als bedeutsame Anforderungen an das Leistungsangebot von Fluggesellschaften ableiten. Darauf aufbauend ist nun zu überprüfen, wie sich strategische Luftverkehrsallianzen auf die Beziehungen zwischen Fluggesellschaften und Reisebüros auswirken. Durch eine Ausweitung des Streckennetzes im Zuge der Code Sharing-Vereinbarungen zwischen den Allianzpartnern können möglicherweise das Gesamtreiseaufkommen gesteigert und das Provisionsvolumen für die Reisebüros entsprechend erhöht werden. Ein positiver Mengeneffekt kann sich auch dann ergeben, wenn strategische Allianzen zu geringeren Flugpreisen führen. In einem solchen Fall müßten die Mengensteigerungen jedoch die aus niedrigeren Preisen resultierenden Mindereinnahmen überkompensieren, um eine Zunahme der Provisionen zu bewirken. ${ }^{317}$ Der Zeitaufwand für die Buchung und Bearbeitung von Tickets könnte auf seiten der Reisebüros darüber hinaus gesenkt werden, wenn die Allianzpartner ihre Vertriebsverträge harmonisieren und damit Transaktionskosten vermindern. ${ }^{318}$ Ein im Rahmen der Expertengespräche befragter Allianzmanager gab beispielsweise an, Reisebüros könnten durch die Bündelung von drei Allianzpartnern in einem Vertrag nahezu 90 Prozent der Reisebedürfnisse ihrer Kunden, d.h. der Fluggäste, decken und die Buchungen vereinfacht abwickeln, anstatt - wie zuvor - mit drei einzelnen Fluggesellschaften zu drei unterschiedlichen Konditionen abzurechnen. ${ }^{319}$

Andererseits liegen Vermutungen nahe, die auf eine mißbräuchliche Ausnutzung von Marktmacht durch die in Allianzen verbündeten Fluggesellschaften gegenüber

317 Für die exakte Bestimmung des aus der Preissenkung resultierenden Umsatzeffekts ist die Kenntnis der Preiselastizităten der Endnutzer notwendig, die als Verhältnis der relativen Änderung der Nachfrage nach einer Flugdienstleistung $\mathrm{i}$ zu der sie auslösenden relativen Änderung des Preises für diese Flugdienstleistung definiert werden kann. Vgl. Meffert, $\mathrm{H}$., Marketing: Grundlagen marktorientierter Unternehmensführung, a.a.O., S. 476.

Eine Harmonisierung der hiermit angesprochenen Konditionenpolitik im Rahmen strategischer Allianzen kann vorbehaltlich der wettbewerbsrechtlichen Zulässigkeit erfolgen. Unter Transaktionskosten sind Kosten zu verstehen, die zum Abschluß einer vertraglichen Vereinbarung führen (z.B. Informations-, Verhandlungs- und Vertragskosten), sowie Kosten der Absicherung, Durchsetzung und ggf. Anpassung der vertraglichen Vereinbarungen. Vgl. Ebers, M., Gotsch, W., Institutionenökonomische Theorien der Organisation, a.a.O., S. 209.

Vgl. Anhang 3. 
den Reisebüros schließen lassen. Eine Bündelung von Allianzpartnern in einem einheitlichen Vertriebsvertrag könnte z.B. zu einer Senkung der Provisions- und Rabattsätze genutzt werden. In Verbindung mit mangelnden Ausweichmöglichkeiten auf andere Fluggesellschaften entstünden den Reisebüros dann Einnahmeeinbußen. Eine solche Bedrohung aus Reisebürosicht besteht jedoch nur bei strategischen Allianzen, die im Besitz einer Ausnahmegenehmigung von kartellrechtlichen Vorschriften sind wie beispielsweise KLM-Northwest Airlines und Lufthansa-United Airlines in den USA sowie Lufthansa-SAS in Europa. ${ }^{320}$ Angesichts eines branchenweit zu verzeichnenden Trends zur Kürzung von Provisionen ${ }^{321}$ dürfte es z.Zt. in Abhängigkeit vom Niveau der allianzseitigen Kürzungen zudem relativ schwerfallen, ein derartiges Verhalten in strategischen Allianzen als mißbräuchlich zu identifizieren.

Neben nachteiligen Auswirkungen strategischer Luftverkehrsallianzen auf die Entwicklung von Provisionen und Rabatten ist ein erhöhter Informations- und damit Zeitaufwand für die Vermittlung von Code Share-Flügen vorstellbar. Die Gründe hierfür liegen zum einen in der relativ hohen Erklärungsbedürftigkeit der von mindestens zwei verschiedenen Fluggesellschaften erbrachten Flugdienstleistung gegenüber den Reisebürokunden und zum anderen in der technischen Systeminfrastruktur, auf der die Interaktionen zwischen Fluggesellschaften und Reisebüros aufbauen. Da derzeit keine gesetzlich festgelegte Pflicht zur Information der Endnutzer von Flugdienstleistungen besteht, bleibt es letztlich den Reisebüromitarbeitern überlassen, ob und in welchem Umfang sie ihre Kunden über das Wesen von Code Share-Flügen aufklären. ${ }^{322}$ Die Ausführungen zur Arbeitsteilung zwischen den Fluggesellschaften haben den Komplexitätsgrad von Code Share-Flugdienstleistungen deutlich werden lassen. Im Sinne einer vollständigen, kundenorientierten Aufklärung müßten Reisebüromitarbeiter demzufolge die

320 Grundsătzlich unterliegen strategische Allianzen, die wie hier in den Bereichen Produktion und Vertrieb gebildet werden, aufgrund ihrer wettbewerbsbeschränkenden Wirkung im deutschen Recht den Regelungen des § 1 GWB (Kartellverbot). Das europäische Recht sieht in Art. 85 III EWGV - ăhnlich wie die $\S \S 2$ bis 8 GWB - die Möglichkeit zur Freistellung vor. Daher ist mit der oben erwähnten Ausnutzung eines solchen Freiraums in bestimmten Bandbreiten zu rechnen. Vgl. Schäfer-Kunz, J., Strategische Allianzen im deutschen und europäischen Kartellrecht, a.a.O., S. 245.

Vgl. z.B. McGee, W.J., Reshaping the Relationship, in: Air Transport World, No. 12, 1997, S. 57-59; Spielberger, M., Provisionskürzungen machen Agenten das Leben schwer, in: fvw, Nr. 1, 1998, S. 9-12.

Vgl. Beyhoff, S., Ehmer, H., Wilken, D., Code-Sharing im internationalen Luftverkehr der Bundesrepublik Deutschland, a.a.O., S. 49. 
jeweils vorliegende Form des Code Sharing identifizieren, die Auswirkungen auf den Verlauf der Flugdienstleistung erfassen und dieses Wissen an ihre Kunden weitergeben. Neben dieser Informationsfunktion ist i.d.R. eine Beratung gefordert, die zentrale Vor- und Nachteile gegenüber konkurrierenden Angeboten verdeutlicht. In dem Spannungsfeld zwischen Zeitdruck und dienstleistungsspezifischer Erklärungsbedürftigkeit ist daher bei einer unterstellten Kundenorientierung der Reisebüros von wachsendem Zeitaufwand und steigenden Prozeßkosten für Code Share-Flugdienstleistungen auszugehen. Sofern die zusätzlichen Kosten nicht durch allianzspezifische Sonderleistungen seitens der Fluggesellschaften kompensiert werden, liegt die Annahme nahe, daß Reisebüros nur ein Mindest$\mathrm{maß}$ an kundenorientierter Information und Beratung bei Code Share-Angeboten gewährleisten oder sogar gänzlich auf entsprechende Hinweise verzichten. Dieser potentielle allianzspezifische Nachteil ist gleichwohl den oben erörterten potentiellen Vorteilen einer Harmonisierung gegenüberzustellen.

Zu den Kunden von Reisebüros zählen neben Endnutzern auch Unternehmen und öffentliche Institutionen - im folgenden als "Firmenkunden" bezeichnet -, deren Mitarbeiter im Zuge ihrer betrieblichen Tätigkeit Flugdienstleistungen in Anspruch nehmen. Darüber hinaus treten Firmenkunden z.T. direkt mit Fluggesellschaften in Interaktion, um Flugscheine zu erwerben. Die Auswirkungen strategischer Luftverkehrsallianzen auf die Beziehungen zwischen Firmenkunden und Fluggesellschaften werden im folgenden erläutert.

\subsubsection{Auswirkungen strategischer Luftverkehrsallianzen auf die Beziehun- gen zu Firmenkunden}

Für die Abwicklung der Buchung und des eigentlichen Erwerbs von Flugscheinen durch Firmenkunden haben sich in Abhängigkeit von nationalen Gesetzgebungen sowie Vereinbarungen zwischen Reisemittler-Dachverbänden und Fluggesellschaften verschiedene Möglichkeiten herausgebildet. ${ }^{323}$ In einigen Ländern kön-

\footnotetext{
323 Wenn Unternehmen als Käufer von Flugscheinen auftreten, die erworbene Flugdienstleistung in den Produktionsprozeß des kaufenden Unternehmens einfließt, mehrere Personen innerhalb der Firmenkundenorganisation am Kauf beteiligt sind und sich dabei an formalisierten Richtlinien orientieren, kann von einer investiven Flugdienstleistung gesprochen werden. Vgl. Backhaus, K., Hahn, C., Das Marketing von investiven Dienstleistungen, in: Handbuch Dienstleistungsmanagement: von der strategischen Konzeption zur praktischen Umsetzung, Hrsg.: Bruhn, M., Meffert, H., Wiesbaden 1998, S. 95. Zur Unterscheidung von investiven und konsumtiven Dienstleistungen vgl. auch Scheuch, F., Dienstleistungsmarketing, München 1982 , S. $57 \mathrm{ff}$. sowie zu weiteren konstitutiven Merkmalen investiven Kaufverhaltens Backhaus, K., Industriegütermarketing, 5., erw. und überarb. Aufl., München 1997, S. 52 u. 4.
} 
nen die Firmenkunden ihre Flugscheine direkt von den Fluggesellschaften erwerben, die bei ihnen mit eigenen Verkaufsstellen, sogenannten implants, physisch vertreten sind. ${ }^{324}$ Dadurch existiert im eigenen Hause ein Zugang zum Computerreservierungssystem, ${ }^{325}$ über das die Buchungen abgewickelt werden. Eine andere Möglichkeit besteht darin, einem Reisebüro die Auswahl und Beschaffung von Flugdienstleistungen zu übertragen. Gemäß einer 1996 in Deutschland durchgeführten Studie beauftragten rund 45 Prozent der zu ihrem Reiseverhalten befragten Firmenkunden ein Reisebüro mit der Vornahme von Buchungen, das in 24 Prozent aller Fälle mit einer Filiale im Firmengebäude vertreten war. ${ }^{326}$ Darüber hinaus können Firmenkunden die Buchung von Flugdienstleistungen in ihrer Reisestelle selbst durchführen. Während die Flugscheine direkt mit der Fluggesellschaft abgerechnet werden, erfolgt die technische Abwicklung der Buchungen über sogenannte Satelliten-Drucker und dezentrale Terminals im Hause der Firmenkunden, die über ein entfernt befindliches Reisebüro mit einem Computerreservierungssystem verbunden sind. ${ }^{327}$ Ferner gelten für Firmenkunden alle in Abbildung 9 wiedergegebenen Möglichkeiten des direkten Flugscheinerwerbs. ${ }^{328}$

Firmenkunden können aufgrund ihres vergleichsweise hohen Bedarfs an Flugdienstleistungen oftmals von Kundenbindungsmaßnahmen profitieren, mit denen die Fluggesellschaften einen möglichst großen Anteil des firmenkundenspezifischen Flugverkehrsaufkommens an sich zu binden versuchen. ${ }^{329}$ Meffert

324 Vgl. Pompl, W., Luftverkehr: eine ökonomische Einführung, a.a.O., S. 174.

325 Ein Computerreservierungssystem besteht aus einer zentralen Datenbank, die das Angebot von Fluggesellschaften und anderen Leistungsanbietern beinhaltet und mit Reisebüros vernetzt ist. Vgl. Weinhold, M., Computerreservierungssysteme im Luftverkehr: Erfahrungen in den USA und Empfehlungen für Europa, Baden-Baden 1995, S. 38.

Vgl. Lettl-Schröder, M., Trends und Prognosen im Geschäftsreisemarkt: die Management Fee setzt sich weiter durch, in: fuw, Nr. 13, 1996, S. 45. Wenn Reisebüros mit ihrer Filiale im Gebäude des Firmenkunden vertreten sind, wird - wie im Fall von Fluggesellschaften im Firmengebăude - ebenfalls von „implants“ gesprochen.

Vgl. Pompl, W., Luftverkehr: eine okonomische Einführung, a.a.O., S. 174. Der oben genannten Studie zufolge verfügen 43 Prozent der befragten Unternehmen über eine eigene Reisestelle. Vgl. Lettl-Schroder, M., Trends und Prognosen im Geschäftsreisemarkt: die Management Fee setzt sich weiter durch, a.a.O., S. 45.

Zu nennen sind beispielsweise die Buchung und der Kauf von Flugscheinen über das Internet. Vgl. Jegminat, G., Bausteine für integrierten Gesamtprozeß, in: fvw, Nr. 27, 1997, S. 9. subsumiert, die auf die Intensivierung bestehender Kundenbeziehungen gerichtet sind und die Abwanderung aktueller Kunden zu Konkurrenten verhindern oder zumindest erschweren sollen. Vgl. Reichheld, F.F., Sasser, W.E., Zero Migration. Dienstleister im Sog der Qualitätsrevolution, in: Harvard Manager, 13. Jg., Nr. 4, 1991, S. $108 \mathrm{ff}$. Der häufig synonym verwendete Begriff des ${ }_{n}$ customer retention“ impliziert darüber hinaus eine selektive Förderung 
und Backhaus (1994) unterscheiden faktische und emotionale Bindungen, die zwischen Anbietern und Kunden bestehen können. Faktische Bindungen werden von den Autoren wiederum in ökonomische, technisch-funktionale und vertragliche Bindungen unterteilt. ${ }^{330}$ Eine faktische Bindung kann demnach durch die ökonomische Vorteilhaftigkeit eines Angebots entstehen. Firmenkunden erhalten von Fluggesellschaften beispielsweise Rabatte, die in Abhängigkeit von dem mit der Airline realisierten Umsatzvolumen gestaffelt sein können. ${ }^{331}$ Durch die Konzentration der Beschaffungsaktivitäten auf möglichst wenige Fluggesellschaften können Firmenkunden folglich ihren Rabattertrag steigern und den zu entrichtenden Kaufpreis für Flugdienstleistungen dadurch senken. Übertragen auf strategische Allianzen, könnte die Bündelung von Flugverkehrsvolumen, das aus Sicht der Firmenkunden bisher mit mehreren Fluggesellschaften einzeln rabattiert wurde, in einer gemeinsamen Allianz-Rabattstaffel zu einer Mengenkonzentration und damit zu einer Steigerung von Rabatterträgen führen. ${ }^{332}$ Strategische Luftverkehrsallianzen wären in diesem Fall mit positiven ökonomischen Auswirkungen für die Firmenkunden verbunden.

Eine faktische Bindung von Firmenkunden an die strategische Allianz durch ökonomische Vorteilhaftigkeit strebt beispielsweise die Deutsche Lufthansa an. Das „Allianz-Plus"-Rabattmodell sieht die Gewährung von Rabatten vor, wenn in dem mit allen Allianzpartnern realisierten Volumen ein jährliches Wachstum erzielt wird. Eine Mindestfördermenge ist nicht vorgegeben. Bei einem Wachstum von einem Prozent beispielsweise erhalten die Firmenkunden eine Rückvergütung von

hochrentabler, aktueller Kundenbeziehungen. Vgl. Meffert, H., Kundenbindung als Element moderner Wettbewerbsstrategien, in: Handbuch Kundenbindungsmanagement, Hrsg.: Bruhn, M., Homburg, C., Wiesbaden 1998, S. 119.

Vgl. Meffert, H., Backhaus, K., Kundenbindung und Kundenmanagement: Instrumente zur Sicherung der Wettbewerbsposition, Dokumentation des Marketing Post Graduate-Seminars am 16./17. Juni 1994 in Münster, Münster 1994. Zwischen den Instrumenten, die zur Erreichung der Kundenbindung eingesetzt werden, bestehen zahlreiche Interdependenzen. Eine okonomische Bindung - z.B. über Rabatte - geht beispielsweise oft mit entsprechenden Verträgen einher, und eine rein technisch-funktionale Bindung ist bei Vernachlässigung der emotionalen Komponenten langfristig wenig sinnvoll.

1997 räumte die Deutsche Lufthansa AG z. B. ab einem Flugumsatz von 50.000 DM einen Rabatt von 1 Prozent ein, ab 2,5 Mio. DM Umsatz 1,5 Prozent und ab 5,0 Mio. DM 2 Prozent. Für das Jahr 1998 wurden die Mindestfördermenge von 50.000 DM auf 100.000 DM heraufgesetzt und die maximale Fördermenge von 20 Mio. DM auf 30 Mio. DM erhöht. Der maximale Fördersatz beträgt z.Zt. 7 Prozent. Vgl. Haas, S., Jetzt sind auch noch die Firmenkunden sauer, a.a.O., S. 8. durch einen gemeinsamen Allianzvertrag voraus. 
0,1 Prozent, bei einem Wachstum von 25 Prozent werden 4 Prozent gewährt. Diese allianzspezifische Förderung wird zusätzlich zur unternehmensspezifischen, volumenbasierten Firmenförderung der Lufthansa angewendet. ${ }^{333} \mathrm{Um}$ von den allianzspezifischen Rabattsätzen profitieren zu können, müssen Firmenkunden demgemäß größere Buchungsanteile mit den Allianzpartnern der Lufthansa realisieren. Bei einem konstanten Bedarf an Flugdienstleistungen ginge ein solches Verhalten jedoch zu Lasten der Lufthansa-spezifischen Rabatterträge. Die Vorteilhaftigkeit dieser allianzbezogenen Kundenbindungsmaßnahme ist folglich aus Sicht der Firmenkunden individuell nach deren Buchungsverhalten zu beurteilen. Zudem erfordert die Ausnutzung allianzbedingter Vorteile ein hohes Maß an Koordination innerhalb des Beschaffungsgremiums ${ }^{334}$ des Firmenkunden, da beispielsweise uninformierte Endnutzer durch ihre Einflußnahme die notwendige Ånderung des Buchungsverhaltens konterkarieren können. Die Anpassung von Reiserichtlinien an die Allianzförderung im Sinne eines übergeordneten „Beeinflussers" ist daher von hoher Bedeutung und wird vom Entscheider auf übergeordneter Ebene angeordnet.

Eine technisch-funktionale Bindung zwischen Firmenkunden und Fluggesellschaften kann aus einer engen Abstimmung technischer Buchungs- und Abrechnungssysteme erwachsen. Zu nennen sind beispielsweise Internet-basierte Anwendungen, die individuell an die Bedürnnisse des Firmenkunden angepaßt werden und im Gegenzug Buchungen mit einer bestimmten Fluggesellschaft

${ }^{333}$ Vgl. Haas, S., Jetzt sind auch noch die Firmenkunden sauer, a.a.O., S. 9. Die getrennten Rabattmodule spiegeln den Wunsch nach Erhaltung der Eigenstăndigkeit wider. Durch eine Bündelung von mehreren Einzel- zu einem gemeinsamen Volumenmodell in der weiter oben beschriebenen Weise ist die Wahrscheinlichkeit eines geänderten Wahlverhaltens seitens der Firmenkunden größer (d.h. Substitution innerhalb der Allianz). Ein solches Modell stellt eine potentielle Gefahr vor allem für diejenige Airline dar, mit der bisher die meisten Buchungen getătigt wurden. Grund: In einem gemeinsamen Volumenmodell trägt die Buchung bei einer bisher wenig genutzten Fluggesellschaft in demselben Maße zur Erzielung von Rabatten bei wie die Buchung bei der bisher hauptsächlich genutzten Airline. In der Folge sind Opportunitătskosten durch entgangene Rabatterträge bei Anbieterwechsel nicht mehr existent. In dem Allianz-Plus-Modell dagegen sichert die Trennung von Einzel- und Allianzförderung die dominante Bedeutung der Lufthansa, wăhrend die Partner in geringerem Maße gefördert werden.

Unter dem Beschaffungsgremium oder auch "buying center" wird im Zusammenhang mit dem organisationalen Kaufverhalten eine Gruppe von Personen verstanden, die mit dem Einkauf von Produkten oder Dienstleistungen für eine Organisation betraut sind. Daher wird auch von einer "Multipersonalităt" des Beschaffungsprozesses im Industriegütermarketing gesprochen. Vgl. z.B. Backhaus, K., Industriegütermarketing, a.a.O., S. 52 u. 4.

Eine Erklärung der Beziehungsstrukturen im buying center des Firmenkunden findet sich im Anhang 5 der vorliegenden Arbeit. 
bevorzugen. ${ }^{335}$ Durch das Angebot von Code Share-Flugdienstleistungen mit Allianzpartnern - unter der Voraussetzung wettbewerbsrechtlicher Zulässigkeit könnte sich das Leistungsspektrum eines solchen Systems aus Sicht der Firmenkunden als größer und attraktiver erweisen. Bei der Abwicklung von Buchungen lassen sich durch die Bündelung der Allianz-Fluggesellschaften in einer einheitlichen Systeminfrastruktur zudem die Transaktionskosten senken und informations- sowie beratungsbezogene Nachteile, die für Reisebüros genannt wurden, vermeiden. Im Beschaffungsgremium des Firmenkunden hängt es wesentlich von den Einkäufern ab, die mit dem System umgehen, inwieweit derartige Vorteile tatsächlich genutzt werden, während diese Frage für Endnutzer von untergeordneter Bedeutung ist. Sofern Reisebüros neben direkten Beziehungen zwischen Firmenkunde und Fluggesellschaft mit der Beschaffung von Flugdienstleistungen betraut sind, ist angesichts ihrer Umgehung mittels eines solchen Systems von entsprechenden reisebüroseitigen Widerständen auszugehen.

Eine weitere Form der Bindung zwischen Firmenkunden und Fluggesellschaften besteht in Verträgen, die - ähnlich wie im Fall von Reisebüros - Konditionen und Förderungsbedingungen zwischen den Vertragsparteien festlegen und daher nicht unabhängig von der ökonomischen und technisch-funktionalen Bindung gesehen werden können. Die Bündelung mehrerer Allianzpartner in einem Vertrag stärkt i.d.R. die Verhandlungsmacht der Fluggesellschaften, die mit den Entscheidern innerhalb des firmenkundenseitigen Beschaffungsgremiums die Vertragsinhalte festlegen. Eine Veränderung der Vertragsinhalte zuungunsten der Firmenkunden könnte die Folge sein. Bei einer Abschätzung der Auswirkungen ist jedoch auch die mittelbare Einflußnahme von Endnutzern und Einkäufern zu berücksichtigen, die beispielsweise ihre Flugerfahrungen oder Erfahrungen mit harmonisierten, störungsarmen Buchungssystemen in die Vertragsgestaltung einbringen. Neben einer Veränderung von Machtpositionen zwischen Firmenkunde und Fluggesell-

335 Gemeinsam mit ihrem Firmenkunden BASF hat die Deutsche Lufthansa beispielsweise den sogenannten Corporate Info Flyway entwickelt. Diese Anwendung basiert auf der InternetTechnologie und ermöglicht es BASF, über ein eigenes Intranet Buchungen im Computerreservierungssystem AMADEUS durchzuführen. Zugang zu diesem System und Buchungsberechtigung haben 500 Sekretärinnen bei BASF, während die physische Ausstellung von Flugtickets über die Firmen-Reisestelle erfolgt. Zudem sind über den Corporate Info Flyway Abfragen des "miles\&more“-Kontos der Endnutzer möglich (Vielfliegerprogramm der Lufthansa). Das Reiseplanungsinstrument "AirPlus" der Lufthansa wird in dieses System integriert und kann mit den spezifischen Reiserichtlinien des Firmenkunden verknüpft werden. Vgl. Jegminat, G., Bausteine für integrierten Gesamtprozeß, in: fvw, Nr. 27, 1997, S. 9. 
schaft kann ein international tätiger Firmenkunde mit anderen Vertragsinhalten konfrontiert werden als bisher, wenn seine ausländische Tochtergesellschaft beispielsweise in die Vertriebszuständigkeit eines Allianzpartners seiner bisher präferierten Fluggesellschaft wechselt. Dabei sind sowohl günstigere als auch ungünstigere Veränderungen möglich, so daß eine generelle Bewertung nicht möglich ist.

Neben den zuvor erörterten Formen faktischer Bindungen können sich emotionale Bindungen zwischen Firmenkunden und Fluggesellschaften entwickeln. Mit der allianzbedingten Arbeitsteilung auf seiten der Fluggesellschaften ist möglicherweise ein Wechsel von personellen Zuständigkeiten verbunden, der die emotionale Bindung zu Entscheidern und Einkäufern im Beschaffungsgremium des Firmenkunden schwächt. Höhere Transaktionskosten aufgrund von Kommunikationsproblemen sind mögliche Folgen für beide Seiten. ${ }^{336}$

Sowohl Reisebüros als auch Firmenkunden stehen lediglich in den ersten beiden Teilprozessen der Reisekette, der Information und Reservierung, in Interaktion mit strategischen Luftverkehrsallianzen. ${ }^{337}$ Endnutzer hingegen erleben die Flugdienstleistung auch über die weiteren Teilprozesse der Reisekette und ermöglichen damit eine weitergehende Untersuchung von Allianz-Flugdienstleistungen. Darüber hinaus besteht in einigen Fällen zwischen Endnutzern und Fluggesellschaften durch das vielfach eingesetzte Instrument der Vielfliegerprogramme eine unmittelbare, individualisierte Beziehung ${ }^{338}$, so daß die Analyse des Verhaltens

336 In ihrer Untersuchung identifizieren Reichheld und Sasser (1991) - u.a. für investive Dienstleistungen - Lerneffekte, die bei einer längerfristigen Interaktion zwischen Anbieter und Nachfrager entstehen und aus Sicht des Anbieters zu einer Reduktion der Kundenbetreuungskosten führen. Vgl. Reichheld, F.F., Sasser, W.E., Zero Migration, a.a.O., S. $109 \mathrm{ff}$.

337 Hinzuzufügen sind strenggenommen die nach der Inanspruchnahme der Flugdienstleistung stattfindenden Interaktionen, die beispielsweise bei endnutzerseitigen Beschwerden $0 . a ̆$. entstehen.

An Vielfliegerprogrammen können nur einzelne, natürliche Personen teilnehmen, wăhrend Unternehmen oder Institutionen nicht teilnahmeberechtigt sind. Der Einfluß auf den Beschaffungsprozeß wird durch Untersuchungen in den USA belegt. Demnach sind für 60 Prozent der Geschäftsreisenden Vielfliegerprogramme bei jeder Flugdienstleistungsbuchung ausschlaggebend für die Wahlentscheidung zugunsten einer bestimmten Fluggesellschaft. Vgl. Beyhoff, S., Vielfliegerprogramme und der Wettbewerb im Luftverkehr, Mitteilung Nr. 94-02 der Deutschen Forschungsanstalt für Luft- und Raumfahrt, Köln 1994, S. 1 u. 8.

Meilengutschriften sind in den USA im Gegensatz zu Deutschland übertragbar. Bei vielen USamerikanischen Unternehmen regeln die Reiserichtlinien daher, daß dienstlich erflogene Boni 
von Endnutzern unter Berücksichtigung der reisebürobezogenen und firmenkundenseitigen Besonderheiten den größtmöglichen Aufschluß über die Wahrnehmung, das Erleben und die Beurteilung strategischer Luftverkehrsallianzen zu geben verspricht.

\subsection{Konzeptionelle Überlegungen und empirische Befunde zu den end- nutzergerichteten Beziehungen strategischer Luftverkehrsallianzen}

\subsubsection{Wahrnehmung strategischer Luftverkehrsallianzen am Beispiel aus- gewählter Informationsinhalte}

Den folgenden Ausführungen liegt die Annahme zugrunde, daß die Wahrnehmung allianzspezifischer Informationsinhalte die Einstellung von Endnutzern gegenüber Luftverkehrsallianzen beeinflußt und damit für die Kaufentscheidung von Relevanz ist. Endnutzer können durch verschiedene externe und interne Stimuli auf strategische Luftverkehrsallianzen aufmerksam werden. ${ }^{339}$ Bei der Untersuchung strategischer Luftverkehrsallianzen aus Sicht von Endnutzern sind grundsätzlich vier Fälle zu unterscheiden (vgl. Abbildung 10). Im ersten Fall nimmt der Endnutzer eine strategische Luftverkehrsallianz weder vor der Inanspruchnahme einer Flugdienstleistung - z.B. durch Werbeanzeigen oder Zeitungsberichte - wahr noch erlebt er sie im Verlauf der Flugdienstleistung durch Interaktionen mit Personen, die an der Erstellung der Leistung beteiligt sind. ${ }^{340}$ Der betreffende Endnutzer kann daher als „Allianz-Ignorant“ bezeichnet werden. Die Untersuchung dieses Wahrnehmungstyps bringt jedoch keine weiteren Erkenntnisse für die Bearbeitung der zweiten Teilzielsetzung der vorliegenden Arbeit.

an Kollegen abzutreten sind, die diese wiederum geschäftich nutzen. Vgl. o.V., Nicht jede Geschäftsreise-Meile bringt Freiflüge, in: Handelsblatt vom 26.5.1997, S. 15.

Externe Stimuli (=Außenreize) sind z.B. Werbeanzeigen, während interne Stimuli von innen her anregen wie beispielsweise der beim Arbeiten auftretende spontane Einfall, eine Flugreise zu buchen. Vgl. Kroeber-Riel, W., Weinberg, P., Konsumentenverhalten, a.a.O., S. 49.

Die Wahrnehmung der strategischen Allianz ist gemåß dem in Kapitel A 3. dieser Arbeit formulierten Begriffsverständnis als Prozeß der Aufnahme, Selektion, Organisation und Interpretation von allianzbezogenen Informationen aufzufassen, der unabhängig von der Absicht, eine Flugdienstleistung buchen zu wollen, stattindet. Zudem findet keine Interaktion mit einem an der Flugdienstleistungserstellung beteiligten Leistungsanbieter statt. Das Erleben der Flugdienstleistung beginnt demgegenüber mit der Informationssuche, in deren Zuge der Endnutzer in Interaktion Z.B. mit einem Reisebüromitarbeiter oder der Telefonistin einer Fluggesellschaft tritt.

Vgl. in ähnlicher Form für Bahnreisen Siefke (1998), bei dem die Phase der Information den ersten Kontaktpunkt im Rahmen der Vor-Reisephase darstellt. Vgl. Siefke, A., Zufriedenheit mit Dienstleistungen, a.a.O., S. $126 f$. 


\begin{tabular}{|c|c|c|}
\hline $\begin{array}{l}\text { Wahrnehmung } \\
\text { Erleben }\end{array}$ & nein & ja \\
\hline nein & $\begin{array}{c}\text { Fall } 1 \\
\text { „Allianz-Ignorant" }\end{array}$ & $\begin{array}{c}\text { Fall } 2 \\
\text { „Allianz-Theoretiker“ }\end{array}$ \\
\hline ja & $\begin{array}{c}\text { Fall } 3 \\
\text { „Allianz-Überraschter“ }\end{array}$ & $\begin{array}{c}\text { Fall } 4 \\
\text { „Allianz-Erfahrener“ }\end{array}$ \\
\hline
\end{tabular}

Abb. 10: Wahrnehmungstypen bei strategischen Luftverkehrsallianzen

Im zweiten Fall wird die Allianz zwar wahrgenommen, aber im Zuge der Flugdienstleistung nicht erlebt. Der Endnutzer setzt sich unabhängig von der Inanspruchnahme einer Flugdienstleistung und ohne Interaktion mit einem Leistungsanbieter durch die Aufnahme und Selektion von Informationen, die beispielsweise über die Gründung einer Allianz Auskunft geben, mit strategischen Luftverkehrsallianzen auseinander und kann als „Allianz-Theoretiker“ benannt werden.

Im dritten Fall nimmt der Endnutzer strategische Luftverkehrsallianzen zunächst nicht wahr, d.h. vor der gezielten Informationssuche faßt er keine allianzbezogenen Informationen auf. Durch die Inanspruchnahme einer Code Share-Flugdienstleistung erlebt er jedoch das Angebot einer strategischen Luftverkehrsallianz, wobei sich das Erleben auf einen, mehrere oder alle Teilprozesse der Reisekette erstrecken kann. Damit erweist sich die strategische Allianz als Überraschung für ihn, so daß vom „Allianz-Überraschten“ gesprochen werden kann. Eine Untersuchung dieses Falls läßt demnach Fragen bezüglich der Wahrnehmung von Allianzen im Vorfeld der Flugdienstleistung offen.

Im vierten Fall nimmt der Endnutzer strategische Luftverkehrsallianzen vor Inanspruchnahme der Flugdienstleistung wahr und erlebt das Leistungsangebot in einem, mehreren oder allen Teilprozessen der Reisekette. Aus diesem Grund gilt er als „Allianz-Erfahrener“. 
Da Wahrnehmung als kognitiver Prozeß an der Aufnahme von Informationen ansetzt, ${ }^{341}$ beschränken sich die folgenden Ausführungen auf die durch externe Stimuli in Gang gesetzte Wahrnehmung allianzspezifischer Informationsinhalte. Die Beschaffenheit von Informationsinhalten kann nicht unabhängig von ihrer Herkunft beurteilt werden, ${ }^{342}$ so daß eine Klassifikation von Informationsquellen sinnvoll erscheint. ${ }^{343}$ Zunächst lassen sich nach dem Merkmal der Herkunft interne und externe Quellen unterscheiden. ${ }^{344}$ Interne Quellen sind im Gedächtnis des Individuums abgespeicherte Informationen, ${ }^{345}$ die beispielsweise auf das zurückliegende Lesen eines Presseartikels oder auf bereits gewonnene Erfahrungen mit einer Allianz-Flugdienstleistung zurückgehen. Sie können durch Außenreize wie z.B. ein Werbeplakat aktiviert werden. Externe Quellen entstammen der sozialen Umwelt des Endnutzers und können nach dem Grad der Mittelbarkeit zum Informationsobjekt - in diesem Fall der Allianz-Flugdienstleistung - in Quellen mit unmittelbaren und mittelbaren Informationen unterschieden werden. Da unmittelbare Informationen erst im Zuge des Erlebens der Allianz-Flugdienstleistung offenbar werden, scheiden sie für die Untersuchung der Wahrnehmung strategischer Luftverkehrsallianzen an dieser Stelle aus.

Die Aufnahme von mittelbaren Informationen über strategische Luftverkehrsallianzen ist demgegenüber nicht an die tatsächliche Inanspruchnahme einer Flugdienstleistung gebunden. Sie kann sich einerseits an Informationen orientieren,

341 Vgl. Kroeber-Riel, W., Weinberg, P., Konsumentenverhalten, a.a.O., S. 51f. Eine Verhaltensrelevanz konzedieren die Autoren den kognitiven Prozessen jedoch nur im Zusammenwirken mit dem auf Emotionen beruhenden, aktivierenden intrapersonalen Teilsystem.

342 Eine von Gierl, Stich und Strohmayr (1997) durchgeführte empirische Analyse belegt, daß Informationen aus einer glaubwürdigen Quelle glaubwürdiger sind als Informationen aus einer weniger glaubwürdigen Quelle. Diese auf den Blick trivial anmutende Erkenntnis hat für den Informationssender weitreichende Implikationen bei der Wahl des Informationsmediums. Vgl. Gierl, H., Stich, A., Strohmayr, M., Einfluß der Glaubwürdigkeit einer Informationsquelle auf die Glaubwürdigkeit der Information, in: Marketing ZFP, 19. Jg., Nr. 1, 1997, S. 30.

Vgl. Katz, R., Informationsquellen der Konsumenten: eine Analyse der Divergenzen zwischen der Beurteilung und Nutzung, Wiesbaden 1983, S. 3.

$344 \mathrm{Zu}$ einer Klassifikation von Informationsquellen vgl. z.B. Gerhard, A., Die Unsicherheit des Konsumenten bei der Kaufentscheidung: Verhaltensweisen von Konsumenten und Anbietern, Wiesbaden 1995, S. 89ff.

345 Analog zum Prozeß der Aufnahme, Verarbeitung und Speicherung von Informationen findet sich in verhaltenswissenschaftlichen Theorieansätzen die modellhafte Unterteilung des menschlichen Gedächtnisses in den sensorischen Informationsspeicher, Kurzzeit- sowie Langzeitspeicher. Vgl. zu einer Darstellung des auf Arbeiten von Atkinson und Shiffrin (1968, 1971) zurückgehenden Ansatzes Kroeber-Riel, W., Weinberg, P., Konsumentenverhalten, a.a.O., S. 225 sowie die dort angegebene Literatur. 
die durch die Partner der Luftverkehrsallianz beispielsweise in Form von TV-Werbespots oder Werbeanzeigen in Printmedien zur Verfügung gestellt werden, so daß von interessengebundenen Informationsquellen gesprochen wird. Andererseits bestehen interessenungebundene, unabhängige Informationsquellen, zu denen z.B. der Verwandten-, Freundes- und Bekanntenkreis des Endnutzers zählen. Der Austausch von Erfahrungen mit Code Share-Flugdienstleistungen oder die Diskussion der Gründung oder Auflösung von strategischen Luftverkehrsallianzen kommen beispielsweise als nicht institutionalisierte Informationsquelle in Frage. ${ }^{346}$ Als nicht interessengebundene, aber institutionalisierte Informationsquellen fungieren Medien, die über Luftverkehrsallianzen und ihre Leistungsangebote berichten, ohne dabei die Interessen der Partner-Fluggesellschaften zu verfolgen. ${ }^{347} \mathrm{Im}$ Vordergrund steht eine möglichst sachorientierte Information über das Thema strategischer Luftverkehrsallianzen. Zu nennen sind beispielsweise Berichte in Zeitungen und Zeitschriften sowie Informationen von Verbraucherverbänden.

Von wesentlicher Bedeutung für die Wahrnehmung strategischer Luftverkehrsallianzen durch Endnutzer ist die Frage, welche Inhalte einer Allianz-Flugdienstleistung überhaupt wahrgenommen werden. Unter Bezugnahme auf interessengebundene Informationsquellen bieten Werbemitteltests einen empirischen Ansatzpunkt zur Beantwortung dieser Frage. Sie stellen Testpersonen vor die Aufgabe der mehrkriteriellen Bewertung von TV-Werbespots, Werbeanzeigen in Printmedien oder sonstigen Werbemitteln, die von den Partner-Fluggesellschaften zur Erreichung kommunikationspolitischer Ziele eingesetzt werden. ${ }^{348}$ In Ermangelung empirischer Ergebnisse derartiger Tests im Zusammenhang mit Luftverkehrsallianzen soll im folgenden exemplarisch eine Werbeanzeige der Star

346 Die Bedeutung des persőnlichen Meinungsaustauschs für die Wahrnehmung strategischer Luftverkehrsallianzen und ihres Leistungsangebots ist nicht zu unterschätzen. Da jedoch fundiertere, kontextspezifische Erkenntnisse hierüber fehlen, wird diese Informationsquelle an dieser Stelle nicht weiter vertieft.

Die Begrifflichkeit ${ }_{n}$ nicht interessengebunden “ kann irreführend sein, da letztlich hinter jedem Bericht bestimmte Interessen wie beispielsweise die Aufklärung von Verbrauchern oder die Information von Kapitalanlegern über die wirtschaftliche Entwicklung der Allianz stehen.

Werbemitteltests können sowohl vor $\left({ }_{n}\right.$ Pre-Tests ${ }^{4}$ ) als auch nach der Schaltung (,Post-Tests“) von Werbemitteln in den Medien durchgeführt werden. Zu einer Beschreibung der Testinhalte und möglicher Verfahren vgl. weiterführend Salcher, E.F., Psychologische Marktforschung, 2., neu bearb. Aufl., Berlin, New York 1995, S. $264 f$. 
Alliance untersucht werden, ${ }^{349}$ die weltweit einheitlich in verschiedenen Printmedien geschaltet wurde und wird (vgl. Abbildung 11). ${ }^{350}$ Die sowohl emotionale als auch informative Elemente aufweisende, in fünf rechteckige Blöcke unterteilte Werbeanzeige ist deutlich erkennbar an Vielflieger gerichtet, die in der Headline direkt als solche angesprochen werden. Kernaussage der Headline ist die Größe des Geltungs- bzw. Akzeptanzbereichs für ein Vielfliegerprogramm, der sich auf sechs Fluggesellschaften erstreckt. Das dominante Bildmotiv der Anzeige zeigt einen Wald mit einem Gelassenheit ausströmenden Mann, der auf einem Stuhl sitzt und von Sonnenstrahlen erleuchtet wird. Das darunter auf schwarzem Grund angeordnete, aus fünf abgerundeten Pfeilspitzen bestehende Zeichenlogo wird durch den Markennamen Star Alliance und den Claim „The airline network for Earth“ ergänzt. Mit Individualität, Geltung, Gelassenheit, Ruhe und Weltbezug läßt sich die hierdurch vermittelte Botschaft zusammenfassen. Der relativ umfangreiche Textteil der Anzeige informiert über zentrale Vorteile, die mit der Nutzung der Star Alliance verbunden sind. Neben Meilensammeln durch gegenseitige Anerkennung der Vielfliegerprogramme, Loungenutzung bei allen sechs Partner-Fluggesellschaften für Business und First Class-Fluggäste sowie Mitglieder im Star Alliance Gold-Club und den Möglichkeiten zum „Abfliegen“ der gesammelten Meilen mit allen sechs Mitglieds-Airlines werden weiterhin ein bevorzugter Checkin sowie die Wartelistenpriorisierung für Star Alliance Gold-Club-Mitglieder herausgestellt. Als fünftes Anzeigenelement findet sich die Fotomontage eines JetFlugzeugs, dessen Profil in sechs Segmente mit dem jeweiligen Corporate Design $^{351}$ der Partner-Fluggesellschaften SAS, Lufthansa, Thai Airways, Varig, Air Canada und United Airlines eingeteilt ist. Auffällig ist die ungewöhnliche Gestalt des Flugzeugs, dessen Länge diejenige bekannter Flugzeugtypen deutlich übertrifft.

349 Eine solche Vorgehensweise stellt lediglich ein Expertenurteil dar und bildet keinen Ersatz für einen Werbemitteltest. Dennoch lassen die Ausführungen Schlüsse auf mögliche wahrnehmungsrelevante Elemente der Informationsquelle "Werbeanzeige“ zu.

Die Gestaltung und zentrale Koordination der Mediaplanung wird von der Werbeagentur Young\&Rubicam im Auftrag der Allianzpartner übernommen.

351

Unter Corporate Design wird der abgestimmte Einsatz der visuellen Elemente der Unternehmenserscheinung verstanden, um die Identităt des Unternehmens zu vermittein. Vgl. Birkigt, K., Stadler, M.-M., Funck, H.J., Corporate Identity: Grundlagen, Funktionen, Fallbeispiele, 7. Aufl., Landsberg/Lech 1994, S. 18ff. Hierzu rechnen vor allem Zeichen, Farbe, Schrift und Typographie, die sich beispielsweise in der Marke ausdrücken. Als Träger des Corporate Design kommen im Luftverkehrsbereich z.B. Flugbegleiter und Flugzeuge, aber auch Flugtickets und Merchandise-Artikel in Frage. 
Be recognised as a frequent flyer on not one, but six airlines.

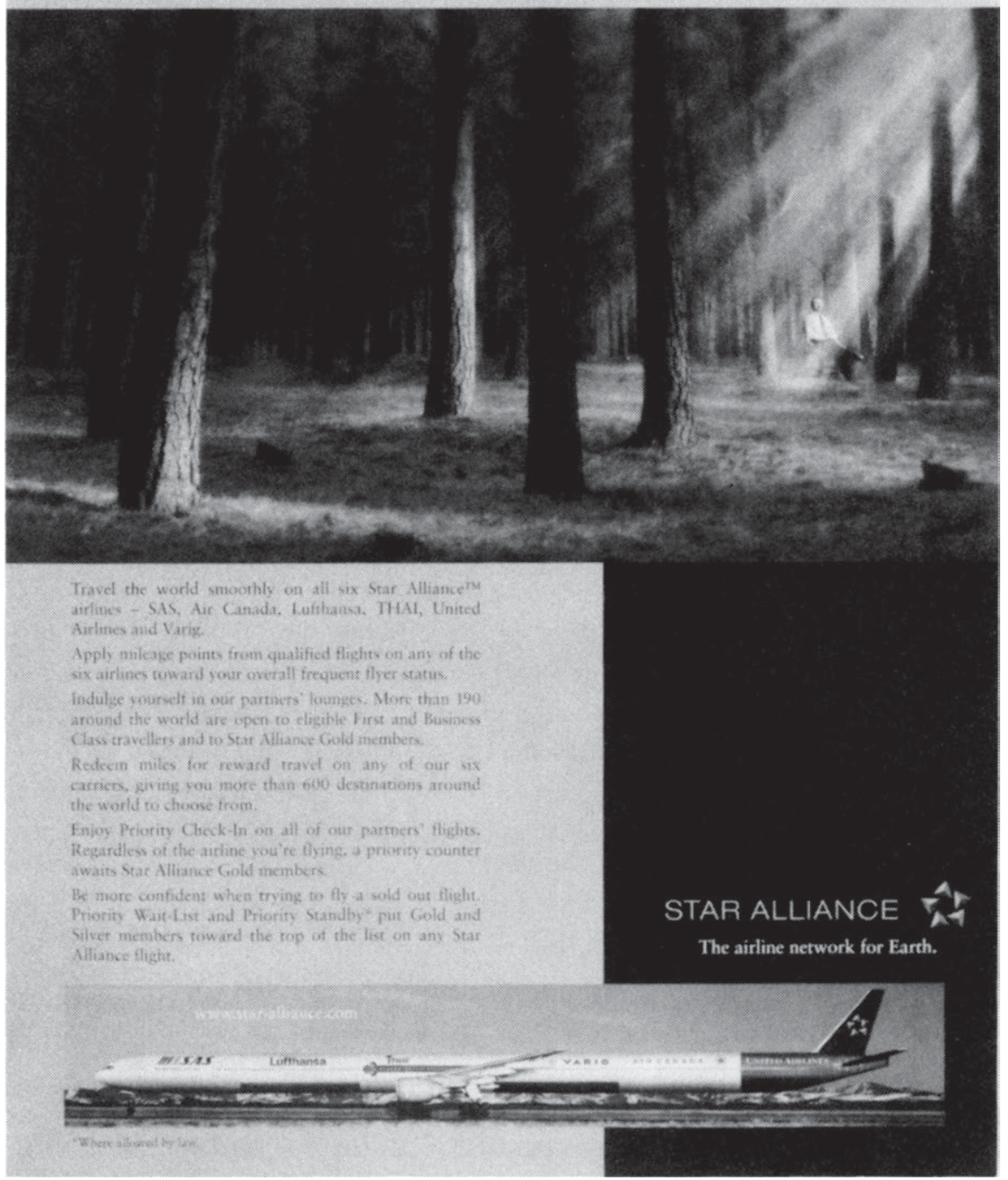

Abb. 11: Werbeanzeige der Star Alliance 
Von zentraler Bedeutung für die Beurteilung dieser Werbeanzeige ist nun das Verständnis der „Produkt"botschaft, d.h. ${ }^{352}$

- welche flugdienstleistungsbezogenen Vorstellungen durch das Betrachten der Anzeige ausgelöst werden,

- welche Eigenschaften der Flugdienstleistung als besonders dominant bewertet werden,

welcher neue und spezifische Nutzen mit der beworbenen Flugdienstleistung verbunden wird und

- ob dieser neue und spezifische Nutzen auch als entsprechend attraktiv und interessant aufgefaßt wird.

Das im Mittelpunkt stehende Bildmotiv des Waldes i.V.m. dem gelassen wirkenden Mann läßt zunächst jeglichen Bezug zu einer Flugdienstleistung vermissen. Erst durch das Lesen der Headline und/oder die Wahrnehmung der übrigen Anzeigenelemente dürfte der Themenbezug deutlich werden. Die Abbildung des Flugzeugs sowie die recht ausführliche Beschreibung von nutzenstiftenden Merkmalen lassen die Absicht erkennen, die komplexe, intangible Allianz-Flugdienstleistung zu visualisieren und zu tangibilisieren, indem "handfeste“ Nutzendimensionen Erwähnung finden (u.a. Belege durch Zahleninformationen wie etwa der Anzahl von Lounges oder Zielflughäfen). Die längenmäßige Verzerrung des Flugzeugrumpfes könnte in Verbindung mit den aneinandergereihten Corporate Design-Elementen von sechs Fluggesellschaften zu einer Verwirrung und Verunsicherung des Betrachters führen. In Verbindung mit dem Textinhalt kann dieser Werbeanzeige gleichwohl ein relativ hoher Informationsgehalt für eine mengenmäßig eher kleine Zielgruppe zugesprochen werden. Die nutzenstiftenden Merkmale beschränken sich jedoch ausschließlich auf den für Statuskunden relevanten Bereich der Zusatzleistungen.

Um komplexere Botschaftsinhalte zu vermitteln, bietet sich zudem die Möglichkeit der Direktwerbung an. ${ }^{353}$ Mittels Direktwerbung können wertmäßig bedeutende

352 Zur Bedeutung der Produktbotschaft und $\mathrm{zu}$ den hiermit verbundenen zentralen Fragestellungen vgl. Salcher, E.F., Psychologische Marktforschung, 2., neu bearb. Aufl., Berlin, New York 1995, S. 270-272.

Direktwerbung stellt die Übertragung einer individuellen Ansprache auf die klassische Werbung dar und kann z.B. in schriftlicher (z.B. Direct Mail) oder fernmündlicher (z.B. Telefonmarketing) Form erfolgen. Angestrebt werden vor allem eine höhere Vertriebseffizienz, höhere Kundenbindung sowie optimale Kundennähe. Vgl. Meffert, H., Marktorientierte 
Zielgruppen wie Vielflieger, die i.d.R. aus geschäftlichem Anlaß reisen, differenziert angesprochen werden. Das zu diesem Zweck benötigte Adressenmaterial steht den Fluggesellschaften beispielsweise über die Datenbank von Vielfliegerprogrammen zur Verfügung. ${ }^{354}$

Endnutzer können strategische Luftverkehrsallianzen darüber hinaus anhand von Informationsbroschüren wahrnehmen. Es ist davon auszugehen, daß über dieses Werbemittel auch komplexere Informationen und Zusammenhänge von den Endnutzern wahrgenommen werden können, da zum einen mehr Raum für die Präsentation allianzspezifischer Informationen zur Verfügung steht und die Lektüre einer allianzspezifischen Informationsbroschüre zum anderen auf eine höhere themengerichtete Aktivierung schließen läßt. ${ }^{355} \mathrm{Um}$ Aussagen über die Wahrnehmung von Inhalten allianzspezifischer Informationsbroschüren zu gewinnen, wird im folgenden exemplarisch auf eine von KLM und Northwest Airlines herausgegebene Broschüre zurückgegriffen. Die mit der Überschrift „An Alliance Of Global Proportions" versehene Informationsbroschüre zeigt auf der Titelseite die vor einem Wolkenhintergrund fotographierten Leitwerke eines KLM- und eines Northwest-Langstreckenjets, die sich in Parkposition befinden. Die Leitwerke lassen unterschiedliche Flugzeugtypen erkennen und heben sich darüber hinaus durch das Markenlogo der jeweiligen Fluggesellschaft voneinander ab. Unter der bereits genannten Überschrift findet sich ein Kreis, dessen Inneres in zwei Halbkreise mit den Markenlogos der Partner aufgeteilt ist. Um den inneren legt sich ein äußerer Kreis, der die Wörter "Worldwide Reliability“ enthält.

Anders als im Fall der Star Alliance-Werbeanzeige hebt die Gestaltung des Titelbilds der Informationsbroschüre stärker auf eine Trennung zwischen den PartnerFluggesellschaften $a b$, da diese jeweils mit einem eigenen Fluggerät visualisiert sind. In der Überschrift, die etwa als „Eine Allianz von globalen Ausmaßen“ über-

Unternehmensführung und Direct Marketing, in: Handbuch Directmarketing, Hrsg.: Dallmer, H., 7. Aufl., Wiesbaden 1997, S. 43 u. 46.

Vgl. Poledna-van Zweden, M., Kundenbindungsprogramme der Deutschen Lufthansa AG, in: Die Nicht-Klassiker unter heutigen Kommunikationsbedingungen, Hrsg.: Belz, C., Tomczak, T., St. Gallen 1995, S. 202.

Die Betrachtungszeit für Anzeigen in Publikumszeitschriften schwankt in Abhängigkeit vom Produktbereich zwischen einer und zwei Sekunden, so daß komplexere Informationen kaum vermittelbar sind. Vgl. Kroeber-Riel, W., Weinberg, P., Konsumentenverhalten, a.a.O., S. 92. Unter Aktivierung verstehen die beiden Autoren eine innere Erregung oder Spannung, die den Organismus mit Energie versorgt und in einen Zustand der Leistungsbereitschaft und -fähigkeit versetzt. Vgl. ebenda, S. 58. 
setzt werden kann, spiegelt sich bereits ein Nutzenversprechen in Form von Globalität, Reichweite und Größe wider. Das kreisförmige "Partnerschaftssiegel “356 ergänzt dieses Versprechen um die Nutzenkomponente "weltweiter Verläßlichkeit“, die auch als Zuverlässigkeit und Vertrauenswürdigkeit interpretiert werden kann. Das Partnerschaftssiegel unterscheidet sich von dem Markenlogo der Star Alliance zum einen durch die parallele Darstellung der Markenlogos der Partner und zum anderen durch die Integration eines Nutzenversprechens als Zeichenbestandteil des Siegels. Im Anschluß an die Titelseite geben 10 Seiten, die aus einer Bild-Text-Mischung bestehen, weiteren Aufschluß über Inhalte der Luftverkehrsallianz. 4 der 15 verwendeten Fotographien stellen Personen dar, die in eine Flugdienstleistung involviert sind. Es handelt sich um 2 Gruppenfotos des fliegenden Personals der beteiligten Partner, 1 Bildmotiv mit Endnutzern an Bord eines Flugzeugs sowie um die Fotographie einer Reisebüromitarbeiterin an einem PC. 5 der 11 Sachmotive enthalten Fotographien von Reisezielen, 4 Fotos zeigen Fluggerät, 1 doppelseitige Abbildung gibt eine Satellitenaufnahme der Erde mit von der Allianz bedienten Städtezielen wieder und 1 Abbildung enthält das Partnerschaftssiegel. Die Auswertung der Textinhalte läßt folgende Kernaussagen zu Tage treten:

1. reibungslose Reiseabläufe durch gemeinsame Abstimmung von Flugplänen und Check-in-Vorgängen am Flughafen,

2. Erweiterung des weltweiten Streckennetzes durch zusätzliche Städteverbindungen,

3. gegenseitige Anerkennung von Meilen in den jeweiligen Vielfliegerprogrammen und spezielle Flugangebote für Vielflieger,

4. Zugang zu Lounges beider Partner für Business und First Class-Passagiere,

5. Durchabfertigung des Gepäcks bis zum Zielort auch bei Umsteigeverbindungen,

6. zweisprachige Betreuung vor, während und nach dem Flug mit den Allianzpartnern,

7. Kombination der individuellen Servicestärken („For example, in Business Class, we put KLM's high quality china, cutlery and glassware together with Northwest's menu options"), ${ }^{357}$

356 In der Broschüre wird auf Seite 3 die Bezeichnung Seal of Partnership“ verwendet.

357 S. 9 der Broschüre. 
8. Selbstdarstellung der Partnerfluggesellschaften mit Schwerpunkt auf Traditionsbewußtsein.

Um aus der Informationsbroschüre verläßliche Aussagen über die endnutzerseitige Wahrnehmung der strategischen Allianz zwischen KLM und Northwest Airlines gewinnen zu können, wären zusätzliche Mediadaten der Broschüre sowie Erkenntnisse aus Werbemitteltests notwendig. ${ }^{358}$ Dennoch lassen sich auch ohne derartige Daten und Ergebnisse erste Anhaltspunkte für potentielle Nutzendimensionen feststellen, die vor allem für die Zielgruppe der Vielflieger und Business sowie First Class-Kunden konzipiert zu sein scheinen. Im Mittelpunkt steht zweifelsohne eine Ausdehnung des bisherigen Leistungsangebots durch die Verbindung von Leistungspotentialen der Partner-Fluggesellschaften (vgl. Punkte 2., 3., 4. und 7.). Die Punkte 1. und 5. sollen dagegen auf eine bessere Beherrschung herkömmlicher Prozesse insbesondere bei Umsteigeverbindungen hinweisen. Die Information über Zweisprachigkeit bei der Passagierbetreuung ist eher als Maßnahme zur Vermeidung von Irritationen zu werten, die durch die Konfrontation eines Niederländers mit einer US-amerikanischen Fluggesellschaft oder eines US-Amerikaners mit einer niederländischen Airline entstehen könnten. Durch Betonung der Unternehmenstradition im Luftverkehrsgeschäft (Punkt 8.) streben die Partner-Fluggesellschaften eine Senkung des wahrgenommenen Risikos beim Kauf einer Flugdienstleistung an, indem langjährige Erfahrung als Signal zur Bildung von Vertrauen genutzt wird. ${ }^{359}$ Die Tradition dient dabei als gemeinsame Schnittmenge oder auch Bindeglied der Allianzpartner.

Wenngleich die Inhalte der Broschüre potentielle Nutzendimensionen der Allianz herausstellen, bleiben Fragen zur konkreten Zusammenarbeit der beiden Fluggesellschaften in den Teilprozessen der Flugdienstleistung offen. Hervorzuheben ist insbesondere die Information über die Trennung von vermarktender und durchführender Fluggesellschaft im Zuge einer Code Share-Flugdienstleistung, die keine Erwähnung findet, obgleich sich weitreichende Implikationen aus dieser Unterscheidung ergeben.

358 Von besonderer Relevanz für die Beurteilung der Wahrnehmung wären die Kontaktmaßzahlen der Allianz-Broschüre, die sich nach Bruhn (1997) in Auflagenhöhe, Kontaktsumme und Reichweite unterscheiden lassen. Vgl. Bruhn, M., Kommunikationspolitik: Grundlagen der Unternehmenskommunikation, München 1997, S. $295 \mathrm{ff}$. 
Werbeanzeigen, Direct Mails und unternehmensseitige Informationsbroschüren geben als interessengebundene Informationsquellen die Perspektive der Fluggesellschaften wieder. Inwieweit die intendierte Einstellungswirkung, die in den dargestellten Beispielen an Vielfliegern ausgerichtet ist, tatsächlich erreicht wird, muß an dieser Stelle offen bleiben. Weiteren Aufschluß versprechen hier die in Kapitel B 2.3 vorzustellenden Ergebnisse der Focusgruppenuntersuchung.

Als in höherem Maße interessenungebundene Quelle setzen sich Presseberichte regelmäßig mit strategischen Luftverkehrsallianzen auseinander. Um Erkenntnisse über die Inhalte dieser Informationsquelle zu gewinnen, wurden 741 Artikel aus deutschsprachigen Zeitungen, Publikumszeitschriften und Fachzeitschriften aus den Jahren 1996, 1997 und 1998 (bis zum 26.5.1998) ausgewertet. ${ }^{360}$ Auswahlkriterium für die Artikel war der Themenschwerpunkt "Luftverkehr" mit den Sparten Fluggesellschaften, Flughäfen und Flugzeugbauern. ${ }^{361}$ Von den 741 Beiträgen beschäftigten sich 120 - entsprechend einem Anteil von 16,19 Prozent - mit dem Thema "strategische Allianzen von Fluggesellschaften". Die Inhalte dieser 120 Artikel teilen sich wie folgt auf unterschiedliche Schwerpunkte auf:

360 Die Analyse beruht zum einen auf Presseberichten, die in der MARIA-Studie "Luftverkehr" abgedruckt sind. Vgl. Gruner+Jahr (Hrsg.), MARIA-Studie Luftverkehr, Hamburg 1997. Zum anderen wurden eigene Recherchen in Printmedien durchgeführt, die für die Beantwortung der Fragestellung als relevant erachtet wurden. Hier sind vor allem Berichte der FAZ (insbesondere Wirtschaftsteil), des Handelsblatts sowie der Fachzeitschrift Fremdenverkehrswirtschaft International (fuw) hervorzuheben. Zum Vorgehen im Rahmen einer Inhaltsanalyse, die auch von Unternehmen als Erfolgskontrolle ihrer Public RelationsAktivitäten eingesetzt wird, vgl. z.B. Bruhn, M., Kommunikationspolitik, a.a.O., S. 597f. sowie die dort angegebene Literatur.

361 Diese relativ weite Abgrenzung des Suchfeldes erweist sich als sinnvoll, da auch Beiträge über Flughăfen und Flugzeugbauer das Thema strategischer Luftverkehrsallianzen behandeln. Flughäfen sind beispielsweise als Drehkreuze von Allianz-Entwicklungen betroffen, wăhrend sich Flugzeugbauer durch die Allianzbildung auf Airline-Seite mit Einkaufsgremien kooperierender Fluggesellschaften konfrontiert sehen. 


\begin{tabular}{|l|c|c|c|c|}
\cline { 2 - 5 } \multicolumn{1}{c|}{} & $\begin{array}{c}\text { Berichte über } \\
\text { Gründungen } \\
\text { oder Auflösun- } \\
\text { gen strategischer } \\
\text { Luftverkehrs- } \\
\text { allianzen }\end{array}$ & $\begin{array}{c}\text { Berichte über } \\
\text { wirtschaftliche } \\
\text { Daten von stra- } \\
\text { tegischen Luft- } \\
\text { verkehrs- } \\
\text { allianzen }\end{array}$ & $\begin{array}{c}\text { Berichte über } \\
\text { rechtliche } \\
\text { Aspekte strate- } \\
\text { gischer Luftver- } \\
\text { kehrsallianzen }\end{array}$ & $\begin{array}{c}\text { Berichte uber } \\
\text { endnutzerbezo- } \\
\text { gene Inhalte von } \\
\text { Luftverkehrs- } \\
\text { allianzen }\end{array}$ \\
\hline Anzahl & 63 & 51 & 35 & 8 \\
\hline Prozent & 52,5 & 42,5 & 29,16 & 6,66 \\
\hline
\end{tabular}

Abb. 12: Inhalte von Presseberichten zu strategischen Luftverkehrsallianzen

In 52,5 Prozent der betrachteten Fälle geben die Artikel Auskunft über neu gegründete oder aufgelöste Partnerschaften zwischen Fluggesellschaften, oft verbunden mit Hintergrundinformationen über die jeweils beteiligten Unternehmen. Darüber hinaus finden sich i.d.R. Hinweise auf die Gestaltung der Zusammenarbeit in Form von Nennungen ausgewählter Code Share-Strecken oder über die Erwähnung intendierter Kapitalbeteiligungen. ${ }^{363}$ Bei 42,5 Prozent der Beiträge stehen wirtschaftliche Daten bestehender Allianzen im Vordergrund, die sich beispielsweise in allianzspezifischen Passagierzahlen, Marktanteilen und der Anzahl gemeinsamer Flüge ausdrücken. ${ }^{364}$ Diese Informationen finden auch Eingang in die Beurteilung der wirtschaftlichen Lage einzelner, an Allianzen beteiligter Fluggesellschaften und lassen Schlüsse auf die Erfolgswirkungen von Partnerschaften zu. $^{365}$ Rechtliche Aspekte strategischer Luftverkehrsallianzen werden in 29,16 Prozent der untersuchten Presseberichte berücksichtigt. Gegenstand sind zum einen die Liberalisierung von Luftverkehrsabkommen zwischen den Staaten

362 Absolute Häufigkeit bezogen auf die Anzahl von 120 Beitrăgen mit Allianz-Inhalt. Da sich ein Beitrag oft mehreren Themenschwerpunkten widmet, addieren sich die Prozentwerte nicht zu einhundert.

363 Vgl. exemplarisch o.V., Swissair gründet europäische Luftverkehrsallianz, in: Handelsblatt vom 31.3.1998, S. 13 sowie o.V., Fluglinie KLM wird Partner von Alitalia, in: FAZ vom 18.12.1997, S. 20.

364 Spaeth (1996) berichtet Z.B. Über die wirtschaftlichen Ergebnisse der bedeutendsten internationalen Allianzen im Luftverkehr. Vgl. Spaeth, A., Ehelust und Ehefrust, in: touristik management, Nr. 3, 1996, S. 27.

$365 \mathrm{Vgl}$. beispielsweise den Bericht über United Airlines bei o.V., Lufthansa-Partner wăchst im Atlantik-Verkehr, in: Handelsblatt vom 22.9.1997, S. 15. 
(„open skies") als Rahmenbedingung für Allianzen und zum anderen die kartellrechtliche Beurteilung strategischer Luftverkehrsallianzen. ${ }^{366}$

Die Lektüre dieser Artikelinhalte informiert den potentiellen Endnutzer von Flugdienstleistungen demnach zwar über managementrelevante Aspekte strategischer Allianzen. Für die Nutzung einer Code Share-Flugdienstleistung, die z.B. von zwei Partnerfluggesellschaften erbracht wird, bergen diese Artikel jedoch kaum relevante Informationen mit Ausnahme des Hinweises auf bestehende Allianz-Konstellationen und ihre Partner. Lediglich 6,66 Prozent der analysierten Beiträge befassen sich mit Aspekten strategischer Luftverkehrsallianzen, die für die Nutzung einer Allianz-Flugdienstleistung relevant sind. Im Mittelpunkt stehen hier Hinweise auf die Gefahr monopolähnlicher Preisbildung und den "Etikettenschwindel" durch die Trennung von vermarktender und durchführender Fluggesellschaft beim Code Sharing, aber auch die Information über Vorteile für den Endnutzer wie die gegenseitige Anerkennung von Vielfliegerprogrammen, Loungezugang und Durchabfertigung des Gepäcks. ${ }^{367}$

Die Wahrnehmung strategischer Luftverkehrsallianzen und ihrer Leistungsangebote anhand von Presseberichten dürfte auf Basis dieser Erkenntnisse eher zu einer Vermehrung des "managementrelevanten" denn des "kundenrelevanten“ Wissens der potentiellen Endnutzer führen. ${ }^{368}$ In überwiegender Ermangelung detaillierter Informationen zum Ablauf einer Code Share-Flugdienstleistung oder möglichen Problembereichen der erlebbaren Zusammenarbeit von Allianzpartnern ist daher von einer betriebswirtschaftlich und juristisch geprägten Wahrnehmung strategischer Luftverkehrsallianzen auszugehen. Sowohl der "Allianz-Theoretiker" als auch der "Allianz-Erfahrene“ überführen Teile der wahrgenommenen Informationen über die Gedächtnisspeicherung in Wissen und bauen eine Einstellung gegenüber strategischen Allianzen auf.

$366 \mathrm{Vgl}$. beispielhaft o.V., Kinnock droht mit dem EuGH, in: Handelsblatt vom 12.3.1998, S. 9; Spaeth, A., Start in den offenen Himmel, in: touristik management, Nr. 4, 1996, S. 54.

367 Vgl. exemplarisch o.V., "Das nenne ich Enteignung“, in: Der Spiegel, Nr. 19, 1998, S. 130f.; Spaeth, A., Ist im Regime des „Offenen Himmels“ alles möglich? in: Das Parlament, Nr. 18, 1998, S. 12; Kolf, F., Airline-Allianz soll nicht nur Kosten senken, in: Handelsblatt vom 15.5.1997, S. 16; Kiani-Kress, R., Heineken-Effekt, in: Wirtschaftswoche, Nr. 49, 1996, S. 134f.

$368 \mathrm{Zu}$ berücksichtigen ist jedoch die Auswahl der hier ausgewerteten Print-Titel, die sich vor allem an Zielgruppen mit höherem Bildungsstand und Einkommen wenden. Diese Zielgruppen sind für Fluggesellschaften als aktuelle oder potentielle Fluggäste allerdings gleichfalls von hohem interesse. 
Lediglich der "Allianz-Erfahrene" konfrontiert dieses Wissen und diese Einstellung mit der tatsächlichen Leistung der strategischen Allianz, in dem er mit Beginn des ersten Teilprozesses der Flugdienstleistung, der Informationssuche, gezielt auf weitere Informationen zugreift. Zusätzlich zu den genannten Quellen, die mittelbare Informationen liefern, gewinnt der Endnutzer Informationen aus dem interaktiven Erleben der Allianz-Flugdienstleistung, die zugleich Basis für die Beurteilung strategischer Luftverkehrsallianzen sind. Im Gegensatz zu inm erlebt der „AllianzÜberraschte" die Flugdienstleistung, ohne zuvor eine strategische Luftverkehrsallianz wahrgenommen zu haben. Der Bekanntheitsgrad strategischer Allianzen liegt in seinem Fall bei 0 Prozent, so daß ex definitione auch keine Einstellung gegenüber Allianzen aufgebaut sein kann. Die im folgenden geführten Überlegungen setzen sich mit dem Erleben der Allianz-Flugdienstleistung in dem Teilprozeß der Informationssuche auseinander und haben sowohl für "Allianz-Erfahrene" als auch für „Allianz-Überraschte“ Gültigkeit.

\subsubsection{Erleben strategischer Luftverkehrsallianzen im Teilprozeß der Infor- mationssuche}

Den Ausgangspunkt für das Erleben einer Allianz-Flugdienstleistung markiert in dem hier zugrunde gelegten Verständnis die gezielte Suche nach Informationen über alternative Leistungsangebote, die geeignet sind, das Bedürfnis des potentiellen Fluggastes zu befriedigen (vgl. Abbildung 13). ${ }^{369}$ Zur Befriedigung des Bedürfnisses stehen dem Endnutzer Ressourcen in Form von Zeit und Geld zur Verfügung, die entweder inm selbst zuzuordnen sind (konsumtive Flugdienstleistung) oder von dem arbeitgebenden Unternehmen bzw. der arbeitgebenden Institution disponiert werden (investive Flugdienstleistung).

$369 \mathrm{Vgl}$. auch zur Darstellung einer Auswahlhandlung bei Verkehrsdienstleistungen Perrey, J., Nutzenorientierte Marktsegmentierung: ein integrativer Ansatz im Verkehrsdienstleistungsbereich, a.a.O., S. 16f. Die hier wiedergegebene Schilderung der Kaufentscheidung lehnt sich an das bereits in Kapitel B 1.1.1 skizzierte Prozeßmodell des Kaufverhaltens in Tradition neobehavioristischer Forschung an. Vgl. weiterführend Gutsche, J., Produktpräferenzanalyse, a.a.O., S. $42 \mathrm{ff}$. 


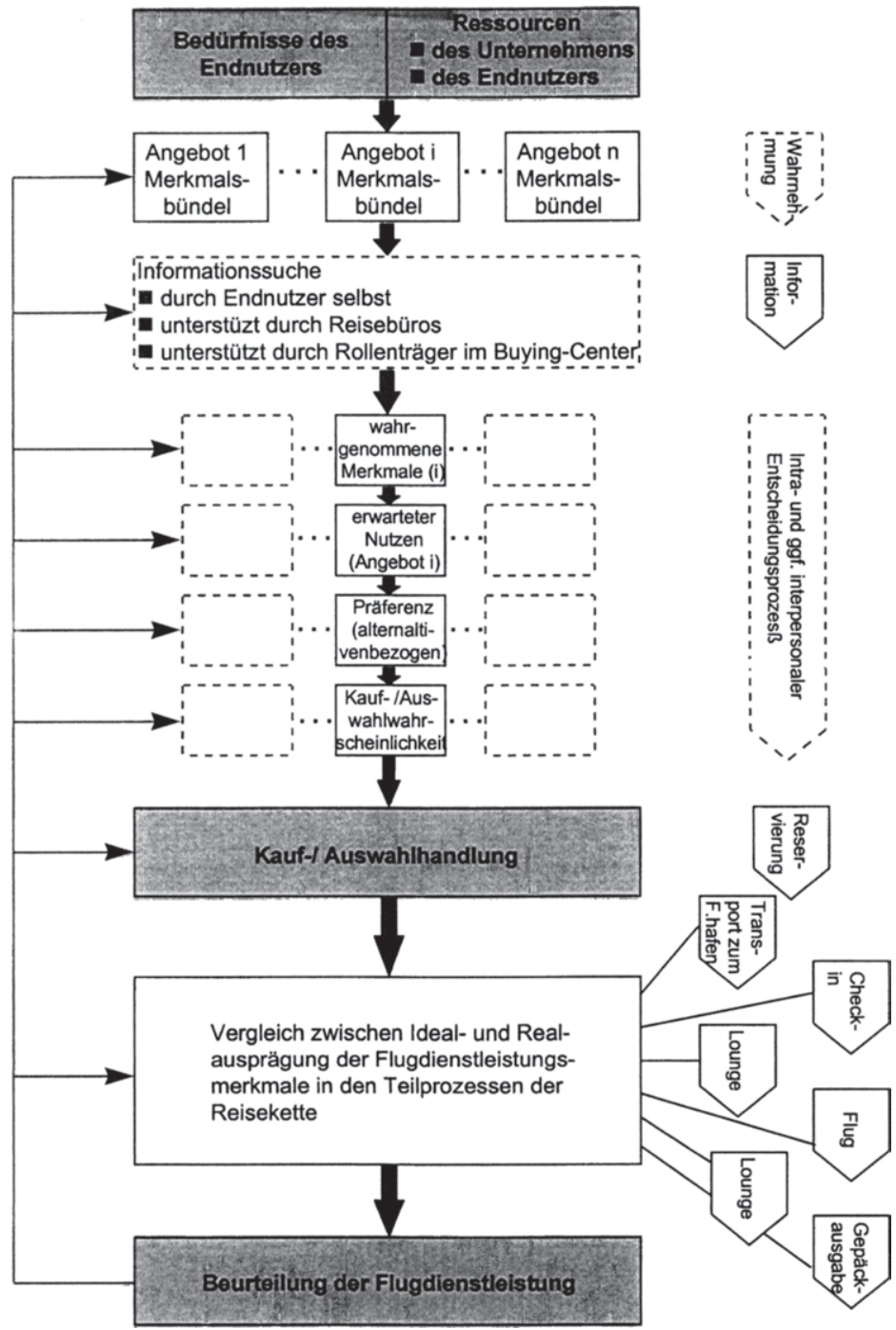

\section{Abb. 13: Vereinfachtes Modell des Auswahlverhaltens und der Beurtei- lung von Flugdienstleistungen durch Endnutzer}

(Quelle: in teilweiser Anlehnung an Gutsche, J., Produktpräferenzanalyse. Ein modelltheoretisches und methodisches Konzept zur Marktsimulation mittels Präferenzerfassungsmodellen, Berlin 1995, S. 42.) 
Die durch die Aktivierung des Bedürfnisses ausgelöste Informationssuche führt zunächst zu einer zielorientierten Auseinandersetzung des Endnutzers mit Informationsquellen, die über alternative Flugdienstleistungsangebote Auskunft geben. Neben den im Zuge der Wahrnehmung erläuterten mittelbaren stehen nun auch unmittelbare Informationsquellen wie ein Beratungsgespräch in einem Reisebüro oder ein Telefonat mit einem Mitarbeiter der Firmen-Reisestelle zur Verfügung. In bezug auf strategische Luftverkehrsallianzen ergeben sich an dieser Stelle einige Besonderheiten, die im folgenden zu erläutern sind. ${ }^{370}$

Um Aufschluß über das Verhalten von Reisebüros als Mittler zwischen Luftverkehrsallianzen und Endnutzern zu gewinnen, führte das US-amerikanische Verkehrsministerium 1994 Stichprobenbuchungen bei Reisebüros durch. In über 30 Prozent der Fälle gaben die Mitarbeiter der Unternehmen keine Hinweise auf den Code Sharing-Charakter bestimmter Flugdienstleistungen. Das gleiche galt für die Fluggesellschaften selbst, bei denen nachgefragt wurde. ${ }^{371}$

Eine im März und April 1995 von der Deutschen Forschungsanstalt für Luft- und Raumfahrt e.V. durchgeführte Untersuchung gelangte zu einem ähnlichen Ergebnis. 40 Reisebüros im Großraum Köln/Bonn/Düsseldorf wurden angerufen und um Auskunft zu einer bestimmten Flugverbindung gebeten, bei der es sich um eine Code Share-Verbindung handelte. Erfolgte durch den Reisebüromitarbeiter keine Information über diesen Sachverhalt, wurde mit Fragen wie „Welche Fluggesellschaft führt diesen Flug durch?" oder "Ist das dann auch Fluggerät und Personal der Fluggesellschaft X?" nachgesetzt. In 80 Prozent der Fälle informierten die Reisebüros die Anrufer überhaupt nicht oder fehlerhaft über den operating carrier. Lediglich 20 Prozent gaben diese Information korrekt weiter. Die wenigsten Reisebüros informierten von sich aus, d.h. ohne gezielte Fragen seitens des Kunden. Ferner schienen viele Reisebüromitarbeiter die Hinweise aus den von innen verwendeten Buchungssystemen nicht zu kennen oder interpretieren zu können. ${ }^{372}$ Einschränkungen dieser Untersuchungsergebnisse sind jedoch mit der geringen

370 Die Erörterung der weiteren Teilprozesse der Auswahl und Beurteilung von Flugdienstleistungen durch Endnutzer erfolgt in Kapitel B 2.2.3, um hier zunăchst die Besonderheiten der Informationssuche herauszustellen. Vgl. Beyhoff, S., Ehmer, H., Wilken, D., Code-Sharing im internationalen Luftverkehr der Bundesrepublik Deutschland, a.a.O., S. 90.

372 Vgl. Beyhoff, S., Ehmer, H., Wilken, D., Code-Sharing im internationalen Luftverkehr der Bundesrepublik Deutschland, a.a.O., S. $90 f$. 
Fallzahl, der regionalen Eingrenzung der Befragungspersonen und der ausschließlich telefonischen Befragungsform verbunden. Zudem ist mittlerweile von Lerneffekten bei Reisebüromitarbeitern im Umgang mit Code Share-Flugdienstleistungen auszugehen, da die Bedeutung derselben seit 1995 kontinuierlich gestiegen ist. Dennoch liegen mit dieser Untersuchung Ergebnisse vor, die zur Erklärung der Irritationen von Fluggästen auf Code Share-Flügen beitragen. Dies gilt in besonderem Maße für den als „Allianz-Überraschter" bezeichneten Fluggasttyp.

Neben der Erklärungsbedürttigkeit des Code Sharing hängen das Informationsund Beratungsverhalten der Reisebüros und damit ein Teil der Informationssuche des Endnutzers wesentlich von der informationstechnologischen Infrastruktur ab. Zur Suche nach Flugplaninformationen, Preisen und Kapazitäten sowie zur Vornahme der Buchungen verwendet die Mehrzahl aller Reisebüros Computerreservierungssysteme. ${ }^{373}$ Diese, häufig im Kapitalbesitz von Fluggesellschaften befindlichen EDV-Systeme ${ }^{374}$ ermöglichen es den dezentralen Nutzern wie Reisebüros, Firmen-Reisestellen sowie Privatpersonen, für gewünschte Flugverbindungen die entsprechenden Angebote unterschiedlicher Fluggesellschaften aufzulisten. Durch das Code Sharing von Allianzpartnern werden Flugdienstleistungen unter dem Markennamen einer Fluggesellschaft, auch als "designator code“ bezeichnet, dargestellt, obwohl der Flug physisch von einer anderen, der PartnerFluggesellschaft durchgeführt wird. Ohne zusätzliche Kennzeichnung könnte ein Reisebüromitarbeiter demzufolge keine Rückschlüsse auf die Arbeitsteilung zwischen den Allianzpartnern ziehen. Aus diesem Grund wurde eine gesetzliche Kennzeichnungspflicht für Code Share-Flugdienstleistungen eingeführt, ${ }^{375}$ die sich in den verschiedenen Computerreservierungssystemen gemäß Abbildung 14 unterschiedlich darstellt. Trotz der bestehenden Kennzeichnungspflicht konnte in einem Expertengespräch durch eine Online-Recherche im ersten Versuch eine

373 In den USA beispielsweise sind 97 Prozent aller Reisebüros mit einem Computerreservierungssystem verbunden. 90 Prozent aller Buchungen mit denselben stellen Flugdienstleistungen dar, die rund 60 Prozent des Gesamtumsatzes dieser Reisebüros ausmachen. Vgl. Lyle, C., Agent blues, in: Airline Business, No. 11, 1995, S. 56.

374 American Airlines implementierte 1964 das erste EDV-gestützte Reservierungssystem SABRE, das auch heute noch zu den führenden Lösungen zählt. Vgl. zur Entstehungsgeschichte McKenney, J.L., Copeland, D.C., Mason, R.O., Waves of Change: Business Evolution through Information Technology, Boston 1995, S. 96ff. Eine Übersicht über Beteiligungs- und Kooperationsverhältnisse zwischen Fluggesellschaften und Computerreservierungssystemen findet sich bei Weinhold, M., Computerreservierungssysteme im Luftverkehr, a.a.O., S. 145.

375 Vgl. Beyhoff, S., Ehmer, H., Wilken, D., Code-Sharing im internationalen Luftverkehr der Bundesrepublik Deutschland, a.a.O., S. 57. 
nicht als solche gekennzeichnete Code Share-Flugverbindung im System ausfindig gemacht werden, so daß systembedingte Fehlinformationen des Reisebürokunden auch in weiteren Fällen nicht auszuschließen sind. ${ }^{376}$

\begin{tabular}{|l|c|c|}
\hline $\begin{array}{l}\text { Name des Computer- } \\
\text { reservierungssystems }\end{array}$ & $\begin{array}{c}\text { Kennzeichnungsart für } \\
\text { Code Sharing }\end{array}$ & Marktanteil weltweit \\
\hline Amadeus & $\star$ & $14 \%$ \\
\hline Galileo & $@$ & $22 \%$ \\
\hline Abacus & $\begin{array}{c}\text { Angabe des operating } \\
\text { carrier }\end{array}$ & $2 \%$ \\
\hline Apollo & ${ }^{*}$ & $8 \%$ \\
\hline Sabre & ${ }^{*}$ & $29 \%$ \\
\hline SystemOne & Angabe des operating \\
carrier & $9 \%$ \\
\hline Worldspan & \multicolumn{2}{|c|}{} \\
\hline Marktanteilssumme & \multicolumn{2}{|c|}{} \\
\hline
\end{tabular}

Abb. 14: Kennzeichnung von Code Sharing-Flugdienstleistungen in groBen Computerreservierungssystemen

(Quelle: Beyhoff, S., Ehmer, H., Wilken, D., Code-Sharing im internationalen Luftverkehr der Bundesrepublik Deutschland, Forschungsbericht Nr. 95-23 der Deutschen Forschungsanstalt für Luft- und Raumfahrt e.V., Köln 1995, S. 58.)

Die in der Abbildung angegebenen Marktanteilswerte verdeutlichen zum einen die Bedeutung der sieben größten Systeme, die 96 Prozent aller weltweiten Buchungen auf sich vereinen, sowie die marktbezogenen Auswirkungen der Kennzeichnungsart für Code Share-Angebote. Lediglich in 14 Prozent der Fälle ist der Name des operating carrier dem Buchungssystem zu entnehmen.

Die Reihenfolge, in der die designator codes der Fluggesellschaften auf dem Bildschirm erscheinen, ist von hoher Relevanz für die Buchung. Schätzungen

376 Vgl. Anhang 4. Es besteht teilweise die Möglichkeit, Code Share-Flugdienstleistungen auch bei nicht vorhandener Kennzeichnung anhand anderer Informationen wie beispielsweise dem angegebenen Fluggerăt zu identifizieren. Dies setzt jedoch die genaue Kenntnis der Flottenzusammensetzung verschiedener Fluggesellschaften voraus. Findet sich bei einem nicht gesondert gekennzeichneten Code Share-Flug der Lufthansa beispielsweise die Fluggerătangabe DH1 (=Turbopropmaschine vom Typ DASH 8) im AMADEUS-System, kann es sich nicht um einen von der Lufthansa selbst operierten Flug handeln, da sie keine derartigen Flugzeuge in ihrer Flotte aufweist. 
gehen davon aus, daß ca. 85 Prozent aller getätigten Flugbuchungen von der ersten Bildschirm-Seite des Computerreservierungssystems stammen. ${ }^{377}$ Mittels Code Sharing können Allianzpartner auf unterschiedliche Weise die Reihenfolge der Darstellung zu ihren Gunsten beeinflussen. ${ }^{378}$ Die Nutzung dieser Möglichkeiten führt mitunter dazu, daß durch nur eine Code Share-Verbindung mehr als eine Bildschirmseite belegt und andere Angebote auf hintere Seiten verdrängt werden. Dieser Effekt („screen padding“) kann auf seiten der Reisebüros zu einer aufwendigen Informationssuche und ergänzendem Nachschlagen führen, um beispielsweise Auskunft über den operating carrier oder den Standort einer Lounge am betreffenden Flughafen geben zu können. ${ }^{379}$ Für den Reisebüromitarbeiter stellt sich daher die Frage, ob er seinem Kunden durch Unterlassung weiterer Suchschritte Informationen vorenthalten soll, um beispielsweise Zeit und damit Kosten zu sparen, oder ob er sich um eine möglichst hohe Informationstransparenz und Beratungsqualität bemühen soll. Im ersten Fall besteht das Risiko, einen nicht angemessen informierten Kunden mit einer für inn überraschenden Flugdienstleistung zu konfrontieren. Unzufriedenheit mit der Beratungsleistung des Reisebüros sowie Kundenabwanderung können die Folge sein. Im zweiten Fall sind Zeitverluste und höhere Kosten der Information und Beratung durch die Reisebüros in Kauf zu nehmen.

Es bleibt festzuhalten, daß Reisebüros im Teilprozeß der Information eine wichtige Rolle einnehmen. Als Mittler zwischen Fluggesellschaft und Endnutzer hängt es in hohem Maße von innen ab, ob und ggf. wie Endnutzer eine strategische Luftverkehrsallianz in diesem Teilprozeß der Reisekette erleben. Da Reisebüros auch für Firmenkunden Aufgaben der Information und Beratung übernehmen, ${ }^{380}$ ist eine mangelnde Informationstransparenz ebenso für Firmenkunden und damit für Geschäftsreisende zu diskutieren. In Abhängigkeit von dem Professionalitätsgrad der Firmen-Reisestelle oder des Sekretariats ist unter Umständen ein noch gravierenderer Grad an informationeller Unterversorgung von Geschäftsreisenden zu erwarten. Bei einem professionell betriebenen Reisemanagement dagegen

377 Vgl. Beyhoff, S., Ehmer, H., Wilken, D., Code-Sharing im internationalen Luftverkehr der Bundesrepublik Deutschland, a.a.O., S. 56.

378 Vgl. im einzelnen Beyhoff, S., Ehmer, H., Wilken, D., Code-Sharing im internationalen Luftverkehr der Bundesrepublik Deutschland, a.a.O., S. 60-68.

379 Vgl. Beyhoff, S., Ehmer, H., Wilken, D., Code-Sharing im internationalen Luftverkehr der Bundesrepublik Deutschland, a.a.O., S. $48 f$.

380 Erinnert sei an den 45-Prozent-Anteil derjenigen Firmenkunden in Deutschland, die Reisebüros mit der Abwicklung ihrer Reisen beauftragen. Vgl. Kapitel B 2.1.2. 
können reisebüroseitige Informations- und Kompetenzdefizite firmenseitig ausgeglichen werden. Zudem sind die Kommunikationsbeziehungen innerhalb des Firmen-Buying Center zu berücksichtigen, die sich z.B. in Erfahrungsberichten des Geschäftsreisenden gegenüber Reisestelle, Vorgesetztem oder Sekretariat niederschlagen. Angesichts einer hohen Erklärungsbedürftigkeit von Code ShareFlugdienstleistungen sowie einer wenig transparenten Systemunterstützung ist die Wahrscheinlichkeit eines Informations- und Beratungsnachteils von AllianzFlugdienstleistungen gegenüber Einzel-Flugdienstleistungen daher hoch. Dies gilt insbesondere für "Allianz-Überraschte" Fluggäste, aber auch für "Allianz-Erfahrene" in Abhängigkeit ihres allianzspezifischen Erfahrungsumfangs und Wissensstandes.

Die damit erörterte Informationssuche bildet gemäß dem Prozeßmodell des Auswahl- und Beurteilungsverhaltens den Ausgangspunkt für intra- und ggf. interpersonale Prozesse, welche die Bewertung und den Vergleich von Leistungsangeboten vor der Auswahl- bzw. Kaufhandlung beinhalten. Im folgenden werden ausgewählte empirische Studien untersucht, die sich auf der Basis von Nutzenerwartungen mit dem Auswahl- und Kaufverhalten von potentiellen Fluggästen auseinandersetzen. Berücksichtigung findet auch eine US-amerikanische Studie, die im speziellen auf strategische Allianzen eingeht. 


\subsubsection{Ausgewählte empirische Befunde zum nutzenbasierten Auswahl- und Kaufverhalten bei Flugdienstleistungen unter besonderer Berücksich- tigung strategischer Luftverkehrsallianzen}

Flugdienstleistungen können als Bündel von Merkmalen und ihren Ausprägungen aufgefaßt werden. ${ }^{381}$ Als Merkmale sind beispielsweise die Verbindungsqualität und der Preis zu nennen, deren Ausprägungen sich in "Direktverbindung vs. Umsteigeverbindung" und „hohes vs. niedriges Preisniveau“ unterteilen lassen. Im Rahmen eines Bewertungsprozesses werden die zur Verfügung stehenden alternativen Leistungsangebote anhand ihrer Merkmale und Merkmalsausprägungen miteinander verglichen und beurteilt. Das Ergebnis dieses Beurteilungsprozesses, der ein rationales Verhalten unterstellt, äußert sich in dem angebotsbezogenen erwarteten Nutzen. ${ }^{382}$ Aufbauend auf den Nutzenerwartungen, die den Leistungsangeboten einzelner Fluggesellschaften und Luftverkehrsallianzen zugeordnet werden, bilden die Endnutzer eine Präferenzrangfolge, die auch in Kauf- bzw. Auswahlwahrscheinlichkeiten ausgedrückt werden kann. ${ }^{383}$

Die Untersuchung des Erlebens und der Beurteilung von Flugdienstleistungen im allgemeinen und von Allianz-Flugdienstleistungen im speziellen setzt vor diesem Hintergrund voraus, daß diejenigen Merkmale identifiziert werden, welche die Quelle der Nutzenstiftung sind. ${ }^{384}$ Dementsprechend bemühen sich Airlines im Rahmen der Marktsegmentierung um die Präzisierung nutzenstiftender Eigenschaften von Flugdienstleistungen. ${ }^{385}$ Aufgrund ihrer inhaltlichen Überschneidung

381 Vgl. z.B. Dieckmann, R., Optimale Preis- und Angebotspolitik für komplexe Produkte: eine Analyse der Güterbündelung unter Berücksichtigung der Verarbeitung von Preisen durch Konsumenten, Frankfurt am Main u.a. 1993, S. 1.

382 Neben äußeren Reizen nehmen auch Werte und Emotionen des Endnutzers Einfluß auf die Beurteilung der Leistungsangebote. Vgl. Perrey, J., Nutzenorientierte Marktsegmentierung: ein integrativer Ansatz im Verkehrsdienstleistungsbereich, a.a.O., S. 17.

${ }^{383}$ Auch bei der vergleichsweise höchsten Kauf- bzw. Auswahlwahrscheinlichkeit ist nicht zwingend von einem tatsächlichen Kauf der Leistung auszugehen. Vgl. hierzu Hahn, C., Conjoint- und Discrete Choice-Analyse als Verfahren zur Abbildung von Präferenzstrukturen und Produktauswahlentscheidungen. Ein theoretischer und computergestützter empirischer Vergleich, Münster 1997, S. 11.

384 Botschen und Mühlbacher (1998) betonen jedoch, daß die dienstleistungsspezifischen Merkmale zumeist nur den funktionalen Teil des Kundennutzens zu erklären vermögen. Sie postulieren daher die zusătzliche Erfassung des individuellen psycho-sozialen Nutzens von Kunden, um Aussagen über das Kaufverhalten ableiten zu können. Vgl. Botschen, G., Mühlbacher, H., Zielgruppenprogramm - Zielgruppenorientierung durch Nutzensegmentierung, in: Handbuch Dienstleistungs-Marketing, Band 1, Hrsg.: Meyer, A., Stuttgart 1998, S. 688. 
geben die solchermaßen erfaßten Nutzenerwartungen Aufschlüsse über die idealerweise vorhandenen Eigenschaften von Flugdienstleistungen.

Zur Segmentierung von Fluggästen auf Basis von Nutzenerwartungen liegen unterschiedliche empirische Studien vor. ${ }^{386}$ In einer 1977 durchgeführten Befragung von Fluggästen auf Nordatlantik-Flugrouten beispielsweise identifizierten die Forscher Robinson und Wind vier Nutzensegmente, die anhand von 12 Merkmalen gebildet wurden, allerdings nur eine bedingte Trennschärfe aufwiesen. Zur Erklärung der Segmentzugehörigkeit trugen insgesamt sechs der zwölf Merkmale bei, die kompositionell erfaßt wurden. ${ }^{387}$ Aus der Anwendung der kompositionellen Methode, bei der die Befragungspersonen zunächst um die Beurteilung von Einzelmerkmalen eines Befragungsobjektes gebeten werden, um diese dann zu einem Gesamturteil zu verdichten, ergab sich in dieser Untersuchung eine hohe Bedeutung der Mehrzahl erfaßter Merkmale („Anspruchsinflation“). Die Nutzensegmente wurden als "Comfort Conscious“ (Komfortbewußte), „Anti Group Flights“ (Gruppenflug-Gegner), „Anti Booking Restrictions“ (Buchungsrestriktionen-Gegner) und „Price Sensitive“ (Preissensible) bezeichnet. Die Nutzenerwartungen der Komfortbewußten drückten sich in vielfältigen Serviceleistungen an Bord des Flugzeugs, möglichst geringen Belästigungen durch Gruppenreisende sowie bequemen Buchungsmöglichkeiten aus. Die Zielgruppe der Gruppenflug-Gegner zeichnete sich durch die dominante abwehrende Haltung gegenüber Gruppenreisenden an Bord des Flugzeugs aus, während das Segment der Buchungsrestriktionen-Gegner vornehmlich an flexiblen Buchungsoptionen interessiert war. Die Zielgruppe der Preissensiblen wies schließlich eine dominant hohe Bedeutung des Merkmals Preis auf, der gegenüber den anderen Merkmalen mehr als 20 Prozentpunkte Bedeutungsunterschied zeigte. ${ }^{388} \mathrm{Im}$ Vergleich zu anderen Unter-

$385 \mathrm{Vgl}$. Söderlund, M., Market orientation and the firm's market intelligence: an empirical study of what airlines want to know about their customers, in: Marketing Today and for the 21st Century, Proceedings of the 24th Annual Conference of the European Marketing Academy, Cergy-Pontoise Cedex 1995, S. 969.

Der Terminus "Nutzensegmentierung" geht auf die Übersetzung des vermutlich von Haley (1968) erstmalig eingeführten Begriffs „Benefit Segmentation“ zurück. Vgl. Haley, R.I., Benefit segmentation. A decision oriented research tool, in: Journal of Marketing, Vol. 32, No. 7, 1968, S. 30-35. Weiterführend vgl. z.B. Perrey, J., Nutzenorientierte Marktsegmentierung: ein integrativer Ansatz im Verkehrsdienstleistungsbereich, a.a.O.

387 Vgl. Robinson, P.J., Wind, Y., Multinational Trade-off Segmentation, in: Moving Ahead with Attitude Research, Hrsg.: Wind, Y., Greenberg, M.G., Chicago 1977, S. $50 \mathrm{ff}$.

388 Vgl. Robinson, P.J., Wind, Y., Multinational Trade-off Segmentation, a.a.O., S. 53. 
suchungen kam dem Preis in der Studie jedoch insgesamt eine überraschend geringe Bedeutung zu.

In einer zwischen 1994 und 1995 durchgeführten Untersuchung nahm Laakmann eine Segmentierung im innerdeutschen Personenluftverkehr vor. ${ }^{389}$ Mittels Conjoint Measurement wurden die fünf Merkmale "Anbieter", "Preis", „Sitze", "Gatebuffet" und „Flexibilität" als clusterbildende Variablen herangezogen. ${ }^{390} \mathrm{Da}$ die ermittelten Merkmalswichtigkeiten insbesondere bezüglich des Preises und der Flexibilität auf Unterschiede zwischen aus geschäftlichem und aus privatem Anlaß reisenden Fluggästen hindeuteten, wurde vor der Nutzensegmentierung eine Aufteilung der Befragten in Geschäfts- und Privatreisende vorgenommen. ${ }^{391}$ Mittels Clusteranalyse konnten daraufhin jeweils drei Nutzensegmente identifiziert werden, die im Fall der Geschäftsreisenden als „Preissensible Commodity-Flieger", "Leistungs- und Komfortorientierte" und "Flexibilitätsorientierte" bezeichnet wurden. Für die Privatreisenden kam Laakmann zu einer nahezu identischen Segmentlösung, deren zweites Segment aufgrund unterschiedlicher Wichtigkeiten im Komfort- und Flexibilitätsmerkmal allerdings geringfügig modifiziert als "Service- und Komfortorientierte" benannt wurde. ${ }^{392}$ Die mit 41 Prozent in der Gruppe der Geschäftsreisenden vertretenen „Preissensiblen Commodity-Flieger" maßen dem Merkmal Preis die höchste Wichtigkeit zu, während die "Leistungsund Komfortorientierten" (42 Prozent) besonderen Wert auf flexible Buchungs-

389 Vgl. Laakmann, K., Value added services als Profilierungsintrument im Wettbewerb, a.a.O., S. $211 \mathrm{ff}$.

390 Das Verfahren der Conjoint-Analyse kennzeichnet die dekompositionelle Methode der Nutzenmessung, bei der die Nutzenbeitrăge der einzelnen Attribute eines Beurteilungsobjektes anhand empirisch erhobener Gesamtnutzenwerte gemessen werden. Für eine weitergehende Darstellung der Methode vgl. Green, P.E., Srinivasan, V., Conjoint Analysis in Consumer Research: Issues and Outlook, in: Journal of Consumer Research, Vol. 5, No. 9, 1978, S. 103ff.; Backhaus, K., u.a., Multivariate Analysemethoden: eine anwendungsorientierte Einführung, 8., verb. Aufl., Berlin u.a. 1996, S. $497 \mathrm{ff}$.

391 Diese Vorgehensweise entspricht einer in der Luftverkehrspraxis nach wie vor zu beobachtenden, nahezu als dogmatisch zu bezeichnenden Trennung zwischen Geschäfts- und Privatreisenden, denen auf der Angebotsseite eine Unterteilung in Beförderungsklassen (Economy, Business und First Class) gegenübersteht. Vgl. auch Diller, H., Deutsche Lufthansa AG - Marktsegmentierung im Luftfahrtgeschäf, in: Fallstudien zum Marketing, Teil 1: Entscheidungssituationen, Hrsg.: Dichtl, E., Wiesbaden 1979, S. 216-219; Friedrich, H., Görgen, W., Marktsegmentierung auf Dienstleistungsmärkten - dargestellt am Beispiel des Marktes für Flugdienstleistungen, Arbeitspapier des Instituts für Marketing- und Distributionsforschung, Köln 1993, S. 40; Flint, P., Will the real United please stand up?, a.a.O., S. 31.

392 Vgl. Laakmann, K., Value added services als Profilierungsintrument im Wettbewerb, a.a.O., S. 227 u. 231. 
möglichkeiten bei angemessenem Preis und Komfort (normale vs. extra breite Sitze) legten. Die "Flexibilitätsorientierten" (17 Prozent) zeichneten sich durch eine Überbetonung der Wichtigkeit flexibler Buchungsoptionen aus, während der Preis mit 48 Prozentpunkten Abstand folgte. Die Merkmalswichtigkeiten stellten sich bei den Privatreisenden segmentspezifisch in ähnlicher Weise dar, ließen jedoch einen höheren Anteil von Preissensiblen (67 Prozent) in der Stichprobe erken nen. ${ }^{393}$ Restriktionen der Untersuchung ergeben sich aus der relativ geringen Anzahl berücksichtigter Merkmale, von denen beispielsweise die Verbindungsqualität oder weitere Serviceattribute fehlen. Einschränkungen der Aussagefähigkeit resultieren zudem aus der national angelegten Befragung, wenngleich von einem bestimmten Anteil erfaßter ausländischer Umsteige-Passagiere auszugehen ist.

Stegmüller (1995) setzte sich in einer Studie mit den Nutzenerwartungen von deutschen, US-amerikanischen und japanischen Fluggästen auf interkontinentalen Flugreisen auseinander und wendete dabei sowohl das kompositionelle als auch dekompositionelle Verfahren zur Messung von Nutzenerwartungen an. ${ }^{394}$ Die folgenden Ausführungen beschränken sich auf die Darstellung der Ergebnisse aus der dekompositionellen Messung. ${ }^{395}$ In die Untersuchung fanden sieben Merkmale Eingang, die mittels Anwendung einer Hybrid-Conjointanalyse zur Bildung von Nutzensegmenten herangezogen wurden. ${ }^{396}$ Es handelte sich um die

393 Vgl. Laakmann, K., Value added services als Profilierungsintrument im Wettbewerb, a.a.O., S. 227-232. Dieser Befund verdeutlicht die Auswirkungen der Ressourcenzuordnung auf den Flugscheinkauf: im Fall einer privat initiierten Flugreise (konsumtive Flugdienstleistung) zahlt der Fluggast selbst, wăhrend eine geschăftlich initiierte Flugreise (investive Flugdienstleistung) i.d.R. vom Arbeitgeber bezahlt wird.

Vgl. Stegmüller, B., Internationale Marksegmentierung als Grundlage für internationale Marketing-Konzeptionen, a.a.O., 1995, S. 306ff.

Diese Einschränkung kann angesichts bestehender Vorbehalte gegenüber der kompositionellen Methode legitimiert werden. Stegmüller selbst weist auf die Problematik der Anspruchsinflation hin, die durch die Vielzahl zu beurteilender Merkmale ohne den Zwang gedanklicher Trade-offs entsteht. Vgl. Stegmüler, B., Internationale Marksegmentierung als Grundlage für internationale Marketing-Konzeptionen, a.a.O., 1995, S. 317. Hahn (1997) kritisiert darüber hinaus die isolierte Merkmalsbeurteilung sowie die mangelnde Einbeziehbarkeit konkurrierender Angebote, so daß eine Einschrănkung auf die dekompositionelle Methode an dieser Stelle einen höheren Erkenntnisgewinn verspricht. Vgl. Hahn, C., Conjoint- und Discrete Choice-Analyse als Verfahren zur Abbildung von Präferenzstrukturen und Produktauswahlentscheidungen, a.a.O., S. 35ff.

Im Rahmen der Hybrid-Conjointanalyse kommen sowohl kompositionelle als auch dekompositionelle Messungen zur Anwendung, welche die Verarbeitung einer höheren Zahl von Merkmalen und Merkmalsausprägungen ermöglichen. Vgl. Green, P.E., Hybrid Models for Conjoint Analysis: An Expository Review, in: Journal of Marketing Research, No. 5, 1984, S. 
Merkmale Sitzkomfort, Preis, Mahlzeiten und Getränkeservice, Bordunterhaltung und Kommnikationsmöglichkeiten, Aufmerksamkeit gegenüber dem Gast an Bord, Flugplan und Qualität der Verbindungen sowie Zusatzangebote. Während die Clusteranalyse in Deutschland und den USA jeweils zu einem 3-Cluster-Ergebnis führte, ergaben sich bei japanischen Fluggästen lediglich zwei Segmente. In Deutschland zeichneten sich alle drei Segmente durch eine dominante Wichtigkeit des Merkmals Preis aus, dem im Segment 1 der Sitzkomfort und die Aufmerksamkeit an Bord folgten. Fluggäste im Segment 2 waren dagegen in deutlich höherem Maße an einem guten Sitzkomfort interessiert, während Flugplan/ Verbindungsqualität an dritter Stelle lagen. Auch in Segment 3 zeigte sich, daß Preis und Sitzkomfort - zwar mit deutlichem Unterschied - die höchsten Merkmalswichtigkeiten darstellten. Die übrigen fünf Merkmale lagen jedoch hinsichtlich ihrer Bedeutung dichter beieinander als in den anderen Segmenten.

Für die US-amerikanischen Fluggäste ergab die Untersuchung insgesamt eine weniger starke Diskrepanz zwischen wichtigsten Merkmalen Preis und Sitzkomfort. Das Segment 2 wies eine hohe Ähnlichkeit zu dem deutschen Segment 2 auf, so daß Stegmüller von "länderübergreifend identischen Nutzensegmenten“ spricht. ${ }^{397}$ Die Fluggäste des Segments 1 werteten dagegen einen hohen Sitzkomfort sogar höher als einen angemessenen Preis, gefolgt von der ihnen entgegengebrachten Aufmerksamkeit an Bord. In dem am preissensibelsten ausgeprägten Segment 3 wiesen die Befragungspersonen dem Sitzkomfort und mit einigem Abstand der Aufmerksamkeit sowie dem Mahlzeiten- und Getränkeservice die höchste Wichtigkeit zu. Das Unterhaltungsangebot an Bord war für alle Segmente gemäß ihrer Bekundung nahezu bedeutungslos.

Gegenüber den deutschen und US-amerikanischen Fluggästen zeigten die japanischen Probanden eine gänzlich abweichende Nutzenstruktur. Während der Preis lediglich das fünftwichtigste Merkmal darstellte, galt dem Flugplan und der Verbindungsqualität die höchste Bedeutung in beiden Segmenten. Im Segment 1 kam daraufhin der Aufmerksamkeit an Bord sowie der Sitzqualität jeweils eine

155f. Angesichts dieses auch von Stegmüller hervorgehobenen Vorteils verwundert die relativ geringe Anzahl von sieben Merkmalen, die für die Clusterbildung herangezogen werden. Vgl. Stegmüller, B., Internationale Marksegmentierung als Grundlage für internationale MarketingKonzeptionen, a.a.O., 1995, S. 232.

397 Stegmüller, B., Internationale Marksegmentierung als Grundlage für internationale MarketingKonzeptionen, a.a.O., 1995, S. 329. 
relativ hohe Wichtigkeit zu, an die sich mit deutlichem Abstand das Mahlzeitenangebot anschloß. Die Fluggäste in Segment 2 unterschieden sich vornehmlich durch eine geringer ausgeprägte Dominanz hinsichtlich des Flugplans und der Verbindungsqualität und die höhere Wichtigkeit des Sitzkomforts vom Segment 1. Die geringste Bedeutung maßen beide Cluster dem Unterhaltungsangebot an Bord sowie den Zusatzangeboten bei. ${ }^{398}$ Als Kritik an dieser Studie ist die Nichtberücksichtigung von buchungsbezogenen Merkmalen hervorzuheben, deren Kaufverhaltensrelevanz in den vorgenannten Studien nachgewiesen werden konnte. Das Merkmal "Flugplan und Qualität der Verbindungen“ bleibt zudem auf einem relativ hohen Abstraktionsniveau und eröffnet damit ungewollte Interpretationsspielräume bei der Auswertung der Befragungsergebnisse.

Eine 1996 von United Airlines in Auftrag gegebene Marktforschungsstudie wählte - ähnlich wie in der Analyse von Laakmann - ein zweistufiges Vorgehen. ${ }^{399}$ Nach einer Unterteilung der befragten Fluggäste in Geschäfts- und Privatreisende wurden auf der Basis von Nutzenerwartungen fünf Segmente unter den Geschäftsreisenden gebildet, die zu 40 Prozent in der Stichprobe vertreten waren. ${ }^{400}$ Das Segment der "Road Warriors" zeichnete sich z.B. durch eine hohe Merkmalswichtigkeit von Serviceleistungen, Sitzkomfort und statusbezogenen Leistungen wie beispielsweise bevorzugtes Einchecken, Loungenutzung und persönliche Ansprache aus. Die Privatreisenden mit einem Stichprobenanteil von 60 Prozent wurden in drei Nutzensegmente unterteilt. Die Nutzenerwartungen im Cluster der „Price Driven Occasionalists" orientieren sich nahezu ausschließlich am Preis von Flugdienstleistungen. ${ }^{401}$

398 Vgl. Stegmüller, B., Internationale Marksegmentierung als Grundlage für internationale Marketing-Konzeptionen, a.a.O., 1995, S. 327-329.

${ }^{309}$ Die Beschreibung der Untersuchung findet sich in steckbriefähnlicher Form bei Flint, P., Will the real United please stand up?, a.a.O., S. 31f. Methodische Details sind dem Beitrag ebenso wenig zu entnehmen wie eine vollstăndige, genauere Erläuterung der segmentspezifischen Merkmalswichtigkeiten. Dennoch können dem Beitrag Erkenntnisse für die Bearbeitung der vorliegenden Problemstellung entnommen werden.

Die Segmente trugen die etwas blumigen Bezeichnungen „Road Warriors“, "Global Travelers“, "Eager Corporate Soldiers", "Reluctant Corporate Soldiers“ sowie "Do-It-Yourself Price Shoppers". Eine Übersetzung unterbleibt daher aus semantischen Gründen. Vgl. Flint, P., Will the real United please stand up?, a.a.O., S. 31.

Die übrigen Privatreisenden-Segmente trugen die Bezeichnung ${ }_{\text {Personal Service Seekers" }}$ und ${ }_{n}$ Mile Collecting Vacationers ${ }^{4}$. Flint, P., Will the real United please stand up?, a.a.O., S. 31. Eine Kritik am Untersuchungsdesign erübrigt sich angesichts der geringen diesbezüglich vorliegenden Angaben. 
Den hiermit erörterten Studien lag keine allianzspezifische Fragestellung zugrunde. Gleichwohl lassen sich aus den Ergebnissen einige Erkenntnisse gewinnen, die für die weitere Bearbeitung der vorliegenden Problemstellung hilfreich sein können:

Im Personenluftverkehr lassen sich auf der Basis individueller Nutzenerwartungen Segmente von Fluggästen bilden, die sich bezüglich der Wichtigkeit bestimmter Leistungsmerkmale von Flugdienstleistungen voneinander unterscheiden.

- Als Quellen der Nutzenstiftung benennen die vier Studien vor allem flexible Buchungsmöglichkeiten, niedrige Preise, umfangreiche Serviceleistungen, Sitzkomfort, Verbindungsqualität und statusbezogene Leistungen.

- Nutzenerwartungen von Fluggästen können länderspezifisch unterschiedlich ausgeprägt sein, wenngleich in einer Studie ein länderübergreifend einheitliches Segment identifiziert werden konnte.

Die Nutzenerwartungen zwischen Privat- und Geschäftsreisenden weisen z.T. hohe Ähnlichkeiten auf. Unterschiede ergeben sich jedoch bei der Wichtigkeit des Merkmals „Preis“, die bei Privatreisenden höher ausgeprägt ist.

Im Wettbewerb gegenüber einzeln operierenden Fluggesellschaften muß sich das Leistungsangebot strategischer Allianzen folglich an den segmentspezifischen Nutzenerwartungen der Fluggäste "messen" lassen, die als anzustrebende Idealausprägung einer Flugdienstleistung verstanden werden können. ${ }^{402} \mathrm{Zu}$ berücksichtigen sind dabei auch länderspezifische Besonderheiten. Diesem endnutzerseitig induzierten Anspruchsspektrum sind nun die anbieterseitigen, allianzbezogenen Leistungspotentiale, -prozesse und -ergebnisse gegenüberzustellen. Eine Verdichtung von Plausibilitätsüberlegungen zum „Fit“ zwischen Leistungsangebot und Nutzenerwartungen i.S. von Idealausprägungen findet sich, differenziert nach den oben dargestellten Studien, in den Abbildungen 15 a) bis d) wieder. Die Ausprägungen für den Fit variieren vereinfacht zwischen

(+) „allianzseitiges Leistungsangebot kommt den Nutzenerwartungen näher als das Leistungsangebot einzeln agierender Fluggesellschaften " und

402 An dieser Stelle wird lediglich auf den Wettbewerb mit einzeln operierenden Fluggesellschaften rekurriert, da die vorgestellten Studien nicht in einem Allianz-Kontext durchgeführt wurden und daher auch keine Rückschlüsse auf spezifische Allianz-Nutzenerwartungen zulassen. 
(-) „allianzseitiges Leistungsangebot wird den Nutzenerwartungen in geringerem Maße gerecht als das Leistungsangebot einzeln agierender Fluggesellschaften".

Für strategische Luftverkehrsallianzen scheint demnach insbesondere ein Fit durch die Weitergabe von Kostensenkungen in Form von Preisvorteilen sowie durch höhere Buchungsflexibilität erreichbar zu sein. Zudem liegen mögliche Potentiale in der Nutzung der partnerindividuellen Kulturen für die Schaffung einer Servicevielfalt und differenzierten Bordbetreuung. Darüber hinaus ist anzunehmen, daß die Erwartungen in bezug auf ein störungsarmes Reisen sowie eine Gewährung von statusbezogenen Sonderleistungen einen hohen potentiellen Fit zur gegenseitigen Statusanerkennung innerhalb von Allianzen sowie einer eng aufeinander abgestimmten Durchführung von Flugdienstleistungen aufweisen. 


\begin{tabular}{|c|c|}
\hline $\begin{array}{l}\text { Merkmale mit nutzenstiftender } \\
\text { Bedeutung }\end{array}$ & $\begin{array}{l}\text { Zu erwartender Fit mit strategischen Luftverkehrsallianzen (Abgleich } \\
\text { von Leistungsangebot und Nutzenerwartungen) }\end{array}$ \\
\hline \multicolumn{2}{|r|}{ Studie von Robinson und Wind (1977) } \\
\hline \multicolumn{2}{|c|}{ Segment 1: „Comfort Conscious“ (Komfortbewußte) } \\
\hline $\begin{array}{l}\text { Serviceangebot an Bord } \\
(20,9 \%)^{403}\end{array}$ & $\begin{array}{l}\text { - Steigerung der Servicevielfalt durch mehrere Partner (+) } \\
\text { - Unterschiedliche Leistungsstandards der Partner (-) }\end{array}$ \\
\hline keine Gruppenreisen $(14,6 \%)$ & - keine pauschale Aussage möglich \\
\hline Preis $(9,1 \%)$ & $\begin{array}{l}\text { Weitergabe von allianzbedingten Kostenvorteilen in Form von Preis- } \\
\text { senkungen }(+) \\
\text { steigende Preise durch Ausnutzung der allianzbedingten Markt- } \\
\text { dominanz (-) }\end{array}$ \\
\hline \multicolumn{2}{|c|}{ Segment 2: „Anti-Group Flights“ (Gruppenflug-Gegner) } \\
\hline keine Gruppenreisen $(44,6 \%)$ & - keine pauschale Aussage möglich \\
\hline Preis $(11,1 \%)$ & $\begin{array}{l}\text { Weitergabe von allianzbedingten Kostenvorteilen in Form von Preis- } \\
\text { senkungen }(+) \\
\text { steigende Preise durch Ausnutzung der allianzbedingten Markt- } \\
\text { dominanz }(-)\end{array}$ \\
\hline Buchungssicherheit $(7,6 \%)$ & $\begin{array}{l}\text { Verunsicherung durch informationstechnologische Abstimmungs- } \\
\text { probleme zwischen Partnern (-) }\end{array}$ \\
\hline \multicolumn{2}{|c|}{ Segment 3: „Anti Booking Restrictions“ (Buchungsrestriktionen-Gegner) } \\
\hline Buchungssicherheit $(22,9 \%)$ & $\begin{array}{l}\text { Verunsicherung durch informationstechnologische Abstimmungs- } \\
\text { probleme zwischen Partnern (-) }\end{array}$ \\
\hline $\begin{array}{l}\text { keine Vorausbuchungsfrist } \\
(19,3 \%)\end{array}$ & - keine pauschale Aussage möglich \\
\hline $\begin{array}{l}\text { flexible Umbuchungsmöglichkei- } \\
\text { ten }(16,3 \%)\end{array}$ & $\begin{array}{l}\text { größere Flexibilität bei Umbuchungen im Allianzsystem durch engere } \\
\text { Abstimmung als im traditionellen Interlining (+) } \\
\text { Verunsicherung durch informationstechnologische Abstimmungs- } \\
\text { probleme zwischen Partnern (-) }\end{array}$ \\
\hline \multicolumn{2}{|c|}{ Segment 4: „Price Sensitive“ (Preissensible) } \\
\hline Preis $(31,8 \%)$ & $\begin{array}{l}\text { Weitergabe von allianzbedingten Kostenvorteilen in Form von Preis- } \\
\text { senkungen }(+) \\
\text { steigende Preise durch Ausnutzung der allianzbedingten Markt- } \\
\text { dominanz (-) }\end{array}$ \\
\hline Buchungssicherheit (10,4\%) & $\begin{array}{l}\text { Verunsicherung durch informationstechnologische Abstimmungs- } \\
\text { probleme zwischen Partnern (-) }\end{array}$ \\
\hline $\begin{array}{l}\text { keine Vorausbuchungsfrist } \\
(8,9 \%)\end{array}$ & - keine pauschale Aussage möglich \\
\hline
\end{tabular}

Legende: $(+)$ nallianzseitiges Leistungsangebot kommt den Nutzenerwartungen năher als das Leistungsangebot einzeln agierender Fluggesellschaften“; (-) „allianzseitiges Leistungsangebot wird den Nutzenerwartungen in geringerem Maße gerecht als das Leistungsangebot einzeln agierender Fluggesellschaften“.

\section{Abb. 15 a): Vergleich zwischen Nutzenerwartungen und Allianz-Leistungs- angebot am Beispiel ausgewählter Studien}

403 Die Prozentwerte geben die Teilnutzenwerte der betreffenden Nutzendimensionen an. 


\begin{tabular}{|c|c|}
\hline $\begin{array}{l}\text { Merkmale mit nutzenstiftender } \\
\text { Bedeutung }\end{array}$ & $\begin{array}{l}\text { Zu erwartender Fit mit strategischen Luftverkehrsallianzen (Abgleich } \\
\text { von Leistungsangebot und Nutzenerwartungen) }\end{array}$ \\
\hline \multicolumn{2}{|c|}{ Studie von Laakmann $(1995)^{404}$} \\
\hline \multicolumn{2}{|c|}{ „Preissensible Commodity-Flieger “ } \\
\hline Preis $(71,72 \%)$ & $\begin{array}{l}\text { Weitergabe von allianzbedingten Kostenvorteilen in Form von Preis- } \\
\text { senkungen }(+) \\
\text { - steigende Preise durch Ausnutzung der allianzbedingten Markt- } \\
\text { dominanz }(-)\end{array}$ \\
\hline $\begin{array}{l}\text { Umbuchungsmöglichkeit } \\
(14,54 \%)\end{array}$ & $\begin{array}{l}\text { - größere Flexibilität bei Umbuchungen im Allianzsystem durch engere } \\
\text { Abstimmung als im traditionellen Interlining (+) } \\
\text { - Verunsicherung durch informationstechnologische Abstimmungs- } \\
\text { probleme zwischen Partnern (-) }\end{array}$ \\
\hline Sitzkomfort $(7,36 \%)$ & $\begin{array}{l}\text { - unterschiedliche Standards der Partner bezüglich Sitzbreite und - } \\
\text { abstand (- bis }+ \text { ) }\end{array}$ \\
\hline \multicolumn{2}{|c|}{ "Leistungs- und Komfortorientierte“ } \\
\hline $\begin{array}{l}\text { Umbuchungsmöglichkeit } \\
(33,42 \%)\end{array}$ & $\begin{array}{l}\text { - größere Flexibilität bei Umbuchungen im Allianzsystem durch engere } \\
\text { Abstimmung als im traditionellen Interlining }(+) \\
\text { - Verunsicherung durch informationstechnologische Abstimmungs- } \\
\text { probleme zwischen Partnern (-) }\end{array}$ \\
\hline Preis $(28,15 \%)$ & $\begin{array}{l}\text { - Weitergabe von allianzbedingten Kostenvorteilen in Form von Preis- } \\
\text { senkungen }(+) \\
\text { - steigende Preise durch Ausnutzung der allianzbedingten Markt- } \\
\text { dominanz (-) }\end{array}$ \\
\hline Sitzkomfort $(18,16 \%)$ & $\begin{array}{l}\text { - unterschiedliche Standards der Partner bezüglich Sitzbreite und - } \\
\text { abstand (- bis +) }\end{array}$ \\
\hline \multicolumn{2}{|l|}{ „Flexibilitätsorientierte“ } \\
\hline $\begin{array}{l}\text { Umbuchungsmöglichkeit } \\
(72,41 \%)\end{array}$ & $\begin{array}{l}\text { - größere Flexibilität bei Umbuchungen im Allianzsystem durch engere } \\
\text { Abstimmung als im traditionellen Interlining (+) } \\
\text { - Verunsicherung durch informationstechnologische Abstimmungs- } \\
\text { probleme zwischen Partnern (-) }\end{array}$ \\
\hline Preis $(24,86 \%)$ & $\begin{array}{l}\text { Weitergabe von allianzbedingten Kostenvorteilen in Form von Preis- } \\
\text { senkungen }(+) \\
\text { - steigende Preise durch Ausnutzung der allianzbedingten Markt- } \\
\text { dominanz (-) }\end{array}$ \\
\hline Gatebuffet $(1,40 \%)$ & $\begin{array}{l}\text { abweichende Standards zwischen den Partnern bezüglich Vorhan- } \\
\text { densein und Ausgestaltung (- bis +) }\end{array}$ \\
\hline
\end{tabular}

Legende: $(+)$ "allianzseitiges Leistungsangebot kommt den Nutzenerwartungen näher als das Leistungsangebot einzeln agierender Fluggesellschaften“; $(-)$ "allianzseitiges Leistungsangebot wird den Nutzenenwartungen in geringerem Maße gerecht als das Leistungsangebot einzeln agierender Fluggesellschaften“.

\section{Abb. 15 b): Vergleich zwischen Nutzenerwartungen und Allianz-Leistungs- angebot am Beispiel ausgewählter Studien}

404 Aus Gründen der Übersichtlichkeit werden lediglich die Nutzensegmente der Geschäftsreisenden wiedergegeben. Diese Einschränkung erfolgt unter dem Wissen, daß die Nutzensegmente der Privatreisenden mit Ausnahme einer stärkeren Bedeutung des Preises ähnliche Nutzenstrukturen aufweisen. 


\begin{tabular}{|c|c|}
\hline $\begin{array}{l}\text { Merkmale mit nutzenstiftender } \\
\text { Bedeutung }\end{array}$ & $\begin{array}{l}\text { Zu erwartender Fit mit strategischen Luftverkehrsallianzen (Abgleich } \\
\text { von Leistungsangebot und Nutzenerwartungen) }\end{array}$ \\
\hline \multicolumn{2}{|c|}{ Studie von Stegmüller $(1995)^{405}$} \\
\hline \multicolumn{2}{|c|}{ Länderübergreifendes Segment 2 (D/USA) } \\
\hline Preis & $\begin{array}{l}\text { Weitergabe von allianzbedingten Kostenvorteilen in Form von Preis- } \\
\text { senkungen }(+) \\
\text { - steigende Preise durch Ausnutzung der allianzbedingten Markt- } \\
\text { dominanz (-) }\end{array}$ \\
\hline Sitzkomfort & $\begin{array}{l}\text { - unterschiedliche Standards der Partner bezüglich Sitzbreite und - } \\
\text { abstand (- bis +) }\end{array}$ \\
\hline \multicolumn{2}{|l|}{ Deutschland, Segment 1} \\
\hline Preis & $\begin{array}{l}\text { Weitergabe von allianzbedingten Kostenvorteilen in Form von Preis- } \\
\text { senkungen }(+) \\
\text { steigende Preise durch Ausnutzung der allianzbedingten Markt- } \\
\text { dominanz (-) }\end{array}$ \\
\hline Sitzkomfort & $\begin{array}{l}\text { - unterschiedliche Standards der Partner bezüglich Sitzbreite und - } \\
\text { abstand (- bis +) }\end{array}$ \\
\hline Aufmerksamkeit an Bord & $\begin{array}{l}\text { - Verständigungsprobleme zwischen Flugbegleitern und Fluggästen bei } \\
\text { unterschiedlichen Nationalităten der Partner (-) } \\
\text { kulturelle Inkompatibilitäten in der Servicephilosophie zwischen den } \\
\text { Partnern (-) } \\
\text { - Bereicherung durch kulturelle Diversität (+) }\end{array}$ \\
\hline \multicolumn{2}{|l|}{ Deutschland, Segment 3} \\
\hline Preis & $\begin{array}{l}\text { Weitergabe von allianzbedingten Kostenvorteilen in Form von Preis- } \\
\text { senkungen (+) } \\
\text { steigende Preise durch Ausnutzung der allianzbedingten Markt- } \\
\text { dominanz (-) }\end{array}$ \\
\hline Sitzkomfort & $\begin{array}{l}\text { unterschiedliche Standards der Partner bezüglich Sitzbreite und - } \\
\text { abstand (- bis +) }\end{array}$ \\
\hline \multicolumn{2}{|l|}{ USA, Segment 1} \\
\hline Sitzkomfort & $\begin{array}{l}\text { unterschiedliche Standards der Partner bezüglich Sitzbreite und - } \\
\text { abstand (- bis +) }\end{array}$ \\
\hline Preis & $\begin{array}{l}\text { Weitergabe von allianzbedingten Kostenvorteilen in Form von Preis- } \\
\text { senkungen (+) } \\
\text { - } \begin{array}{l}\text { steigende Preise durch Ausnutzung der allianzbedingten Markt- } \\
\text { dominanz (-) }\end{array}\end{array}$ \\
\hline Aufmerksamkeit an Bord & s. Deutschland, Segment 1 \\
\hline
\end{tabular}

Legende: (+) „allianzseitiges Leistungsangebot kommt den Nutzenerwartungen năher als das Leistungsangebot einzeln agierender Fluggesellschaften“; (-) „allianzseitiges Leistungsangebot wird den Nutzenerwartungen in geringerem Maße gerecht als das Leistungsangebot einzeln agierender Fluggesellschaften“.

\section{Abb. 15 c): Vergleich zwischen Nutzenerwartungen und Allianz-Leistungs- angebot am Beispiel ausgewählter Studien}

405 Da Teilnutzenwerte in dieser Untersuchung nicht angegeben sind, sind die Merkmale nach ihrer Wichtigkeit geordnet. Im länderübergreifenden Segment 2 beispielsweise bildet der Preis das dominante Merkmal, gefolgt vom Sitzkomfort. Die übrigen Merkmale weisen einen deutlichen Abstand zu diesen beiden Merkmalen auf und sind daher nicht in der Tabelle wiedergegeben. 


\begin{tabular}{|c|c|}
\hline $\begin{array}{l}\text { Merkmale mit nutzenstiftender } \\
\text { Bedeutung }\end{array}$ & $\begin{array}{l}\text { Zu enwartender Fit mit strategischen Luftverkehrsallianzen (Abgleich } \\
\text { von Leistungsangebot und Nutzenerwartungen) }\end{array}$ \\
\hline \multicolumn{2}{|c|}{ Studie von Stegmüller (1995) (Forts.) } \\
\hline \multicolumn{2}{|l|}{ Japan, Segment 1} \\
\hline $\begin{array}{l}\text { Flugplan und Verbindungsqua- } \\
\text { lität }\end{array}$ & $\begin{array}{l}\text { Erweiterung des Streckennetzes/Erhöhung der Anzahl Flüge auf } \\
\text { bestehenden Strecken durch Aufnahme von Code Share-Verbindun- } \\
\text { gen }(+) \\
\text { - One-Stop-Check-in, Gepäck-Durchabfertigung, abgestimmte Flugzei- } \\
\text { ten, räumliche Nähe der Partner am Flughafen (+) } \\
\text { - Abstimmungsprobleme zwischen Partnern (-) }\end{array}$ \\
\hline Aufmerksamkeit an Bord & $\begin{array}{l}\text { - Verständigungsprobleme zwischen Flugbegleitern und Fluggästen bei } \\
\text { unterschiedlichen Nationalităten der Partner (-) } \\
\text { - kulturelle Inkompatibilitäten in der Servicephilosophie zwischen den } \\
\text { Partnern (-) } \\
\text { - Bereicherung durch kulturelle Diversităt (+) }\end{array}$ \\
\hline Sitzkomfort & $\begin{array}{l}\text { - unterschiedliche Standards der Partner bezüglich Sitzbreite und - } \\
\text { abstand (- bis +) }\end{array}$ \\
\hline $\begin{array}{l}\text { Mahlzeitenangebot/Getränke- } \\
\text { service }\end{array}$ & $\begin{array}{l}\text { - kulturell bedingte Unterschiede im Mahlzeitenangebot der Partner } \\
\text { (eher -) } \\
\text { - unterschiedliche Standards beim Getränkeservice (- bis +) }\end{array}$ \\
\hline \multicolumn{2}{|l|}{ Japan, Segment 2} \\
\hline $\begin{array}{l}\text { Flugplan und Verbindungsqua- } \\
\text { lität }\end{array}$ & - s. Japan, Segment 1 \\
\hline Sitzkomfort & - s. Japan, Segment 1 \\
\hline Aufmerksamkeit an Bord & - s. Japan, Segment 1 \\
\hline $\begin{array}{l}\text { Mahlzeitenangebot/Getränke- } \\
\text { service }\end{array}$ & - s. Japan, Segment 1 \\
\hline \multicolumn{2}{|c|}{ Studie von United Airlines, dokumentiert bei Flint (1997) } \\
\hline \multicolumn{2}{|c|}{ Geschäftsreisenden-Segment „Road Warriors“ } \\
\hline Serviceleistungen an Bord & $\begin{array}{l}\text { - Steigerung der Servicevielfalt durch mehrere Partner (+) } \\
\text { - Unterschiedliche Leistungsstandards der Partner (-) }\end{array}$ \\
\hline Sitzkomfort & $\begin{array}{l}\text { - unterschiedliche Standards der Partner bezüglich Sitzbreite und } \\
\text {-abstand (- bis }+)\end{array}$ \\
\hline statusbezogene Leistungen & $\begin{array}{l}\text { - gegenseitige Anerkennung der Vielfliegerprogramme }(+) \\
\text { - Zugang zu Lounges der Partner-Airlines (+) } \\
\text { - Anerkennung von Statuskunden innerhalb der Allianz (+) }\end{array}$ \\
\hline \multicolumn{2}{|c|}{ Privatreisende „Price Driven Occasionalists“ } \\
\hline Preis & $\begin{array}{l}\text { Weitergabe von allianzbedingten Kostenvorteilen in Form von Preis- } \\
\text { senkungen }(+) \\
\text { - } \begin{array}{l}\text { steigende Preise durch Ausnutzung der allianzbedingten Markt- } \\
\text { dominanz }(-)\end{array}\end{array}$ \\
\hline
\end{tabular}

Legende: $(+)$ „allianzseitiges Leistungsangebot kommt den Nutzenerwartungen näher als das Leistungsangebot einzeln agierender Fluggesellschaften“; $(-)$ nallianzseitiges Leistungsangebot wird den Nutzenerwartungen in geringerem Maße gerecht als das Leistungsangebot einzeln agierender Fluggesellschaften“.

\section{Abb. 15 d): Vergleich zwischen Nutzenerwartungen und Allianz-Leistungs- angebot am Beispiel ausgewählter Studien}


Neben Potentialen zur Übererfüllung deuten andere Überlegungen auf eine mögliche Untererfüllung von Nutzenerwartungen gegenüber dem konkurrierenden Angebot von Einzel-Flugdienstleistungen hin. Zunächst sind in diesem Zusammenhang steigende Preise durch die Ausnutzung der allianzbedingten Marktdominanz zu nennen, die das Leistungsangebot der Allianz insbesondere für das Segment preissensibler Fluggäste unattraktiver wirken lassen dürften. Bezüglich des Serviceangebots und der Betreuung an Bord könnten unterschiedliche Leistungsstandards der Partner-Fluggesellschaften, sprachliche Verständigungsschwierigkeiten sowie abweichende Servicephilosophien einem Fit zu den endnutzerseitigen Nutzenerwartungen vor allem der Service- und Komfortorientierten abträglich sein. Die Sicherstellung einer optimal aufeinander abgestimmten Flugplanung sowie flexibler Buchungsmöglichkeiten schließlich ist möglicherweise durch Koordinationsschwierigkeiten der Partner sowie informationstechnologische Inkompatibilitäten und Reibungsverluste gefährdet. Diese Überlegung wiegt um so schwerer, als sich die Folgen solcher Schnittstellenprobleme beispielsweise in Wartezeiten, verpaßten Anschlußfügen und fehlgeleitetem Gepäck ausdrücken können. ${ }^{406}$

Um nun auch empirisch überprüften Aufschluß über die Relevanz von Leistungsmerkmalen für das Auswahlverhalten von Fluggästen zwischen Allianz- und NichtAllianzflugdienstleistungen zu erhalten, wurde 1994 eine Studie vom US-amerikanischen Verkehrsministerium in Auftrag gegeben. ${ }^{407}$ Unter Anwendung der Discrete-Choice Analyse, die - basierend auf einer dekompositionellen Leistungsbeurteilung - das Ergebnis eines individuellen Wahlverhaltens als diskrete 0/1 Entscheidung (Auswahl oder Nichtauswahl) prognostiziert, ${ }^{408}$ wurde die Bedeutung verschiedener Flugdienstleistungsmerkmale für die Kaufentscheidung unter-

406 Bereits eingangs wurde auf die Bedeutung von Verspätungen, Gepäckhandling und Kundenbeschwerden eingegangen. Eine Bewertung findet in den USA in Form der "triple crown“ statt: Pünktlichkeit („Anteil Flüge, die nicht spăter als 15 Minuten nach Plan gestartet oder gelandet sind“), Gepäckhandling („Anzahl Gepäckschäden/-fehlleitung pro 1.000

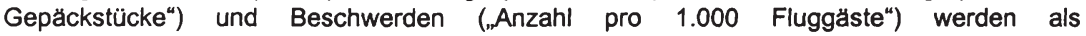
Qualitätskennzahl erfaßt.

Die folgenden Ausführungen beziehen sich auf die Seiten $82 \mathrm{ff}$. bei Gellman Research Associates (Hrsg.), A Study of International Airline Code Sharing, Washington 1994.

408 Zur näheren Erläuterung des Verfahrens der Discrete Choice-Analyse vgl. z.B. Hahn, C., Conjoint- und Discrete Choice-Analyse als Verfahren zur Abbildung von Präferenzstrukturen und Produktauswahlentscheidungen, a.a.O., S. 83ff.; Balderjahn, I. Marktreaktionen von Konsumenten. Ein theoretisch-methodisches Konzept zur Analyse der Wirkung marketingpolitischer Instrumente, Berlin 1993, S. $117 \mathrm{ff}$. 
sucht. Im Gegensatz zu den vorgenannten Untersuchungen stand in diesem Fall weniger die Segmentierung des Marktes für Flugdienstleistungen im Vordergrund, die mit diesem Verfahren auch nicht zu leisten wäre, ${ }^{409}$ als vielmehr die Abschätzung von Marktanteilsveränderungen durch das Leistungsangebot strategischer Luftverkehrsallianzen. Als unabhängige Variablen wählten die Forscher sechs Merkmale aus, die mit inren Merkmalsausprägungen aus der Darstellung in Abbildung 16 hervorgehen.

\begin{tabular}{|l|l|}
\hline \multicolumn{1}{|c|}{ Merkmal } & \multicolumn{1}{c|}{ Merkmalsausprägung(en) } \\
\hline Verbindungsqualität (1) & $\begin{array}{l}\text { 1. Code Share-Direktverbindung } \\
\text { 2. Direktverbindung Einzelfluggesellschaft } \\
\text { 3. Code Share-Umsteigeverbindung } \\
\text { 4. Umsteigeverbindung mit Zubringerflug- } \\
\text { gesellschaft } \\
\text { 5. Übrige Umsteigeverbindungen (konven- } \\
\text { tionelle IATA Interline Agreements) }\end{array}$ \\
\hline Zeitabstände zwischen Flügen (2) & $\begin{array}{l}\text { gering (= viele tägliche Abflugtermine) bis } \\
\text { hoch (= wenige tägliche Abflugtermine) }\end{array}$ \\
\hline Durchschnittspreis (3) & niedrig bis hoch \\
\hline $\begin{array}{l}\text { Angebot an First und Business Class-Sitz- } \\
\text { plätzen (4) }\end{array}$ & \begin{tabular}{l} 
gering bis hoch \\
\hline Flugreisezeit (5)
\end{tabular} \\
\hline Drehkreuzfunktion des Flughafens (6) & $\begin{array}{l}\text { kurz bis lang } \\
\text { ja (= Abflug- oder Zielflughafen ist Dreh- } \\
\text { kreuz der Fluggesellschaft) oder nein (= } \\
\text { Abflug- Oder Zielflughafen ist kein Dreh- } \\
\text { kreuz der Fluggesellschaft) }\end{array}$ \\
\hline
\end{tabular}

Abb. 16: Konzeptualisierung kaufverhaltensrelevanter Merkmale und Merkmalsausprägungen von Flugdienstleistungen in der Studie von Gellman Research Associates

(Quelle: in Anlehnung an Gellman Research Associates (Hrsg.), A Study of International Airline Code Sharing, Washington 1994, S. 93)

$409 \mathrm{Vgl}$. Perrey, J., Nutzenorientierte Marktsegmentierung: ein integrativer Ansatz im Verkehrsdienstleistungsbereich, a.a.O., S. 88f. 
Als abhängige Variable diente die Auswahl (nominal skaliert 1) oder Nichtauswahl (0) einer bestimmten Flugdienstleistung, die in aggregierter Form Rückschlüsse auf streckenbezogene Marktanteile geben sollte. Die Ausprägungen des Merkmals Verbindungsqualität sind in der Reihenfolge ihrer Vorziehenswürdigkeit wiedergegeben, die von den Forschern angenommen und in der empirischen Untersuchung auch bestätigt wurde. Demnach werden Code Share-Direktverbindunge ${ }^{410}$ allen anderen Ausprägungen der Verbindungsqualität vorgezogen. Für diese Tatsache werden zum einen die Listungseffekte in den Computerreservierungssystemen der Reisebüros verantwortlich gemacht. Zum anderen begründen die Autoren der Studie diesen Befund mit allianzspezifischen Vorteilen für Endnutzer wie beispielsweise der gegenseitigen Anerkennung von Vielfliegerprogrammen und dem Zugang zu Lounges der Partnerfluggesellschaften. ${ }^{411}$ In verstärktem Maße trifft diese Argumentation auf den Vergleich zwischen Code ShareUmsteigeverbindungen ${ }^{412}$ und Zubringer- sowie auf IATA-Interline-Agreements basierenden Umsteigeverbindungen zu. ${ }^{413}$ Den Allianzpartnern wird bei Code Share-Umsteigeverbindungen demzufolge eine höhere Kompetenz und Zuverlässigkeit bei der Durchabfertigung von Gepäck zugesprochen als im Fall von Zubringern oder konventionellem Interlining. Darüber hinaus bilden das nur einmal erforderliche Einchecken sowie die räumliche Nähe der von den Partnern genutzten Flugsteige weitere Gründe für die Vorziehenswürdigkeit einer Code Share-Umsteigeverbindung. ${ }^{414}$

Mit dem Zeitabstand zwischen Flügen berücksichtigt die Untersuchung ein Merkmal, das bei hoher Ausprägung eine negative Wirkung auf die Auswahlentscheidung hat. Ein großer zeitlicher Abstand zwischen den Flügen einer Airline

410 Eine Code Share-Direktverbindung liegt vor, wenn ein Flug mit gemeinsamer Flugnummer in den Flugplaninformationen der Partnerfluggesellschaften erscheint und von lediglich einer Fluggesellschaft tatsächlich durchgeführt wird, ohne daß ein Umsteigen der Passagiere notwendig ist.

411 Vgl. Gellman Research Associates (Hrsg.), A Study of International Airline Code Sharing, a.a.O., S. 90.

412 Eine Code Share-Umsteigeverbindung liegt vor, wenn Passagiere mindestens einmal umsteigen müssen, um ihr Ziel zu erreichen. Der eigentliche Flug kann dabei entweder von lediglich einem Allianzpartner oder aber von mehreren Partnern arbeitsteilig durchgeführt werden.

413 Umsteigeverbindungen mit Zubringerfluggesellschaften zeichnen sich durch ihre Abstimmung mit dem Angebot der großen Fluggesellschaften an deren Drehkreuzen aus, haben aber keine gemeinsame Flugnummer.

414 Vgl. Gellman Research Associates (Hrsg.), A Study of International Airline Code Sharing, a.a.O., S. 90. 
auf einer bestimmten Flugstrecke birgt für den Endnutzer das Risiko, im Fall von Unregelmäßigkeiten des Flugverkehrs - z.B. Verspätungen, Streichungen - eigene Termine mangels Ausweichoptionen zu verpassen. Zudem reduziert ein zeitbezogen geringes Flugangebot grundsätzlich die Anzahl der zur Verfügung stehenden individuellen Wahlmöglichkeiten. Der negative Zusammenhang zwischen einem hohen Zeitabstand und der bekundeten Kaufentscheidung konnte in der Studie empirisch bestätigt werden. Die Erweiterung des Flugangebots einer Airline durch zusätzliche Code Share-Flugdienstleistungen mit Allianzpartnern führt nach den Erkenntnissen der Forscher zu einer Bevorzugung von Allianz-Flugdienstleistungen gegenüber Nicht-Allianzflugdienstleistungen.

Das Merkmal Durchschnittspreis wurde mit Ausprägungen zwischen gering und hoch sowie einer bei zunehmender Höhe vermuteten negativen Auswirkung auf die Kaufentscheidung berücksichtigt. Diese Annahme bestätigte sich in der Auswertung der empirischen Daten. Ergebnisse bezüglich einer allianzspezifischen Ausprägung dieses Merkmals fehlen jedoch in der Studie, da sich keine Tendenzaussage der Form "Wenn eine Allianz-Flugdienstleistung vorliegt, ist der Preis des Flugscheins niedriger als bei einer Nicht-Allianz-Flugdienstleistung" treffen ließ.

Mit dem Angebot an First und Business Class-Sitzen sollte ein Merkmal zur Erfassung der Servicequalität in das Forschungsdesign einfließen, dessen angenommene positive Wirkung auf die Wahl einer Flugdienstleistung empirisch erhärtet werden konnte. Ausgangspunkt der Überlegungen bildete die Signalwirkung eines hohen Anteils von komfortablen First und Business Class-Sitzplätzen in Flugzeugen für die Beurteilung der Servicequalität einer Fluggesellschaft. ${ }^{415}$ Auch bezüglich dieses Merkmals unterstellten die Forscher keine für Allianz-Flugdienstleistungen spezifische Ausprägung.

Dem Merkmal der streckenbezogenen Flugreisezeit, gemessen als Differenz zwischen planmäßiger Abflug- und planmäßiger Ankunftszeit, ${ }^{416}$ wurde bei

415 An dieser Stelle ist zu bezweifeln, ob die von den Forschern vorgenommene Auszählung der beförderungsklassenspezifischen Sitzplatzkapazitäten in den entsprechenden Flugzeugen geeignet ist, die endnutzerseitige Beurteilung dieses Merkmals valide abzubilden. Zum Vorgehen vgl. Gellman Research Associates (Hrsg.), A Study of International Airline Code Sharing, a.a.O., S. 91. zusătzlich die Übergangszeiten, die durch den Wechsel des Flugzeugs erforderlich sind. 
zunehmender Länge eine negative Auswirkung auf den Kauf einer Flugdienstleistung zugesprochen. Diese Annahme wurde empirisch bestätigt. Eine Reduktion der Flugreisezeit durch die Abstimmung von Flugplänen, optimiertes Schnittstellenmanagement und die räumliche Nähe der Flugsteige an Flughäfen zwischen den Allianzpartnern führte laut der Studie bei Code Share-Umsteigeverbindungen zu einer Präferenz von Allianz-Flugdienstleistungen gegenüber Nicht-AllianzFlugdienstleistungen. Dieses Merkmal ist demnach nicht unabhängig von der Verbindungsqualität zu sehen.

Mit dem Vorliegen einer Drehkreuzfunktion des Abflug- oder Zielflughafens bezogen die Forscher ein nominales Merkmal in ihre Untersuchung ein, dessen Kaufverhaltensrelevanz aus der drehkreuzbedingten Marktpräsenz einer Fluggesellschaft erklärt wurde. Annahmegemäß beeinflußt z.B. der Zielflughafen Amsterdam die Auswahl zugunsten einer Flugdienstleistung der niederländischen Airline KLM positiv, da hier eine starke Marktposition dieser Fluggesellschaft vorliegt. Auf den in die Analyse einbezogenen Flugverbindungen konnte ein positiver Einfluß dieses Merkmals für neun Fluggesellschaften und deren Drehkreuze empirisch nachgewiesen werden, während im Fall von British Airways mit London/Heathrow eine negative Auswirkung festgestellt wurde. Eine Erklärung für diesen Befund lassen die Autoren vermissen. ${ }^{417}$ Durch die gegenseitige Verknüpfung ihrer Drehkreuze beeinflussen die Partner von Luftverkehrsallianzen demzufolge die Präferenz der Endnutzer zu ihren Gunsten.

Zusammenfassend sind nach diesen empirischen Befunden strategische Luftverkehrsallianzen grundsätzlich geeignet, mit ihrem partnerschaftlich abgestimmten Leistungsangebot eine größere Nähe zu der als ideal empfundenen Flugdienstleistung zu erzeugen als einzeln agierende Fluggesellschaften. Diese Aussage gilt jedoch nur für folgende Nutzendimensionen:

417 Vgl. Gellman Research Associates (Hrsg.), A Study of International Airline Code Sharing, a.a.O., S. 92. 
Verbindungsqualität von Flügen (durch bessere Abstimmung der Partner),

- Zeitabstand zwischen Flügen (durch Angebot zusätzlicher Flugverbindungen),

- Flugreisezeit (durch Optimierung der Flugplankoordination und räumliche Nähe an Flughäfen) sowie

- Vorliegen einer Drehkreuzfunktion (durch Vernetzung flughafenbezogener Präsenzvorteile).

Einschränkungen bei der Interpretation dieser Befunde ergeben sich aus dem zugrunde liegenden Forschungsansatz, der trotz empirisch bestätigter Relevanz Zweifel an der Geeignetheit des Attributs "Angebot an First und Business Class Sitzplätzen" zur Abbildung der Servicequalität einer Fluggesellschaft aufkommen läßt, wenngleich sich diese nicht als potentieller allianzspezifischer Vorteil herausgestellt hat. Darüber hinaus ist die Unabhängigkeit der Merkmale „Verbindungsqualität" und „Flugreisezeit" in Frage zu stellen.

Neben diesen Kritikpunkten können die Untersuchungsergebnisse aufgrund einer mangelnden Segmentbildung der Auskunftspersonen nicht als Grundlage für eine segmentspezifische Marktbearbeitung herangezogen werden. ${ }^{418}$ Zudem kapriziert sich die Studie auf einen Auswahl-/Nicht-Auswahl-Vergleich zwischen AllianzFlugdienstleistungen und solchen Flugdienstleistungen, die von einzeln agierenden Fluggesellschaften angeboten werden. Ein Vergleich zwischen den Leistungsangeboten konkurrierender Luftverkehrsallianzen ist daher nicht möglich. Aufschlüsse über die segmentspezifische Beurteilung von Allianz-Flugdienstleistungen gibt die im folgenden zu erörternde Focusgruppenbefragung, die sich zudem mit der Problematik der Abgrenzung von Allianz-Flugdienstleistungen sowohl gegenüber Einzel- als auch Allianzkonkurrenzangeboten auseinandersetzt.

418 Die wesentliche Ursache hierfür liegt in der verwendeten Methode der Discrete-ChoiceAnalyse, deren Einsatz angesichts der mit der Untersuchung verfolgten Zielsetzung allerdings auch zweckkonform ist. Bei diesem Verfahren erfolgt die Schätzung der Nutzenwerte aufgrund der geringen Anzahl an Eingangsdaten auf der Gruppenebene, so daß eine einheitliche Nutzenstruktur der Nachfrager unterstellt wird. Vgl. die Diskussion bei Perrey, J., Nutzenorientierte Marktsegmentierung: ein integrativer Ansatz im Verkehrsdienstleistungsbereich, a.a.O., S. 88f. und die dort angegebene Literatur. 


\subsection{Ergebnisse einer Focusgruppenbefragung zur Beurteilung strate- gischer Luftverkehrsallianzen durch das Endnutzersegment der „Vielflieger"}

\subsubsection{Anlage der empirischen Untersuchung}

Die vorausgegangenen Ausführungen haben verdeutlicht, daß sich bereits vor der eigentlichen Inanspruchnahme einer Allianz-Flugdienstleistung Einstellungen gegenüber dem Leistungsangebot strategischer Luftverkehrsallianzen bilden können. Dies gilt sowohl für die Wahrnehmungstypen „Allianz-Theoretiker" als auch für „Allianz-Erfahrene“. Der im folgenden zu erörternden Untersuchung liegt die Annahme zugrunde, daß Endnutzer im Zuge der Informationssuche Nutzenerwartungen i.S. von Idealausprägungen bilden, anhand derer die Flugdienstleistungen sowohl einzeln agierender Fluggesellschaften als auch gemeinsam agierender Airlines in Luftverkehrsallianzen bewertet werden. Führt die Bewertung zum Kauf bzw. zur Buchung einer Allianz-Flugdienstleistung, verschaffen sich die Endnutzer im Zuge des Erlebens der weiteren Teilprozesse ein Bild von deren Realausprägungen. Der Abgleich zwischen der Ideal- und Realausprägung der Allianz-Flugdienstleistung als Beurteilung derselben gibt schließlich wertvolle Hinweise auf die strategische Positionierung von Luftverkehrsallianzen. Die umfassendsten Aussagen zur Beurteilung strategischer Luftverkehrsallianzen lassen sich aus einer Untersuchung von „Allianz-Erfahrenen“ ableiten, die Allianzen sowohl wahrnehmen als auch erleben.

Aufbauend auf diesen Überlegungen wurde im Zeitraum zwischen August und September $1996 \mathrm{im}$ Auftrag einer strategischen Luftverkehrsallianz eine Befragung von Fluggästen durchgeführt, ${ }^{419}$ die sich als "Vielflieger" bezeichnen lassen. Diese Benennung orientiert sich an dem Merkmal der Reisehäufigkeit, die als ein Filterkriterium bei der Auswahl der Befragungspersonen fungierte. Jede Befragungsperson unternahm mindestens 6 geschäftlich veranlaßte Flugreisen pro Jahr. Innerhalb der letzten 24 Monate mußten die Befragungspersonen zudem mindestens 4 interkontinentale Flugreisen, die geschäftlich veranlaßt waren, unternommen haben, von denen 2 nicht länger als ein Jahr zurückliegen durften. Eine dieser interkontinentalen Flugreisen mußte dabei mit dem Wechsel der Fluggesellschaft verbunden sein (Umsteigeverbindung).

419 Die folgenden Ausführungen beziehen sich auf die dem Verfasser vorliegende Dokumentation der empirischen Untersuchung, die sich jedoch auf einem relativ hohen Aggregationsniveau bewegt. Detailliertere Erkenntnisse, die an einigen Stellen wünschenswert gewesen wären, lassen sich daher nicht wiedergeben. 
Befragt wurden insgesamt 150 Personen, die zu 18 Focusgruppen ${ }^{420}$ mit einer durchschnittlichen Gruppenstärke von 8 Personen zusammengefaßt wurden. Die Focusgruppeninterviews wurden in 9 Großstädten durchgeführt, die über die 3 Kontinente Amerika, Europa und Asien verteilt sind. Es handelte sich im einzelnen um San Francisco, Chicago, Toronto, London, Frankfurt, Kopenhagen, São Paulo, Bangkok und Tokyo. Pro Befragungsort wurden 2 Gruppen gebildet und befragt. Gruppe 1 wurde als "allianzloyale Gruppe“ bezeichnet, deren Mitglieder die Mehrzahl ihrer Flugreisen mit einer der auftraggebenden Fluggesellschaften, i.d.R. derjenigen ihrer Heimatländer, unternahmen. Zugleich sollten mindestens 50 Prozent der Gruppe bereits mit einer der Allianzpartner-Fluggesellschaften geflogen sein. Gruppe 2 („Wettbewerber-Gruppe“) setzte sich dagegen aus Mitgliedern zusammen, welche die Mehrzahl ihrer interkontinentalen Flugreisen mit einer anderen Airline als der für das Heimatland „zuständigen" Fluggesellschaft unternahmen. Gleichzeitig mußte die Hälfte von ihnen bereits mit einem Wettbewerber eines der auftraggebenden Allianzpartner geflogen $\operatorname{sein}^{421}$ und Erfahrun-

420 Focusgruppeninterviews, auch als Gruppenexploration bezeichnet, beinhalten eine gleichzeitige Befragung mehrerer Personen, denen während des Gesprächs die Kommunikation und Interaktion untereinander erlaubt sind. Vgl. Meffert, H., Marketingforschung und Käuferverhalten, a.a.O., S. 230.

Im Anwendungsfeld der Marktforschung werden Focusgruppeninterviews vornehmlich zur wegbereitenden, qualitativen Exploration, zur Ideengenerierung und -selektion sowie der Erforschung komplexer, schlecht strukturierter Sachverhalte eingesetzt. Im Vordergrund steht die Gewinnung qualitativer Daten wie beispielsweise Motive, Einstellungen, Gedanken und Gefühle, um weniger zu einer quantitativen Erfassung als vielmehr zu einem besseren Verständnis des Untersuchungsobjekts zu gelangen. Vgl. weiterführend Drayton, J.L., Fahad, G.A., Tynan, A.C., The Focus Group: A Contoversial Research Technique, in: Graduate Management Research, Winter 1989, S. 36f.; Salcher, E.F., Psychologische Marktforschung, 2., neu bearb. Aufl., Berlin, New York 1995, S. 44f. Zu einer Beschreibung des Ablaufs von Focusgruppeninterviews vgl. Greenbaum, T.L., The handbook for focus group research, New York 1993. Angesichts der bislang kaum vorhandenen empirischen Erkenntnisse zu strategischen Luftverkehrsallianzen und des als hoch anzusehenden Komplexitătsgrads des hier betrachteten Untersuchungsobjekts erscheint die Auswahl dieser Befragungsmethode nachvollziehbar. Einschränkungen der Aussagefähigkeit ergeben sich jedoch vor allem aus der mangelnden Reprăsentativităt der gewonnenen Daten, wie die Beschreibung der Stichprobe verdeutlicht, aus moglichen Verzerrungen im Antwortverhalten, die durch die Gruppeninteraktion verursacht werden, sowie dem qualitativen Charakter der gewonnenen Informationen, der eine statistische Auswertung stark limitiert oder gănzlich verhindert. Vgl. Tuckel, P. Leppo, E., Kaplan, B., A View From the Other Side of the Mirror, in: Marketing Research, Vol. 5, No. 4, 1995, S. 24ff.; Whipple, T.W., Mapping Focus Group Data, in: Marketing Research, Vol. 6, No. 1, 1996, S. 16ff.; Mendes de Almeida, P.F., A Review of Group Discussion Methodology, in: European Research, Vol. 8, No. 3, 1980, S. 114; Fahad, G.A., Group discussions: a misunderstood technique, in: Journal of Marketing Management, Vol. 1, No. 3, 1986, S. 319.

Für jeden Befragungsort wurden die in Betracht zu ziehenden Wettbewerber ex ante definiert. 
gen mit den Allianzpartnern der von ihnen bevorzugten Airline besitzen. Für beide Gruppen galt, daß die Zielorte der interkontinentalen Flugreisen in mindestens 2 der 6 vorab definierten Hauptverkehrsmärkte liegen mußten. ${ }^{422}$

Mindestens zwei Drittel der Mitglieder beider Gruppen mußten ferner überwiegend in der Business oder First Class geflogen sein. Bis zu ein Drittel der Gruppenmitglieder konnten demnach aufgrund von Reiserichtlinien überwiegend Economy Class genutzt haben. Voraussetzung war zudem, daß keine Befragungsperson grundsätzlich ablehnend gegenüber einer der auftraggebenden Fluggesellschaften eingestellt war. ${ }^{423}$ Das Durchschnittsalter der Befragungspersonen betrug 45 Jahre, und die Stichprobe bestand zu 92 Prozent aus Männern und 8 Prozent aus Frauen. Insgesamt 94 Prozent der Stichprobe setzten sich aus acht unterschiedlichen Nationalitäten zusammen.

Der empirischen Studie lagen vier aufeinander aufbauende Teilzielsetzungen zugrunde. In der ersten Teilzielsetzung galt es, die grundsätzlich mit einer Flugdienstleistung in Verbindung stehenden Bedürfnisse der Befragungspersonen zu eruieren, um anhand dieser fundamentalen Bedürfnisse die Einstellungen der Vielflieger zu strategischen Luftverkehrsallianzen zu explorieren. Aufbauend auf den fundamentalen Bedürfnissen verfolgte die Studie daraufhin die zweite Teilzielsetzung, die aus Sicht der Befragungspersonen zentralen Nutzenerwartungen an Flugdienstleistungen zu identifizieren und die strategischen Luftverkehrsallianzen zugesprochene Fähigkeit zur Erfüllung derselben im Vergleich zu einzeln agierenden Fluggesellschaften zu ermitteln. Daraufhin bestand eine dritte Teilzielsetzung der Untersuchung in der Konzipierung alternativer Positionierungsansätze für eine fiktive Allianz, die auf den zuvor gewonnenen Erkenntnissen aufbauen sollten. Als vierte Teilzielsetzung beinhaltete die Studie die Ableitung einer Positionierungsempfehlung für die Allianz des Auftraggebers, indem explorierte Idealausprägungen mit den durch die Befragungspersonen empfundenen Realausprägungen abgeglichen wurden, um auf diese Weise eine Grundlage für die Ableitung von Umsetzungsmaßnahmen der strategischen Positionierung zu schaffen.

422 Als Hauptverkehrsmärkte wurden Nordamerika, Südamerika, Europa, Afrika, Mittlerer Osten sowie Asien zugrunde gelegt.

423 Dieses Merkmal war nicht erfüllt, wenn eine der auftraggebenden Fluggesellschaften als Antwort auf folgende Frage genannt wurde: „Gibt es Fluggesellschaften, mit denen Sie nicht fliegen würden?" 


\subsubsection{Fundamentale Bedürfnisse von Vielfliegern und ihre Einstellungen gegenüber strategischen Luftverkehrsallianzen}

Die in den Focusgruppeninterviews gewonnenen Erkenntnisse lassen drei fundamentale Bedürfnisse der befragten Vielflieger in den Vordergrund treten. Das einer in Anspruch genommenen Flugdienstleistung zugrunde liegende Bedürfnis kann als Mobilitätsbedürfnis bezeichnet werden, das sich in dem Wunsch ausdrückt, von einem Punkt $A$ nach $B$ oder $C$ sowie üblicherweise wieder zurück nach $A$ zu gelangen. Die Befragungspersonen äußerten darüber hinaus das Bedürfnis, im Zuge einer Flugdienstleistung ihren eigenen Ressourceneinsatz in Form von Zeit, Geld und Frustrationstoleranz möglichst weitgehend zu reduzieren, so daß von einem Problemfreiheitsbedürfnis gesprochen werden kann. Als Selbstverwirklichungsbedürfnis identifizierte die Studie schließlich den subjektiv im Verlauf einer Flugdienstleistung empfundenen Mangel an Komfort, Abwechslungsreichtum und Produktivität. Die Befragungspersonen wurden daraufhin um die Äußerung ihrer Einstellung gegenüber strategischen Luftverkehrsallianzen gebeten. Die Antworten ließen zum einen den Einfluß der Medienberichterstattung, aber auch eigene Erfahrungen mit Code Share-Flugdienstleistungen erkennen. Die Zitate "Airlines are in it for the money", „....all I get is miles" oder "I thought I was on American where I'm a Platinum member, and it turned out to be Canadian...I needed to change my flight plan and no one at American could help me “424 belegten eine von Skepsis geprägte Einstellung gegenüber Allianzen, die offenbar als ökonomische Vorteilssuche, "Marketing-Trick“ und Irritation bewertet wurden.

Im Anschluß an die Bekundung ihrer Einstellung wurden die Vielflieger mit einem Vergleich der Leistungsfähigkeit einzeln agierender Fluggesellschaften und strategischer Luftverkehrsallianzen zur Befriedigung der drei Bedürfnisdimensionen konfrontiert. Die Befragten schätzten Einzelfluggesellschaften und Allianzen hinsichtlich ihrer Fähigkeit, das Mobilitätsbedürfnis zu erfüllen, in gleichem Maße als hoch ein. Dem Problemfreiheitsbedürfnis wurden Luftverkehrsallianzen in den Augen der Vielflieger in mittlerem bis hohem Maße gerecht, während einzeln agierenden Airlines hier eine nur mittlere bis geringe Kompetenz zugemessen wurde.

424 Die Äußerung „Platinum member“ gibt Aufschluß über den bei American Airlines maximal erreichbaren Kundenstatus im Vielfliegerprogramm "Aadvantage“, für den eine jährliche Flugleistung von 50.000 Meilen notwendig ist. Vgl. Rodrian, H.W., Der Meilen-Bonus macht aus dem Flug einen Gewinn, in: Handelsblatt vom 17.11.1997, S. 41. 
Eine Befriedigung des Selbstverwirklichungsbedürfnisses sprachen die Befragungspersonen dagegen lediglich ausgewählten Einzelfluggesellschaften zu. Die Fähigkeit von Luftverkehrsallianzen, diesem Bedürfnis gerecht zu werden, wurde als gering eingestuft.

Damit ergab sich für strategische Allianzen lediglich in bezug auf das Problemfreiheitsbedürfnis ein konkreter Ansatzpunkt für eine Dominanzposition mit Differenzierungspotential gegenüber konkurrierenden Einzel-Flugdienstleistungen. Hinsichtlich der Eignung zur Befriedigung des Selbstverwirklichungsbedürfnisses offenbarte sich demgegenüber ein in der Einstellung der Vielflieger verankerter Leistungsnachteil strategischer Allianzen gegenüber einzeln agierenden Fluggesellschaften. Die Ursache für diese Befunde wurde vornehmlich in einem zum Untersuchungszeitpunkt noch relativ geringen Wissens- bzw. Erfahrungsstand im Umgang mit dem Leistungsangebot strategischer Luftverkehrsallianzen gesehen.

Um weitergehende Aufschlüsse über die Beurteilung des Allianz-Flugdienstleistungsangebots zu erhalten, wurden daraufhin die Nutzenerwartungen der Vielflieger exploriert und einer Bewertung zugeführt.

\subsubsection{Erfüllung zentraler Nutzenerwartungen von Vielfliegern durch strate- gische Luftverkehrsallianzen}

Die Diskussionen in den Focusgruppen ergaben insgesamt sechs Nutzendimensionen von Flugdienstleistungen, die von den befragten Vielfliegern als besonders relevant angesehen wurden. In ihrer idealen Ausprägung handelte es sich um folgende Nutzenerwartungen:

weltweiter Sicherheitsstandard,

nöchstes Komfortniveau,

- weltweites Streckennetz mit flexiblen Buchungsoptionen,

- nahtloses Reisen,

- weltweite Vielfliegervorteile sowie

- individualisierte Betreuung. ${ }^{425}$

425 Diese Nutzenerwartungen weisen damit teilweise hohe Überschneidungen zu den Ergebnissen der in Kapitel B 2.2.3 vorgestellten empirischen Studien zur Nutzensegmentierung bei Flugdienstleistungen auf. 
Der idealerweise gegebene weltweite Sicherheitsstandard wurde in den Gruppenexplorationen mit den Vielfliegern in zwei Komponenten aufgeteilt. Die als "Zutrauen, Vertrauen" ("confidence“) bezeichnete Komponente gab das zwischen Fluggast und Fluggesellschaft wachsende Vertrauensverhältnis wieder, das zu einem Abbau von Unsicherheit im Vorfeld einer Flugdienstleistung beiträgt. Der Bedeutungsinhalt wurde durch Zitate wie "You never know what you'll fly on" und "It's critical that you trust the airlines" reflektiert. Als zweite Komponente beschrieben die Vielflieger ein Gefühl der Seelenruhe („peace of mind“), die an Bord eines von innen als sicher eingestuften Flugzeugs empfunden wurde. Bei der Beurteilung der Fähigkeit strategischer Luftverkehrsallianzen zur Erfüllung dieser Nutzenerwartung wurde von den Befragungspersonen ein möglicher allianzspezifischer Halo-Effekt hervorgehoben. ${ }^{426}$ Die Vielflieger stellten damit auf die Signalwirkung einer Allianz für die Sicherheitsbewertung einzelner Partner-Airlines ab, die zu einer Senkung des erhöhten wahrgenommenen Risikos bei der Inanspruchnahme bisher nicht genutzter Fluggesellschaften beiträgt.

Der Komfort an Bord des Flugzeugs wurde von den Befragungspersonen vornehmlich über die Sitzqualität und Beinfreiheit definiert. Ein höchstmögliches Komfortniveau korrespondierte dabei mit dem Selbstverwirklichungsbedürfnis der Vielflieger, welche die Luftverkehrsallianzen als tendenziell unterlegen gegenüber einzeln agierenden Fluggesellschaften beurteilten, dieses Bedürfnis zu befriedigen. Dieser Befund bestätigt die oben geführten Plausibilitätsüberlegungen, nach denen aus partnerbedingt unterschiedlichen Servicestandards negative Beurteilungen resultieren können. Offenbar scheint ein einheitlich hohes Komfortniveau bedeutsamer zu sein als ein diesbezüglicher Variantenreichtum zwischen den Partnern-Fluggesellschaften.

Die Nutzenerwartung „weltweites Streckennetz mit flexiblen Buchungsoptionen" stellte die Idealausprägung einer Eigenschaft dar, die in den Focusgruppeninterviews in weitere drei Komponenten unterteilt wurde. Zum einen bekundeten die befragten Vielflieger in Anlehnung an das oben genannte Problemfrei-

426 Unter Halo-Effekt wird die Beeinflussung der Wahrnehmung bestimmter Leistungseigenschaften durch eine generelle Einstellung zu der Leistung verstanden. Im Beispielfall könnte eine positive Einstellung zu einer bestimmten Luftverkehrsallianz die negative Einzelwahrnehmung des Sicherheitsmerkmals einer Partner-Airline positiv beeinflussen. Vgl. z.B. Meffert, H., Marketingforschung und Käuferverhalten, a.a.O., S. 62. 
heitsbedürfnis den Wunsch nach einem zeitsparenden Reiseablauf. Aus dem Angebot allianzseitig geschaffener Zusatzflüge auf bestimmten Strecken resultierte für sie unmittelbar ein Nutzenzuwachs, da Terminverlegungen oder Verspätungen auf diese Weise abgefedert werden konnten. Zum anderen maßen sie einem erhöhten Angebot in Form von zusätzlichen Strecken und Verbindungen pro Strecke eine unmittelbar streßreduzierende Wirkung bei - genannt wurden insbesondere das als lästig empfundene Warten und „Herumsitzen“ an Flugsteigen oder in Lounges. Damit bestätigen sich wiederum die Plausibilitätsüberlegungen, denen zufolge der Verbindungsqualität eine zentrale Bedeutung beim Fit zwischen Leistungsangebot und Nutzenerwartung zukam. Darüber hinaus hoben die Befragungspersonen in den Focusgruppeninterviews Spareffekte durch die Inanspruchnahme spezieller "Around the World Tickets" hervor, die von einigen Luftverkehrsallianzen angeboten werden. Zudem wurde der Wegfall zusätzlicher Kosten bei einer Umbuchung innerhalb des Allianzsystems explizit als positive Eigenschaft mit nutzenstiftender Wirkung herausgestellt.

Ohne Überschneidungsfreiheit zur Verbindungsqualität/Buchungsflexibilität aufzuweisen, wurde ein nahtloses Reisen („seamless travel“) als Nutzenerwartung identifiziert. Unter diesem Merkmal wurde die Auflösung aller schnittstellenbedingten Probleme subsumiert, die im Zuge einer Flugdienstleistung und insbesondere einer Umsteigeverbindung auftreten können. Als allianzspezifische Vorteile bei der Erfüllung dieser Nutzenerwartung werteten die Vielflieger die nur einmal notwendige Gepäckaufgabe bei Code Share-Flugdienstleistungen, die - verbunden mit einem verantwortungsvollen Gepäckhandling - zu einer individuellen Entlastung beitrug. Auch die mit einem nahtlosen Reisen verbundene Zeitersparnis nahm Bezug auf das Problemfreiheitsbedürfnis der Befragungspersonen. Genannt wurden insbesondere Zeitvorteile und Ersparnis von Ärger durch die Schaffung einer größeren räumlichen Nähe der Partner-Flugsteige an Flughäfen sowie das einmalige Check-in bei einer Allianz-Flugdienstleistung. Zudem hoben die Vielflieger die mit einer besseren Schnittstellenabstimmung seitens der Partner einher gehende Erleichterung und Streßreduzierung für sie selbst hervor. Stellvertretend sei folgendes Zitat angeführt: „Why not let them [the airlines, Anm. d. Verf.] handle things so I can relax and unwind“. Die in Kapitel B 2.2.3 in Erwägung gezogene negative Auswirkung durch partnerschaftliche Abstimmungsprobleme bleibt bei dieser Eigenschaft demnach unberücksichtigt, während sich die vermuteten positiven Ausprägungen empirisch bestätigen. 
Mit weltweiten Vielfliegervorteilen erfaßte die Untersuchung eine Nutzenerwartung, die in den Studien zur Nutzensegmentierung nur peripher berührt wurde. ${ }^{427}$ Für die in den Focusgruppeninterviews befragten Vielflieger bestanden bei dieser Nutzenerwartung die höchsten Potentiale strategischer Luftverkehrsallianzen, der Idealausprägung nahe zu kommen und sich gegenüber einzeln agierenden Fluggesellschaften abzugrenzen. Als Grund hierfür wurde auf die Möglichkeit hingewiesen, Privilegien wie Loungenutzung, Priorisierung auf Wartelisten sowie bevorzugtes Einchecken von bisher einer Fluggesellschaft auf dann mehrere Partner-Airlines ausdehnen zu können. Neben diesen prozeßbezogenen Eigenschaften hoben die Befragungspersonen das schnellere Erreichen kritischer Meilengrenzen sowie größere Streckennetze zum "Abfliegen" von Meilen mit Allianzpartnern hervor. Derartige Privilegien wurden von den Befragungspersonen oft als Belohnung oder Kompensation für die berufliche Tätigkeit gewertet wurden ("rewards are my treat...light at the end of the tunnel"). In diesem Fall sprachen die Befragten auch von „tangible rewards".

Abweichungen im Antwortverhalten ergaben sich diesbezüglich für britische und asiatische Vielflieger, die in größerem Maße am Sammeln von Meilen interessiert waren als die übrigen Focusgruppenteilnehmer. Begründet wurde dies im Fall der Briten mit einer größeren Streuung der Buchungen auf mehrere verschiedenen Fluggesellschaften und bei den Asiaten mit der relativen Neuigkeit dieses Kundenbindungsinstruments, das in ihrem Heimatmarkt für einige Strecken und Tarife keine Gültigkeit besaß.

Mit der Nutzenerwartung einer individualisierten Betreuung verbanden die Vielflieger schließlich einen persönlichen Service an Bord, der idealerweise proaktiv von den Flugbegleitern ausging und von einer unaufdringlichen Aufmerksamkeit geprägt war. Im Gegensatz zum Komfort bezog sich diese Eigenschaft wesentlich auf das Personal der Fluggesellschaft, wenngleich die Auswirkungen persönlicher Betreuung ähnliche Züge wie ein höchstmöglicher Komfort oder nahtloses Reisen trugen und sich damit am Problemfreiheitsbedürfnis und z.T. auch am Selbstverwirklichungsbedürfnis orientierten. Genannt wurden Entspannung, Erleichterung und Leichtigkeit als Komponenten einer ideal ausgeprägten individualisierten Betreuung. Einzeln agierende Fluggesellschaften wurden tendenziell als besser in

427 Sie findet Berücksichtigung als „statusbezogene Leistungen“ im Geschäftsreisenden-Segment der "Road Warriors ${ }^{u}$ in der Studie von United Airlines. Vgl. Kapitel B 2.2.3. 
der Lage eingeschätzt, diese Nutzenerwartung zu erfüllen. Damit ergibt sich eine gewisse Konsistenz zu den Plausibilitätsüberlegungen, die im Zuge der Untersuchung Stegmüllers mit dem Merkmal „Aufmerksamkeit an Bord“ geführt wurden. Kulturell bedingte Inkompatibilitäten der Servicephilosophie oder auch Sprachprobleme könnten hier als Ursachen einer Inferiorität von Allianzen gewertet werden.

Überblickartig ergibt sich damit die in Abbildung 17 zusammengefaßte Beurteilung des Erfüllungsgrads strategischer Luftverkehrsallianzen bezüglich der sechs identifizierten Nutzenerwartungen. Mit Ausnahme des Komfortniveaus und der individualisierten Betreuung beurteilten die befragten Vielflieger strategische Luftverkehrsallianzen demnach als überlegen gegenüber einzeln agierenden Fluggesellschaften.

\begin{tabular}{|l|l|}
\cline { 2 - 2 } \multicolumn{1}{l|}{} & Erfüllungsgrad durch strategische Luftverkehrsallianzen \\
\hline $\begin{array}{l}\text { Weltweiter Sicherheits- } \\
\text { standard }\end{array}$ & $\begin{array}{l}\text { Hoch. Grund: Allianzen können von einem Halo-Effekt profi- } \\
\text { tieren, der mit einem partnerübergreifenden Sicherheitssie- } \\
\text { gel zu vergleichen ist. }\end{array}$ \\
\hline Höchstes Komfortniveau & $\begin{array}{l}\text { Gering. Grund: Unterschiedliche Komfortniveaus werden } \\
\text { schlechter beurteilt als Komfort „aus einer Hand“. }\end{array}$ \\
\hline $\begin{array}{l}\text { Weltweites Streckennetz } \\
\text { mit flexiblen Buchungs- } \\
\text { optionen }\end{array}$ & $\begin{array}{l}\text { Hoch. Grund: Zusätzliche Streckenverbindungen und enge } \\
\text { Buchungsabstimmungen werden als einzeln agierenden } \\
\text { Airlines mit kleinerem Streckennetz überlegen beurteilt. }\end{array}$ \\
\hline Nahtloses Reisen & $\begin{array}{l}\text { Hoch. Grund: Abstimmung der Allianzpartner wird mit den } \\
\text { Vorteilen des einmaligen Eincheckens, kürzeren Wegen und } \\
\text { partnerschaftlicher Gepäckweiterleitung verbunden. }\end{array}$ \\
\hline $\begin{array}{l}\text { Weltweite Vielfliegervor- } \\
\text { teile }\end{array}$ & $\begin{array}{l}\text { Sehr hoch. Grund: Privilegien werden auf mehrere Airlines } \\
\text { ausgedehnt, ohne einer bestimmten Fluggesellschaft ver- } \\
\text { haftet bleiben zu müssen. }\end{array}$ \\
\hline $\begin{array}{l}\text { Individualisierte Betreu- } \\
\text { ung }\end{array}$ & $\begin{array}{l}\text { Gering bis sehr gering. Grund: Inkompatibilitäten der Ser- } \\
\text { vicephilosophie bei länderspezifisch geprägten Erwartungen } \\
\text { lassen bei einzeln agierenden Fluggesellschaften weniger } \\
\text { Schwierigkeiten erwarten. }\end{array}$ \\
\hline
\end{tabular}

Abb. 17: Beurteilung des allianzbezogenen Erfüllungsgrads zentraler Nutzenerwartungen durch Vielflieger

Um Aussagen über die relative Bedeutung der damit erörterten Nutzenerwartungen zueinander zu gewinnen, wurde daraufhin eine Rangreihung nach den Häu- 
figkeiten der "sehr wichtig"- und "wichtig"-Nennungen vorgenommen. Sie ergab eine dominante Wichtigkeit des weltweiten Sicherheitsstandards (166 Nennungen), gefolgt vom höchsten Komfortniveau (130), dem weltweiten Streckennetz mit flexiblen Buchungsmöglichkeiten (129) und dem nahtlosen Reisen (122). Mit einigem Abstand schlossen sich Vielfliegervorteile (82) und die individualisierte Betreuung (63) an. Angesichts der mangelnden Repräsentativität der Daten und der mit einer solchen Erfassung verbundenen Anspruchsinflation sind diese Wichtigkeiten jedoch mit Vorsicht zu interpretieren. Nicht zuletzt aus diesem Grunde wurden zusätzliche Marktforschungsdaten bemüht, um die Wichtigkeiten allianzspezifischer Nutzenerwartungen zu erfassen. Die Auswertung empirischer Daten einer Tracking-Studie, in deren Zuge regelmäßig Befragungen an Bord von Flugzeugen der Allianzpartner durchgeführt wurden, ergab ein von den Focusgruppenergebnissen abweichendes Bild. 92 Prozent der befragten Fluggäste nannten die Möglichkeit, Meilen mit der Partnerfluggesellschaft sammeln zu können, als sehr wichtige nutzenstiftende Eigenschaft einer strategischen Luftverkehrsallianz. Mit deutlichem Abstand schlossen sich die Merkmale "komfortables Reisen" (67 Prozent), „zusätzliche Anzahl Flüge pro bestehender Strecke und zusätzliche Ziele“ (66 Prozent) sowie der „Zugang zu Lounges der Partner-Airlines“ (59 Prozent) an.

Im nächsten Untersuchungsschritt wurden auf Basis dieser Erkenntnisse vier verbale Positionierungskonzepte für eine fiktive strategische Luftverkehrsallianz entwickelt und auf ihre Akzeptanz bei den Focusgruppen-Teilnehmern geprüft.

\subsubsection{Ableitung und Überprüfung alternativer Positionierungsansätze am Beispiel einer fiktiven Luftverkehrsallianz}

Um konkrete Aufschlüsse über die Dominanzfunktion einer Luftverkehrsallianz und über ihre Differenzierungsfähigkeit gegenüber einzeln agierenden Fluggesellschaften zu erhalten, ${ }^{428}$ wurden den Vielfliegern vier unterschiedliche verbale Stimuli präsentiert. Die Reaktionen in den Gruppendiskussionen wurden erfaßt und zu prägnanten Beschreibungen verdichtet. Diese Vorgehensweise kann daher nicht mit einer Positionierung im herkömmlichen Sinne verglichen werden, in deren Zuge Objekte auf der Grundlage von Eigenschafts- oder Ähnlichkeitsbeurtei-

${ }^{428}$ Vgl. Woratschek, H., Positionierung - Analysemethoden, Entscheidungen, Umsetzung, in: Handbuch Dienstleistungs-Marketing, Band 1, Hrsg.: Meyer, A., Stuttgart 1998, S. 698 u. S. $705 f$. 
lungen im Wahrnehmungsraum von Befragungspersonen positioniert werden, wobei möglichst repräsentative Stichproben zugrunde liegen und multivariate Analysemethoden zur Anwendung gelangen. ${ }^{429}$

Als Stimulus 1 fungierte der aus semantischen Gründen nicht übersetzte Satz "We're Putting the World on Your Schedule". Diese Aussage führte nach dem Bekunden von 130 Befragungspersonen dazu, eine Luftverkehrsallianz als möglichen Problemlösungsanbieter Betracht zu ziehen. Hervorgehoben wurden die mit diesem Satz verbundene Möglichkeit zur effizienten Zeitnutzung, die aus zusätzlichen Flugzielen und Verbindungen pro Strecke erwachsenden Flexibilitätsspielräume bei der Reiseplanung sowie die bequeme Regelung komplexer Reiseabläufe aus der Hand „eines" Leistungsanbieters. Damit korrespondierte dieser Stimulus mit dem Problemfreiheitsbedürfnis. U.a. wurden folgende Aussagen zitiert: "You spend less time waiting...you are in control", "Time is money“, "I want my trip to be as trouble-free as possible".

Als zweiter Stimulus wurde die Wortfolge „Simpler, More Seamless International Travel“" eingesetzt. Dieser Aussage maßen 112 Vielflieger eine entsprechende Problemlösungskompetenz bei. Einige Zitate ließen eine lehrbuchhafte Verbindung dieser Aussage mit dem Wesen strategischer Luftverkehrsallianzen erkennen und damit die Prägung durch Presseberichte deutlich werden ("Isn't this the point of an alliance?", „Not coming out of one system and into another"). Im Mittelpunkt der Reaktionen stand jedoch die von den Vielfliegern mit dieser Wortfolge verbundene Fähigkeit einer Allianz, eine von Streß und Ärgernissen freie Flugdienstleistung zu erstellen, in deren Zuge alle potentiellen Probleme proaktiv durch den Anbieter gelöst werden.

Die Phrase „We'd Recognize You Anywhere in the World" kam als dritter Stimulus zum Einsatz und vereinte die allianzspezifischen Assoziationen von 95 Befragungspersonen auf sich. Während schon zwischen Stimulus 1 und 2 gewisse Überschneidungen der Assoziationen feststellbar waren, traten an dieser Stelle nahezu identische Aussagen wie bei Stimulus 2 auf, die sich auf den - hier jedoch statusabhängigen - störungsfreien Ablauf einer Flugdienstleistung bezogen. Daneben verbanden die Vielflieger vor allem auf ihren Status abgestimmte

429 Vgl. Backhaus, K., u.a., Multivariate Analysemethoden: eine anwendungsorientierte Einführung, 8., verb. Aufl., Berlin u.a. 1996, S. $432 f f$. 
Sonderbehandlungen mit der Phrase weltweiter Anerkennung. Die Aussagen lassen auf eine rational basierte Vorteilssuche schließen, die sich bei britischen und asiatischen Befragungspersonen stärker auf das Sammeln von Meilen und den Erhalt von Bonusflügen konzentrierte als auf die Erlangung statusbezogener Vorteile wie bevorzugtes Check-in oder Wartelistenpriorisierung.

Der vierte Stimulus stellte das Merkmal der Diversität von Allianzen in den Vordergrund und lautete „You Get the Best of Each...and the Best of All“. 80 Vielflieger assoziierten diesen Satz mit Luftverkehrsallianzen und konnten sich vorstellen, von einer Vereinigung der partnerschaftlichen Stärken auch als Endnutzer zu profitieren. Die Zitate, die sich zwecks Beleg dieser Aussage in der Dokumentation finden, wirken jedoch eher halbherzig und ungenau: „The whole thing has people behind it working for you, so it's got to be good!" oder "More heads the better. They can share ideas. They won't try and hide it if they discover something good as they probably do now". Bei der Vereinigung von Stärken konnten nationalitätsbedingte Einflüsse festgestellt werden, die den Partnerfluggesellschaften herkunftslandbedingte Leistungsvorteile beimaßen. Als Beispiel wurde die Betreuungsqualität an Bord asiatischer Fluggesellschaften hervorgehoben. Andere Vielflieger sahen für Airlines ihrer eigenen Nationalität Chancen, die eigene Leistungsfähigkeit durch die Allianz mit anderen Fluggesellschaften zu steigern.

Abschließend empfahl die Studie eine an "tangiblen Leistungsmerkmalen“ ausgerichtete Positionierung für die Allianz, ${ }^{430}$ um später unter der Voraussetzung einer gestiegenen Bekanntheit und gewachsener endnutzerseitiger Erfahrungen im Umgang mit Allianz-Flugdienstleistungen einen stärker emotional geprägten Positionierungsansatz zu verfolgen. Aufbauend auf den Erkenntnissen der bisherigen Untersuchungsschritte wurde im vierten Teil der Studie ein Positionierungsansatz für die Allianz der Auftraggeber vorgeschlagen.

\subsection{5 „Worldwide Recognition“ als vielfliegerorientierter Positionierungs- ansatz für eine strategische Luftverkehrsallianz}

Der Vorschlag, eine gemeinsame, auf Nutzenerwartungen aufbauende Allianzmarke zu schaffen, bildete den Ausgangspunkt des letzten Untersuchungsschrit-

${ }^{430}$ Mit "tangibel" ist in diesem Zusammenhang "handfest”, „unmittelbar ersichtlich", aber auch "meßbar" gemeint. Nach dieser Auffassung wäre beispielsweise der weltweite Loungezugang tangibel, wăhrend eine „Kombination der individuellen Stärken der Partner" eher als intangibel zu bezeichnen wäre. 
tes. ${ }^{431}$ Zunächst wurde das Merkmal der individualisierten Betreuung aufgrund des als gering bis sehr gering beurteilten allianzseitigen Erfüllungsgrades ausgesondert, um dann anhand der übrigen fünf explorierten Nutzenerwartungen die Kerneigenschaften der Allianzmarke zu definieren. ${ }^{432}$ Dabei wurde eine Nutzenhierarchie entwickelt, deren Fundament in dem Merkmal „weltweiter Sicherheitsstandard" bestand. Auf diesem Fundament bauten die übrigen Nutzenerwartungen "konsistentes Komfortniveau“, „weltweites Streckennetzes/flexible Buchungsoptionen“, "nahtloses Reisen vom Anfang bis zum Ende" sowie "Vielfliegervorteile Status/Privilegien" auf. Für jedes Merkmal wurde ein aus Endnutzerperspektive formulierter Leitsatz entwickelt, der in Abbildung 18 wiedergegeben ist.

431 Diese Überlegung erweist sich als richtig, zumal angesichts der nebeneinander geführten,

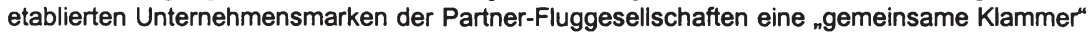
fehlt. Meffert (1994) geht in seinem entscheidungsorientierten Ansatz der Markenpolitik bereits implizit von der Existenz einer Marke aus, für die Ziele, Positionierung, Strategie sowie Detailentscheidungen und Anpassungsentscheidungen zu bestimmen bzw. Zu treffen sind. Vgl. Meffert, H., Entscheidungsorientierter Ansatz der Markenpolitik, a.a.O., S. 175.

An dieser Stelle sei ausdrücklich auf die spitze Zielgruppendefinition hingewiesen (vgl. die Anlage der Untersuchung), auf die das Allianz-Flugdienstleistungsangebot ausgerichtet wurde. 


\section{Leitsatz:}

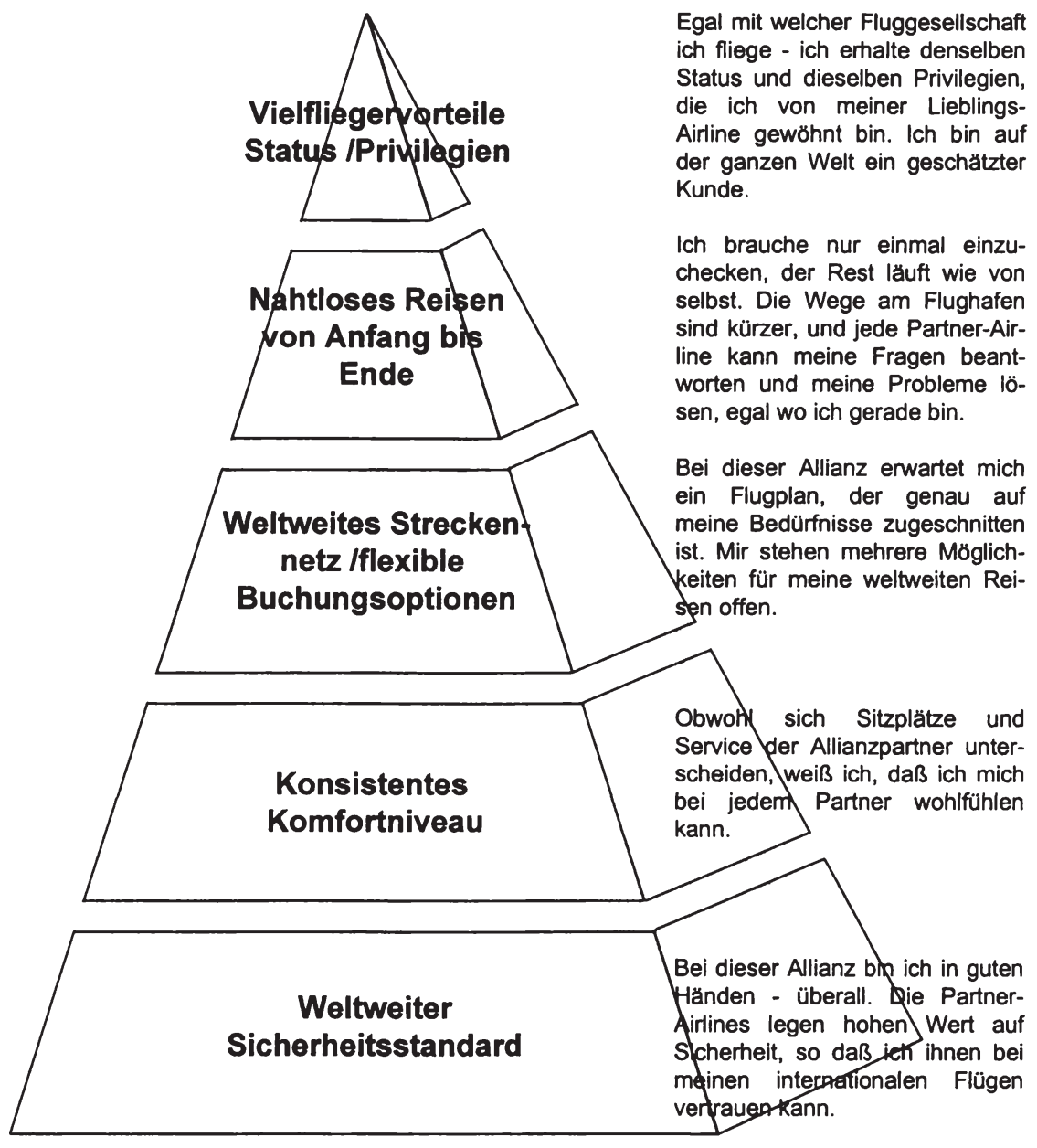

\section{Abb. 18: Kerneigenschaften der Allianzmarke (Idealposition)}

Im Anschluß an die solchermaßen bestimmte Idealposition wurden die Vielflieger um die Beurteilung der Real-Ausprägungen dieser Kerneigenschaften am Beispiel der Auftraggeber-Allianz gebeten. Dabei dienten konkurrierende Luftverkehrsallianzen explizit als Vergleichsobjekte. Die fundamentale Eigenschaft der 
Auftraggeber-Allianz, die Nutzenerwartung eines weltweiten Sicherheitsstandards zu erfüllen, wurde dabei weder als dominantes Merkmal identifiziert noch für signifikant besser oder schlechter gehalten als im Fall anderer Luftverkehrsallianzen. Insgesamt werteten die Befragungspersonen die Allianz jedoch als eine „Mischung aus sicheren Fluggesellschaften“.

Sehr knapp fiel die Beurteilung der Realausprägung des Merkmals „konsistentes Komfortniveau“ aus, für das die Vielflieger in den Gruppendiskussionen weder eine dominante Position konstatierten noch Unterschiede zu den konkurrierenden strategischen Luftverkehrsallianzen ausmachten.

Mit relativ geringem Abstand zur Idealposition beurteilten die befragten Vielflieger die Real-Ausprägung des Merkmals "weltweites Streckennetz/flexible Buchungsoptionen“, durch das sich die Luftverkehrsallianz zudem deutlich von den Wettbewerbs-Allianzen differenzieren konnte. In bezug auf ein „nahtloses Reisen vom Anfang bis zum Ende" attestierten die Befragten der Luftverkehrsallianz demgegenüber keine Dominanz. Im Vergleich zu den übrigen Allianzen wurde darüber hinaus deutlich, daß einige Vielflieger offenbar aufgrund schlechter Erfahrungen Zweifel an der Eignung der Allianz zur Befriedigung des Problemfreiheitsbedürfnisses hatten.

Hinsichtlich der Kerneigenschaft „Vielfliegervorteile Status/Privilegien“ maßen die Befragungspersonen der Luftverkehrsallianz zum Untersuchungszeitpunkt keine dominante Position in ihrem eigenen Wahrnehmungsraum zu. Zugleich wurde die mangelnde Differenzierung gegenüber anderen Allianznetzwerken hervorgehoben, in denen ebenfalls eine gegenseitige Anerkennung der partnerschaftlichen Vielfliegerprogramme praktiziert wurde. Potential zur Abgrenzung gegenüber anderen Allianzen sahen die Befragungspersonen dagegen in einer Übertragbarkeit von Statusmerkmalen zwischen den Partner-Fluggesellschaf ten. $^{433}$

Um schließlich die zwischen Ideal- und Real-Ausprägungen der Kerneigenschaften bestehenden Unterschiede bei möglichst großem Differenzierungspotential gegenüber dem Wettbewerb zu überwinden, schlugen die Forscher auf Basis der

433 Dieser Hinweis beruht vermutlich auf einem eigennützigen Streben der Befragungspersonen, die durch derartige Äußerungen entsprechende Änderungen zu initiieren hoffen. 
gewonnenen Erkenntnisse die Anwendung eines als "Worldwide Recognition“ bezeichneten Positionierungsansatzes vor. Im Kern dieses Konzepts stand die Aussage des persönlichen (Wieder-)Erkennens ("recognition“) von Vielfliegern in allen Teilen der Welt ("worldwide"). Die in dem Positionierungskonzept enthaltenen Vorschläge für ausgewählte Maßnahmen lassen sich im Zusammenhang mit den identifizierten Ideal-Real-Unterschieden der Nutzenerwartungen darstellen (vgl. Abbildung 19).

\begin{tabular}{|l|l|l|l|}
\hline Nutzenerwartung & Befund zur Dominanz & $\begin{array}{l}\text { Befund zur Differenzie- } \\
\text { rung }\end{array}$ & $\begin{array}{l}\text { Maßnahmen zur Profi- } \\
\text { lierung }\end{array}$ \\
\hline $\begin{array}{l}\text { Weltweiter Sicherheits- } \\
\text { standard }\end{array}$ & keine Dominanz & keine Differenzierung & keine Angabe \\
\hline $\begin{array}{l}\text { Konsistentes Komfort- } \\
\text { niveau }\end{array}$ & keine Dominanz & keine Differenzierung & keine Angabe \\
\hline $\begin{array}{l}\text { Weltweites Strecken- } \\
\text { netz/flexible Buchungs- } \\
\text { optionen }\end{array}$ & hohe Dominanz & deutliche Differenzierung & keine Angabe \\
\hline $\begin{array}{l}\text { Nahtloses Reisen vom } \\
\text { Anfang bis zum Ende }\end{array}$ & keine Dominanz & $\begin{array}{l}\text { leicht negative Differen- } \\
\text { zierung aufgrund } \\
\text { schlechter Erfahrungen }\end{array}$ & $\begin{array}{l}\text { Ermöglichung eines ent- } \\
\text { spannten Reisens durch } \\
\text { bevorzugte Behandlung } \\
\text { seitens des Kontaktper- } \\
\text { sonals } \\
\text { konsistente Abläufe bei } \\
\text { Standardvorgängen } \\
\text { (Check-in, Kofferaufgabe, } \\
\text { Mitnahmevorschriften) } \\
\text { Erzeugung von Vertraut- } \\
\text { heit durch gemeinsame } \\
\text { Umgangsnormen }\end{array}$ \\
\hline $\begin{array}{l}\text { Vielfliegervorteile Sta- } \\
\text { tus/Privilegien }\end{array}$ & keine Dominanz & $\begin{array}{l}\text { kaum Differenzierung } \\
\text { aufgrund von Nach- } \\
\text { ahmungen; Differenzie- } \\
\text { rungs“potential“ bei } \\
\text { weltweiter Statusaner- } \\
\text { kennung }\end{array}$ & $\begin{array}{l}\text { Schaffung eines gemein- } \\
\text { samen Markennamens, } \\
\text { um Orientierung und } \\
\text { Identifizierbarkeit zu } \\
\text { ermöglichen } \\
\text { Reduzierung der Vielflie- } \\
\text { gerprogramme auf ein } \\
\text { allianzweites Programm } \\
\text { Obertragbarkeit des Kun- } \\
\text { denstatus (Akzeptanz } \\
\text { durch Partner-Airlines) }\end{array}$ \\
\hline
\end{tabular}

\section{Abb. 19: Ansatzpunkte für die Positionierung am Beispiel einer strate- gischen Luftverkehrsallianz}

Die der Studie zu entnehmenden Vorschläge für die Ergreifung von Maßnahmen zur Erreichung der angestrebten strategischen Positionierung der Allianz stellen 
ersichtlich auf die Erfüllung von Nutzenerwartungen ab, die einen sehr spezifischen Bezug zur Gruppe der Vielflieger aufweisen. Wenngleich sich die vorliegenden Ausführungen damit auf ein mengenmäßig eher kleines Segment von Fluggästen beziehen, wurde bereits früher auf die hohe wertmäßige Bedeutung von Vielfliegern für Fluggesellschaften hingewiesen. Die Auswahl dieser Gruppe für die Focusgruppenbefragung dürfte zudem vor dem Hintergrund ihres Erfahrungswissens erfolgt sein, das zur Beurteilung des Untersuchungsobjektes notwendig ist. Offen bleibt jedoch insgesamt die konkrete Erfassung des allianzspezifischen (Zusatz-)Nutzens über ein geeignetes Meßinstrumentarium, so daß die Diskussion von Ansatzpunkten zur Ausgestaltung des Marketing-Instrumentariums auf lediglich qualitativen Daten aufbauen kann. 


\section{Nachfragergerichtete Ausgestaltung strategischer Luftverkehrsallian- zen}

Die in der empirischen Analyse deutlich gewordene Differenz zwischen Ideal- und Realposition der strategischen Allianz markiert den Ausgangspunkt für die Ausgestaltung des Marketing-Instrumentariums, das zur Überwindung dieser Differenz eingesetzt wird. ${ }^{434}$ Die Kerninhalte des "Worldwide Recognition“-Konzepts sind im vorliegenden Fall als strategische Leitlinien zu verstehen, an denen sich die Umsetzung der Positionierung in konkrete Marketingmaßnahmen orientiert. Den Ausgangspunkt bildet dabei die Erörterung der Allianz-Markenpolitik, da ihr vielfach ein instrumenteübergreifender Charakter zugesprochen wird. ${ }^{435}$ Die übrigen Marketing-Instrumente, zu denen die Leistungs-, Distributions-, Kontrahierungsund Kommunikationspolitik zählen, richten sich im Sinne eines integrierten Markenkonzepts an der Allianz-Markenpolitik aus. ${ }^{436}$

Angesichts des Dienstleistungscharakters von Flugdienstleistungen ist zudem eine Erweiterung des traditionellen Marketing-Mix um die Dimensionen Personal („Personnel“), Prozeßmanagement („Process") und physische Einrichtungen („Physical Facilities") für das Marketing von Luftverkehrsallianzen zu diskutie ren. ${ }^{437}$ Die Würdigung des Dienstleistungspersonals als eigenständiges "Marketing-Instrument" trägt der Tatsache Rechnung, daß die Mitarbeiter von Dienstleistungsunternehmen durch ihre Interaktion mit den Endnutzern als eine

434 Zur Abfolge von Prozeßschritten bei der Positionierung vgl. z.B. Becker, J., Konzeptionelle Grundfragen der Positionierung, in: Positionierung: Kernentscheidung des Marketing, Hrsg.: Tomczak, T., Rudolph, T., Roosdorp, A., St. Gallen 1996, S. 18.

Vgl. Angehrn, O., Markenpolitik, in: Handwörterbuch der Absatzwirtschaft, Hrsg.: Tietz, B., Stuttgart 1974, Sp. 1227; Meffert, H., Bruhn, M., Markenstrategien im Wettbewerb. Empirische Untersuchungen zur Akzeptanz von Hersteller-, Handels- und Gattungsmarken (No Names), Wiesbaden 1984, S. 12; Arnold, D., The handbook of brand management, Reading 1992, S. 6. Vgl. Meffert, H., Marketing: Grundlagen marktorientierter Unternehmensführung, a.a.O., S. 789.

Dieser Vorschlag geht ursprünglich auf Magrath (1986) zurück, der die Erweiterung mit den Besonderheiten des Dienstleistungsmarketing gegenüber Konsum-/Investitionsgütermarketing begründet. Vgl. Magrath, A.J., When Marketing Services, 4 Ps Are Not Enough, in: Business Horizons, Vol. 29, No. 3, 1986, S. 44-50. Payne (1993) argumentiert ähnlich und schlägt eine Erweiterung um die Dimensionen "People ${ }^{4}$, ${ }_{\text {Processes“ }}$ und "Customer Service" vor, von denen die beiden erstgenannten deckungsgleich mit Magraths "Personnel" und "Process" sind. Unscharf bleibt in Paynes Begrifflichkeit allerdings die inhaltliche Konkretisierung der Dimension "Customer Service", hinter der sich nach seinem Verstăndnis ein steigendes Anspruchsniveau der Kunden sowie die Notwendigkeit zum Aufbau von Kundenbeziehungen verbergen. Eine Einordnung in die traditionelle Leistungs- und Kommunikationspolitik erscheint hier ohne weiteres möglich. Vgl. Payne, A., The essence of services marketing, Englewood Cliffs 1993, S. $25 f$. 
wichtige Schnittstelle zum Absatzmarkt fungieren. ${ }^{438}$ Dies trifft auch auf die Mitarbeiter von Fluggesellschaften zu, die im Verlauf der Reisekette, insbesondere während des eigentlichen Fluges, mit den Fluggästen in Kontakt stehen. Aus diesem Grund beteiligt beispielsweise die US-amerikanische Fluggesellschaft Southwest Airlines die Vielflieger unter ihren Kunden an den Einstellungsgesprächen und der Auswahl von Bewerbern für das Kabinenpersonal. ${ }^{439}$ Der Nutzung des Fähigkeitenpotentials und der sozialen Kompetenz von Mitarbeitern wird ein hoher Stellenwert beigemessen, so daß eine über die vier traditionellen MixBereiche hinausgehende Erörterung der Personaldimension unter Fokussierung auf die marketingrelevanten Aspekte als gerechtfertigt angesehen werden kann. $^{440}$

Eine Erweiterung des Marketing-Instrumentariums um Prozeßmanagement und physische Einrichtungen dürfte dagegen kaum von zusätzlichem Erkenntnisgewinn sein, da beide Dimensionen als integrale Bestandteile der Leistungspolitik von Dienstleistungsunternehmen und damit auch von strategischen Luftverkehrsallianzen anzusehen sind. ${ }^{441} \mathrm{Im}$ Luftverkehr können speziell das Netzmanagement (Abstimmung von Flugplänen) und das Produkt- und Servicemanagement (Abstimmung boden- und bordbezogener Prozesse) unterschieden werden.

Zunächst gilt es, durch die Erörterung der Allianz-Markenpolitik den gemeinsamen Orientierungsrahmen für die Ausgestaltung der weiteren Marketing-Instrumente festzulegen.

438 Schlesinger und Heskett (1992) führen beispielsweise an, daß mehr als 65 Prozent der Kunden von Dienstleistern abwandern, weil sie das Personal als innen gegenüber nachlässig und gleichgültig empfinden. Vgl. Schlesinger, L.A., Heskett, J.L., Dem Kunden dienen - das müssen viele Dienstleister erst noch lernen, in: Harvard Manager, 14. Jg., Nr. 1, 1992, S. 109.

Vgl. Heskett, J.L, u.a., Putting the Service-Profit Chain to Work, in: Harvard Business Review, Vol. 72, No. 2, 1994, S. 172.

$440 \mathrm{Zu}$ einem ăhnlichen Vorgehen vgl. Meffert, H., Bruhn, M., Dienstleistungsmarketing: Grundlagen-Konzepte-Methoden, mit Fallbeispielen, a.a.O., S. 289.

441 Vgl. Meyer, A., Dullinger, F., Leistungsprogramm von Dienstleistungs-Anbietern, in: Handbuch Dienstleistungs-Marketing, Band 1, Hrsg.: Meyer, A., Stuttgart 1998, S. 713. Meffert und Bruhn (1997) lehnen die Erweiterung um diese beiden Dimensionen mit dem Hinweis ab, daß lediglich Aktivităten zur Verhaltensbeeinflussung des Kunden im Marketing-Mix zu berücksichtigen seien. Vgl. Meffert, H., Bruhn, M., Dienstleistungsmarketing: GrundlagenKonzepte-Methoden, mit Fallbeispielen, a.a.O., S. 289. 


\subsection{Ausgestaltung der allianzspezifischen Markenpolitik}

\subsubsection{Markenpolitische Zielsetzungen und rechtliche Rahmenbedingungen der Allianz-Markenpolitik}

Eine Marke kann als ein in der Psyche von Konsumenten verankertes, unverwechselbares Vorstellungsbild von einem Produkt oder einer Dienstleistung verstanden werden. Die zugrunde liegende markierte Leistung wird dabei in einem möglichst großen Absatzraum in gleichartigem Auftritt und in gleichbleibender oder verbesserter Qualität über einen längeren Zeitraum hinweg angeboten. ${ }^{442}$ Ausgehend von diesem Begriffsverständnis erfüllt die Markierung des AllianzFlugdienstleistungsangebots wichtige Funktionen für die Nachfrager. Sie erleichtert zunächst die Identifikation der Allianz-Leistungen und schafft damit die Basis für eine Identifikation der Nachfrager mit der in Anspruch genommenen Flugdienstleistung. Zudem fungiert die Allianzmarke als Orientierungshilfe bei der Auswahl von Flugdienstleistungen. ${ }^{443}$ Beide Funktionen scheinen aufgrund des hoch wahrscheinlichen Informations- und Beratungsnachteils von Allianz-Flugdienstleistungen von besonderer Bedeutung für strategische Luftverkehrsallianzen zu sein. ${ }^{444}$

Unter der Voraussetzung einer entsprechenden Bekanntheit und Reputation ermöglicht eine Marke den Aufbau von Vertrauen und kann als Beweis der Kompetenz und Sicherheit zum Abbau eines erhöhten wahrgenommenen Risikos beitragen. ${ }^{445}$ Während die Bedeutung dieser Funktionen angesichts der immateriellen Bestandteile von Flugdienstleistungen und ihres sicherheitssensiblen Charakters nicht zu unterschätzen ist, ${ }^{446}$ dürfte ihre Erfüllung durch eine neu

442 Vgl. Meffert, H., Marketing: Grundlagen marktorientierter Unternehmensführung, a.a.O., S. 785. Diese Definition weist sowohl Bestandteile der Legaldefinition von Marken auf als auch hersteller- und wirkungsbezogene Verständnisinhalte.

443 Vgl. Meffert, H., Burmann, C., Abnutzbarkeit und Nutzungsdauer von Marken - ein Beitrag zur steuerlichen Behandlung von Warenzeichen, Arbeitspapier Nr. 117 der Wissenschaftlichen Gesellschaft für Marketing und Unternehmensführung e.V., Hrsg.: Meffert, H., Backhaus, K., Münster 1998, S. 5.

444 Dies gilt sowohl in bezug auf die reservierungssysteminhärenten Kennzeichnungsprobleme als auch im Hinblick auf die inhaltliche Konkretisierung von allianzspezifischen Nutzendimensionen.

$445 \mathrm{Vgl}$. Meffert, H., Die Bedeutung der Marke für das Image und das Erscheinungsbild von Dienstleistungsunternehmen, in: LeitWerk, Nr. 7, 1989, S. 7; Gerhard, A., Die Unsicherheit des Konsumenten bei der Kaufentscheidung: Verhaltensweisen von Konsumenten und Anbietern, Wiesbaden 1995, S. 129.

446 Die besondere Relevanz der Nutzenerwartung "Sicherheit“ im Luftverkehr bestätigt neben den Focusgruppenergebnissen z.B. Pompl (1991) in einem „Polaritătenprofil der 
gebildete Allianzmarke eher längerfristig zu enwarten sein, da die notwendigen Lernprozesse auf seiten der Nachfrager i.d.R. mit hohem Zeitbedarf einher gehen. ${ }^{447}$ Schließlich kann eine Allianzmarke bei entsprechender Positionierung eine Image- oder auch Prestigefunktion im sozialen Umfeld des Endnutzers von Allianz-Flugdienstleistungen erfüllen. ${ }^{448}$ Diese Überlegung findet in deutlicher Weise ihren Niederschlag in der "Worldwide Recognition“-Positionierung der Allianz, die auf das hochwertige Segment der Vielflieger ausgerichtet ist und deren Markenkern explizit statusbezogene Eigenschaften aufweist.

Aus diesen Funktionen einer Marke ergeben sich für die Partner-Fluggesellschaften der hier betrachteten Allianz Ansatzpunkte zur Ausgestaltung einer gemeinsamen Markenpolitik, unter der grundsätzlich alle Entscheidungen und Maßnahmen zusammengefaßt werden, die mit der Markierung von Produkten und Dienstleistungen zusammenhängen. ${ }^{449}$ Im Gegensatz zu einzeln agierenden Fluggesellschaften sind einige Besonderheiten zu beachten, die bereits in der Phase der markenpolitischen Zielsetzung der Allianz deutlich werden. ${ }^{450}$ Fluggesellschaften verfolgen mit dem Eingehen strategischer Allianzen individuelle Ziele, die von denjenigen ihrer Partner abweichen können. Eine gemeinsame Markenpolitik für die strategische Allianz erfordert indessen eine einheitliche markenstra-

Produktanforderungen“, demgemäß das Merkmal Sicherheit von dominanter Bedeutung ist, sowie Leonhardt-Weber (1990), die zwischen subjektiv empfundener Sicherheit sowie statistisch meßbarer, objektiver Sicherheit unterscheidet. Vgl. Pompl, W., Luftverkehr: eine ökonomische Einführung, a.a.O., S. 88; Leonhardt-Weber, B., Die Entwicklung der Qualitătsmerkmale im Verkehr, a.a.O., S. 29.

Vgl. Meffert, H., Burmann, C., Abnutzbarkeit und Nutzungsdauer von Marken - ein Beitrag zur steuerlichen Behandlung von Warenzeichen, a.a.O., S. 11.

An dieser Stelle sei noch einmal auf das Begriffsverstăndnis des Markenimage im Sinne von Meffert und Burmann (1996) verwiesen, die darunter die "Gesamtheit aller subjektiven Vorstellungen einer Person von der Marke hinsichtlich der wahrgenommenen Eigenschaften und der Eignung dieser Marke zur Befriedigung der rationalen und emotionalen Bedürfnisse des Individuums“ verstehen. Meffert, H., Burmann, C., Identitătsorientierte Markenführung Grundlagen für das Management von Markenportfolios, a.a.O., S. 34. Die oben genannte Image- oder Prestigefunktion ergibt sich aus der Abstrahlung wünschenswerter Eigenschaften der Marke auf den Markennutzer (z.B. Vorzeigen einer Vielflieger-Status-Kundenkarte einer großen Fluggesellschaft zur Demonstration von Kosmopolitentum oder beruflichem Erfolg).

Der entscheidungsorientierte Ansatz der Markenpolitik geht von einer Bewertung von Alternativen anhand vorgegebener Ziele in einer gegebenen Entscheidungssituation aus. Von zentraler Bedeutung sind die mit unterschiedlichen Gestaltungsalternativen der Markenpolitik verbundenen Auswirkungen auf das Nachfrager- und Wettbewerbsverhalten sowie das eigene Unternehmen. Vgl. Meffert, H., Entscheidungsorientierter Ansatz der Markenpolitik, a.a.O., S. $174 f$. 
tegische Zielsetzung, so daß ein diesbezüglicher Konsens der beteiligten Allianzpartner von essentieller Bedeutung ist. Wenngleich eine explizite Nennung der markenstrategischen Ziele in der Focusgruppenanalyse unterbleibt, kann angesichts des Positionierungsansatzes "Worldwide Recognition" auf folgende Ziele der allianzspezifischen Markenpolitik geschlossen werden: ${ }^{451}$

- Schaffung einer weltweiten Markenbekanntheit für die strategische Luftverkehrsallianz als Voraussetzung für die Erreichung der übrigen markenpolitischen Zielsetzungen,

- Bildung einer Präferenz, insbesondere bei Vielfliegern, zugunsten des AllianzLeistungsangebots gegenüber konkurrierenden Angeboten,

- Aufbau eines durch hohe Sicherheit, Komfort, Angebotsumfang und -dichte, ${ }^{452}$ reibungslose Abläufe und weltweit gültige Statusleistungen geprägten AllianzMarkenimage als Grundlage für ein von Vertrauen geprägtes Verhältnis zu den Nachfragern,

- Ermöglichung einer differenzierten Marktbearbeitung, die sich im vorliegenden Fall auf das Segment der Vielflieger konzentriert. ${ }^{453}$

Zur Erreichung dieser Zielsetzungen haben die Partner-Fluggesellschaften die allianzspezifische Markenstrategie festzulegen. Die Entscheidung darüber, ob die Partner ihre eigene Firmenmarke neben der Allianzmarke weiterführen oder sie gegen eine übergreifende, gemeinsame Allianzmarke „eintauschen“, wird dabei zum einen von rechtlichen Bedingungen beeinflußt und zum anderen von den unternehmensseitigen Gegebenheiten der beteiligten Partner determiniert.

451 Vgl. auch Meffert, H., Strategien zur Profilierung von Marken, in: Marke und Markenartikel als Instrumente des Wettbewerbs, Hrsg.: Dichtl, E., Eggers, W., München 1992, S. 131; Bruhn, M., Begriffsabgrenzungen und Erscheinungsformen von Marken, in: Handbuch Markenartikel, Stuttgart 1994, S. 23 .

Unter dem Angebotsumfang wird hier die Anzahl der angeflogenen Destinationen, unter der Angebotsdichte die Anzahl der Flüge pro Streckenverbindung pro Tag verstanden.

Diese Ziele erweisen sich damit als konsistent zu den in Kapitel B 1.2 herausgearbeiteten Zielsetzungen, die Fluggesellschaften mit der Bildung strategischer Allianzen verfolgen. So kann die Schaffung der Markenbekanntheit aus dem Allianzziel "Erhöhung des Bekanntheitsgrades“ abgeleitet werden, während das Ziel der Präferenzbildung zur Erreichung der Marktanteilssteigerung/-sicherung sowie zum Aufbau von Markteintrittsbarrieren beitrăgt. Die Verbesserung des Markenimage wird in einer gemeinsamen Markenpolitik durch den Aufbau einer Allianzmarke angestrebt, wăhrend das markenstrategische Ziel der Ermöglichung einer differenzierten Marktbearbeitung eng mit dem Marktanteilsziel verbunden ist. 
Die bilateralen Luftverkehrsabkommen, auf denen nach wie vor ein Großteil des internationalen Luftverkehrs beruht, fordern fast ausnahmslos, daß sich die zu designierenden Fluggesellschaften im Eigentum oder unter überwiegender Kontrolle von Angehörigen des Designierungsstaates befinden. Zudem müssen die Flugzeuge der zu designierenden Airlines im Eigentum von Inländern stehen und in das nationale Luftfahrzeugregister eingetragen sein. ${ }^{454}$ Der Grund für diese Vorschriften ist in der Sicherstellung des im internationalen Luftrecht gültigen "genuine link" zu sehen, der eine „echte Bindung" zwischen Registerstaat und Luftfahrzeug verlangt. ${ }^{455}$ Diese Bindung manifestiert sich in der Verwendung einer Marke, mit der u.a. auch die eingesetzten Flugzeuge markiert werden.

In dem hier zugrunde liegenden Fall verwendeten die an der Allianz beteiligten Partner-Fluggesellschaften bislang ihren eigenen Unternehmensnamen als Marke, so daß nach dem Markeninhalt auch von Firmenmarken gesprochen werden kann. ${ }^{456}$ Die im Rahmen der strategischen Allianz zum Einsatz gekommenen Flugzeuge konnten folglich anhand der Firmenmarke eindeutig der jeweiligen Fluggesellschaft und Nationalität zugeordnet werden. Gleiches gilt für alle anderen Kontaktsubjekte und -objekte der beteiligten Airlines wie beispielsweise Flugpersonal oder Flugschalter am Flughafen. Der Grundsatz des "genuine link“ wurde durch die strategische Allianz daher nicht verletzt. ${ }^{457}$

Die Verwendung einer gemeinsamen Allianzmarke bei gleichzeitiger Aufgabe der Firmenmarken der Partner würde eine zweifelsfreie Zuordnung von Fluggerät zu einer bestimmten Fluggesellschaft erschweren bzw. verhindern und der intendierten Wirkung des "genuine link“ zuwiderlaufen. Daher ist aus rechtlichen Gründen die Weiterführung der Firmenmarke parallel zur Allianzmarke zwingend erforderlich.

454 Vgl. Reckewerth, S., Die Zusammenarbeit der Luftverkehrsgesellschaften nach europăischem und US-amerikanischen Recht, a.a.O., S. 41.

455 Der "genuine link“ (echte Bindung) soll dem Flaggenstaat eine moglichst effektive Kontrolle in technischer, administrativer, sozial- und arbeitsrechtlicher Hinsicht erlauben. Vgl. Reckewerth, S., Die Zusammenarbeit der Luftverkehrsgesellschaften nach europäischem und USamerikanischen Recht, a.a.O., S. 41.

Vgl. Stauss, B., Markierungspolitik bei Dienstleistungen - Die "Dienstleistungsmarke“, in: Handbuch Dienstleistungsmanagement: von der strategischen Konzeption zur praktischen Umsetzung, Hrsg.: Bruhn, M., Meffert, H., Wiesbaden 1998, S. 567.

457 Die rechtlich vorgeschriebenen Eigentumsverhältnisse wurden durch die Allianz ebenfalls nicht berührt, da keine wechselseitigen Kapitalbeteiligungen vorlagen. 
Unabhängig von dieser rein rechtlichen Argumentation dürfte die freiwillige Aufgabe der eigenen Firmenmarke durch eine Fluggesellschaft nicht zuletzt vor dem Hintergrund der Markenwertdiskussion ${ }^{458}$ äußerst unwahrscheinlich, wenn nicht gar undenkbar sein. Der "Ersatz" einer etablierten Marke durch eine neu gebildete Allianz-„Phantasiemarke" wäre mit einer Eliminierung des in der Marke kumulierten Vertrauenskapitals bei einem gleichzeitigen zusätzlichen Mittelabfluß für den Aufbau der neuen Marke verbunden. Unter der Annahme einer Bilanzierungsfähigkeit von Marken, die im US-amerikanischen und britischen Recht beim entgeltlichen Erwerb von Markenrechten gegeben ist, hätte ein solcher Schritt bilanzielle Abschreibungen auf den Markenwert zur Folge. ${ }^{459}$

Aus rechtlichen und ökonomischen Gründen ist der Fortbestand der einzelnen Firmenmarken der Partner-Fluggesellschaften daher als einschränkende Nebenbedingung bei der Wahl der markenstrategischen Option für die strategische Luftverkehrsallianz anzusehen.

\subsubsection{Festlegung der Markenstrategie für die Luftverkehrsallianz}

Nach Meffert (1994) erfolgt die Festlegung der Markenstrategie in einem dreidimensionalen Wettbewerbsraum, der sich aus den Dimensionen des vertikalen, horizontalen und internationalen Wettbewerbs bildet. ${ }^{460}$ Da Absatzmittler wie beispielsweise Reisebüros keine Flugdienstleistungen unter eigener Marke anbieten und daher auch nicht in unmittelbarer Konkurrenz zu Fluggesellschaften oder

${ }^{458}$ In der Literatur haben sich unterschiedliche Ansätze zur Erfassung und Definition des Markenwerts herausgebildet, die in finanz- und marketingorientierte Ansätze unterschieden werden können. Wăhrend die finanzorientierte Begriffsbestimmung auf den Wert einer Marke als finanzielle Größe (z.B. Substanzwert, Ertragswert) abstellt, haben sich marketingseitig verschiedene verhaltenswissenschaftlich geprägte Definitionen herausgebildet (z.B. Nutzenerwartung an ein markiertes Produkt gegenüber der Nutzenenwartung an ein unmarkiertes Produkt). Vgl. Irmscher, M., Markenwertmanagement: Aufbau und Erhalt von Markenwissen und -vertrauen im Wettbewerb; eine informationsökonomische Analyse, Frankfurt am Main u.a. 1997, S. 57ff.

Im deutschen Recht spiegelt sich der Wert einer Marke in dem als Geschafftswert bezeichneten Unterschiedsbetrag zwischen Buch- und Substanzwert eines Unternehmens wider. Vgl. Meffert, H., Burmann, C., Abnutzbarkeit und Nutzungsdauer von Marken - ein Beitrag zur steuerlichen Behandlung von Warenzeichen, a.a.O., S. 1ff. sowie Moorhouse, M., Brand Accounting, in: Brand Valuation: Establishing a True and Fair View, Hrsg.: Murphy, J., London 1989, S. 143-153; Wild, K., Everitt, H., The Accountancy Perspective in the UK, in: Brand Valuation: Establishing a True and Fair View, Hrsg.: Murphy, J., London 1989, S. 55-68.

Vgl. Meffert, H., Entscheidungsorientierter Ansatz der Markenpolitik, a.a.O., S. 180. 
Luftverkehrsallianzen stehen, erübrigt sich im folgenden die Erörterung der vertikalen Wettbewerbsdimension. ${ }^{461}$

Im horizontalen Wettbewerb haben die Allianzpartner zu entscheiden, in welcher markenstrategischen Form sie die von innen gebildete Allianz gegenüber ihren Wettbewerbern profilieren. ${ }^{462}$ Die Auswahlmöglichkeiten zwischen den üblicherweise diskutierten Optionen der Einzel-, Mehr-, Familien- und Dachmarkenstrategien sowie Markentransfer- und Tandemmarkenstrategie ${ }^{463}$ engen sich angesichts der rechtlich-ökonomischen Restriktion sowie dem positionierungsbedingten Anspruch einer nach weltweiter Wiedererkennung strebenden Markierung auf die Wahl einer Dachmarkenstrategie ein. Da die Allianzmarke auf bereits etablierte Firmenmarken der Partner-Fluggesellschaften trifft, die ihrerseits als Dachmarke für eine Reihe weiterer Leistungen wie beispielsweise Linienflugdienste, Catering oder Lufffrachtaktivitäten fungieren, ${ }^{464}$ sind verschiedene Ebenen eines Markenportfolios zu unterscheiden (vgl. Abbildung 20). ${ }^{465}$

461 Im vertikalen Wettbewerb wird - i.d.R. bezogen auf Konsumgüter - zwischen Hersteller-, Handels- und Gattungsmarken differenziert. Nach Schenk (1994) unterscheiden sich Herstellermarken ("Markenartikel“) und Handelsmarken ("Eigenmarken") lediglich durch die jeweilige Markeneignerschaft und durch die Disposition über die Gestaltung der Marke voneinander, während Gattungsmarken aufgrund ihrer ökonomischen Konzeption und ihres warenzeichenrechtlichen Schutzes als eine Variante der Handelsmarken zu betrachten sind. Vgl. Schenk, H.-O., Handels- und Gattungsmarken, in: Handbuch Markenartikel: Anforderungen an die Markenpolitik aus Sicht von Wissenschaft und Praxis, Hrsg.: Bruhn, M., Stuttgart 1994, S. 62f.

Die von einem Lufthansa CityCenter-Reisebüro unter der Marke "Lufthansa“ angebotenen Flugdienstleistungen sind demnach nicht als Handelsmarke aufzufassen, da der Markeneigner die Lufthansa AG ist. Vielmehr handelt es sich aus Sicht der Fluggesellschaft Lufthansa bei den CityCenter-Reisebüros um eine Maßnahme der Vorwärtsintegration. Auch Gattungsmarken sind im Luftverkehr nicht beobachtbar und daher im weiteren nicht von Relevanz.

Vgl. Meffert, H., Strategien zur Profilierung von Marken, a.a.O., S. 137. Unter Profilierung wird mit Ceyp (1996) die „Planung, Durchsetzung und Kontrolle aller Marketing-Mix-Aktivităten [verstanden, Anm. d. Verf.], die auf die Erreichung einer Präferenz in der Wahrnehmung der Abnehmer gerichtet sind“. Ceyp, M., Ökologieorientierte Profilierung im vertikalen Marketing dargestellt am Beispiel der Elektrobranche, Frankfurt am Main u.a. 1996, S. 4. Durch die Einbeziehung des Präferenz-Begriffs werden die Vorziehenswürdigkeit eines Objektes gegenüber anderen Objekten und damit die Wettbewerbsorientierung in der Definition berücksichtigt.

Vgl. zu einer Übersicht Bruhn, M. (Hrsg.), Handbuch Markenartikel: Anforderungen an die Markenpolitik aus Sicht von Wissenschaft und Praxis, Stuttgart 1994, S. 463-582; zur Tandemmarkenstrategie vgl. Stauss, B., Dienstleistungsmarken, in: Handbuch Markenartikel: Anforderungen an die Markenpolitik aus Sicht von Wissenschaft und Praxis, Hrsg.: Bruhn, M., Stuttgart 1994, S. 88.

464 Treffende Beispiele finden sich U.a. bei British Airways, die mehr als 150 Marken in ihrem Portfolio aufweist und deren Mehrzahl in ein Dachmarkenkonzept integriert. Hausleitner, M., 
Allianz-Ebene

Dachmarkenstrategie

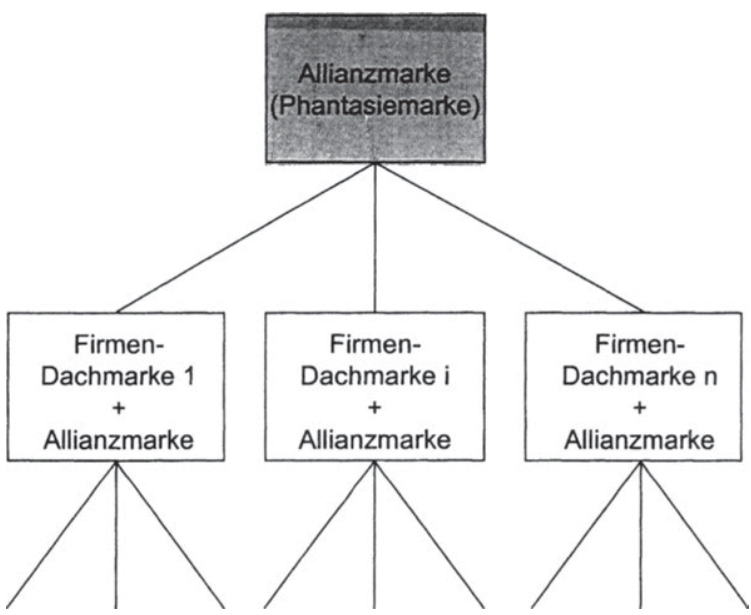

Weitere Dienstleistungsmarken, die unter dem Firmendach der einzelnen Partner geführt werden

(z.B. Linienflugdienste, Catering, Luftfracht,

\section{Abb. 20: Markenhierarchie der strategischen Luftverkehrsallianz}

(Quelle: Meffert, H., Perrey, J., Mehrmarkenstrategien - Ansatzpunkte für das Management von Markenportfolios, in: Perspektiven moderner Markenführung, Hrsg.: Esch, F.-R. (im Druck))

Auf der sogenannten Allianz-Ebene werden demnach alle Leistungen der strategischen Luftverkehrsallianz unter der Allianzmarke zusammengefaßt, die als Dachmarke dient. ${ }^{466} \mathrm{Da}$ der Allianzmarke kein einzelnes Unternehmen mit einem

Viele Identităten führen zum Marken-Brei, in: Marketing Journal, Nr. 4, 1995, S. 263. Swissair bediente sich bei der jüngsten Markenrestrukturierung ebenfalls eines Dachmarkenkonzepts („SAirGroup“). Vgl. Eggen, B., Markenmanagement am Beispiel der SAirGroup, in: Thexis, Nr. 3, 1998, S. 27.

Laforet und Saunders (1994) unterscheiden die Hierarchieebenen "Corporate Dominant", vergleichbar mit der Dachmarke, "Mixed Brands“ i.S. einer gleichzeitigen Markierung mit zwei Marken, von denen eine i.d.R. eine Firmenmarke darstellt, sowie „Brand Dominant", die der Einzelmarke entspricht. Vgl. Laforet, S., Saunders, J., Managing Brand Portfolios: How The Leaders Do It, in: Journal of Advertising Research, Vol. 34, No. 5, 1994, S. 68. Meffert und Perrey unterscheiden eine Makro-, Mikro- und Marktebene. Vgl. Meffert, H., Perrey, J., Mehrmarkenstrategien - Ansatzpunkte für das Management von Markenportfolios, in: Perspektiven moderner Markenführung, Hrsg.: Esch, F.-R. (im Druck).

Zur Dachmarkendefinition vgl. Meffert, H., Strategien zur Profilierung von Marken, a.a.O., S. 144. 
Firmennamen zugrunde liegt, kann in bezug auf den Markeninhalt von einer Phantasiemarke gesprochen werden, die gleichwohl Hinweise auf das Leistungsversprechen der Marke geben kann. ${ }^{467}$ Die jüngst eingeführte Allianzmarke "Oneworld" ist ein Beispiel für eine Wortmarke mit dem Leistungsversprechen weltweiter Präsenz, während "Star Alliance" als Wortmarke keine Rückschlüsse auf den Leistungsinhalt zuläßt. Die ehemalige "Global Excellence“-Allianz zwischen Delta Air Lines, Swissair und Singapore Airlines dagegen deutete einen weltweiten Bezug mit herausragender Qualität an, ohne daß ein Objektbezug erkennbar war ("was wird exzellent hergestellt?"). Die vor kurzem bekanntgegebene Allianzmarke "Wings" um Northwest Airlines und KLM deutet dagegen den Tätigkeitsbezug zum Fliegen an, enthält jedoch kein darüber hinaus gehendes Leistungsversprechen.

Auf der Firmen-Ebene unter der Allianz-Dachmarke sind die Firmenmarken der einzelnen Partner-Fluggesellschaften angesiedelt. Da die gemeinsamen Aktivitäten im Rahmen der strategischen Allianz auf ein bestimmtes Strategisches Geschäftsfeld ausgerichtet sind, die Partner-Fluggesellschaften trotz der parallelen Allianz-Markierung noch getrennt wahrnehmbar sind und die Markenführung der einzelnen Firmenmarken durch die Unternehmen getrennt erfolgt, kann auf dieser Ebene von einer Mehrmarkenstrategie mit der Einschränkung einer parallelen Allianz-Markierung gesprochen werden. ${ }^{468}$ Die Allianz-Fluggesellschaften verfolgen im vorliegenden Fall wie oben erwähnt ihrerseits zudem eine Dachmarkenstrategie, so daß es sich um eine Mehr-Dachmarkenstrategie mit paralleler Allianz-Markierung handelt. ${ }^{469}$

467 Zur Systematisierung nach dem Kriterium des Markeninhalts vgl. Stauss, B., Markierungspolitik bei Dienstleistungen - „Die Dienstleistungsmarke“, in: Handbuch Dienstleistungs-Marketing, Band 1, Hrsg.: Meyer, A., Stuttgart 1998, S. 567.

468 Neben diesen drei konstitutiven Merkmalen einer Mehrmarkenstrategie arbeiten Meffert und Perrey (1998) zudem noch die Unterscheidbarkeit der Produkte und Dienstleistungen anhand zentraler Leistungsmerkmale bzw. der Ausgestaltung der Marketinginstrumente heraus. Vgl. Meffert, H., Perrey, J., Mehrmarkenstrategien - Ansatzpunkte für das Management von Markenportfolios, a.a.O. Ähnlich wie der Marktauftritt kann die Erfüllung des Merkmals unterscheidbarer Produkte bzw. Dienstleistungen nur subjektiv aus Sicht des Konsumenten beurteilt werden. Mangels spezifischer empirischer Erkenntnisse zu diesem Problem ist daher auf die Plausibilitătsüberlegung zurückzugreifen, daß sich in den Jahren vor der Allianzmitgliedschaft bei den einzelnen Partnern eine einzelunternehmensspezifische Identität herausgebildet hat, die zunächst auch im Allianznetzwerk Bestand hat und sich sowohl in der Flugdienstleistung als auch im Marktauftritt manifestiert und damit die geforderte Unterscheidbarkeit erfüllt.

Die parallele Firmen- und Allianzmarkierung ist mit der "Mixed Brand"-Hierarchieebene bei Laforet und Saunders (1994) vergleichbar, wenngleich die beiden Autoren nicht auf die 
Eine Beurteilung dieser Allianzmarkenstrategie hat sich an ihrer Eignung zur Erreichung der markenpolitischen Zielsetzungen der Luftverkehrsallianz sowie an den unternehmensseitigen Bedingungen der Allianz zu orientieren. Im Aufbau einer Phantasie-Dachmarke für die Allianz besteht zunächst die Chance, die Inhalte der Positionierungsempfehlung "reißbrettartig“ umzusetzen und zudem die national geprägten Markenidentitäten der Partner-Fluggesellschaften zugunsten einer übergeordneten Instanz im Sinne einer Meta-Identität zumindest teilweise zu überwinden, um auf diese Weise die Basis für eine weltweite Markenpräsenz zu schaffen. Aufgrund einer mangelnden Einzelunternehmenspersönlichkeit hinter der Allianzmarke dürfte eine zentrale Herausforderung darin liegen, diese MetaIdentität über bloße Nutzenerwartungen hinaus mit Charakter zu füllen. Der Chance einer weltweiten Markenpräsenz steht zudem das Streben der beteiligten Partner-Fluggesellschaften nach einer Profilierung der eigenen Identität entgegen. Zudem sind von den Allianzpartnern die Kosten für den Aufbau der neuen Allianzmarke zu tragen, so daß hierfür abfließende Ressourcen nicht mehr für den Einsatz im eigenen Unternehmen zur Verfügung stehen.

Für die Wahl einer Allianz-Dachmarke spricht wiederum die Überlegung, die bisher überwiegend an Einzelfluggesellschaften orientierte Präferenzbildung von Nachfragern auf die Luftverkehrsallianz übertragen zu können. Eine Parallelmarkierung durch die Kombination aller beteiligten Firmenmarken im Sinne eines CoBranding, ${ }^{470}$ wie es beispielsweise von der Lufthansa und ihren Partnern vor der Gründung der Star Alliance praktiziert wurde, ${ }^{471}$ ist demgegenüber mit dem Risiko

Möglichkeit einer weiteren Dachmarkenbildung auf dieser Ebene eingehen. Vgl. Laforet, $\mathrm{S}$., Saunders, J., Managing Brand Portfolios: How The Leaders Do It, a.a.O., S. 68f.

Unter Co-Branding wird die Markierung eines bereits mit einer Marke versehenen Produktes oder einer Dienstleistung mit einem oder mehreren zusätzlichen Markenzeichen verstanden, wobei sich die Rechte an den zusätzlichen Markenzeichen im Besitz einer anderen oder mehrerer anderer Organisation(en) befinden. Vgl. Ohlwein, M., Schiele, T.P., Co-Branding, in: WiSt, Nr. 11, 1994, S. 577. Einzug in die Marketing-Diskussion hat diese Form der parallelen Markierung verstärkt seit der Einführung sogenannter co-branded Kreditkarten gehalten, die das Ergebnis einer Kooperation zwischen einem Finanzdienstleistungs- und einem NichtFinanzdienstleistungsunternehmen darstellen. Für den Kunden ist die Beteiligung beider Partnerunternehmen optisch und inhaltlich eindeutig zu erkennen (z.B. BahnCard der Deutschen Bahn AG mit integrierter Visa-Card). Vgl. Hüttermann, M.U., Chancen und Risiken des Co-Branding: Co-Branding - Marketing-Strategie für Kreditinstitute dargestellt am Beispiel der Kreditkarte, Hamburg 1992, S. 20.

Vgl. Klein, H., Internationaler Wettbewerb der Allianzen, a.a.O., S. 22. 
einer Irritation der Nachfrager verbunden. Von einer Dachmarke geht stattdessen eine ordnende Wirkung aus.

In engem Zusammenhang mit der Präferenzbildung steht das Ziel des Markenimage-Aufbaus für die Allianz, der an den Nutzenerwartungen der "Worldwide Recognition"-Positionierung ausgerichtet ist. Angesichts der parallelen Markierung entstehen zwischen den Firmenmarken und der Allianz-Dachmarke sogenannte Imagetransfereffekte, die sowohl positiv als auch negativ sein können. ${ }^{472}$ Entscheidend für die Untersuchung derartiger Effekte ist der situative Kontext, in dem der einzelne Fluggast seine Idealvorstellung von einer Flugdienstleistung mit der Realausprägung abgleicht und der betreffenden Fluggesellschaft positive oder negative Urteile zuordnet. Bei einer parallelen Markierung stellen sich nun die Fragen,

- ob die Urteile nur der Firmenmarke, nur der Allianz-Dachmarke oder beiden Marken und dann in welchem Verhältnis zugeordnet werden,

- ob ggf. selektiv einer der beiden Marken nur die positiven und der anderen Marke nur die negativen Urteile zugeordnet werden,

- ob ggf. "Dritt-Effekte“ dergestalt auftreten, daß Assoziationen, erlebte Probleme oder besonders zufriedenstellende Erlebnisse einer Partner-Fluggesellschaft zugeordnet werden, die gar nicht an der Leistungserstellung beteiligt war. ${ }^{473}$

Diese Fragen gewinnen noch an Komplexität, da Allianz-Flugdienstleistungen von zwei oder sogar mehr Partner-Fluggesellschaften arbeitsteilig durchgeführt werden und dem Endnutzer daher ein unmittelbarer Leistungsvergleich zwischen den Airlines möglich ist. Eine Erreichung der Markenimage-Zielsetzung für die AllianzDachmarke ist demnach davon abhängig, ob die Partner-Fluggesellschaften

472 Meffert und Heinemann (1990) betonen in ihrer gestaltungsorientierten Definition die positiven Imagetransfereffekte: Imagetransfer ist eine „Unternehmensaktivität zur Übertragung positiver Imagebestandteile (...) von einer Hauptmarke eines Produktbereichs auf ein anderes Produkt einer anderen Produktkategorie über das Vehikel des gemeinsamen Markennamens und zur Rückübertragung und Verstärkung dieser positiven Imagebestandteile von der Transfermarke auf die Hauptmarke“. Meffert, H., Heinemann, G., Operationalisierung des Imagetransfers Begrenzung des Transferrisikos durch Ähnlichkeitsmessung, in: Marketing ZFP, Nr. 1, 1990, S. 6. Im hier vorliegenden Fall beschränken sich die Ausführungen jedoch auf Effekte innerhalb derselben Leistungskategorie.

473 Zur Beantwortung dieser Fragen sind geeignete Methoden zur Erfassung der Imagetransfereffekte heranzuziehen. Vgl. stellvertretend Mayerhofer, W., Imagetransfer - Die Nutzung von Erlebniswelten für die Positionierung von Ländern, Produktgruppen und Marken, Wien 1995. 
bereits einzeln über positionierungsadäquate Imageeigenschaften verfügen und ob sich zwischen den Firmenmarken und der Allianz-Dachmarke positive Imagetransfereffekte vollziehen. Die Ergebnisse der Focusgruppenbefragung deuten darauf hin, daß die Vielflieger die hier betrachtete Allianz als Mischung aus sicheren Fluggesellschaften beurteilten und damit das Vorliegen solcher Effekte bestätigten. $^{474}$

Ob die Allianz-Dachmarke schließlich geeignet ist, das Ziel einer differenzierten, auf Vielflieger konzentrierten Marktbearbeitung zu erreichen, muß an dieser Stelle bezweifelt werden. Da das Segment der Vielflieger in der Anlage der Focusgruppenuntersuchung weltweit definiert und erfaßt wurde, wäre der Aufbau einer getrennt von den Firmenmarken operierenden, auf das Vielfliegersegment ausgerichteten Allianzmarke die deutlich überlegene strategische Option zur Erreichung dieses Ziels. Unternehmensseitig stünden einer solchen Lösung jedoch die bereits aufgeführten rechtlichen und ökonomischen Gründe entgegen. Die hier gewählte Allianz-Dachmarkenstrategie stellt bildlich gesprochen die Ummantelung bestehender Firmenmarken dar, die angesichts ihrer eigenen Markenportfolios ohnehin bereits mit der Gefahr einer Deprofilierung konfrontiert sind.

Zusammenfassend kann die Dachmarkenstrategie der Luftverkehrsallianz als Grundlage für die Schaffung einer weltweiten Markenpräsenz und -präferenz gewertet werden, die allerdings auf den Konsens und die Ressourcen aller beteiligten Partner angewiesen ist. Als komplex sind die zu erwartenden Imagetransfereffekte einzustufen, die zugleich maßgeblich über den Erfolg der Allianz-Markenpolitik entscheiden. ${ }^{475}$ Nicht förderlich ist die Dachmarkenstrategie für die Umsetzung einer spezifisch vielfliegerorientierten Marktbearbeitung, wobei rechtliche und ökonomische Gründe hier eine angemessenere Lösung verhindern.

Mit der Auswahl einer Dachmarkenstrategie zur Erreichung der markenpolitischen Zielsetzungen der Allianz ist zugleich eine Vorentscheidung für die Festlegung der internationalen markenstrategischen Dimension getroffen. Grundsätzlich kann

474 Vgl. Kapitel B 2.3.5.

475 Müller (1994) fordert beispielsweise, daß eine "starke, gesunde Originalmarke“ die notwendige Voraussetzung für eine erfolgreiche Dachmarkenstrategie bildet. Idealerweise sollte sie seiner Meinung nach Marktführer oder Marktzweiter sein. Vgl. Müller, G.-M., Dachmarkenstrategien, in: Handbuch Markenartikel: Anforderungen an die Markenpolitik aus Sicht von Wissenschaft und Praxis, Hrsg.: Bruhn, M., Stuttgart 1994, S. 503. 
zwischen multinationalen Markenstrategien, Mischstrategien und globalen Markenstrategien unterschieden werden. ${ }^{476}$ Der multinationale Gedanke eines auf länderindividuellen Markenkonzepten aufbauenden Marktauftritts liefe dem weltweiten Anspruch des „Worldwide Recognition“-Konzepts zuwider und ist daher für die vorliegende Allianz nicht als relevant anzusehen. Auch die Mischstrategien stellen keine geeignete strategische Option für die Allianz dar, da sie auf länderindividuelle Gegebenheiten abstellen und eine Bündelung oder Modularisierung des Leistungsangebots vorsehen. ${ }^{477}$ Eine globale Markenstrategie dagegen beinhaltet im Kern eine weltweit schutzfähige, mit einer einheitlichen Identität ausgestattete Marke, die in ihrer "Reinform“ weltweit mit einem identischen Marktauftritt in bezug auf Positionierung und Marketing-Instrumenteeinsatz verbunden ist. ${ }^{478}$ Die globale Markenstrategie weist damit die vergleichsweise höchste Übereinstimmung mit dem Positionierungsansatz "Worldwide Recognition" auf, wenngleich einige Besonderheiten zu berücksichtigen sind. Die geographische Reichweite der Allianzmarke ist explizit auf eine Weltmarktabdeckung ausgerichtet, während die Zielgruppendefinition eine Fokussierung auf das Segment der Vielflieger vorsieht. Daher muß strenggenommen von einer globalen Nischenstrategie gesprochen werden. Zudem ergeben sich allianzseitig Variationen in der Gestaltung des Leistungsangebots, die auf die partnerindividuellen Unterschiede zurückzuführen sind. Das Bekenntnis zur Beibehaltung dieser Leistungsdifferenzierung drückt sich in dem Leitsatz zum konsistenten Komfortniveau der Allianz aus, nach dem sich "Sitzplätze und Service der Allianzpartner unterscheiden“. ${ }^{49}$ Von der "Reinform" einer globalen Markenstrategie trennt die Allianz ferner die Art der Parallelmarkierung von Firmen- und Allianzmarke.

476 Vgl. zu dieser Unterteilung Meffert, H., Marketing: Grundlagen marktorientierter Unternehmensführung, a.a.O., S. 808ff. Ähnlich Becker (1994), der unter dem Aspekt der geopolitischen Reichweite nationale, internationale und Weltmarken unterscheidet. Vgl. Becker, J., Typen von Markenstrategien, in: Handbuch Markenartikel: Anforderungen an die Markenpolitik aus Sicht von Wissenschaft und Praxis, Hrsg.: Bruhn, M., Stuttgart 1994, S. 488.

Bei der Strategie der "konzeptionellen Bündelung“ im Sinne Waltermanns (1989) wird innerhalb homogener Ländergruppen jeweils eine einheitliche Positionierung vorgenommen, wăhrend bei der "modularen Strategie" eine international identische Basispositionierung länderspezifisch ergänzt wird. Vgl. Waltermann, B., Internationale Markenpolitik und Produktpositionierung: markenpolitische Entscheidungen im europäischen Automobilmarkt, Wien 1989, S. 74.

Vgl. Becker, J., Typen von Markenstrategien, in: Handbuch Markenartikel: Anforderungen an die Markenpolitik aus Sicht von Wissenschaft und Praxis, Hrsg.: Bruhn, M., Stuttgart 1994, S. 490. 
Mit der solchermaßen bestimmten Allianzmarkenstrategie sind die Rahmenbedingungen für die Ausgestaltung der übrigen Marketing-Instrumente vorgezeichnet, die - beginnend mit der allianzseitigen Leistungspolitik - im folgenden erörtert wird.

\subsection{Leistungspolitik der strategischen Luftverkehrsallianz}

Im Rahmen ihrer gemeinsamen Leistungspolitik legen die Partner-Fluggesellschaften der Allianz das Leistungsprogramm fest, mit dem die Nutzenerwartungen der Endnutzer - hier speziell der Zielgruppe "Vielflieger" - erfüllt werden sollen. ${ }^{480}$ Die Flugpläne der beteiligten Airlines geben in komprimierter Form Auskunft über das Leistungsprogramm und enthalten jeweils saisonal gültige Angaben zu den Abflugs- und Zielorten, zu den Abflugs- und Ankunftszeiten, zum eingesetzten Fluggerät und dessen Kapazität sowie zur Streckenführung. ${ }^{481}$ Flugpläne spiegeln demnach die Kombination unterschiedlicher Potentialfaktoren wider, bei denen es sich vor allem um Luftverkehrsrechte, Start- und Landerechte sowie Fluggerät handelt. Bei der hier betrachteten strategischen Allianz führen die Partner ihr eigenes Leistungspotential mit demjenigen ihrer Partner zu einer gemeinsamen Leistungsbereitschaft zusammen, ${ }^{482}$ die sich an den Nutzenerwartungen der Vielflieger zu orientieren hat. Die Gestaltung dieser Leistungsbereitschaft, die sich letztlich in abgestimmten Flugplänen ausdrückt, ist Aufgabe des Allianz-Netzmanagement. ${ }^{483}$

Diese grundsätzliche Leistungsbereitschaft ergänzen die Allianzpartner um weitere Faktoren, welche die angebotene Flugdienstleistung inhaltlich konkretisieren und von dem Leistungsangebot anderer Fluggesellschaften oder Luftverkehrsallianzen abgrenzbar machen. Zu nennen sind beispielsweise die Kabinengestaltung der Flugzeuge, das Mahlzeitenangebot sowie die Loungeausstattung am

480 Zur Leistungspolitik von Dienstleistungsunternehmen vgl. z.B. Meffert, H., Bruhn, M., Dienstleistungsmarketing: Grundlagen-Konzepte-Methoden, mit Fallbeispielen, a.a.O., S. 292.

481

482 in der Dienstleistungsproduktion, in: Handbuch Dienstleistungsmanagement: von der strategischen Konzeption zur praktischen Umsetzung, Hrsg.: Bruhn, M., Meffert, H., Wiesbaden 1998, S. 143f.

483

Vgl. Weber, G., Erfolgsfaktoren im Kerngeschäft von europäischen Luftverkehrsgesellschaften, a.a.O., S. 54. Organisatorisch hat sich der Aufgabeninhalt des Netzmanagement in der Bildung entsprechender Vorstandsressorts, Bereiche und Hauptabteilungen/Abteilungen vieler Fluggesellschaften niedergeschlagen. Vgl. auch die beispielhaft dargestellte strukturelle Anbindung einer strategischen Allianz an eine bestehende Organisationsstruktur in Abbildung 8, Kapitel B 1.4.2. 
Flughafen. Die Ausrichtung dieser Faktoren an den Nutzenerwartungen der Vielflieger obliegt dem Produkt- und Servicemanagement der Allianz, das an den Teilprozessen der Reisekette ansetzt.

weltweiter Sicherheitsstandard

- weltweites Streckennetz

nahtloses Reisen

konsistentes Komfortniveau

- flexible Buchungsoptionen

- nahtloses Reisen

- Vielfliegervorteile Status/Privilegien
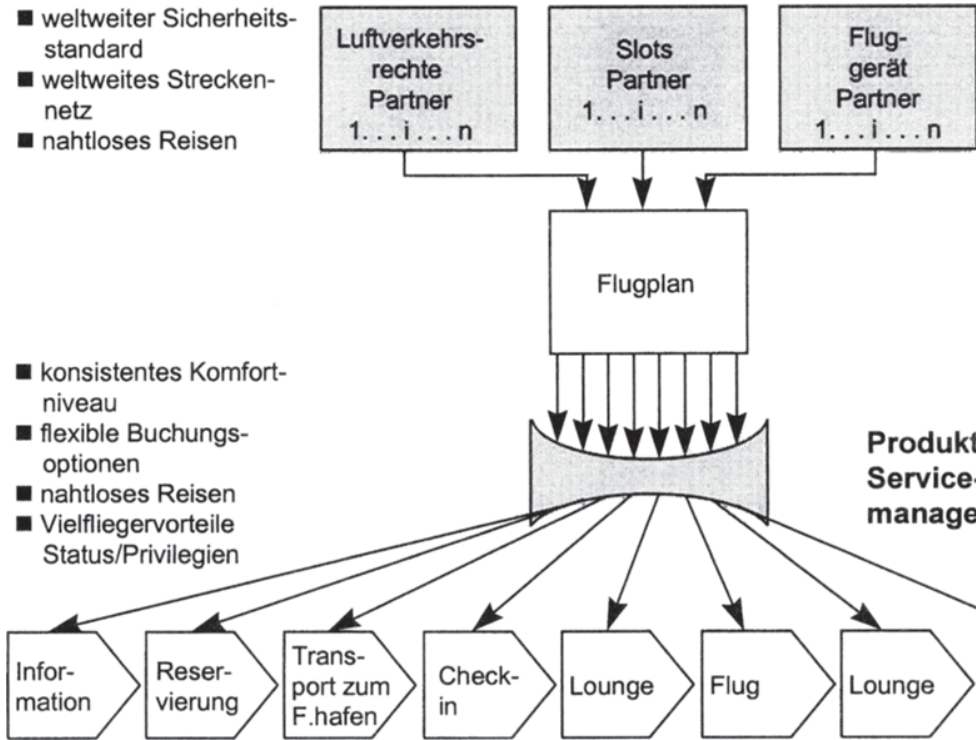

Netzmanagement

\section{Abb. 21: Nutzenorientierte Ausgestaltung der Allianz-Leistungspolitik}

Abbildung 21 verdeutlicht den Zusammenhang zwischen den beiden Submixbereichen der Leistungspolitik und ordnet innen die in der Focusgruppenuntersuchung explorierten Nutzenerwartungen der Vielflieger zu. Die nutzenorientierte Ausgestaltung des Netzmanagement ist Gegenstand des folgenden Kapitels. 


\subsubsection{Allianzseitiges Netzmanagement durch gegenseitige Abstimmung von Flugplänen}

Die an der strategischen Allianz beteiligten Partner-Fluggesellschaften bringen jeweils ein eigenes, von innen bedientes Streckennetz in die Allianz ein, das durch geographische Abflug- und Zielorte definiert ist. Den Flugstrecken werden wiederum bestimmte Kapazitäten zugeordnet, die sich aus dem eingesetzten Fluggerät sowie der Bedienungshäufigkeit ergeben. ${ }^{484}$ Zunächst sind der technische Zustand des eingesetzten Fluggeräts sowie der Ausbildungsstand des CockpitPersonals auf die Erfüllung der Nutzenerwartung eines weltweiten Sicherheitsstandards auszurichten. Eine objektive, statistisch meßbare Sicherheit kann durch kontinuierliche Wartungs- und Instandhaltungsarbeiten, regelmäßige Ersatzbeschaffungen von Fluggerät sowie Schulungen des Cockpit-Personals erreicht werden. Darüber hinaus ist die von den Vielfliegern empfundene subjektive Sicherheit zu berücksichtigen, ${ }^{485}$ die sich in der Einschätzung bestimmter Merkmale wie beispielsweise dem optischen Zustand der Flugzeuge ausdrückt. Da der technische Zustand des Fluggeräts und die Qualifikation des Cockpit-Personals letztlich nicht oder nur zu prohibitiv hohen Kosten durch den Vielflieger selbst beurteilt werden können, ${ }^{486}$ kommt dem Aufbau von Vertrauen eine zentrale Bedeutung zu. Idealerweise können die Allianzpartner bereits auf ein in den jeweiligen Firmenmarken enthaltenes Vertrauenspotential zurückgreifen, das im Zuge gemeinsamer Aktivitäten auf die Allianz übertragen wird. Ein großes Sicherheitsgefälle zwischen den Partnem wäre der Erfüllung der Nutzenerwartung „weltweiter Sicherheitsstandard" daher abträglich. Steigt ein Passagier beispielsweise auf einem Code Share-Flug von American Airlines in ein Flugzeug dessen Allianzpartners China Airlines um, erhöht sich statistisch gesehen sein Risiko, mit der Maschine abzustürzen, um das 76-fache. ${ }^{487}$

484 Zur Gestaltung des Streckenangebots vgl. z.B. Neu, M., Marketing-Strategien für den internationalen Wettbewerb von Luftfahrtgesellschaften, Würzburg 1989, S. 111.

485 Vgl. Leonhardt-Weber, B., Die Entwicklung der Qualitătsmerkmale im Verkehr, a.a.O., S. 29.

486 Die Beurteilbarkeit von Güter- und Dienstleistungseigenschaften ist Gegenstand informationsökonomischer Überlegungen. Bei der Sicherheit von Flugdienstleistungen kann demnach von einem hohen Anteil an Vertrauenseigenschaften ausgegangen werden. Vgl. z.B. Kaas, K.P., Busch, A., Inspektions-, Erfahrungs- und Vertrauenseigenschaften von Produkten, in: Marketing ZFP, 18. Jg., Nr. 4, 1996, S. 244.

487

Als Berechnungsgrundlage dient der statistisch ermittelte Wert „Tote pro eine Million Flüge von 1969-1994", der für American Airlines 0,15 und für China Airlines 11,43 ausweist. Vgl. Kolf, F., Lange Zeit blind vertraut, in: Wirtschaftswoche, Nr. 8, 1996, S. 88. American Airlines und China Airlines führen seit Dezember 1997 Code Sharing auf den Strecken Taipeh-Los Angeles, 
Die Ergebnisse der Focusgruppenbefragung belegen, daß die strategische Luftverkehrsallianz bezüglich der Nutzendimension "weltweiter Sicherheitsstandard“ weder eine dominante noch differenzierende Position innehat. Von kritischer Bedeutung ist daher die Etablierung eines allianzweiten Sicherheitsstandards, der sich in der gemeinsamen Dachmarke ausdrückt. Die Voraussetzungen hierfür scheinen angesichts der Beurteilung der Allianz als "Mischung sicherer Fluggesellschaften" grundsätzlich gegeben zu sein. Restriktionen bei der Umsetzung des Allianz-Sicherheitsstandards resultieren gleichwohl aus wenig oder nicht beeinflußbaren Faktoren wie der Luftraumüberwachung oder der Flughafensicherheit, die den sicherheitsrelevanten Teil der Infrastruktur für Fluggesellschaften zur Verfügung stellen. ${ }^{488}$

Um nun der vielfliegerseitigen Nutzenerwartung eines weltweiten Streckennetzes zu genügen, sind die partnerschaftlichen Streckennetze theoretisch so miteinander zu kombinieren, daß sich eine einhundertprozentige Abdeckung aller Verkehrsmärkte ergibt. Ein Vorteil besteht dabei in der Kombination starker Heimatmarktpositionen der Partner-Fluggesellschaften, die sich beispielsweise in der hohen Präsenz an Drehkreuzen und in einer ausgeprägten Kenntnis der lokalen Marktverhältnisse ausdrücken. ${ }^{489}$ Idealerweise ist es dann für die Vielflieger möglich, an jedem Flughafen Zugang zu mindestens einer der Partner-Fluggesellschaften zu erhalten und die gewünschte Streckenverbindung direkt oder durch die Kombination von mehreren, im Allianznetzwerk bedienten Teilstrecken in Anspruch nehmen zu können. Eine derartige Maximal-Erfüllung der Nutzen-

Taipeh-San Francisco sowie auf inneramerikanischen Verbindungen durch. Vgl. Gallacher, J., Hold your horses, in: Airline Business, No. 6, 1998, S. 58.

Sinton (1995) erkennt eine besondere Herausforderung bei der Etablierung von Sicherheitsstandards in gewachsenen Sicherheits-Infrastrukturen, die von den Fluggesellschaften als gegeben akzeptiert werden müssen. Vgl. Sinton, L., Seamless Security Screening, in: Air Transport Progress, Hrsg.: Goold, I., London 1995, S. 195.

Die Bedeutung von Drehkreuzen, die im sogenannten hub-and-spoke-System die Nabe (hub) bilden, in welcher der aus den Speichen (spokes) zuströmende Nebenverkehr gesammelt und auf Hauptstrecken verteilt wird, geht aus den Marktanteilen großer Fluggesellschaften an Großflughäfen hervor. So vereinigt American Airlines in ihrem Hub Dallas Fort Worth 63 Prozent des gesamten dortigen Passagieraufkommens auf sich, während United Airlines in Denver 78 Prozent und Delta Air Lines in Atlanta 85 Prozent aller Passagiere befördern. Vgl. Flint, P. Will four go into two? in: Air Transport World, No. 6, 1998, S. 40. Für Thai Airways erweisen sich die mit dem Drehkreuz Bangkok verbundenen Schwierigkeiten (Kapazitätsbegrenzungen sowie technische Probleme) als positionsgefăhrdend innerhalb der Star Alliance. Vgl. Ballantyne, T., Walker, K., Thai is hardly Star choice, in: Airline Business, No. 7, 1997, S. 12. 
erwartung "weltweites Streckennetz/flexible Buchungsoptionen" ist jedoch ohne gleichzeitige Berücksichtigung des Oberziels Gewinn ökonomisch wenig sinnvoll, da nicht alle theoretisch denkbaren Verkehrsströme gewinnbringend zu bedienen sind. ${ }^{490}$ Weber (1997) beispielsweise ermittelt anhand der Anzahl von weltweit 5.000 Verkehrsflughäfen die theoretische Gesamtzahl von $x^{*}(x-1)=24.995 .000$ Verkehrsströmen, die sich jedoch durch ökonomische und marktstrategische Überlegungen auf handhabbare ca. 10.000 bis 20.000 Verkehrsströme reduzieren läßt. ${ }^{491}$ Die Auswahl der im Zuge der Allianz bedienten Flugstrecken hat sich daher an der aktuellen und potentiellen Bedeutung bestimmter Verkehrsströme für die Zielgruppe der Vielflieger - gemessen an ihrem Anteil an der Gesamtzahl Passagiere in diesen Verkehrsströmen - zu orientieren. Durch die Zusammenführung von streckenspezifischen Umsatz- und Kostendaten im Rahmen der Luftverkehrsallianz sowie durch die Auswertung von Daten aus den Vielfliegerprogrammen der Partner-Fluggesellschaften können die erfolgskritischen Verkehrsströme ermittelt werden, an denen sich die Gestaltung des Allianz-Streckennetzes zu orientieren hat. Aus Sicht einer einzelnen Partner-Airline kann eine Entscheidung des Allianz-Netzmanagement durchaus negative Folgen für weiterhin im Alleingang bediente Flugstrecken haben. In solchen Fällen tritt das Einzel-Optimum hinter dem Allianz-Gesamtoptimum zurück. ${ }^{492}$

Bezüglich der damit festgelegten Streckennetzbreite ist es der betrachteten Luftverkehrsallianz offenbar bereits gelungen, eine dominante Position bei den Viel-

490 Die ökonomische Vorteilhaftigkeit der Streckenbedienung wird maßgeblich durch die Streckenlänge (steigende Stückkosten bei sinkender Länge) und den Sitzladefaktor (steigende Stückkosten bei sinkendem Sitzladefaktor) beeinflußt und läßt insbesondere Kurzstrecken sowie wenig nachgefragte Strecken unvorteilhaft erscheinen. Vgl. Oum, T.H., Winning Airlines: productivity and cost competitiveness of the world's major airlines, Norwell 1998, S. 143. Desungeachtet finden sich im Luftverkehr Fälle, in denen verlustbringende Strecken von Fluggesellschaften dauerhaft bedient werden, da sich entweder ökonomisch positive Netzeffekte durch Zubringerfunktionen ergeben oder die Strecke gehalten wird, um Wettbewerber zu attackieren bzw. um den Markteintritt neuer Wettbewerber zu verhindern.

Vgl. Weber, G., Erfolgsfaktoren im Kerngeschäft von europäischen Luftverkehrsgesellschaften, a.a.O., S. 59. OKonomische Restriktionen ergeben sich Weber zufolge durch eine Mindestentfernung von 250 km Luftlinie, unterhalb derer Flüge nur als Zubringerflüge vertretbar sind. Nach absatzmarktgerichteten Überlegungen erweist sich dagegen die Bedienung bestimmter Strecken angesichts des Heimatmarktes von Fluggesellschaften als nicht sinnvoll (z.B. Bedienung Tokio-New York durch eine europäische Airline).

In den Expertengesprächen offenbarte sich bezüglich des dort praktizierten AllianzNetzmanagement ein wechselseitiger Prozeß des "Gebens und Nehmens“ von Strecken und Bedienungszuständigkeiten, der nicht zuletzt durch Verhandlungsgeschick und entsprechende Kontrollmaßnahmen gestaltet wurde. Vgl. Anhang 1. 
fliegern einzunehmen und sich darüber hinaus deutlich gegenüber den konkurrierenden Allianzen abzugrenzen. Neben der Breite des Netzes ist eine auf die vielfliegerseitig gewünschte Flexibilität angelegte Streckennetztiefe anzustreben, welche die Bedienungshäufigkeit strategisch bedeutsamer Flugverbindungen erhöht. Auch in dieser Hinsicht haben die Allianzpartner augenscheinlich durch zusätzliche Code Share-Verbindungen eine dominante und differenzierende Position bei den Vielfliegern erreicht. Als limitierende Faktoren eines Allianz-Netzmanagement sind vor allem die Anzahl und die Qualität verfügbarer Luftverkehrsrechte sowie die Anzahl und die zeitliche Lage von Start- und Landerechten zu nennen, die eine Kapazitätsbeschränkung darstellen. ${ }^{493}$

Eine weitere Aufgabe des Allianz-Netzmanagement besteht in der Ermöglichung eines nahtlosen Reisens durch eine entsprechende Anpassung der Flugpläne der Allianzpartner. Dabei ist zunächst eine möglichst hohe Anzahl an Code ShareDirektverbindungen in der Allianz anzustreben, deren Vorziehenswürdigkeit in der Untersuchung von Gellman Research Associates empirisch belegt werden konnte. ${ }^{494}$ Direktverbindungen vermeiden den bei Umsteigeflügen notwendigen Flugzeugwechsel und verkürzen die Reisezeit. Zudem lassen sich weitere Vorteile nutzen, die im Zuge des Produkt- und Servicemanagement von Allianzen erörtert werden. Für die Erreichung eines nahtlosen Reisens ist auf seiten des Netzmanagement ferner die Optimierung von Übergangszeiten, die bei Umsteigeverbindungen entstehen, von entscheidender Bedeutung. Die Ankunfts- und Abflugzeiten von Flügen innerhalb des Allianz-Netzwerks sind so aufeinander abzustimmen, daß den Vielfliegern am Umsteigeflughafen einerseits genügend Zeit zum Erreichen des Anschlußfluges bleibt, andererseits jedoch die Wartezeit bis zum Abflug möglichst gering gehalten wird. Bei der Planung derartiger nahtloser Übergänge sollten die Allianzpartner gemeinsam sogenannte time banks oder Zeitknoten an Flughäfen belegen, ${ }^{495}$ innerhalb derer Start- und Landevorgänge mög-

493 Diese Kapazitätsbeschränkung kann auch durch die gegenseitige Erschließung von Slots oder Luftverkehrsrechten in Allianzen nur in Grenzen umgangen werden. Nach Lüking (1993) hängt die für das Netzmanagement bedeutungsvolle Kapazităt eines Verkehrsstroms generell von der Kapazităt des jeweils am wenigsten leistungsfähigen Flughafens oder Luftraumsektors ab. Diese Kapazităt kann beispielsweise über die Kennziffer der „praktischen Kapazităt“ gemessen werden, welche die Höchstzahl der Starts und Landungen pro Zeiteinheit auf tolerierbarem Sicherheits- und Verspätungsniveau beinhaltet. Vgl. Lüking, J., Angebotsplanung und Fluggastverhalten im überlasteten Luftverkehrssystem, Bern u.a. 1993, S. 33 u. 35. Vgl. Kapitel B 2.2.4. R., Luftverkehr, a.a.O., S. 171. Zu den Auswirkungen von Flugverschiebungen im Knoten eines 
lichst ineinander übergreifend abgewickelt werden. Vormachtstellungen der Partner-Airlines an ihren Drehkreuzen können in diesem Zuge genutzt werden, da eine große Anzahl an Start- und Landerechten eine entsprechend flexible Disposition ermöglicht. Als Restriktionen erweisen sich wiederum Kapazitätsengpässe an Flughäfen sowie situative Faktoren wie Verspätungen, welche die Erfüllung der Nutzenerwartung eines störungsfreien Reisens konterkarieren können (z.B. verpaßter Anschlußflug).

Die Herausforderung an die strategische Luftverkehrsallianz, die Nutzenerwartung des nahtlosen Reisens zu erfüllen, ist als hoch einzustufen, da die befragten Vielflieger diesbezüglich einen relativen Wettbewerbsnachteil der Allianz konstatierten. ${ }^{496}$ Durch eine „reibungslose“ Abstimmung boden- und bordbezogener Prozesse müssen die Allianzpartner demnach zu einer Überwindung des bestehenden Wettbewerbsnachteils beitragen und zugleich die Erfüllung der weiteren Nutzenerwartungen seitens der Vielflieger anstreben. Diese Aufgaben stellen sich dem im folgenden zu erläuternden Produkt- und Servicemanagement der Allianz.

\subsubsection{Produkt- und Servicemanagement der strategischen Luftverkehrs- allianz}

Grundsätzlich bieten sich in jedem Teilprozeß der Reisekette Ansatzpunkte für eine vielfliegerorientierte Leistungserstellung. Da die meisten einzeln agierenden Fluggesellschaften bereits ein nach Zielgruppen differenziertes Leistungsprogramm anbieten, ${ }^{497}$ über dessen Erstellung sie selbst die Prozeßkontrolle innehaben, liegen die zentralen Herausforderungen für Luftverkehrsallianzen in der Festlegung eines gemeinsamen Rahmens für das Allianz-Leistungsprogramm, der den einzelnen Partnern ausreichende Differenzierungsspielräume offenläßt, sowie

Umsteigeflughafens vgl. Weber, G., Erfolgsfaktoren im Kerngeschaft von europäischen Luftverkehrsgesellschaften, a.a.O., S. 75.

496

497

Vgl. Kapitel B 2.3.5.

$\mathrm{Zu}$ nennen sind beispielsweise (1) die Unterteilung der Flugzeugkabine in unterschiedliche Beförderungsklassen (i.d.R. Economy, Business, First Class), in denen u.a. die Betreuung, der Sitzkomfort und das Mahlzeitenangebot variieren sowie (2) die Staffelung von Vielfliegerprivilegien nach der Anzahl geflogener Meilen, die zugleich über die Zugangsberechtigung für Lounges entscheiden. British Airways beispielsweise hat vier differenzierte Leistungsbündel entwickelt, die unter den Bezeichnungen „World Traveller" $/$ "Euro Traveller", "Club World"/"Club Europe", "First Class“ und "Concorde" mit unterschiedlichen, prozeßbezogenen Leistungsniveaus auftreten. Vgl. Mei-Pochtler, A., Markenmanagement für Dienstleistungs-Anbieter, in: Handbuch Dienstleistungs-Marketing, Band 1, Hrsg.: Meyer, A., Stuttgart 1998, S. 676. 
in der Harmonisierung von Prozessen, deren Kontrolle auf die Allianzebene gehoben wird.

In den Teilprozessen der Information und Reservierung bestehen hohe Interdependenzen zwischen dem Produkt- und Servicemanagement sowie der AllianzDistributions- und -Kommunikationspolitik. Die Einrichtung eines telefonischen 24Stunden-Allianzinformationsservice beispielsweise, der Vielfliegern für Flugplanauskünfte, Bonusprogramm-Informationen oder Problemlösungen zur Verfügung steht, bildet eine Maßnahme zur Unterstützung des nahtlosen Reisens und ist daher nicht nur auf den ersten Teilprozeß der Information begrenzt. Ein solcher Dienst setzt voraus, daß die in den Call Centern ${ }^{498}$ eingesetzten Mitarbeiter der Allianzpartner stets auf einheitlich hohem Informationsstand sind und mindestens über fließende Sprachkenntnisse in allen Sprachen der beteiligten Partner verfügen. Zudem sind Airline-„Spezialisten“ zu definieren, die auf alle fluggesellschaftsspezifischen Fragen zu einer bestimmten Partner-Airline antworten können. Die Relevanz einer solchen Einzelzuständigkeit ergibt sich aus den bereits gewachsenen Beziehungen zwischen Vielfliegern und einzelnen Fluggesellschaften der Allianz. ${ }^{499}$ Weitere Herausforderungen für die Allianz ergeben sich aus der Abstimmung der anbieterseitigen Informationsinhalte mit den Reisebüros und Firmen-Reisestellen, um ein zur Verunsicherung führendes Informationsgefälle zwischen den Absatzstufen zu vermeiden, sowie in der Abgleichung telefonisch vermittelter Auskünfte mit den Inhalten, die über die Marktkommunikation der Allianz verbreitet werden. .00

Im Teilprozeß der Reservierung obliegt es dem Allianz-Produkt- und Servicemanagement daraufhin, eine durchgehende Reservierung vom Start- bis zum Zielort eines Fluges auch dann zu ermöglichen, wenn mehrere Umsteigevorgänge mit einer solchen Flugdienstleistung verbunden sind, um dem Anspruch des nahtlosen Reisens zu genügen. Zu berücksichtigen sind u.a. individuelle Sitz-

498 Call-Center sind organisatorische Einrichtungen zur telefonischen Betreuung von aktuellen und potentiellen Kunden. Vgl. z.B. Ceyp, M., Call Center als Querschnittsinstrument im Marketing, in: IfM-News, Nr. 1, 1998, S. 19.

Das in Kapitel B 2.3.2 wiedergegebene Zitat eines Vielfliegers, der sich als American AirlinesKunde auf einem Code Share-Flug mit Canadian Airlines mit Schwierigkeiten bei der Änderung seiner Reisepläne konfrontiert sah, unterstreicht exemplarisch die Bedeutung von Einzelzustăndigkeiten, solange keine optimale Integration zwischen den Partnern erreicht ist.

500 Idealerweise sind die Mitarbeiter im Call Center mit den Inhalten von Allianz-Werbekampagnen vertraut, bevor diese in Werbeträgern geschaltet werden. 
platzpräferenzen insbesondere derjenigen Fluggäste, die quasi routinemäßig auf einer bestimmten Strecke fliegen. ${ }^{501}$ Die mit einer Reservierung innerhalb einer Luftverkehrsallianz verbundenen Probleme treten anhand des folgenden Beispiels zu Tage. Nimmt ein Vielflieger einen Tag vor Inanspruchnahme seiner Flugdienstleistung eine Umbuchung auf eine Partner-Airline vor, wird diese Änderung in vielen Fällen nicht rechtzeitig von dem Abflugkontrollsystem der betreffenden Partner-Fluggesellschaft erfaßt. ${ }^{502}$ Folgen sind Schwierigkeiten bei der weiteren Reiseführung sowie eine kaum individualisierte Betreuung des Kunden, da Detailinformationen über dessen Präferenzen fehlen. ${ }^{503}$ Ausschlaggebend für die Erfüllung der hiervon gleichermaßen betroffenen Erwartung eines nahtlosen Reisens und weltweiter Vielfliegervorteile ist daher die Implementierung einer gemeinsamen Allianz-Informationstechnologie, die eine partnerübergreifende Kontrolle aller relevanten Prozesse ermöglicht. Wenngleich eine auf einer gemeinsamen Datenbasis aufbauende, einheitliche Allianz-Informationstechnologie als strategischer Wettbewerbsvorteil gegenüber anderen Allianzen angesehen werden kann, stehen einer solchen Integration das Individualisierungsstreben der Partner ${ }^{504}$ und das Risiko möglicher Allianz-Austritte entgegen. Derartige Überlegungen finden bei der Star Alliance beispielsweise inren Niederschlag in der Entwicklung einer Internet-basierten Schnittstelle "Starnet", welche die unterschiedlichen IT-Systeme der sechs Partner kompatibel machen soll. Neben wechselseitigen Zugriffsmöglichkeiten können die einzelnen Partner nach dem bisheri-

\footnotetext{
501 In einem Expertengespräch wies der Manager einer großen Fluggesellschaft explizit auf das Erfahrungswissen von Vielfliegern hin, die - bezogen auf eine einzelne Airline - genau über die innen zustehenden Leistungen informiert sind und diese auch einfordern. Vgl. Anhang 2.

Dieses Phänomen wird auch als "no-record“ bezeichnet und ist auf systembedingte Abstimmungsmängel zurückzuführen. Zu einer detaillierten Erklärung vgl. Schmidt, A., Computerreservierungssysteme im Luftverkehr: Darstellung, Entwicklung und wettbewerbliche Beurteilung, Hamburg 1995, S. 24.

Vgl. Pommes, C. de, Are you IT-compatible?, a.a.O., S. 27.

Investitionen in eine gemeinsame Allianz-Informationstechnologie werden von den einzelnen Partnern die zu erwartenden Einzahlungen aus der Allianz gegenübergestellt. Im Fall der Star Alliance fiel die Entscheidung zuungunsten eines gemeinsamen Systems aus: "It's a huge project and we all have big investments in our own, customized systems. The international benefits are not worth all the money and effort. One hundred million dollars in added revenue from Lufthansa is a relatively small number in a $\$ 16$ billion company." (Zitat des IT-Leiters von United Airlines, abgedruckt bei Feldman, J.M., Making Alliances Work, in: Air Transport World, No. 6, 1998, S. 34 .
} 
gen Stand der Planungen mit ihren eigenen Systemen und Sprachen unabhängig voneinander weiter operieren. ${ }^{505}$

Neben den informationstechnologischen Aspekten ist im Teilprozeß der Reservierung eine Markierung der relevanten Kontaktsubjekte und -objekte mit der Allianzmarke zu berücksichtigen. ${ }^{506}$ Bei einer telefonischen Reservierung über Call Center könnte beispielsweise ein entsprechender Jingle für die Allianz entwickelt werden. In Reisebüros sind Anstecknadeln mit dem Allianz-Logo für die Reisebüromitarbeiter denkbar, während der Schriftverkehr mit Reisebüros, Firmen-Reisestellen und Vielfliegern auf Briefpapier mit der Allianz-Markierung erfolgen könnte. Auch die auf Tickets, Kofferanhängern und Aufklebern neben der Firmenmarke angebrachte Allianzmarke erfült wichtige Orientierungsfunktionen, auf die in gleicher Weise im weiteren Verlauf der Flugdienstleistung zu achten ist.

Eine zentrale Bedeutung im Rahmen des Produkt- und Servicemanagement kommt darüber hinaus der Entwicklung einer allianzweiten Vielfliegerkarte zu, die ihrem Besitzer bei allen Partner-Fluggesellschaften denselben Status und dieselben Privilegien zugesteht, die er von seiner bisher präferierten Fluggesellschaft gewöhnt war. Nur durch eine vollständige Harmonisierung der bisher getrennt definierten Bedingungen, die dem Allianz-Vielfliegerprogramm zugrunde liegen, kann das Nutzenversprechen der fluggesellschaftsunabhängigen, weltweiten Wiedererkennung innerhalb der Allianz umgesetzt werden. ${ }^{507}$ Demnach sind durch das Allianz-Produkt- und Servicemanagement die Konditionen für den Erhalt von Freiflügen, sonstigen Vergütungen, für das Erreichen eines bestimmten Status sowie für den Verfallszeitraum der erflogenen Bonusmeilen festzusetzen. Abbildung 22 verdeutlicht die Problematik am Beispiel der strategischen Allianz

505 Vgl. Walker, K., Where's the glue? in: Airline Business, No. 7, 1998, S. 31. Meffert (1995) weist auf die Kostennachteile solcher Insellösungen hin, da die Unternehmen Investitionen in „Adapter" leisten müssen, die keine produktiven, sondern Konvertierungsleistungen erbringen. Zudem sind derartige Lösungsansătze mit Flexibilitătsnachteilen verbunden. Vgl. Meffert, J.P.H., Strategische Neuorientierung der Computerindustrie durch Standards, in: Thexis, Nr. 1, 1995, S. 33.

Zur physischen Markierung von Dienstleistungen vgl. Meffert, H., Bruhn, M., Dienstleistungsmarketing: Grundlagen-Konzepte-Methoden, mit Fallbeispielen, a.a.O., S. 324. Deutlich wird an dieser Stelle einmal mehr der instrumenteübergreifende Charakter der Markenpolitik.

507 Kritiker einer solchen Vorgehensweise heben den Verlust einer individuellen Wiedererkennung durch eine bestimmte Fluggesellschaft und deren Mitarbeiter hervor. Vgl. Gallacher, J., Power to the plans, in: Airline Business, No. 8, 1997, S. 37. 
zwischen Air France und Continental Airlines. Die Mitglieder in den Vielfliegerprogrammen dieser beiden Fluggesellschaften können durch Flüge mit der jeweiligen Airline in der Economy Class Meilen sammeln, die bei Buchung der Business Class mit dem Faktor 2,0 bzw. 1,25 sowie bei Nutzung der First Class mit dem Zähler 3,0 bzw. 1,5 multipliziert werden. Bei Erreichen einer kritischen Meilengrenze werden Freitickets oder Upgrades gewährt (z.B. Höherstufung von der Business in die First Class), und zusätzlich ist mit einer bestimmten Anzahl geflogener Meilen pro Jahr ein bestimmter Status verbunden.

\begin{tabular}{|c|c|c|c|c|c|c|c|c|}
\hline & \multicolumn{2}{|c|}{ Prămien } & \multicolumn{2}{|c|}{ Status } & \multicolumn{4}{|c|}{ Meilenerwerb } \\
\hline & Freitickets & Upgrades & $\begin{array}{l}\text { 1. Stufe } \\
\text { ab Jah- } \\
\text { res- } \\
\text { leistung } \\
\text { von... }\end{array}$ & $\begin{array}{l}\text { 2. Stufe } \\
\text { ab Jah- } \\
\text { res- } \\
\text { leistung } \\
\text { von... }\end{array}$ & $\begin{array}{c}\text { Multipli- } \\
\text { kator } \\
\text { Business } \\
\text { Class }\end{array}$ & $\begin{array}{l}\text { Multipli- } \\
\text { kator First } \\
\text { Class }\end{array}$ & $\begin{array}{c}\text { Bonus bei } \\
\text { Beitritt }\end{array}$ & $\begin{array}{l}\text { Kredit- } \\
\text { karten- } \\
\text { option }\end{array}$ \\
\hline $\begin{array}{l}\text { Air } \\
\text { France } \\
\text { Frequen- } \\
\text { ce Plus }\end{array}$ & ja & ja & $\begin{array}{l}30.000 \\
\text { Meilen }\end{array}$ & $\begin{array}{l}60.000 \\
\text { Meilen }\end{array}$ & 2,0 & 3,0 & $\begin{array}{l}3.000 \\
\text { Meilen }\end{array}$ & Amexco \\
\hline $\begin{array}{l}\text { Continen- } \\
\text { tal Air- } \\
\text { lines One } \\
\text { Pass }\end{array}$ & ja & ja & $\begin{array}{l}20.000 \\
\text { Meilen }\end{array}$ & $\begin{array}{l}35.000 \\
\text { Meilen }\end{array}$ & 1,25 & 1,5 & $\begin{array}{l}2.500 \\
\text { Meilen }\end{array}$ & Amexco \\
\hline $\begin{array}{l}\text { Implika- } \\
\text { tionen für } \\
\text { die Alli- } \\
\text { anz }\end{array}$ & \multicolumn{2}{|c|}{$\begin{array}{l}\text { kein Anpassungs- } \\
\text { bedarf }\end{array}$} & \multicolumn{4}{|c|}{$\begin{array}{l}\text { Festlegung einheitlicher Meilengrenzen und } \\
\text { Multiplikatoren notwendig }\end{array}$} & $\begin{array}{l}\text { Festle- } \\
\text { gung } \\
\text { einer ein- } \\
\text { heitlichen } \\
\text { Meilen- } \\
\text { zahl }\end{array}$ & $\begin{array}{l}\text { kein } \\
\text { Anpas- } \\
\text { sungs- } \\
\text { bedarf }\end{array}$ \\
\hline
\end{tabular}

Abb. 22: Ermittlung des Harmonisierungsbedarfs am Beispiel gemeinsamer Vielfliegerprogramme

(Quelle: in Anlehnung an Rodrian, H.W., Der Meilen-Bonus macht aus dem Flug einen Gewinn, in: Handelsblatt vom 17.11.1997, S. 41)

Bereits dieses Beispiel mit lediglich zwei Allianzpartnern belegt den Harmonisierungsbedarf bei einer Zusammenführung der Vielfliegerprogramme. Inwieweit ein Fluggast durch eine vollständige Harmonisierung der Programme besser-, schlechter- oder gleichgestellt wird, hängt im vorliegenden Fall vom Einigungsniveau der Partner sowie von dem individuellen Reiseverhalten des Vielfliegers ab. 
Neben der formalen Harmonisierung gilt es, die mitarbeiterseitige Kenntnis und Akzeptanz des allianzweiten Vielfliegerprogramms sicherzustellen, um negative Überraschungseffekte seitens der Fluggäste zu vermeiden. In der partnerübergreifenden Statusanerkennung, die auf der operativen Ebene wesentlich von den Mitarbeitern der Allianzpartner getragen wird, sahen die in den Focusgruppen befragten Vielflieger ein deutliches Potential zur Abgrenzung gegenüber konkurrierenden Allianzen, so daß offenbar eine höhere Informationstransparenz innerhalb der Allianz geschaffen werden muß.

Im Teilprozeß des Transports zum Flughafen, der durch öffentliche Verkehrsmittel oder den eigenen Pkw erfolgt, besteht die Hauptaufgabe des Allianz-Produkt- und Servicemanagement daraufhin in der Gestaltung eines Orientierungssystems in der Flughafenumgebung, das ggf. durch bevorzugte Parkmöglichkeiten für diejenigen Vielflieger ergänzt wird, die im Besitz einer entsprechenden AllianzKundenkarte sind. Am Check-in gilt es, auch bei Umsteigeverbindungen eine Durchabfertigung der Fluggäste und ihres Gepäcks zu gewährleisten, um der Nutzenerwartung eines nahtlosen Reisens zu entsprechen. Åhnlich dem Teilprozeß der Reservierung, erweisen sich informationstechnologische Gegebenheiten auch hier als Engpaßfaktor. Schätzungen zufolge ist die Etablierung gemeinsamer Check-in-Prozesse zur Realisierung einer solchen Durchabfertigung im Fall der Star Alliance mit Kosten in Höhe von 100 Millionen Dollar sowie einem Umsetzungszeitraum von zwei bis drei Jahren verbunden. ${ }^{508}$ Zusätzlich erweisen sich die infrastrukturellen Bedingungen der Flughäfen, die i.d.R. informationstechnologische "Insellösungen" darstellen, häufig als Barriere bei der Erreichung der Allianzziele. ${ }^{509}$ Gleiches gilt für die Schaffung einer räumlichen Nähe zwischen Abfertigungsschaltern, Lounges und Abfluggates, die sich insbesondere auf Großflughäfen zeit- und streßreduzierend für die Vielflieger erweist. ${ }^{510}$

Die Bemühungen der Allianzpartner, ein nahtloses Reisen sowie weltweite Vielfliegervorteile zu ermöglichen, gelangen auch bei der Vereinheitlichung von

\footnotetext{
508 Vgl. Feldman, J.M., Making Alliances Work, a.a.O., S. 33.

509 Vgl. Pommes, C. de, Are you IT-compatible?, a.a.O., S. 29.

510 I.d.R. nutzen Fluggesellschaften bestimmte Areale von Flughäfen über mehrere Jahre, gestalten diese z.T. auch selbst und sind vertraglich an die Nutzung derselben gebunden, so daß die räumliche Zentrierung unterschiedlicher Fluggesellschaften auch hier mit entsprechenden Hemmnissen verbunden ist. Vgl. Feldman, J.M., Making Alliances Work, a.a.O., S. 33.
} 
Gepäckmitnahmevorschriften an ihre Grenzen, die vornehmlich rechtlicher Natur sind. Während in den USA die Mitnahme von zwei Handgepäckstücken auch in der Economy Class üblich ist, untersagen europäische Luftverkehrsbehörden i.d.R. die Mitnahme von mehr als einem Bordgepäckstück. ${ }^{511}$ Auch die vom Verband asiatisch-pazifischer Fluggesellschaften verabschiedete Resolution begrenzt die Anzahl des Bordgepäcks auf ein Stück und legt darüber hinaus genaue Maßstäbe an Abmessungen (max. $115 \mathrm{~cm}$ ) und Gewicht (max. $7 \mathrm{~kg}$ ) an. ${ }^{512}$

Das Produkt- und Servicemanagement der strategischen Allianz hat im Teilprozeß der Loungenutzung, die dem eigentlichen Flug sowohl vor- als auch nachgelagert sein kann, daraufhin eine allianzweit einheitliche Zugangsbestimmung zu definieren, die in engem Zusammenhang mit der oben erörterten Allianz-Vielfliegerkarte zu sehen ist. Die Vielfliegerkarte kann als verbrieftes Leistungsversprechen bzw. Nutzungsrecht angesehen werden, mittels dessen die Vielflieger auf eine erweiterte Anzahl an Lounges zugreifen können. Da in der Focusgruppenbefragung zunächst kaum Differenzierungspotential in der erweiterten Nutzungsbasis identifiziert wurde, scheint wiederum die personalisierte Ansprache der Statuskunden durch die Allianzmitarbeiter in höherem Maße zur Erfüllung der Nutzenerwartungen „Vielfliegervorteile Status/Privilegien“ geeignet zu sein. Unterstützend ergibt sich die Notwendigkeit eines klaren Orientierungssystems mit entsprechender Allianz-Markierung. Dennoch drücken die Untersuchungsergebnisse der Vielfliegerbefragung in der Nutzenerwartung „konsistentes Komfortniveau" deutlich aus, daß eine partnerindividuelle Gestaltung des Lounge-Interieurs bei Wahrung eines bestimmten Qualitätsstandards durchaus begrüßt wird. ${ }^{513}$

Die Beibehaltung einer unternehmensindividuellen Kabinengestaltung der Flugzeuge, die in der Allianz zum Einsatz kommen, erweist sich auch im Teilprozeß des eigentlichen Fluges prinzipiell als geeignet, die Nutzenerwartung eines konsistenten Komfortniveaus zu erfüllen. Es sind jedoch allianzweit gültige Qualitätsstandards zu definieren, die z.B. Mindestniveaus für den Sauberkeitszustand

511 Vgl. das im Anhang 1 dokumentierte Expertengespräch mit dem Vertreter einer großen Fluggesellschaft.

512 Vgl. Nelms, D.W., Improving the tube, in: Air Transport World, No. 3, 1998, S. 80.

$513 \mathrm{Vgl}$. Kapitel B 2.3.5 sowie weitere Ergebnisse, die ein in sich homogenes Erscheinungsbild als erfolgversprechend ansehen. Vgl. Nelms, D.W., Improving the tube, a.a.O., S. 78. 
der Kabine sowie der sanitären Anlagen festschreiben. ${ }^{514}$ Die Fixierung bestimmter Sitzmaße im Sinne eines Allianz-Standards dürfte dagegen angesichts der zu erwartenden Umrüst- und Opportunitätskosten für einzelne Partner kaum auf allianzinterne Akzeptanz stoßen. ${ }^{515}$ Für die Erreichung eines konsistenten Komfortniveaus sowie eine nahtlosen Reisens sind darüber hinaus gemeinsame Normen im Umgang mit den Vielfliegern von Bedeutung. Es ist jedoch fraglich, inwieweit sich bestimmte personale Verhaltensmuster, die zudem einem eher langfristigen kulturellen Einfluß unterworfen sind, in Richtung eines gemeinsamen Allianz-Standards - von einheitlichen Begrüßungs- und Verabschiedungsformeln abgesehen - verändern lassen. Da die unterschiedliche Unternehmensidentität der Partner-Fluggesellschaften auch in den Uniformen des Bordpersonals zum Ausdruck kommt, ist es notwendig, z.B. durch Anstecknadeln mit dem Logo der Allianzmarke zu einer Orientierung von Fluggästen beizutragen. Zudem haben die Auswahl und Ausbildung des Bordpersonals unter Berücksichtigung der Sprachkenntnisse der Bewerberinnen und Bewerber zu erfolgen, um eine landessprachliche Betreuung der Vielflieger zu ermöglichen. Hier besteht eine Schnittstelle zu dem später zu erörternden Instrument der Personalpolitik.

Im Teilprozeß der Gepäckausgabe besteht die Aufgabe des Produkt- und Servicemanagement schließlich in der Sicherstellung einer koordinierten Gepäckweiterleitung sowie einer allianzweit zuvorkommenden, individualisierten Behandlung von Problemfällen wie beispielsweise Gepäckschäden oder Gepäckfehlleitungen. Die Einrichtung einer telefonischen "Hotline“, die Allianzkunden in Problemfällen zur Verfügung steht, könnte mit dem eingangs erwähnten 24-Stunden-Allianzinformationsservice verbunden werden. Gemeinsame Standards („Auslieferung des Gepäcks innerhalb von 20 Minuten“) werden in ihrer Einhaltung entscheidend von der informationstechnologischen Infrastruktur beeinflußt, die zwischen den Partnern und den jeweiligen Flughäfen besteht. ${ }^{516}$

514 Ein in den Expertengesprächen erfaßtes Unternehmen wendete einen auf der Bewertung mehrerer Leistungsmerkmale beruhenden Qualitătsindex an, in den u.a. auch der Zustand der Kabine, der Sitze und der WCs einging. Vgl. Anhang 2. Sofern mehrere Allianzpartner derartige Qualitätsmessungen anwenden, bietet sich bei begrenztem Aufwand die Möglichkeit, einen gemeinsamen Standard zur Steuerung der Allianz-Flugqualităt zu etablieren und kontinuierlich zu überprüfen.

Schătzungen zufolge resultiert die Entfernung von zehn Sitzplätzen, die mit einem Platzgewinn von etwa einem Zoll zusätzlicher Beinfreiheit einhergeht, in jährlichen Umsatzverlusten von 500.000 (mittelgroße Fluggesellschaft) bis 15 Millionen US-Dollar (große Fluggesellschaft). Vgl. Nelms, D.W., Improving the tube, a.a.O., S. 74.

516 Vgl. Feldman, J.M., Making Alliances Work, a.a.O., S. 33. 
Während sich die Aufgaben des Allianz-Produkt- und Servicemanagement damit auf die koordinierte Leistungserstellung im Zuge der gesamten Reisekette beziehen, konzentriert sich die Distributionspolitik auf alle Entscheidungen, die mit dem Weg des Flugscheins im Sinne eines verbrieften Leistungsversprechens von den Allianz-Mitgliedern zum Endnutzer zusammenhängen. ${ }^{517}$

\subsection{Ausgestaltung der Distributionspolitik der strategischen Luftverkehrs- allianz}

Bei der Abstimmung der allianzseitigen Distributionspolitik haben sich die PartnerFluggesellschaften an den zuvor definierten markenpolitischen Zielsetzungen zu orientieren, um einen integrierten Marktauftritt zu gewährleisten. ${ }^{518}$ Diese Zielsetzungen können daher als Kriterien für die Festlegung der vertikalen Absatzkanalstruktur dienen, die im folgenden zu diskutieren ist.

Die Nutzung des indirekten Fremdvertriebs, bei dem die Reisebüros eine bedeutende Stellung einnehmen, ist unter Berücksichtigung des Ziels einer weltweiten Markenbekanntheit mit dem Vorteil eines vergleichsweise hohen Distributionsgrads verbunden. ${ }^{519} \mathrm{Da}$ das im Flugplan enthaltene Allianz-Leistungsangebot Eingang in die Computerreservierungssysteme findet, auf die wiederum die Absatzmittler zugreifen, ergibt sich dieser Vertriebsweg quasi „automatisch“ für das Allianz-Flugdienstleistungsangebot. ${ }^{520}$ Als ökonomisch vorteilhaft erweisen

517 Die Notwendigkeit dieser begrifflichen Eingrenzung ergibt sich aus der dienstleistungsspezifischen Besonderheit von Flugdienstleistungen, die strenggenommen nur bei Anwesenheit des externen Faktors (Fluggast und dessen Verfügungsobjekt(e)) vertrieben werden können. Der Flugschein indessen speichert das Leistungsversprechen für einen bestimmten Zeitraum, so daß Vertrieb und Inanspruchnahme entkoppelt werden. Vgl. Meffert, H., Bruhn, M., Dienstleistungsmarketing: Grundlagen-Konzepte-Methoden, mit Fallbeispielen, a.a.O., S. 424.

Anders dagegen Zeithaml und Bitner (1996), die unter dem Begriff der Distribution die Zwischenschaltung von Mittlern ("intermediaries“) diskutieren, welche die Leistungserstellung für den Dienstleistungsanbieter übernehmen (z.B. Franchisepartner). Bei einer derartigen Begriffsauffassung stellt sich jedoch die Frage, ob nicht vielmehr von einer horizontalen Anbieter-Partnerschaft als von einer vertikalen Anbieter-Absatzmittler-Beziehung zu sprechen ist. Vgl. Zeithaml, V.A., Bitner, M.J., Services Marketing, New York u.a. 1996, S. 335. Vgl. Meffert, H., Marketing: Grundlagen marktorientierter Unternehmensführung, a.a.O., S. 558. Es sei noch einmal auf den hohen Anteil der Reisebüros an der Gesamtzahl Flugbuchungen hingewiesen, der in den USA bei ca. 80 Prozent und in Europa zwischen 80 und 90 Prozent liegt.

Die Einspeisung des eigenen Flugdienstleistungsangebots in Computerreservierungssysteme ist mittlerweile zum Standard im Luftverkehr geworden. Vgl. Schmidt, A., 
sich beim indirekten Fremdvertrieb zudem die systembedingten Listungseffekte von Code Share-Flugdienstleistungen sowie die Möglichkeit, auf das Außendienst-Know-how der Allianzpartner in deren Heimatmärkten zurückgreifen zu können. Entscheidend ist dabei die Motivation des Außendienstes, das neben den Einzel-Flugdienstleistungen vertriebene Allianz-Angebot als gleichberechtigt oder bevorzugt im Absatzkanal durchzusetzen. ${ }^{521}$ Über den Reisebürovertrieb erhalten die Partner-Fluggesellschaften darüber hinaus Zugang zu demjenigen Teil der Firmenkunden, die ihre Flugbuchungen durch Reisebüros vornehmen lassen, so daß die Erreichung des Markenbekanntheitsziels auch mittelbar für die Kernzielgruppe der Vielflieger als vorteilhaft beurteilt werden kann. ${ }^{522}$

Die Bildung einer Präferenz bei den Vielfliegern für das Allianz-Leistungsangebot kann durch den indirekten Fremdvertrieb dagegen nur eingeschränkt erreicht werden. Der Grund hierfür liegt vor allem in der Schwierigkeit, eine relativ komplexe, erklärungsbedürftige Dienstleistung wie den Code Share-Flug über ein Massenvertriebsmedium wie z.B. Reisebüros für ein eng abgegrenztes Marktsegment zu profilieren. Die auf die oben genannten Listungseffekte zurückzuführenden Absatzzahlen von Code Share-Flugdienstleistungen dürfen dabei nicht mit dem aus einer individuellen Präferenz für das Allianz-Angebot resultierenden Absatzvolumen gleichgesetzt werden. ${ }^{523}$ Daran schließt sich zugleich eine nur bedingte Eignung des indirekten Fremdvertriebs an, das Ziel eines Markenimage-Aufbaus im Sinne des „Worldwide Recognition"-Ansatzes zu erreichen.

Computerreservierungssysteme im Luftverkehr: Darstellung, Entwicklung und wettbewerbliche Beurteilung, Hamburg 1995, S. 68.

521

Die Mitgliedsgesellschaften der Star Alliance haben beispielsweise Handbücher entwickelt, um u.a. den Außendienst über das Produkt- und Serviceangebot der Allianz zu informieren. Als schwierige Aufgabe erwies sich insbesondere die Erläuterung der Bedeutung der Luftverkehrsallianz für das eigene Unternehmen: „They [the sales staff, Anm. d. Verf.] aren't exposed. So, the goal this year is to win the hearts and minds of everyone, showing that their pay depends on Star and the effect of that extra passenger in Des Moines." Zitat des Regionalleiters für den Atlantik-Verkehr von United Airlines, Graham Atkinson, abgedruckt bei Feldman, J.M., Making Alliances Work, a.a.O., S. 33.

522 Die in der Focusgruppenbefragung erfaßten Vielflieger waren durch Filterkriterien explizit als Geschäftsreisende ausgewählt und stehen demnach in engem Zusammenhang mit dem Buying Center von Firmenkunden.

523 Zur Isolierung der angebotsbedingten und nachfragerinduzierten Effekte wäre eine Marktsimulation mit entsprechenden Parametern notwendig. Zugleich ist damit die von Gellman Research Associates (1994) durchgeführte Studie zu kritisieren, in der beide Effekte im Merkmal der Verbindungsqualităt vermischt werden und daher keine differenzierte Erklärung der empirischen Befunde zur Vorziehenswürdigkeit von Code Share-Flugdienstleistungen zulassen. 
Die für eine derartige Profilierung notwendige detaillierte Kommunikation konkurriert mit dem Angebotsportfolio weiterer Fluggesellschaften, die ihrerseits entweder einzeln oder wiederum in Allianzen um eine eigenständige Positionierung bei den Reisebüros und den Endnutzern bemüht sind. Vor dem Hintergrund eines grundsätzlich für Code Share-Flugdienstleistungen anzunehmenden Informationsnachteils scheint es aus Sicht der Allianz daher angebracht zu sein, die Informationstransparenz in diesem Absatzkanal zu erhöhen, bevor der Imageaufbau über die vielfliegerseitigen Nutzendimensionen betrieben wird. Auf diese Maßnahme ist im Rahmen der Kommunikationspolitik näher einzugehen.

Der indirekte Fremdvertrieb ist schließlich im Vergleich zu einem firmenkundenund endnutzergerichteten Direktvertrieb unterlegen, das Ziel einer differenzierten Marktbearbeitung zu erreichen, da systembedingt kein Reisebüro vom Vertrieb des Allianz-Leistungsangebots ausgeschlossen werden kann und damit die Grundlage für eine selektive oder exklusive Absatzmittlerstrategie fehlt. ${ }^{524}$ Zusammenfassend bietet der indirekte Fremdvertrieb von Allianz-Flugdienstleistungen zwar die Chance, eine weltweite Markenbekanntheit der Luftverkehrsallianz aufzubauen. Von entscheidender Bedeutung ist jedoch die Bereitschaft der Außendienstmitarbeiter der Partner-Fluggesellschaften, das Leistungsangebot der Allianz gegenüber den Reisebüros transparent zu machen und loyalitätsbezogene Denkschemata zumindest partiell zu überwinden. Die nur bedingte Eignung des indirekten Fremdvertriebs zur Erreichung von Präferenz-, Image- und Differenzierungszielen ist daher durch den Direktvertrieb von Flugscheinen an Firmenkunden und Vielflieger auszugleichen bzw. überzukompensieren.

Während der Direktvertrieb an Firmenkunden nicht geeignet ist, eine weltweit hohe Markenbekanntheit der Allianzmarke aufzubauen, ${ }^{525}$ ergeben sich Vorteile durch eine differenzierte Ansprache des Vielflieger-Segments, das aufgrund des geschäftsbedingten Reiseanlasses in den Kontext von Firmenkunden eingebunden ist. Da die Wertigkeit von Firmenkunden für die Partner-Fluggesellschaften der Allianz von hoher Bedeutung ist, bereitet die Identifizierung der relevanten

524 Bei der selektiven Distribution werden die Absatzmittler vornehmlich nach qualitativen Kriterien ausgewăhit (z.B. Geschäftslage), wăhrend der exklusive Vertrieb zusătzlich eine quantitative Einschränkung vorsieht. Vgl. Meffert, H., Marketing: Grundlagen marktorientierter Unternehmensführung, a.a.O., S. 568.

Der Grund hierfür liegt in der geringen Anzahl an Firmenkunden, gemessen an der Gesamtzahl Fluggăste in der Welt. 
Firmenkunden kaum Schwierigkeiten. Über die physische Nähe der Fluggesellschaften zu ihren Firmenkunden, die im Fall eigener Verkaufsstellen der Airlines im Hause des Kunden gegeben ist, sowie über die Nutzung bestehender Vereinbarungen wie beispielsweise Firmenförderungsmaßnahmen bestehen grundsätzlich gute Voraussetzungen für die Beeinflussung der Buying Center-Mitglieder. Die tatsächliche Umsetzung dieser Profilierungschance hängt jedoch entscheidend von der Bereitschaft der Allianzmitglieder ab, partnerübergreifende kontrahierungs- und kommunikationspolitische Maßnahmen im Sinne der angestrebten Allianz-Position zu entwickeln, auf die in den entsprechenden Kapiteln einzugehen sein wird.

Auch für den Direktvertrieb von Flugscheinen an Vielflieger lassen sich zunächst Vorteile einer differenzierten Marktbearbeitung hervorheben. Vielfliegerprogramme stellen in diesem Fall die logistische Infrastruktur dar und ermöglichen beispielsweise den Flugscheinversand per Post an den Vielflieger selbst oder die Abwicklung des Ticketkaufs über elektronische Ticketsysteme. ${ }^{526}$ Demnach ist auch für den Aufbau des Allianzmarkenimage und der Präferenz der Vielflieger zugunsten der Allianz der vergleichsweise höchste Zielerreichungsgrad bei dieser Vertriebsform zu erwarten. Entscheidend ist wiederum der partnerübergreifende, konsistente Allianzmarkenauftritt im Zusammenhang mit allen Distributionsmaßnahmen. Als eher gering ist der Beitrag des vielfliegergerichteten Direktvertriebs zur Erreichung einer weltweiten Markenbekanntheit der Allianz zu bewerten, da der Vertriebsfokus lediglich auf der aktuellen Kundengruppe der Vielflieger sowie auf den durch Mund-zu-Mund-Propaganda erreichten potentiellen Endnutzern liegt, deren Anteil jedoch im Vergleich zu den über Reisebüros potentiell erreichbaren Endnutzern als gering einzustufen ist.

526 United Airlines beispielsweise implementiert das von ihr entwickelte Electronic Ticketing System in der Star Alliance, das den physischen Versand von Flugscheinen für die ausgewählte Gruppe der Vielflieger überflüssig macht. Die Vertriebsbindung wird entsprechend über das elektronische Ticket erreicht, mit dem Reisebüros andere Absatzmittler überflüssig werden. Vgl. Feldman, J.M., Making Alliances Work, a.a.O., S. 35. 


\begin{tabular}{|c|c|c|c|}
\hline $\begin{array}{l}\text { Vertriebsform } \\
\text { markenpolitische Ziel- } \\
\text { setzung }\end{array}$ & $\begin{array}{l}\text { indirekter Fremdver- } \\
\text { trieb, insbesondere } \\
\text { Reisebüros }\end{array}$ & $\begin{array}{l}\text { Direktvertrieb an Fir- } \\
\text { menkunden }\end{array}$ & $\begin{array}{c}\text { Direktvertrieb an Viel- } \\
\text { flieger }\end{array}$ \\
\hline $\begin{array}{l}\text { weltweite Markenbe- } \\
\text { kanntheit }\end{array}$ & $\begin{array}{c}\mathbf{+ +} \\
\text { hoher Distributionsgrad }\end{array}$ & $\begin{array}{c}- \\
\begin{array}{c}\text { mengenmäßig geringe } \\
\text { Bedeutung }\end{array}\end{array}$ & $\begin{array}{c}\text { - } \\
\text { nur auf aktuelle Vielflieger } \\
\text { gerichtet }\end{array}$ \\
\hline $\begin{array}{l}\text { Erzeugung von Präfe- } \\
\text { renz }\end{array}$ & $\begin{array}{c}\text { - } \\
\text { Massenvertrieb erschwert } \\
\text { individuelle Profilierung }\end{array}$ & $\begin{array}{c}+ \\
\text { gute Voraussetzungen } \\
\text { durch bestehende Bezie- } \\
\text { hungen }\end{array}$ & $\begin{array}{c}++ \\
\text { individuelle Ansprache }\end{array}$ \\
\hline $\begin{array}{l}\text { Markenimage } \\
\text { „Worldwide Recogni- } \\
\text { tion“ }\end{array}$ & $\begin{array}{l}- \\
\text { durch Massenvertrieb nur } \\
\text { schwierig kommunizierbar }\end{array}$ & $\begin{array}{l}\text { O bis + } \\
\text { abhängig von kontrahie- } \\
\text { rungs- und kommunika- } \\
\text { tionspolitischen Maß- } \\
\text { nahmen }\end{array}$ & $\begin{array}{l}\text { + bis ++ } \\
\text { gezielter Imageaufbau } \\
\text { durch individualisierte } \\
\text { Maßnahmen möglich }\end{array}$ \\
\hline $\begin{array}{l}\text { differenzierte Markt- } \\
\text { bearbeitung }\end{array}$ & $\begin{array}{c}-- \\
\begin{array}{l}\text { Zugang zu Vielfliegern } \\
\text { kaum möglich }\end{array}\end{array}$ & $\begin{array}{l}+ \\
\text { gut möglich in Abhängig- } \\
\text { keit von Buying Center- } \\
\text { Strukturen }\end{array}$ & $\begin{array}{c}\mathbf{+ +} \\
\begin{array}{c}\text { Einzelkundenmarketing } \\
\text { möglich }\end{array}\end{array}$ \\
\hline
\end{tabular}

Legende: ++ ${ }_{n}$ sehr gut geeignet"; + ${ }_{n}$ gut geeignet"; 0 indifferent"; - ${ }_{n}$ weniger gut geeignet"; - ${ }_{n}$ nicht geeignet"

\section{Abb. 23: Geeignetheit alternativer Vertriebsformen zur Erreichung alli- anzmarkenpolitischer Zielsetzungen}

Als Nachteil des Direktvertriebs an Vielflieger sind die vergleichsweise hohen Kosten zu nennen, die durch eine individualisierte, allianzbezogene Ansprache entstehen, zumal diese Vertriebsform nicht substitutiv, sondern komplementär zum indirekten Fremdvertrieb eingesetzt wird. ${ }^{527}$ Abbildung 23 enthält eine zusammenfassende Gegenüberstellung der erörterten Vertriebsformen bezüglich ihrer Eignung zur Erreichung der markenpolitischen Ziele der strategischen Luftverkehrsallianz. ${ }^{528}$ Ein kombinierter Einsatz der Vertriebsformen unter Nutzung ihrer spezifischen Vorteile scheint demnach besonders adäquat zu sein, zur „Worldwide Recognition"-Positionierung der Allianz beizutragen. Dabei darf aber nicht der Handlungsbedarf übersehen werden, der im Aufbau einer allianzweit

527 Vgl. Meffert, H., Marketing: Grundlagen marktorientierter Unternehmensführung, a.a.O., S. 603.

528 Die Bewertung der vorgestellten Vertriebsformen ist als subjektiv geprägtes Expertenurteil zu betrachten. 
einheitlich hohen Informationstransparenz des Leistungsangebots insbesondere gegenüber Reisebüros und Firmenkunden besteht.

Mit der Kontrahierungspolitik verfügen die Allianzpartner über ein Instrument, mittels dessen die herausgearbeiteten Nachteile des indirekten Fremdvertriebs abgeschwächt werden können. Dieser Aspekt fließt in die sich anschließenden Ausführungen ein, in deren Zuge die zentralen Entscheidungstatbestände der Preisfestlegung und Konditionenbestimmung der strategischen Luftverkehrsallianz erörtert werden.

\subsection{Absatzmarktorientierte Ausrichtung der Allianz-Kontrahierungspolitik}

\subsubsection{Problemfelder einer gemeinsamen Preissetzung}

Das Kontrahierungsmix umfaßt grundsätzlich alle vertraglich festgelegten Vereinbarungen über das Entgelt der angebotenen Leistung (Preispolitik), über mögliche Rabatte sowie die mit der Zahlung und Lieferung zusammenhängenden Bedingungen (Konditionenpolitik). ${ }^{529}$ Zwischen den übrigen Instrumenten des Marketing-Mix und dem Kontrahierungsmix besteht eine enge Verbindung, da der Nutzen des Leistungsangebots mit dem Preis desselben verglichen wird und eine Kaufhandlung nur dann zustande kommt, wenn die Differenz aus Sicht des potentiellen Käufers positiv ausfällt. Der Preis kann daher auch als „Reflektor“ des Marketing-Mix bezeichnet werden. ${ }^{530}$

Die an der Allianz beteiligten Fluggesellschaften stehen zunächst vor der Aufgabe, die Preise für das von innen gemeinsam erstellte Leistungsangebot festzulegen. Der damit verbundene Entscheidungsprozeß wird von einer Reihe im folgenden zu erörternder externer - zu nennen sind vor allem rechtliche Rahmenbedingungen sowie branchen- und absatzmarktbezogene Besonderheiten - und interner Variablen beeinflußt, unter denen insbesondere die unternehmensspezifischen Gegebenheiten der einzelnen Partner-Fluggesellschaften hervorstehen.

Die Durchführung von Code Share-Flugdienstleistungen, die ein wesentlicher Bestandteil der hier betrachteten strategischen Allianz sind, könnte nach europäischem Recht die Merkmale des Art. 85 I EWGV erfüllen, nach dem Unternehmensvereinbarungen, Beschlüsse von Unternehmensvereinigungen sowie

\footnotetext{
529 Vgl. Meffert, H., Marketing: Grundlagen marktorientierter Unternehmensführung, a.a.O., S. 467.

530

Vgl. Simon, H., Preismanagement: Analyse-Strategie-Umsetzung, a.a.O., S. 4.
} 
abgestimmte Verhaltensweisen verboten sind, wenn sie eine Aufteilung der Märkte bezwecken oder bewirken und darüber hinaus geeignet sind, den Handel innerhalb der Europäischen Union zu beeinträchtigen. ${ }^{531}$ Dieser Tatbestand ist im vorliegenden Fall grundsätzlich erfült, da die Allianzpartner u.a. internationale Streckenverbindungen arbeitsteilig bedienen, deren Start- und/oder Zielorte in der Europäischen Union liegen, so daß von einer produktbezogenen Aufteilung des Marktes gesprochen werden kann. ${ }^{532}$ Art. 85 III EWGV sieht jedoch im Einzelfall bei Vorliegen entsprechender Gründe die Möglichkeit der Nichtanwendung dieses Kartellverbots vor. Gründe für die Nichtanwendung des Kartellverbots sind beispielsweise gegeben, wenn

das Code Sharing der Erschließung neuer Verbindungen oder der Aufrechterhaltung von Diensten auf verkehrsschwachen Strecken dient,

- die im Zuge der Allianz gemeinsam bedienten Streckenverbindungen kein Zugangshindernis für andere Unternehmen darstellen,

- Ersatzrouten vorhanden sind, auf welche die Fluggäste im Falle überhöhter Preise oder anderer übermäßig ungünstiger Bedingungen ausweichen kön nen. ${ }^{53}$

Auch auf seiten der US-amerikanischen Gesetzgebung werden Code ShareDienste prinzipiell als eine Form der Aufteilung des Marktes behandelt, können jedoch im Zuge einer Einzelfallprüfung unter Berücksichtigung von Verbrauchervorteilen ebenfalls vom Kartellverbot ausgenommen werden ( nity"). ${ }^{534}$ Das Vorliegen einer derartigen Ausnahmegenehmigung für die von der Allianz bedienten Strecken ist daher die notwendige rechtliche Bedingung, ohne die eine gemeinsame Preisgestaltung in der Allianz nicht möglich ist. ${ }^{535}$

531 Vgl. Reckewerth, S., Die Zusammenarbeit der Luftverkehrsgesellschaften nach europäischem und US-amerikanischen Recht, a.a.O., S. 288.

Die produktbezogene Marktaufteilung drückt sich z.B. in der Übernahme aller Teilprozesse bezogen auf eine bestimmte Streckenverbindung - bis zum Beginn des eigentlichen Fluges durch die Fluggesellschaft $A$ aus, wăhrend Fluggesellschaft $B$ den eigentlichen Flug sowie die sich anschließenden Teilprozesse übernimmt.

Vgl. Reckewerth, S., Die Zusammenarbeit der Luftverkehrsgesellschaften nach europäischem und US-amerikanischen Recht, a.a.O., S. 289.

Vgl. Reckewerth, S., Die Zusammenarbeit der Luftverkehrsgesellschaften nach europäischem und US-amerikanischen Recht, a.a.O., S. 289ff.

Gleiches gilt für sogenannte Poolabkommen im Rahmen strategischer Luftverkehrsallianzen, welche die Sammlung gemeinsam erwirtschafteter Einnahmen und ggf. auch entstandener Kosten in einem Pool vorsehen und diese gemäß der erbrachten Leistungen auf die Partner verteilen. Zur rechtlichen Behandlung vgl. Reckewerth, S., Die Zusammenarbeit der 
Unter der Annahme, daß für die hier betrachtete Luftverkehrsallianz eine Ausnahmegenehmigung vom Kartellverbot besteht, unterliegen die für das gemeinsame Leistungsangebot festzusetzenden Preise der Tarifhinterlegungspflicht bei den staatlichen Aufsichtsbehörden der beteiligten Partner. ${ }^{536}$ Unter Tarifen werden dabei bindende, nach Leistungsarten geordnete und längerfristig gültige Verzeichnisse der Preise und Beförderungsbedingungen von Fluggesellschaften verstanden. ${ }^{537}$ Diese branchenbezogene Besonderheit, von der eine flexibilitätsbeschränkende Wirkung auf die Preispolitik der Fluggesellschaften ausgeht, wird jedoch in zunehmendem Maße durch den Vertrieb sogenannter GraumarktTickets in ihrer Bedeutung eingeschränkt. ${ }^{538}$ Das Angebot von Flugscheinen zu Preisen unterhalb der genehmigten Tarife oder die Umgehung von tariflichen Anwendungsbestimmungen haben dazu geführt, daß sich der graue Markt mittlerweile fest etabliert hat und von vielen Linienfluggesellschaften für eine flexible Preispolitik genutzt wird. ${ }^{539}$

Unter Kenntnis dieser luftverkehrsspezifischen Rahmenbedingungen setzt die Allianz-Preispolitik am Segment der Vielflieger an. Von besonderer Relevanz erweist sich das den Vielfliegern eigene Merkmal des geschäftlichen Reiseanlasses, der mit einer anzunehmenden deutlich geringeren Preiselastizität der Nachfrage einhergeht als dies bei Privatreisenden der Fall ist. Der Grund hierfür liegt in der Übernahme der Flugkosten durch das arbeitgebende Unternehmen. Seitens der Fluggesellschaften steht den unterschiedlichen Preisbereitschaften

Luftverkehrsgesellschaften nach europäischem und US-amerikanischen Recht, a.a.O., S. $250 \mathrm{ff}$.

Den Staaten in der Europäischen Union wird die Interventionsmogglichkeit bei einem für ungerechtfertigt hoch oder niedrig befundenen Tarif eingeräumt. Vgl. Ehmer, H., Ein Jahr Binnenmarkt im Luftverkehr, in: Internationales Verkehrswesen, Nr. 9, 1994, S. 513.

Vgl. Pompl, W., Luftverkehr: eine ökonomische Einführung, a.a.O., S. 111. Jeder Tarif - z.B. Normaltarif, Sondertarif - unterliegt bestimmten Anwendungsbestimmungen (z.B. Gültigkeit für eine bestimmte Beförderungsklasse; Umbuchungsbedingungen; Mindestaufenthalt) und gibt die Preisforderung für den genannten Leistungsumfang sowie die Beförderungsbedingungen wieder. Durch die Veröffentlichungspflicht soll den potentiellen Nachfragern eine Kenntnisnahme ermöglicht werden. Vgl. Sterzenbach, R., Luftverkehr, a.a.O., S. 281.

$\mathrm{Vgl}$. auch die Ausführungen zu den luftverkehrsspezifischen Rahmenbedingungen in Kapitel B 1.1.2.

539 Sterzenbach (1996) nennt für das Jahr 1993 ein Marktvolumen von „mindestens drei Milliarden DM" für Graumarkt-Flugscheine in Deutschland. Vgl. Sterzenbach, R., Luftverkehr, a.a.O., S. 291. 
der Fluggäste i.d.R. eine Differenzierung des Leistungsangebots gegenüber, ${ }^{540}$ die sich am Boden beispielsweise in bevorzugten Check-in-Prozeduren für Statuskunden ausdrückt und an Bord von Flugzeugen in der Unterteilung der Kabine in verschiedene Beförderungsklassen ihren Niederschlag findet. Das beobachtbare Merkmal der Beförderungsklassenwahl floß daher im vorliegenden Fall als Auswahlkriterium in die Focusgruppenbefragung ein, indem mindestens zwei Drittel der Focusgruppenmitglieder überwiegend in der Business oder First Class geflogen sein mußten. Eine daher anzunehmende verhältnismäßig niedrige Preiselastizität der Vielflieger, die mit dem „Worldwide Recognition“-Ansatz der strategischen Allianz erreicht werden sollen, spricht demnach grundsätzlich für die Verfolgung einer Hochpreisstrategie, die mit der markenpolitischen Zielsetzung eines durch hohe Sicherheit, Komfort, Angebotsumfang und -dichte, reibungslose Abläufe sowie weltweite Statusleistungen geprägten Allianzmarkenimage zudem überaus konsistent ist.

Für eine gemeinsame Allianz-Preispolitik ist jedoch nicht allein die Preiselastizität der Nachfrage von Bedeutung. Fluggesellschaften streben vielmehr eine Abstimmung von Preisen und Kapazitäten an, da sie zum einen hohe Fixkostenanteile aufweisen und - bedingt durch die nur diskretionär variierbare Flugzeuggröße - über unflexible Kapazitäten disponieren. Zum anderen verfallen nicht in Anspruch genommene Sitzplätze und tragen nicht mehr zur Deckung der Fixkosten bei. Zudem werden Flugdienstleistungen im voraus gebucht und bergen damit das Risiko eines Nichterscheinens ("no show“) des potentiellen Nutzers. Darüber hinaus sind im Luftverkehr zeitliche Nachfrageschwankungen zu beobachten, die sowohl tageszeitlich, wochenzeitlich als auch saisonal auftreten können. Um diese vielfältigen Bestimmungsfaktoren im Rahmen ihrer Preispolitik zu berücksichtigen, haben viele Fluggesellschaften sogenannte Yield Management-Systeme entwickelt, ${ }^{541}$ die im Kern eine gewinnmaximierende Preis-Kapazitäts-Steuerung beinhalten. ${ }^{542}$ Im Sinne der Allianz-Positionierung gilt es nun zu

540 Vgl. Nagle, T.T., Holden, R.K., Larsen, G., Pricing - Praxis der optimalen Preisfindung, Berlin u.a. 1998, S. 200.

541 Mitunter wird im Luftverkehr auch von "Revenue Management"-Systemen gesprochen. Vgl. z.B. Feldman, J.M., Getting serious on pricing, in: Air Transport World, No. 10, 1994, S. 56ff.; Curry, R.E., Revenue management using consumer choice models, in: Aeronomics Incorporated, First Quarter, 1994, S. $1 \mathrm{ff}$.

Vgl. Daudel, S., Vialle, G., Yield-Management: Ertrăge optimieren durch nachfrageorientierte Angebotssteuerung, Frankfurt/Main, New York 1992, S. 35. Gelegentlich wird auch von umsatzmaximierender Preis-Mengen-Steuerung gesprochen, da sich unter der Annahme sehr niedriger Grenzkosten - z.B. die Kosten eines zusătzlichen Passagiers in einem nicht 
vermeiden, daß partnerindividuelle, durch eigene Yield Management-Systeme ermittelte Preise für Allianz-Flugdienstleistungen verlangt werden, da auf diese Weise das Ziel eines gemeinsam aufzubauenden, weltweit einheitlichen AllianzMarkenimage konterkariert würde. ${ }^{543}$ Daraus ergibt sich die Forderung nach einer Integration der Yield Management-Systeme der Partner, um für die Zielgruppe der Vielflieger eine zur Allianz-Positionierung konsistente, den Kapazitätseinsatz optimierende Preisbildung zu erreichen.

Abbildung 24 gibt exemplarisch den Aufbau eines Yield Management-Systems wieder, das bei Fluggesellschaften zum Einsatz kommt. Die Erläuterung der Funktionsweise aus der Angebotsperspektive ist notwendig, um die Schwierigkeiten erklären zu können, die sich aus dem Postulat eines aus Sicht der Endnutzer konsistenten Preissystems ergeben. Das Yield Management-System wird von fünf Informationsquellen mit Daten versorgt. ${ }^{544}$ Externe Informationsquellen geben in Form von Veranstaltungsankündigungen (z.B. Messen, Konferenzen) Hinweise auf zu erwartende Nachfrage nach Flugdienstleistungen. Interne Informationsquellen enthalten u.a. die Vertragsinhalte, die zwischen der Fluggesellschaft und ihren Firmenkunden sowie Reisebüros bestehen. Über eine Buchungsdatenbank kann die Airline auf Vergangenheitsdaten zurückgreifen, während das Computerreservierungssystem sowie die an den Flughäfen geführten Check-in-Systeme Gegenwartsdaten der Nutzung von Flugdienstleistungen zur Verfügung stellen. Aus einer Kombination dieser Daten wird mittels mathematischer Algorithmen eine Prognose über den $\mathrm{zu}$ erwartenden zeitlichen und mengenmäßigen Buchungsverlauf, die Nachfragestruktur (z.B. Verhältnis Geschäfts- zu Privatreisenden) sowie die zu erwartende Stornierungsrate erstellt.

vollbesetzten Flugzeug - der umsatz- und der gewinnmaximale Preis annăhern. Vgl. Simon, $H_{\text {., }}$ Preismanagement: Analyse-Strategie-Umsetzung, a.a.O., S. 568.

543 Der Preis wäre dann nicht mehr der Reflektor einer in sich konsistenten Leistung, sondern ließe vielmehr Rückschlüsse auf möglicherweise unterschiedliche Leistungsniveaus zwischen den Partnern zu. Eine solche Wirkung stünde dem Positionierungsansatz „Worldwide Recognition" diametral gegenüber. Auch Sander (1997) betont die Bedeutung der von ihm als "instrumentelle Kompatibilităt" bezeichneten Abstimmung des Preisniveaus mit den übrigen Marketing-Instrumenten. Vgl. Sander, M., Internationales Preismanagement: eine Analyse preispolitischer Handlungsalternativen im internationalen Marketing unter besonderer Berücksichtigung der Preisfindung, Heidelberg 1997, S. 60.

544 Die folgenden Ausführungen beziehen sich auf Enzweiler, T., Wo die Preise laufen lernen, in: Manager Magazin, Nr. 3, 1990, S. 247. 


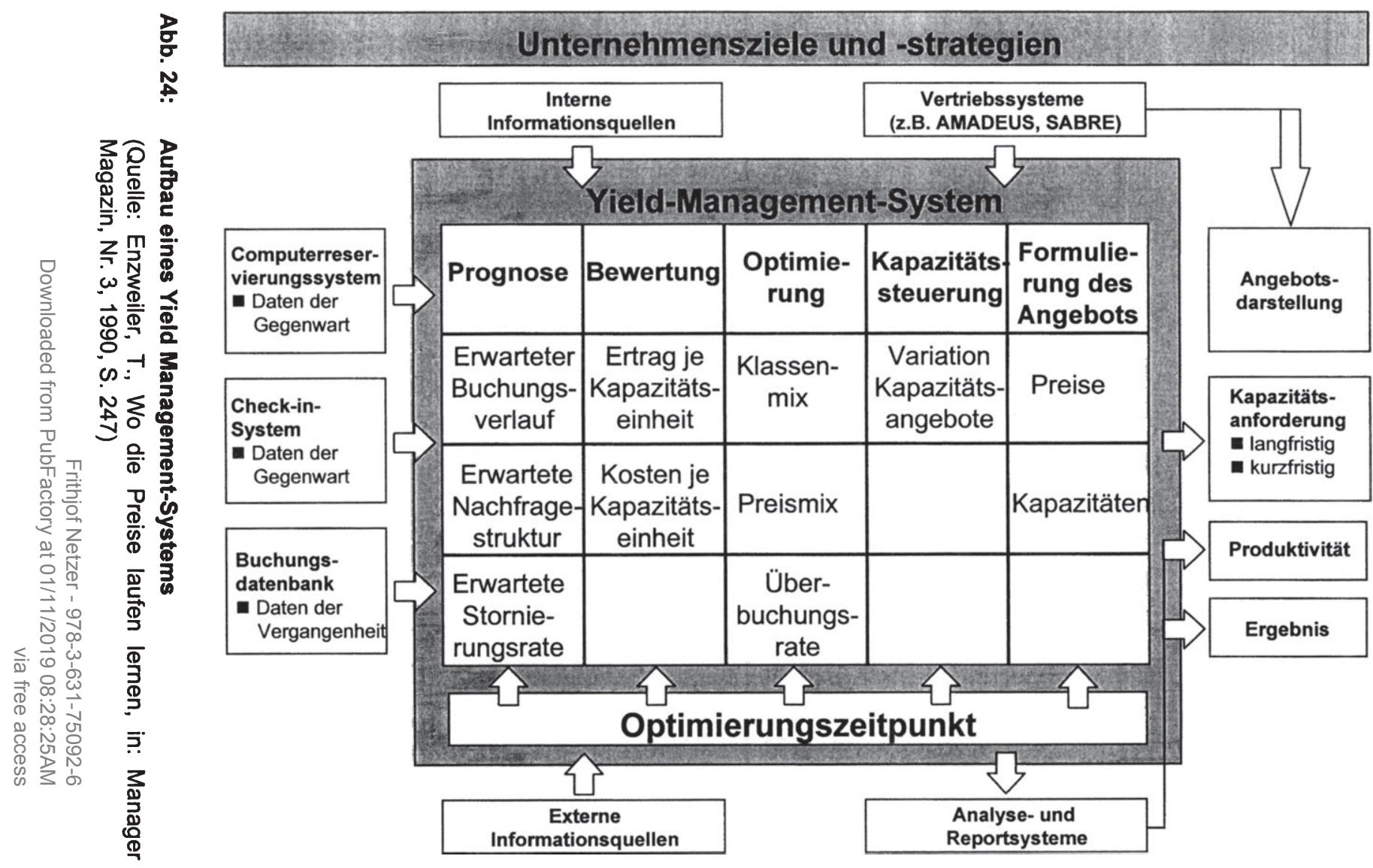


Daraufhin wird die Prognose mit Preisen sowie mit den variablen Kosten bewertet. Hierauf baut die Optimierung auf, deren Ergebnis die Festlegung bestimmter Buchungsklassen ist, denen spezifische Preise zugeordnet sind. Die Überbuchungsrate berücksichtigt das mögliche Nichterscheinen von Passagieren, die ein Flugticket gebucht haben. Ausgehend von dieser Optimierung kann die Airline ihr Kapazitätsangebot durch Variation des eingesetzten Fluggeräts steuern. Das solchermaßen formulierte Angebot wird mit den entsprechenden Preisen und Kapazitäten in den Computerreservierungssystemen dargestellt und kann z.B. durch Reisebüros abgerufen und verkauft werden. Interne Analyse- und Reportsysteme ermöglichen schließlich die Berechnung von Kennziffern wie beispielsweise Durchschnittserlös pro Passagier-Kilometer.

Für die Ausgestaltung einer konsistenten Allianz-Preispolitik ergibt sich an dieser Stelle die Frage, inwieweit die von den Partnern eingesetzten Yield ManagementSysteme kompatibel sind und einer Integration zugeführt werden können. Dies gilt zunächst für die Vergleichbarkeit der erfaßten Daten, die vor dem Hintergrund der jeweiligen Unternehmenssituation zu interpretieren sind. Der unternehmensspezifische geographische Heimatmarkt jeder Partner-Airline, ${ }^{545}$ ihre Positionierung im Heimatmarkt, ihre Flottenstruktur sowie ihre Kostensituation ${ }^{546}$ beeinflussen die Qualität der Eingangsdaten in entscheidendem Maße. Daher ist von einer nur bedingten Vergleichbarkeit der Daten auszugehen. Neben dieser inhaltlichqualtitativen Dimension erweisen sich technische Inkompatibilitäten einer Integration der Yield Management-Systeme als hinderlich, die i.d.R. isoliert entwickelt wurden und daher über keine gemeinsame informationstechnologische Plattform verfügen. ${ }^{547}$

545 Unterschiede zwischen den Heimatmärkten der Partner-Fluggesellschaften lassen sich auf makro- und mikrő̈konomische Faktoren zurückführen. Zu makroökonomischen Faktoren zăhlen beispielsweise das Marktvolumen, das Rechtssystem und die kulturelle Entwicklung, während unter den mikrőkonomischen Faktoren vor allem der Wettbewerb (z.B. Wettbewerbsintensität zwischen den etablierten Fluggesellschaften), die Distribution und die Konsumenten (z.B. Kaufverhalten) subsumiert werden. Vgl. Meffert, H., Bolz, J., Internationales Marketing-Management, a.a.O., S. 42ff. Zu einer Diskussion der Bedeutung derartiger externer Determinanten für die Preispolitik vgl. Sander, M., Internationales Preismanagement: eine Analyse preispolitischer Handlungsalternativen im internationalen Marketing unter besonderer Berücksichtigung der Preisfindung, Heidelberg 1997, S. $29 \mathrm{ff}$.

Das Stückkostenniveau zwischen den Fluggesellschaften variiert z.T. sehr deutlich. Gemessen am Stückkostenniveau von American Airlines im Jahre 1990 (Index $=100$ ) belief sich der Indexwert von USAir 1995 auf 132, derjenige von American Airlines selbst auf 95, der Wert von SAS betrug 192 und derjenige von Singapore Airlines 65. Vgl. Oum, T.H., Winning Airlines: productivity and cost competitiveness of the world's major airlines, Norwell 1998, S. $140 \mathrm{ff}$.

Vgl. Walker, K., Where's the glue? in: Airline Business, No. 7, 1998, S. 31. 
Einer Integration der Yield Management-Systeme im Rahmen der Allianz stehen schließlich vor allem die Eigeninteressen der beteiligten Partner-Fluggesellschaften entgegen. Da sich durch den Einsatz dieser komplexen Preis-KapazitätsSteuerung bedeutende ökonomische Ergebniseffekte realisieren lassen, ${ }^{548}$ sind die Partner einer auf rechtlicher Selbständigkeit und wirtschaftlich weitgehender Selbständigkeit fußenden strategischen Allianz nur in sehr begrenztem Maße oder aber überhaupt nicht bereit, ihre informationstechnologische Leistungskompetenz auf diesem äußerst sensiblen Gebiet mit anderen Airlines zu teilen. ${ }^{549}$ Eigene Austrittsüberlegungen oder die Furcht vor dem Austritt eines Partners, der von der IT-Kompetenz eines anderen Partners profitiert hat und nun zum Konkurrenten wird, begründen ebenfalls die auf Wahrung eigener Yield Management-Systeme bedachte Haltung innerhalb von Allianzen. ${ }^{550}$

Für die Ausgestaltung der gemeinsamen Preispolitik läßt sich zusammenfassend folgern, daß eine allianzweit konsistente Preisfestlegung im Sinne des „Worldwide Recognition“-Ansatzes zu empfehlen wäre. Da die Partner-Fluggesellschaften jedoch über Yield Management-Systeme verfügen, die auf die unternehmensspezifischen Gegebenheiten abgestimmt sind und zudem eine hohe individuelle, erfolgskritische Bedeutung und damit Sensitivität besitzen, kann die Allianz-Preispolitik lediglich eine Kompromißlösung im Sinne der Vernetzung von Einzeloptimierungen darstellen. ${ }^{551}$ Damit ergibt sich im Hinblick auf die Positionierung der Allianz eine Inkonsistenz zwischen der Preispolitik und den übrigen Mixbereichen, so daß keine langfristige preisliche Abgrenzung des Allianz-Angebots gegenüber den Einzelangeboten der beteiligten Partner-Fluggesellschaften erreicht wird. ${ }^{52}$ Gleiches gilt für die preisliche Positionierung gegenüber den Wettbewerbern. Zudem ergibt sich die Frage, inwieweit der für eine Allianz-Flug-

548 Schätzungen zufolge realisiert American Airlines durch den Einsatz seines Yield ManagementSystems einen jährlichen Zusatzumsatz in Höhe von 500 Millionen US-Dollar. Vgl. Simon, H., Dolan, R.J., Profit durch Power Pricing: Strategien aktiver Preispolitik, Frankfurt/Main, New York 1997, S. 273.

Vgl. Walker, K., Where's the glue? in: Airline Business, No. 7, 1998, S. 30. Vgl. Feldman, J.M., Making Alliances Work, a.a.O., S. 33.

551 Es finden folglich diejenigen Preise Anwendung, die durch das Yield Management-System des jeweiligen operating carrier ermittelt werden. nur für bestimmte Ziele im Flugplan der Allianz Gültigkeit besitzen. Für die hier im Mittelpunkt stehende Gruppe der Vielflieger sind derartige Angebote i.d.R. von untergeordneter Bedeutung. 
dienstleistung zu entrichtende Preis der Anforderung einer wahrgenommenen „Preisehrlichkeit" im Sinne eines ausgewogenen Preis-/Leistungsverhältnisses genügt. ${ }^{553}$ Informations- und Abstimmungsmängel könnten in Verbindung mit gleichbleibenden oder sogar erhöhten Preisniveaus zu einer aus Endnutzersicht merklich verringerten Preisgerechtigkeit des Allianz- gegenüber dem Einzelangebot führen.

In engem Zusammenhang mit der Preispolitik ist die Festlegung der Konditionen zu sehen, die für Absatzmittler, Firmenkunden und Fluggäste Gültigkeit besitzen. Die Abstimmung dieses Instruments im Rahmen der strategischen Luftverkehrsallianz ist Gegenstand des folgenden Kapitels.

553 Vgl. Diller, H., Preisehrlichkeit - Eine neue Zielgröße im Preismanagement des Einzelhandels, in: Thexis, 14. Jg., Nr. 2, 1997, S. 17. 


\subsubsection{Abstimmung der Konditionenpolitik in der Luftverkehrsallianz}

Der Anspruch einer weltweiten Wiedererkennung ist auf der Ebene der Konditionengestaltung nur konsequent umzusetzen, wenn die beteiligten Partner-Fluggesellschaften unter der Prämisse kartellrechtlicher Genehmigung einheitliche, für die Allianz gültige Rahmenbedingungen im Umgang mit Absatzmittlern, Firmenkunden und Fluggästen definieren.

Mit der Gewährung von Rabatten steht Fluggesellschaften ein Mittel zur Verfügung, das geeignet ist, das Empfehlungs- und Verkaufsverhalten von Absatzmittlern sowie das Auswahlverhalten von Firmenkunden zu ihren Gunsten zu beeinflussen. ${ }^{554} \mathrm{Um}$ eine auf das Allianz-Leistungsangebot fokussierte Verhaltensbeeinflussung zu erreichen, ist die Einführung zusätzlicher Rabatte auf Code Share-Flugdienstleistungen notwendig. Dabei stellt sich die Frage, inwieweit die an der Allianz beteiligten Partner-Fluggesellschaften bereit sind, zusätzliche Rabattzahlungen zu leisten, die sich unmittelbar erlösschmälernd auswirken. Zudem sind die individuell zu erwartenden Verlagerungen von bisher einzeln mit den Partner-Fluggesellschaften realisierten Umsatzvolumina in Richtung auf Allianz-Umsatzvolumina abzuschätzen. ${ }^{555}$

Nicht zuletzt unter Berücksichtigung von Eigeninteressen hat die Deutsche Lufthansa beispielsweise das Allianz-Plus-Rabattmodell für Firmenkunden neben ihrer eigenen volumenbasierten Förderung eingeführt. Eine Verlagerung zum Allianz-Leistungsangebot müssen sich die Firmenkunden durch entgangene Rabatte bei der Lufthansa „erkaufen“, so daß insbesondere bei den im Heimatmarkt der Lufthansa ansässigen Firmenkunden keine nennenswerten Verlagerungen zu erwarten sind..$^{556}$

Für Absatzmittler, Firmenkunden und Fluggäste ist darüber hinaus eine möglichst allianzeinheitliche Gestaltung der in den Tarifen festgelegten Buchungsbedin-

554 Damit kann zugleich die Schwăche des indirekten Fremdvertriebs bei der Erreichung einer Präferenzbildung bei den Vielfliegern abgemildert werden.

Der Umfang dieses Verlagerungseffektes bemißt sich wesentlich durch die Kreuzpreiselastizităt, die zwischen den einzeln angebotenen Leistungen und dem AllianzLeistungsangebot besteht und Rückschlüsse auf die reisebüroseitig empfundene Substituierbarkeit beider Leistungen zuläßt. Zur Definition vgl. Simon, H., Preismanagement: Analyse-Strategie-Umsetzung, a.a.O., S. 93.

Vgl. Kapitel B 2.1.2. 
gungen anzustreben. Dasselbe gilt für Graumarkt-Tickets, die von der Allianz in Umlauf gebracht werden. Das Fehlen allianzweit einheitlich definierter Konditionen könnte beispielsweise dazu führen, daß für einen Vielflieger unvorherzusehende Umbuchungs- oder Stornogebühren anfallen und damit die angestrebte Positionierung einer u.a. durch flexible Buchungsoptionen und nahtloses Reisen gekennzeichneten Allianz konterkariert würde.

Als weitere Maßnahme im Rahmen der strategischen Luftverkehrsallianz ist die konditionenmäßige Harmonisierung der eingesetzten Vielfliegerprogramme zu nennen, auf deren Problemfelder bereits unter dem Produkt- und Servicemanagement der Allianz hingewiesen wurde. Die im Zuge von Vielfliegerprogrammen ausgegebenen Kundenkarten stellen strenggenommen eine Schnittmenge zwischen dem kontrahierungs- und kommunikationspolitischen Instrumentarium dar, da sie neben ihrer preislichen Anreizwirkung zugleich als Medium der Direktkommunikation von Fluggesellschaften fungieren. Ihre kommunikationsbezogenen Einsatzmöglichkeiten im Rahmen von Luftverkehrsallianzen fließen in die sich anschließenden Überlegungen zur allianzseitigen Kommunikationspolitik ein.

\subsection{Ausgestaltung der Allianz-Kommunikationspolitik}

\subsubsection{Festlegung einer Kommunikationsstrategie für die Luftverkehrsallianz}

Unter dem Begriff der Kommunikationspolitik werden grundsätzlich alle Entscheidungen subsumiert, die auf die Gestaltung der Kommunikation von Unternehmen gerichtet sind. ${ }^{557}$ Über die Kommunikation, die als Übermittlung von Informationen und Bedeutungsinhalten definiert werden kann, versuchen Unternehmen, die Meinungen, Einstellungen, Erwartungen und Verhaltensweisen bestimmter Adressaten so zu steuern, daß die unternehmensspezifischen Zielsetzungen in möglichst hohem Maße erreicht werden. ${ }^{558}$

Die gemeinsame Gestaltung von Kommunikationsaktivitäten durch mehrere Unternehmen, die dabei ihre Eigenständigkeit wahren, komplementäre Zielsetzungen verfolgen und eigene Beiträge einbringen, wird in der Literatur unter dem

557 Vgl. Diller, H., Kommunikationspolitik, in: Vahlens Großes Marketinglexikon, Hrsg.: Diller, H., München 1992, S. 546.

558 Vgl. Meffert, H., Marketing: Grundlagen der Absatzpolitik, 7., überarb. u. erw. Aufl., Wiesbaden 1986, S. 443. 
Begriff der kooperativen Marketing-Kommunikation diskutiert. ${ }^{559}$ Die im folgenden zu erörternde Allianz-Kommunikationspolitik ist der Unterform der Gruppenkommunikation zuzurechnen, da sie durch mehrere einzeln erkennbare Anbieter identischer, i.d.R. substitutiver Leistungen gestaltet wird. ${ }^{560}$

Den Ausgangspunkt einer entscheidungsorientierten Allianz-Kommunikationspolitik ${ }^{561}$ bilden die auf den Focusgruppenergebnissen aufbauenden markenpolitischen Zielsetzungen der hier betrachteten Luftverkehrsallianz. ${ }^{562}$ Die aus innen abzuleitenden kommunikativen Zielsetzungen sind nach Inhalt (z.B. „Erhöhung des ungestützten Bekanntheitsgrades der Allianz"), Ausmaß (z.B. „von derzeit 20 Prozent auf 30 Prozent"), Zeitbezug (z.B. „innerhalb der nächsten 12 Monate") und Segmentbezug (z.B. "bei allen Vielfliegern weltweit") zu operationalisieren, ${ }^{563}$ um die Basis für eine spätere Soll-Ist-Analyse zu schaffen. Für die Operationalisierung des Allianzziels, eine Präferenz bei den Vielfliegern zu erzeugen, bieten

$559 \mathrm{Vgl}$. Lindemann, M., Kooperative Marketing-Kommunikation: kommunikationspolitische Zusammenarbeit von Konsumgüter-Anbietern, Bergisch Gladbach, Köln 1993, S. 18; Berndt, R., Kooperative Werbung - Organisation, Planung und Vorteilhaftigkeit kooperativer Werbemaßnahmen, in: WiSt, Nr. 1, 1985, S. 1.

Vgl. Lindemann, M., Kooperative Marketing-Kommunikation: kommunikationspolitische Zusammenarbeit von Konsumgüter-Anbietern, a.a.O., S. 54. Unter dem etwas irreführenden Begriff „identisch“ versteht Lindemann Produkte einer Warengattung. Vgl. ebenda, S. 36. Bei Purtschert (1988) findet sich die Zugehörigkeit zur selben Branche als Abgrenzungskriterium zu anderen Formen der kooperativen Marketing-Kommunikation wie beispielsweise der Gemeinschaftskommunikation. Vgl. Purtschert, R., Möglichkeiten und Grenzen der Gemeinschaftswerbung, in: ZfB, Nr. 4, 1988, S. 522.

Die hier betrachtete Luftverkehrsallianz erfült das Merkmal der einzelnen Erkennbarkeit, da die Firmenmarken der Partner neben der gemeinsamen Allianzmarkierung stehen. Zudem kann das Merkmal der Substituierbarkeit der angebotenen Flugdienstleistungen als weitestgehend gegeben angesehen werden, da die praktizierte Arbeitsteilung der Partner-Fluggesellschaften ansonsten auf massiven Widerstand der Passagiere stoßen würde. Von einer vollständigen Substituierbarkeit kann bei bestehenden Leistungsdifferenzen indessen nicht gesprochen werden, so daß die obige Definition von Lindemann zu relativieren ist.

Zum entscheidungsorientierten Ansatz der Kommunikationspolitik vgl. Meffert, H., Marketing: Grundlagen marktorientierter Unternehmensführung, a.a.O., S. 668.

Die Ausrichtung der gemeinsamen Kommunikationsaktivităten an diesen Zielsetzungen trăgt zugleich zur Erreichung eines möglichst konsistenten Erscheinungsbildes der Allianz bei. Auf diese Weise wird der Forderung nach einer integrierten Marktkommunikation begegnet. Vgl. Bruhn, M., Kommunikationspolitik, a.a.O., S. 96.

Zur Operationalisierung von Kommunikationszielen vgl. Pepels, W., KommunikationsManagement: Marketing-Kommunikation vom Briefing bis zur Realisation, 2. Aufl., Stuttgart 1996, S. $110 \mathrm{ff}$. 
sich beispielsweise Wiederkaufraten an, während das Markenimage-Ziel durch Methoden der Einstellungsmessung operationalisiert werden kann. ${ }^{564}$

Die Kommunikationsziele bestimmen die Ausrichtung der Kommunikationsstrategie, die Meffert (1998) in fünf Strategiedimensionen unterteilt: ${ }^{565}$

Objektdimension,

- Zielungsdimension,

- Instrumentedimension,

- Mediadimension und

- Gestaltungs- und Botschaftsdimension.

Bezüglich der Objektdimension können die Allianzpartner den Schwerpunkt auf eine eher leistungsbezogene oder eine eher unternehmensbezogene Kommunikation legen. Da die Allianzmarke im Positionierungsansatz ausschließlich über Nutzendimensionen definiert wurde, kann kommunikativ von einer Leistungsmarke gesprochen werden, hinter welcher verschiedene Fluggesellschaften mit ihren eigenen Firmenmarken stehen. Eine unternehmensbezogene Kommunikation ist für die Allianz demgegenüber mit der kaum lösbaren Aufgabe verbunden, in einer identitătslosen, neu geschaffenen Allianzmarke die Identitäten der Partner-Fluggesellschaften zu einer homogenen Einzelidentität zu verschmelzen. Aus diesem Grund ist eine leistungsbezogene Kommunikation für die Luftverkehrsallianz vorzuziehen.

Die anschließend festzulegende Zielungsdimension kann in vier Unterdimensionen gegliedert werden. Über die personale Zielung legen die Allianzpartner fest, ob eine eher einzel- oder massengerichtete Kommunikation erfolgt. Da die Gruppe der Vielflieger einen mengenmäßig relativ kleinen Anteil an den Flugreisenden repräsentiert, ist in diesem Fall die individualisierte einer massengerichteten Ansprache vorzuziehen. Die zeitliche Zielung gibt daraufhin an, ob die Kommunikationsaktivitäten der Allianz punktuell pulsierend oder eher kontinuier-

564 Anwendung bei der Messung von Images findet z.B. das klassische semantische Differential, über das Assoziationen zu Objekten mittels gegensătzlicher Eigenschaftswörter auf RatingSkalen gemessen werden. Vgl. Kroeber-Riel, W., Weinberg, P., Konsumentenverhalten, a.a.O., S. 197.

565 Vgl. Meffert, H., Marketing: Grundlagen marktorientierter Unternehmensführung, a.a.O., S. $689 f$. 
lich eingesetzt werden sollen. ${ }^{566}$ Diesbezüglich lassen sich aus dem Lebenszyklus-Konzept erste Gestaltungshinweise für die Marktkommunikation der Allianzmarke ableiten ${ }^{567}$ Wenn sich die hier betrachtete Allianzmarke im Anfangsstadium der Markteinführung befindet, scheint eine zunächst pulsierende Einführungskommunikation sinnvoll zu sein, die beim Übergang in eine andere Lebenszyklusphase durch eine eher kontinuierliche Kommunikation abgelöst wird. ${ }^{568}$ Maßgeblich für eine solche Entscheidung ist jedoch die Kenntnis der Kommunikationselastizität der Nachfrage, die länderspezifisch variieren kann. ${ }^{569}$ Eine generell gültige Empfehlung kann daher an dieser Stelle nicht gegeben werden. Hinsichtlich der sich anschließenden räumlichen Zielung bedingt der "Worldwide Recognition“-Ansatz eine Ausrichtung der Kommunikationsaktivitäten auf den Weltmarkt.

Als dominante Zielgruppen unterscheidet Meffert schließlich strenggenommen Marktstufen, wenn zwischen einer überwiegend endnutzergerichteten („Pull-Strategie") und einer überwiegend absatzmittlergerichteten Kommunikation („PushStrategie") zu wählen ist. ${ }^{570} \mathrm{Im}$ vorliegenden Fall sind zudem Firmenkunden in der

566 Von pulsierenden Kommunikationsaktivităten wird gesprochen, wenn das Kommunikationsbudget periodenweise gleichförmig herauf- und herabgesetzt wird, wăhrend kontinuierliche Aktivităten eine gleichmäßige Verteilung des Budgets auf die Perioden vorsehen. Vgl. Schmalen, H., Kommunikationspolitik: Werbeplanung, 2., überarb. und erw. Aufl., Stuttgart, Berlin, Köln 1992, S. 83.

567 Das u.a. in der strategischen Marketingplanung diskutierte Lebenszykluskonzept strebt eine Typologisierung strategisch relevanter Situationen an, aus denen Handlungsempfehlungen für das Marketing z.B. von Produkten, Marken oder Unternehmen abgeleitet werden sollen. Der Aussagewert dieses Konzeptes in nicht unumstritten, da die zugrunde liegenden Annahmen u.a. von einer kontinuierlichen zeitlichen Entwicklung und einem situativen Determinismus ausgehen. Vgl. Meffert, H., Marketing-Management: Analyse, Strategie, Implementierung, a.a.O., S. 46.

Schürmann (1993) konnte in einer 1990/1991 durchgeführten Untersuchung von 350 Markenartikeln signifikant positive Umsatz- und Marktanteilswirkungen der absoluten Werbebudgethöhe und des Werbedrucks in der Einführungsphase nachweisen, die in den darauffolgenden Phasen abnahmen bzw. keine signifikante Zusammenhänge mehr erkennen ließen. In der Reifephase wurde ein signifikant positiver Zusammenhang zwischen kontinuierlicher Werbung und dem Umsatzwachstum identifiziert. Vgl. Schürmann, U., Erfolgsfaktoren der Werbung im Produktlebenszyklus: ein Beitrag zur Werbewirkungsforschung, Frankfurt am Main u.a. 1993, S. $156 f$.

Die Kommunikationselastizităt gibt an, um wieviele Einheiten sich die die abhăngige Variable z.B. der Absatz von Flugscheinen - ăndert, wenn das Kommunikationsbudget um eine Einheit variiert wird. Vgl. Bruhn, M., Kommunikationspolitik, a.a.O., S. 282. Zum Einfluß des Länderkontextes vgl. z.B. Müller, S., Kornmeier, M., Grenzen der Standardisierung im Internationalen Marketing, in: Jahrbuch der Absatz- und Verbrauchsforschung, Nr. 1, 1996, S. 18.

Vgl. Meffert, H., Marketing: Grundlagen marktorientierter Unternehmensführung, a.a.O., S. 690. 
Marktkommunikation zu berücksichtigen. Die Entscheidung für eine Schwerpunktsetzung zugunsten einer bestimmten Marktstufe kann nur anhand geeigneter Kriterien vollzogen werden, die auf die spezifischen Kontextfaktoren der Allianzpartner abzustimmen sind. Unter der Annahme, daß über die Mitgliedschaft in Bonusprogrammen der Partner-Fluggesellschaften oder der Allianz selbst eine gute Erreichbarkeit der Vielflieger sichergestellt ist, können mit einer endnutzergerichteten, personalisierten Zielung wesentlich differenziertere Botschaftsinhalte transportiert werden als über eine absatzmittlergerichtete Kommunikation..$^{571}$

Durch einen Akzent auf der vielfliegergerichteten Direktkommunikation ließe sich z.B. die Transparenz des komplexen Allianz-Leistungsangebots erhöhen, so daß Überraschungseffekte auf seiten der entsprechend informierten Fluggäste reduziert oder vermieden werden können. ${ }^{572}$ Diese Chance erscheint gerade vor dem Hintergrund der erörterten Informationsnachteile von Allianz-Leistungsangeboten für die Dominanz der personalisierten Zielung zu sprechen. Parallel ist eine direkte Ansprache der Firmenkunden anzustreben, die sowohl zur Intensivierung bestehender als auch zum Aufbau neuer Geschäftsbeziehungen genutzt werden kann. Gleiches gilt für die reisebürogerichtete „Push-Kommunikation“, über die auch der Nachteil einer relativ geringen Gesamtmarktdurchdringung der vorgenannten Kommunikationsstufen kompensiert werden kann.

Aufbauend auf diesen Überlegungen haben die Allianzpartner daraufhin eine Auswahl der einzusetzenden Kommunikationsinstrumente vorzunehmen. ${ }^{573} \mathrm{Um}$ der in der Literatur erhobenen Forderung nach einem integrativen Instrumenteein-

571 Vgl. Wiencke, W., Cards\&Clubs als Dialogmarketing-Instrument, in: Handbuch Directmarketing, Hrsg.: Dallmer, H., 7. Aufl., Wiesbaden 1997, S. 335.

572 Grenzen sind einer allianzweiten Direktkommunikation durch rechtliche Rahmenbedingungen in den Heimatländern der Allianzpartner sowie durch länderspezifisch variierende Reaktionsmuster der Vielflieger auf die Direktansprache gesetzt. Vgl. Miller, R.N., Multinational Direct Marketing - On the Brink of the New Millenium, in: Handbuch Directmarketing, Hrsg.: Dallmer, H., 7. Aufl., Wiesbaden 1997, S. 71.

573 Unter einem Kommunikationsinstrument werden mehrere ähnliche Kommunikationsmaßnahmen zu einem "Bündel" zusammengefaßt. Vgl. Bruhn, M., Kommunikationspolitik, a.a.O., S. 3. Ein personalisiertes Anschreiben an einen Vielflieger, in dem dieser über Leistungsvorteile der Allianz informiert wird (=Kommunikationsmaßnahme), ist beispielsweise dem Instrument der Direktkommunikation zuzurechnen. Dabei werden zugleich die Interdependenzen zwischen den im Rahmen der Zielung getroffenen Entscheidungen und der Instrumenteauswahl deutlich. 
satz Rechnung zu tragen, ${ }^{574}$ gilt es, eine Kombination ausgewählter Kommunikationsinstrumente auf die Erreichung der Allianzziele auszurichten. In der Phase der Allianzmarkeneinführung kann die klassische Werbung ${ }^{575}$ beispielsweise als Leitinstrument fungieren und über entsprechenden Mediaeinsatz zum Aufbau und zur Erhöhung der Allianzmarkenbekanntheit beitragen, da in Abhängigkeit von den eingesetzten Medien hohe Reichweiten und Kontaktzahlen erzielbar sind. ${ }^{576}$ In gleicher Weise bietet es sich an, klassische Werbung für die Erreichung des Markenimage-Ziels der Allianz zu verwenden. ${ }^{577}$

Ein ergänzender Einsatz der Direktkommunikation erscheint sinnvoll, um eine Interaktion zwischen der Allianz und den Adressaten der Kommunikation herzustellen, die mittels klassischer Werbung nicht möglich ist. In diesem Zusammenhang ist auch die Nutzung von Multimedia-Anwendungen in der Direktkommunikation naheliegend, ${ }^{578}$ da gerade im Luftverkehr auf eine gut entwickelte informationstechnologische Infrastruktur zurückgegriffen werden kann. Eine neuere Untersuchung belegt, daß 109 der insgesamt 255 IATA-Mitgliedsfluggesellschaften im Internet vertreten sind. Zu den Inhalten, die von Interessenten im Wege

574 Bruhn (1993) unterscheidet zwischen der inhaltlichen, formalen und zeitlichen Integration von Kommunikationsaktivităten. Das Bestreben, die verschiedenen Kommunikationsinstrumente aufeinander abzustimmen, fällt in den Bereich der inhaltlichen Integration und wird weitergehend als "instrumentelle Integration“ bezeichnet. Vgl. Bruhn, M., Integrierte Kommunikation als Unternehmensaufgabe und Gestaltungsprozeß, in: Effizientes Kommunikations-Management, Hrsg.: Bruhn, M., Dahlhoff, H.D., Stuttgart 1993, S. 5.

Unter klassischer Werbung wird der zielgerichtete kommunikative Beeinflussungsprozeß mit Hilfe von Massenkommunikationsmitteln in verschiedenen Medien verstanden. Zu „klassischen" Medien zählen z.B. Fernsehen, Printmedien sowie Hörfunk. Daher kann der Einsatz von Kommunikationsinstrumenten nicht losgelöst von den zur Verfügung stehenden Medien betrachtet werden. Vgl. Meffert, H., Marketing: Grundlagen marktorientierter Unternehmensführung, a.a.O., S. 692.

Vgl. Pepels, W., Kommunikations-Management: Marketing-Kommunikation vom Briefing bis zur Realisation, a.a.O., S. 322ff.

577 Da die Allianzpartner unterschiedliche Heimatmärkte aufweisen, ist die länderspezifische Mediastruktur zu beachten. In Japan beispielsweise betrug der Anteil der TV-Werbung am nationalen Werbeaufkommen 1995 43,1 Prozent, in den USA 37,3 Prozent und in Deutschland 22,0 Prozent. Neben der unterschiedlichen Medialandschaft sind u.a. international divergierende rechtliche Nutzungsbeschränkungen von Medien zu beachten. Vgl. . Meffert, $H$., Bolz, J., Internationales Marketing-Management, a.a.O., S. $203 \mathrm{f}$.

578 Multimedia-Kommunikation beinhaltet nach Bruhn (1997) die „zielgerichtete, systematische Planung, Entwicklung, Distribution und Kontrolle eines computergestützten, interaktiven und multimodalen Kommunikationssystems als zeitunabhăngige Plattform eines zweiseitigen, von den individuellen Informations- und Unterhaltungsbedürfnissen des Rezipienten gesteuerten Kommunikationsprozesses mit dem Ziel der Vermittlung unternehmensgesteuerter Botschaften“. Bruhn, M., Multimedia-Kommunikation: systematische Planung und Umsetzung eines interaktiven Marketinginstruments, München 1997, S. 8. 
einer dialoggeführten Internet-Navigation abgerufen werden können, zählen u.a. Informationen zu Mitnahmevorschriften, Kabinengestaltung, Vielfliegerprogrammen, Flugplänen, Tarifen, eingesetztem Fluggerät sowie zum Mahlzeiten- und Unterhaltungsangebot. Nur ein geringer Anteil der Fluggesellschaften bietet hingegen Buchungsmöglichkeiten an. ${ }^{579}$ Damit besteht auch für die strategische Luftverkehrsallianz die Chance, komplexe Informationsinhalte aufzubrechen und sie einer individuellen, interaktiven Nutzung durch Vielflieger, Firmenkunden und Reisebüros zugänglich zu machen.

Die damit bereits angesprochene Mediadimension, die sich in klassische elektronische Medien (z.B. TV), Printmedien (z.B. Tageszeitung) und neue elektronische Medien (Z.B. Internet) unterteilen läßt, ${ }^{580}$ hängt in ihrer Ausgestaltung durch die Allianz von externen Faktoren wie den Heimatmarktgegebenheiten der Allianzpartner sowie von internen Faktoren wie der Instrumenteauswahl ab, so daß hier keine spezifische Empfehlung gegeben werden kann. Angesichts der bisher diskutierten Dimensionen der Kommunikationsstrategie ist jedoch von einem kombinierten Einsatz aller drei Media-Formen auszugehen.

Bezüglich der Gestaltungs- und Botschaftsdimension haben die Allianzpartner schließlich abzustimmen, "was" den Adressaten "wie" kommunikativ zu vermitteln ist. Ausgangspunkt ist das "Positioning Statement", in dem der Angebotsanspruch der Allianz - in diesem Fall der Anspruch einer weltweiten Wiedererkennung - und die Anspruchsbegründung - z.B. Airline-Präsenz in der ganzen Welt - zum Ausdruck kommen. Die Umsetzung dieser Positionierungsinhalte in Bilder, Worte, Schriften und Zeichen sowie ggf. in Bewegungen und Farben erfolgt über die "Creative Platform". Im Mittelpunkt der Creative Platform steht das von den Allianzpartnern definierte Nutzenversprechen, das sich in den Kerneigenschaften der Allianzmarke ausdrückt. ${ }^{581}$ Eher rational geprägte Gestaltungselemente und Botschaftsinhalte sind angesichts des frühen zeitlichen Entwicklungsstadiums der Allianzmarke sowie des komplexen Leistungsinhalts vorzuziehen, um das Kom-

579 Vgl. Ludwig, E., Travella, R., Online-Distribution und E-Commerce bei Fluggesellschaften, in: Alternative Formen der Distribution - Electronic Commerce, Online Distribution und Direct Distribution, Hrsg.: Belz, C., Tomczak, T., Thexis Fachbericht für Marketing Nr. 1, 1998, S. $42 f$. Vgl. Meffert, H., Marketing: Grundlagen marktorientierter Unternehmensführung, a.a.O., S. 689.

${ }^{581}$ Neben dem Nutzenversprechen fließen der Nutzenbeweis und die Stilkomponente in die Creative Platform ein. Vgl. weiterführend Pepels, W., Kommunikations-Management: Marketing-Kommunikation vom Briefing bis zur Realisation, a.a.O., S. $274 \mathrm{ff}$. 
munikationsobjekt zunächst zu tangibilisieren und gegenüber konkurrierenden Angeboten abzugrenzen.

Zusammenfassend können die von der Allianz abzustimmenden Entscheidungsdimensionen in einem Profil dargestellt werden (vgl. Abbildung 25), das gleichwohl nur eine statische Momentaufnahme wiedergibt und in Abhängigkeit der Kontextbedingungen entsprechend anzupassen ist. Die Bewertungen auf den von Meffert (1998) vorgeschlagenen bipolaren Rating-Skalen lassen subjektive Ermessensspielräume des Entscheiders bzw. Experten zu und sind daher lediglich als mögliche Ausprăgungsformen der Kommunikationsstrategie der Allianz zu werten. ${ }^{582}$

582 Vgl. Meffert, H., Marketing: Grundlagen marktorientierter Unternehmensführung, a.a.O., S. 690. 


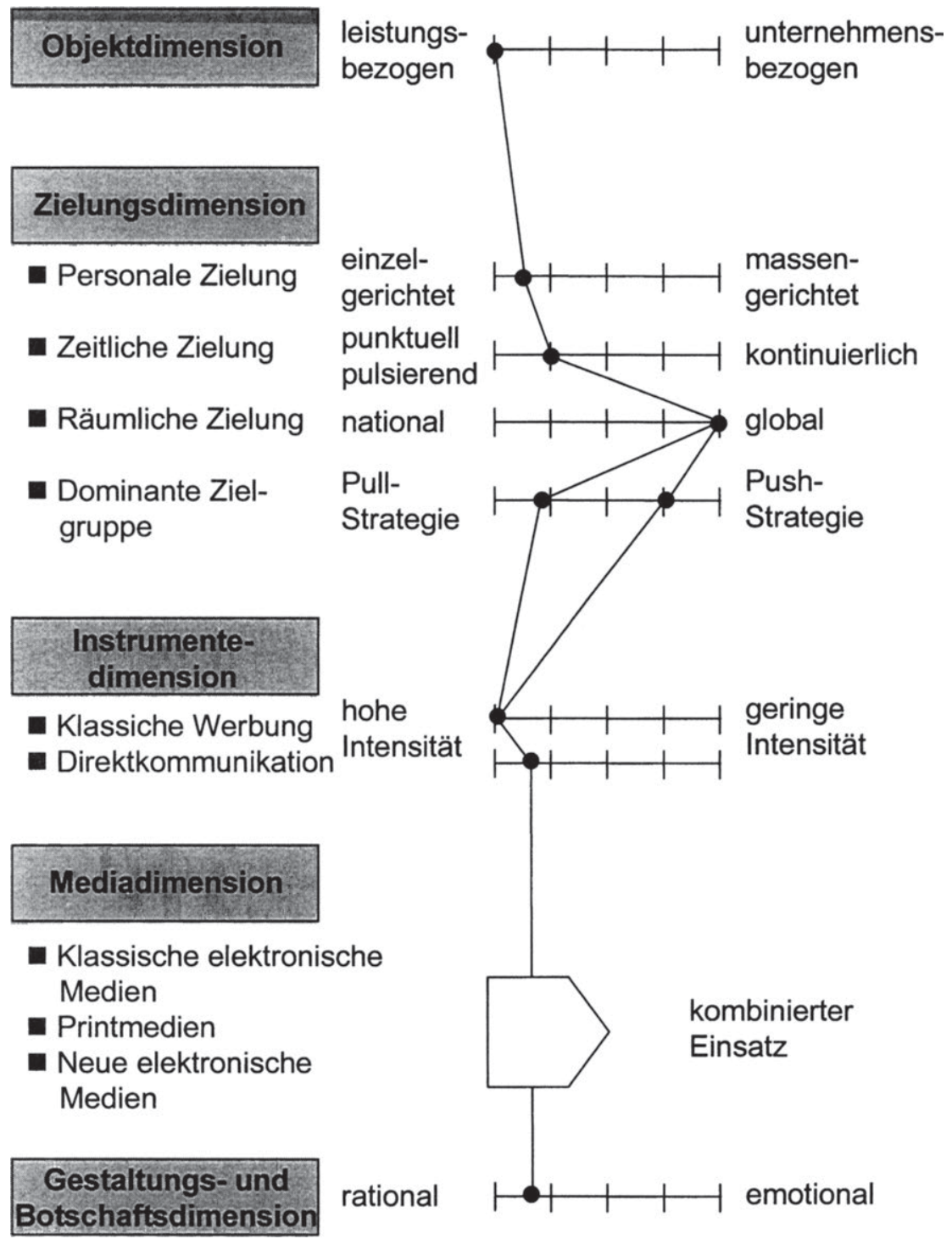

Abb. 25: Exemplarisches Profil der Allianz-Kommunikationsstrategie (Quelle: in Anlehnung an Meffert, H., Marketing: Grundlagen marktorientierter Unternehmensführung: Konzepte-Instrumente-Praxisbeispiele, 8., vollst. neubearb. und erw. Aufl., Wiesbaden 1998, S. 690) 


\subsubsection{Problemfelder der Budgetierung}

Über die Umsetzung der Kommunikationsstrategie in entsprechende aktionsfähige Handlungen entscheidet nicht zuletzt die Höhe des gemeinsam festzulegenden Budgets. Die Ermittlung des notwendigen Budgetumfangs im hier erörterten Fall der Allianz-Kommunikation unterscheidet sich grundsätzlich nicht von dem Vorgehen bei einer individuellen Marktkommunikation. ${ }^{583}$ Es ergibt sich jedoch die Notwendigkeit, die von den einzelnen Allianzpartnern zu tragenden Anteile am Gesamtbudget festzulegen.

Gemäß dem koalitionstheoretischen Gedankengut darf aus Sicht des einzelnen Partnerunternehmens der zu leistende Kostenbeitrag maximal so hoch sein, daß gerade noch ein individueller Vorteil aus der Gruppenkommunikation gezogen werden kann. Die Obergrenze ist erreicht, wenn dieser Vorteil durch die zu leistenden Budgetbeiträge gerade ausgeglichen ist. Der Bestimmung der Untergrenze der Budgetanteile liegt zunächst die Annahme zugrunde, daß die Summe der Budgetanteile dem Gesamtbudget der Allianz-Kommunikation entsprechen muß. Leisten alle Partner-Fluggesellschaften außer einer Airline Beiträge in Höhe ihrer individuellen Obergrenze, muß der Beitrag dieser einen Fluggesellschaft die verbleibenden Kosten decken. Die Höhe dieses Beitrags spiegelt die Untergrenze der Budgetanteile wider. In diesem Fall kommt der gemeinsam erzielte Vorteil nur einem Partner allein zugute. ${ }^{584}$

Während die Überlegungen zur Bestimmung der Obergrenze noch nachvollziehbar sind, obwohl sich hier Schwierigkeiten bei der Quantifizierung des individuellen Vorteils ergeben, basiert die Ermittlung der Untergrenze auf der Annahme einer einseitig asymmetrischen Leistungsbereitschaft, die angesichts gemeinsamer Budgetverhandlungen zwischen den Partnern als realitätsfremd erscheint. Die Ausführungen zur Ergebnisverteilung in strategischen Allianzen haben deutlich werden lassen, daß gerade unmittelbar ergebniswirksame Abstimmungsprozesse einer wachsamen Kontrolle aller Allianzpartner unterliegen und das Ausscheren eines einzelnen Partners daher eher die Ausnahme bleiben dürfte.

$583 \mathrm{Zu}$ einer Übersicht und Bewertung verschiedener Verfahren vgl. z.B. Barzen, D., MarketingBudgetierung, Frankfurt/Main u.a. 1990.

$584 \mathrm{Vgl}$. zu diesen Überlegungen Lindemann, M., Kooperative Marketing-Kommunikation: kommunikationspolitische Zusammenarbeit von Konsumgüter-Anbietern, a.a.O., S. 244. 
Um derartige Friktionen bereits im vorhinein zu vermeiden, ist es angebracht, ein geeignetes Prinzip zur Verteilung des Gesamtbudgets auf die Partner-Fluggesellschaften zu entwickeln. Wenn zu enwarten ist, daß alle beteiligten Partner im Zuge der Allianz-Kommunikation die gleichen Kosten - z.B. für die Schaltung von Allianz-Werbeanzeigen in Printmedien ihres Heimatmarktes - verursachen und in gleicher Weise von dem Nutzen der Gruppenkommunikation profitieren (z.B. in Form von zusätzlichen Umsatzerlösen), empfiehlt sich eine Gleichverteilung des Gesamtbudgets auf die einzelnen Allianzpartner. Eine derartige Verteilungsregel ist mit der Notwendigkeit verbunden, den individuell aus Sicht einer einzelnen Airline aus der Gruppenkommunikation erzielten Nutzen meßtechnisch zu isolieren und zu bewerten. Da die Partnerunternehmen parallel zur Allianzkommunikation individuelle Kommunikationsaktivitäten durchführen, stellt sich diese Aufgabe als überaus kompliziert dar. Aus diesem Grund bietet sich die Verwendung von Indikatoren an, die Aufschlüsse über den potentiellen Nutzen des einzelnen Allianzpartners geben. Lindemann (1993) schlägt daher die Verwendung des Umsatzvolumens oder des Marktanteils der Partnerunternehmen vor, nach denen das Gesamtbudget der Allianz-Kommunikation auf die Partner geschlüsselt wird. ${ }^{585}$ Ein solcher Verteilungsmodus ist zwar in hohem Maße operational und praxistauglich, kann aber zu einem Dominanzanspruch der umsatz- bzw. marktanteilsstärksten Allianzpartner führen und sich in einer entsprechenden Prägung der Kommunikationsstrategie niederschlagen. ${ }^{586}$

Eine nach erfolgter Budgetierung im Markt durchgesetzte Allianz-Kommunikation ist schließlich im Sinne des entscheidungsorientierten Planungsansatzes bezüglich ihres Kommunikationserfolgs zu bewerten, indem die Ist-Werte der Allianzmarkenbekanntheit, der Wiederkaufraten und des Allianzmarkenimage erfaßt und den Soll-Werten gegenübergestellt werden. Zu diesem Zweck können die aus der Werbewirkungsforschung bekannten Methoden unter Berücksichtigung der mit innen verbundenen Restriktionen angewendet werden. ${ }^{587}$ Aus Sicht des einzelnen

\footnotetext{
585 Vgl. Lindemann, M., Kooperative Marketing-Kommunikation: kommunikationspolitische Zusammenarbeit von Konsumgüter-Anbietern, a.a.O., S. 246.

586

Schmalen (1992) berichtet in ăhnlicher Weise über Beobachtungen von EinzelhandelsWerbegemeinschaften, in denen "große Beitragszahler" eine dominierende Rolle zu übernehmen versuchen. Vgl. Schmalen, H., Kommunikationspolitik: Werbeplanung, 2., überarb. und erw. Aufl., Stuttgart, Berlin, Köln 1992, S. 222.

Vgl. überblickartig Schub von Bossiazky, G., Psychologische Marketingforschung: qualitative Methoden und ihre Anwendung in der Markt-, Produkt- und Kommunikationsforschung, München 1992, S. 40ff.; Kroeber-Riel, W., Strategie und Technik der Werbung, 4. Aufl., Stuttgart 1993, S. 97.
} 
Partnerunternehmens nimmt die Schwierigkeit der Erfolgskontrolle durch die Form der Gruppenkommunikation zu, da die Wirkungszusammenhänge aufgrund des parallelen Markenauftritts von Firmen- und Allianzmarke an Komplexität gewinnen und damit höhere Anforderungen an das meßtechnische Instrumentarium stellen als im Fall der Individualkommunikation. ${ }^{588}$

Neben einer instrumentell ausgerichteten Wirkungskontrolle kommunikationspolitischer Aktivitäten bietet sich in dem hier betrachteten Dienstleistungskontext von Fluggesellschaften auch die Auswertung von „Primärdaten" an, die von dem Kundenkontaktpersonal an das Management der Allianzpartner weitergeleitet werden. Die marketingrelevanten Gestaltungsprobleme der Personalpolitik der strategischen Luftverkehrsallianz sind Gegenstand des folgenden Kapitels.

\subsection{Relevanz der Personalpolitik für die absatzmarktgerichtete Gestaltung der Luftverkehrsallianz}

Die exponierte Bedeutung der Personalpolitik für Dienstleistungsunternehmen wie Fluggesellschaften ergibt sich aus der Interaktivităt zwischen den Mitarbeitern des Anbieters und den Kunden im Zuge des Dienstleistungserstellungsprozesses. Hervorgehoben wurde dabei der Zusammenhang zwischen der Motivation des Personals, der Kundenzufriedenheit und dem ökonomischen Erfolg. ${ }^{589}$ Die nicht zuletzt durch derartige Forschungsergebnisse geförderte Besinnung von Unternehmen auf ihre Mitarbeiter führte in der Folge dazu, daß das Gedankengut des Marketing auf innengerichtete Prozesse übertragen wurde.

Der Ansatz des internen Marketing stellt die Mitarbeiterorientierung als Handlungsmaxime neben die Kundenorientierung und ist nach Bruhn (1995) um die systematische Optimierung unternehmensinterner Prozesse mit Instrumenten des Marketing- und Personalmanagement bemüht. Durch eine konsequente Kundenund Mitarbeiterorientierung soll das Marketing als interne Denkhaltung durchgesetzt werden, um die marktgerichteten Unternehmensziele effizienter zu errei-

588 Vgl. Lindemann, M., Kooperative Marketing-Kommunikation: kommunikationspolitische Zusammenarbeit von Konsumgüter-Anbietern, a.a.O., S. 251.

Vgl. Heskett, J.L., Managing in the Service Economy, Boston 1986, S. 117ff.; Heskett, J.L., u.a., Putting the Service-Profit Chain to Work, in: Harvard Business Review, Vol. 72, No. 2, 1994, S. 164ff.; Baron, S., Harris, K., Services Marketing. Text and Cases, Houndsmill u.a. 1995 , S. $126 f f$. 
chen. ${ }^{590}$ Wagner (1997) hebt demgegenüber die Bedeutung der Mitarbeiterzufriedenheit für "jedwede betriebliche Funktionserfüllung" hervor und interpretiert sie als "notwendige Bedingung für eine erfolgreiche absatzmarktorientierte Füh rung". ${ }^{591}$ Diesem Verständnis soll in den sich anschließenden Ausführungen gefolgt werden, deren Fokus auf den allianzspezifischen Problembereichen der Personalpolitik liegt.

Den in Kundenkontakt stehenden Mitarbeitern der Partner-Fluggesellschaften kommt die Aufgabe zu, das über die Kerneigenschaften der Allianzmarke definierte Nutzenversprechen im Zuge der Interaktion mit den Vielfliegern entsprechend umzusetzen. Implizit weist jede Kerneigenschaft mindestens eine personalbezogene Inhaltskomponente auf, so daß sich hieraus die zentralen Anforderungen an das Kundenkontaktpersonal der Luftverkehrsallianz ableiten lassen. Demnach wollen die Vielflieger

sich überall in guten Händen wissen,

- sich bei jeder Partner-Fluggesellschaft wohlfühlen können,

- flexiblem Servicepersonal gegenüberstehen,

- auf jede Frage von allen Partner-Fluggesellschaften eine adäquate Antwort erhalten,

Hilfe bei der Lösung von Problemen erhalten und

auf der ganzen Welt ein geschätzter Kunde sein.

Bei näherer Betrachtung unterscheidet sich diese Aufstellung kaum von Anforderungskatalogen, die üblicherweise für die Einstellung von Kundenkontaktpersonal entwickelt werden. ${ }^{592}$ Die zentrale Herausforderung für die Partner-Fluggesellschaften der Allianz besteht jedoch darin, das durch Arbeitsverträge an jeweils nur

$590 \mathrm{Vgl}$. Bruhn, M., Internes Marketing als Forschungsgebiet der Marketingwissenschaft, in: Internes Marketing. Integration der Kunden- und Mitarbeiterorientierung, Hrsg.: Bruhn, M., Wiesbaden 1995, S. 22.

591 Vgl. Wagner, H., Marktorientierte Unternehmensführung versus Orientierung an Mitarbeiterinteressen, Shareholder-Value und Gemeinwohlverpflichtung, in: Marktorientierte Unternehmensführung: Reflexionen - Denkanstoßße - Perspektiven, Hrsg.: Bruhn, M., Steffenhagen, H., Wiesbaden 1997, S. 93.

Bei Meffert und Bruhn (1997) finden sich u.a. die Anforderungskriterien "Einfühlungsvermögen“, „Fähigkeit, zuzuhören“, "geistige Flexibilităt", "Kommunikationsfähigkeit" und "Beharrungsvermogen“, die sich ohne Schwierigkeiten den oben genannten Anforderungen zuordnen lassen. Vgl. Meffert, H., Bruhn, M., Dienstleistungsmarketing: Grundlagen-Konzepte-Methoden, mit Fallbeispielen, a.a.O., S. 457. 
eine Fluggesellschaft gebundene Personal auf eine Dienstleistungserstellung auszurichten, von der auch andere Fluggesellschaften profitieren, ohne direkte Entgeltzahlungen an die entsprechenden Mitarbeiter zu leisten. ${ }^{593}$ In Abhängigkeit von der Loyalität des Mitarbeiters zum Unternehmen, die sich beispielsweise in der zeitlichen Zugehörigkeit ausdrückt, kann die auf Unternehmensleitungsebene verabschiedete Zusammenarbeit mit den Allianzpartnern bisher gültige Wertemuster in Frage stellen. In den Expertengesprächen wurde deutlich, daß sich insbesondere bei zuvor bestehenden Wettbewerbsverhältnissen der Partner-Airlines Reaktanzen im Mitarbeiterkreis gegenüber einer näheren Zusammenarbeit auf der operativen Ebene - z.B. am Check in - entwickeln. Die zwischen Northwest Airlines und Continental Airlines geschlossene Allianz beispielsweise scheiterte zunächst an Streiks der Piloten, die in der Allianz Nachteile für ihre eigene Situation ausmachten. ${ }^{594}$ Zusätzlich dürften sich die durch Synergienutzung in Form von Rationalisierungsmaßnahmen entstehenden Risiken eines Arbeitsplatzverlustes negativ auf die Bereitschaft der Mitarbeiter auswirken, die oben genannten Anforderungen zu erfüllen. Der eingangs erörterte Zusammenhang zwischen Mitarbeiterzufriedenheit und Kundenzufriedenheit verdeutlicht damit zugleich die Gefahr, den Nutzenerwartungen der Vielflieger in geringerem Maße zu entsprechen als im Falle eines Alleingangs.

Unter besonderer Berücksichtigung der Bindung von Personal an das arbeitgebende Unternehmen stellt Sattelberger (1997) seinen Vorschlägen zur Gestaltung der Personalpolitik eine Matrix voran, die sich aus den Dimensionen "Commitment für den Arbeitgeber“ und „Anhänglichkeitsverhalten“ ergibt (vgl. Abbildung 26). ${ }^{595}$

593 Als indirekte Zahlungen können die anteiligen Gewinne aus der Luftverkehrsallianz gewertet werden, die jedoch nicht unmittelbar ersichtlich sind, sondern durch geeignete interne Kommunikationsaktivităten transparent gemacht werden müssen.

Vgl. o.V., Northwest-Piloten gegen Allianz mit Continental, in: Handelsblatt vom 18.12.1997, S. 20.

Vgl. Sattelberger, T., Personalmanagement in virtuellen Unternehmen, Dokumentation des Gastvortrags am Betriebswirtschaftlichen Institut für Anlagen- und Systemtechnologien der Westfălischen Wilhelms-Universităt Münster am 28.1.1997. Auf diese Quelle beziehen sich auch die folgenden Ausführungen. 


\begin{tabular}{|c|c|c|c|}
\hline $\begin{array}{l}\text { Commit- } \\
\text { ment für } \\
\text { den }\end{array}$ & hoch & $\begin{array}{l}\text { Portfolio-Workers } \\
\text { (Partner auf Zeit) }\end{array}$ & $\begin{array}{l}\text { Wertorientierte Loyalisten } \\
\text { (Getreuer Ekkehard) }\end{array}$ \\
\hline $\begin{array}{l}\text { Arbeit- } \\
\text { geber }\end{array}$ & niedrig & $\begin{array}{c}\text { Taktisch kalkulierende } \\
\text { Überläufer/Abtrünnige } \\
\text { (Schnäppchenjäger) }\end{array}$ & $\begin{array}{l}\text { Gewohnheitsmitarbeiter } \\
\text { (Trittbrettfahrer) }\end{array}$ \\
\hline & & niedrig & hoch \\
\hline & & \multicolumn{2}{|c|}{ Anhänglichkeitsverhalten } \\
\hline
\end{tabular}

Abb. 26: Typologisierung von Mitarbeitern

(Quelle: Sattelberger, T., Personalmanagement in virtuellen Unternehmen, Dokumentation des Gastvortrags am Betriebswirtschaftlichen Institut für Anlagen- und Systemtechnologien der Westfälischen Wilhelms-Universität Münster am 28.1.1997)

Bei einem niedrigen Commitment im Sinne des persönlichen Einsatzwillens, der mit einer geringen Anhänglichkeit einhergeht („Taktisch kalkulierende Überläufer/Abtrünnige"), erscheint der personalpolitische Aufwand durch die Allianzpartner als zu hoch, um eine Verhaltensbeeinflussung im Hinblick auf die Erfüllung der vielfliegerseitigen Nutzenerwartungen zu bewirken. Sogenannte "Gewohnheitsmitarbeiter" sind demgegenüber durch Anreizinstrumente wie z.B. allianzspezifische Zusatzvergütungen zu einer Verhaltensänderung zu motivieren. Die Bindung von sogenannten Portfolio-Workers gelingt tendenziell durch ein interessantes Arbeitsumfeld, das bei Allianzen möglicherweise aus einem höheren interkulturellen Grad der Teams resultiert, während die unternehmensübergreifende Denkhaltung bei den „Wertorientierten Loyalisten“ vornehmlich über gezielte interne Kommunikationsmaßnahmen (z.B. regelmäßige Teilnahme an Allianzmeetings auf der Arbeitsebene) gefördert werden kann.

Für eine Ausrichtung der Mitarbeiter an dem Nutzenversprechen der Allianz sind vor diesem Hintergrund diejenigen Mitarbeiter auszuwählen, die eine positive Grundeinstellung gegenüber den Allianzpartnern aufweisen und die im Sinne 
einer Vorbildfunktion innerhalb des eigenen Unternehmens eingesetzt werden können. Gruppeninteraktionen, die unter Beteiligung der Mitarbeiter aller PartnerFluggesellschaften durchgeführt werden, bieten die Möglichkeit zur Verbesserung des gegenseitigen Verständnisses und zur Entstehung von Beziehungsnetzwerken, die sich wiederum stabilisierend auf die Allianz-Partnerschaft auswirken und diesen Effekt auch nach außen tragen können. 


\section{Würdigung und Ausblick}

\section{Zusammenfassende Würdigung der Untersuchungsergebnisse}

Den Ausgangspunkt der vorliegenden Untersuchung bildete die Beobachtung, daß Unternehmen mit anderen Unternehmen derselben Marktstufe strategische Allianzen eingehen, um ihre eigene Position im Wettbewerb zu verbessern. Der internationale Luftverkehr ist derartigen Tendenzen in besonderem Maße ausgesetzt, da die aus knappen Luftverkehrsrechten und Slots resultierenden Restriktionen durch strategische Allianzen abgeschwächt und die ökonomischen Ergebnisse der beteiligten Fluggesellschaften positiv beeinflußt werden können. Ein solches, an zusätzlichem Gewinn aus gemeinsamem Ressourceneinsatz orientiertes Verhalten reflektiert die Erkenntnisse der Koalitionstheorie und lenkt das Augenmerk zugleich auf die Frage der optimalen Gestaltung strategischer Luftverkehrsallianzen.

Im Zuge der gemeinsamen Erstellung von Flugdienstleistungen bedienen sich Luftverkehrsallianzen unterschiedlicher, aus der Organisationsforschung bekannter Formen der Arbeitsteilung, die sich an den Teilprozessen der Reisekette ausrichten. Charakteristisch ist eine dynamisch wechselnde, kombinierte Form artund mengenmäßiger Arbeitsteilung innerhalb der Allianz, während die dauerhafte Spezialisierung einer Partner-Fluggesellschaft auf lediglich ausgewählte Teilprozesse wie z.B. den eigentlichen Flug bei den hier betrachteten Allianzen nicht feststellbar war. Der Grund hierfür liegt in dem Wunsch der beteiligten Fluggesellschaften, die eigenen Fähigkeiten zur Erstellung aller Teilprozesse der Reisekette zu bewahren und die rechtliche sowie weitgehende wirtschaftliche Selbständigkeit aufrechtzuerhalten.

Zur Lösung des aus der unternehmensübergreifenden Arbeitsteilung erwachsenden Koordinationsproblems kommt dem Instrument der Selbstabstimmung eine besondere Bedeutung zu, da diese eine dezentrale, eher beziehungs- denn weisungsbezogene Abstimmung komplexer Prozesse ermöglicht. Neben der Arbeitsteilung schaffen die Partner-Fluggesellschaften durch die Einbindung der Allianzaktivitäten in ihre eigene Leitungsgliederung sowie durch geeignete Verfahren zur Verteilung der Allianzgewinne die organisationalen Voraussetzungen dafür, daß die Leistungen der Allianz gemeinsam am Absatzmarkt angeboten werden können. 
Während die Abstimmung allianzinterner Prozesse die notwendige Bedingung für einen gemeinsamen Erfolg darstellt, entscheidet letztlich die Orientierung des Allianz-Leistungsangebots an den Bedürfnissen der Nachfrager und den Angeboten der Wettbewerber über den langfristigen Erfolg der Allianz. Vor dem Hintergrund positiver ökonomischer Ergebniswirkungen von Luftverkehrsallianzen, die sich bereits kurzfristig in zunehmender Produktivität und steigenden Gewinnen ausdrücken, scheint die absatzmarktseitige outside-in-Perspektive bisher jedoch zugunsten einer angebotsseitigen inside-out-Perspektive strategischer Allianzen vernachlässigt worden zu sein. Möglicherweise ist nicht zuletzt in dieser unzureichenden Berücksichtigung der Absatzmarktorientierung ein Grund für das häufige Scheitern strategischer Luftverkehrsallianzen zu sehen, das sich in „Überlebensraten" von 26 Prozent (1992-1995) bei rein vertraglich vereinbarten und 73 Prozent bei kapitalbasierten Allianzen ausdrückt.

Dieser Befund motivierte eine eingehendere Untersuchung der endnutzerseitigen Wahrnehmung, des Erlebens und der Beurteilung von Leistungsangeboten strategischer Luftverkehrsallianzen. Auswertungen von Inhalten ausgewählter Informationsquellen legen die Vermutung nahe, daß Endnutzer durch die Berichterstattung in Printmedien sowie durch kommunikative Maßnahmen seitens der Allianzen selbst kaum über das Wesen von Code Share-Flugdienstleistungen informiert werden, die den Kern von Luftverkehrsallianzen bilden. Auch im Zuge des Erlebens von Allianz-Flugdienstleistungen bestehen hohe potentielle Informationsnachteile gegenüber den Leistungsangeboten von einzeln operierenden Fluggesellschaften, zumal die Beratung durch vermittelnde Reisebüros oft von Informationsdefiziten, Zeitmangel und buchungssysteminhärenten Schwächen geprägt ist. Im Fall eines investiven Flugscheinerwerbs wird die Informationsqualität zusätzlich von den spezifischen Strukturen im Buying Center des Firmenkunden beeinflußt.

Um Aufschluß über die Eignung des Leistungsangebots von Luftverkehrsallianzen zur Erfüllung fluggastseitiger Nutzenerwartungen zu erhalten, wurden in der Literatur dokumentierte, empirisch validierte Nutzendimensionen von Flugdienstleistungen der potentiellen Leistungsfähigkeit strategischer Allianzen gegenübergestellt. Als zentrale Nutzenerwartungen arbeiteten die nicht allianzspezifisch angelegten Studien flexible Buchungsmöglichkeiten, niedrige Preise, umfangreiche Serviceleistungen, Sitzkomfort, Verbindungsqualität und statusbezogene Leistungen heraus. Als zentrales Ergebnis einer allianzspezifisch angelegten Studie 
konnte die Überlegenheit strategischer Luftverkehrsallianzen bei der Erfüllung einiger Nutzendimensionen nachgewiesen werden, bei denen es sich um die Verbindungsqualität, das Angebot zusätzlicher Flüge, die Flugreisezeit sowie das Vorliegen einer Drehkreuzfunktion handelt. Demnach sind Luftverkehrsallianzen grundsätzlich geeignet, Vorteile durch eine partnerschaftliche Abstimmung der Reiseabläufe und durch die gegenseitige Nutzung starker Heimatmarktpositionen zu realisieren.

In einer Focusgruppenuntersuchung wurden daraufhin Vielflieger bezüglich ihrer allianzspezifischen Erfahrung befragt. Sie stuften strategische Luftverkehrsallianzen nur bei der Erfüllung des Problemfreiheitsbedürfnisses im Sinne eines störungsfreien Reiseablaufes als potentiell überlegen gegenüber einzeln agierenden Fluggesellschaften ein. Das Mobilitätsbedürfnis wurde als ähnlich gut und das Selbstverwirklichungsbedürfnis - der Wunsch nach Komfort, Abwechslungsreichtum und Produktivität beim Fliegen - als eher schlechter erfüllbar beurteilt.

Ansatzpunkte für eine strategische Positionierung der betrachteten Allianz boten sich in der Ableitung von fünf nutzenorientierten Kerneigenschaften in ihrer Idealausprägung, die wiederum Überschneidungen zu den oben genannten Nutzendimensionen aufweisen. Als wichtigste Kerneigenschaft ließen sich Vielfliegervorteile mit entsprechenden Statusleistungen und Privilegien identifizieren, gefolgt von nahtlosem Reisen, einem weltweiten Streckennetz mit flexiblen Buchungsoptionen, einem konsistenten Komfortniveau sowie einem weltweiten Sicherheitsstandard. Eine Einschätzung der im Mittelpunkt stehenden strategischen Allianz in bezug auf ihre Realpositionen dieser Merkmale ergab eine hohe Dominanz und deutliche Differenzierungsmöglichkeit lediglich bei der Erfüllung der Nutzenerwartung "weltweites Streckennetz/flexible Buchungsoptionen“. Differenzierungspotential ordneten die Befragungspersonen der Allianz hinsichtlich der Vielfliegervorteile mit entsprechenden Statusleistungen und Privilegien zu. Eine sogar leicht negative Differenzierung zu konkurrierenden Angeboten zeigte sich aufgrund schlechter Erfahrungen der Vielflieger bei der Nutzendimension des nahtlosen Reisens.

Aufbauend auf diesen Erkenntnissen konnte die Ausrichtung der MarketingInstrumente der betrachteten Allianz im Sinne einer auf weltweiter, persönlicher Wiedererkennung („Worldwide Recognition“) beruhenden Positionierung diskutiert werden. Den Ausgangspunkt bildete die Etablierung einer Allianzmarke für das 
gemeinsame Leistungsangebot der Partner-Fluggesellschaften. Die Wahl der Allianz-Markenstrategie unterliegt im Luftverkehr rechtlichen Restriktionen, welche die Aufgabe der eigenen Firmenmarke zugunsten einer unternehmensübergreifenden Allianzmarke untersagen. Unabhängig von den rechtlichen Restriktionen sprechen auch ökonomische Erwägungen, nach denen sich in der Firmenmarke die Identität des Unternehmens und das kumulierte Vertrauenskapital der Kunden widerspiegeln, gegen eine solche Lösung. Um dennoch von der integrierenden Wirkung einer gemeinsamen Marke zu profitieren, erscheint die Einführung einer neuen Allianz-Dachmarke sinnvoll, unter der die Firmenmarken der Partner-Fluggesellschaften parallel weitergeführt werden. Risiken dieser ausschließlich über nutzenbasierte Kerneigenschaften definierten Dachmarke liegen gleichwohl in ihrer Identitätslosigkeit, die eine persönliche Identifizierung der Fluggäste mit dieser Marke erschwert, sowie in den nur schwierig einschätzbaren Imagetransfereffekten, die zwischen den beteiligten Firmenmarken und der Allianzmarke wirken.

Die gemeinsame Leistungspolitik der Allianz baut insbesondere auf einer Verknüpfung der Streckennetze der Partner-Fluggesellschaften auf, über die im Idealfall eine "lückenlose", Ökonomisch sinnvolle weltweite Marktabdeckung erreicht werden kann. Der Befund zur Dominanz und Differenzierung bezüglich der Nutzenerwartung „weltweites Streckennetz/flexible Buchungsoptionen“ läßt für die betrachtete Allianz bereits eine erreichte vorteilhafte Positionierung erkennen. Die auf dem gemeinsamen Streckennetzmanagement aufbauende Abstimmung des Produkt- und Serviceangebots vollzieht sich im Spannungsfeld zwischen dem notwendigen $M a ß$ vereinheitlichter Prozesse und dem vielfliegerseitigen Wunsch nach fluggesellschaftsspezifischer Individualităt. Die Sicherstellung harmonisierter Leistungsprozesse erfordert eine unternehmensübergreifende Abstimmung der informationstechnologischen Infrastruktur, die sich angesichts etablierter „Insellösungen“ auf seiten der Partner als überaus schwierig erweist. Die vielfliegerseitig gewünschte Individualität von Produkt- und Serviceleistungen wie beispielsweise Loungeausstattung, Kabinendesign und persönlicher Service an Bord hingegen ist unter Einhaltung eines gemeinsam definierten Qualitätsstandards verhältnismäßig unkompliziert umzusetzen. Gleiches gilt für die Harmonisierung der gemeinsamen Vielfliegerprogramme, in denen die Allianzpartner über ein bedeutendes Instrument zur Erfüllung der Nutzenerwartung "Vielfliegervorteile Status/Privilegien" verfügen. 
Beim Vertrieb sehen sich die Allianzpartner in Abhängigkeit von dem gewählten Absatzkanal unterschiedlichen Herausforderungen gegenüber. Reisebüros, über die länderspezifisch variierend rund 80 bis 95 Prozent aller Flugscheine distribuiert werden, bieten zwar ein hohes Marktdurchdringungspotential für AllianzFlugdienstleistungen. Zweifel kommen jedoch hinsichtlich der Eignung von Reisebüros auf, bei der Zielgruppe der Vielflieger eine entsprechende Markenpositionierung aufzubauen, da Reisebüromitarbeiter angesichts der Komplexität des AllianzLeistungsangebots und gleichzeitig bestehenden Intransparenzen der Buchungssysteme oft überfordert sind. Demgegenüber eröffnet der Direktvertrieb an Firmenkunden über die Nutzung bestehender Geschäftsbeziehungen der PartnerFluggesellschaften wesentlich differenziertere Möglichkeiten, die Worldwide Recognition-Positionierung umzusetzen. Durch die Zusammenlegung ihrer Bonusprogramme besteht für die Allianzpartner darüber hinaus die Möglichkeit, gegenseitig Zugang zu den wertmäßig besonders bedeutsamen Vielfliegern zu erlangen und Flugscheine auch direkt an die Endnutzer zu vertreiben. Diese Vertriebsform ist lediglich auf aktuelle Stammkunden gerichtet und daher in Kombination mit den vorgenannten Absatzkanälen einzusetzen.

Die Kontrahierungspolitik der Allianz kann nur unter der Prämisse kartellrechtlicher Genehmigung gemeinsam von den Allianzpartnern gestaltet werden. Einer einheitlichen Allianz-Preisbildung stehen die Bestrebungen der einzelnen Partner entgegen, durch den Einsatz unternehmensspezifischer Yield ManagementSysteme zu einer Preis-Kapazităts-Optimierung zu gelangen. Als Preise für die Allianz-Flugdienstleistungen ergeben sich daher die einzeln optimierten Preise der jeweils den Flug durchführenden Partner-Airline („operating carrier"). Damit entsteht eine Inkonsistenz zu der angestrebten einheitlichen Markenpositionierung der Allianz. Gleiches gilt für die Abstimmung der Konditionen innerhalb der Luftverkehrsallianz, da die Partner-Fluggesellschaften bestrebt sind, insbesondere ihre eigene Heimatmarktposition durch Rabatte gegenüber Reisebüros und Firmenkunden zu stärken. Die für Endnutzer besonders relevanten Buchungsbedingungen, die in den Tarifen festgelegt werden, bedürfen indessen einer unternehmensübergreifenden Vereinheitlichung, um Irritationen der Nutzer etwa bei Umbuchungen oder Stornierungen zu vermeiden.

Die Aufgabe der gemeinsamen Allianz-Kommunikationspolitik besteht in der kommunikativen Umsetzung der zentralen Nutzendimensionen der Allianzmarke. Es enweist sich als schwierig, konktrete Handlungsempfehlungen abzuleiten, da 
bei der Abstimmung der Kommunikationspolitik die unterschiedlichen kommunikativen Positionierungen der einzelnen Partner-Fluggesellschaften zu berücksichtigen sind. Dennoch lassen sich Tendenzaussagen zur positionierungskonformen Ausgestaltung der Allianz-Kommunikation treffen. Demnach erscheint eine leistungsbezogene, globale, an Einzelpersonen (Vielflieger) ausgerichtete Kommunikation adäquat zu sein, die durch den kombinierten Einsatz klassischer Werbung und Direktkommunikation zur Übermittlung eher rationaler Botschaftsinhalte gekennzeichnet ist. Die Festlegung des Allianzbudgets erfordert aufgrund ihrer unmittelbaren Ergebniswirksamkeit einen transparenten Verteilungsschlüssel, der beispielsweise in Kennzahlen wie Umsatz- oder Marktanteilsgrößen der beteiligten Partner gefunden werden kann.

Im Bereich der Personalpolitik stehen die Allianzpartner vor der Herausforderung, ihre Mitarbeiter, die i.d.R. einer einzelunternehmensbezogenen Loyalität verhaftet sind, zu dem erforderlichen Commitment für die Allianzaktivitäten zu motivieren. Der Erfüllung dieser Aufgabe stehen zum einen Ängste vor einem möglichen Arbeitsplatzverlust durch die Allianz entgegen und zum anderen das Beharren auf bisher gewohnten Denk- und Verhaltensmustern, in denen jetzige Partner noch immer als Wettbewerber angesehen werden. Je nach der Höhe des Commitment für den Arbeitgeber und dem „Anhänglichkeitsverhalten" der Mitarbeiter können verschiedene Instrumente wie beispielsweise allianzspezifische Zusatzvergütungen oder Allianzmeetings eingesetzt werden, um das Spannungsfeld zwischen Einzelunternehmens- und Allianzloyalität zu überwinden. 


\section{Ansatzpunkte zur Erfassung des Nutzenbeitrags von Allianz-Flug- dienstleistungen}

Bei der Wahl einer Flugdienstleistung können Nachfrager grundsätzlich zwischen dem Leistungsangebot einzeln agierender Fluggesellschaften und dem Angebot strategischer Luftverkehrsallianzen wählen. Wie die zurückliegenden Ausführungen aufgezeigt haben, bergen gemeinsam erbrachte Allianz-Flugdienstleistungen aus Sicht der Nachfrager sowohl potentielle komparative Vorteile, die sich in höheren individuellen Nutzenbeiträgen ausdrücken, als auch potentielle komparative Nachteile, von denen beispielsweise aufgrund von anbieterseitigen Informations- oder Koordinationsmängeln eine nutzenmindernde Wirkung ausgeht.

Aus der Sicht von Luftverkehrsallianzen ist es vor diesem Hintergrund von erfolgskritischer Bedeutung, die für die Kaufentscheidung zentralen Nutzenerwartungen an eine Flugdienstleistung zu identifizieren und im Hinblick auf ihre allianzspezifische Gestaltungsnotwendigkeit und ihr allianzspezifisches Gestaltungspotential zu überprüfen. Um Erkenntnisse zu gewinnen, die über den explorativen Charakter der oben vorgestellten Focusgruppenuntersuchung hinausgehen, ist ein Meßinstrumentarium heranzuziehen, welches in der Lage ist, den allianzspezifischen Nutzenbeitrag konkret zu analysieren und einer Messung zugänglich zu machen. Zu diesem Zweck läßt sich ein vierstufiges Vorgehen heranziehen. Ausgehend von einer Konzeptualisierung bzw. Festlegung der in eine solche Analyse einzubeziehenden Nutzendimensionen gilt es, ein geeignetes Meßverfahren zu bestimmen, mit dem die Nutzenerwartungen der Nachfrager gegenüber diesen Dimensionen ermittelt werden können. Nach der im anschließenden Schritt vorzunehmenden verfahrenstechnischen Schätzung dieser Nutzenerwartungen lassen sich dann zielgerichtete Empfehlungen hinsichtlich der Gestaltung des allianzspezifischen Leistungsspektrums (etwa auf der Ebene einzelner Nachfragersegmente) vornehmen.

Die Konzeptualisierung der Nutzendimensionen von Flugdienstleistungen umfaßt die Festlegung der in eine solche Untersuchung einzubeziehenden allianzspezifischen und einzelfluggesellschaftsbezogenen Merkmale. In diesem Zusammenhang ist zu beachten, daß die ausgewählten Nutzendimensionen der Anforderung einer hinreichenden Konkretisierbarkeit genügen. Diese ist gegeben, wenn sich der von den betreffenden Merkmalen gestiftete Nutzen präzisieren und objektivieren läßt, wie dies beispielsweise beim Preis oder bei der Reisezeit der Fall ist. 
Merkmale wie z.B. die Freundlichkeit des Servicepersonals sind für eine Präzisierung und Objektivierung in dieser Form hingegen kaum zugänglich und daher eher über subjektive, assoziative Nutzenvorstellungen („sympathisch“, „aufmerksam") zu beschreiben und zu konkretisieren. ${ }^{596}$ Damit lassen sich aus den Ergebnissen einer Nutzenwahrnehmung der Nachfrager gegenüber solchen Merkmalen gleichwohl nur mit einigen Problemen zielgerichtete Handlungsempfehlungen im Sinne einer konkreten Ausgestaltung der Leistungsparameter von Flugdienstleistungen ableiten. Im Hinblick auf den Gestaltungsaspekt des Angebotsspektrums von Flugdienstleistungen gewinnt eine Erfassung der Nutzenerwartungen von Nachfragern daher um so mehr an Aussagekraft, je stärker es im Rahmen der Konzeptualisierung der Nutzendimensionen gelingt, auf solche Merkmale zurückzugreifen, die sich als physikalisch-funktionale Leistungsbestandteile von Flugdienstleistungen charakterisieren bzw. konkretisieren lassen.

Zur allianzspezifischen Konzeptualisierung der Nutzendimensionen kann auf die im Rahmen dieser Arbeit vorgestellten Ergebnisse empirischer Untersuchungen zurückgegriffen werden. Abbildung 27 enthält die wesentlichen, hier identifizierten Merkmale, von denen eine nutzenstiftende Wirkung ausgeht. Die Darstellung der Merkmale erfolgt in der Rangfolge nach ihrer/ihrem allianz- und einzelfluggesellschaftsspezifischen Gestaltungsnotwendigkeit/-potential.

Das Merkmal Preis ist durch den exemplarisch für eine bestimmte Strecke in unterschiedlichen Niveaus vorzugebenden Geldbetrag präzisierbar. Die Charakterisierung des Sicherheitsniveaus bereitet angesichts der Mehrzahl möglicher Schlüsselgrößen, an denen Fluggäste ihre Beurteilung ausrichten, einige Schwierigkeiten. Beispielhaft kann der optisch durch den Fluggast erfaßbare gute oder schlechte Zustand des Flugzeugs als Merkmalsausprägung herangezogen werden. Insgesamt nimmt diese Eigenschaft indes den Charakter eines Hygienefaktors ein, so daß auf eine Aufnahme in das Merkmalsset i.d.R. verzichtet werden kann bzw. sollte. Dem Komfortniveau lassen sich etwa die Ausprägungen der Sitzbreite und des Sitzabstandes zum Vordersitz zuordnen. Die Servicequalität an Bord kann durch die Aufmerksamkeit und Freundlichkeit der Flugbegleiter präzisiert werden, wobei wiederum subjektive Einflüsse bei der Beurteilung durch

598 Vgl. zur Konzeptualisierung von Nutzendimensionen im Verkehrsdienstleistungsbereich weiterführend Perrey, J., Nutzenorientierte Marktsegmentierung: ein integrativer Ansatz im Verkehrsdienstleistungsbereich, a.a.O., S. 49. 
die Fluggäste zu beachten sind, die ggf. zu Verzerrungen der späteren Ergebnisse einer Nutzenschätzung führen können.

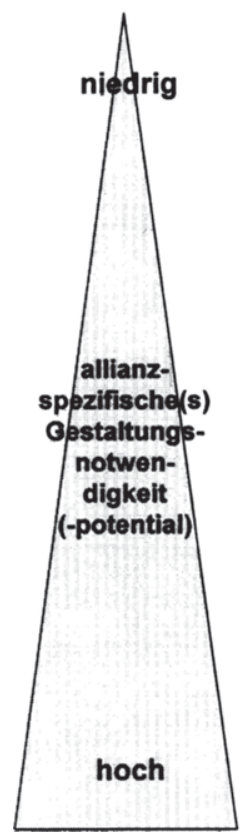
1. Preis
Vorgabe alternativer Preisniveaus
2. Sicherheitsniveau
Flugzeug in optisch einwandfreiem Zustand vs. Flugzeug überholungsbedürftig

3. Komfortniveau

breite Sitze mit großem Sitzabstand vs. schmale Sitze mit geringem Sitzabstand

4. Servicequalităt an Bord aufmerksamer, freundlicher Flugbegleiter vs. unfreundlicher, unaufmerksamer Flugbegleiter

5. Zeitliche Verbindungsqualităt kurze Aufenthaltsdauer vs. lange Aufenthaltsdauer auf Umsteigeflughăfen

6. Răumliche Verbindungsqualităt Vorgabe alternativer Längenangaben von Wegen, die an Umsteigeflughäfen zurückzulegen sind

7. Streckennetz weltweit vs. regional

8. Vielfllegervorteile Status und Privilegien bei mehreren Fluggesellschaften vs. bei einer Fluggesellschaft anerkannt

9. Anbieterwechsel im Zuge der Flugdienstleistung ist ein/kein Wechsel der Fluggesellschaft notwendig

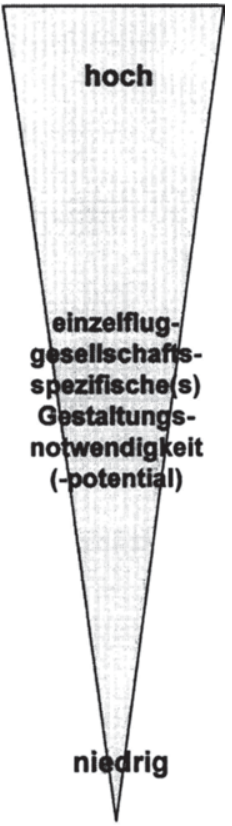

Abb. 27: Konzeptualisierung von Nutzendimensionen zur Erfassung allianzspezifischer Nutzenbeiträge

Bei der zeitlichen Verbindungsqualität handelt es sich um ein Merkmal, das durch die Angabe der Zeit, die zwischen zwei Flügen liegt, in Minuten oder Stunden metrisch erfaßt werden kann. Die räumliche Verbindungsqualität kann dagegen in Entfernungsmaßen angegeben werden, die an Umsteigeflughäfen zurückzulegen sind. In der Realität sind einem derartigen Objektivierungsversuch jedoch Grenzen gesetzt, da sich das Empfinden der Fluggäste für Entfernungen auch an der physischen Belastung z.B. bei einem Terminalwechsel mit Gepäckaufnahme und/oder dem individuellen räumlichen Gefühl anstelle von metrischen Größen ausrichten kann. Zu erwägen sind daher mögliche Visualisierungshilfen, die Flugreisende etwa in unterschiedlichen Umsteigesituationen abbilden und zur Erklärung der Merkmalsausprägungen beitragen können. 
Das Merkmal Streckennetz kann durch die Ausprägungen der weltweiten versus regionalen Präsenz der Fluggesellschaft bzw. Allianz konkretisiert werden. Alternativ wäre auch die Vorgabe von Länderschwerpunkten denkbar. In der Nutzendimension Vielfliegervorteile ist zu präzisieren, worin die Vorteile für den vielfliegenden Fluggast liegen. Als mögliche Ausprägungen lassen sich daher die Anerkennung des besonderen Kundenstatus (z.B. Platinum Member bei American Airlines) sowie die Gewährung von Privilegien (z.B. bevorzugtes Einchecken) bei entweder nur einer Airline oder bei mehreren Allianz-Fluggesellschaften auswählen.

Bei dem Merkmal Anbieterwechsel handelt es sich um die Notwendigkeit des Fluggastes, im Zuge seiner Flugdienstleistung die Fluggesellschaft wechseln zu müssen. Dies kann bereits zu Beginn der Flugreise erforderlich sein (z.B. Code Sharing), ist jedoch insbesondere bei Umsteigeverbindungen sowohl im traditionellen Interlining als auch im Code Sharing möglich. Mit diesem Merkmal gelingt es indes nicht vollständig, die hinter der Auskunft der Befragungsperson stehenden intrapersonellen Beweggründe für die Ablehnung oder Präferenz eines Anbieterwechsels zu identifizieren, da sich diese vermutlich in hohem Maße an der Markenidentität der betreffenden Fluggesellschaft orientieren. Der tatsăchliche Nutzenbeitrag (positiver oder negativer Nutzen) eines solchen Anbieterwechsels wird sich daher allenfalls ansatzweise mit Hilfe eines Meßinstrumentariums zur Erfassung der Nutzenerwartungen im Flugdienstleistungsbereich valide ermitteln lassen.

Die auf eine derartige oder ähnliche Weise konzeptualisierten Nutzendimensionen sind daraufhin in einem zweiten Schritt in ein geeignetes Instrumentarium zur Messung der Nutzenerwartungen von Nachfragern zu überführen. Zu diesem Zweck bieten sich das bereits kurz erläuterte Verfahren der traditionellen ConjointAnalyse oder andere Ansätze der dekompositionellen Nutzenmessung an. Die letztliche Auswahl eines solchen Verfahrens ist unter sorgfältiger Analyse der mit derartigen Ansätzen verbundenen Stärken und Schwächen vorzunehmen und erfordert auch hinsichtlich der konkreten Ausgestaltung des Untersuchungsablaufs ein hohes methodisches Know-how. ${ }^{597}$ Unter Berücksichtigung der sich aus der Konzeptualisierung ergebenden Restriktionen und der methodenimma-

597 Vgl. dazu stellvertretend Perrey, J., Nutzenorientierte Marktsegmentierung: ein integrativer Ansatz im Verkehrsdienstleistungsbereich, a.a.O., S. 65ff. 
nenten Schwächen des ausgewählten Meßverfahrens erfolgt in einem dritten Schritt die Schätzung der Nutzenwerte, die den statistischen Anforderungen der Objektivität, Reliabilität und Validität genügen muß. ${ }^{598}$ Aufbauend auf den damit gewonnenen Erkenntnissen lassen sich schließlich in einem vierten Schritt Überlegungen zur nutzengerechten Ausgestaltung des Flugdienstleistungsangebots vornehmen. Eine besondere Bedeutung nimmt dabei die Kanalisierung des Nutzenbeitrags einzelfluggesellschaftsbezogener und allianzspezifischer Leistungsparameter ein. Aufbauend auf der Durchführung einer Nutzensegmentierung lassen sich beispielsweise segmentspezifische Aussagen über Preisbereitschaften von Fluggästen gegenüber allianzspezifischen Leistungsparametern ableiten, die als hilfreiche Grundlage einer "bedürfnisorientierten“ Konzeption von AllianzFlugdienstleistungen fungieren können.

Angesichts einer weiter zunehmenden Verbreitung strategischer Allianzen im Luftverkehr ist insgesamt davon auszugehen, daß sich bestehende Nutzendifferenzen zwischen einzeln und in der Allianz erbrachten Flugdienstleistungen tendenziell verringern werden. In einem Wettbewerb von Allianznetzwerken gilt es daher zu untersuchen, ob und ggf. inwieweit sich Fluggäste den anbieterseitig geschaffenen Tatsachen gemeinsamer Leistungserstellung entziehen und auf ein ausschließlich einzeln erbrachtes Angebot ausweichen können. Aus der Anbieterperspektive stellt sich in diesem Zusammenhang die erfolgskritische Frage, auf welche Weise das durch die eigene Identität geprägte Profil der Einzelfluggesellschaft im Allianznetzwerk erhalten werden kann.

598 Vgl. zu einer Darstellung statistischer Methoden und Kennziffern zur Beurteilung von Reliabilităt und Validităt Homburg, C., Giering, A., Konzeptualisierung und Operationalisierung komplexer Konstrukte. Ein Leitfaden für die Marketingforschung, in: Marketing ZFP, 18. Jg., Nr. 1,1996, S. $5 \mathrm{ff}$. 


\section{Implikationen für das Management von Allianzen unter besonderer Berücksichtigung von Controllingaspekten}

Da die Allianzaktivitäten potentiell der Wahrnehmung durch Absatzmittler, Firmenkunden und Endnutzer unterliegen und Allianz-Flugdienstleistungen zudem von den Endnutzern erlebt und beurteilt werden, erweist sich die absatzmarktorientierte Gestaltung des Allianz-Leistungsangebots als ein zentraler Erfolgsfaktor ${ }^{599}$ strategischer Luftverkehrsallianzen.

Die mit der Absatzmarktorientierung einhergehende Notwendigkeit, alle von der Allianz betroffenen Leistungserstellungsprozesse mit den Allianzpartnern abzustimmen, konfrontiert die beteiligten Fluggesellschaften mit gegenseitigen Zugriffen auf bisher einzeln genutzte Leistungspotentiale (z.B. Bodenstationen, Flugzeuge) sowie mit Eingriffen in bisher einzeln erbrachte Leistungsangebote. Die solchermaßen entstehenden Verflechtungen erfordern die Implementierung eines Allianz-Controlling, das die Partner-Fluggesellschaften zum einen mit den relevanten internen und absatzmarktbezogenen Informationen versorgt (Informationsversorgungsfunktion) und zum anderen die Planung und Kontrolle der gemeinsamen Aktivitäten anhand von Soll-Ist-Vergleichen ermöglicht, welche die Basis für Abweichungsanalysen bilden (Planungs- und Kontrollfunktion). ${ }^{600}$ Abbildung 28 verdeutlicht den Aufbau des Allianz-Controlling, ${ }^{601}$ dessen Ausgangspunkt die mit der Allianz verfolgten Ziele bilden.

599 Patt (1988) unterscheidet qualitative strategische Erfolgsfaktoren, die als Unternehmensgrundsătze bzw. Grundtugenden unternehmerischen Handelns überdurchschnittlich erfolgreiche Unternehmen von anderen unterscheiden, sowie quantitative strategische Erfolgsfaktoren, unter denen Situationen, Strukturen oder Leistungsfaktoren mit signifikantem Einfluß auf den Unternehmens- oder Geschäftsfelderfolg verstanden werden. Vgl. Patt, P.-J., Strategische Erfolgsfaktoren im Einzelhandel: eine empirische Analyse am Beispiel des Bekleidungsfachhandels, Frankfurt am Main u.a. 1988, S. 6f. Die Absatzmarktorientierung ist demnach den qualitativen Erfolgsfaktoren zuzurechnen.

Nach Hahn (1996) kann Controlling als informationelle Sicherung ergebnisorientierter Unternehmensführung verstanden werden. Das Entscheiden und Handeln der Unternehmung hier: der strategischen Luftverkehrsallianz - soll durch die Aufbereitung von Führungsinformationen ergebnisorientiert ausgerichtet werden. Vgl. Hahn, D., PuK, Controllingkonzepte: Planung und Kontrolle, Planungs- und Kontrollsysteme, Planungs- und Kontrollrechnung, 5., überarb. und erw. Aufl., Wiesbaden 1996, S. 175.

$\mathrm{Zu}$ einer Gegenüberstellung ausgewăhlter Controlling-Konzepte in der deutschsprachigen Literatur vgl. Hahn, D., PuK, Controllingkonzepte: Planung und Kontrolle, Planungs- und Kontrollsysteme, Planungs- und Kontrollrechnung, a.a.O., S. 187. 


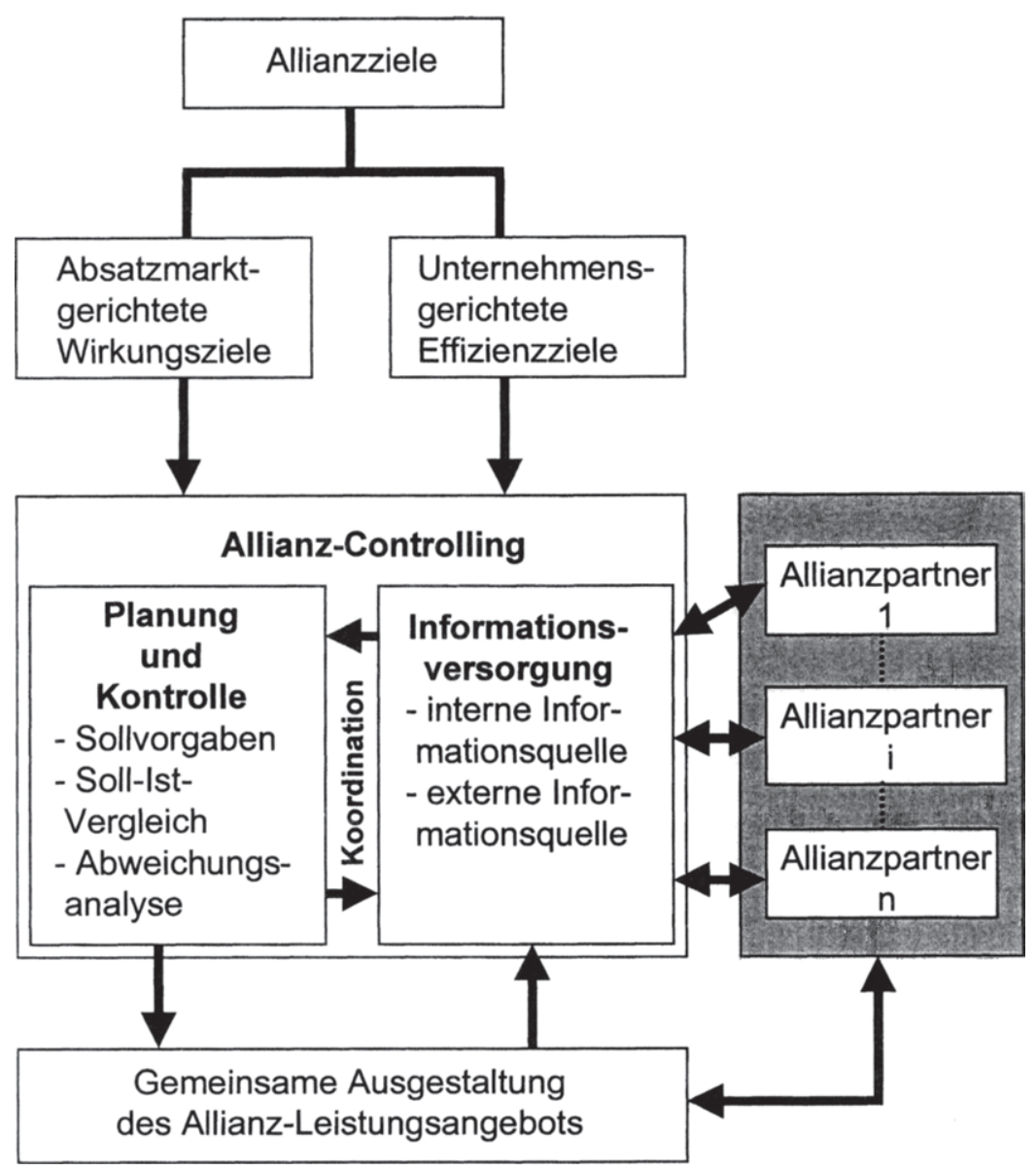

\section{Abb. 28: Aufbau des Allianz-Controlling}

Wenngleich in der Literatur eine primäre Ausrichtung des Controlling auf Wertziele im Sinne monetärer, quantitativer Größen wie z.B. Umsatz oder Gewinn konstatiert wird, ${ }^{602}$ erscheint es aufgrund der Mittel-Zweck-Beziehung zwischen psychographischen Größen wie dem Bekanntheitsgrad sowie Markenimage und den vorgenannten ökonomischen Größen sinnvoll zu sein, das Allianz-Controlling auch

602 Vgl. Meffert, H., Marketing: Grundlagen marktorientierter Unternehmensführung, a.a.O., S. 1037. 
auf operationalisierte psychographische Zielgrößen auszurichten. ${ }^{603}$ Als unternehmensgerichtete Effizienzzielgröße kann wiederum auf Produktivitätskennziffern wie den Sitzladefaktor zurückgegriffen werden.

Der zentrale Unterschied zwischen dem Allianz-Controlling und dem Controlling eines Einzelunternehmens liegt in der Erfassung und Zurechnung des allianzspezifischen Erfolgs. Sofern keine geeigneten Ansätze zur Berechnung des allianzspezifischen Erfolgsbeitrags existieren, die zudem das für die Partnerunternehmen wichtige Merkmal der intersubjektiven Nachprüfbarkeit erfüllen, setzt ein effizientes Allianzcontrolling ein hohes Maß an wechselseitigem Vertrauen voraus. Darüber hinaus müssen die Partnerunternehmen bereit sein, den anderen Allianzpartnern Informationen aus eigenen internen Quellen (z.B. Streckenergebnisrechnungen) sowie eigenen externen Quellen (z.B. Berichtsbände von Marktforschungsinstituten) zugänglich zu machen und damit die Basis für eine gemeinsame Informationsversorgung zu schaffen. ${ }^{604}$ Diese Notwendigkeit impliziert das Risiko der Verschleierung oder Verfälschung von Daten durch einzelne Partner, die um deren Geheimhaltung bemüht sind. Im Gegensatz zum Controlling bei einzelnen Unternehmen erfordern die Vorgabe von Sollwerten sowie die Kontrolle und Abweichungsanalyse bei Allianzen darüber hinaus den Entscheidungskonsens der Partner, zumal aufgrund der rechtlichen und weitgehenden wirtschaftlichen Unabhängigkeit keine formale Weisungsmacht zwischen den Partnern besteht, um Vorgaben durchzusetzen.

Da die Allianzpartner eigene Ressourcen in die strategische Allianz einbringen, die auch von anderen Partnern genutzt werden, sind sie an einem möglichst echtzeitorientierten Abgleich der eigenen Beiträge mit den ihnen zurechenbaren Erlösen interessiert. Einerseits wollen sie Informationen über die Wirtschaftlichkeit ihres Allianzengagements erhalten, andererseits streben sie eine als "gerecht" empfundene Ergebnisverteilung zwischen den Partnern an. Aus diesen Gründen

603 Wiedmann (1994) betont, daß sich die Markenführung am Aufbau und der Absicherung langfristiger Erfolgspotentiale statt an kurzfristiger Gewinnerzielung orientiert und schlägt den Markenwert als operationalisierte Zielgrőße für ein strategisches Markencontrolling vor, in den Indikatoren wie der Marktanteil oder die Markenloyalităt eingehen. Vgl. Wiedmann, K.-P., Strategisches Markencontrolling, in: Handbuch Markenartikel: Anforderungen an die Markenpolitik aus Sicht von Wissenschaft und Praxis, Hrsg.: Bruhn, M., Stuttgart 1994, S. 1320.

Neben Informationen, die bei den einzelnen Partnerunternehmen vorliegen, kann ggf. auf gemeinsam durchgeführte Studien wie im Fall der hier erörterten Focusgruppenbefragung zurückgegriffen werden. 
erweisen sich schnelle Rückkopplungen über Soll-Ist-Abweichungen als bedeutsam („Feed-back-Prinzip“), um rechtzeitige Anpassungsmaßnahmen einleiten zu können. Zugleich sieht das Allianz-Controlling im Sinne einer zukunftsorientierten Steuerung die Antizipation möglicher Abweichungen von den Sollvorgaben vor, indem frühzeitig Informationen über Einflüsse erfaßt werden, die potentiell zu Planungsänderungen führen („Feed-forward-Prinzip“). ${ }^{605}$ Als Beispiel sind Nachrichten über Allianz-Neugründungen oder -Auflösungen zu nennen, die eine wettbewerbliche Änderung im eigenen Allianz-Netzwerk implizieren können. ${ }^{606}$

Um eine komprimierte Übersicht über das Allianz-Controlling zu erhalten, lassen sich die zielbezogenen Soll- und Istwerte den von der Allianz abgedeckten Verkehrsströmen zuordnen (vgl. Abbildung 29).

\begin{tabular}{|c|c|c|c|c|c|c|c|c|}
\hline & \multicolumn{7}{|c|}{ strategische Allianz } & \multirow{2}{*}{$\begin{array}{c}\text { Haupt- } \\
\text { wettbew. } \\
\text { wie links } \\
\text { (Beobach } \\
\text { tungen) }\end{array}$} \\
\hline & $\begin{array}{l}\text { Umsatz- } \\
\text { erlose } \\
\text { (Soll/list) }\end{array}$ & $\begin{array}{c}\text { relativer } \\
\text { Markt- } \\
\text { anteil } \\
\text { (Soll/list) }\end{array}$ & $\begin{array}{l}\text { Bekannt- } \\
\text { heitsgrad } \\
\text { (Soll/lst) }\end{array}$ & $\begin{array}{l}\text { Marken- } \\
\text { image } \\
\text { (Sollilst) }\end{array}$ & $\begin{array}{l}\text { Loyalităts- } \\
\text { rate } \\
\text { (Soll//st) }\end{array}$ & $\begin{array}{l}\text { Kosten } \\
\text { (Soll/list) }\end{array}$ & $\begin{array}{l}\text { Sitzlade- } \\
\text { faktor } \\
\text { (Soll/list) }\end{array}$ & \\
\hline \multirow{2}{*}{\multicolumn{9}{|c|}{$\begin{array}{l}\text { Hauptver- } \\
\text { keths- } \\
\text { strom A } \\
\text { Strecke } \\
A_{1} \\
A_{1} \\
A_{n}\end{array}$}} \\
\hline & & & & & & & & \\
\hline \multirow{2}{*}{\multicolumn{9}{|c|}{$\begin{array}{l}\text { Hauptver- } \\
\text { kehrs- } \\
\text { strom B } \\
\text { Strecke } \\
B_{1} \\
B_{1} \\
B_{n}\end{array}$}} \\
\hline & & & & & & & & \\
\hline$\ldots$ & & & & & & & & \\
\hline
\end{tabular}

\section{Abb. 29: Erfassungsraster für das Allianz-Controlling}

605 Die konsequente Umsetzung des Feed-forward-Prinzips mündet in einer Frühwarnfunktion des Controlling. Vgl. Meffert, H., Marketing: Grundlagen marktorientierter Unternehmensführung, a.a.O., S. 1043.

606 Die strategische Allianz zwischen Delta Air Lines und United Airlines konfrontiert die Deutsche Lufthansa beispielsweise mit der Tatsache, daß sich ein Allianzpartner (United) mit einer Fluggesellschaft verbündet (Delta), die zugleich Partner eines europäischen Hauptwettbewerbers (Swissair) der Lufthansa ist. 
Während sich die Zielgrößen Umsatzerlöse, relativer Marktanteil (Marktanteil des/der operating carrier im Vergleich zum stärksten Wettbewerber), Kosten sowie Sitzladefaktor auf diejenigen Partner-Fluggesellschaften beziehen, die als operating carrier den eigentlichen Flug durchführen, sind der Bekanntheitsgrad, das Markenimage sowie die Loyalitätsrate auf die gemeinsame Allianzmarke ausgerichtet. ${ }^{607}$ Um Aussagen über die Differenzierung der Allianz gegenüber den Wettbewerbern treffen zu können, empfiehlt es sich, die Ausprägungen der Zielgrößen bei den jeweiligen Hauptwettbewerbern abzuschätzen und ggf. bei der Vorgabe von Soll-Werten zu berücksichtigen.

Für die Partner-Fluggesellschaften besteht damit die Möglichkeit, den individuellen ökonomischen Wert ihrer Mitgliedschaft in der Allianz zu bestimmen, Aufschluß über die geographische Verteilung dieses Werts zu erlangen und durch den direkten Vergleich mit den Ergebnissen der Partner sowie ggf. der Wettbewerber Rückschlüsse auf die eigene Position innerhalb der Allianz und im Wettbewerb zu ziehen ${ }^{608}$ Schwierigkeiten sind dagegen mit einer Ökonomisierung der Wechselwirkungen zwischen den psychographischen Zielgrößen der Allianz und denjenigen der einzelnen Partner-Fluggesellschaften verbunden. ${ }^{609}$

Abschließend kann das Allianz-Controlling zwar unterstützend zu einer Optimierung der Allianzergebnisse beitragen. Zugleich werden jedoch die Grenzen einer gemeinsamen Abstimmung deutlich, die strategische Allianzen nur als Zwischenlösung auf dem Weg zu einer vollständigen Integration der Aktivitäten erscheinen lassen, wie dies etwa bei Fusionen der Fall ist.

607 Auf einem geringeren Aggregationsniveau können zudem leistungsprozeßbezogene Größen wie beispielsweise Abflugs- und Ankunftspünktlichkeit sowie Wartezeit am Check-in in das Allianz-Controlling einfließen, die wiederum Einfluß auf die übergeordneten psychographischen Ziele haben.

Eine derartige statische Momentaufnahme ist durch geeignete Prämissen hinsichtlich möglicher Umweltzustände (Szenarien) zu dynamisieren. Vgl. Z.B. Altwegg, R., Strategiebewertung und Ermittlung des Synergiewertes bei Kooperationen: ein strategischer Kooperationsbewertungsansatz, a.a.O., S. 135ff.

$\mathrm{Zu}$ Bewertungsproblemen auf der niedrigeren Aggregationsebene der Leistungsprozesse vgl. z.B. Bruhn, M., Georgi, D., Kundenbezogene Wirtschaftlichkeitsanalyse des Qualitătsmanagements für Dienstleistungen: Konzept, Modellrechnung und Fallbeispiel, in: Marketing ZFP, Nr. 2, 1998, S. 98 . 


\section{Ausblick}

Die Auseinandersetzung mit dem Forschungsgegenstand strategischer Allianzen offenbart eine Fülle von Problemstellungen, von denen in der vorliegenden Arbeit lediglich ein Ausschnitt vertieft werden konnte. Dementsprechend ergeben sich sowohl in konzeptioneller als auch in methodischer Hinsicht verschiedene Ansatzpunkte für weiterführende Forschungsarbeiten.

- Die durch die Focusgruppenbefragung von 150 Vielfliegern gewonnenen Erkenntnisse sind nicht geeignet, auf die Gesamtheit aller Fluggäste übertragen zu werden. Es erscheint daher nicht zuletzt angesichts einer wachsenden Bedeutung von Allianz-Flugdienstleistungsangeboten vielversprechend, größerzahlige Untersuchungsdesigns zu entwickeln und ins Feld zu führen, um auch weitere Zielgruppen in die Überlegungen zur absatzmarktorientierten Allianzgestaltung einzubeziehen. Zugleich ist im Sinne des oben angeführten Vorschlags ein Design zu entwickeln, das in der Lage ist, den allianzspezifischen (Zusatz-)Nutzen meßtechnisch zu erfassen und Preisbereitschaften zuzuordnen, die Hinweise auf die nachfragerseitige Relevanz der betreffenden Nutzendimensionen geben können.

Aufgrund der hohen Bedeutung von Absatzmittlern für die Distribution von Allianz-Flugdienstleistungen bietet sich die Möglichkeit, der vorhandenen Forschungslücke hinsichtlich des allianzspezifischen Informations- und Beratungsverhaltens von Mitarbeitern der Absatzmittlerinstitutionen durch geeignete Formen empirischer Forschung wirksam zu begegnen. Erkenntnisse aus Testkäufen, Panel-Befragungen oder Tiefeninterviews mit Mitarbeitern könnten zu einem besseren Verständnis der vertikalen Beziehungen strategischer Allianzen im Luftverkehr beitragen. Zudem bieten sich hier Ansatzpunkte, die fortschreitende empirische Erfassung und Untersuchung des Zusammenhangs zwischen Kundenzufriedenheit und Mitarbeiterzufriedenheit in eine entsprechende Studie zu integrieren.

Aus einem gemeinsamen Markenauftritt der Allianzpartner resultieren Imagetransfereffekte, deren Intensität, Richtung und Wirkung von hoher Bedeutung für die individuellen Markenimages der Partner sein können. Die Konzeptualisierung und Operationalisierung dieser Beziehungen stellen eine anspruchsvolle Aufgabe im Rahmen der weiterführenden Marketingforschung dar, in 
deren Zuge auf bereits vorliegende Ansätze der Ähnlichkeitsmessung von Objekten sowie auf Erkenntnisse der vergleichenden internationalen Marketingforschung zurückgegriffen werden könnte.

- Es wurde deutlich, daß der informationstechnologischen Infrastruktur bei der operativen Abstimmung der gemeinsamen Leistungserstellungsprozesse im Rahmen strategischer Luftverkehrsallianzen eine zentrale Bedeutung zukommt. An dieser Schnittstelle zwischen Marketing und Informatik ergeben sich Ansatzpunkte für die Entwicklung kundengerichteter Systeme, die zugleich in der Lage sind, bestehende informationstechnologische Insellösungen zu überbrücken und eine optimale Kompatibilität zwischen den Allianzpartnern herzustellen.

- Die vorliegende Untersuchung konzentriert sich ausschließlich auf strategische Allianzen im Personenluftverkehr. Die in der Focusgruppenuntersuchung exemplarisch erfaßte strategische Allianz weist hinreichende Ähnlichkeiten in ihrer Gestaltung zu den direkt konkurrierenden Luftverkehrsallianzen auf, so daß eine Übertragung der hier gewonnenen Erkenntnisse auf alle weltweit präsenten Allianznetzwerke möglich ist. Um Parallelen oder Unterschiede zu anderen Branchen bezüglich der Auswirkungen einer gemeinsamen Leistungserstellung auf die nachfragerseitige Wahrnehmung, das Erleben und die Beurteilung strategischer Allianzen aufzudecken, wäre die Ausdehnung des Branchenfokus wünschenswert. 


\section{Anhangverzeichnis}

Anhang 1 Zusammenfassung des Gespräches mit dem

Managing Director Strategic Alliances der

Auskunftsairline A am 15. Juli 1997

Anhang 2 Zusammenfassung des Gespräches mit dem Leiter

der Netzplanung der Auskunftsairline B am 4.

September 1997

Anhang 3 Zusammenfassung des Gespräches mit dem General

254

Manager für Deutschland und Österreich der

Auskunftsairline C am 17. September 1997

Anhang 4 Record of the expert interview with the Director of

Industry Affairs Answering Airline D, December 17 , 1997

Anhang 5 Grundzüge des Kaufverhaltens im buying center von

Firmenkunden 


\author{
Anhang 1 \\ Zusammenfassung des Gespräches mit dem \\ Managing Director Strategic Alliances der Auskunftsairline A $^{1}$ \\ am 15. Juli 1997
}

1. Entstehungsgründe strategischer Allianzen im Luftverkehr

2. Ziele strategischer Allianzen

3. Kriterien zur Auswahl der Partner

4. Marktstrategische Entscheidungen

5. Rollenverteilung in strategischen Allianzen

6. Implementierung strategischer Allianzen

7. Steuerung und Kontrolle in strategischen Allianzen

\title{
1. Entstehungsgründe strategischer Allianzen im Luftverkehr
}

Die strategischen Allianzen der Auskunftsairline A im Passagebereich sind in drei unterschiedlich geführte Allianzen zu unterteilen: zum einen in das Allianznetzwerk 1 mit den Partnern Airline B, Airline C, Airline D, Airline E und Airline F (ab 1.10.97). Die Aufnahme zusätzlicher Partner ist geplant. Weitere strategische Allianzen bilden das Allianznetzwerk 2 mit den Franchisepartnern Airline G, Airline $\mathrm{H}$ und Airline I sowie die Allianzen unter der Bezeichnung „Allianznetzwerk 3 “, zu dem u.a. Airline J zählt. Wenngleich diese drei Allianzformen nachfragerseitig ähnlich wahrgenommen werden, stellen sie sich aus Sicht der Auskunftsairline A sowohl hinsichtlich ihrer innen- als auch außengerichteten Führung völlig unterschiedlich dar. Um diese Unterschiedlichkeit auch nach außen zu signalisieren, werden kommunikative Maßnahmen wie beispielsweise die Markteinführung der Dachmarke „Allianznetzwerk 1“ eingesetzt. Für die Partnerschaft in dem Allianznetzwerk

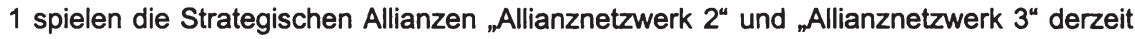
keine Rolle, um den ohnehin hohen Komplexitätsgrad des Allianzmanagement nicht noch weiter zu erhöhen, zumal dann bis zu vierzig verschiedene Fluggesellschaften in Verhandlungen einzubeziehen wären.

Aktuelle Tendenzen deuten auf eine zweigleisige Entwicklung im internationalen Wettbewerb hin. Neben allianzierten Netzwerk-Fluggesellschaften, die ein full-service-

1 Der Name des Gesprächspartners sowie die Namen der Fluggesellschaften sind aus Gründen der Vertraulichkeit anonymisiert. 
Angebot aufweisen, werden sich voraussichtlich Nischenanbieter im Alleingang positionieren, die sich - ähnlich wie Southwest Airlines - auf einen Punkt-zu-PunktVerkehr konzentrieren und vor allem über den Preis profilieren. Als allianzbegünstigende Faktoren sind für die Auskunftsairline $A$ vor allem kundenseitige Faktoren wie die wachsende globale Mobilität sowie rechtliche Faktoren wie die Deregulierung des Luftverkehrs mit neuen verkehrsrechtlichen Rahmenbedingungen ausschlaggebend. Im Mittelpunkt der kundenseitigen Anforderungen an eine Flugreise steht der Wunsch nach einem Produkt "aus einer Hand“. Während das traditionelle Interlining bei Umsteigeverbindungen zwischen zwei Fluggesellschaften bisher zwar eine Durchabfertigung des Gepäcks bis an den Zielort ermöglichte, wurde der einmalige Personen-Check-in vom Ausgangsort bis zum Zielort nur selten praktiziert. Folglich kam es bei dem Kernprodukt "Transport von A nach B“ zu einem Systembruch, sofern mehr als eine Fluggesellschaft an der Leistungserstellung beteiligt war. Darüber hinausgehende Ansatzpunkte einer Fluggesellschaft-übergreifenden Zusammenarbeit bestanden im Rahmen des Interlining nicht. Das Eingehen von strategischen Partnerschaften wie dem Allianznetzwerk 1 ermöglicht demgegenüber das Angebot von Leistungen, die zusătzlich zu einem besser abgestimmten Kermprodukt offeriert werden. Neben einer personenbezogenen Durchabfertigung können Mitglieder in Vielfliegerprogrammen ihre mit den Allianznetzwerk 1-Partnern geflogenen Meilen auf dem von ihnen präferierten Meilenkonto gutschreiben lassen und somit sowohl eher die für Bonusflüge notwendige Meilengrenze erreichen als auch Statusmeilen sammeln, um den Status eines privilegierten Top-Kunden zu erlangen. Zudem steht ein größeres Streckennetz für das Abfliegen der Bonusmeilen zur Verfügung. Überdies stehen Business- und First ClassKunden sowie Statuskunden weltweit 179 Lounges an den Flughäfen offen, um Warteund Übergangszeiten zu überbrücken.

Allianzfördernde Wirkung ging auch von den Aktivitäten der Wettbewerber aus, die sich um Allianzpartner bemühten. Nachdem die strategische Partnerschaft zwischen Airline $\mathrm{C}$ und Airline $\mathrm{K}$ gegen Ende der achtziger Jahre den vielleicht deutlichsten Impuls für weitere Allianzvorhaben in der Luftverkehrsindustrie gegeben hatte, ging die Auskunftsairline A mit Airline $L$ eine Code-Share-Allianz ein. Eine wichtige Zielsetzung war die Gewinnung von Erfahrungen in geographischer Nähe zum wichtigen USamerikanischen Markt. Daran schloß sich ein Versuch der Partnerschaft mit Airline $M$ an, der jedoch scheiterte, obgleich formal ähnliche Voraussetzungen wie bei der fruchtbaren Zusammenarbeit mit Airline $L$ gegeben waren. Gründe für das Scheitern sind in erster Linie in inneren Akzeptanzbarrieren der Mitarbeiter zu suchen. Während in weiten Teilen des involvierten Management die Bereitschaft zur Partnerschaft vorhanden war, stellte es 
sich gerade bei einer hochkompetitiven und selbstbewußten Fluggesellschaft wie Airline $M$ als schwierig heraus, die Auskunftsairline A-Mitarbeiter zur Kooperation mit einem über Jahre geprägten "Feindbild" zu bewegen. Vielmehr erwies sich eine hohe Loyalität der Mitarbeiter gegenüber dem eigenen Arbeitgeber als abträglich für die Funktionsfähigkeit der Allianz. Zudem wurden die mit der Allianz angestrebten wirtschaftlichen Ziele nicht erreicht.

Ähnlich wie in diesem Fall wurden die Partnerschaften zwischen Airline $C$ und Airline $K$ sowie British Airways und Airline $E$ beendet. Etwas später fanden neue Verhandlungen zwischen Air France und Airline $K$ sowie Alitalia und Airline $K$ statt, führten jedoch zunächst nicht zum Erfolg. Die Allianz zwischen Northwest Airlines und der niederländischen KLM galt demgegenüber - unter anderem begünstigt durch eine relativ frühzeitig gewährte Antitrust Immunity - lange Zeit als Musterbeispiel für eine erfolgreiche Kooperation. Mittlerweile ergeben sich jedoch auch bei dieser Allianz gravierende Probleme, deren Ursachen überwiegend in einer unterschiedlichen Philosophie des Management liegen. Während KLM an der Entwicklung einer langfristigen Perspektive für die Fluggesellschaften interessiert ist, verfolgt das Topmanagement von Northwest in erster Linie finanzielle Ziele. Die Kapitalbeteiligung von KLM in Verbindung mit der Besetzung von Board-Positionen bei Northwest intensiviert diese Konflikte, die überdies öffentlich ausgetragen werden und zu einer erheblichen Verunsicherung und Demotivation der Mitarbeiter auf beiden Seiten beitragen.

\section{Ziele strategischer Allianzen}

Hinsichtlich der Ziele von Allianzpartnern lassen sich seitens der Fluggesellschaften vor allem Wirtschaftlichkeits- und Wachstumsziele nennen, die unter Wahrung der eigenen Unabhängigkeit verfolgt werden. Airlines sehen sich im internationalen Luftverkehr vor allem drei wachstumsbegrenzenden Faktoren gegenüber:

1. Verfügbarkeit von Verkehrsrechten

2. Verfügbarkeit von Start- und Landerechten sowie

3. Dichte des aktuellen Angebots auf den Flugstrecken.

Mittels strategischer Allianzen, denen eine marktteilnehmerbezogene Strategie zugrunde liegt, sollen diese Begrenzungsfaktoren umgangen werden, um Wachstumspotentiale zu erschließen. Beispielsweise können über Gemeinschaftsflüge im Rahmen strategischer Allianzen Verkehrsrechte der Partner genutzt werden, Zugang zu Start- und Landerechten gefunden und die Streckenplanungen gemeinsam optimiert werden, sofern Antitrust-Immunität besteht. Ausgehend von dieser Zielsetzung sind demzufolge die in Frage kommenden Partner nach bestimmten Auswahlkriterien zu bewerten. 


\section{Kriterien zur Auswahl der Partner}

Die wichtigsten Kriterien zur Auswahl der Allianzpartner lassen sich vereinfacht in netzspezifische und unternehmensspezifische Kriterien unterteilen. Wichtigste Voraussetzung einer strategischen Partnerschaft ist die gegenseitige Ergänzung der Streckennetze, d.h. die Komplementarität der in die Allianz eingebrachten Flugverbindungen. Zum einen soll vermieden werden, daß sich die Partner gegenseitig konkurrenzieren, zum anderen ist gerade im Fall ähnlicher Streckennetze die rechtliche Genehmigung von Allianzen durch die Kartellbehörden wesentlich problematischer. So ist es der Airline $E$ und Auskunftsairline $A$ auf der von beiden Fluggesellschaften gleichermaßen beflogenen Strecke Frankfurt-Washington untersagt, sich auf dieselbe Weise miteinander abzustimmen wie auf den Strecken, für die eine Ausnahmegenehmigung vom Kartellrecht besteht.

Da es das Ziel der Auskunftsairline A ist, in den wichtigsten Märkten der Welt präsent zu sein, muß der Allianzpartner zudem über angemessene Marktanteile in mindestens einem der Schlüsselmärkte und damit über leistungsfähige Verkehrsdrehkreuze verfügen. Angestrebt werden derzeit je nach Region ca. zwanzig Prozent AllianzMarktanteil am Verkehrsaufkommen. Das partnerindividuelle Ziel der Maximierung des eigenen Marktanteils tritt gegenüber dem gemeinsamen Ziel zurück, da sich über große Allianz-Marktanteile ein entsprechender Rückfluß in das eigene Netz errechnet. Als Schlüsselmärkte gelten aus Sicht der Auskunftsairline A die Triademärkte Amerika, Europa und Asien sowie die Verbindungen dieser Märkte untereinander, so daß sich rein rechnerisch sechs Kernmärkte ergeben. In diesen Kernmärkten vollziehen sich neunzig Prozent des gesamten Weltluftverkehrs. Damit tritt ein geozentrisches Verständnis der Globalisierung an die Stelle einer ethnozentrischen Auffassung, nach der aus dem Heimatmarkt heraus unterschiedliche Ziele in der Welt bedient wurden. Fluggesellschaften wie die Auskunftsairline A, die im Vergleich mit US-amerikanischen Carriern nur einen geographisch kleinen Heimatmarkt aufweisen, verfügen daher über größere Erfahrungen mit anderen Kulturen, da bereits alle Flüge von mehr als zwei Stunden Dauer international sind.

Als unternehmensspezifische Auswahlkriterien, anhand derer potentielle Partner analysiert werden, sind insbesondere das Ausmaß an einzubringender Vertriebsstärke, die finanzielle Stabilität und die fachliche Kompetenz des Management ausschlaggebend. Die "Chemie" zwischen den Partnern bildet schließlich eine wichtige Voraussetzung für die Funktionsfähigkeit der Allianz, wie zurückliegende Erfahrungen z.B. mit Airline M 
belegen konnten. Vor dem Hintergrund dieser netz- und unternehmensspezifischen Auswahlkriterien boten sich für die Auskunftsairline $A$ als Partner im US-amerikanischen Markt zunächst die drei großen Fluggesellschaften Airline $E$, Airline $M$ und Airline $N$ an. Während im Fall der Airline N vor allem rechtliche Gründe der Genehmigung einer Allianz entgegengestanden hätten - zum Zeitpunkt der Partnerwahl wies diese Fluggesellschaft noch ein sehr umfangreiches Deutschland-Angebot auf -, lagen die Hubs von Airline $M$ mit Chicago und Dallas Fort Worth relativ weit westlich, so daß der aufkommenstarke Ostteil der USA nur umständlich hätte bedient werden können. Airline $E$ konnte demgegenüber Washington D.C. als Drehkreuz mit günstigerer geographischer Lage einbringen. Zudem stellt Airline E - gemeinsam mit Northwest Airlines - den stärksten Anbieter im Transpazifik-Verkehr und damit einem weiteren Schlüsselmarkt dar. Darüber hinaus besitzt Airline $\mathrm{E}$ historisch bedingt Verkehrsrechte in Japan, mittels derer der asiatische Raum bedient werden kann. Somit ergänzten sich die starke Europa-Position der Auskunftsairline A mit diesen Marktpositionen, während im Nordatlantik-Verkehr die Kräfte gebündelt wurden. Mit Airline $C$ konnten die Marktstellung in Europa gestärkt und der Nordatlantik- sowie Asienverkehr ergänzt werden. Airline D erhöht ihrerseits den Anbieterdruck auf asiatischer Seite. Derzeit werden noch Partner für den südpazifischen Raum gesucht, um das "globale Puzzle“ zu komplettieren.

Aus Sicht der Airline E stellte sich die Partnerwahl ähnlich dar. Aufgrund ihrer jeweiligen Marktpositionen boten sich zunächst British Airways, Air France und die Auskunftsairline A als potentiell interessante Partnerkandidaten an. Mit British Airways bestanden bereits negative Erfahrungen aus der Allianz, für deren Scheitern letztlich vor allem die relativ intensive Konkurrenzierung auf der Nordatlantikstrecke über das Drehkreuz LondonHeathrow ausschlaggebend war. Bezüglich einer möglichen Partnerschaft mit Air France erwiesen sich der hohe staatliche Einfluß und der eher protektionistische Charakter der Air France-Führung für eine strategische Partnerschaft als hinderlich, so daß sich die Auskunftsairline $A$ als beste Wahl darstellte, zumal auch die Größenverhältnisse beider Fluggesellschaften die Einhaltung eines Kräftegleichgewichts versprachen. Nachdem sich die Beziehung zwischen Airline $E$ und Auskunftsairline $A$ in ihrer Entwicklung als überaus erfolgreich gestaltete, boten sich für Airline $\mathrm{M}$ als "Folger" der Allianzbildung noch Air France und British Airways an. Die mit beiden Unternehmen geführten Verhandlungen mündeten bekanntermaßen in der angestrebten Partnerschaft mit British Airways. Rahmenbedingung der Allianzbildung ist schließlich die rechtliche Durchführbarkeit des Gemeinschaftsvorhabens, die bereits im Vorfeld der Verhandlungen juristisch geprüft wird. 
Mit der zunehmenden Verfestigung von strategischen Luftverkehrsallianzenn, die sich im Wettbewerb gegenüberstehen, bilden sich allianzspezifische „Aufnahmebedingungen“ heraus. Eine Bedingung für die Aufnahme in das Allianznetzwerk 1 ist beispielsweise das Bestehen von bilateralen Beziehungen zu jeder der aktuell sechs PartnerFluggesellschaften, so daß - vergleichbar mit dem Atomium - ein multilaterales Beziehungsgeflecht entsteht. Die Airline $F$ konnte aufgrund ihrer mangelnden Partnerschaft mit Airline $E$ daher bislang nicht Partner des Allianznetzwerks 1 werden und mußte erst die bestehende Partnerschaft mit Airline $\mathrm{N}$ zugunsten einer Zusammenarbeit mit Airline $E$ aufgeben, um sich für das Allianznetzwerk 1 zu qualifizieren. Bestehende bilaterale Beziehungen wie beispielsweise zur Auskunftsairline A blieben von dieser Bedingung unberührt.

Dem Auswahlprozeß liegen i.d.R. bereits bestimmte marktstrategisch relevante Entscheidungstatbestände zugrunde, die im Zuge der partnerschaftlichen Zusammenarbeit zu einer Strategie zusammengefaßt und implementiert werden.

\section{Marktstrategische Entscheidungen}

Die im Rahmen einer strategischen Allianz zu fällenden Entscheidungen lassen sich im wesentlichen in drei Hierarchieebenen unterteilen. Als marktstrategische Basisentscheidung ist die Zusammenlegung bzw. Abstimmung der Flugpläne auf multilateraler Ebene anzusehen. Diese Entscheidung ist von grundlegender Bedeutung, da erst dann der Umfang des gemeinsamen Leistungsangebotes (Flugplan) definiert und das Entscheidungsfeld eingegrenzt ist. Darauf aufbauend sind im Rahmen von marktstrategischen Detailentscheidungen die marktorientierten Systeminfrastrukturen wie Computerreservierungssysteme, Check-in-Systeme sowie Hintergrund-Systeme wie Gepäckrückverfolgung aufeinander abzustimmen, um einen Systembruch an den kundensensiblen Übergängen zwischen den beteiligten Fluggesellschaften zu vermeiden. An dieser Stelle ist auch die räumliche Nähe der Flughafen-Terminals der Partner zu nennen, deren idealtypische Realisierung oft jedoch nur mittel- bis langfristig möglich ist. Grund hierfür sind die baulichen Vorbedingungen sowie rechtliche Faktoren. Ein weiteres Beispiel für die notwendige Systemabstimmung ist die Verwendung von Buchungsklassen in den Reservierungssystemen. Viele Fluggesellschaften verwenden individuell verschiedene Buchungsklassen, die mit einem bestimmten Wert (Preis) verbunden sind. Im Fall einer Systeminkompatibilität wäre folglich das Flugticket einer bestimmten Buchungsklasse nicht auf die Partner-Airline übertragbar (Flugscheinvermerk "not endorsable") und liefe damit dem Ziel der Schnittstellenharmonisierung zuwider. Diese Subsysteme der Flugreise-Systeminfrastruktur ermöglichen einen aus Kundensicht 
"nahtlosen" Reiseverlauf ( Fluggesellschaften eine unabdingbare Voraussetzung zur Schaffung von Kundenzufriedenheit darstellt - im Sinne der Führungstheorien vergleichbar mit einem Hygienefaktor. Die kundenorientierte marktliche Durchsetzung des AllianzZusatznutzens setzt schließlich die Umsetzung der vorgenannten Entscheidungstatbestände voraus. Der kundenorientierte Zusatznutzen des Allianznetzwerks 1 soll vor dem Hintergrund abgestimmter Streckennetze und Systeminfrastrukturen vor allem durch die fluggastbezogene Durchabfertigung, die Harmonisierung der Vielflieger- und Statuskundenprogramme sowie die gegenseitige Loungenutzung erreicht werden.

Auf der operativen Ebene läßt sich am Beispiel der gegenseitig anerkannten Vielfliegerprogramme darstellen, wie die Beiträge der einzelnen Partner miteinander verrechnet werden. Verfliegt ein Fluggast beispielsweise die mit der Auskunftsairline A Vielflieger-Karte enworbenen Meilen mit einem Flug der Airline E, erhält Auskunftsairline A eine Rechnung von Airline $E$ und vice versa. Im einzelnen werden derartige Vorgänge in einer Saldenbilanz erfaßt und gesammelt, die einzelnen Strecken mit einem bestimmten cent-per-mile-Wert bewertet und am Ende des festgesetzten Abrechnungszeitraums wird der Differenzbetrag ermittelt (sog. Netto-Swap).

Die Implementierung der strategischen Entscheidungen erfolgt in Form wechselseitiger Abstimmungsprozesse, innerhalb derer die beteiligten Partnerunternehmen verschiedene Rollen einnehmen.

\section{Rollenverteilung in strategischen Allianzen}

Innerhalb der strategischen Allianz der beteiligten Unternehmen bilden sich bereits von Anbeginn, aber auch im Zuge der Zusammenarbeit unterschiedliche Rollen heraus, welche die beteiligten Unternehmen ausfüllen. Zu nennen ist zunächst die regionale Führungsrolle, die von den Fluggesellschaften in ihren jeweiligen Heimatmärkten übernommen wird. Im Fall des Allianznetzwerks 1 übernimmt Airline $E$ die Führungsrolle in den USA, Airline B in Kanada, Auskunftsairline A und Airline $C$ in Europa und sowie Airline $D$ im asiatischen Bereich. Neben diesen regional definierten Rollen beeinflußt die globale Vertriebsstärke, die beispielsweise aus dem hohen Beitrag einer Fluggesellschaft zum Verkehrsaufkommen der Allianz resultiert, die multilateralen Verhandlungen. Derartige implizite Machtasymmetrien können nicht wie im Fall von Fusionen oder Akquisitionen ausgenutzt werden, um eigene Ziele zu realisieren. Vielmehr ist der Verhandlungsprozeß zwischen gleichberechtigten Partnern ausschlaggebend für die Ent- 
scheidungsfindung. Nicht zu leugnen ist hingegen ein deutlicher Führungseinfluß eines oder zweier dominierender Unternehmen, wenn der durch eine bestimmte Entscheidung zu leistende Beitrag maßgeblich über demjenigen der anderen Partner liegt.

\section{Implementierung strategischer Allianzen}

Der Prozeß der Implementierung der marktstrategischen Entscheidungen vollzieht sich zunächst im Sinne eines „top-to-down-Ansatzes“ von der multilateralen Vorstandsentscheidung (Alliance Development Board) über das Alliance Development Committee (ADC) bis hin zur Ebene der Fachgruppen, die mit der operativen Umsetzung und Durchsetzung betraut sind. Bei Unstimmigkeiten zwischen den Fachgruppen werden analog im Sinne eines "bottom-up-Ansatzes" das ADC als klärende Instanz und in einigen Fällen auch das Alliance Development Board hinzugezogen. Die Entscheidungen, die im Rahmen einer Allianz zu fällen sind, lassen sich als Schnittmenge der jeweiligen Entscheidungsgesamtmenge der Partner abbilden. Die Entscheidungen der Schnittmenge können sich in bezug auf die Individualentscheidungen grundsätzlich indifferent, komplementär oder konfliktär verhalten. Im Falle konfliktärer Beziehungen ist zu entscheiden, wie die Prioritäten zwischen Allianz- und Individualentscheidungen festzulegen sind und welche Rahmenbedingungen das Entscheidungsfeld ggf. einengen.

Ein Beispiel für eine konfliktäre Schnittmengenentscheidung ist die Regelung der Mitnahme von Bordgepäck zwischen den Partnern Airline E und Auskunftsairline A. Während in den USA die Mitnahme von zwei Handgepäckstücken auch in der Economy Class üblich ist und von den Fluggesellschaften akzeptiert wird, untersagen europäische Luftverkehrsbehörden i.d.R. die Mitnahme von mehr als einem Bordgepäckstück. Für die kooperierenden Fluggesellschaften stellt sich an der Schnittstelle zwischen den USA und Deutschland die Frage, wie mit diesem Konflikt umgegangen wird. Eine Nivellierung auf den europäischen Standard würde für Airline $E$ in dieser Dimension Einbußen der Wettbewerbsfähigkeit auf dem inneramerikanischen Markt bedeuten; für Auskunftsairline A ergäben sich keine Auswirkungen. Eine Einigung auf den US-amerikanischen Standard wäre aus rechtlichen Gründen in Europa nicht möglich, bedeutete aber theoretisch eine Steigerung der Wettbewerbsfähigkeit für die Auskunftsairline A. Folglich muß das gemeinsame Ziel einer Schnittstellenharmonisierung gegenüber den Vor- bzw. Nachteilen des Status Quo abgewogen werden. Da es für das hier beschriebene Entscheidungsproblem kein Optimierungsmodell gibt, bedarf es des Improvisationsgeschicks des Boden- bzw. Kabinenpersonals im Umgang mit den betroffenen Fluggästen, um den entstehenden Systembruch abzumildern bzw. in Ausnahmefällen sogar zu heilen. 
Neben rechtlichen Bestimmungen stellt das finanzielle Volumen, das mit den Entscheidungen in Zusammenhang steht, einen bedeutenden Einflußfaktor allianzspezifischer Entscheidungen dar. Als erschwerend wirkt sich oft die Tatsache aus, daß erforderliche Investitionen - z.B. in die Umschulung von Mitarbeitern bei der Entscheidung für ein bestimmtes EDV-System des Partners - nicht symmetrisch auf die beteiligten Partner verteilt sind. In derartigen Entscheidungssituationen werden neben dem Umfang des finanziellen Volumens auch die Wichtigkeit der Entscheidung für die Nachfrager sowie die Auswirkungen auf das nicht-allianzspezifische Angebot berücksichtigt. Die hohe Komplexität solcher Entscheidungssituationen hat in der Vergangenheit oft zu einem Scheitern vor allem rein vertraglich ausgestalteter Allianzen geführt, so daß eine präzise Herausarbeitung und Operationalisierung kritischer Entscheidungstatbestände vor Eingehen einer strategischen Allianz von erfolgskritischer Bedeutung sind. Erfahrungen der Auskunftsairline A haben zugleich gezeigt, daß die vollständige rechtliche Absicherung einer strategischen Allianz aufgrund der Vielschichtigkeit auftretender Effekte nicht möglich ist. Daher ermöglichen die der Allianz zugrunde liegenden Verträge stets einen relativ einfachen Ausstieg aus der Partnerschaft, der ohnehin bei einem bestehenden Wunsch kaum vermeidbar wäre. Verträgen kommt vor allem eine protektionistische Funktion zu, die der präventiven Beschreibung von Konfliktfällen dient. Eine zu starke Orientierung der Partner am vertraglichen Regelwerk führte im Extremfall zur Handlungsunfähigkeit der Organisation.

Bei der unternehmensspezifischen Umsetzung und Durchsetzung der strategischen Allianzentscheidungen bei der Auskunftsairline A kommt dem Auskunftsairline A-internen Alliance Coordination Team eine funktionsübergreifende Koordinationsfunktion zu. Erschwert wird die Koordinationsaufgabe insbesondere dann, wenn der oben dargestellte Fall konfliktärer Zielwirkungen vorliegt, d.h. die Linienfunktionen eigene Ziele unter die Allianzziele - zumindest teilweise - unterordnen müssen. Daher ist den Allianzkoordinatoren ein hohes Maß an informierenden und motivierenden Aktivitäten zu eigen, um unternehmensinterne Durchsetzungsbarrieren zu senken. Wenn es gelingt, den Mitarbeitern den positiven Effekt einer allianzbezogenen Entscheidung für das Gesamtunternehmen gegenüber dem punktuellen Nachteil der betroffenen Funktion deutlich zu machen, ist bereits ein Großteil der notwendigen Überzeugungsarbeit geleistet. Die Akzeptanz der Koordinationsfunktion im Unternehmen Auskunftsairline A wird nicht zuletzt durch die hohe wirtschaftliche Bedeutung und den Markterfolg der strategischen Allianzen für die Auskunftsairline A erhöht. 
Im Zuge der Koordination der allianzspezifischen Aufgaben ist es wichtig, das den Entscheidungen zugrunde liegende Gesamtbild zu vermitteln, um das Verständnis für Zusammenhänge zwischen betrieblichen und allianztypischen Vorgängen zu fördern. Dieses Gesamtverständnis ist umso wichtiger, als die Verhandlungen zwischen den Allianzpartnern oft wechselseitige Zugeständnisse bedingen, die i.d.R. zu einem größeren Systemgewinn führen. Der Wunsch nach einer Absenkung der Prorates seitens eines Partners, der geringere Netzressourcen in die Partnerschaft einbringt, kann beispielsweise zunächst zu einem unmittelbarem Gewinnrückgang in dem betreffenden Streckenabschnitt der Auskunftsairline A und damit der zuständigen Abteilung führen. Falls dieser Wunsch jedoch mit einem Angebot verbunden ist, das per saldo den Gewinn der Auskunftsairline A insgesamt erhöht, wird dieses "Gegengeschäft" geschlossen, und es ist Aufgabe des Allianzkoordinators, den Nettoeffekt für das Gesamtunternehmen gegenüber der betroffenen Abteilung zu verdeutlichen.

Die Durchsetzung der Allianzziele auf der Mitarbeiterebene stößt neben rational begründeten Hemmnissen auf emotionale Barrieren, die im Fall der Auskunftsairline A vor allem in der traditionell hohen Loyalität begründet sind. Wie u.a. Erfahrungen mit gescheiterten Allianzen in der Vergangenheit gezeigt haben, empfanden viele Mitarbeiter die Zusammenarbeit mit ehemaligen Konkurrenten als illoyal und behinderten daher die Umsetzung der Allianzentscheidungen. Durch die positiven Erfahrungen mit den Allianzpartnern hat sich diese Mentalität in einem schrittweisen Prozeß spürbar geändert und zu einer größeren Offenheit gegenüber unternehmensexternen Organisationen geführt.

\section{Steuerung und Kontrolle in strategischen Allianzen}

Die Überprüfung des partnerschaftlichen Leistungsinputs in die Allianz vollzieht sich im Rahmen eines kontinuierlichen, wechselseitigen Prozesses. Werden nicht vereinbarte Ungleichgewichte oder Abweichungen von Zielvereinbarungen festgestellt, wird die Methode der moral suasion angewandt, um den bzw. die Partner wieder entsprechend auszurichten. Die moral suasion muß in besonderer Weise Rücksicht auf die kulturellen Unterschiede der Partner nehmen, da gerade die Äußerung von Kritik von hoher Sensibilität ist. Wird die Abweichung eines einzelnen Allianzpartners von den anderen Partnern in gleicher Weise diagnostiziert, erfolgt in multilateralen Gesprächen eine klare Adressierung des Problemgegenstandes, um zu einer Lösung desselben zu gelangen.

Von maßgeblicher Bedeutung für die Kontrolle und Steuerung der strategischen Allianz ist die Komplexität, die durch die Abstimmung der Allianzentscheidungen mit den 
Allianzpartnern sowie den eigenen Unternehmensentscheidungen entsteht. Neben den monolateralen, d.h. rein isolierten Auskunftsairline A-Entscheidungen sind bilaterale und schließlich multilaterale Entscheidungen zu koordinieren. Erhöht wird der Koordinationsumfang zudem durch die Netzeffekte, die durch die individuellen regionalen Allianzen der jeweiligen Allianznetzwerk 1-Partner entstehen. Seitens der Auskunftsairline A sind u.a. Allianznetzwerk 2 und Allianznetzwerk 3 zu nennen, seitens Airline E u.a. Airline E Express. Diese Komplexität erfordert eine hohe Informationsverarbeitungskapazität und -kompetenz der an der Koordination beteiligten Mitarbeiter, so daß der Einsatz von unterstützenden Instrumenten wie Intranet und standardisierten Protokollen erforderlich wird. Um den Koordinationsaufwand handhabbar zu halten, werden die individuellen regionalen Kooperationspartner zur Zeit noch nicht in das gesamte Allianz-System integriert, wenngleich ihre Existenz gleichsam als "virtuelles" Vorteilsargument durch die großen Fluggesellschaften genutzt wird.

Die interne Organisation der regionalen Partnerschaften liegt in der Kompetenz des Netzmanagement und ist nach regionalen Aspekten dezentralisiert. In Frankreich ist beispielsweise Airline $\mathrm{O}$, in Italien Airline $\mathrm{J}$ der jeweilige Partner. Kennzeichnend für diese Kooperationen ist u.a. der geringere Umfang einbezogener Funktionen der Linie, während das Allianznetzwerk 1 sämtliche Auskunftsairline A-Funktionen abdeckt. 


\section{Anhang 2}

\section{Zusammenfassung des Gespräches mit dem Leiter der Netzplanung der Auskunftsairline B am 4. September $1997^{2}$}

1. Gründe für strategische Allianzen mit regionalen Fluggesellschaften

2. Franchising als enge Form der strategischen Allianz

3. „Allianznetzwerk 1"-Konzept als eigenständige Allianz mit regionalen Partnergesellschaften

4. Einflußfaktoren auf die Formen strategischer Allianzen

5. Ausgestaltung des externen Marketing von strategischen Allianzen

6. Auswirkungen strategischer Allianzen auf die Kunden

\section{Gründe für strategische Allianzen mit regionalen Fluggesellschaften}

Als Gründe für den Einsatz eines Franchisekonzepts im Regionalluftverkehr waren für die Auskunftsairline B vor allem externe wettbewerbsbezogene und unternehmensinterne Faktoren ausschlaggebend. Die Airline $C$, einer der Hauptwettbewerber, drängte mit Franchiseangeboten in den europäischen Markt (z.B. Airline C Express), die sich durch hohe Flexibilität und niedrige Kosten auszeichneten. Diese Entwicklung wurde zum Anlaß genommen, über ein eigenes Auskunftsairline B-Franchising nachzudenken. Ausgangspunkt bildete die marktstrategische Überlegung, auch Strecken mit niedrigerem Verkehrsaufkommen sowohl in Deutschland als auch im angrenzenden Ausland zu bedienen, um die Zubringerfunktion in der eigenen Hand zu halten und gegenüber Wettbewerbern aus dem Ausland entsprechende Markteintrittsbarrieren in diesen Marktsegmenten zu errichten. Für den Flugbetrieb auf Strecken mit relativ geringen Entfernungen, niedrigem oder mittlerem Verkehrsaufkommen sowie flughafenbedingten Restriktionen bietet sich aus technischen Gründen der Einsatz von Turboprop-Fluggerät an. Dieses Fluggerät weist auf Kurzstrecken deutliche Stückkostenvorteile gegenüber JetFlugzeugen auf und eröffnet auch unter operativ kritischen Bedingungen (z.B. verkürzte Lande-/Startbahn) Einsatzvorteile. Da in der Auskunftsairline B-Konzernflotte vor Jahresfrist die letzten Turboprop-Maschinen gegen Jet-Flugzeuge ersetzt wurden, bot sich in der Strategischen Allianz mit kleineren Partnern in diesen Marktsegmenten eine geeignete Lösung.

2 Der Name des Gesprächspartners sowie die Namen der Fluggesellschaften sind aus Gründen der Vertraulichkeit anonymisiert. 


\section{Franchising als enge Form der strategischen Allianz}

Die Suche geeigneter Partner richtet sich nach wirtschaftlichen Zielen, wie Kostenniveau und finanzielle Stabilität des Carriers, als auch qualitativen Zielen, wie Zuverlässigkeit, Pünktlichkeit, Servicequalität, als auch nach dem Komfort des eingesetzten Fluggeräts. Darüber hinaus wird berücksichtigt, inwieweit eine Erweiterung des Streckennetzes mit Hilfe des Partners erzielt werden kann, damit dem Kunden weitere Auskunftsairline BProdukte/-Flugverbindungen angeboten werden können. Airline $D$ bot sich angesichts ihrer DASH-8-Flotte zur Bedienung der selektierten Strecken an und brachte angesichts einer regional fokussierten Bekanntheit die Bereitschaft mit, unter Auskunftsairline BMarkierung zu operieren. Die Form des Franchising gewährt dem Franchisenehmer - in diesem Fall Airline D - dabei ein hohes Maß unternehmerischer Selbständigkeit, die sich beispielsweise in der eigenverantwortlichen Kapazitätsplanung ausdrückt (Nutzung der lokalen Marktkompetenz). Für die Auskunftsairline B entstehen durch den Franchisevertrag Komplexitätskosten durch die Betreuung der Franchisenehmer. Im Gegensatz zu geübten, auf Erfahrungen beruhenden internen Abläufen wie beispielsweise beim Einsatz einer Auskunftsairline B-eigenen Boeing 737 entstehen durch den Betrieb einer DASH-8 bei einem externen Leistungserbringer höhere Prozeßkosten in Form von Abstimmungskosten. Diese höheren Prozeßkosten werden jedoch durch den Stückkostenvorteil und den marktstrategischen Vorteil der Nischenbesetzung ausgeglichen.

Die Gestaltung des Marktauftritts des Auskunftsairline B Franchising „Allianznetzwerk 2“ hat zum Ziel, eine möglichst große Nähe zu den Auskunftsairline BLeistungsstandards zu erreichen. Die wichtigste Maßgröße bildet zu diesem Zweck das innere Bild, das Auskunftsairline B-Kunden von einer Flugreise mit der Auskunftsairline B haben. Dieses innere Bild setzt sich aus einer Mehrzahl von Merkmalen zusammen, die das Leistungsangebot („Package ${ }^{4}$ ) der Auskunftsairline B beschreiben und zusammengefaßt die Erwartung der Kunden an die Dienstleistung widerspiegeln. Die Zufriedenheit der Flugreisenden mit der Leistung des Franchisenehmers hängt demzufolge auch davon ab, inwieweit die Erwartung von Auskunftsairline B-Standards durch die tatsächlich erlebte Dienstleistung durch den Franchisenehmer erfüllt wird.

Ein phasenspezifischer Vergleich offenbart Unterschiede zwischen dem Auskunftsairline B-Produkt und dem Franchising-Produkt. In den Auskunftsairline B-Flugplaninformationen weist ein entsprechender Vermerk zunächst daraufhin, daß bestimmte Flüge von PartnerFluggesellschaften durchgeführt werden. Erst ein Abgleich des Airline-Codes oder eine Nachfrage beim Reisemittler gibt Aufschluß über den Namen der Fluggesellschaft, die 
den Flug tatsächlich durchführt. Für Airline $D$ bringt diese Tatsache den unmittelbar ersichtlichen Vorteil des weltweiten Auskunftsairline B-Vertriebs mit sich. Durch den weltweiten Vertrieb im Computerreservierungssystem wird Reisebüros als auch anderen Absatzmittlern das Airline D-Produkt über die Auskunftsairline B-Flugnummer weltweit zur Verfügung gestellt. Darüber hinaus werden durch den Auskunftsairline B-Vertrieb verkaufsunterstützende Maßnahmen eingeleitet, z.B. Marketingkommunikation, Verkaufsaktionen und Vertriebsförderung. Die hier genannten Vertriebsleistungen, als auch Leistungen bzgl. Marketingkommunikation - wie Frequent Flyer-Programme, Werbung, Public Relations und vor allem die Nutzung der Marke Auskunftsairline B - sind aus Kundensicht die Kernmerkmale des Franchise-Konzepts. Der Check-In-Prozeß vollzieht sich an Auskunftsairline B-Schaltern, und am Flugsteig bieten die FranchisePartner dieselbe Leistungspalette an wie beim Auskunftsairline B-Produkt (Tageszeitungen, kalte Getränke, Kaffee/Tee). Das Fluggerät ist am Leitwerk mit dem Allianznetzwerk 2-Logo markiert (gelbe Sterne im Kreis auf dunkelblauem Grund) und trägt die Aufschrift „Allianznetzwerk $2^{4}$ in der Auskunftsairline B-Schriftart. Der Auftritt der Flugbegleiter sowie das Serviceangebot an Bord orientieren sich am Produkt des Auskunftsairline B Kont-Produkts, sind jedoch nicht identisch. Eine Standardisierung des Bordprodukts wird vor allem durch die Einklassen-Aufteilung (wie Kont-Produkt Auskunftsairline B) sowie den Einkauf der Cateringleistungen bei der Konzerngesellschaft Catering erreicht. Kundenbefragungen belegen, daß die Gesamtleistung der FranchisingFlugreise dem Auskunftsairline B Kont-Produkt sehr nahe kommt. Das Auskunftsairline B Kont-Produkt wird als Referenz-Maßgröße herangezogen, da das Auskunftsairline BKernprodukt ("Classic") eine Mischung aus Kontinental- und Interkontinental-Produkt darstellt und daher nur eingeschränkt mit ausschließlich regionalen Flugreisen zu vergleichen ist.

Kernstück der Vereinbarung zwischen Franchisegeber und -nehmer bildet das Leistungspaket der Auskunftsairline B, das dem Franchisenehmer zu Verfügung gestellt wird. Wichtige Eckpunkte sind:

- Vergabe einer Auskunftsairline B Code-Share-Flugnummer und damit Zugang zu weltweiter Vertriebsbasis

- Übernahme des Ground Handling und sonstiger Betreuungsaufgaben an allen betroffenen Flughäfen durch Auskunftsairline B

- Verhaltensmaßgaben durch die Auskunftsairline B im Kundenumgang (z.B. bei Gepäckverlust)

- Regelung der Durchabfertigung auf Auskunftsairline B-Anschlußflüge und damit Zugang zu weltweitem Streckennetz (z.B. Flug von Milwaukee nach Chicago (Airline 
E), Chicago nach Frankfurt (Auskunftsairline B) und Frankfurt nach Augsburg (Airline D))

- Anrechnung der mit Airline $D$ abgeflogenen Meilen auf das Vielfliegerprogramm der Auskunftsairline B

- Gewährung von system- und marketingbezogenen Beratungsleistungen (z.B. Einräumung der Nutzung von Flugplanbewertungsmodellen oder Revenue Management-Systemen, Produkt-Layout etc.)

Im Gegenzug entrichtet der Franchisenehmer für das inm gewährte Leistungspaket passagierbezogene Franchisegebühren, die sich aus den verschiedenen Leistungspaket-Komponenten zusammensetzen. Als Betrag wird entweder eine absolut pro Passagier festgesetzte Summe oder ein Prozentsatz von einer variablen Erfolgsgröße vereinbart. Die für jeden Franchisenehmer individuell definierte Franchisegebühr hängt wesentlich vom Umfang des eingebrachten Streckennetzes sowie insgesamt von der Verhandlungsmachtposition des Franchisenehmers ab.

\section{3. „Allianznetzwerk 1"-Konzept als eigenständige Allianz mit regionalen Partnergesellschaften}

Eine weitaus geringer standardisierte Form der Allianz stellt das Allianznetzwerk 1Konzept dar, unter dem Code Share-Verträge mit vorwiegend regional operierenden Fluggesellschaften abgeschlossen werden. Airline $F$ führt den eigentlichen Betrieb des Fluges durch, stellt das Personal und trägt das unternehmerische Risiko. Diese Kooperationsform entspricht dem Wunsch des kleinen Partners, der die eigene Unternehmens- und Markenidentität bewahren will, zumal kein eigenes Logo für das Allianznetzwerk 1-Konzept geschaffen wurde. Der Schriftzug im Auskunftsairline BDesign „Allianznetzwerk 1“ identifiziert den Partner jedoch eindeutig als Auskunftsairline B-Partner. Im Rahmen der Allianz mit Airline $F$ werden vorwiegend Flugreisende aus dem norditalienischen Einzugsgebiet nach München und vice versa befördert. Als günstig erweist sich auch in diesem Fall der Einsatz von ATR-42-Maschinen durch Airline F, da das Passagieraufkommen hauptsächlich aus kleineren Flughäfen generiert wird.

Der Wunsch nach Erhaltung der Eigenständigkeit hält regionale Fluggesellschaften mitunter davon ab, als Franchisenehmer im Allianznetzwerk 2 und damit in engerer Form als beim Allianznetzwerk 1-Konzept zu kooperieren. Airline $F$ beispielsweise lag das Angebot vor, im Allianznetzwerk 2 zu operieren. Das Management lehnte diesen Vorschlag jedoch ab, da ein zu großer Verlust der eigenen Identität befürchtet wurde. Der Fortbestand der Strategischen Allianz in Form des Allianznetzwerk 1-Konzepts blieb 
davon unberührt, zumal die Parallelmarkierung (Co-Branding) weiterhin eine Verbindung von regionaler Kompetenz (Airline $F$ gilt in Norditalien als guter Markenname) und globaler Kompetenz (Auskunftsairline B) signalisiert. Der Partner trägt demzufolge ein höheres unternehmerisches Risiko, die Marke Auskunftsairline B wird nicht ganzheitlich zur Verfügung gestellt, auch das Leistungspaket ist eingeschränkter und damit die Form der Zusammenarbeit weniger eng. Dementsprechend agiert der Partner eigenständiger.

Für die Allianzpartner ergeben sich im Fall des Allianznetzwerk 1-Konzepts andere Vertragsinhalte als im Fall des Franchising:

Marketing- und systembezogene Beratungsleistungen sind nicht eingeschlossen

- Auskunftsairline B erläßt keine Richtlinien hinsichtlich des Markenauftritts bzw. des Produkt- und Serviceniveaus

- Der Partner wird nicht berechtigt, die Marke Auskunftsairline B im Außenauftritt zu nutzen

Marketing- und Kommunikationsleistungen, die gezielt die individuelle Partnerschaft fördern, werden nicht geleistet

- Der Partner kann einen Vielfliegerprogramm-Vertrag abschließen. Dies bedeutet, daß der Partner - Z.B. Airline F - die Kosten eines Bonusfluges im Auskunftsairline B-Netz gemäß seines Anteils an den kumulierten Flugmeilen zu tragen hat. In der Praxis stellt sich dieses Prozedere in Form einer Saldenbilanz dar, in der die mit dem jeweiligen Vielfliegerprogramm geflogenen Meilen sowie der Bonusflug gegenübergestellt und aufgerechnet werden. Aufgrund der höheren Streckenattraktivität fällt diese Bilanz für die Auskunftsairline B i.d.R. günstiger aus, d.h. die Kosten des Bonusfluges, den ein Airline F-Fluggast im Streckennetz der Auskunftsairline B wahrnimmt, werden Airline F angelastet. Im Allianznetzwerk 2 wird die Teilnahme am Vielfliegerprogramm demgegenüber als festgesetzter Anteil an der passagierbezogenen Franchisegebühr abgerechnet.

Hinsichtlich der innengerichteten Führung gestaltet sich die Beziehung zu einem Allianznetzwerk 1-Carrier als eher unregelmäßig und relativ lose, während für das Allianznetzwerk 2 bilaterale Gespräche bei der Auskunftsairline B in ca. 2-3monatigen Abständen einen regelmäßigen Informationsaustausch mit den Franchisenehmern sicherstellen. Zudem wird in derartigen Meetings mit den Allianznetzwerk 2Fluggesellschaften diskutiert, welche Maßnahmen zu ergreifen sind, falls es Schwachstellen gibt. Die Interaktion zwischen Auskunftsairline B und Airline D beispielsweise erweist sich als wesentlich intensiver als im falle von anderen Agreements, die im Kern die zu erbringende Flugleistung von A nach B festschreiben und 
kaum weiteren Regelungsbedarf im laufenden Betrieb mit sich bringen. Meetings zwischen Auskunftsairline $B$ und Airline $F$ finden folglich in längeren Abständen - teilweise einmal jährlich - statt und haben vor allem die Beziehungspflege zum Inhalt. Angesichts der größeren Unabhängigkeit des Partners werden fachliche Aspekte zudem weniger deterministisch erörtert, d.h. Auskunftsairline B tritt eher als Diskussions- bzw. Sparringpartner auf.

\section{Einflußfaktoren auf die Formen strategischer Allianzen}

Neben der Eignung des Streckennetzes, das der Partner betreibt, sind folgende wesentliche Einflußfaktoren für die Allianzform Franchise wichtig:

- Qualitativer Standard des Partners, bezogen auf Zuverlässigkeit, Sicherheit, Service und Komfort des eingesetzten Flugzeugs.

- Wirtschaftliche Kriterien: Kostenniveau und finanzielle Stabilität/Kapitalgeber.

- Unternehmerische Führung, d.h. der Verkehrsbetrieb besteht länger als ein Jahr und es existiert ein unternehmerisches Führungssystem.

Ein Vergleich zwischen dem deutschen und britischen Markt offenbart in diesem Punkt zentrale strukturbedingte Unterschiede. Während die föderale Struktur in Deutschland zur Entstehung dezentraler Verkehrsknotenpunkte wie Hamburg, Düsseldorf, Köln, Frankfurt, Stuttgart, München und Berlin geführt hat, erweist sich der britische Luftverkehrsmarkt als wesentlich zentralistischer. Airline $C$ hat sich z.B. vor allem auf die Verkehrsdrehscheibe London konzentriert und bedient neben Zielen im Interkontinentalbereich sowie Kontinentaleuropa mit eigenem Fluggerät vor allem die Strecken London-Manchester vv. sowie London-Birmingham vv. Innerbritische Strecken mit geringerem Aufkommen wurden daher oft an kleinere Fluggesellschaften in Form des Franchising oder wet lease vergeben. Nicht zuletzt vor diesem Hintergrund hat Airline $C$ bis zu 11 Allianzpartner von und nach London, während Auskunftsairline B derzeit ausgewählte Städteverbindungen im Kontinentalbereich mit zwei Franchisepartnern (Airline $D$, Airline $G$ ) und vier Airlines im Allianznetzwerk 1-Konzept (Airline F, Airline $\mathrm{H}$, Airline I, Airline J) bedient.

In den USA bestehen im Vergleich dazu auch Allianzformen, bei denen kleine Fluggesellschaften lediglich eine Gebühr für den Zugang zum Computerreservierungsprogramm entrichten und das Fluggerät mit dem Namenszug des größeren Partners gestalten, ohne daß ein wet lease agreement oder ein Franchisingvertrag zugrunde liegt. Dies hat - nicht zuletzt aufgrund der geringeren Betreuungsleistungen des größeren Partners - höhere Fluktuationen unter den kleineren Fluggesellschaften zur Folge. Treten auf seiten der kleineren Partner Schwierigkeiten wie 
z.B. überhöhte Unpünktlichkeit oder Gepäckschäden auf, kann der größere Partner die Flugnummer und damit die Grundlage der Zusammenarbeit entziehen.

Eine Analyse des Streckennetzes am Beginn einer Partnerwahl beinhaltet auch wettbewerbsrechtliche Aspekte, da im Fall einer hohen Überschneidung der Streckennetze eine Wettbewerbsbeziehung zwischen den potentiellen Partnern vorliegt und damit Genehmigungspflicht für die strategische Allianz besteht. Ist die Strategische Allianz wettbewerbsrechtlich unbedenklich bzw. genehmigt, führt die für das Auskunftsairline B Kont-Produkt zuständige Konzerngesellschaft ein Produktaudit, ein technisches Audit und Flugbetriebsaudit durch. Bei Erfüllung der Qualitätskriterien gelangt das Allianzvorhaben per Vorstandsvorlage schließlich in den Auskunftsairline Binternen Entscheidungsprozeß $B$. Hinsichtlich der internen organisatorischen Anbindung werden die Allianzformen Allianznetzwerk 2 und Allianznetzwerk 1 derzeit vom Netzmanagement betreut. Es wurde keine eigenständige Organisationseinheit gebildet, da diese die Komplexitätskosten in einem nicht vertretbaren Maße erhöht hätte. Vielmehr setzt sich ein Team von Mitarbeitern derzeit schwerpunktmäßig mit dem wesentlich umfangreicheren und komplexeren Allianznetzwerk 3 und anderen internationalen strategischen Allianzen auseinander, das zukünftig auch das Kont-Produkt der Auskunftsairline B und die regionalen Allianzen (Allianznetzwerk 2, Allianznetzwerk 1) betreuen soll. Durch diese Teambildung unter der Bezeichnung "Netzplanung, Kooperation und Partnerschaft" soll eine höhere Integration der Aktivitäten in das gesamte Streckennetz erreicht werden. Eine Zusammenfassung dieser heterogenen Allianzformen ist möglich, da die zugrunde liegenden Geschäftsprozesse in allen Fällen gleich sind.

Durch die Partnerschaft der Auskunftsairline B in mehreren Allianzen entstehen mitunter Konflikte. Die von Airline $G$ im Code Share mit der Auskunftsairline B beflogenen Strecken tangieren z.B. teilweise Strecken, die traditionell von Airline $K$ - einem Allianznetzwerk 3-Partner der Auskunftsairline $B$ - bedient werden. In derartigen Fällen wird der Konflikt in bilateralen Gesprächen zwischen Auskunftsairline B und den betroffenen Allianzpartnern offen diskutiert und im Dialog gelöst.

\section{Ausgestaltung des externen Marketing von strategischen Allianzen}

Ausgangspunkt ist die Netzplanung, die - etwa vergleichbar mit der Produktionsprogrammplanung in Industriebetrieben - aufbauend auf geographischen sowie marktbezogenen und unternehmensinternen Daten festlegt, auf welchen Strecken mit welchen Kapazitäten geflogen werden soll. Dementsprechend finden sich in den an 
den Heimatmarkt Deutschland angrenzenden Staaten verschiedene regionale Partner, die im vertraglich vereinbarten Umfang Strecken für Auskunftsairline B bedienen. Zu nennen sind beispielsweise für Norditalien Airline $F$, für Frankreich Airline $H$ (potentieller Partner im Allianznetzwerk 1), im Benelux-Markt Airline J, Airline $G$ in Dänemark, Airline L in Großbritannien sowie Airline $\mathrm{K}$ in Skandinavien. Da sich die Größendimensionen bei den beiden letztgenannten Partnern beträchtlich von den kleineren Regionalfluggellschaften unterscheiden, werden diese Allianzen maßgeblich von dem Management des Allianznetzwerks 3 betreut.

Im Zuge der Teamarbeit ist zwecks Umsetzung der durch die Netzplanung geschaffenen Fakten in das externe Marketing eine Koordination mit den Fachbereichen erforderlich, die von der Allianz betroffen sind. Zu nennen seien beispielhaft die Fachbereiche für

- Reservierungssysteme sowie Vertrieb und Förderungsprogramme im Bereich der Distributionspolitik,

- Vielfliegerprogramm, Qualitätsaudit und Stationsmanagement im Bereich der Produktund Servicepolitik,

- Marketingkommunikation und Werbung.

Durch die Vergabe einer Gemeinschaftsflugnummer öffnet Auskunftsairline B den Allianzpartnern Zugang zu dem Computerreservierungssystem und schafft damit eine gemeinsame Vertriebsbasis. Eine Kontrolle über die Distributionsaktivitäten der Partner wird durch den zunehmenden Anteil von Online-Buchungen reduziert, da Endkunden dann direkt in Interaktion mit dem Leistungserbringer Flugscheine buchen.

Die Preis- und Tarifpolitik ist abhängig von der gewählten Kooperationsform. Beim wet lease werden die Auskunftsairline B-Preise und Konditionen vollständig übernommen, da Auskunftsairline $B$ auch das wirtschaftliche Risiko trägt. Beim Franchise werden die Auskunftsairline B-Tarifarten und die Konditionen übernommen. Die Auskunftsairline BPreise sind allerdings unverbindliche Preisempfehlungen, für die eine Zustimmung des Partners erforderlich ist. Durch die Einbindung in das globale Netz, die Zubringerfunktion, ist eine Angleichung an das Auskunftsairline B-Tarifsystem zu empfehlen. Im Code Sharing definiert jeder der beteiligten Partner für seine eigene Flugnummer die anzuwendenden Preise und Konditionen. Hier sind also Unterschiede zwischen dem Auskunftsairline B-Tarif- und Preissystem und dem Tarif- und Preissystem des Partners grundsätzlich möglich. 
Negative Auswirkungen auf den Markenwert der Auskunftsairline B durch eine Vielzahl unterschiedlicher Allianzpartner, die als Allianznetzwerk 2 oder Allianznetzwerk 1 markiert sind, sollen durch regelmäßige Qualitätsaudits vermieden werden. Kunden bewerten bei einem Code Share-Flug naturgemäß nicht die eigene Leistung der Auskunftsairline B, sondern vielmehr die Kompetenz der Auskunftsairline B bei der Auswahl leistungsfähiger Fluggesellschaften, die wiederum Auswirkungen auf das Markenimage der Auskunftsairline B hat. Dementsprechend ergeht bei einem Unterschreiten der Mindestqualitätsanforderungen eine Mahnung an den Partner, dessen Vertrag dann aufgelöst wird, wenn die Ursachen der Mängel nicht beseitigt werden.

\section{Auswirkungen strategischer Allianzen auf die Kunden}

Eine Analyse der Kundenreaktionen auf strategische Allianzen im Luftverkehr offenbart einen Lernprozeß, der sukzessive zu geringeren Überraschungsmomenten bei der Konfrontation mit diesem Phänomen führt. Die Praxiserkenntnisse zeigen, daß eine zunächst abwehrende Haltung gegenüber einer Allianzform wie dem Franchising oder dem wet lease sukzessive durch positive eigene Erfahrungen abgebaut werden kann. Die bereits zitierten Kundenbefragungen ergeben beispielsweise gerade im Servicebereich hohe Zufriedenheitswerte für die Franchisenehmer und damit auch die Auskunftsairline B. Zudem führt die Summe der Flüge zum Aufbau von Routinewissen bei den Fluggästen, so daß Überraschungseffekte oder Unsicherheiten im Sinne flugreiseablaufbedingter Probleme im Zeitablauf abnehmen werden, wie auch das Beispiel des USamerikanischen Marktes zeigt. Dort stellt der Einsatz auch kleinster, streckenadäquat eingesetzter Maschinen oder ein in bestimmten Grenzen variierender Außenauftritt von Allianzpartnern keine kundenseitige Akzeptanzbarriere dar.

Der Lernprozeß der Kunden findet demgemäß insbesondere bei den Stammkunden statt, die mengenmäßig ca. 10\%-15\% der Auskunftsairline B-Kunden ausmachen und dabei mehr als $50 \%$ des Bruttoumsatzes der Auskunftsairline B generieren. Vielflieger zeichnen sich durch einen besonders guten Informationsstand aus, der sich zum einen auf die flugreisebezogenen Details auswirkt - z.B. räumliche Lage des Gates, Name der operierenden Fluggesellschaft, Flugzeugtyp - und zum anderen den Umfang der innen zustehenden Leistungen umfaßt. Als wichtigstes Instrument zur Nutzung "verbriefter" Leistungen dient diesen Kunden die Kundenkarte, die in unterschiedlichen Ausprägungen - mit und ohne Status, Art des Status - unterschiedliche Anspruchsniveaus definiert (z.B. Gratisflüge bei erreichtem Meilenvolumen, standardmäßig bevorzugtes Einchecken, Priorisierung bei Wartelisten etc.). 


\section{Anhang 3}

\section{Zusammenfassung des Gespräches mit dem General Manager für Deutschland und Österreich der Auskunftsairline $\mathrm{C}^{3}$ am 17. September 1997}

1. Kooperationserfahrungen der Auskunftsairline $C$

2. Strategische Ausgestaltung der Auskunftsairline C-Airline D-Allianz

3. Operative Ausgestaltung der Auskunftsairline C-Airline D-Allianz

4. Barrieren der Harmonisierung

\section{Kooperationserfahrungen der Auskunftsairline $C$}

Die Liberalisierung des europäischen Luftverkehrsmarktes führte u.a. dazu, daß in der Branche geeignete Partner gesucht wurden, um die eigene Wettbewerbsfähigkeit zu stärken. Eine Bedrohung der Wettbewerbsfähigkeit wurde vor allem in dem Abbau bisher bestehender, nationalstaatlicher Markteintrittsbarrieren gesehen. Auskunftsairline $C$ initiierte Ende der achtziger Jahre gemeinsam mit Airline $E$ und Airline $F$ das Allianznetzwerk 1, das daraufhin mit der niederländischen Airline $G$ weiter ausgebaut werden sollte. Inhalt der Planungen war eine Fusion der vier beteiligten Fluggesellschaften (Projektname „Allianznetzwerk $2^{4}$ ), die im Sinne der USamerikanischen Entwicklung nach dem Carter Act von 1978 durch Zusammenlegung und gemeinsame Nutzung von Ressourcen die Leistungsfähigkeit steigern sollte. Die Erfahrungen im Zuge dieses ambitiösen Projektes ließen jedoch einmal mehr die Unterschiede zwischen den USA und Europa deutlich werden. Ein stark privatwirtschaftlich ausgerichteter Luftverkehrsmarkt in den USA war und ist bis heute nicht mit der von staatlichen Fluggesellschaften geprägten Situation in Europa vergleichbar. Dementsprechend waren Diskussionen um eine gemeinsame Namensgebung und Markierung der zu verschmelzenden Allianznetzwerk 2-Gesellschaft stark von nationalen Emotionen geprägt, die schließlich neben anderen Gründen ausschlaggebend für ein Scheitern von Allianznetzwerk 2 waren.

Zuvor hatte Auskunftsairline $\mathrm{C}$ bereits versucht, die belgische Airline $\mathrm{H}$ sowie die Airline $\mathrm{I}$ zu akquirieren. Dies geschah wiederum aus der Erkenntnis, bei einem wachsenden

3 Der Name des Gesprächspartners sowie die Namen der Fluggesellschaften sind aus Gründen der Vertraulichkeit anonymisiert. 
Wettbewerbsdruck nicht ohne starke Partner in Europa und auf anderen Kontinenten bestehen zu können. Diese Versuche scheiterten jedoch ebenso wie eine Allianz mit Airline J, die 1989 eingegangen wurde. Angesichts dieser Erfahrungen beschloß Auskunftsairline $C$ daraufhin, mit einer marktführenden europäischen Fluggesellschaft zu kooperieren. Als mögliche Partner boten sich demzufolge Airline $\mathrm{K}$ und Airline $\mathrm{D}$ an. Für die Wahl der Airline D sprachen vor allem das Marktvolumen dessen HeimatLuftverkehrsmarktes sowie dessen dezentrale bzw. föderale Struktur, die es der Airline $D$ zugleich erschwert, die fünf Hauptverkehrsflughäfen gegenüber ausländischen Wettbewerbern zu verteidigen. Im Gegensatz zu dieser Marktsituation wickelt sich in Großbritannien ca. $80 \%$ des Gesamtverkehrsaufkommens über London ab, und ähnlich verhält es sich in Frankreich mit den Strecken Paris-Lyon und Paris-Marseille. Neben diesen Kriterien ergänzte sich Skandinavien mit Deutschland zu einer geographischen Nord-Süd-Achse in Mitteleuropa, so daß die räumliche Marktabdeckung der beiden Fluggesellschaften eine hohe Komplementarität aufwies und damit Airline $D$ für Auskunftsairline $\mathrm{C}$ zum präferierten Partner wurde.

\section{Strategische Ausgestaltung der Auskunftsairline C-Airline D-Allianz}

Die Entscheidung und damit der Beginn der Planung der Allianz zwischen Auskunftsairline $C$ und Airline $D$ fiel vor eineinhalb Jahren bei einem Gedankenaustausch der Vorstandsvorsitzenden beider Unternehmen. Es wurde schnell deutlich, daß die Erwartungen an eine Allianz sehr homogen waren, so daß mit der Entscheidung für eine Kooperation schneeballartig ein Prozeß der Implementierung derselben in Gang gesetzt wurde. Ein wichtiges Bindeglied neben dem kulturellen Fit bildete der Wettbewerbsdruck, der beide Partner zur Allianz zusammengeführt hatte. Die gute bilaterale Beziehung zwischen Auskunftsairline $C$ und Airline $D$ führte sukzessive zu einer weiteren Vernetzung der Aktivitäten mit Airline L, Airline M, Airline $N$ und Airline O. Im Rahmen des Allianznetzwerks 3 fußen dennoch $70 \%$ der Geschäftstätigkeiten von Auskunftsairline C auf der Verbindung zu Airline D.

Die Arbeitsteilung zwischen den Allianznetzwerk 3-Partnern wird in Form des sogenannten Landlord- oder auch Lead Partner-Prinzips geregelt, das den jeweiligen Fluggesellschaften in deren Heimatmarkt die Wahrnehmung eines Großteils der flugreisebezogenen Aktivitäten für die Partner überträgt. Zwischen den Partnern werden jeweils für ein Jahr gültige Entscheidungskompetenzen verteilt, die sich auf die Wahrnehmung allianzübergreifender Funktionen wie beispielsweise Streckennetzplanung, Marketingkommunikation und Produktgestaltung beziehen. Durch die Rotation der Entscheidungskompetenzen sollen zum einen Erfahrungswissen im 
unternehmensübergreifenden Allianzmanagement gefördert und zum anderen dem Entstehen dauerhafter Machtungleichgewichte vorgebeugt werden. In Skandinavien beispielsweise werden die Arbeitsverträge der bisher von Airline $D$ angestellten Mitarbeiter Zug um Zug auf die Auskunftsairline $C$ transferiert. Gleiches gilt in umgekehrter Weise für die Auskunftsairline C-Mitarbeiter in Deutschland. Ab dem 1. Januar 1998 wird Auskunftsairline C zudem die Etatverantwortung für die Airline DUmsätze in Skandinavien übernehmen. In Deutschland ist Airline $D$ für die Abfertigung der Auskunftsairline C-Kunden an den sieben Stationen zuständig, an denen Auskunftsairline $C$ bisher vertreten war. Gleiches gilt für die sieben Reservierungsbüros der Auskunftsairline $C$ in Deutschland. In diesem Zuge wurden bisher insgesamt 100 Mitarbeiter von Auskunftsairline $C$ auf Airline $D$ übergeleitet.

Die strategische Zielsetzung sieht mittel- bis langfristig eine vollständige Integration der gemeinsamen Aktivitäten in den betroffenen Märkten vor. Lediglich ein Management Team wird jeweils in einem bestimmten Umfang Repräsentanz- und Schnittstellenfunktionen für Auskunftsairline $C$ in Deutschland bzw. Airline $D$ in Skandinavien übernehmen. Ab 1. Januar 1998 setzt Airline D jeweils ein derartiges Team in Norwegen, Schweden und Dänemark ein, während mittelfristig nur noch ein Team in Stockholm stellvertretend als Mittler zwischen dem Auskunftsairline C- und dem Airline DManagement fungieren soll.

In der Umsetzung gestaltet sich die Allianz zwischen Airline $D$ und Auskunftsairline $C$ wie ein Joint Venture. Die Umsatzerlöse des gemeinsamen Streckennetzes zwischen Deutschland und Skandinavien werden ebenso wie die Kosten im Verhältnis 50:50 geteilt. Damit ähnelt diese Kooperationsform den traditionellen Poolabkommen, stellt aber einen "revenue/cost-pool" dar, in dem Umsatzerlöse und Kosten bilanzartig gegenübergestellt und geteilt werden. Da diese Form der Abrechnung ein hohes Maß an Vertrauen voraussetzt, gilt zwischen den beiden Partnern eine sogenannte open book policy, d.h. die Höhe der anfallenden Kosten und deren Komponenten werden dem Partner im Zuge der gemeinsamen Planung zugänglich gemacht. Mit den anderen Allianznetzwerk 3Partnern besteht kein cost pool, so daß auch keine open book policy betrieben wird. Vielmehr dominieren hier Code Share-Flüge, bei denen die Streckenerlöse dem jeweiligen operating carrier zufließen.

Auch mit der Airline $D$ existieren neben dem Joint Venture weitere Code ShareAbkommen, in denen Flüge aus Kopenhagen zu Zielen in Afrika, dem Mittleren Osten oder Asien über die Airline D-Drehkreuze Frankfurt und München verteilt werden. Werden 
solche Code Share-Flüge aufgrund einer Umsteigeverbindung beispielsweise von beiden Partnern operiert, teilen sich die Fluggesellschaften den Ertrag des Fluges im Verhältnis der entsprechenden Streckenlänge (Prorating). Über die zusätzliche Gewährung eines Kommissionsbetrages für den initiating carrier, bei dem der Flug gebucht wurde, wird nachgedacht, um damit einen Teil der Vertriebskosten abzudecken.

\section{Operative Ausgestaltung der Auskunftsairline C-Airline D-Allianz}

Auf der Ebene der Absatzmittler - Reisebüros und Firmenkunden - nimmt Auskunftsairline $\mathrm{C}$ als Carrier am "P“-Programm teil, in dem das Konditionensystem der Airline $\mathrm{D}$ geregelt ist. Insgesamt beruhen mittlerweile ca. $60 \%$ aller Auskunftsairline C-Umsätze, die durch Verkehrsaufkommen zwischen Deutschland und Skandinavien erzielt werden, auf vollständig harmonisierten Abläufen und Preisen zwischen Airline D und Auskunftsairline C. Im Gegensatz dazu setzt Auskunftsairline $C$ auf anderen Strecken wie beispielsweise der Nordatlantikroute oder Fernost ein differenziertes Preissystem ein, das sich von demjenigen der anderen Allianznetzwerk 3-Partner unterscheidet. Eine derartige Preisdifferenzierung beruht auf der unterschiedlichen Wichtigkeit von Leistungsmerkmalen für die Kunden (Nutzensegmente). Während eine hohe kundenseitige Zeitsensibilität im Geschäftsreiseverkehr zwischen Skandinavien und Deutschland harmonisierte Preise auf Airline D-Niveau erlaubt, senkt Auskunftsairline C die Preise im Interkontinentalbereich unter das Niveau von Allianznetzwerk 3-Partnern wie Airline $L$ oder Airline $D$, da dann wiederum Produktmerkmale wie die Art des Fluggeräts, Komfort an Bord u.a. eine größere Bedeutung für die Kunden einnehmen und das Zielsegment der Auskunftsairline $C$ hier vor allem über preispolitische Maßnahmen angesprochen werden kann. Die Abstimmung der Preisniveaus in dem Allianznetzwerk 3 stell daher eine große Herausforderung dar, da zu große Preisdifferenzen dem Gedanken eines gemeinsamen Markendaches widersprechen und zu geringe Preisdifferenzen die Aufgabe bestimmter Kundensegmente zur Folge haben.

Nachfragerseitig belegen Befragungen, daß ca. 70\% aller Auskunftsairline C-Fluggäste über die Zusammenarbeit zwischen Airline $D$ und Auskunftsairline $C$ sowie die Integration der beiden Systeme informiert sind. Dieser Wert verdeutlicht, daß der Aufbau von Wissen über derartige Kooperationen bei den Kunden äußerst schwierig und mit zeitlichem Aufwand verbunden ist. Ähnlich verhält es sich mit der Etablierung der Dachmarke Allianznetzwerk 3, deren Bekanntheitsgrad noch nicht sehr hoch ist. Der Erfolg dieser Marke wird in hohem Maße von der gelungenen Kommunizierung des Kundennutzens abhängen. In dieser Hinsicht kommt der gegenseitigen Anerkennung der Vielfliegerprogramme zur Zeit eine zentrale Bedeutung $\mathrm{zu}$, verbunden mit der 
weltweiten Anerkennung von Statuskunden und dem Loungezugang. Mit dem sogenannten Around-the-World-Ticket hat das Allianznetzwerk 3 zudem ein Angebot in den Markt eingeführt, das die Leistungsfähigkeit und die allianzspezifischen Vorteile der verbundenen Fluggesellschaften demonstrieren soll. Dieses Ticket besitzt Gültigkeit für 600 Destinationen in 108 Ländern auf 5 Kontinenten, die von den Partnern angeflogen werden. Die Preise sind in drei Kategorien eingeteilt, die sich nach der Beförderungsklasse und der Entfernung richten, und die Flüge werden mit Bonuspunkten auf die Vielfliegerprogramme angerechnet.

Weitere Kundenreaktionen zeigen, daß die Auskunftsairline C-Fluggäste die Integration mit der Airline $D$ überaus begrüßen. Auf seiten der Firmenkunden wird demgegenüber Kritik an dem vergleichsweise hohen Preisniveau geäußert, das nach der Allianzbildung etabliert wurde. Andererseits stellen sinkende Transaktionskosten durch die Bündelung der Allianz-Fluggesellschaften im „P“-Vertrag einen absatzmittlerbezogenen Vorteil dar, der auch entsprechend positiv von den Reisebüros und Firmenkunden wahrgenommen wird. Derzeit sind Airline D, Auskunftsairline $C$ und Airline L sowohl im Agenten- als auch im Firmenkundenbereich in „P"-Verträgen integriert. Durch die Bündelung aller Allianznetzwerk 3-Partner in standardisierten „P“-Verträgen könnten bei vielen Firmen $90 \%$ der Reisebedürfnisse gedeckt und vereinfacht abgewickelt werden.

Zentrale Herausforderungen an das Allianzmanagement erwachsen auf operativer Ebene vor allem aus der Harmonisierung der Informations- und Abwicklungssysteme für Buchungen, Check-in-Prozeduren, Gepäckweiterleitung etc. Zugleich ergeben sich aus der Kooperation jedoch auch Vorteile, die vor allem im Einkauf unmittelbar wirksam werden. So konnten beispielsweise bei dem gemeinsamen Einkauf von 14.000 Personal Computern 10 Millionen DM eingespart werden. Gleiches gilt für den Kauf von Papier, den Auskunftsairline $\mathrm{C}$ auch für Airline $\mathrm{D}$ übernimmt. Weitere Einsparungspotentiale ergeben sich mittel- bis langfristig aus einer Vereinheitlichung der Flotte, so daß Ersatzteile günstiger beschafft und Synergien bei der gemeinsamen Wartung des Fluggeräts ausgenutzt werden können.

Derzeit gehen zehn Arbeitsgruppen der Frage nach, welche Möglichkeiten sich zu einer weiteren Vernetzung der Geschäftsaktivitäten in allen Funktionen bieten. Neben einem gemeinschaftlichen kommunikativen Auftritt, der in Deutschland maßgeblich von der Airline D geführt wird, bilden gemeinsame Preisaktionen mit der Airline D, die durch die kartellrechtliche Ausnahmegenehmigung zulässig sind, ein weiteres Beispiel für kooperative externe Marketingmaßnahmen, die - in Abhängigkeit von dem jeweils 
relevanten Markt - in Europa vor allem Airline $\mathrm{K}$, Airline $\mathrm{G}$ und Airline $\mathrm{P}$ sowie im Nordatlantik-Verkehr alle US-amerikanischen Carrier treffen sollen.

\section{Barrieren der Harmonisierung}

Hinsichtlich der Harmonisierung des Inflight-Produkts ist es das erklärte Ziel der Allianznetzwerk 3-Partner, auf den gemeinschaftlich beflogenen Strecken das jeweils beste Produkt anzubieten. Einer ganzheitlichen Harmonisierung werden auch in Zukunft weiterhin bestimmte Hemmnisse gegenüberstehen:

- Es bestehen Unterschiede in den Beförderungsklassen. Schon innerhalb des Airline D-Konzerns bietet das Airline D Kont-Produkt nur eine Klasse und das Kontinentalprodukt zwei Klassen an, während das Interkontinentalprodukt mit drei Klassen aufwartet. Auskunftsairline $C$ bietet demgegenüber auf allen Flügen zwei Klassen an und besitzt keine First Class.

- Die Flotten der Allianzpartner weisen bezüglich des eingesetzten Fluggeräts deutliche Unterschiede auf, die sich dementsprechend auf die Wahrnehmung des Fluges durch den Fluggast auswirken. Auf Kurz- und Mittelstrecken setzt Airline $D$ beispielsweise Maschinen des Typs Airbus 319, 320 und 321, Boeing 737 sowie Canadair und Avro ein, während Auskunftsairline $C$ überwiegend mit McDonnell Douglas 80 und 90 , DC-9 und Fokker operiert. An Schnittstellen mit Airline $L$ treffen Umsteigepassagiere wiederum auf Boeing 727, 737 und Airbus 320, die inneramerikanisch eingesetzt werden.

- Die Innenausstattung der eingesetzten Flugzeuge weist unternehmensspezifische Unterschiede auf, die Bestandteil der jeweiligen Corporate Identity sind. Zu nennen sind neben "harten" Faktoren wie der Sitzausstattung, der farblichen Ausgestaltung und der Bekleidung der Flugbegleiter auch "weiche" Faktoren wie Servicekultur im Umgang mit den Fluggästen, Umfang des Unterhaltungsangebots an Bord sowie Getränke- und Mahlzeitenangebot.

Ein deutscher Fluggast, der beispielsweise von Skandinavien nach Deutschland in der Business Class fliegt, erhält an Bord der Auskunftsairline $C$ derzeit eine deutsche Tageszeitung, während er auf einem Airline D-Flug zusätzlich auch zwischen verschiedenen Zeitschriften wählen kann. Harmonisierungsmaßnahmen bedeuten daher umfangreiche Detailarbeit und erfordern Zeit, bis das gewünschte Niveau erreicht ist. Dennoch erlangen die Partner des Allianznetzwerks 3 gegenüber den Wettbewerbern Zeitvorteile bei der Implementierung dieser Maßnahmen. Trotz der Harmonisierungsbestrebungen sind alle Allianzpartner an einer Bewahrung ihrer eigenen, auch länderkulturell geprägten Identität interessiert und stellen diese auch weiterhin 
offen heraus, so daß sich ein Zielkonflikt zwischen Harmonisierung und Differenzierung ergibt.

Bei der Durchsetzung der Harmonisierung auf Mitarbeiterebene sind vor allem arbeitsrechtliche Bedingungen zu beachten. Der Bildung "gemischter" Crews steht beispielsweise der Widerstand der Gewerkschaften gegenüber. Die Zusammenarbeit der Fluggesellschaften am Boden ist dagegen leichter durchzusetzen, wenngleich insbesondere in der Anfangsphase der Auskunftsairline C-Airline D-Allianz innere Widerstände der Mitarbeiter gegenüber einer Zusammenarbeit mit vormaligen Konkurrenten bestanden. Befürchtungen um den Verlust des eigenen Arbeitsplatzes durch die Zusammenlegung von Aktivitäten wurde mit der Garantie von Arbeitsplätzen am bisherigen Einsatzort begegnet. Zudem konnte in $90 \%$ aller Fälle auch das Gehaltsniveau aufrechterhalten werden. 


\section{Anhang 4}

\section{Record of the expert interview with the Director of Industry Affairs Answering Airline D, December 17, $1997^{4}$}

\section{Background of the Answering Airline D - Airline E alliance}

2. Internal relations in alliances

3. Customer orientation in strategic alliances

4. Marketing measures towards travel agents and firms

5. Marketing measures towards travelers

6. Competitive strategy

7. Monitoring the partnership

\section{Background of the Answering Airline D - Airline $E$ alliance}

The most important motive for Answering Airline $D$ to ally with Airline $E$ was the existence of mutual needs. Being a regional carrier, Answering Airline $D$ operates at a considerably lower cost level than Airline $E$ does concerning the service of regional routes. For instance, Airline E started to operate Munich-Trieste in 1988 in order to feed the Munich hub. This service turned out not to be profitable for Airline $E$ but at the same time it was strategically meaningful to maintain the feeder function of this city pair. At that time, Answering Airline $D$ already had the regional market competence and presence in northern Italy and appeared to be an adequate partner to take over the feeder function for Airline $E$ in the future. The German airline at the same time was able to offer access to its world-wide network with all its distribution power. As a result of this match Answering Airline D offered the opportunity to become Airline E's code-share partner.

Before the cooperation Airline E operated Munich-Triest vv. through Airline E's continental product using Fokker 50 aircraft twice a day. Subsequently Answering Airline D managed to generate more travel activities on this route and increased the number of flights from then two to three times a day operating aircraft of the ATR-42 type. The reason for this can be found in a better market penetration. About 45 per cent of the Triest-Munich traffic derives from Italy with the rest coming from abroad. While the 55 per cent of foreign

4 Der Name des Gesprächspartners sowie die Namen der Fluggesellschaften sind aus Gründen der Vertraulichkeit anonymisiert. 
traffic stagnated at its previous level Answering Airline D was able to increase the Italian traffic by exploiting the specifically regional market competence. For instance, Answering Airline $D$ offered more flexible schedules fitting better into the traffic structures in Munich. By this means Italian travelers with destinations in Europe could partly be drawn from the Italian competitor, Airline F operating from Milan, to the Triest based Answering Airline D which could offer the connection to a global network. Joining the Airline $E$ and Answering Airline D salesforce in northern Italy gave another momentum to the cooperation.

A similar development took place at the Verona-Munich route which saw an increase from one flight a day to three flights a day (workdays) and an additional Verona-Frankfurt connection. To put it in a nutshell, Airline $E$ benefits from Answering Airline D's feeder services and Answering Airline D benefits from Airline E's world-wide distribution power.

\section{Internal relations in alliances}

Partnering with other airlines brings up the question whether to standardize the common product or not. Basically, each airline has an own identity which is unlikely to be given up during the partnership. An exception might be the franchise concept "Team Lufthansa" in which the brand of the operating franchisee carrier diminishes. Product standards are tightly linked to the Airline $E$ continental product standard and only leave minor possibilities for an own identity based branding.

At the contrary Airline E nowadays accepts Answering Airline D's diverse identity and regards cultural differences between them as an asset rather than a problem. Partner specific differences become evident for the customer in all phases of interaction between traveler and airline. For instance, while ground handling for Answering Airline $D$ in Munich is taken over by Airline $E$ it is done the other way around in Triest. Furthermore, on board of Answering Airline $D$ there is a clear difference in the whole product which is branded as "settimocielo" and which sets concise quality and catering standards. At least one of the Answering Airline D flight attendants speaks the language of the partner carrier's home market, i.e. German.

Building a distinct market position with a strong brand identity requested continuous marketing investments. But these market investments are recognized to be the central point of difference compared to carriers like Airline G. While a market provider can offer aircraft and route capacity as well as a good market penetration, established brand proposition and local competence a capacity provider offers mainly aircraft and route 
capacity at a competitive price level. Thus a market provider can offer an added value in terms of marketing to any potential partner.

Answering Airline D sees its role as a market provider as an important arm to protect itself against substitution. The company's competence in the Italian market led for example to initiating a Verona-Paris connection which in the beginning had been evaluated critically by Airline $\mathrm{E}$. Nowadays it proves to be a very profitable route and complements the Airline E network. Similar objections might exist against other potentially interesting destinations. Verona-Brussels for instance could cause feeder effects to the hub of Airline E's competitor Airline $\mathrm{H}$. This effect would have to be compared to the net revenue it would add to the partners' network. It becomes evident that the joint planning process has to be open minded, rational and based on mutual trust.

Apart from bilateral relations between Answering Airline $D$ and Airline $E$ there are contacts with other members in the alliance like Airline $G$ or Airline I. These contacts emerge for instance from European Regional Airlines conferences where carriers of similar size have the opportunity to meet each other and exchange experiences. Although it is often thought about a closer cooperation in the field of maintenance most carriers prefer to stay on their own in the end. Few examples can be seen - Airline $\mathrm{J}$ entered a cooperation to have its SAAB 2000 maintained by another airline.

On the operating level, at the beginning of the cooperation with Answering Airline D, Airline $E$ implemented their own customer service index at Answering Airline $D$ in order to measure the quality level of the Italian partner. The costs for this instrument were borne by Airline $\mathrm{E}$. Since the results showed quality figures above the 85 per cent benchmark in a period of two years Airline $E$ decided to give up this measure of control in December 1996. In May 1997 Answering Airline D re-implemented this instrument on its own with the same questions and proceedings. Results of the monthly quality monitor showed a very good perceived overall flight impression and cabin quality. Airport and booking information was ranked rather moderately at the same time. The latter issues are difficult to overcome since neither ground handling in Munich nor booking procedures in the German market can be controlled adequately by Answering Airline D. Central airport-related problems which are often mocked by customers in Munich have been poor information policy concerning delays and mishandling of baggage. Those facts do have negative impact on Answering Airline D's image. 
Answering Airline D does not employ people in handling processes in Munich so it is difficult to influence this quality dimension directly. Therefore it was important for Answering Airline $D$ to know about the cooperation awareness among their passengers which lies around an increasing percentage of 55-60 per cent between May and October 1997 compared to an average of 53 per cent in 1996.

Concerning the participation in Airline E's frequent flier program the internal procedures follow a determined pattern. Answering Airline $D$ is requested to pay a certain amount for the miles which are flown on Answering Airline $D$ by use of the frequent flier card. For instance, the Triest-Munich wv. connection adds up to 2000 miles which are booked on the customer's frequent flier account. Airline $E$ receives a monetary equivalent for this by Answering Airline $D$. When it comes to burn the miles the traveler gets his incentive flight for free on the Airline $E$ network. Answering Airline $D$ receives an internally prorated sum by Airline $E$ if the traveler uses an Answering Airline $D$ flight for his free travel. While Airline $E$ by this means capitalizes its global route network Answering Airline $D$ benefits from participating in an existing frequent flier program.

\section{Customer orientation in strategic alliances}

For Answering Airline $D$ the customer plays the key role in planning a cooperation. Consequently an airline alliance has to offer an added value to its customers which have to be divided into travelers, travel agencies and major firms. Added values mainly derive from the following customer benefits:
a) additional frequencies
b) additional destinations
c) better fare structure
d) frequent flier program with multiple options

Regarding points a) and b) the above mentioned examples have already demonstrated the effect of cooperating with each other. Lower fares of point c) can be achieved by offering online tickets which are not regulated by IATA (.gray market“). Traveling from Triest to Denver and back for example would then cost DM 1.200,- compared to a regular fare of approximately DM 1.800 ,- in the Italian market. Triest-Denver would include the cooperation between three partners, namely Answering Airline D (Triest-Munich), Airline $\mathrm{E}$ (Munich-Frankfurt-Chicago) and Airline K (Chicago-Denver). While EU deregulation measures allow the publication of such fares and thus enable customers to perceive this price benefit, in the non-EU business customers have a lower chance to perceive those 
advantages. Nevertheless, word-of-mouth communication among travelers must not be underestimated. Undoubtedly the price is a most important buying criterion.

Since the beginning code-share flights have again and again provoked different reactions of travelers. Negative reactions result in dissatisfaction caused by expectations which remain unfulfilled. Travelers booked on a certain airline expect if experienced a certain product consisting of individually perceived, lived and remembered items. Such items can be found along the travel chain during which the traveler interacts with different companies, among them the airline itself. If these expectations are not met the customer rests surprised. This might happen along a code-share flight when the traveler finds himself in the hands of a partner airline. After the moment of surprise it depends on the partner's product whether the customer turns satisfied or dissatisfied. Answering Airline $D$ 's experiences show that Airline $E$ customers appreciate the Answering Airline D product.

\section{Marketing measures towards travel agents and firms}

In order to avoid any worry causing surprise effect the alliance partners pursue a dual strategy directed to both travelers and travel agents/firms. Answering Airline $D$ uses an information policy emphasizing a high degree of transparency. In this effort travel agencies play a key role since they use and transmit the information given by airlines to their customers. Consequently there is a constant flow of information between the airline's sales force and the travel agent. Distribution is organized due to the country, i.e. in Germany Answering Airline D is integrated within the "PP-System" which contains terms and conditions for travel agents and firms. In Italy Airline $E$ is marketed through Answering Airline D's overriding contract on the jointly offered routes. Commission is paid by the operating carrier so Answering Airline $D$ pays for Triest-Munich and Airline $E$ pays for the same travelers from Munich to outbound destinations. The exchange of distribution cost figures is done regularly.

One major issue regarding the work of travel agents is the competence towards their customers. An empirical research done on this topic back in 1995 revealed a serious lack of knowledge about code-share flights on the side of employees in travel agencies. Possible reasons lie in the lack of time caused by stress situations in customer interactions as well as in code-share specific issues. Travel agencies receive their flight information by computer reservation systems that show the airline code and the flight number. Code-share flights are marked additionally by an asterix or a different sign so that the agent can recognize it as a code-share flight. The question which carrier actually 
operates the flight can sometimes only be answered by additional search activities in the system or in flight schedules.

Franchise agreements for example only show the franchiser's code which is marked by an asterix in the AMADEUS system. The computer reservation system would show the Augsburg-Frankfurt route as follows:

$\begin{array}{llllll}1^{*} & \text { LH9621 } & \text { AGB } & \text { FRA } & 0605 & 0710 \\ 2^{*} & \text { LH9623 } & \text { AGB } & \text { FRA } & 0950 & 1055 \\ 3^{*} & \text { LH9625 } & \text { AGB } & \text { FRA } & 1330 & 1435 \\ 4 & \text { LH9627 } & \text { AGB } & \text { FRA } & 1745 & 1850\end{array}$

The fourth flight even does not carry the asterix and thus suggests that Airline $E$ (code LH) operates this flight at its own. Flights $1^{*}$ to $3^{*}$ cause additional effort to find out the name of the actually operating carrier which is Airline G. Code-share agreements like Answering Airline D-Airline $E$ at the contrary show two different airline codes for one and the same flight number. In the Triest-Munich example it is as follows:

$\begin{array}{llllll}\text { 1: } & \text { LH9086 } & \text { TRS } & \text { MUC } & 0800 & 0910 \\ 2 & \text { EN9086 } & \text { TRS } & \text { MUC } & 0800 & 0910\end{array}$

This flight is only operated by Answering Airline D (code EN) but appears in the first line as a Airline $E$ code-share flight (code $\mathrm{LH}$ ) marked by : . It becomes evident that the complexity of code-sharing is an information barrier uneasy to overcome by the airlines. Answering Airline D applies information campaigns every two to three weeks through its sales force.

In corporate business Answering Airline D grants special fares both on their flights and on Airline $E$ flights to important key accounts. For instance, a Genoa-based key customer of Answering Airline D receives special fares on Genoa-Munich-Moscow. Answering Airline D operates Genoa-Munich and persuaded Airline $E$ to grant a special tariff on Munich-Moscow because by cooperation this customer could be drawn away from the competitor Swissair. 


\section{Marketing measures towards travelers}

Referring to travelers Answering Airline D tries to respect different expectations towards traveling. These expectations can be expressed by benefit structures that prevail in the traveler's mind. These benefit structures are yet influenced by several factors like the distance of a flight and whether it is a connecting flight or a city pair. For instance, Answering Airline $D$ is respecting the flexibility issue by adapting its schedules to Airline E's hub structure and the passengers' needs. On medium haul flights travelers tend to ask for comfortable seating, diversified food and beverage more than they do on short haul flights. These needs are met by constantly renewing and replacing the ATR-42 fleet currently several ATR-42 320 have been replaced by the new ATR-42 500 type. The new types operate mainly on Answering Airline D's medium haul flights like Verona-Parigi and Verona-Barcelona and feature much more comfortable seats as well as a reduced noise level on board.

Furthermore the price is set at a competitive level in order to satisfy the price oriented customer segment. By figures around 35 to 45 per cent of Answering Airline D's customers make up business travelers paying full fares. These travelers are characterized by their flight patterns that leave only little flexibility. At the contrary leisure travelers show much less rigid time restrictions in planning their trip so that measures like minimum stay or book in advance in connection with lower prices are adequate to target them. It has to be respected though that business travelers turn into leisure travelers and vice versa every now and then.

Within the cooperation with Airline $E$ the German partner does the pricing for the Munich destination. Also the flights operated by Answering Airline $D$ from Verona to Paris and Verona to Barcelona are done by code-share with Airline $E$ and add up to Airline E's worldwide route network. Thus a better market penetration without building up own Answering Airline $D$ sales forces in France or Spain is achieved by offering additional city pairs to Airline $E$ that does not need to build up own flight operations. The mutual benefit for both partners can be seen easily.

Additionally Answering Airline $D$ takes part in the frequent flier program which is an attractive add-on since Answering Airline $D$ has not had an own frequent flyer program before the cooperation. This marketing tool raises customer loyalty and supports plans to widen the network of Answering Airline D. Italian travelers are drawn on frequent flier by on board announcements which inform about the number of miles that can be collected 
by the actual flight. Flight attendants provide customers with application forms during the flight if asked for.

\section{Competitive strategy}

According to Answering Airline D's philosophy it is hardly possible to keep up a sustainable competitive advantage which is only cost or price related. It seems rather essential to assure a market leadership on as many origin-destination markets as possible in order to compensate possible price attacks by cross subsidizing the attacked route. Moreover a market leadership has to be built upon different customer related benefits such as number and quality of frequencies, access to lounges, frequent flier programs and others.

Airline alliances have to fulfill existing needs better than their competitors do. This implies competition between alliances and single operating carriers as well as competition between alliances. At the same time competition between the alliance partners themselves in some areas of their cooperation has to be taken into account. Compared to earlier years alliances provide a product which is shaped to the needs of a seamless travel. Single operating carriers then had to interline with other airlines and still have to nowadays. Such interline agreements result in trips in which flights are connected rather by chance than by purpose. Travelers are forced to accept waiting times at airports and have restricted options choosing alternative connections. Luggage - by few exceptions cannot be checked through to the final destination with a traditional interline ticket.

At the contrary partners in an alliance try to coordinate their schedules in order to minimize waiting times for customers. In practice it means to harmonize arrivals and departures within certain time banks or knots. This coordination is done for each airport in which partners cooperate. Actually every airline alliance functions this way and makes it difficult to achieve a sustainable competitive advantage. Again creating market power by building dense partner networks turns out to become the most meaningful strategic issue. Competitive alliance forces arise in Italy mainly by the recently formed Airline L-Airline $F$ alliance which is likely to be integrated into the well established Airline L-Airline $M$ alliance. Concerning the above mentioned example travelers could choose a flight from Malpensa to Minneapolis which is the hub of Airline $M$ and on to Denver again. This strategic move immediately puts the Triest-Denver connection under competitive pressure. 
The Airline E-Answering Airline D alliance has never faced any antitrust law problems since there has never been any joint capacity cutting or price increasing behavior. At the contrary both partners even increased capacities and provided a wider range of travel opportunities to their customers.

\section{Monitoring the partnership}

Monitoring concerns both external and internal results of the cooperation. An important tool in order to monitor customer benefits is the above mentioned customer service index. Internal outcomes of the partnership are measured by the monthly financial results. These results are monitored route by route but the cooperation effect is not measured separately. The reason for this is the high degree of integration of the cooperation in Answering Airline D's daily business and undoubtedly the partnership with Airline E plays a big role in it. Partnership issues are discussed in bilateral talks with Airline $E$ headquarters every now and then although one would prefer a more regularly established kind of meeting. This fact is recognized to be a rather weak point within the cooperation. Contents of those bilateral meetings are analyzing figures, short term and mid term planning and further developmental steps.

Aside the headquarters contacts Answering Airline $D$ makes use of having interaction with the Italian based Airline $E$ representatives every week. The contact is held mainly with a Airline $\mathrm{E}$ expatriate situated in Venice who is responsible for Northeast Italy. 


\section{Anhang 5}

\section{Grundzüge des Kaufverhaltens im buying center von Firmenkunden}

Firmenkunden treten als Käufer von Flugscheinen auf, wenn beispielsweise Verkaufsverhandlungen im Ausland die Nutzung des Verkehrsmittels Flugzeug erforderlich machen. Die erworbene Flugdienstleistung fließt in den Produktionsprozeß des Unternehmens ein. Damit handelt es sich bei der Flugdienstleistung um eine investive Dienstleistung. Der investive Charakter tritt noch durch weitere Merkmale zu Tage. Die Nachfrage nach dem Flugschein kann als abgeleitete Nachfrage ${ }^{5}$ bezeichnet werden. Zudem sind i.d.R. mehrere Personen innerhalb der Firmenkundenorganisation an der Beschaffung beteiligt (Endnutzer, Sekretariat, Firmen-Reisestelle u.a.), der Beschaffungsprozeß orientiert sich häufig an formalisierten Richtlinien (hier speziell: Reiserichtlinien) und es sind oft weitere Organisationen wie Reisebüros in den Beschaffungsprozeß eingeschaltet, der daher auch als organisationales Beschaffungsverhalten bezeichnet wird. $^{6}$ Darüber hinaus weisen investive Dienstleistungen auch auf Anbieterseite die Merkmale der Multiorganisationalität und Multipersonalität auf und sind zudem durch ein erhöhtes Maß an interaktiver Vermarktung gekennzeichnet. $^{7}$

Unter Berücksichtigung dieser Besonderheiten haben sich verschiedene Forschungsansätze um die modellhafte Erfassung des organisationalen Beschaffungsverhaltens bemüht. ${ }^{8}$ In Anlehnung an das von Webster und Wind (1972) entwickelte Rollenkonzept ${ }^{9}$ können auch bei Firmenkunden unterschiedliche Rollen beim

5 Die Nachfrage kann als abgeleitet bezeichnet werden, da der Beschaffung der Flugdienstleistung eine andere Nachfrage vorgelagert ist. Dies wird aus dem Beispiel der Verkaufsverhandlungen deutlich, welche die Nachfrage nach einer Flugdienstleistung erzeugen.

6 Vgl. Ritzerfeld, U., Marketing-Mix-Strategien in Investitionsgütermärkten: Entwicklung und Simulation marktstrukturspezifischer Strategien, Wiesbaden 1993, S. $7 \mathrm{f}$.

7 Vgl. Backhaus, K., Industriegütermarketing, 5., erw. und überarb. Aufl., München 1997, S. 3-5. Im weiteren hebt Backhaus die Internationalität und Bedeutung von Dienstleistungen für Industriegüter hervor, die im hier vorliegenden Zusammenhang nahezu per se auch für Flugdienstleistungen kennzeichnend sind, sowie den staatlichen Einfluß auf den Beschaffungsprozeß, der in Form von gesetzlichen Regelungen hier ebenfalls gegeben ist.

$8 \quad \mathrm{Zu}$ einem Überblick über ausgewählte Ansätze zur Erfassung und Erklärung des organisationalen Beschaffungsverhaltens vgl. Backhaus, K., Industriegütermarketing, 5., erw. und überarb. Aufl., München 1997, S. 49ff. Backhaus konstatiert, daß Kaufverhaltensansätze wie Z.B. das S-O-R-Modell nicht zweckadäquat für die Erklärung von Problemen des Industriegütermarketing sind.

9 Vgl. Webster, F.E., Wind, Y., Organizational Buying Behavior, Englewood Cliffs 1972, S. $78 \mathrm{ff}$. 
Kauf von Flugdienstleistungen identifiziert werden. In Abbildung 1 ist eine solche Rollenverteilung im Beschaffungsgremium eines Firmenkunden exemplarisch dargestellt. ${ }^{10}$

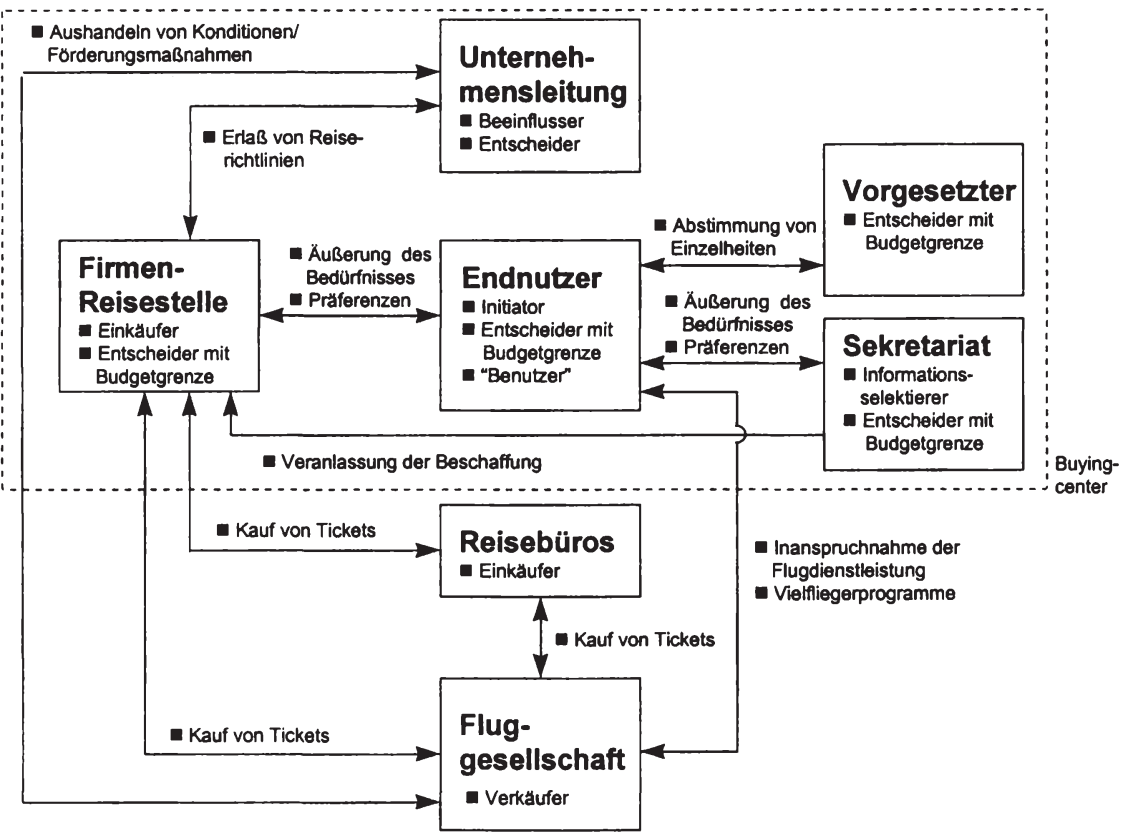

Abb. 1: Zusammensetzung des Buying Center bei Firmenkunden von Fluggesellschaften

Es ist anzunehmen, daß zunächst bei einem Mitarbeiter - in der Abbildung als "Endnutzer" dargestellt - innerhalb der Organisation des Firmenkunden das Bedürfnis entsteht, in persönlichen Kontakt mit einem Geschäftspartner zu treten, der an einem anderen Ort befindlich ist. Dieser Mitarbeiter setzt als "Initiator" den organisationalen Beschaffungsprozeß in Gang. ${ }^{11}$ Als "Benutzer"12 der Flugdienstleistung kommt inm im

10 Die Rollenverteilung des Buying Centers bildet nur einen Teil des situativen Kontextes ab, der bei organisationalen Beschaffungsvorgängen zu berücksichtigen ist. Backhaus (1997) bezieht Umwelteinflüsse, Organisationsmerkmale, Kauftyp, Beschaffungsprozeß sowie die Zusammensetzung der Verkaufsgremien („selling center“) in den Kontext mit ein. Vgl. Backhaus, K., Industriegütermarketing, 5., erw. und überarb. Aufl., München 1997, S. 52.

11 Die Rolle des Initiators wird von Bonoma (1982) in dem Konzept von Webster und Wind ergänzt. Vgl. Bonoma, T.V., Major Sales: Who Really Does the Buying? in: Harvard Business 
Verlauf des Beschaffungsprozesses insbesondere dann eine Schlüsselstellung zu, wenn er aufgrund seiner hierarchischen Position im Unernehmen oder in der öffentlichen Institution seine individuellen Präferenzen bei der Wahl der Fluggesellschaft durchsetzen kann. Im Luftverkehr besteht über Vielfliegerprogramme zudem eine direkte Bindung zwischen Endnutzer und Fluggesellschaft, die von hoher Bedeutung für die Beschaffungsentscheidung ist. Als „Beeinflusser“ der Beschaffungsentscheidung kann eine leitende Organisationseinheit auftreten, die über die Formulierung von Reiserichtlinien Vorschriften für die Auswahl von Verkehrsmitteln und Anbietern festlegt. ${ }^{13}$ Die Rolle des „Entscheiders“, der aufgrund seiner Machtposition letztlich über die Beschaffung bestimmt, wird häufig auf der Unternehmensleitungsebene angesiedelt. ${ }^{14} \mathrm{Je}$ nach firmenkundenspezifischer Reisehäufigkeit und je nach Wert der einzelnen Flugdienstleistung dürtten im vorliegenden Fall auch nachgelagerte Organisationseinheiten in festgelegten Budgetgrenzen über die einzelne Beschaffung entscheiden. Demnach sind Vorgesetzte, die Geschäftsreisenden selbst sowie Sekretariate und Firmen-Reisestellen in variierendem Umfang als Entscheider vorstellbar, was sich in der Abbildung durch die gesetzten Klammern ausdrückt. ${ }^{15}$ Sekretariaten kommt darüber hinaus die Rolle von "Informationsselektierern“ zu, die im Beschaffungsprozeß Aufgaben der Vorauswahl zwischen Verkehrsmitteln und Anbietern übernehmen und durch die (Nicht-)Weitergabe von Informationen einen indirekten Einfluß

Review, Vol. 60, No. 3, 1982, S. 113ff. Vorstellbar ist auch die Anordnung einer Geschäftsreise durch den Vorgesetzten, der dann die Rolle des Initiators inne hătte.

12 Die Übersetzung des von Webster und Wind (1972) verwendeten Begriffs „User" in „Benutzer" ist inhaltlich als deckungsgleich mit dem im Rahmen dieser Arbeit eingesetzten Begriff "Endnutzer" anzusehen.

13 Reiserichtlinien stellen ein formalisiertes Regelwerk dar, das einen Rahmen für die Nutzung aller mit einer dienstlich veranlaßten Reise zusammenhängenden Produkte und Dienstleistungen festlegt. Die Inhalte variieren i.d.R. in Abhängigkeit vom jeweiligen Unternehmen oder der jeweiligen Institution, umfassen jedoch oft wirtschaftlichkeitsorientierte Regeln zur Wahl des Verkehrsmittels, der Unterbringung und der Obergrenze von Reisenebenkosten. Zu einer beispielhaften Ausgestaltung von Reiserichtlinien vgl. Haas, S., Mitarbeiter auf die gleiche Spur bringen, in: fww, Nr. 11, 1998, S. $36 f$. Vgl. Backhaus, K., Industriegütermarketing, 5., erw. und überarb. Aufl., München 1997, S. 65.

15 Eine 1994 vom Spiegel-Verlag dokumentierte Studie über das Geschäftsreiseverhalten von Deutschen ergab, daß in durchschnittlich 30 Prozent der erfaßten Fälle die Entscheidung über die Wahl der Fluggesellschaft vom Reisenden selbst getroffen wurde. In weiteren 7 Prozent der Fälle konnten die Reisenden zwar selbst entscheiden, mußten sich jedoch an vorgegebenen Restriktionen orientieren. $\mathrm{Zu} 13$ Prozent wurde die Auswahlentscheidung vom Vorgesetzten getroffen, in 6 Prozent der Fălle wăhlten das Sekretariat oder andere Mitarbeiter des Reisenden die Fluggesellschaft aus. Reisestellen waren in 3 Prozent der Fälle Entscheider über die Fluggesellschaft. Vgl. Spiegel-Verlag (Hrsg.), Spiegel-Dokumentation: Geschäftsreisen 1994, Hamburg 1994, S. 83. Diese Ergebnisse beinhalten jedoch lediglich bekundete Verhaltensweisen, die durch ein am Prestige orientiertes Antwortverhalten verzerrt sein können („ich entscheide selbst“), da die Reisenden befragt wurden. 
auf die Entscheidung ausüben können. Als „Einkäufer" der Flugdienstleistung agieren i.d.R. die Firmen-Reisestelle mit direktem oder indirektem (über Reisebüros) Kontakt zur Fluggesellschaft sowie Reisebüros, die im Auftrag des Firmenkunden handeln.

Es ist davon auszugehen, daß die am Beschaffungsprozeß beteiligten Rollenträger unterschiedliche Anforderungen an das Angebot von Fluggesellschaften haben, da Leistungsangebote jeweils aus der spezifischen Rollenperspektive ${ }^{18}$ beurteilt werden. Während Unternehmensleitung, Firmenreisestelle sowie Vorgesetzter vornehmlich an „hard facts" wie Flugtarifhöhe, Terminkompatibilität des Flugplans mit demjenigen des Mitarbeiters sowie zuverlässigen Buchungsmöglichkeiten interessiert sein dürtten, ${ }^{17}$ belegen Untersuchungen, daß die Endnutzer im buying center - von den Fluggesellschaften als Geschäftsreisende bezeichnet - eine im Vergleich zu Privatreisenden geringe Preiselastizität aufweisen. Die Ursache für diesen Befund ist leicht ersichtlich, da das arbeitgebende Unternehmen und nicht der Endnutzer selbst den Preis für den Flugschein entrichtet. ${ }^{18}$ Privatreisende sind demgegenüber nicht einen investiven Kontext eingebunden, sondern lassen die Annahme von Flugdienstleistungen als konsumtive Dienstleistung im Vordergrund stehen.

16 Das Verhalten von Rollenträgern wird in der Sozialpsychologie als Prozeßergebnis des Sendens und Empfangens von Rollen interpretiert. Demnach bilden sich bestimmte Rollenerwartungen, die mit dem Ziel der Beeinflussung an den Rollenempfänger kommuniziert werden („role sending“). Dieser setzt die an inn gerichteten Erwartungen in Abhängigkeit von seiner Wahrnehmung ( stark rollengeprăgt sein kann („role behavior"). Organisatorische Einflußgrößen sowie Persönlichkeitsfaktoren und interpersonale Beziehungen beeinflussen diesen Prozeß, in dem dynamische Rollenentwicklungen sowie Inhalte von Rollensendungen nicht abgebildet werden. $\mathrm{Zu}$ einer Darstellung vgl. Staehle, W.H., Management: eine verhaltenswissenschaftliche Perspektive, 7. Aufl., München 1994, S. 255-257.

Bei dieser Einschătzung handelt es sich lediglich um eine Expertenmeinung.

Pompl (1991) führt Elastizităten für Privat- und Geschäftsreisende an, die beispielsweise zwischen 1962 und 1975 bei Abflügen aus Großbritannien bei 2,2 bis 2,6 (Privatreisende) versus 0,9 (Geschäftsreisende) lagen. Vgl. Pompl, W., Luftverkehr: eine okonomische Einführung, 2., aktualisierte und erw. Aufl, Berlin u.a. 1991, S. 101. 


\section{Literaturverzeichnis}

Abell, D.E., Defining the Business: The Starting Point of Strategic Planning, Englewood Cliffs 1980.

Adam, D., Planung und Entscheidung: Modelle - Ziele - Methoden, 4., vollst. überarb. u. wesentlich erw. Aufl., Wiesbaden 1996.

Ahlert, D., Distributionspolitik. Das Management des Absatzkanals, 3. Aufl., Stuttgart 1996.

Alchian, A.A., Specifity, Specialization, and Coalitions, in: Journal of Institutional and Theoretical Economics, Vol. 140, 1984, S. 34-49.

Aldrich, H., Whetten, D.A., Organization-sets, action-sets, and networks: making the most of simplicity, in: Handbook of organizational design, Vol. 1, Hrsg.: Nystrom, P.C., Starbuck, W.H., Oxford u.a. 1981, S. 385-405.

Altwegg, R., Strategiebewertung und Ermittlung des Synergiewertes bei Kooperationen: ein strategischer Kooperationsbewertungsansatz, Basel 1995.

Andritzky, K., Die Operationalisierbarkeit von Theorien zum Konsumentenverhalten, Berlin 1976.

Angehrn, O., Markenpolitik, in: Handwörterbuch der Absatzwirtschaft, Hrsg.: Tietz, B., Stuttgart 1974, Sp. 1227-1234.

Arnold, D., The handbook of brand management, Reading 1992.

Aulakh, P.S., Kotabe, M., Sahay, A., Trust and Performance in Cross-Border Marketing Partnerships: A Behavioral Approach, in: Journal of International Business Studies, Special Issue, 1996, S. 1005-1032.

Backhaus, K., Büschken, J., Voeth, M., Internationales Marketing, 2., überarb. und erg. Aufl., Stuttgart 1998.

Backhaus, K., Hahn, C., Das Marketing von investiven Dienstleistungen, in: Handbuch Dienstleistungsmanagement: von der strategischen Konzeption zur praktischen Umsetzung, Hrsg.: Bruhn, M., Meffert, H., Wiesbaden 1998, S. 93-114.

Backhaus, K., Industrial marketing - State of the art in Germany, in: Industrial Marketing, Hrsg.: Backhaus, K., Wilson, D.T., Berlin 1986, S. 3-14.

Backhaus, K., Industriegütermarketing, 5., erw. und überarb. Aufl., München 1997.

Backhaus, K., Investitionsgüter-Marketing - Theorieloses Konzept mit Allgemeinheitsanspruch? in: zfbf, 44. Jg., Nr. 9, 1992, S. 771-791. 
Backhaus, K., Meyer, M., Strategische Allianzen und strategische Netzwerke, in: WiSt, Nr. 7, 1993, S. 330-334.

Backhaus, K., Piltz, K., Strategische Allianzen - eine neue Form kooperativen Wettbewerbs? In: Strategische Allianzen, Hrsg.: Backhaus, K., Piltz, K., Sonderheft Nr. 27 der Zfbf, 1990, S. 1-10.

Backhaus, K., u.a., Multivariate Analysemethoden: eine anwendungsorientierte Einführung, 8., verb. Aufl., Berlin u.a. 1996.

Backhaus, M., Voeth, M., Strategische Allianzen - Herausforderungen neuer Kooperationsformen, in: Stabilität und Effizienz hybrider Organisationsformen, Hrsg.: Wagner, H., Jäger, W., Münster 1995, S. 63-83.

Balderjahn, I. Marktreaktionen von Konsumenten. Ein theoretisch-methodisches Konzept zur Analyse der Wirkung marketingpolitischer Instrumente, Berlin 1993.

Ballantyne, T., Walker, K., Thai is hardly Star choice, in: Airline Business, No. 7 , 1997, S. 12.

Barney, J.B., Gaining and Sustaining Competitive Advantage, Reading, Mass. u.a. 1997.

Baron, S., Harris, K., Services Marketing. Text and Cases, Houndsmill u.a. 1995.

Barton, C., u.a., Is there a future for Europe's airlines? in: The McKinsey Quarterly, No. 4, 1994, S. 29-40.

Barzen, D., Marketing-Budgetierung, Frankfurt/Main u.a. 1990.

Bass, F.M., Pessemier, E.A., Lehmann, D.R., An Experimental Study of Relationships between Attitudes, Brand Preference and Choice, Institute Paper No. 307, Krannert Graduate School of Industrial Administration, Purdue University, Layette 1971.

Becker, J., Konzeptionelle Grundfragen der Positionierung, in: Positionierung: Kernentscheidung des Marketing, Hrsg.: Tomczak, T., Rudolph, T., Roosdorp, A., St. Gallen 1996, S. 12-23.

Becker, J., Typen von Markenstrategien, in: Handbuch Markenartikel: Anforderungen an die Markenpolitik aus Sicht von Wissenschaft und Praxis, Hrsg.: Bruhn, M., Stuttgart 1994, S. 463-498.

Berndt, R., Kooperative Werbung - Organisation, Planung und Vorteilhaftigkeit kooperativer Werbemaßnahmen, in: WiSt, Nr. 1, 1985, S. 1-7.

Beyhoff, S., Ehmer, H., Wilken, D., Code-Sharing im internationalen Luftverkehr der Bundesrepublik Deutschland, Forschungsbericht Nr. 95-23 der Deutschen Forschungsanstalt für Luft- und Raumfahrt e.V., Köln 1995. 
Beyhoff, S., Vielfliegerprogramme und der Wettbewerb im Luftverkehr, Mitteilung Nr. 94-02 der Deutschen Forschungsanstalt für Luft- und Raumfahrt, Köln 1994.

Birkelbach, R., Qualitätsmanagement in Dienstleistungscentern: Konzeption und typenspezifische Ausgestaltung unter besonderer Berücksichtigung von Verkehrsflughäfen, Frankfurt am Main u.a. 1993.

Birkigt, K., Stadler, M.-M., Funck, H.J., Corporate Identity: Grundlagen, Funktionen, Fallbeispiele, 7. Aufl., Landsberg/Lech 1994.

Blancke, W., Evolution und strategische Allianzen: Der Einfluß von strategischen Allianzen auf den Wettbewerb, Bayreuth 1994.

Blankenburg Holm, D., Eriksson, K., Johanson, J., Business Networks and Cooperation in International Business Relations, in: Journal of International Business Studies, Special Issue, 1996, insb. S. 10331053.

Blattberg, R.C., Deighton, J., Aus rentablen Kunden vollen Nutzen ziehen, in: Harvard Business Manager, 19. Jg., Nr. 1, 1997, S. 24-32.

Bleicher, K., Das Konzept integriertes Management, 4., rev. und erw. Aufl., Frankfurt/M., New York 1996.

Bleicher, K., Der Strategie-, Struktur- und Kulturfit Strategischer Allianzen als Erfolgsfaktor, in: Wegweiser für Strategische Allianzen: Meilen- und Stolpersteine bei Kooperationen, Hrsg.: Bronder, C., Pritzl, R., Wiesbaden 1992, S. 267-292.

Bleicher, K., Marketing im Spannungsfeld von Wettbewerbs- und Potentialorientierung, in: Marktorientierte Unternehmensführung: Reflexionen - Denkanstöße - Perspektiven, Hrsg.: Bruhn, M., Steffenhagen, H., Wiesbaden 1997, S. 38-55.

Bleicher, K., Organisation: Strategien - Strukturen - Kulturen, 2., vollständig neu bearb. und erw. Aufl., Wiesbaden 1991.

Bliss, C., Integriertes Komplexitätsmanagement - Ansätze und Lösungsmöglichkeiten, Arbeitspapier Nr. 115 der Wissenschaftlichen Gesellschaft für Marketing und Unternehmensführung e.V., Hrsg.: Meffert, H., Backhaus, K., Münster 1998.

Bongartz, U., Unternehmensspezifische Ressourcen und strategische Gruppen im US-Luftverkehrsmarkt, in: ZfB, 68. Jg., Nr. 4, 1998, S. 381-407.

Bonoma, T.V., Major Sales: Who Really Does the Buying? in: Harvard Business Review, Vol. 60, No. 3, 1982, S. 111-119.

Bonus, H., The Cooperative Association as a Business Enterprise: A Study in the Economics of Transactions, in: Zeitschrift für die gesamte Staatswissenschaft, 142. Jg., 1986, S. 310-339. 
Borys, B., Jemison, D.B., Hybrid arrangements as strategic alliances: Theoretical issues in organizational combinations, in: Academy of Management Review, Vol. 14, 1989, S. 234-249.

Botschen, G., Mühlbacher, H., Zielgruppenprogramm - Zielgruppenorientierung durch Nutzensegmentierung, in: Handbuch DienstleistungsMarketing, Band 1, Hrsg.: Meyer, A., Stuttgart 1998, S. 681-692.

Brockhoff, K., Produktpolitik, 3. Aufl., Stuttgart u.a. 1993.

Bronder, C., Pritzl, R., Ein konzeptioneller Ansatz zur Gestaltung und Entwicklung strategischer Allianzen, in: Wegweiser für strategische Allianzen: Meilen- und Stolpersteine bei Kooperationen, Hrsg.: Bronder, C., Pritzl, R., Frankfurt am Main 1992, S. 17-44.

Bronder, C., Pritzl, R., Leitfaden für strategische Allianzen, in: Harvard Manager, 13. Jg., Nr. 1, 1991, S. 44-53.

Bruhn, M. (Hrsg.), Handbuch Markenartikel: Anforderungen an die Markenpolitik aus Sicht von Wissenschaft und Praxis, Stuttgart 1994.

Bruhn, M., Begriffsabgrenzungen und Erscheinungsformen von Marken, in: Handbuch Markenartikel, Stuttgart 1994, S. 3-41.

Bruhn, M., Integrierte Kommunikation als Unternehmensaufgabe und Gestaltungsprozeß, in: Effizientes Kommunikations-Management, Hrsg.: Bruhn, M., Dahlhoff, H.D., Stuttgart 1993, S. 1-33.

Bruhn, M., Internes Marketing als Forschungsgebiet der Marketingwissenschaft, in: Internes Marketing. Integration der Kunden- und Mitarbeiterorientierung, Hrsg.: Bruhn, M., Wiesbaden 1995, S. 13-61.

Bruhn, M., Kommunikationspolitik: Grundlagen der Unternehmenskommunikation, München 1997.

Bruhn, M., Multimedia-Kommunikation: systematische Planung und Umsetzung eines interaktiven Marketinginstruments, München 1997.

Bruhn, M., Georgi, D., Kundenbezogene Wirtschaftlichkeitsanalyse des Qualitätsmanagements für Dienstleistungen: Konzept, Modellrechnung und Fallbeispiel, in: Marketing ZFP, Nr. 2, 1998, S. 98-108.

Bühner, R., Betriebswirtschaftliche Organisationslehre, 6., verb. und erg. Aufl., München und Wien 1992.

Ceyp, M., Call Center als Querschnittsinstrument im Marketing, in: IfM-News, Nr. 1, 1998, S. 19-24.

Ceyp, M., Ökologieorientierte Profilierung im vertikalen Marketing dargestellt am Beispiel der Elektrobranche, Frankfurt am Main u.a. 1996.

Chandler, A., Strategy and Structure: Chapters in the History of the Industrial Enterprise, Cambridge, Mass., London 1984 (13., unveränd. Nachdruck der 1. Aufl. von 1962). 
Churchill, G.A., Suprenaut, C., An Investigation into the Determinants of Customer Satisfaction, in: Journal of Marketing Research, Vol. 19, No. 11, 1982, S. 491-504.

Cohen, A., Transatlantische Allianz zwischen British Airways und American Airlines, Neuer Vorstoß auf Europas Luftverkehrsmärkte, in: fuw, Nr. 14, 1996, S. 70.

Corsten, H., Stuhlmann, S., Zur Mehrstufigkeit in der Dienstleistungsproduktion, in: Handbuch Dienstleistungsmanagement: von der strategischen Konzeption zur praktischen Umsetzung, Hrsg.: Bruhn, M., Meffert, H., Wiesbaden 1998, S. 142-162.

Curry, R.E., Revenue management using consumer choice models, in: Aeronomics Incorporated, First Quarter, 1994, S. 1-6.

Daudel, S., Vialle, G., Yield-Management: Erträge optimieren durch nachfrageorientierte Angebotssteuerung, Frankfurt/Main, New York 1992.

Davidow, W.H., Malone, M.S., The virtual corporation: Structuring and revitalizing the corporation for the $21^{\text {st }}$ century, New York 1992.

Deiß, G., Aufgaben und Gestaltungsprobleme betrieblicher Reisestellen, in: Betriebswirtschaftliche Studien zum Personenverkehr und Tourismus, Hrsg.: Brauer, K.M., Berlin 1985, S. 83-101.

Dieckmann, R., Optimale Preis- und Angebotspolitik für komplexe Produkte: eine Analyse der Güterbündelung unter Berücksichtigung der Verarbeitung von Preisen durch Konsumenten, Frankfurt am Main u.a. 1993.

Diegruber, J., Erfolgsfaktoren nationaler europäischer Linienluftverkehrsgesellschaften im Markt der 90er Jahre, St. Gallen 1991.

Dill, W.R., Environment as an influence on managerial autonomy, in: Administrative Science Quarterly, Vol. 3, 1958, S. 409-443.

Diller, H., Deutsche Lufthansa AG - Marktsegmentierung im Luftfahrtgeschäft, in: Fallstudien zum Marketing, Teil 1: Entscheidungssituationen, Hrsg.: Dichtl, E., Wiesbaden 1979, S. 206-238.

Diller, H., Kommunikationspolitik, in: Vahlens Großes Marketinglexikon, Hrsg.: Diller, H., München 1992, S. 546-548.

Diller, H., Preisehrlichkeit - Eine neue Zielgröße im Preismanagement des Einzelhandels, in: Thexis, 14. Jg., Nr. 2, 1997, S. 16-21.

Doganis, R., Flying off Course: The Economics of International Airlines, 2. Aufl., London, New York 1991. 
Dollinger, M.J., Golden, P.A., Saxton, T., The Effect of Reputation on the Decision to Joint Venture, in: Strategic Management Journal, Vol. 18, No. 2, 1997, S. 127-140.

Drayton, J.L., Fahad, G.A., Tynan, A.C., The Focus Group: A Contoversial Research Technique, in: Graduate Management Research, Winter 1989, S. 34-51.

Dreher, A., Marketingorientierung als Unternehmensphilosophie: Phänomen und empirische Erfassung, Wiesbaden 1995.

Dussauge, P., Garrette, B., Determinants of success in international strategic alliances: evidence from the global aerospace industry, in: Journal of International Business Studies , Third Quarter 1995, S. 506.

Dyer, D., Schlesinger, L., Northwest Airlines: Strategic Alliance and Strategic Position, Harvard Business School Case, Boston, Mass. 1997.

Ebers, M., Gotsch, W., Institutionenökonomische Theorien der Organisation, in: Organisationstheorien, Hrsg.: Kieser, A., 2., überarb. Aufl., Stuttgart, Berlin, Köln 1995, S. 185.

Eggen, B., Markenmanagement am Beispiel der SAirGroup, in: Thexis, Nr. 3, 1998, S. 27-28.

Ehmer, H., Ein Jahr Binnenmarkt im Luftverkehr, in: Internationales Verkehrswesen, Nr. 9, 1994, S. 512-517.

Eisele, J., Erfolgsfaktoren des Joint-Venture-Management, Wiesbaden 1995.

Engelhardt, W.H., Kleinaltenkamp, M., Reckenfelderbäumer, M., Leistungsbündel als Absatzobjekte: Ein Ansatz zur Überwindung der Dichotomie aus Sach- und Dienstleistungen, in: zfbf, 45. Jg., Nr. 5, 1993, S. 395-426.

Enzweiler, T., Wo die Preise laufen lernen, in: Manager Magazin, Nr. 3, 1990, S. 246-253.

EU-Kommission (Hrsg.), Wirkung und Wirksamkeit des Binnenmarktes, Dokumenten-Nr. MEMO/96/103, Luxemburg 1996.

Fahad, G.A., Group discussions: a misunderstood technique, in: Journal of Marketing Management, Vol. 1, No. 3, 1986, S. 315-327.

Feldman, J.M., Getting serious on pricing, in: Air Transport World, No. 10, 1994, S. 56-60.

Feldman, J.M., Making Alliances Work, in: Air Transport World, No. 6, 1998, S. 2735.

Flint, P. Will four go into two? in: Air Transport World, No. 6, 1998, S. 39-42.

Flint, P., Will the real United please stand up? In: Air Transport World, No. 8, 1997, S. 26-34.

Franko, L.G., Joint venture survival in multinational corporations, New York 1971. 
Frentz, M. H., Ex Occidente Nox? "Corporate Restructuring, Mergers \& Acquisitions" vs. strategischer Allianzen in der liberalisierten Luftverkehrsindustrie, in: Journal für Betriebswirtschaft , 43. Jg., Nr. 1, 1993, S. 20-44.

Frese, E., Grundlagen der Organisation: Konzept - Prinzipien - Strukturen, 6., überarb. Aufl., Wiesbaden 1995.

Freter, H., Marktsegmentierung, Stuttgart 1983.

Freyer, W., Tourismus: Einführung in die Fremdenverkehrsökonomie, 3., erg. und aktualisierte Aufl., München, Wien 1991.

Friedrich, H., Görgen, W., Marktsegmentierung auf Dienstleistungsmärkten dargestellt am Beispiel des Marktes für Flugdienstleistungen, Arbeitspapier des Instituts für Marketing- und Distributionsforschung, Köln 1993.

Gahl, A., Die Konzeption strategischer Allianzen, Berlin 1991.

Gallacher, J., Airline Alliances: Partners for now, in: Airline Business, No. 6, 1997, S. 26-67.

Gallacher, J., Hold your horses, in: Airline Business, No. 6, 1998, S. 42-81.

Gallacher, J., Power to the plans, in: Airline Business, No. 8, 1997, S. 34-37.

Gälweiler, A., Unternehmenssicherung und strategische Planung, in: zfbf, 28. Jg., 1976, S. 362-379.

Gellman Research Associates (Hrsg.), A Study of International Airline Code Sharing, Washington 1994.

General Accounting Office (Hrsg.), International Aviation: Airline Alliances Produce Benefits, but Effect on Competition is Uncertain, Report No. 95-99, Washington 1995.

Ghemawat, P., Sustainable Advantage, in: Harvard Business Review, Vol. 64, No. 5, 1986, S. 53-58.

Gierl, H., Stich, A., Strohmayr, M., Einfluß der Glaubwürdigkeit einer Informationsquelle auf die Glaubwürdigkeit der Information, in: Marketing ZFP, 19. Jg., Nr. 1, 1997, S. 27-31.

Giesen-Netzer, I., Implementierung von Rücknahme- und Recyclingsystemen bei Gebrauchsgütern, Frankfurt am Main u.a. 1998.

Gomes-Casseres, B., The alliance revolution: the new shape of business rivalry, Cambridge, London 1996.

Göpfert, I., Interlining, Code-Sharing und Trucking, in: WiSt, Heft 9, 1994, S. 460462.

Green, P.E., Hybrid Models for Conjoint Analysis: An Expository Review, in: Journal of Marketing Research, No. 5, 1984, S. 155-169. 
Green, P.E., Srinivasan, V., Conjoint Analysis in Consumer Research: Issues and Outlook, in: Journal of Consumer Research, Vol. 5, No. 9, 1978, S. 103-123.

Greenbaum, T.L., The handbook for focus group research, New York 1993.

Grenier, R., Metes, G., Enterprise networking: working together apart, o.O., 1992.

Gruner+Jahr (Hrsg.), MARIA-Studie Luftverkehr, Hamburg 1997.

Gulati, R., Alliances and Networks, in: Strategic Management Journal, Vol. 19, No. 4, 1998, S. 293-317.

Gutsche, J., Produktpräferenzanalyse. Ein modelltheoretisches und methodisches Konzept zur Marktsimulation mittels Präferenzerfassungsmodellen, Berlin 1995.

Haas, S., Auch die Briten halten die Reisebüros jetzt kurz, in: fuw, Nr. 27, 1997, S. 11.

Haas, S., Jetzt sind auch noch die Firmenkunden sauer, in: fuw, Nr. 27, 1997, S. 8-9.

Haedrich, G., Tomczak, T., Produktpolitik, Stuttgart u.a. 1996.

Hahn, C., Conjoint- und Discrete Choice-Analyse als Verfahren zur Abbildung von Präferenzstrukturen und Produktauswahlentscheidungen. Ein theoretischer und computergestützter empirischer Vergleich, Münster 1997.

Hahn, D., PuK, Controllingkonzepte: Planung und Kontrolle, Planungs- und Kontrollsysteme, Planungs- und Kontrollrechnung, 5., überarb. und erw. Aufl., Wiesbaden 1996.

Håkansson, H. (Hrsg.), Industrial marketing and purchasing of industrial goods An interaction approach, New York 1982.

Håkansson, H., Easton, G., Markets as networks: Editorial introduction, in: International Journal of Research in Marketing, Vol. 13, 1996, S. 407-413.

Håkansson, H., Industrial technological development: A network approach, London u.a. 1987.

Haley, R.I., Benefit segmentation. A decision oriented research tool, in: Journal of Marketing, Vol. 32, No. 7, 1968, S. 30-35.

Hamel, G., Competition for Competence and Interpartner Learning Within International Strategic Alliances, in: Strategic Management Journal, Summer Special Issue, 1991, S. 83-103.

Hamel, G., Doz, Y.L., Prahalad, C.K., Collaborate with Your competitors - and Win, in: Harvard Business Review, Vol. 67, No. 1, 1989, S. 133-139.

Harbison, J.R., Pekar, P., Cross-Border Alliances in the Age of Collaboration, Working Paper, Hrsg.: Booz, Allen \& Hamilton, Los Angeles 1997. 
Harrigan, K.R., Strategies for joint ventures, Lexington, Mass. 1985.

Hätty, H., Der Markentransfer, Heidelberg 1989.

Hausleitner, M., Viele Identitäten führen zum Marken-Brei, in: Marketing Journal, Nr. 4, 1995, S. 262-264.

Heinen, E., Führung als Gegenstand der Betriebswirtschaftslehre, in: Betriebswirtschaftliche Führungslehre: ein entscheidungsorientierter Ansatz, Hrsg.: Heinen, E., Wiesbaden 1978, S. 19-44.

Henard, J., Das Tarifkorsett reißt an allen Nähten, in: FAZ vom 13.06.1986, S. R 7.

Henke, N., Wettbewerbsvorteile durch Integration von Geschäftsaktivitäten: ein zeitablaufbezogener wettbewerbsstrategischer Analyseansatz unter besonderer Berücksichtigung des Einsatzes von Kommunikationsund Informationssystemen (KIS), Frankfurt am Main u.a. 1995.

Hentschel, B., Dienstleistungsqualität aus Kundensicht: vom merkmals- zum ereignisorientierten Ansatz, Wiesbaden 1992.

Herrmann, A., Produktwahlverhalten: Erläuterung und Weiterentwicklung von Modellen zur Analyse des Produktwahlverhaltens aus marketingtheoretischer Sicht, Stuttgart 1992.

Heskett, J.L., Managing in the Service Economy, Boston 1986.

Heskett, J.L, u.a., Putting the Service-Profit Chain to Work, in: Harvard Business Review, Vol. 72, No. 2, 1994, S. 164-174.

Hildebrandt, K., Thai Airways baut Kooperation mit Lufthansa und United aus und strukturiert um, in: fuw, Nr. 17, 1996, S. 49-50.

Hinthorne, T., Predatory Capitalism, Pragmatism, And Legal Positivism In The Airlines Industry, in: Strategic Management Journal, Vol. 17, No. 4, 1996, S. 251-270.

Homburg, C., Giering, A., Konzeptualisierung und Operationalisierung komplexer Konstrukte. Ein Leitfaden für die Marketingforschung, in: Marketing ZFP, 18. Jg., Nr. 1, 1996, S. 5-24.

Hunt, M.S., Competition in the major home appliance industry, Diss., Harvard University 1972.

Hüttermann, M.U., Chancen und Risiken des Co-Branding: Co-Branding Marketing-Strategie für Kreditinstitute dargestellt am Beispiel der Kreditkarte, Hamburg 1992.

Hwang, P., Burgers, W.P., The Many Faces of Multi-Firm Alliances, in: California Management Review, Vol. 39, 1997, No. 3, S. 101-117.

Irmscher, M., Markenwertmanagement: Aufbau und Erhalt von Markenwissen und -vertrauen im Wettbewerb; eine informationsökonomische Analyse, Frankfurt am Main u.a. 1997. 
Jäckel, K., Kooperationsstrategien im Linienluftverkehr vor dem Hintergrund zunehmender Integrationsentwicklung in Europa, Bergisch Gladbach, Köln 1991.

Jahrfeld, M., Der Weg zum Mehrwert bleibt verschlungen, in: fuw, Nr. 23, 1997, S. 14-15.

Jarillo, J.C., On Strategic Networks, in: Strategic Management Journal, Vol. 9, No. 1, 1988, S. 31-41.

Jay, 1964, zitiert in: Aldrich, H., Whetten, D.A., Organization-sets, action-sets, and networks: making the most of simplicity, in: Handbook of organizational design, Vol. 1, Hrsg.: Nystrom, P.C., Starbuck, W.H., Oxford u.a. 1981, S. 385-400.

Jegminat, G., Bausteine für integrierten Gesamtprozeß, in: fvw, Nr. 27, 1997, S. 9. Jegminat, G., Bausteine für integrierten Gesamtprozeß, in: fvw, Nr. 27, 1997, S. 9. Jegminat, G., Die Airline-Allianz von KLM und Northwest: aus transatlantischer Achse globales Netz geformt, in: fww, Nr. 13, 1996, S. 65.

Jegminat, G., Strategische Allianzen nur die zweitbeste Lösung, in: fvw, Nr. 25, 1997, S. 78.

Jennings, M., Poisoned Pals? In: Airline Business, No. 7, 1996, S. 22-24.

Jeschke, K., Nachkaufmarketing: Kundenzufriedenheit und Kundenbindung auf Konsumgütermärkten, Frankfurt am Main u.a. 1995.

Jones, L., Euro agents fight change, in: Airline Business, No. 5, 1997, S. 18.

Kaas, K.-P., Marketing als Bewältigung von Informations- und Unsicherheitsproblemen im Markt, in: Die Betriebswirtschaft, 50. Jg., 1990, S. 539-548.

Kaas, K.P., Busch, A., Inspektions-, Erfahrungs- und Vertrauenseigenschaften von Produkten, in: Marketing ZFP, 18. Jg., Nr. 4, 1996, S. 243-252.

Kaas, K.-P., Runow, H., Wie befriedigend sind die Ergebnisse der Forschung zur Verbraucherzufriedenheit? In: DBW, 44. Jg., Nr. 3, 1984, S. 451-460.

Kartte, W., Wettbewerbspolitische und wettbewerbsrechtliche Probleme Strategischer Allianzen, in: Wegweiser für Strategische Allianzen: Meilenund Stolpersteine bei Kooperationen, Hrsg.: Bronder, C., Pritzl, R., Wiesbaden 1992, S. 401-422.

Katz, R., Informationsquellen der Konsumenten: eine Analyse der Divergenzen zwischen der Beurteilung und Nutzung, Wiesbaden 1983.

Kenter, M.E., Die Steuerung ausländischer Tochtergesellschaften: Instrumente und Effizienz, Frankfurt am Main, Bern, New York 1985.

Khanna, T., Gulati, R., Nohria, N., The Dynamics of Learning Alliances: Competition, Cooperation, and Relative Scope, in: Strategic Management Journal, Vol. 19, No. 3, 1998, S. 193-210. 
Kiani-Kress, R., Heineken-Effekt, in: Wirtschaftswoche, Nr. 49, 1996, S. 134-135.

Kieser, A., Anleitung zum kritischen Umgang mit Organisationstheorien, in: Organisationstheorien, Hrsg.: Kieser, A., 2., überarb. Aufl., Stuttgart, Berlin, Köln 1995, S. 1-30.

Kieser, A., Kubicek, H., Organisation, 3., völlig neu bearb. Aufl., Berlin, New York 1992.

Killing, J.P., How to make a global joint venture work, in: Harvard Business Review, Vol. 60, No. 3, 1982, S. 120-127.

Klein, H., Allianzen - Herausforderungen und Strategien aus Sicht der Deutschen Lufthansa AG, in: Internationales Verkehrswesen, Nr. 12, 1996, S. 12-16.

Klein, H., Dienstleistung als Hidden Champion, Vortragsunterlagen der Deutschen Lufthansa AG am 5. November 1997 an der Westfälischen WilhelmsUniversität Münster.

Klein, H., Internationaler Wettbewerb der Allianzen, in: Allianzen bei Verkehrsdienstleistungen - Perspektiven vor dem Hintergrund sich ändernder Wirtschaftsbedingungen, Dokumentationspapier Nr. 112 der Wissenschaftlichen Gesellschaft für Marketing und Unternehmensführung e.V., Hrsg.: Meffert, H., Backhaus, K., Münster 1997, S. 12-25.

Klein, H., Qualitätsmanagement der Deutschen Lufthansa AG, in: Dienstleistungsqualität: Konzepte-Methoden-Erfahrungen, Hrsg.: Bruhn, M., Stauss, B., 2. Auflage, Wiesbaden 1995, S. 479-493.

Klein, B., Leffler, K., The Role of Market Forces in Assuring Contractual Performance, in: Journal of Political Economy, Vol. 90, 1981, S. 615641.

Kloyer, M., Management von Franchisenetzwerken: eine Resource-DependencePerspektive, Wiesbaden 1995.

Knyphausen-Aufseß, D. zu, Theorie der strategischen Unternehmensführung: state of the art und neue Perspektiven, Wiesbaden 1995.

Kogut, B., A study of the life cycle of joint ventures, in: Cooperative strategies in international business, Hrsg.: Contractor, F.J., Lorange, P., Lexington, Mass. 1988, S. 169-186.

Kolf, F., Airline-Allianz soll nicht nur Kosten senken, in: Handelsblatt vom 15.5.1997, S. 16.

Kolf, F., Lange Zeit blind vertraut, in: Wirtschaftswoche, Nr. 8, 1996, S. 88-90.

Kotabe, M., Swan, K.S., The role of strategic alliances in high-technology new product development, in: Strategic Management Journal, Vol. 16, No. 8 , 1995, S. 621-636. 
Krahn, H., Markteintrittsbarrieren auf dem deregulierten US-amerikanischen Luftverkehrsmarkt: Schlußfolgerungen für die Luftverkehrspolitik der Europäischen Gemeinschaft, Frankfurt am Main u.a. 1994.

Kreikebaum, H., Strategische Unternehmensplanung, 6., überarb. und erw. Aufl., Stuttgart, Berlin, Köln 1997

Kreutzer, R., Lead-Country-Konzept, in: Wirtschaftswissenschaftliches Studium, Nr. 8, 1987, S. 416-419.

Kroeber-Riel, W., Strategie und Technik der Werbung, 4. Aufl., Stuttgart 1993.

Kroeber-Riel, W., Weinberg, P., Konsumentenverhalten, 6., völlig überarb. Aufl., München 1996.

Kutschker, M., Mößlang, A., Kooperationen als Mittel der Internationalisierung von Dienstleistungsunternehmen, in: DBW, 56. Jg., Nr. 3, 1996, S. 319337.

Laakmann, K., Value added services als Profilierungsintrument im Wettbewerb: Analyse, Generierung und Bewertung, Frankfurt am Main u.a. 1995

Laaser, C., Ausländische Erfahrungen mit Deregulierungsexperimenten im Verkehrswesen, Kieler Arbeitspapiere Nr. 270, Kiel 1986.

Laforet, S., Saunders, J., Managing Brand Portfolios: How The Leaders Do It, in: Journal of Advertising Research, Vol. 34, No. 5, 1994, S. 64-76.

Langner, S.J., The Allocation of Slots in the Airline Industry: A Transaction Cost Economics Analysis, Baden-Baden 1996.

Leonhardt-Weber, B., Die Entwicklung der Qualitätsmerkmale im Verkehr: eine Analyse vor dem Hintergrund der technischen, wirtschaftlichen und gesellschaftlichen Entwicklung, München 1990.

Lettl-Schröder, M., Trends und Prognosen im Geschäftsreisemarkt: die Management Fee setzt sich weiter durch, in: fvw, Nr. 13, 1996, S. 45-46.

Levere, J., Agents of change, in: Airline Business, No. 8, 1997, S. 52-54.

Levitt, T., The Globalization of Markets, in: Harvard Business Review, Vol. 61, No. 6, 1983, S. 92-102

Lindemann, M., Kooperative Marketing-Kommunikation: kommunikationspolitische Zusammenarbeit von Konsumgüter-Anbietern, Bergisch Gladbach, Köln 1993.

Lindquist, J., The role of alliances in the strategy of airlines, Präsentationsunterlagen der Boston Consulting Group im Rahmen der Airline Alliances Conference, London 1995.

Lode, R., Formen der Unternehmenskooperation: Betriebswirtschaftliche Überlegungen und Ansätze zur Begriffsbildung der Unternehmenskooperation und zur Systematisierung ihrer Formen, Münster 1974. 
Ludwig, E., Travella, R., Online-Distribution und E-Commerce bei Fluggesellschaften, in: Alternative Formen der Distribution Electronic Commerce, Online Distribution und Direct Distribution, Hrsg.: Belz, C., Tomczak, T., Thexis Fachbericht für Marketing Nr. 1, 1998, S. 39-68.

Lufthansa AG (Hrsg.), Lufthansa Imagemonitor: Awareness and judgement of alliances between a US airline and a foreign airline, November 1995.

Lüking, J., Angebotsplanung und Fluggastverhalten im überlasteten Luftverkehrssystem, Bern u.a. 1993.

Lutz, V., Horizontale strategische Allianzen: Ansatzpunkte zu ihrer Institutionalisierung, Hamburg 1993.

Lyle, C., Agent blues, in: Airline Business, No. 11, 1995, S. 56-60.

Magrath, A.J., When Marketing Services, 4 Ps Are Not Enough, in: Business Horizons, Vol. 29, No. 3, 1986, S. 44-50.

Malik, F., Strategie des Managements komplexer Systeme, 5. Aufl., Bern 1996.

Manzanec, J., Strukturmodelle des Konsumentenverhaltens, Wien 1978.

Mattsson, L.-G., Management of strategic change in a "markets-as-networks" perspective, in: The management of strategic change, Hrsg.: Pettigrew, A.M., Oxford 1987, S. 234-260.

Mayerhofer, W., Imagetransfer - Die Nutzung von Erlebniswelten für die Positionierung von Ländern, Produktgruppen und Marken, Wien 1995.

McGee, W.J., Reshaping the Relationship, in: Air Transport World, No. 12, 1997, S. 57-59.

McKenney, J.L., Copeland, D.C., Mason, R.O., Waves of Change: Business Evolution through Information Technology, Boston 1995.

Meffert, H., Marketing: Grundlagen der Absatzpolitik, 7., überarb. u. erw. Aufl., Wiesbaden 1986.

Meffert, H., Marketing: Grundlagen marktorientierter Unternehmensführung: Konzepte-Instrumente-Praxisbeispiele, 8., vollst. neubearb. und enw. Aufl., Wiesbaden 1998.

Meffert, H., Marketingforschung und Käuferverhalten, 2., vollst. überarb. und erw. Aufl., Wiesbaden 1992.

Meffert, H., Marketing-Management: Analyse, Strategie, Implementierung, Wiesbaden 1994.

Meffert, $\mathrm{H}$., Die Bedeutung der Marke für das Image und das Erscheinungsbild von Dienstleistungsunternehmen, in: LeitWerk, Nr. 7, 1989, S. 1-11.

Meffert, H., Die virtuelle Unternehmung: Perspektiven aus der Sicht des Marketing, in: Marktleistung und Wettbewerb, Festschrift zum 65. 
Geburtstag von W.H. Engelhardt, Hrsg.: Backhaus, K., u.a., Wiesbaden 1997, S. 115-141.

Meffert, H., Dienstleistungsphilosophie und -kultur, in: Handbuch DienstleistungsMarketing, Band 1, Hrsg.: Meyer, A., Stuttgart 1998, S. 121-138.

Meffert, H., Entscheidungsorientierter Ansatz der Markenpolitik, in: Handbuch Markenartikel: Anforderungen an die Markenpolitik aus Sicht von Wissenschaft und Praxis, Hrsg.: Bruhn, M., Stuttgart 1994, S. 174197.

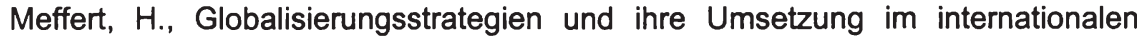
Wettbewerb, in: DBW, 49.Jg., Heft 4, 1989, S. 445-463.

Meffert, H., Kundenbindung als Element moderner Wettbewerbsstrategien, in: Handbuch Kundenbindungsmanagement, Hrsg.: Bruhn, M., Homburg, C., Wiesbaden 1998, S. 115-133.

Meffert, H., Marktorientierte Unternehmensführung und Direct Marketing, in: Handbuch Directmarketing, Hrsg.: Dallmer, H., 7. Aufl., Wiesbaden 1997, S. 33-52.

Meffert, H., Strategien zur Profilierung von Marken, in: Marke und Markenartikel als Instrumente des Wettbewerbs, Hrsg.: Dichtl, E., Eggers, W., München 1992, S. 129-156.

Meffert, H., Vertikales Marketing und Marketingtheorie, in: Konflikt und Kooperation in Absatzkanälen: ein Beitrag zur verhaltensorientierten Marketingtheorie, Hrsg.: Steffenhagen, H., Wiesbaden 1975, S. 1520.

Meffert, H., Backhaus, K., Kundenbindung und Kundenmanagement: Instrumente zur Sicherung der Wettbewerbsposition, Dokumentation des Marketing Post Graduate-Seminars am 16./17. Juni 1994 in Münster, Münster 1994.

Meffert, H., Bolz, J., Internationales Marketing-Management, 3., überarb. und erg. Aufl., Stuttgart, Berlin, Köln 1998.

Meffert, H., Bruhn, M., Dienstleistungsmarketing: Grundlagen-KonzepteMethoden, mit Fallbeispielen, 2., überarb. und erw. Aufl., Wiesbaden 1997.

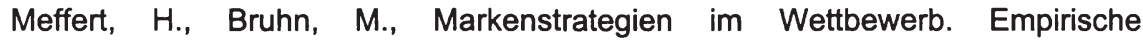
Untersuchungen zur Akzeptanz von Hersteller-, Handels- und Gattungsmarken (No Names), Wiesbaden 1984.

Meffert, H., Burmann, C., Abnutzbarkeit und Nutzungsdauer von Marken - ein Beitrag zur steuerlichen Behandlung von Warenzeichen, Arbeitspapier Nr. 117 der Wissenschaftlichen Gesellschaft für Marketing und Unternehmensführung e.V., Hrsg.: Meffert, H., Backhaus, K., Münster 1998. 
Meffert, H., Burmann, C., Identitätsorientierte Markenführung - Grundlagen für das Management von Markenportfolios, Arbeitspapier Nr. 100 der Wissenschaftlichen Gesellschaft für Marketing und Unternehmensführung e.V., Hrsg.: Meffert, H., Wagner, H., Backhaus, K., Münster 1996.

Meffert, H., Heinemann, G., Operationalisierung des Imagetransfers, in: Marketing ZFP, Nr. 1, 1990, S. 5-10.

Meffert, H., Netzer, F., Formen strategischer Netzwerke und Implikationen für das Marketing, Arbeitspapier Nr. 109 der Wissenschaftlichen Gesellschaft für Marketing und Unternehmensführung e.V., Hrsg.: Meffert, H., Backhaus, K., Münster 1997.

Meffert, H., Perrey, J., Nutzensegmentierung im Verkehrsdienstleistungsbereich theoretische Grundlagen und empirische Erkenntnisse am Beispiel des Schienenpersonenverkehrs, in: Tourismus Journal, 1. Jg., Nr. 1, 1997, S. 13- 40.

Meffert, J.P.H., Strategische Neuorientierung der Computerindustrie durch Standards, in: Thexis, Nr. 1, 1995, S. 32-37.

Mei-Pochtler, A., Markenmanagement für Dienstleistungs-Anbieter, in: Handbuch Dienstleistungs-Marketing, Band 1, Hrsg.: Meyer, A., Stuttgart 1998, S. 665-678.

Mendes de Almeida, P.F., A Review of Group Discussion Methodology, in: European Research, Vol. 8, No. 3, 1980, S. 114-120.

Meurer, J., Führung von Franchisesystemen: Führungstypen - Einflußfaktoren Verhaltens- und Erfolgswirkungen, Wiesbaden 1997.

Meyer, A., Dullinger, F., Leistungsprogramm von Dienstleistungs-Anbietern, in: Handbuch Dienstleistungs-Marketing, Band 1, Hrsg.: Meyer, A., Stuttgart 1998, S. 711-735.

Meyer, M., Ökonomische Organisation der Industrie: Netzwerkarrangements zwischen Markt und Unternehmung, Wiesbaden 1995.

Miles, R.E., Snow, C.C., Organizations: New Concepts for New Forms, in: California Management Review, Vol. 28, No. 3, 1986, S. 62-73.

Miller, R.N., Multinational Direct Marketing - On the Brink of the New Millenium, in: Handbuch Directmarketing, Hrsg.: Dallmer, H., 7. Aufl., Wiesbaden 1997, S. 67-78.

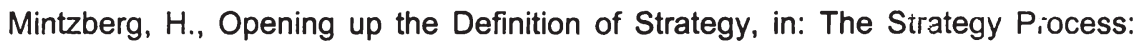
Concepts, Contexts, \& Cases, Hrsg.: Quinn, J.B., Mintzberg, H., James, R.M., Englewood Cliffs, New Jersey 1988, S. 13-20.

Mintzberg, H., Patterns in Strategy Formulation, in: Management Science, Vol. 24, No. 9,1978 , S. 934-948. 
Moorhouse, M., Brand Accounting, in: Brand Valuation: Establishing a True and Fair View, Hrsg.: Murphy, J., London 1989, S. 143-153.

Mowery, D.C., Oxley, J.E., Silverman, B.S., Strategic Alliances and Interfirm Knowledge Transfer, in: Strategic Management Journal, Vol. 17, Winter Special Issue, 1996, S. 77-91.

Müller, G.-M., Dachmarkenstrategien, in: Handbuch Markenartikel: Anforderungen an die Markenpolitik aus Sicht von Wissenschaft und Praxis, Hrsg.: Bruhn, M., Stuttgart 1994, S. 499-511.

Müller, S., Kornmeier, M., Grenzen der Standardisierung im Internationalen Marketing, in: Jahrbuch der Absatz- und Verbrauchsforschung, Nr. 1, 1996, S. 4-29.

Nagle, T.T., Holden, R.K., Larsen, G., Pricing - Praxis der optimalen Preisfindung, Berlin u.a. 1998.

Nalebuff, B., Brandenburger, A., Coopetition - kooperativ konkurrieren: mit der Spieltheorie zum Unternehmenserfolg, Frankfurt am Main, New York 1996.

Nelms, D.W., Improving the tube, in: Air Transport World, No. 3, 1998, S. 73-81.

Neu, M., Marketing-Strategien für den internationalen Wettbewerb von Luftfahrtgesellschaften, Würzburg 1989.

Nickel, F., Stationsmanagement von Luftverkehrsgesellschaften - eine systemanalytische Betrachtung und empirische Untersuchung der Stationsmanagement-Systeme internationaler Luftverkehrsgesellschaften, Karlsruhe 1994.

Nitschke, U.K., Mediatisierte interne Kommunikation in internationalen Unternehmungen - Möglichkeiten und Auswirkungen von Informations- und Kommunikationssystemen für globale Netzwerkorganisationen, Bamberg 1996.

Nohria, N., Eccles, R.G. (Hrsg.), Networks and organizations: structure, form, and action, Boston 1992.

Nohria, N., Is a Network Perspective a Useful Way of Studying Organizations? In: Networks and organizations: structure, form, and action, Hrsg.: Nohria, N., Eccles, R.G., Boston 1992, S. 1-22.

Nystrom, P.C., Starbuck, W.H. (Hrsg.), Handbook of organizational design, Vol. 1, Oxford u.a. 1981.

o.V., „Das nenne ich Enteignung“, in: Der Spiegel, Nr. 19, 1998, S. 130-132.

o.V., Flughafen Frankfurt bietet in Südafrika, in: FAZ vom 29.11.1997, S. 21.

o.V., Fluglinie KLM wird Partner von Alitalia, in: FAZ vom 18.12.1997, S. 20.

o.V., Kinnock droht mit dem EuGH, in: Handelsblatt vom 12.3.1998, S. 9. 
o.V., Lufffahrt: Neue weltweite Allianz, in: Spiegel Homepage, www.spiegel.de/homepage/home/link-network_wir.html vom 21.09.1998.

o.V., Lufthansa und Singapore Airlines kooperieren, in: FAZ vom 25.11.1997, S. 29.

o.V., Lufthansa will höhere Dividende zahlen, in: Handelsblatt vom 12.3.1998, S. 1.

o.V., Lufthansa-Partner wächst im Atlantik-Verkehr, in: Handelsblatt vom 22.9.1997, S. 15.

o.V., Nicht jede Geschäftsreise-Meile bringt Freiflüge, in: Handelsblatt vom 26.5.1997, S. 15.

o.V., Northwest-Piloten gegen Allianz mit Continental, in: Handelsblatt vom 18.12.1997, S. 20.

o.V., Streit um die Allianz von British Airways und American Airlines, in: FAZ vom 16.1.1997, S. 17.

o.V., Streit um die Allianz von British Airways und American Airlines, in: FAZ vom 16.1.1997, S. 17

o.V., Swissair gründet europäische Luftverkehrsallianz, in: Handelsblatt vom 31.3.1998, S. 13.

o.V., The World Airline Report, in: Air Transport World, No. 7, 1997, S. 85-139.

o.V., Trio kommt auf $80 \%$ Marktanteil, in: Handelsblatt vom 27.4.1998, S. 13.

o.V., Vehemente Kritik an Auflagen der EU, in: Handelsblatt vom 9.7.1998, S. 14.

o.V., World Airline Report, in: Air Transport World, No. 7, 1997, S. 62.

Odell, M., Whitaker, R., A picture of health, in: Airline Business, No. 5, 1997, S. 28-38.

Ogger, G., König Kunde: angeschmiert und abserviert, Hamburg 1996.

Ohlwein, M., Schiele, T.P., Co-Branding, in: WiSt, Nr. 11, 1994, S. 577-578.

Ohmae, K., Die Macht der Triade, Wiesbaden 1985.

Osterloh, M., Frost, J., Prozessmanagement als Kernkompetenz: wie Sie Business Reengineering strategisch nutzen können, Wiesbaden 1996.

Oum, T.H., Winning Airlines: productivity and cost competitiveness of the world's major airlines, Norwell 1998.

Papadopoulos, N., What product and country images are and are not, in: ProductCountry Images: Impact and Role in International Marketing, Hrsg.: Papadopoulos, N., Binghamton 1993, S. 3-38. 
Parasuraman, A., Zeithaml, V.A., Berry, L.L., A Conceptual Model of Service Quality and Its Implications for Future Research, in: Journal of Marketing, Vol. 49, No. 4, 1985, S. 41-50.

Patt, P.-J., Strategische Erfolgsfaktoren im Einzelhandel: eine empirische Analyse am Beispiel des Bekleidungsfachhandels, Frankfurt am Main u.a. 1988.

Payne, A., The essence of services marketing, Englewood Cliffs 1993.

Pepels, W., Kommunikations-Management: Marketing-Kommunikation vom Briefing bis zur Realisation, 2. Aufl., Stuttgart 1996.

Perlmutter, H.V., Heenan, D.A., Cooperate to compete globally, in: Harvard Business Review, Vol. 64, No. 2, 1986, S. 136-152.

Perlmutter, H.V., The Tortuous Evolution of the Multinational Corporation, in: Columbia Journal of World Business, No. 4, 1969, S. 9-18.

Perrey, J., Nutzenorientierte Marktsegmentierung: ein integrativer Ansatz zum Zielgruppenmarketing im Verkehrsdienstleistungsbereich, Wiesbaden 1998.

Peters, T., Waterman, R.H., Auf der Suche nach Spitzenleistungen. Was man von den bestgeführten US-Unternehmen lernen kann, Landsberg/Lech 1983.

Petersen, R., The price of loyalty, in: Airline Business, No. 8, 1997, S. 38-40.

Pfeffer, J., Organizations and Organization Theory, Boston u.a. 1982.

Pfeiffer, R. (Hrsg.), Systemdenken und Globalisierung: Folgerungen für die lernende Organisation im internationalen Umfeld, Berlin 1997.

Picot, A., Dietl, H., Franck, E., Organisation: eine ökonomische Perspektive, Stuttgart 1997.

Picot, A., Reichwald, R., Wigand, R.T., Die grenzenlose Unternehmung: Information, Organisation und Management, 2. Aufl., Wiesbaden 1996.

Piepelow, V., Die europäischen Linienfluggesellschaften im Wettbewerb: eine Analyse mittels des Konzepts strategischer Gruppen, Frankfurt am Main u.a. 1997.

Pierer, H. von, Mirow, M., Das Management strategischer Allianzen, in: Handbuch Unternehmungsführung, Hrsg.: Corsten, H., Reiß, M., Wiesbaden 1995, S. 705-717.

Poledna-van Zweden, M., Kundenbindungsprogramme der Deutschen Lufthansa AG, in: Die Nicht-Klassiker unter heutigen Kommunikationsbedingungen, Hrsg.: Belz, C., Tomczak, T., St. Gallen 1995, S. 198-206.

Pommes, C. de, Are you IT-compatible? In: Airline Business, No. 7, 1998, S. 2629. 
Pompl, W., Luftverkehr: eine ökonomische Einführung, 2., aktualisierte und erw. Aufl, Berlin u.a. 1991.

Pompl, W., Touristikmanagement 2: Qualitäts-, Produkt-, Preismanagement, Berlin u.a. 1996.

Pons-Großwörterbuch Deutsch-Englisch, Englisch-Deutsch, 2. Aufl., Stuttgart, Dresden 1993.

Porter, M.E., Competitive Advantage, New York, London 1985.

Porter, M.E., Competitive Strategy: Techniques for Analyzing Industries and Competitors, New York, London 1980.

Porter, M.E., Fuller, M.B., Coalitions and global strategy, in: Competition in global industries, Hrsg.: Porter, M.E., Boston, Mass. 1986, S. 315-343.

Preiss, K., Goldman, S.L., Nagel, R.N., Cooperate to Compete: Building Agile Business Relationships, New York u.a. 1996.

Pugh, D.S., u.a., Dimensions of Organization Structure, in: Administrative Science Quarterly, Vol. 13, No. 1, 1968, S. 65-105.

Purtschert, R., Möglichkeiten und Grenzen der Gemeinschaftswerbung, in: ZfB, Nr. 4, 1988, S. 521-534.

Rangan, U.S., Yoshino, M.Y., Forging Alliances: A Guide to Top Management, in: The Columbia Journal of World Business, Fall 1996, S. 6-13.

Rao, A.R., Qu, L., Ruekert, R.W., Brand Alliances as Information About Unobservable Product Quality, MSI Working Paper No. 97-100, Cambridge, Mass. 1997.

Reckewerth, S., Die Zusammenarbeit der Luftverkehrsgesellschaften nach europäischem und US-amerikanischen Recht, Köln u.a. 1993.

Reichheld, F.F., Sasser, W.E., Zero Migration. Dienstleister im Sog der Qualitätsrevolution, in: Harvard Manager, 13. Jg., Nr. 4, 1991, S. 108-116.

Ritzerfeld, U., Marketing-Mix-Strategien in Investitionsgütermärkten: Entwicklung und Simulation marktstrukturspezifischer Strategien, Wiesbaden 1993.

Robinson, P.J., Wind, Y., Multinational Trade-off Segmentation, in: Moving Ahead with Attitude Research, Hrsg.: Wind, Y., Greenberg, M.G., Chicago 1977, S. 50-57.

Rodrian, H.W., Der Meilen-Bonus macht aus dem Flug einen Gewinn, in: Handelsblatt vom 17.11.1997, S. 41.

Rößger, E., Hünermann, K., Einführung in die Luftverkehrspolitik, Zürich 1965. O'Connor, W.E., An introduction to airline economics, 5. Aufl., Westport 1995. 
Salcher, E.F., Psychologische Marktforschung, 2., neu bearb. Aufl., Berlin, New York 1995.

Sander, M., Die Bestimmung und Steuerung des Wertes von Marken. Eine Analyse aus Sicht des Markeninhabers, Heidelberg 1994.

Sander, M., Internationales Preismanagement: eine Analyse preispolitischer Handlungsalternativen im internationalen Marketing unter besonderer Berücksichtigung der Preisfindung, Heidelberg 1997.

Sanz, F.L., The EC air transport in the international legal framework of civil aviation, in: Der Luftverkehr in der Europäischen Union: die Lücke im Binnenmarkt, Hrsg.: Rogalla, D., Schweren, K., Baden-Baden 1994, S. 15-21.

Sattelberger, T., Personalmanagement in virtuellen Unternehmen, Dokumentation des Gastvortrags am Betriebswirtschaftlichen Institut für Anlagenund Systemtechnologien der Westfälischen Wilhelms-Universität Münster am 28.1.1997.

Sattler, H., Markenbewertung, in: Zeitschrift für Betriebswirtschaft, 65. Jg., Nr. 6, 1995, S. 663-682.

Schäfer-Kunz, J., Strategische Allianzen im deutschen und europäischen Kartellrecht, Frankfurt am Main u.a. 1995.

Schäfer-Kunz, J., Strategische Allianzen im deutschen und europäischen Kartellrecht, Frankfurt am Main u.a. 1995.

Schanz, G., Methodologie für Betriebswirte, 2., überarb. u. erw. Aufl., Stuttgart 1988.

Schenk, H.-O., Handels- und Gattungsmarken, in: Handbuch Markenartikel: Anforderungen an die Markenpolitik aus Sicht von Wissenschaft und Praxis, Hrsg.: Bruhn, M., Stuttgart 1994, S. 57-78.

Scheuch, F., Dienstleistungsmarketing, München 1982.

Schlesinger, L.A., Heskett, J.L., Dem Kunden dienen - das müssen viele Dienstleister erst noch lernen, in: Harvard Manager, 14. Jg., Nr. 1, 1992, S. 106-116.

Schmalen, H., Kommunikationspolitik: Werbeplanung, 2., überarb. und erw. Aufl., Stuttgart, Berlin, Köln 1992.

Schmengler, H.J., Thieme, M., Die Bedeutung eines Bonusprogramms im Marketing einer Luftverkehrsgesellschaft, in: Marketing ZFP, Nr. 2, 1995, S. 130-135.

Schmidt, A., Computerreservierungssysteme im Luftverkehr: Darstellung, Entwicklung und wettbewerbliche Beurteilung, Hamburg 1995.

Schmidt, S., Strategische Allianzen im Luftverkehr - Erfolgsorientiertes Management europäischer Flug-Carrier, Trier 1993. 
Schräder, A., Management virtueller Unternehmungen: organisatorische Konzeption und informationstechnische Unterstützung flexibler Allianzen, Frankfurt/M., New York 1996.

Schreyögg, G., Organisation: Grundlagen moderner Organisationsgestaltung, Wiesbaden 1996.

Schub von Bossiazky, G., Psychologische Marketingforschung: qualitative Methoden und ihre Anwendung in der Markt-, Produkt- und Kommunikationsforschung, München 1992.

Schulte-Zurhausen, M., Organisation, München 1995.

Schürmann, U., Erfolgsfaktoren der Werbung im Produktlebenszyklus: ein Beitrag zur Werbewirkungsforschung, Frankfurt am Main u.a. 1993.

Schwamborn, S., Strategische Allianzen im internationalen Marketing: Planung und portfolioanalytische Beurteilung, Wiesbaden 1994.

Shaw, S., Airline Marketing\&Management, 3. Aufl., London 1990.

Shostack, G.L., Planning the Service Encounter, in: The Service Encounter: Managing Employee/Customer Interaction in Service Businesses, Hrsg.: Czepiel, J.A., Solomon, M.R., Surprenant, C.F., Lexington 1985, S. 243-253.

Siefke, A., Zufriedenheit mit Dienstleistungen: ein phasenorientierter Ansatz zur Operationalisierung und Erklärung der Kundenzufriedenheit im Verkehrsbereich auf empirischer Basis, Frankfurt am Main u.a. 1998.

Simon, H., Dolan, R.J., Profit durch Power Pricing: Strategien aktiver Preispolitik, Frankfurt/Main, New York 1997.

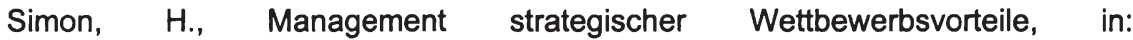
Wettbewerbsvorteile und Wettbewerbsfähigkeit, Hrsg.: Simon, H., Stuttgart 1988, S. 1-17.

Simon, H., Preismanagement: Analyse-Strategie-Umsetzung, 2. Aufl., Wiesbaden 1992.

Sinton, L., Seamless Security Screening, in: Air Transport Progress, Hrsg.: Goold, I., London 1995, S. 195-196.

Skapinker, M., BA-American air alliance faces a further setback, in: Financial Times vom 8.10.1998, S. 17.

Söderlund, M., Market orientation and the firm's market intelligence: an empirical study of what airlines want to know about their customers, in: Marketing Today and for the 21st Century, Proceedings of the 24th Annual Conference of the European Marketing Academy, CergyPontoise Cedex 1995, S. 959-978.

Spaeth, A., Ehelust und Ehefrust, in: touristik management, Nr. 3, 1996, S. 27.

Spaeth, A., Ist im Regime des „Offenen Himmels" alles möglich? in: Das Parlament, Nr. 18, 1998 S. 12. 
Spaeth, A., Start in den offenen Himmel, in: touristik management, Nr. 4, 1996, S. 54.

Spiegel-Verlag (Hrsg.), Spiegel-Dokumentation: Geschäftsreisen 1994, Hamburg 1994.

Spielberger, M., Provisionskürzungen machen Agenten das Leben schwer, in: fvw, Nr. 1, 1998, S. 9-12.

Spielberger, M., Reisebüro-Vertrieb wird an seinem Nutzen gemessen, in: fuw, Nr. 23, 1997, S. 12-13.

Staehle, W.H., Management: eine verhaltenswissenschaftliche Perspektive, 7. Aufl., München 1994.

Stauss, B., "Augenblicke der Wahrheit" in der Dienstleistungserstellung, in: Dienstleistungsqualităt: Konzepte, Methoden, Erfahrungen, Hrsg.: Bruhn, M., Stauss, B., 2. Aufl., Wiesbaden 1995, S. 379-399.

Stauss, B., Dienstleistungsmarken, in: Handbuch Markenartikel: Anforderungen an die Markenpolitik aus Sicht von Wissenschaft und Praxis, Hrsg.: Bruhn, M., Stuttgart 1994, S. 79-103.

Stauss, B., Markierungspolitik bei Dienstleistungen - „Die Dienstleistungsmarke“, in: Handbuch Dienstleistungs-Marketing, Band 1, Hrsg.: Meyer, A., Stuttgart 1998, S. 560-580.

Stegmüller, B., Internationale Marksegmentierung als Grundlage für internationale Marketing-Konzeptionen, Bergisch Gladbach, Köln 1995.

Steinmann, H., Scherer, A.G., Die multinationale Unternehmung als moralischer Aktor - Bemerkungen zu einigen normativen Grundlagenproblemen des interkulturellen Managements, in: Interkulturelles Management: Theoretische Fundierung und funktionsbereichsspezifische Konzepte, Hrsg.: Engelhard, J., Wiesbaden 1997, S. 23-53.

Steinmann, H., Schreyögg, G., Management: Grundlagen der Unternehmensführung; Konzepte - Funktionen - Fallstudien, 4., überarb. und erw. Aufl., Wiesbaden 1997.

Sterzenbach, R., Luftverkehr: betriebswirtschaftliches Lehr- und Handbuch, München, Wien 1996.

Sydow, J., Finanzdienstleistungsnetzwerke: zur Organisation einer ökonomischen Institution des Kontraktgütermarketings, in: Kontrakte, Geschäftsbeziehungen, Netzwerke - Marketing und Neue Institutionenökonomik, Sonderheft der zfbf, Nr. 35, 1995, Hrsg.: Kaas, K.-P., S. 139-157.

Sydow, J., Strategische Netzwerke: Evolution und Organisation, Wiesbaden 1992.

Taucher, G., Der dornige Weg strategischer Allianzen, in: Harvard Manager, 10. Jg., Nr. 3, 1988, S. 86-91. 
Trommsdorff, V., Konsumentenverhalten, 3., überarb. und erw. Aufl., Stuttgart, Berlin, Köln 1998.

Tuckel, P. Leppo, E., Kaplan, B., A View From the Other Side of the Mirror, in: Marketing Research, Vol. 5, No. 4, 1995, S. 24-27.

Turnball, P.W., Valla, J.-P., Strategies for industrial marketing, London 1986.

United States of America (Hrsg.), Public Law 95-504, Oct. 24, 1978, S. 17051754.

Verchère, I., The air transport industry in crisis: solving over-capacity and financing new equipment, London 1994.

Wagner, H., Marktorientierte Unternehmensführung versus Orientierung an Mitarbeiterinteressen, Shareholder-Value und Gemeinwohlverpflichtung, in: Marktorientierte Unternehmensführung: Reflexionen - Denkanstöße - Perspektiven, Hrsg.: Bruhn, M., Steffenhagen, H., Wiesbaden 1997, S. 87-102.

Walker, K., Where's the glue? in: Airline Business, No. 7, 1998, S. 30-31.

Waltermann, B., Internationale Markenpolitik und Produktpositionierung: markenpolitische Entscheidungen im europäischen Automobilmarkt, Wien 1989.

Weber, G., Erfolgsfaktoren im Kerngeschäft von europäischen Luftverkehrsgesellschaften, Bamberg 1997.

Webster, F.E., Wind, Y., Organizational Buying Behavior, Englewood Cliffs 1972.

Weinhold, M., Computerreservierungssysteme im Luftverkehr: Erfahrungen in den USA und Empfehlungen für Europa, Baden-Baden 1995.

Whipple, T.W., Mapping Focus Group Data, in: Marketing Research, Vol. 6, No. 1, 1996, S. 16-21.

Whitaker, R., A system approach, in: Airline Business, No. 1, 1996, S. 40-43.

Wiedmann, K.-P., Strategisches Markencontrolling, in: Handbuch Markenartikel: Anforderungen an die Markenpolitik aus Sicht von Wissenschaft und Praxis, Hrsg.: Bruhn, M., Stuttgart 1994, S. 1319-1329.

Wiencke, W., Cards\&Clubs als Dialogmarketing-Instrument, in: Handbuch Directmarketing, Hrsg.: Dallmer, H., 7. Aufl., Wiesbaden 1997, S. 331-344.

Wild, K., Everitt, H., The Accountancy Perspective in the UK, in: Brand Valuation: Establishing a True and Fair View, Hrsg.: Murphy, J., London 1989, S. 55-68.

Wilken, D., Code-Sharing im Luftverkehr Deutschlands, in: Internationales Verkehrswesen, Nr. 7/8, 1996, insb. S. 25-29.

Williams, G., The airline industry and the impact of deregulation, 2. Aufl., Aldershot 1996.

Williamson, O.E., The Economic Institutions of Capitalism, New York 1985. 
Woratschek, H., Positionierung - Analysemethoden, Entscheidungen, Umsetzung, in: Handbuch Dienstleistungs-Marketing, Band 1, Hrsg.: Meyer, A., Stuttgart 1998, S. 693-710.

Youssef, W.A.H., Causes and Effects of International Airline Equity Alliances, Ann Arbor 1992.

Zeithaml, V.A., Bitner, M.J., Services Marketing, New York u.a. 1996. 


\section{SCHRIFTEN ZUM MARKETING}

Band 1 Friedrich Wehrle: Strategische Marketingplanung in Warenhäusern. Anwendung der Portfolio-Methode. 1981. 2. Auflage. 1984.

Band 2 Jürgen Althans: Die Übertragbarkeit von Werbekonzeptionen auf internationale Märkte. Analyse und Exploration auf der Grundlage einer Befragung bei europaweit tätigen Werbeagenturen. 1982.

Band 3 Günter Kimmeskamp: Die Rollenbeurteilung von Handelsvertretungen. Eine empirische Untersuchung zur Einschätzung des Dienstleistungsangebotes durch Industrie und Handel. 1982.

Band 4 Mantred Bruhn: Konsumentenzufriedenheit und Beschwerden. Erklärungsansätze und Ergebnisse einer empirischen Untersuchung in ausgewählten Konsumbereichen. 1982.

Band 5 Heribert Meffert (Hrsg.): Kundendienst-Management. Entwicklungsstand und Entscheidungsprobleme der Kundendienstpolitik. 1982.

Band 6 Ralf Becker: Die Beurteilung von Handelsvertretern und Reisenden durch Hersteller und Kunden. Eine empirische Untersuchung zum Vergleich der Funktionen und Leistungen. 1982.

Band 7 Gerd Schnetkamp: Einstellungen und Involvement als Bestimmungsfaktoren des sozialen Verhaltens. Eine empirische Analyse am Beispiel der Organspendebereitschaft in der Bundesrepublik Deutschland. 1982.

Band 8 Stephan Bentz: Kennzahlensysteme zur Erfolgskontrolle des Verkaufs und der MarketingLogistik. Entwicklung und Anwendung in der Konsumgüterindustrie. 1983.

Band 9 Jan Honsel: Das Kaufverhalten im Antiquitätenmarkt. Eine empirische Analyse der Kaufmotive, ihrer Bestimmungsfaktoren und Verhaltenswirkungen. 1984.

\section{SCHRIFTEN ZU MARKETING UND MANAGEMENT}

Band 10 Matthias Krups: Marketing innovativer Dienstleistungen am Beispiel elektronischer Wirtschaftsinformationsdienste. 1985.

Band 11 Bernd Faehsler: Emotionale Grundhaltungen als Einflußfaktoren des Käuferverhaltens. Eine empirische Analyse der Beziehungen zwischen emotionalen Grundhaltungen und ausgewählten Konsumstrukturen. 1986.

Band 12 Ernst-Otto Thiesing: Strategische Marketingplanung in filialisierten Universalbanken. Integrierte Filial- und Kundengruppenstrategien auf der Grundlage erfolgsbeeinflussender Schlüsselfaktoren. 1986.

Band 13 Rainer Landwehr: Standardisierung der internationalen Werbeplanung. Eine Untersuchung der Prozeßstandardisierung am Beispiel der Werbebudgetierung im Automobilmarkt. 1988.

Band 14 Paul-Josef Patt: Strategische Erfolgsfaktoren im Einzelhandel. Eine empirische Analyse am Beispiel des Bekleidungsfachhandels. 1988. 2. Auflage. 1990.

Band 15 Elisabeth Tolle: Der Einfluß ablenkender Tätigkeiten auf die Werbewirkung. Bestimmungsfaktoren der Art und Höhe von Ablenkungseffekten bei Rundfunkspots. 1988.

Band 16 Hanns Ostmeier: Ökologieorientierte Produktinnovationen. Eine empirische Analyse unter besonderer Berücksichtigung ihrer Erfolgseinschätzung. 1990.

Band 17 Bernd Büker: Qualitätsbeurteilung investiver Dienstleistungen. Operationalisierungsansätze an einem empirischen Beispiel zentraler EDV-Dienste. 1991.

Band 18 Kerstin Ch. Monhemius: Umweltbewußtes Kaufverhalten von Konsumenten. Ein Beitrag zur Operationalisierung, Erklärung und Typologie des Verhaltens in der Kaufsituation. 1993. 
Band 19 Uwe Schürmann: Erfolgsfaktoren der Werbung im Produktlebenszyklus. Ein Beitrag zur Werbewirkungsforschung. 1993.

Band 20 Ralf Birkelbach: Qualitätsmanagement in Dienstleistungscentern. Konzeption und typenspezifische Ausgestaltung unter besonderer Berücksichtigung von Verkehrsflughäfen. 1993.

Band 21 Simone Frömbling. Zielgruppenmarketing im Fremdenverkehr von Regionen. Ein Beitrag zur Marktsegmentierung auf der Grundlage von Werten, Motiven und Einstellungen. 1993.

Band 22 Marcus Poggenpohl: Verbundanalyse im Einzelhandel auf der Grundlage von Kundenkarteninformationen. Eine empirische Untersuchung von Verbundbeziehungen zwischen Abteilungen. 1994.

Band 23 Kai Bauche: Segmentierung von Kundendienstleistungen auf investiven Märkten. Dargestellt am Beispiel von Personal Computern. 1994.

Band 24 Ewald Werthmöller: Räumliche Identität als Aufgabenfeld des Städte- und Regionenmarketing. Ein Beitrag zur Fundierung des Placemarketing. 1995.

Band 25 Nicolaus Müller: Marketingstrategien in High-Tech-Märkten. Typologisierung, Ausgestaltungsformen und Einflußfaktoren auf der Grundlage strategischer Gruppen. 1995.

Band 26 Nicolaus Henke: Wettbewerbsvorteile durch Integration von Geschäftsaktivitäten. Ein zeitablaufbezogener wettbewerbsstrategischer Analyseansatz unter besonderer Berücksichtigung des Einsatzes von Kommunikations- und Informationssystemen (KIS). 1995.

Band 27 Kai Laakmann: Value-Added Services als Profilierungsinstrument im Wettbewerb. Analyse, Generierung und Bewertung. 1995.

Band 28 Stephan Wöllenstein: Betriebstypenprofilierung in vertraglichen Vertriebssystemen. Eine Analyse von Einflußfaktoren und Erfolgswirkungen auf der Grundlage eines Vertragshändlersystems im Automobilhandel. 1996.

Band 29 Michael Szeliga: Push und Pull in der Markenpolitik. Ein Beitrag zur modellgestützten Marketingplanung am Beispiel des Reifenmarktes. 1996.

Band 30 Hans-Ulrich Schröder: Globales Produktmanagement. Eine empirische Analyse des Instrumenteeinsatzes in ausgewählten Branchen der Konsumgüterindustrie. 1996.

Band 31 Peter Lensker: Planung und Implementierung standardisierter vs. differenzierter Sortimentsstrategien in Filialbetrieben des Einzelhandels. 1996.

Band 32 Michael H. Ceyp: Ökologieorientierte Profilierung im vertikalen Marketing. Dargestellt am Beispiel der Elektrobranche. 1996.

Band 33 Mark Unger: Die Automobil-Kaufentscheidung. Ein theoretischer Erklärungsansatz und seine empirische Überprüfung. 1998.

Band 34 Ralf Ueding: Management von Messebeteiligungen. Identifikation und Erklärung messespezifischer Grundhaltungen auf der Basis einer empirischen Untersuchung. 1998.

Band 35 Andreas Siefke: Zufriedenheit mit Dienstleistungen. Ein phasenorientierter Ansatz zur Operationalisierung und Erklärung der Kundenzufriedenheit im Verkehrsbereich auf empirischer Basis. 1998.

Band 36 Irene Giesen-Netzer: Implementierung von Rücknahme- und Recyclingsystemen bei Gebrauchsgütern. 1998.

Band 37 Frithjof Netzer: Strategische Allianzen im Luftverkehr. Nachfragerorientierte Problemfelder ihrer Gestaltung. 1999. 


\section{Efficient Consumer Response (ECR)}

\section{Basisstrategien und Grundtechniken, zentrale Erfolgsfaktoren sowie globaler Implementierungsplan}

Frankfurt/M., Berlin, Bern, New York, Paris, Wien, 1997, 3., akt. u. erw. Aufl. 1998. 287 S., zahlr. Abb. u. Fallbeispiele

Europäische Hochschulschriften: Reihe 5, Volks- und Betriebswirtschaft. Bd. 2062

ISBN 3-631-33228-9 • br. DM 65.-*

Dieses Buch hat richtungsweisende Akzente in der ECR-Diskussion gesetzt und sich innerhalb kürzester Zeit als Standardwerk in Praxis und Wissenschaft etabliert. Auch in der nun vorliegenden dritten, aktualisierten und erweiterten Auflage soll der immer wichtiger werdende ECR-Ansatz, der eine partnerschaftlich angelegte Zusammenarbeit zwischen Handel und Industrie zum Wohle des Verbrauchers postuliert, in seiner Gesamtheit vorgestellt und analysiert werden.

"Mit dieser Grundlagenarbeit, die mit keiner Seite die wertvolle Zeit des Lesers verschwendet, ist es dem Autor nicht nur gelungen, ECR umfassend, kritisch und fundiert zu untersuchen, sondern es konnten vielmehr auch wichtige Lösungsvorschläge und Anregungen geliefert werden, die den Unternehmen von großem Wert sein dürften."

(1) Alexander M. Lintner, Geschäftsführer Roland Berger \& Partner "Eine sehr detaillierte und gleichermaßen verständliche und eingängige Ausarbeitung." Lebensmittel Zeitung "Ich bin froh, diese Schrift in Händen zu haben."

Prof. Dr. Lothar Müller-Hagedorn, Universität Köln

"Dieses Werk ist für unsere Mitglieder sicherlich von großem Interesse."

Angelika Wiesgen-Pick, Geschäftsführerin Bundesverband der Deutschen

Spirituosenindustrie e.V., Bonn

"Umfassend, verständlich und auf Deutsch."

manager magazin

"Ein wichtiger Beitrag zum Thema ECR, der sicher vielen Managern eine wichtige

Entscheidungshilfe sein kann."

Prof. Dr. H.-P. Liebmann, Universität Graz

"Eine hervorragende Arbeit. Nicht nur phantastisch präsentiert, sondern auch inhaltlich ausgezeichnet."

Wolfgang K. A. Disch, Herausgeber MARKETING JOURNAL, Hamburg

Aus dem Inhalt: ECR-Historie - ECR-Basisstrategien und Grundtechniken · Zentrale ECRErfolgsfaktoren - Globaler ECR-Implementierungsplan

Frankfurt/M - Berlin · Bern · New York · Paris · Wien

Auslieferung: Verlag Peter Lang AG

Jupiterstr. 15, CH-3000 Bern 15

Telefax (004131) 9402131

*inklusive Mehrwertsteuer

Preisänderungen vorbehalten 